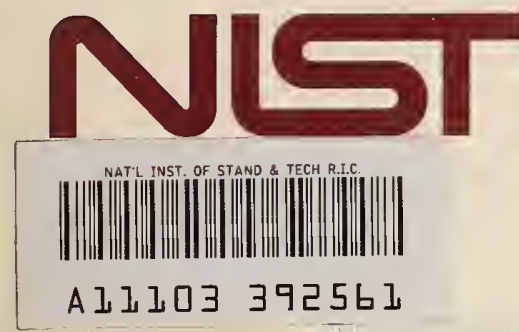

UNITED STATES

DEPARTMENT OF COMMERCE

NATIONAL INSTITUTE OF STANDARDS

AND TECHNOLOGY

\section{REFERENCE}

NIST PUBLICATIONS

\title{
Characterization of Clocks and Oscillators
}

Edited by

D.B. Sullivan

D.W. Allan

D.A. Howe

F.L. Walls

$-Q C-$

100

.45753

\#1337

1990 
NATIONAL INSTITUTE OF STANDARDS \& TECHNOLOGY

Research Information Center

Gaithersburg, MD 20899 


\section{Characterization of Clocks and Oscillators}

Edited by

D.B. Sullivan

D.W. Allan

D.A. Howe

F.L. Walls

Time and Frequency Division

Center for Atomic, Molecular, and Optical Physics

National Measurement Laboratory

National Institute of Standards and Technology

Boulder, Colorado 80303-3328

This publication covers portions of NBS Monograph 140 published in 1974. The purpose of this document is to replace this outdated monograph in the areas of methods for characterizing clocks and oscillators and definitions and standards relating to such characterization.

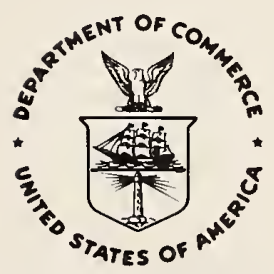

U.S. DEPARTMENT OF COMMERCE, Robert A. Mosbacher, Secretary NATIONAL INSTITUTE OF STANDARDS AND TECHNOLOGY, John W. Lyons, Director 
National Institute of Standards and Technology Technical Note 1337 Natl. Inst. Stand. Technol., Tech. Note 1337, 352 pages (Jan. 1990) CODEN:NTNOEF 


\section{PREFACE}

For many years following its publication in 1974, "TIME AND FREQUENCY: Theory and Fundamentals," a volume edited by Byron E. Blair and published as NBS Monograph 140, served as a common reference for those engaged in the characterization of very stable clocks and oscillators. Monograph 140 has gradually become outdated, and with the recent issuance of a new military specification, MIL-0-55310B, which covers general specifications for crystal oscillators, it has become especially clear that Monograph 140 no longer meets the needs it so ably served in earlier years. During development of the new military specification, a process involving discussion and input from many quarters, a key author of the specification, John Vig of the US Army Electronics Technology and Devices, urged the National Bureau of Standards (now the National Institute of Standards and Technology, NIST) to issue a revised publication to serve as reference for the characterization of clocks and oscillators. With NIST having agreed to this task, the framers of the military specification used the nomenclature "NBS Monograph 140R" in their document, anticipating a revised $(\mathrm{R})$ volume which had not yet been prepared.

Considering the availability of a number of newer books in the time and frequency field, the rewriting of a major volume like Monograph 140 seemed inappropriate. The real need has not been for rework of everything in Monograph 140, but only for those parts which provide reference to definitions and methods for measurement and characterization of clocks and oscillators, subjects which are fully covered in a number of papers distributed through a variety of conference proceedings, books, and journals. For the near term, we concluded that the most effective procedure would be to collect a representative set of these papers into one reference source with introductory comments which permit the reader to quickly access material required to meet particular needs. Thus, we arrived at this particular collection. The editors' challenge has been to select representative papers, to organize them in a convenient manner, and to deal with errata and notation inconsistencies in a reasonable manner. In the longer term, the material in this volume needs to be more completely integrated. This task would profitably await further developments in the area of phase noise measurements.

Donald B. Sullivan

David W. Allan

David A. Howe

Fred L. Walls

Boulder, Colorado

February 28, 1990 



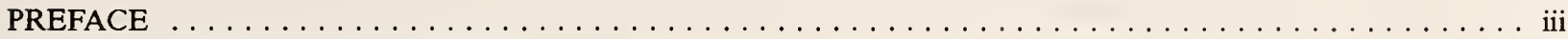

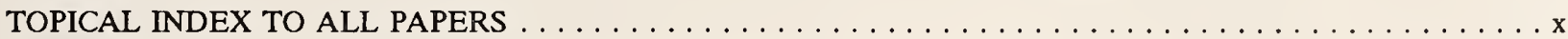

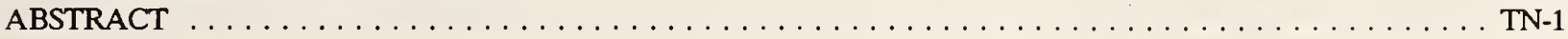

\section{A. INTRODUCTION}

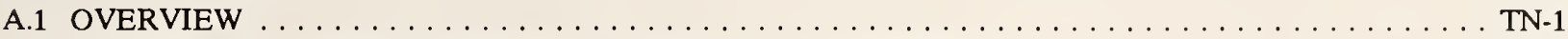

A.2 COMMENTS ON INTRODUCTORY AND TUTORIAL PAPERS $\ldots \ldots \ldots \ldots \ldots$

A.3 COMMENTS ON PAPERS ON STANDARDS AND DEFINITIONS . . . . . . . . . . . . . . TN-3

A.4 COMMENTS ON SUPPORTING PAPERS $\ldots \ldots \ldots \ldots \ldots \ldots \ldots \ldots \ldots$

A.5 GUIDE TO SELECTION OF MEASUREMENT METHODS $\ldots \ldots \ldots \ldots \ldots \ldots$

A.6 RELATIONSHIP OF THE MODIFIED ALLAN VARIANCE TO THE ALLAN VARIANCE . . . . . . TN-9

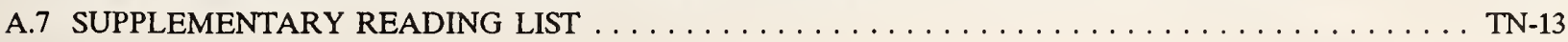

\section{B. INTRODUCTORY AND TUTORIAL PAPERS}

B.1 Properties of Signal Sources and Measurement Methods

D.A. Howe, D.W. Allan, and J.A. Barnes

35th Annual Symposium on Frequency Control, 1981 Proceedings . . . . . . . . . . . . . . . . . . . . . TN-14

I. The Sine Wave and Stability . . . . . . . . . . . . . . . . . . . . . TN-14

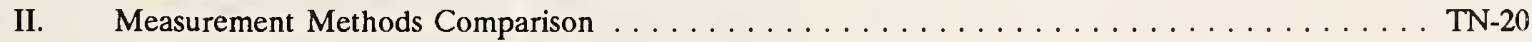

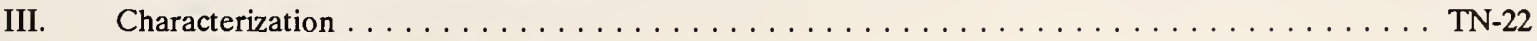

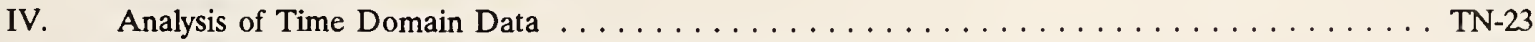

V. Confidence of the Estimate and Overlapping Samples . . . . . . . . . . . . . . . TN-26

VI. Maximal Use of the Data and Determination of the Degrees of Freedom . . . . . . . . . . TN-28

VII. Example of Time Domain Signal Processing and Analysis . . . . . . . . . . . . . . . . TN-30

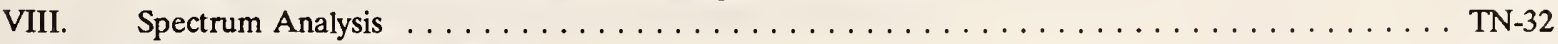

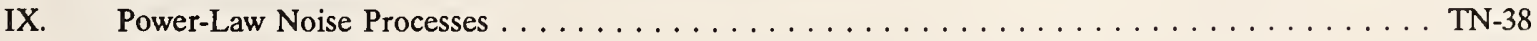

X. Pitfalls in Digitizing the Data . . . . . . . . . . . . . . . . . . . . . . TN-39

XI. Translation From Frequency Domain Stability Measurement to Time Domain Stability

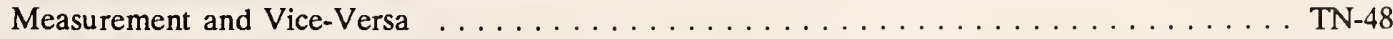

XII. Causes of Noise Properties in a Signal Source $\ldots \ldots \ldots \ldots \ldots \ldots \ldots$

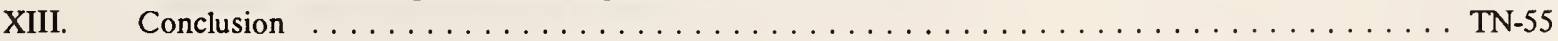

B.2 Frequency and Time - Their Measurement and Characterization

Samuel R. Stein

Precision Frequency Control, Volume 2, Chapter 12, 1985 . . . . . . . . . . . . . . . . . . . . TN-61

12.1 Concepts, Definitions, and Measures of Stability . . . . . . . . . . . . . . . . TN-63

12.1.1 Relationship Between the Power Spectrum and the Phase Spectrum . . . . . . . . . . . . TN-65

12.1.2 The IEEE Recommended Measures of Frequency Stability . . . . . . . . . . . . . . . . TN-65

12.1.3 The Concepts of the Frequency Domain and the Time Domain . . . . . . . . . . . . TN-73

12.1.4 Translation Between the Spectral Density of Frequency and the Allan Variance . . . . . . TN-73

12.1.5 The Modified Allan Variance $\ldots \ldots \ldots \ldots \ldots \ldots \ldots \ldots \ldots \ldots$

12.1.6 Determination of the Mean Frequency and Frequency Drift of an Oscillator . . . . . . . . TN-77

12.1.7 Confidence of the Estimate and Overlapping Samples . . . . . . . . . . . . . . . TN-80

12.1.8 Efficient Use of the Data and Determination of the Degrees of Freedom . . . . . . . . . TN-83

12.1.9 Separating the Variances of an Oscillator and the Reference . . . . . . . . . . . . . TN-86 


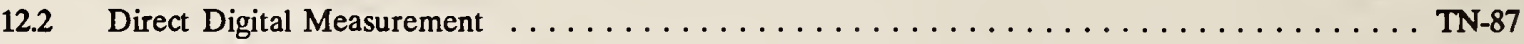

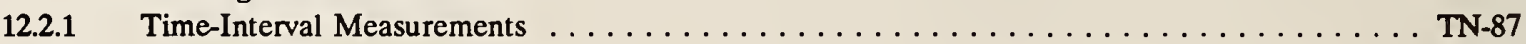

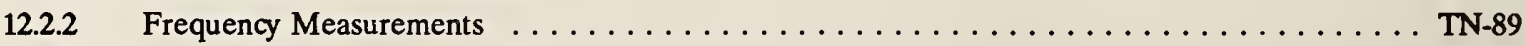

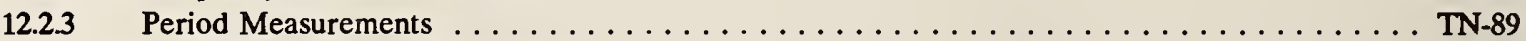

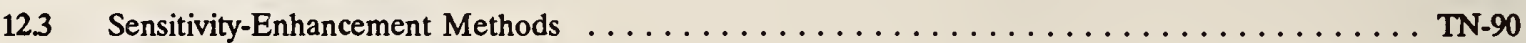

12.3.1 Heterodyne Techniques . . . . . . . . . . . . . . . . . . . . . . . . . . . . . . . . . . . . . . . . . . . .

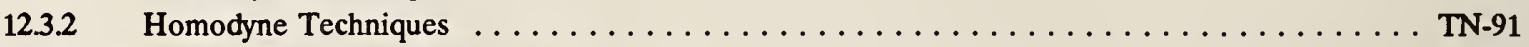

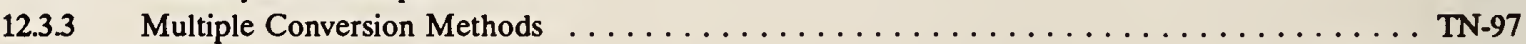

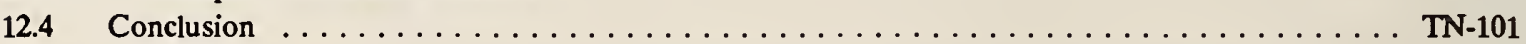

B.3 Time and Frequency (Time-Domain) Characterization, Estimation, and Prediction of Precision

Clocks and Oscillators

David W. Allan

IEEE Transactions on Ultrasonics, Ferroelectrics, and Frequency Control, 1987 . . . . . . . . . . . TN-121

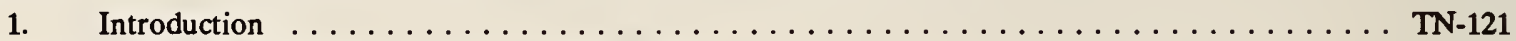

2. Systematic Models for Clocks and Oscillators . . . . . . . . . . . . . . . . . . . TN-121

3. Random Models for Clocks and Oscillators . . . . . . . . . . . . . . . . . . . TN-123

4. Time-Domain Signal Characterization . . . . . . . . . . . . . . . . . . . . . TN-123

5. Time and Frequency Estimation and Prediction . . . . . . . . . . . . . . . TN-126

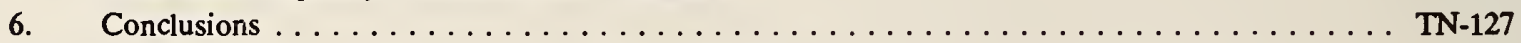

B.4 Extending the Range and Accuracy of Phase Noise Measurements

F.L. Walls, A.J.D. Clements, C.M. Felton, M.A. Lombardi and M.D. Vanek

$42 n d$ Annual Symposium on Frequency Control, 1988 Proceedings . . . . . . . . . . . . . . . . . . . T TN-129

I. Introduction $\ldots \ldots \ldots \ldots \ldots \ldots \ldots \ldots \ldots \ldots \ldots \ldots \ldots \ldots$

II. Model of a Noisy Signal . . . . . . . . . . . . . . . . . . . . . . . . . . . . . TN-129

III. Two Oscillator Method . . . . . . . . . . . . . . . . . . . . . . . . TN-130

IV. The New NBS Phase Noise Measurement Systems . . . . . . . . . . . . . . . . T T

V. Conclusion $\ldots \ldots \ldots \ldots \ldots \ldots \ldots \ldots \ldots \ldots \ldots \ldots \ldots \ldots \ldots$

\section{PAPERS ON STANDARDS AND DEFINITIONS}

\section{C.1 Standard Terminology for Fundamental Frequency and Time Metrology}

David Allan, Helmut Hellwig, Peter Kartaschoff, Jacques Vanier, John Vig, Gernot M.R. Winkler, and Nicholas Yannoni

42nd Annual Symposium on Frequency Control, 1988 Proceedings . . . . . . . . . . . . . . . . . . . TN-139

1. Introduction $\ldots \ldots \ldots \ldots \ldots \ldots \ldots \ldots \ldots \ldots \ldots \ldots \ldots \ldots$

2. Measures of Frequency and Phase Instability $\ldots \ldots \ldots \ldots \ldots \ldots \ldots \ldots$

3. Characterization of Frequency and Phase Instabilities . . . . . . . . . . . . . . . TN-139

4. Confidence Limits of Measurements . . . . . . . . . . . . . . . . . . . . TN-140

5. Recommendations for Characterizing or Reporting Measurements of Frequency and

Phase Instabilities $\ldots \ldots \ldots \ldots \ldots \ldots \ldots \ldots \ldots \ldots \ldots \ldots$ TN-141 


\section{C.2 Characterization of Frequency Stability}

James A. Barnes, Andrew R. Chi, Leonard S. Cutler, Daniel J. Healey, David B. Leeson,

Thomas E. McGunigal, James A. Mullen, Jr., Warren L. Smith, Richard L. Sydnor,

Robert F.C. Vessot, and Gernot M.R. Winkler

IEEE Transactions on Instrumentation and Measurement, $1971 \ldots \ldots \ldots \ldots \ldots \ldots \ldots \ldots$ TN-146

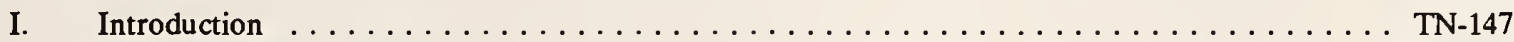

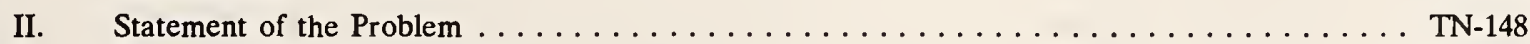

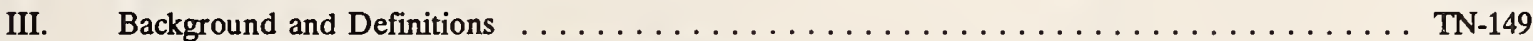

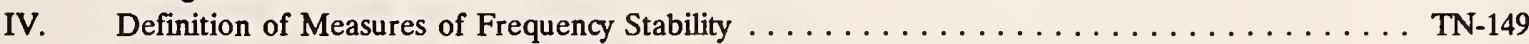

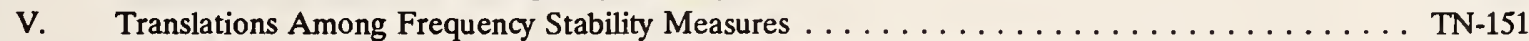

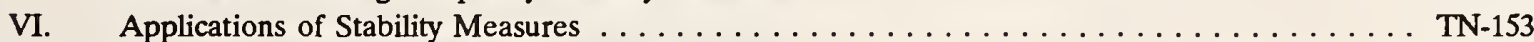

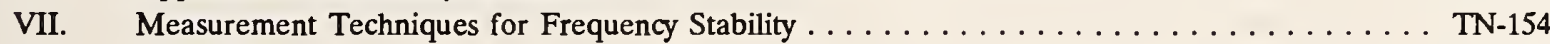

VIII. Conclusions . . . . . . . . . . . . . . . . . . . . . . . . . . . . . . . . . . . TN-157

C. 3 Characterization of Frequency and Phase Noise

Comité Consultatif International Des Radiocommunications (CCIR)

Report 580 of the CCIR, 1986

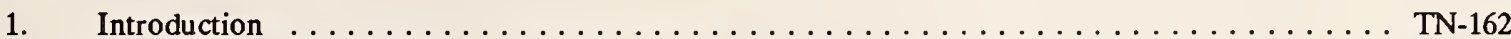

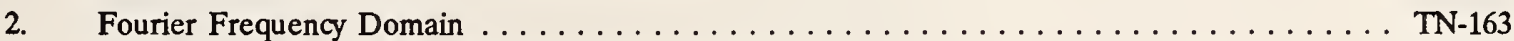

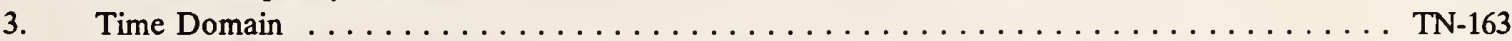

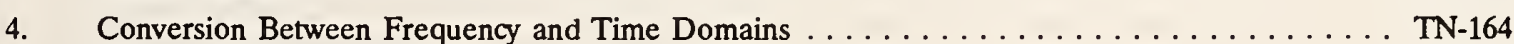

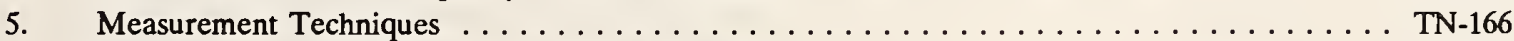

6. Confidence Limits of Time Domain Measurements $\ldots \ldots \ldots \ldots \ldots \ldots \ldots \ldots \ldots$ TN-166 $\ldots \ldots \ldots$

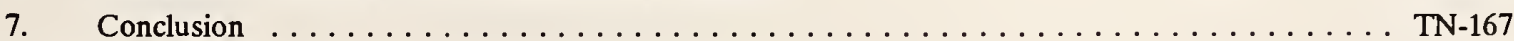

\section{SUPPORTING PAPERS}

D.1 Characterization and Measurement of Time and Frequency Stability

P. Lesage and C. Audoin

Radio Science, 1979

1. Introduction $\ldots \ldots \ldots \ldots \ldots \ldots \ldots \ldots \ldots \ldots \ldots \ldots \ldots \ldots \ldots$

2. Definitions: Model of Frequency Fluctuations $\ldots \ldots \ldots \ldots \ldots \ldots \ldots \ldots \ldots$

3. Noise Processes in Frequency Generators . . . . . . . . . . . . . . . . . . . . . TN-173

4. Measurements in the Frequency Domain . . . . . . . . . . . . . . . . . . . TN-174

5. Measurements in the Time Domain . . . . . . . . . . . . . . . . . . . . . . TN-177

6. Characterization of Frequency Stability in the Time Domain . . . . . . . . . . . . . TN-178

7. Characterization of Frequency Stability via Filtering or Frequency Noise . . . . . . . . . . TN-182

8. Spectral Analysis Inferred From Time Domain Measurements . . . . . . . . . . . . . TN-183

9. Structure Functions of Oscillator Fractional Phase and Frequency Fluctuations . . . . . . . . T TN-184

10. Power Spectral Density of Stable Frequency Sources . . . . . . . . . . . . . . . . TN-185

11. Conclusion $\ldots \ldots \ldots \ldots \ldots \ldots \ldots \ldots \ldots \ldots \ldots \ldots \ldots$ 
D.2 Phase Noise and AM Noise Measurements in the Frequency Domain Algie L. Lance, Wendell D. Seal, and Frederik Labaar Infrared and Millimeter Waves, Vol. 11, 1984

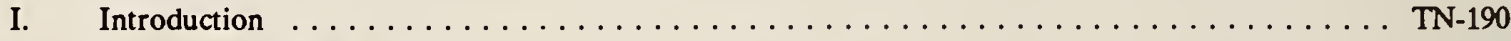

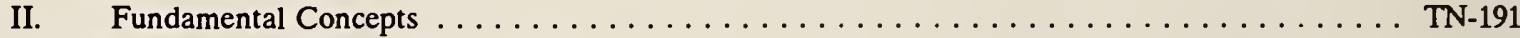

A. Noise Sidebands . . . . . . . . . . . . . . . . . . . . . . . . . . . TN-194

B. Spectral Density . . . . . . . . . . . . . . . . . . . . . . . . . . . . TN-194

C. Spectral Densities of Phase Fluctuations in the Frequency Domain . . . . . . . . . . . . TN-197

D. Modulation Theory and Spectral Density Relationships . . . . . . . . . . . . . . . . . TN-199

E. Noise Processes . . . . . . . . . . . . . . . . . . . . . . . . . . . . . TN-202

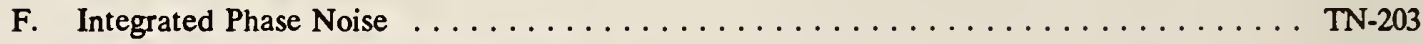

G. AM Noise in the Frequency Domain . . . . . . . . . . . . . . . . . . . . TN-205

III. Phase-Noise Measurements Using the Two-Oscillator Technique . . . . . . . . . . . . . . TN-206

A. Two Noisy Oscillators . . . . . . . . . . . . . . . . . . . . . . . TN-208

B. Automated Phase-Noise Measurements Using the Two-Oscillator Technique . . . . . . . . TN-209

C. Calibration and Measurements Using the Two-Oscillator System . . . . . . . . . . . TN-210

IV. Single-Oscillator Phase-Noise Measurement Systems and Techniques . . . . . . . . . . . TN-218

A. The Delay Line as an FM Discriminator . . . . . . . . . . . . . . . . . TN-219

B. Calibration and Measurements Using the Delay Line as an FM Discriminator . . . . . . TN-225

C. Dual Delay-Line Discriminator . . . . . . . . . . . . . . . . . . . . . . TN-232

D. Millimeter-Wave Phase-Noise Measurements . . . . . . . . . . . . . . . . . TN-234

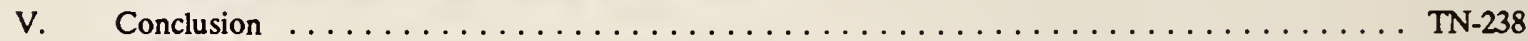

D.3 Performance of an Automated High Accuracy Phase Measurement System

S. Stein, D. Glaze, J. Levine, J. Gray, D. Hilliard, D. Howe, and L. Erb

36 th Annual Symposium on Frequency Control, 1982 Proceedings . . . . . . . . . . . . . . . . . TN-241

1. Review of the Dual Mixer Time Difference Technique . . . . . . . . . . . . . . . . . TN-241

2. Extended Dual Mixer Time Difference Measurement Technique . . . . . . . . . . . . . . TN-242

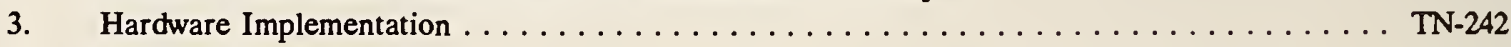

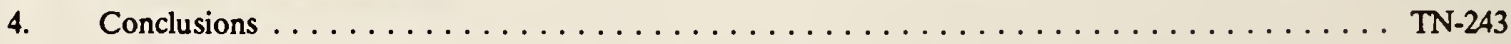

D.4 Biases and Variances of Several FFT Spectral Estimators as a Function of Noise Type and Number of Samples F.L. Walls, D.B. Percival and W.R. Irelan 43rd Annual Symposium on Frequency Control, 1989 Proceedings . . . . . . . . . . . . . . . . . . . . TN-248

I. Introduction $\ldots \ldots \ldots \ldots \ldots \ldots \ldots \ldots \ldots \ldots \ldots \ldots \ldots \ldots \ldots$

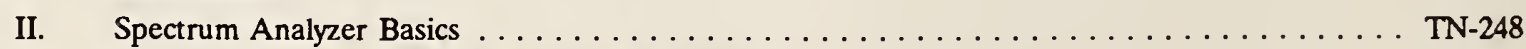

III. Expected Value and Bias of Spectral Estimates . . . . . . . . . . . . . . . . . . TN-249

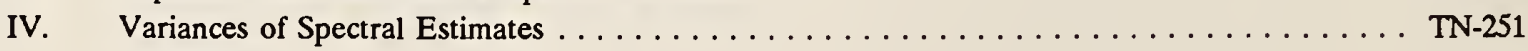

V. Conclusions .................................. TN-252 
D.5 A Modified "Allan Variance" with Increased Oscillator Characterization Ability David W. Allan and James A. Barnes

35th Annual Symposium on Frequency Control, 1981 Proceedings

1. Introduction

2. Definition of "Allan Variance" and Related Concepts . . . . . . . . . . . . . . . . TN-254

3. Development of the Modified Allan Variance . . . . . . . . . . . . . . . . . . . . . TN-255

4. Comparisons, Tests, and Examples of Usage of the Modified Allan Variance . . . . . . . . . TN-256

5. Conclusion $\ldots \ldots \ldots \ldots \ldots \ldots \ldots \ldots \ldots \ldots \ldots \ldots \ldots \ldots \ldots$

D.6 Characterization of Frequency Stability: Analysis of the Modified Allan Variance and

Properties of Its Estimate

Paul Lesage and Théophane Ayi

IEEE Transactions on Instrumentation and Measurement, 1984

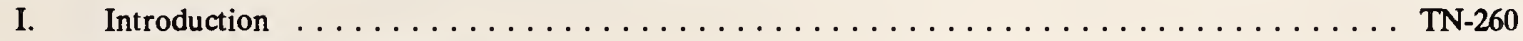

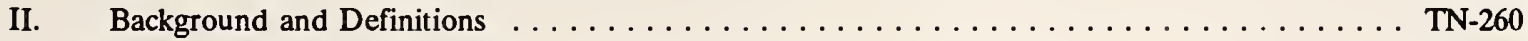

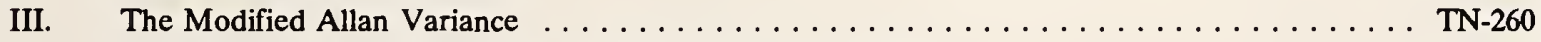

IV. Uncertainty on the Estimate of the Modified Allan Variance . . . . . . . . . . . . TN-262

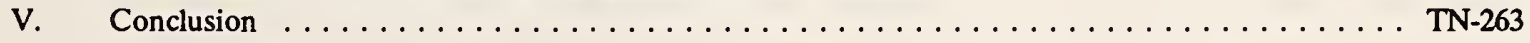

D.7 The Measurement of Linear Frequency Drift in Oscillators

James A. Barnes

15th Annual PTTI Meeting, 1983 Proceedings

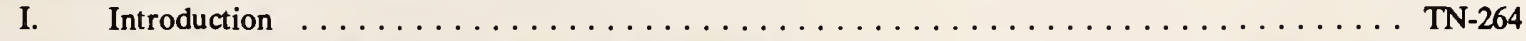

II. Least Squares Regression of Phase on a Quadratic $\ldots \ldots \ldots \ldots \ldots \ldots \ldots$

III. Example . . . . . . . . . . . . . . . . . . . . . . . . . . . TN-266

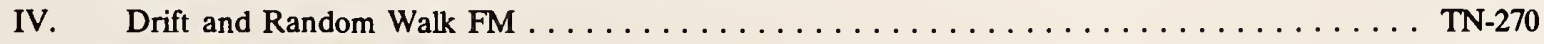

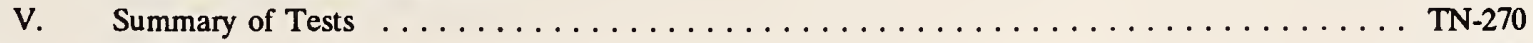

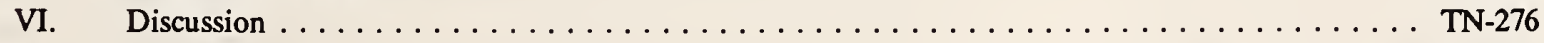

VII. Conclusions $\ldots \ldots \ldots \ldots \ldots \ldots \ldots \ldots \ldots \ldots \ldots \ldots \ldots \ldots$

D.8 Variances Based on Data With Dead Time Between the Measurements

James A. Barnes and David W. Allan

NIST Technical Note 1318, 1990

1. Introduction $\ldots \ldots \ldots \ldots \ldots \ldots \ldots \ldots \ldots \ldots \ldots \ldots \ldots \ldots \ldots \ldots \ldots$

2. The Allan Variance . . . . . . . . . . . . . . . . . . . . . . . . . . . . . TN-298

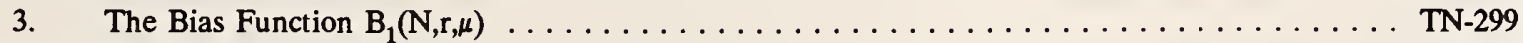

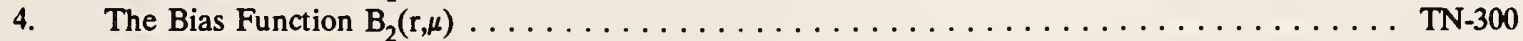

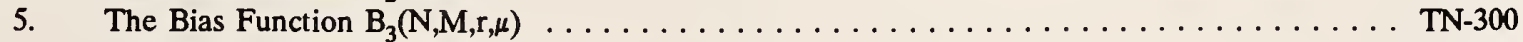

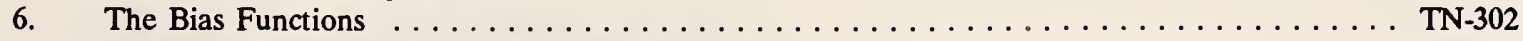

7. Examples of the Use of the Bias Functions $\ldots \ldots \ldots \ldots \ldots \ldots \ldots$

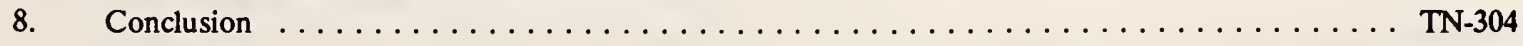

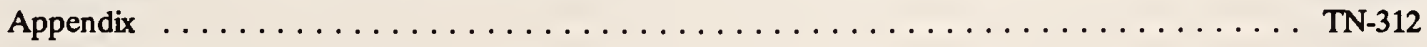

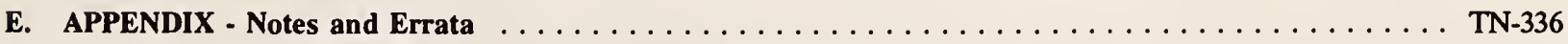


The following index is designed for quick location of substantial discussion of a particular topic. The page numbers are those where the topic is first cited in the reference.

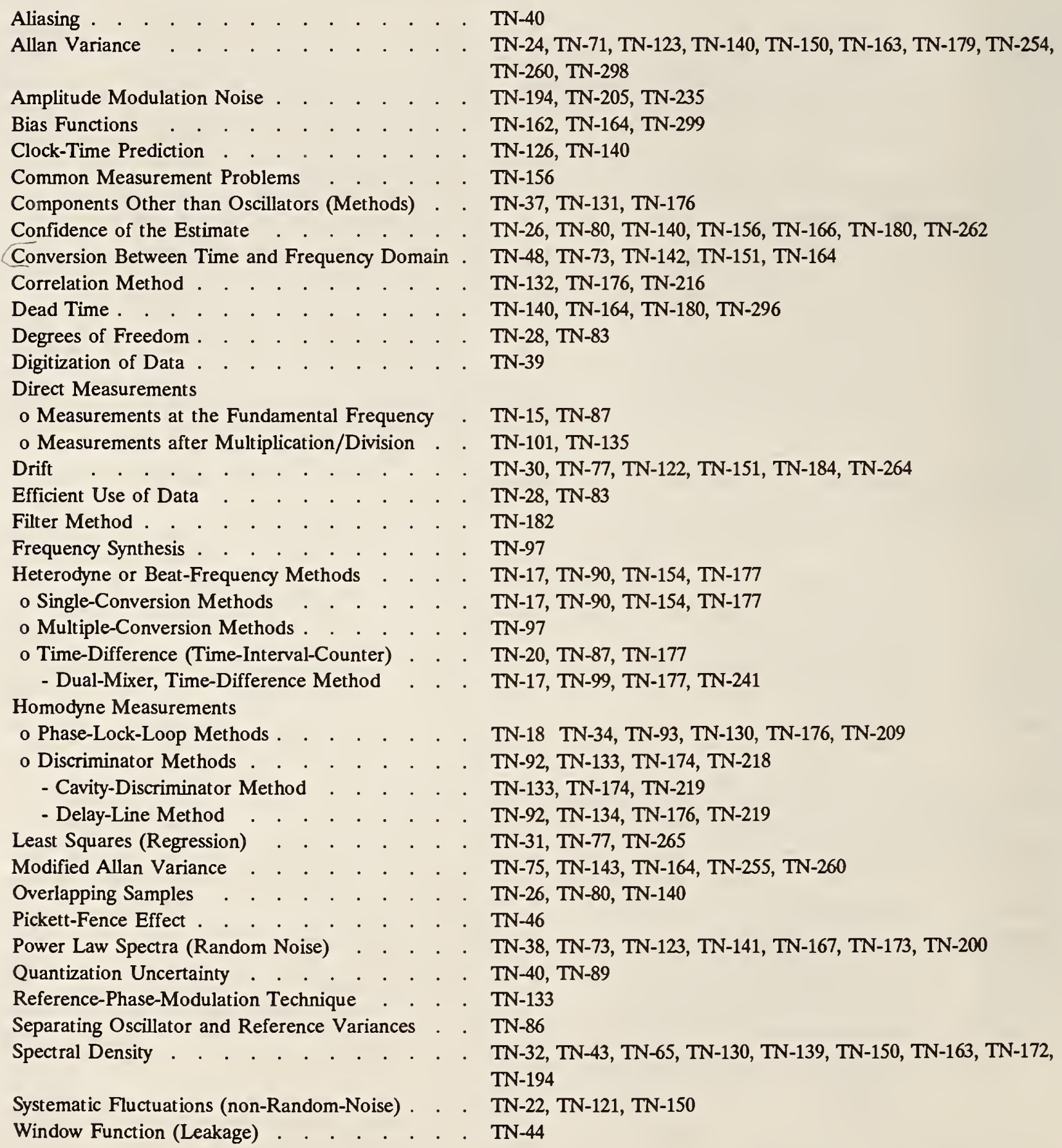




\title{
CHARACTERIZATION OF CLOCKS AND OSCILLATORS
}

\author{
D.B. Sullivan, D.W. Allan, D.A. Howe and F.L. Walls, Editors \\ Time and Frequency Division \\ National Institute of Standards and Technology \\ Boulder, Colorado 80303
}

This is a collection of published papers assembled as a reference for those involved in characterizing and specifying high-performance clocks and oscillators. It is an interim replacement for NBS Monograph 140, Time and Frequency: Theory and Fundamentals, an older volume of papers edited by Byron E. Blair. This current volume includes tutorial papers, papers on standards and definitions, and a collection of papers detailing specific measurement and analysis techniques. The discussion in the introduction to the volume provides a guide to the content of the papers, and tables and graphs provide further help in organizing methods described in the papers.

Key words: Allan variance, clocks, frequency, oscillators, phase noise, spectral density, time, two-sample variance.

\section{A. INTRODUCTION}

\section{A.1 OVERVIEW}

The papers in this volume are organized into three groups: Introductory and Tutorial Papers, Papers on Definitions and Standards, and Supporting Papers. The three sections (A.2, A.3, and A.4) immediately following this introduction provide overviews of each of the three groups of papers with comments on each paper. The arrangement of the papers in this particular order is somewhat arbitrary, since, for example, the first two papers under Supporting Papers could be included with the Introductory and Tutorial Papers, while paper B.4 could easily be placed with the Supporting Papers. Our rationale for the first group of papers (discussed in more detail in section A.2) is that, taken as a group, they provide reasonably complete coverage of the concepts used in characterizing clocks and oscillators. Several of the papers, taken individually, are good introductory papers, but, for this publication, need to be complemented with additional material to provide coverage of an appropriate range of topics.

The second group (section C) of three papers discussed in section A.3 were specifically written to address definitions and standards. This is a particularly important section, since consistency in specification of performance can only be achieved if manufacturers and users refer to the same measurement and characterization parameters.

The Supporting Papers in section D provide additional discussion of topics introduced in the first group. The first papers in this group (D.1 and D.2) also provide good introductory material which might be used with section $B$ to gain a better understanding of the concepts.

Section A.5 provides a table and graph designed to help the reader select a measurement method to meet a particular need. To make this useful, it was kept simple and must therefore be used with care. Such tabular information can never be arranged well enough to anticipate all 
of the wide range of measurement situations which might be encountered. However, it can serve as a starting point for the decision-making process.

Section A. 6 contains some new material which should be helpful in understanding the relationship between the Allan variance and the modified Allan variance. Since these ideas are unpublished, we include them here rather than with the papers on those topics. This section is followed by a reading list with references to major articles and books which can be used as supplementary resources. Because some of the papers include extensive reference lists, we have limited our list to works which are either very comprehensive or only recently published. Particularly extensive reference lists are included with papers B.1, B.2, C.1, C.3, D.1, and D.2.

Since notation and definitions have changed over the period bridged by these papers, we have highlighted problem areas on the papers with an asterisk $\left({ }^{*}\right)$. A note directs the reader to the Appendix where the particular problem is discussed. We have also used this device to highlight inconsistencies and the usual typographic and other errors which creep into the literature. The page numbers of the original publications are retained, but we have also used a continuous page numbering to simplify location of items in the volume.

The topical index on page xi organizes much of the material in the papers under a few key subject headings. This index provides a shortcut to locating material on a particular topic.

\section{A.2 COMMENTS ON INTRODUCTORY AND TUTORIAL PAPERS}

Paper B.1 in this section, by Howe, Allan, and Barnes, was originally prepared and presented as a tutorial paper and has been used with success as an introductory paper in our annual Time and Frequency Seminar. This paper is now 9 years old, so there are a substantial number of notes which relate to updates in notation. The paper is nevertheless highly readable and introduces many of the key measurement methods, providing circuit diagrams with enough specific detail to be useful in real laboratory situations. Furthermore, it includes discussion and examples on handling of data which are useful for practical application of the concepts. The paper presents a particularly useful discussion of the pitfalls encountered in digitizing data, a problem which is often overlooked.

The second paper (B.2) by Stein is more advanced and those familiar with the general concepts may find it a better starting point. This and other papers in this collection cite earlier IEEE recommendations on measures of frequency stability and, while much of this has not changed, there is a new IEEE standard (paper C.1). In general, the reader should consult the overview and papers of section $\mathrm{C}$ if there is any question concerning definitions or terminology. Paper B.2 is quite comprehensive, introducing topics (not covered in paper B.1) such as the modified Allan variance, the delay-line-phase-noise-measurement system, and the use of frequency synthesis to reach frequencies far from normally available reference frequencies.

The materials in papers B.1 and B.2, aside from differences in level of presentation, are organized in quite different ways. The Howe-Allan-Barnes paper goes directly to the measurement concepts and then describes the means for analyzing the output data and understanding the confidence of the measurements. On the other hand, the Stein paper carefully lays out the theoretical background needed to analyze the data before introducing the measurement concepts. Both papers cover time-domain and frequency-domain measurements.

Paper B.3 by Allan reviews the concepts of the two-sample or Allan variance and the modified Allan variance showing how classical statistical methods fail to usefully describe the time-domain performance of good oscillators. The Allan variance concept is also introduced in 
papers B.1 and B.2, and the modified Allan variance is described further in papers D.4 and D.5. The presentation in paper B. 3 is particularly useful in that it discusses general aspects of performance of different types of oscillators (quartz, rubidium, hydrogen, and cesium) providing the basis for prediction of time errors, a topic which may prove useful to those who must develop system specifications.

Paper B.4 by Walls, Clements, Felton, Lombardi, and Vanek adds to the discussion of frequency-domain measurements providing information on methods which can be used to increase the dynamic range for both carrier frequency and for Fourier frequencies up to 10 percent from the carrier. With some aerospace hardware now carrying phase-noise specifications, this is an important addition to the literature.

\section{A.3 COMMENTS ON PAPERS ON STANDARDS AND DEFINITIONS}

The first paper in this group (paper C.1) outlines the standard terminology now used for fundamental frequency and time metrology. This document was widely circulated for comment during the draft stage and, with its acceptance by IEEE as a standard, supersedes the earlier reference (paper C.2) which had served as the foundation for characterization of frequency stability. This latter paper is included because it is so widely cited, and the reader will probably be confronted with specifications based on its recommendations. Paper C.2 contains additional material on applications of stability measures and measurement techniques including a useful discussion of some of the common hazards in measurements. Paper C.1 restricts itself to very concise statements of the definitions.

The reader will note that the updated terminology in the first paper (C.1) varies in a number of minor ways from the earlier paper (C.2). A notable addition to definitions is the introduction of script "ell", $\mathscr{L}(\mathrm{f})$, which has become an important measure of phase noise. This quantity was previously defined as the ratio of the power in one sideband, due to phase modulation to the total signal power. For Fourier frequencies far from the carrier, this quantity can be simply related to the usual spectral densities which are the quantities that are generally measured, but the relation breaks down in the important region near the carrier. To resolve this problem, the new standard defines the approximate relation between $\mathscr{L}(\mathrm{f})$ and spectral density as being exact and applicable for any Fourier frequency.

The third paper in this group (paper C.3), from the 1986 report of the International Radio Consultative Committee (CCIR), presents the definitions and terminology which have been accepted for international use by this body. The material in this particularly readable document is fairly consistent with the IEEE standard and would be useful to those involved in specification of performance for international trade. A number of minor changes to this document have been recommended by different delegations to the CCIR and these will likely be made in their next publication.

\section{A.4 COMMENTS ON SUPPORTING PAPERS}

Papers D.1 and D.2 are included in this collection for a number of reasons. First, they provide alternative introductions to the general topic of oscillator characterization. And second, they include material not fully covered by introductory papers, B.1 and B.2. 
The first of these (D.1) by Lesage and Audoin covers most of the same ground as papers B.1 and B.2, but, in addition, includes a nice discussion of characterization of frequency stability via filtering of phase or frequency noise, a method which may be especially useful for rapid, automated measurements where high accuracy is not required. Furthermore, this paper discusses characterization of stable laser sources, a topic not covered in any of the other reference papers.

Paper D.2 by Lance, Seal, and Labaar limits itself to discussion of the measurement of phase noise and amplitude-modulation (AM) noise. The paper presents a detailed discussion of delay-line measurement methods which can be used if a second reference oscillator is not available. The delay-line concept is also introduced in B.2 and B.4, but in much less detail. While the delay-line method is less sensitive (for lower Fourier frequency) than two-oscillator methods, it is easier to implement. The paper contains many good examples which the reader will find useful.

A complete discussion of AM noise is beyond the scope of this volume. AM noise is usually ignored in the measurement and specification of phase noise in sources under the assumption that the AM noise is always less than the phase noise. This assumption is generally true only for Fourier frequencies close to the carrier. At larger Fourier frequencies the normalized AM noise can be the same order of magnitude as the phase noise. In systems with active amplitude leveling, the normalized AM noise can be higher than the phase noise. Under this condition the AM to PM conversion in the rest of the system may degrade the overall phase noise performance. For these reasons, we cannot ignore amplitude noise altogether. Paper D.2 provides a useful discussion of amplitude noise. Note 1 in appendix $E$ provides further information on definitions, notation and, in particular, the specification of added phase noise and amplitude noise for signal-handling components.

The next contribution (D.3) provides substantially more detail on the extension of the time-domain, dual-mixer concept for highly accurate time and time-interval measurements. The basic dual-mixer ideas are included in papers B.1 and B.2.

Paper D.4, published recently, provides the first quantitative treatment of confidence estimates for phase-noise measurements. To the best of our knowledge, this is the only available treatment of this important subject. We expect to see additional papers on this topic in the future.

Paper D.5 discusses the modified Allan variance in more detail than the introductory papers B.1, B.2, and B.3. It is followed by the Lesage-Ayi paper (D.6) which provides analytical expressions for the standard set of power-law noise types and also includes discussion of the uncertainty of the estimate of the modified Allan variance.

Linear frequency drift in oscillators is treated by Barnes in paper D.7. As noted in this paper, even with correction for drift, the magnitude of drift error eventually dominates all time uncertainties in clock models. Drift is particularly important in certain oscillators (e.g., quartz oscillators) and a proper measure and treatment of drift is essential. As with other topics treated by this group of papers, introductory papers B.1, B.2, and B.3 present some discussion of frequency drift, but D.7 is included because it contains a much more comprehensive discussion of the subject.

The final paper (D.8) by Barnes and Allan contains the most recent treatment of measurements made with dead times between them. Paper C.2 introduced the use of bias functions, $B_{1}$ and $B_{2}$, which can be used to predict the Allan variance for one set of parameters based on another set (for the power-law noise models). This last paper extends those ideas, introducing a third bias function, $B_{3}$, which can be used to translate the Allan variance between cases where 
dead time is accumulated at the end and where dead time is distributed between measurements, a useful process for many data-acquisition situations.

\section{A.5 GUIDE TO SELECTION OF MEASUREMENT METHODS}

The table and graph in this section are quick guides to performance limits of the different methods as well as indications of advantages and disadvantages of each. The ideas presented here are drawn from papers B.1 and B.4, but we have modified and expanded the material to make it more comprehensive. The table has been kept simple, so the suggested methods should be viewed as starting points only. They cannot possibly cover all measurement situations.

For a given measurement task, it is often best to start with a quick, simple measurement which will then help to define the problem. For example, faced with the need to characterize an oscillator, a good starting point might be to feed the output of that oscillator along with the output of a similar, but more stable, oscillator into a good mixer and then look at the output. If the two can be brought into quadrature by tuning one of the oscillators or by using a phaselocked loop, then the output can be fed to a spectrum analyzer to get an immediate, at least qualitative idea, of the performance of the oscillator. This mixing process, which brings the fluctuations to baseband where measurement is much more straightforward, is basic to many of the measurement methods. A large number of measurement problems can probably be resolved with this simple, single-conversion, heterodyne arrangement. If the simplest approach is insufficient, then some of the more advanced methods outlined below can be used.

There are many ways to go about categorizing the various measurement methods. Since this volume is aimed at practical measurements, we choose to use the characteristics of the measurement circuit as the basis for sorting. In this arrangement we have (1) direct measurements where no signal mixers are used, (2) heterodyne measurements where two unequal frequencies are involved, and (3) homodyne measurements where two equal frequencies are involved. These methods are listed below.

\section{Direct Measurements}

1. Measurements at the Fundamental Frequency

2. Measurements after Multiplication/Division

II. Heterodyne Measurements

1. Single-Conversion Methods

2. Multiple-Conversion Methods

3. Time-Difference Method

a. Dual-Mixer, Time-Difference Method

III. Homodyne Measurements

1. Phase-Lock-Loop Methods (two oscillators)

a. Loose-Phase-Lock-Loop Method

b. Tight-Phase-Lock-Loop Method

2. Discriminator Methods (single oscillator)

a. Cavity-Discriminator Method

b. Delay-Line Method 


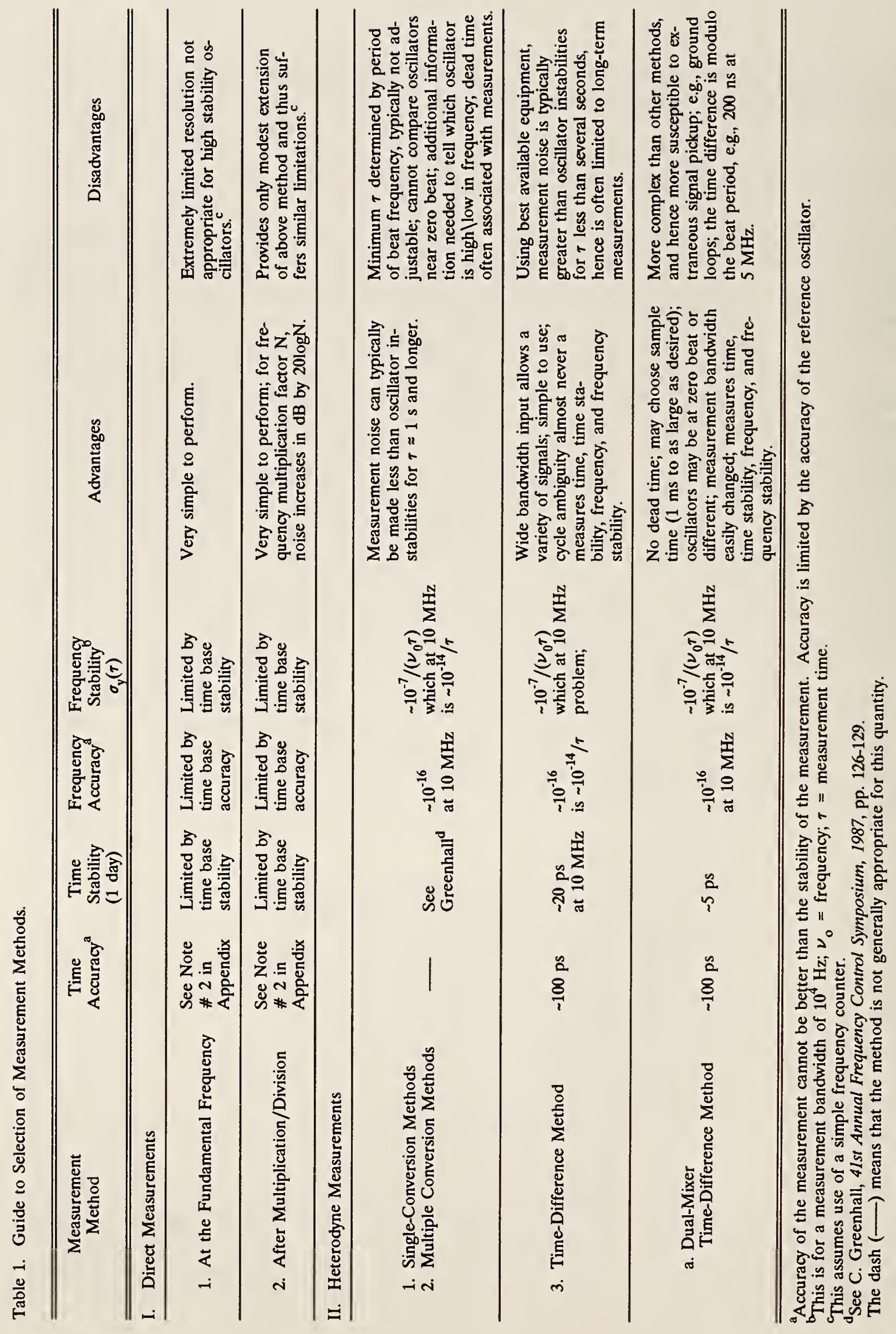




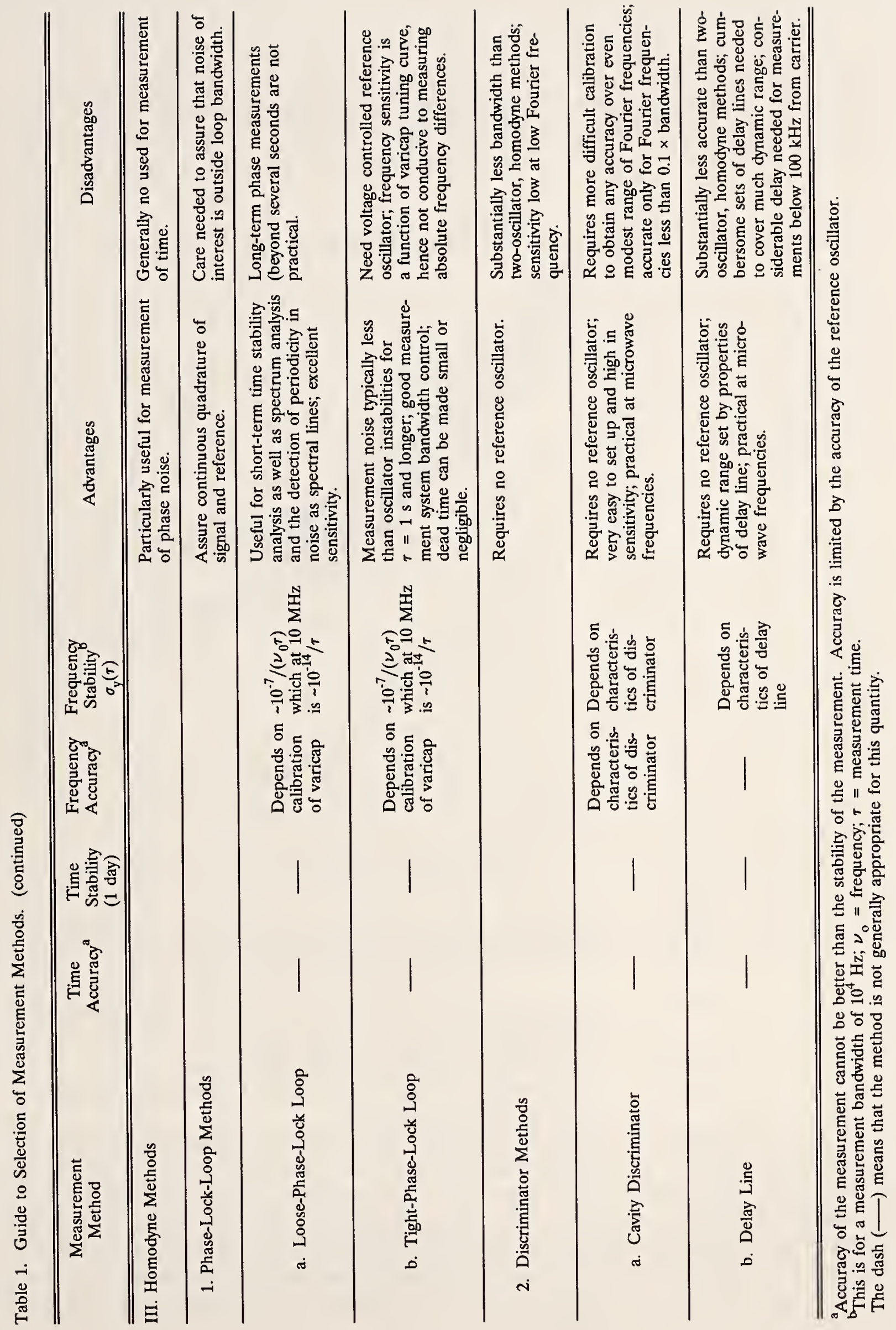




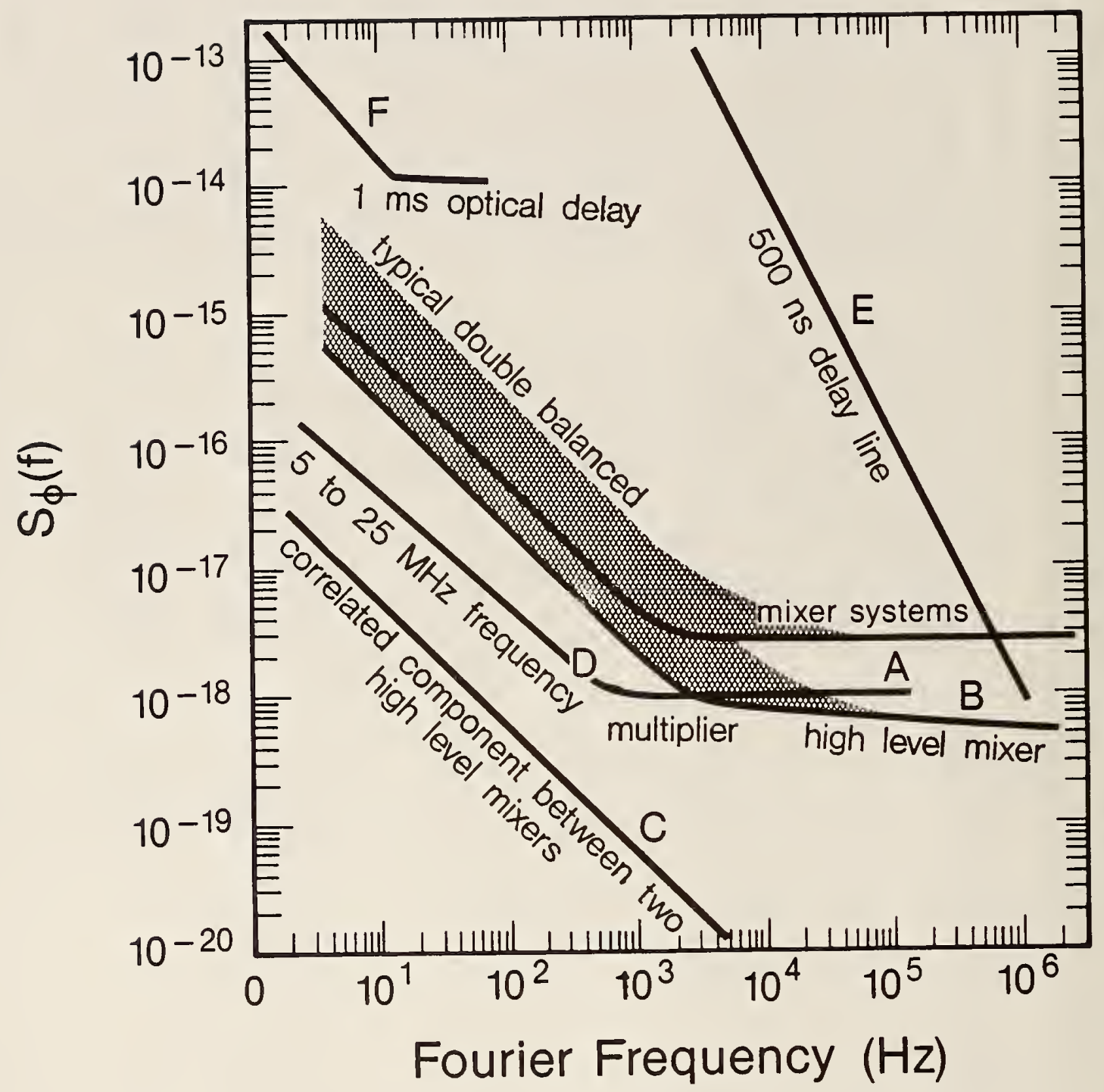

Figure 1. Comparison of nominal lower noise limits for different frequency-domain measurement methods.

Curve A. The noise limit (resolution), $\mathbf{S} \phi(\mathrm{f})$, of typical double-balanced mixer systems at carrier frequencies from $0.1 \mathrm{MHz}$ to $26 \mathrm{GHz}$.

Curve B. The noise limit, $S \phi(f)$, for a high-level mixer.

Curve C. The correlated component of $S \phi(f)$ between two channels using high-level mixers.

Curve D. The equivalent noise limit, $\mathrm{S} \phi(\mathrm{f})$, of a 5 to $25 \mathrm{MHz}$ frequency multiplier.

Curve E. Approximate phase noise limit for a typical delay-line system which uses a $500 \mathrm{~ns}$ delay line.

Curve F. Approximate phase noise limit for a delay-line system which achieves a $1 \mathrm{~ms}$ delay through encoding the signal on an optical carrier and transmitting it across a long optical fiber to a detector. 
Table 1 organizes the measurement methods in the above manner giving performance limits, advantages and disadvantages for each. Figure 1 following the table provides limitations for phase noise measurements as a function of Fourier frequency. The reader is again reminded that the table is highly simplified giving nominal levels that can be achieved. Exceptions can be found to almost every entry.

\section{A.6 RELATIONSHIP OF THE MODIFIED ALLAN VARIANCE TO THE ALLAN VARIANCE}

In sorting through this set of papers and other published literature on the Allan Variance, we were stimulated to further consider the relationship between the modified Allan variance and the Allan variance. The ideas which were developed in this process have not been published, so we include them here. Paper D.6, "Characterization of Frequency Stability: Analysis of the Modified Allan Variance and Properties of Its Estimate," by Lesage and Ayi adds new insights and augments the Allan and Barnes paper (D.5), "A Modified Allan Variance with Increased Oscillator Characterization Ability." In this section we extend the ideas presented in these two papers and provide further clarification of the relationship between the two variances, both of which are sometimes referred to as two-sample variances.

Figure 2 shows the ratio $\left[\bmod \sigma_{\mathrm{y}}(\tau) / \sigma_{\mathrm{y}}(\tau)\right]^{2}$ as a function of $\mathrm{n}$, the number of time or phase samples averaged together to calculate $\bmod \sigma_{\mathrm{y}}(\tau)$. This ratio is shown for power-law noise spectra (indexed by the value of $\alpha$ ) running from $\mathrm{f}^{2}$ to $\mathrm{f}^{2}$. These corrected results have a somewhat different shape for $\alpha=-1$ than those presented in either paper D.5 or paper D.6. Furthermore, this figure also shows the dependence on bandwidth for the case where $\alpha=1$. For all other values of $\alpha$ shown, there is no dependence on bandwidth. Table 2 gives explicit values for the ratio as a function of $\mathrm{n}$ for low $\mathrm{n}$ as well as the asymptotic limit for large $\mathrm{n}$. For $\alpha=1$, the asymptotic limit of the ratio is considerably simplified from that given in papers D.5 and D.6. With these results it is possible to easily convert between $\bmod \sigma_{y}(\tau)$ and $\sigma_{y}(\tau)$ for any of the common power-law spectra.

The information in figure 2 and table 2 was obtained directly from the basic definitions of $\sigma_{y}(\tau)$ and $\bmod \sigma_{y}(\tau)$ using numerical techniques. The results of the numerical calculations were checked against those obtained analytically by Allan and Barnes (D.5) and Lesage and Ayi (D.6). For $\alpha=2,0$ and -2 the results agree exactly. For $\alpha=1$ and -1 the analytical expressions are really obtained as approximations. The numerical calculations are obviously more reliable. Details of our calculations can be found in a NIST report [1]. A useful integral expression (not commonly found in the literature) for $\bmod \sigma_{\mathrm{y}}^{2}(\tau)$ is

$$
\operatorname{modo}_{y}^{2}(\tau)-\frac{2}{n^{4} \pi^{2} \tau_{0}^{2}} \int_{0}^{f_{k}} \frac{s_{y}(f) \sin ^{6}(\pi \tau f)}{f^{2} \sin ^{2}\left(\pi \tau_{0} f\right)} d f .
$$

Figure 2 and table 2 show that, for the fractional frequency fluctuations, mod $\sigma_{y}(\tau)$ always yields a lower value than $\sigma_{y}(\tau)$. In the presence of frequency modulation (FM) noise with $\alpha \geq 0$, the improvement is very significant for large $\mathrm{n}$. This condition has been examined in detail by Bernier [2]. For white FM noise, $\alpha=0$, the optimum estimator for time interval $\tau$ is to use the value of the times or phases separated by $\tau$ to determine frequency. This is analogous to the algorithm for calculating $\sigma_{y}(\tau)$ which yields the one-sigma uncertainty in the estimate 
$\stackrel{\Xi}{\Xi} \stackrel{\stackrel{\Xi}{\Xi}}{\Xi}$

ᄃ $\frac{0}{2}$

ซ్

틀.

苋导

.

。

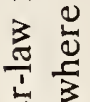

เ

동

ธี 11

है

80

눙 오

I.

㸃

$\$ 2$

$2 \div$

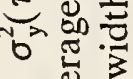

용

F

글

ठ ह

\&

늠

象 。

ह

怘

ते ญै

है है ह

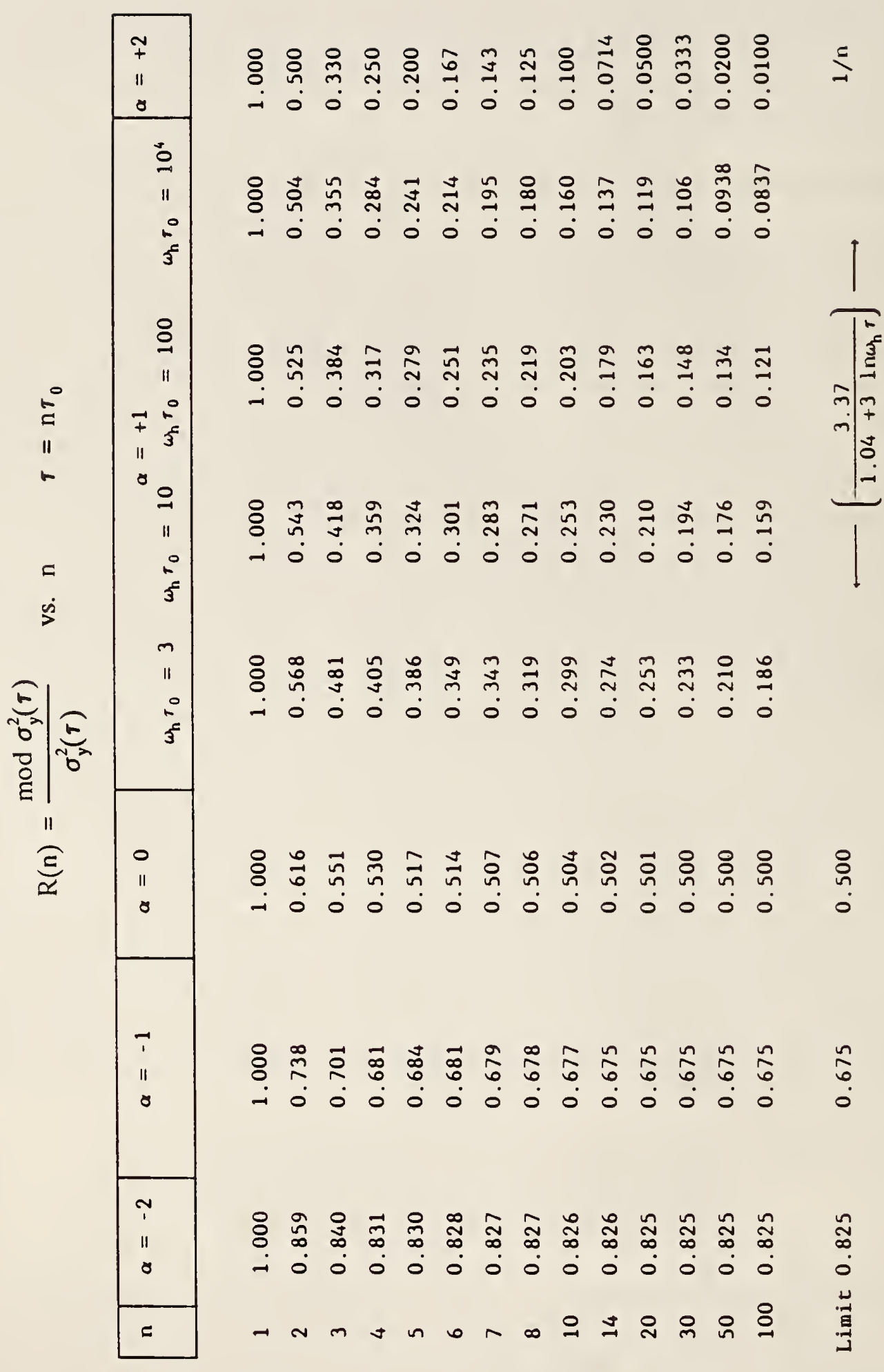




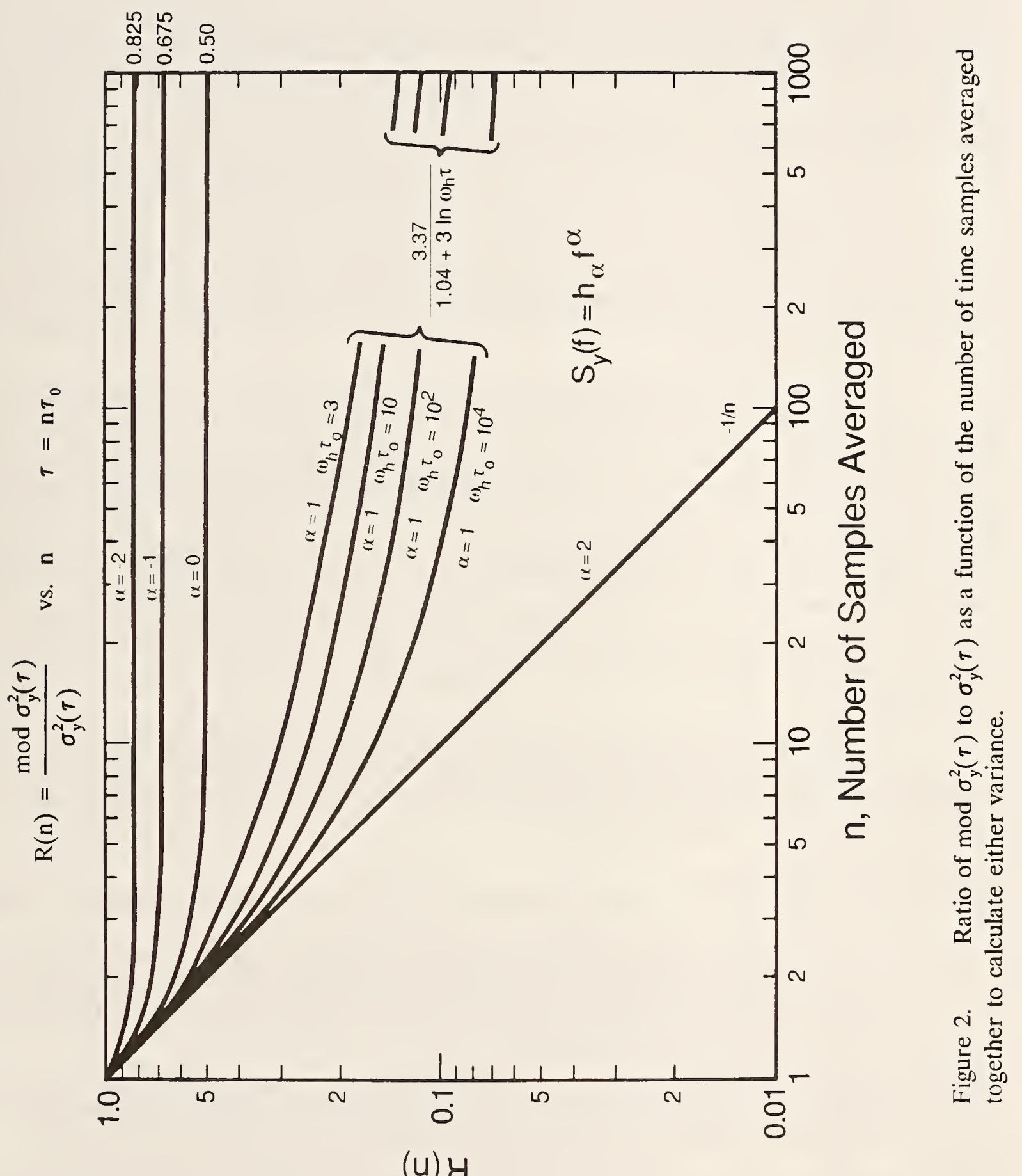


of the frequency measured in this manner over the interval $\tau$. The estimate of frequency, obtained by averaging the phase or time data, is degraded by about 10 percent from that obtained by using just the end points [3]. This is analogous to the algorithm for calculating mod $\sigma_{\mathrm{y}}(\tau)$ which, in this case, underestimates the uncertainty in measuring frequency by $\sqrt{2}$. For white phase modulation (PM) noise, $\alpha=2$, the optimum estimator for frequency is obtained by averaging the time or phase data over the interval $\tau$. This is analogous to the algorithm for calculating mod $\sigma_{y}(\tau)$ which yields the one-sigma uncertainty in the estimate for frequency measured in this manner. This estimate for frequency is $\sqrt{ }$ better than that provided by $\sigma_{\mathrm{y}}(\tau)$.

Based on these considerations, it is our opinion that mod $\sigma_{y}(\tau)$ can be profitably used much more often than it is now. The presence of significant high-frequency FM or PM noise in the measurement system, in an oscillator, or in an oscillator slaved to a frequency reference, is very common. The use of $\bmod \sigma_{y}(\tau)$ in such circumstances allows one to more quickly assess systematic errors and long-term frequency stability. In other words, a much more precise value for the frequency or the time of a signal (for a given measurement interval) can be derived using $\bmod \sigma_{y}(\tau)$ when $n$ is large.

The primary reasons for using $\sigma_{y}(\tau)$ are that it is well known, it is simple to calculate, it is the most efficient estimator for FM noise $(\alpha \leq 0)$, and it has a unique value for all $\tau$. The advantages of $\bmod \sigma_{\mathrm{y}}(\tau)$ are cited in the above paragraph. There are some situations where a study of both $\sigma_{y}(\tau)$ and mod $\sigma_{y}(\tau)$ can be even more revealing than either one. The disadvantages of using mod $\sigma_{y}(\tau)$ are that it is more complex to calculate and thus requires more computer time and it has not been commonly used in the literature, so interpretation of the results is more difficult to reconcile with published information. Another concern sometimes raised is that mod $\sigma_{y}(\tau)$ does not have a unique value in regions dominated by FM noise $(\alpha \geq 0)$. With rapidly increasing computer speeds, the computational disadvantage is disappearing. The corrected and expanded information presented in figure 2 and table 2 addresses the concern about uniqueness.

The primary disadvantage of using $\sigma_{y}(\tau)$ is that the results can be too conservative. That is, if the level of high-frequency FM noise is high, then the results are biased high, and it can take much longer (often orders of magnitude longer) to characterize the underlying low-frequency performance of the signal under test.

\section{References to Section A.6}

[1] Walls, F. L., John Gary, Abbie O'Gallagher, Roland Sweet and Linda Sweet, "Time Domain Frquency Stability Calculated from the Frequency Domain Description: Use of the SIGINT Software Package to Calculate Time Domain Frequency Stability from the Frequency Domain," NISTIR 89-3916, 1989.

[2] Bernier, L.G., Theoretical Analysis of the Modified Allan Variance, Proceedings of the 41st Frequency Control Symposium, IEEE Catalogue No. 87CH2427-3, 116, 1987.

[3] Allan, D.W. and J.E. Gray, Comments on the October 1970 Metrologia Paper "The U.S. Naval Observatory Time Reference and Performance of a Sample of Atomic Clocks," International Journal of Scientific Metrology, 7, 79, 1971. 


\section{A.7 SUPPLEMENTARY READING LIST}

$\underline{\text { Books }}$

Gerber, Eduard A. and Arthur Ballato (editors), Precision Frequency Control, Volumes 1 and 2 (Academic Press, New York, 1985).

Jespersen, James and Jane Fitz-Randolph, From Sundials to Atomic Clocks, (Dover Publications, New York, 1982).

Kartaschoff, P., Frequency and Time, (Academic Press, New York, 1978).

Kruppa, Venceslav F. (editor), Frequency Stability: Fundamentals and Measurement, (IEEE Press, New York, 1983).

Randall, R.B., Frequency Analysis, (Brüel and Kjær, Denmark, 1987).

Vanier, J. and C. Audoin, The Quantum Physics of Atomic Frequency Standards, Volumes 1 and 2 (Adam Hilger, Bristol, England, 1989).

Special Issues of Journals devoted to Time and Frequency Topics

Proceedings of the IEEE, Vol. 54, February, 1966.

Proceedings of the IEEE, Vol. 55, June, 1967.

Proceedings of the IEEE, Vol. 74, January, 1986.

IEEE Transactions on Ultrasonics, Ferroelectrics, and Frequency Control, Vol. UFFC-34, November, 1987.

$\underline{\text { Regular Publications dealing with Time and Frequency Topics }}$

IEEE Transactions on Ultrasonics, Ferrolectrics and Frequency Control.

Proceedings of the Annual Symposium on Frequency Control, IEEE.

Proceedings of the Annual Precise Time and Time Interval (PTTI) Applications and Planning Meeting, available from the U.S. Naval Observatory.

Proceedings of the European Frequency and Time Forum. 
From: Proceedings of the 35th Annual Symposium on Frequency Control, 1981.

PROPERTIES OF SIGNAL SOURCES AND MEASUREMENT METHODS

D. A. Howe, O. W. All an, and J. A. Barnes

Time and Frequency Division National Bureau of Standards Boulder, Colorado 80303

Summary

This paper is a review of prequency stability measurement techniques and of noise properties of frequency sources.

First, a historical development of the usefulness of spectrum analysis and time domain measurements will be presented. Then the rationale will be stated for the use of the two-sample (Allan) variance rather than the classical variance. Next, a range of measurement procedures will be outlined with the trade-offs given for the various techniques employed. Methods of interpreting the measurement results will be given. In particular, the five commonly used noise models (white PM, flicker PM, white FM, flicker FM, and random walk FM) and their causes will be discussed. Methods of characterizing systematics will also be given. Confidence intervals on the various measures will be discussed. In addition, we will point out methods of improving this confidence interval for a fixed number of data points.

Topics will be treated in conceptual detail. Only light (fundamental) mathematical treatment will be given.

Although traditional concepts. will be detailed, two new topics will be introduced in this paper: (1) accuracy limitations of digital and computer-based analysis and (2) optimizing the results from a fixed set of input data.

The final section will be devoted to fundamental (physical) causes of noise in commonly used frequency standards. Also transforms from time to frequency domain and vice-versa will be given.

Key Words. Frequency stability; Oscillator noise modeling; Power law spectrum; Time-domaín stability; Frequency-domain stability; White noise; Flicker noise.

\section{Introduction}

Precision oscillators play an important role in high speed communications, navigation, space tracking, deep space probes and in numarous other important applications. In this paper, we will review some precision methods of measuring the frequency and frequency stability of precision oscillators. Development of topics does not rely heavily on mathematics. The equipment and set-up for stability measurements are outlined. Examples and typical results are presented. Physical interpretations of comnon noise processes are discussed. A table is provided by which typical frequency domain stability characteristics may be translated to time domain stability characteristics and vice-versa.

\section{THE SINE WAVE ANO STABILITY}

A sine wave signal generator produces a voltage that changes in time in a sinusoidal way as shown in figure 1.1. The signal is an oscillating signal because the sine wave repeats itself. A cycle of the oscillation is produced in one period "T". The phase is the angle " $\Phi$ " within a cycle corresonding to a particular time " $t$ ".

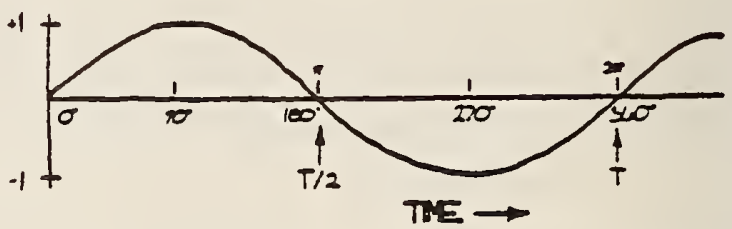

FIGURE 1.1

It is convenient for us to express angles in radians rather than in units of degrees, and positive zero-crossings will occur at even multiples of $\pi$-radians. The frequency " $v "$ is the number of cycles in one second, which is the reciprocal of period (seconds per cyele). The expression describing the voltage " $V$ " out of a sine wave signal generator is given by $V(t)=V_{p}$ $\sin [\phi(t)]$ where $V_{p}$ is the peak voltage amplitude. Equivalent expressions are

$$
v(t)=v_{p} \sin \left(2 \pi \frac{t}{T}\right)
$$


and

$$
V(t)=V_{p} \sin (2 \pi v t)
$$

Consider figure 1.2. Let's assume that the maximum value of " $V$ " equals 1 , hence " $V$ " $=1$. We say that the voltage "V( $t)$ " is nomalized to unity. If we know the frequency of a signal and if the signal is a sine wave, then we can determine the incremental change in the period " $T$ " (denoted by $\Delta t)$ at a particular angle of phase.

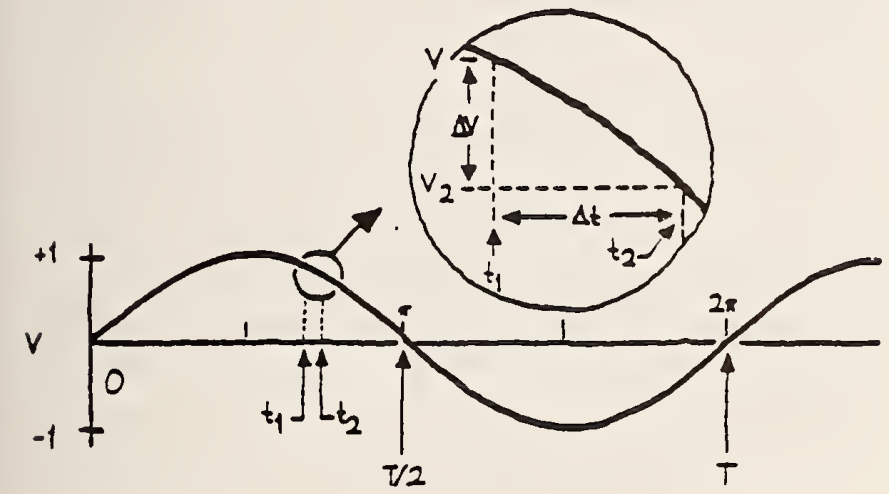

FIGURE 1.2

Note that no matter how big or small $\Delta t$ may be, we can determine $\Delta V$. Let us look at this from another point of view. Suppose we can measure $\Delta V$ and $\Delta t$. From this, there is a sine wave at a unique minimum frequency corresponding to the given $\Delta V$ and $\Delta t$. For infinitesimally small $\Delta t$, this frequency is called the instantaneous frequency at this $t$. The smaller the interval $\Delta t$, the better the approximation of instantaneous frequency at $t$.

When we speak of oscillators and the signals they produce, we recognize that an oscillator has some nominal frequency at which it operates. The "frequency stability" of an oscillator is a term used to characterize the frequency fluctuations of the oscillator signal. There is no formal definition for "frequency. stability". However, one usually refers to frequency stability when com paring one oscillator with another. As we shall see later, we can define particular aspects of an oscillator's output then draw conclusions about its relative frequency stability. In general terms,
"Frequency stability is the degree to which an oscillating signal produces the same value of frequency for any interval, $\Delta t$, throughout a specified period of time".

Let's examine the two waveforms shown in figure 1.3. Frequency stability depends on the amount of time involved in a measurement. Of the two oscillating signals, it is evident that " 2 " is more stable than " 1 " from time $t_{1}$ to $t_{3}$ assuming the horizontal scales are linear in time.

\section{UNTRE HEOENCY}

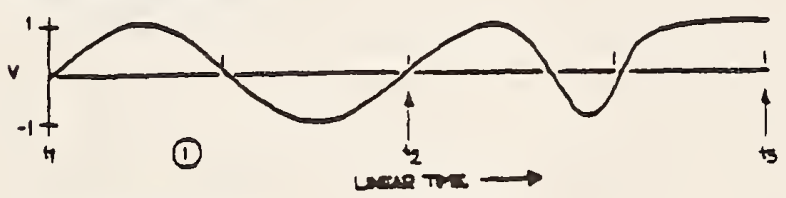

SRE ROEICY

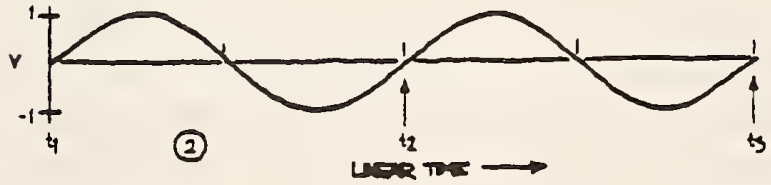

FIGURE 1.3

From time $t_{1}$ to $t_{2}$, there may be some question as to which of the two signals is more stable, but it's clear that from time $t_{2}$ to $t_{3}$, signal " 1 " is at a different frequency from that in interval $t_{1}$ to $t_{2}$.

If we want an oscillator to produce a particular frequency $v_{0}$, then we're correct in stating that if the oscillator signal frequency deviates from $v_{0}$ over any interval, this is a result of something which is undesirable. In the design of an oscillator, it is important to consider the sources of mechanisms which degrade the oscillator's frequency stability. All undesirable mechanisms cause random (noise) or systematic processes to exist along with the sine wave signal of the oscillator. To account for the noise components at the output of a sine wave signal generator, we can express the output as

$$
V(t)=\left[V_{0}+\varepsilon(t)\right] \sin \left[2 \pi v_{0} t+\phi(t)\right] .
$$


where $v_{0} \equiv$ nominal peak voltage amplitude, $\varepsilon(t) \equiv$ deviation of amplitude from nominal,

$v_{0} \equiv$ nominal fundamental frequency, $\phi(t) \equiv$ deviation of phase from nominal.

Ideally " $\varepsilon$ " and " $\phi$ " should equal zero for all time. However, in the real world there are no perfect oscillators. To determine the extent of the noise components " $\varepsilon$ " and " $\phi$ ", we shall turn our attention to measurement techniques.

The typical precision oscillator, of course, has a very stable sinusoidal voltage output with a frequency $u$ and a period of oscillation $T$, which is the reciprocal of the frequency $(v=I / T)$. One goal is to measure the frequency and/or the frequency stability of the sinusoid. Instability is actuaily measured, but with little confusion it is often called stability in the literature. Naturally, fluctuations in frequency correspond to fluctuations in the period. Almost all frequency measurements, with very few exceptions, are measurements of phase or of the period fluctuations in an oscillator, not of frequency, even though the frequency may be the readout. As an example, most frequency counters sense the zero (or near zero) crossing of the sinusoidal voltage, which is the point at which the voltage is the most sensitive to phase fluctuations.

One must also realize that any frequency measurement involves two oscillators. In some instances, one oscillator is in the counter. It is impossible to purely measure only one oscillator. In some instances one oscillator may be enough better than the other that the fluctuations measured may be considered essentially those of the latter. However, in general because frequency measurements are always dual, it is useful to define:

$$
y(t)=\frac{v_{1}-v_{0}}{v_{0}}
$$

as the fractional frequency difference or deviation of oscillator one, $v_{1}$, with respect to a reference oscillator $v_{0}$ divided by the nominal frequency $v_{0}$. Now, $y(t)$ is a dimensionless quantity and useful in describing oscillator and clock performance; e.g., the time deviation, $x(t)$, of an oscillator over a period of time $t$, is simply given by:

$$
x(t)=\int_{0}^{t} y\left(t^{\prime}\right) d t^{\prime}
$$

Since it is impossible to measure instantaneous frequency, any frequency or fractional frequency measurement always involves some sample time, st or " $\tau$ "--some time window through which the oscillators are observed; whether it's a picosecond, a second, or a day, there is always some sample time. So when determining a fractional frequency, $y(t)$, in fact what is happening is that the time deviation is being measured say starting at some time $t$ and again at a later time, $t+\tau$. The difference in these two time deviations, divided by $\tau$ gives the average fractional frequency over that period $\tau$ :

$$
\vec{y}(t)=\frac{x(t+\tau) \cdot x(t)}{\tau} .
$$

Tau, $\tau$, may be called the sample time or averaging time; e.g., it may be determined by the gate time of a counter.

What happens in many cases is that one samples a number of cycles of an oscillation during the preset gate time of a counter; after the gate time has elapsed, the counter latches the value of the number of cycles so that it can be read out, printed, or stored in some other way. Then there is a delay tine for such processing of the data before the counter ams and starts again on the next cycle of the oscillation. During the delay time (or process time), information is lost. We have chosen to call it dead time and in some instances it becowes a problem. Unfortunately for data processing in typical oscillators the effects of dead time often hurt most when it is the hardest to avoid. In other words, for times that are short compared to a second when it is very difficult to avoid dead time, that is usually where dead time can make a significant difference in the data analysis. Typically for many oscillators, if 
the sample time is long compared to a second, the dead time makes littie difference in the data analysis, unless it is excessive. ${ }^{1}$ New equipment or techniques are now available which contribute zero or negligible dead time. 2

In reality, of course, the sinusoidal output of an oscillator is not pure, but it contains noise fluctuations as well. This section deais with the measurement of these fluctuations to determine the quality of a precision signal source.

We will describe five different methods of measuring the frequency fluctuations in precision oseillators.

\subsection{Common Methods of Measuring Frequency Sta- bility}

A. Beat frequency method

The first system is called a heterodyne frequency measuring method or beat frequency method. The signal from two independent oscillators are fed into the two ports of a double balanced mixer as illustrated in figure 1.4.

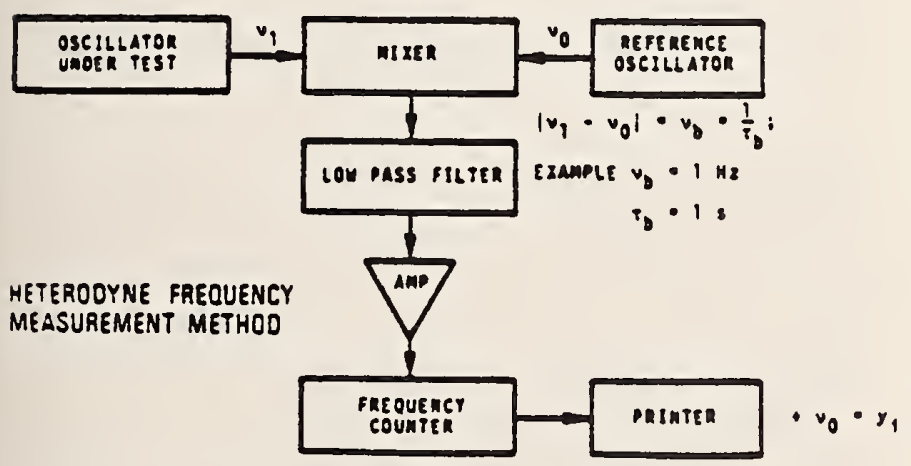

FIGURE 1.4

The difference frequency or the beat frequency, $v_{b}$, is obtained as the output of a low pass filter which follows the mixer. This beat frequency is then amplified and fed to a frequency counter and printer or to some recording device. The fractional frequency is obtained by dividing $v_{b}$ by the nominal carrier frequency $v_{0}$. This system has excellent precision; one can measure essentially all state-of-the-art oscillators.
B. Dual mixer time difference (DTMD) system

This system shows some significant promise and has just begun to be exploited. A block diagram is shown is figure 1.5 .

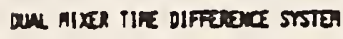
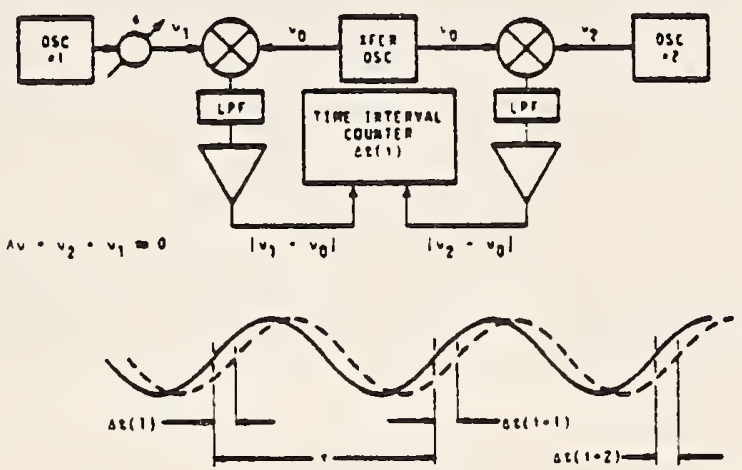

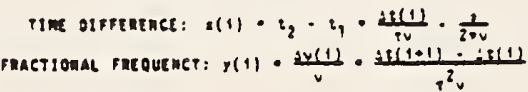

FIGURE 1.5

To preface the remarks on the OMTD, it should be mentioned that if the time or the time fluctuations can be measured directly, an advantage is obtained over just measuring the frequency. The reason is that ons can calculate the frequency from the time without dead time as well as know the time behavior. The reason, in the past, that frequency has not been inferred from the time (for sample times of the order of several seconds and shorter) is that the time difference between a pair of oscillators operating as clocks could not be measured with sufficient precision (commercialiy the best that is available is $10^{-11}$ seconds). The system described in this section demonstrates a precision of $10^{-13}$ seconds. Such precision opens the door to making time measurements as well as frequency and frequency stability measuements for sample times as short as a few milliseconds and longer, all without dead time.

In figure 1.5, oscillator 1 could be considered under test and oscillator 2 could be considered the reference oscillator. These signais go to the ports of a pair of double balanced mixers. Another oscillator with separate symmetric buffered outputs is fed to the remaining other two 
ports of the pair of double balanced mixers. This common oscillator's frequency is offset by a desired amount from the other two oscillators. Then two different beat frequencies come out of the two rixers as shown. These two beat frequencies will be out of phase by an amount proportional to the time difference between oscillator 1 and 2-excluding the differential phase shift that may be inserted. Further, the beat frequencies differ in frequency by an amount equal to the frequency difference between oscillators 1 and 2 .

This measurement technique is very useful where one has oscillator 1 and oscillator 2 on the same frequency. This is typical for atomic standards (cesium, rubidium, and hydrogen frequency standards).

Illustrated at the bottom of figure 1.5 is what might represent the beat frequencies out of the two mixers. A phase shifter may be inserted as illustrated to adjust the phase so that the two beat rates are nominally in phase; this adjustment sets up the nice condition that the noise of the common oscillator tends to cancel (for certain types of noise) when the time difference is determined. After amplifying these beat signals, the start port of a time interval counter is triggered with the positive zero crossing of one beat and the stop port with the positive zero crossing of the other beat. Taking the time difference between the zero crossings of these beat frequencies, one measures the time difference between oscillator 1 and oscillator 2, but with a precision which has been amplified by the ratio of the carrier frequency to the beat frequency (over that normally achievable with this sane time interval counter). The time difference $x(i)$ for the $i^{\text {th }}$ measurement between oscillators $I$ and 2 is given by eq (1.5).

$$
x(i)=\frac{\Delta t(i)}{\tau_{b} v_{0}}-\frac{\phi}{2 \pi v_{0}}+\frac{k}{v_{0}}
$$

where $\Delta t(i)$ is the $i^{\text {th }}$ time difference as read on the counter, $\tau_{b}$ is the beat period, $v_{o}$ is the nominal carrier frequency, $\$$ is the phase delay in radians added to the signal of oscillator 1 , and $k$ is an integer to be determined in order to remove the cycle ambiguity. It is only important to know $k$ if the absolute time difference is desired; for frequency and frequency stability measurements and for time fluctuation measurements, $k$ may be assumed zero unless one goes through a cycle during a set of measurements. The fractional frequency can be derived in the nomal way from the time fluctuations.

$$
y_{1,2}(i, \tau)=\left\{\begin{array}{l}
\frac{v_{1}(i, \tau)-v_{2}(i, \tau)}{v_{0}} \\
\frac{x(i+1)-x(i)}{\tau} \\
\frac{\Delta t(i+1)-\Delta t(i)}{\tau_{b}^{2} v_{0}}
\end{array}\right.
$$

In eqs (1.5) and (1.6), assumptions are made that the transfer (or common) oscillator is set at a lower frequency than oscillators $I$ and 2 , and that the voltage zero crossing of the beat $v_{1}-v_{c}$ starts and that $v_{2}-v_{c}$ stops the time interval counter. The fractional frequency difference may be averaged over any integer multiple of $\tau_{b}$ :

$$
y_{1,2}\left(i, m \tau_{b}\right)=\frac{x(i+m)-x(i)}{m \tau_{b}}
$$

where $m$ is any positive integer. If needed, $\tau_{b}$ can be made to be very small by having very high beat frequencies. The transfer (or common) oscillator may be replaced with a low phase-noise frequency synthesizer, which derives its basic reference frequency from oscillator 2 . In this set-up the nominal beat frequencies are simply given by the amount that the output frequency of the synthesizer is offset from $v_{2}$. Sample times as short as a few milliseconds are easiliy obtained. Logging the data at such a rate can be a problem without special equipment. The latest NBS time scale measurement system is based on the OMTO and is yieiding an excellent cost benefit ratio.

\section{Loose ohase lock loop method}

This first type of phase lock loop method is illustrated in figure 1.6. The signal from an oscillator under test is fed into one port of a 
mixer. The signal from a reference oscillator is fed into the other port of this mixer. The signals are in quadrature, that is, they are 90 degrees out

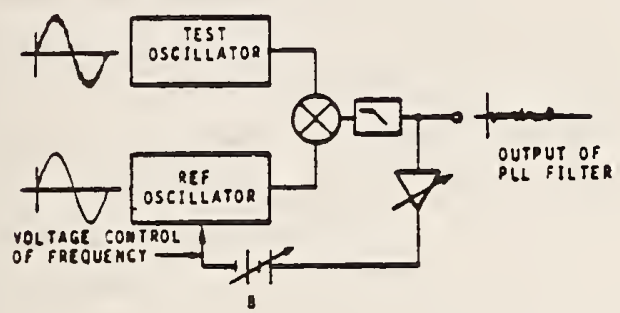

FIGURE 1.6

of phase so that the average voltage out of the mixer is nominaliy zero, and the instantaneous voltage fluctuations correspond to phase fluctuations rather than to amplitude fluctuations between the two signals. The mixer is a key element in the system. The advent of the Schottky barrier diode was a significant breakthrough in making low noise precision stability measurements. The output of this mixer is fed through a low pass filter and then amplified in a feedback loop, causing the voltage controlled oseillator (reference) to be phase loeked to the test oscillator. The attack time of the loop is adjusted such that a very loose phase lock (long time constant) condition exists. This is discussed later in section VIII.

The attack time is the time it takes the servo system to make $70 \%$ of its ultimate correction after being slightly disturbed. The attack time is equal to $1 / \pi w_{h}$, where $w_{h}$ is the servo bandwidth. If the attack time of the loop is about a second then the voltage fluctuations will be proportional to the phase fluctuations for sample tiaes shorter than the attack time. Depending on the coefficient of the tuning capacitor and the quality of the oscillators involved, the amplification used may vary significantly but may typicaliy range from 40 to $80 \mathrm{~dB}$ via a good low noise amplifier. In turn this signal can be fed to a specturm analyzer to measure the fourier components of the phase fluctuations. This system of frequencydomain analysis is discussed in sections VIII to $x$.
It is of particular use for sample times shorter than one second (for Fourier frequencies greater than $1 \mathrm{~Hz}$ ) in analyzing the characteristics of an oscillator. It is specifically very useful if one has discrete side bands such as $60 \mathrm{~Hz}$ or detailed structure in the spectrum. How to characterize precision oscillators using this technique will be treated in detail later in section IX and XI.

One may also take the output voltage from the above amplifier and feed it to an $A / D$ converter. This digital output becomes an extremely sensitive measure of the short term time or phase fluctuations between the two oscillators. Precisions of the order of a picosecond are easily achievable.

\section{Tight phase lock loop method}

The second type of phase lock loop method (shown in figure 1.7) is essentially the same as the first in figure 1.6 except that in this case the loop is in a tight phase lock condition; i.e., the attack time of the loop should be of the order of a few milliseconds. In such a case, the phase fluctuations are being integrated so that the voltage output is proportional to the frequency

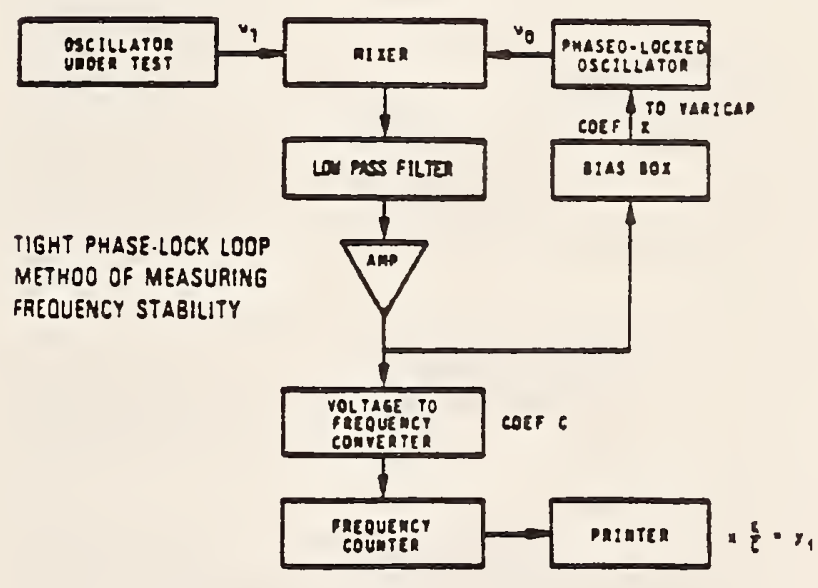

FIGURE 1.7

fluctuations between the two oscillators and is no longer proportional to the phase fluetuations for sample times longer than the attack time of the loop. A bias box is used to adjust the voltage on the varicap to a tuning point that is fairly linear and of a reasonable value. The voltage 
fluctuations prior to the bias box (biased slightly away from zero) may be fed to a voltage to frequency converter which in turn is fed to a frequency counter where one may read out the frequency fluctuations with great amplification of the instabilities between this pair of oscillators. The frequency counter data are logged with a data logging device. The coefficient of the varicap and the coefficient of the voltage to frequency converter are used to determine the fractional frequency fiuctuations, $y_{i}$, between the oscillators, where $i$ denotes the $i^{\text {th }}$ measurement as shown in figure 1.7. It is not difficult to achieve a sensitivity of a part in $10^{14}$ per $\mathrm{Hz}$ resolution of the frequency counter, so one has excellent precision capabilities with this system.

\section{E. Time difference method}

The last measurement method we will illustrate is very commoniy used, but typically does not have the measurement precision more readily available in the first four methods illustrated above. This method is called the time difference method, and is shown in figure 1.8. Because of the wide

The Interw coum

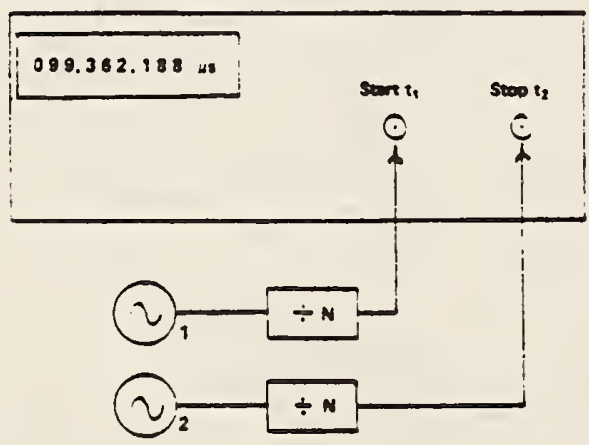

FIGURE 1.8

bandwidth, needed to measure fast rise-time pulses, this method is limited in signal-to-noise ratio. However, some counters are commercially available allowing one to do signal averaging or to do precision rise-time comparison (precision of time difference measurements in the range of $10 \mathrm{~ns}$ to 10 ps are now available). Such a method yields a direct measurement of $x(t)$ without any translation, conversion, or multiplication factors. Caution should be exercised in using this technique even if adequate measurement precision is available because it is not uncomon to have significant instabilities in the frequency dividers shown in figure 1.8--of the order of several nanoseconds. The technology exists to build better frequency dividers than are commonly available, but manufacturers have not yet availed themselves of state-ofthe-art techniques in a cost beneficial manner. A trick to by-pass divider problems is to feed the oscillator signals directly into the time interval counter and observe the zero voltage crossing into a well matched impedance. (In fact, in all of the above methods one needs to pay attention to impedance matching, cable lengths and types, and connectors). The divided signal can be used to resolve cycle ambiguity of the carrier, otherwise the carrier phase at zero volts may be used as the time reference. The slope of the signal at zero volts is $2 \pi v_{0} / \tau_{1}$, where $\tau_{1}=1 / v_{1}$ (the period of oscillation). For $V_{p}=1$ volt and a $5 \mathrm{MHz}$ signal, this slope is $3 \mathrm{~m}$ volts/ns, which is a very good sensitivity.

\section{MEASUREMENT METHOOS COMPARISON}

When making measurements between a pair of frequency standards or clocks, it is desirable to have less noise in the measurement systen than the composite noise in the pair of standards being measured. This places stringent requirements on measurement systems as the state-of-the-art of precision frequency and time standards has advanced to its current level. As will be shown, perhaps one of the greatest areas of disparity between measurement system noise and the noise in current standards is in the area of time difference measurements. Commercial equipment can measure time differences to at best $10^{-11} \mathrm{~s}$, but the time fluctuations second to second of state-of-the-art standards is as good as $10^{-13} \mathrm{~s}$.

The disparity is unfortunate because if time differences between two standards could be measured with adequate precision then one may also know the time fiuctuations, the frequency differences, and the frequency fluctuations. In fact, one can set 


\begin{tabular}{|c|c|c|c|c|c|}
\hline \multirow{2}{*}{$\begin{array}{l}\text { Hierachy } \\
\text { Status }\end{array}$} & \multirow{2}{*}{$\begin{array}{l}\text { Exanplo of } \\
\text { Measurement } \\
\text { Metnod }\end{array}$} & \multicolumn{4}{|c|}{ Dota deducible iron the mesurement } \\
\hline & & $\operatorname{rise}$ & oting & Freq. & bFreq. \\
\hline 1 & $\begin{array}{l}\text { Dul Mixer Ties diff. } \\
\text { or } \\
\text { Tiee Interval Counter }\end{array}$ & $x(t)=\frac{\Delta q}{\tau v}$ & $\begin{array}{l}6 x(t, z)= \\
x(t+\tau)-x(t)\end{array}$ & $\begin{array}{l}y(t, \tau)= \\
\frac{6 \times(\tau, \tau)}{1}\end{array}$ & $\begin{array}{c}6 y(t, \tau) x \\
y(t+\tau, \tau)-y(t, \tau)\end{array}$ \\
\hline 2 & $\begin{array}{l}\text { Loose Phase-locked } \\
\text { referenca oscillator }\end{array}$ & can't ceesure & $\begin{array}{l}6 \times(t, \tau)= \\
\frac{60(t, \tau)}{2 \pi}\end{array}$ & $\omega$ & 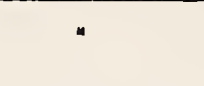 \\
\hline 3 & $\begin{array}{l}\text { Hetorodyne or beat } \\
\text { Prequency }\end{array}$ & can't masure & con't asesure & $\begin{array}{c}|y(t, \tau)|= \\
\frac{v_{\text {beot }}}{v_{0}}\end{array}$ & “ \\
\hline 4 & $\begin{array}{l}\text { Tight phase-locked } \\
\text { reference oscillator }\end{array}$ & can't masure & can't mesure & ean't mesure & $\begin{array}{c}6 y(\varepsilon, r) \\
c \cdot 6 V\end{array}$ \\
\hline
\end{tabular}

TABLE $2 . I$

\begin{tabular}{|c|c|c|c|c|c|c|c|}
\hline $\begin{array}{l}\text { Mosureseont } \\
\text { noenod }\end{array}$ & $\lim _{\text {aceuracy }}^{\text {ilm }}$ & $\begin{array}{l}\text { Ties } \\
\text { stadility }\end{array}$ & $\begin{array}{l}\text { Eroquency } \\
\text { oceuracy } \\
\text { (I day) }\end{array}$ & $\begin{array}{l}\text { Erequency } \\
\text { subol lity }\end{array}$ & Aoveneagess & Discavantuges & $\begin{array}{l}\text { poroxicate } \\
\text { Cost (ks) }\end{array}$ \\
\hline $\begin{array}{l}\text { Ties Intorval } \\
\text { Councor }\end{array}$ & $-1000 \mathrm{ps}$ & 10ps & -10.28 & $\underset{x=1}{-1.10-11}$ & 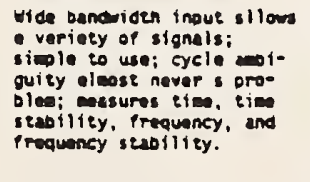 & 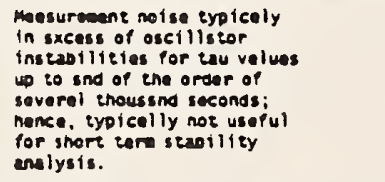 & 1 to 7 \\
\hline Heterodyne or & $\infty$ & $\cdots$ & $-10-10$ & $\sum_{i=1}^{-1.10-13}$ & 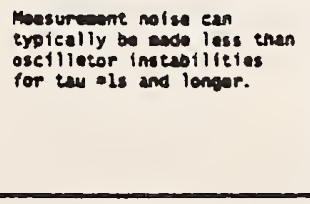 & 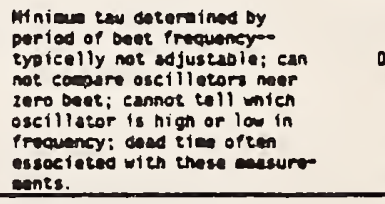 & 0.2 to 1 \\
\hline $\begin{array}{l}\text { Tigne ponaser } \\
\text { lockere roferoneo" } \\
\text { oscillineor }\end{array}$ & $\cdots$ & $\cdots$ & - & $x_{x \rightarrow-1}^{-1.20-22}$ & 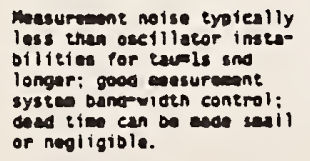 & 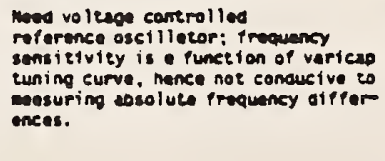 & 20 \\
\hline
\end{tabular}


up an interesting hierarchy of kinds of measurement systens: 1) those that can measure time, $x(t) ; 2$ ) those that can measure changes in time or time fluctuations $\delta x(t) ; 3)$ those that can measure frequency, $v\left(y \equiv\left(v-v_{0}\right) / v_{0}\right)$; and 4) those that can measure changes in frequency or frequency fluctuations, $\delta v\left(\delta y \equiv \delta v / v_{0}\right)$. As depicted in table 2.1, if a measurement system is of status 1 in this hierarchy, i.e., it can measure time, then time fluctuations, frequency and frequency fluctuations can be deduced. However, if a measurement system is only capable of measuring time fluctuations (status 2 - table 2.1), then time cannot be deduced, but frequency and frequency fluctuations can. If frequency is being measured (status 3 table 2.1), then neither time nor time fluctuations may be deduced with fidelity because essentially all commercial frequency measuring devices have "dead time" (technology is at a point where that is changing with fast data processing speeds that are now available). Dead time in a frequency measurement destroys the opportunity of integrating the fractional frequency to get to "true" time fluctuations. Of course, if frequency can be measured, then trivially one may deduce the frequency fluctuations. Finally, if a system can only measure frequency fluctuations istatus 4 table 2.1), then neither time, nor time fluctuations, nor frequency can be deduced from the data. If the frequency stability is the primary concern then one may be perfectly happy to employ such a measurement system, and similarly for the other statuses in this measurement hierarchy. Obviously, if a measurement method of Status 1 could be employed with state-of-the-art precision, this would provide the greatest flexibility in data processing. From section 1, the dual mixer time difference system is purported to be such a method.

Tabie 2.2 is a comparison of these different measurement methods. The values entered are nominal; there may be unique situations where significant departures are observed. The time and frequency stabilities listed are the nominal second to second ms values. The accuracies listed are taken in an absolute sense. The costs listed are nominal estimates in 1981 dollars.
Figure 2.1 is a diagram indicating the sample time regions over which the various methods

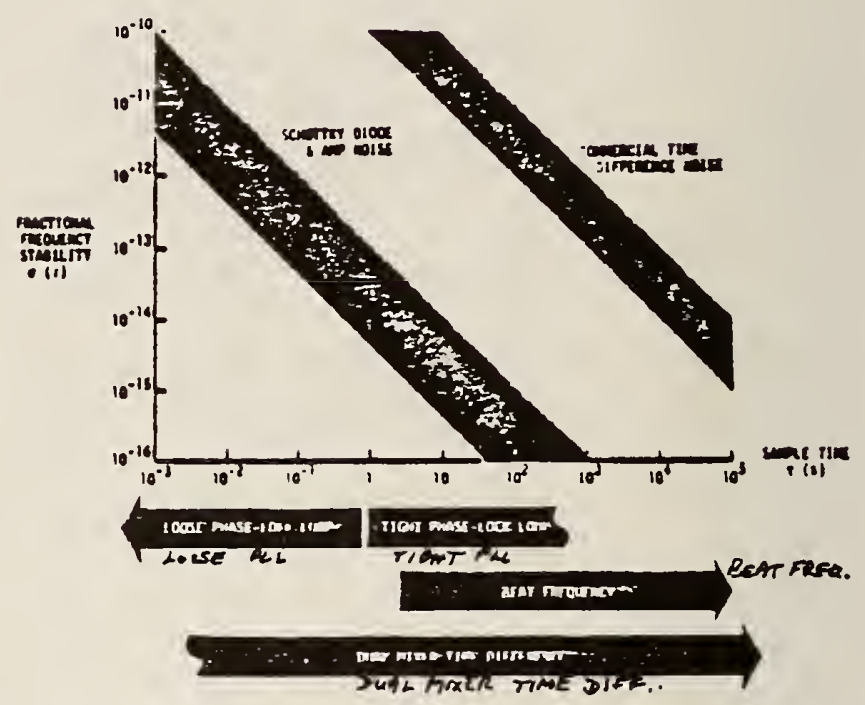

FIGURE 2.1

are most appropriately appiied. The large diagonally oriented area indicates the typical noise limits of the measurement technique (at particular values of sample time indicated on the horizontal scale).

\section{CHARACTERIZATION}

Given a set of data of the fractional frequency or time fluctuations between a pair of oscillators, it is useful to characterize these fluctuations with reasonable and tractable models of performance. In so doing for many kinds of oscillators, it is useful to consider the flucuations as those that are random (may only be predicted statistically) and those that are nonrandon (e.g., systematics - those that are environmentally induced or those that have a causal effect that can be determined and in many cases can be predicted).

\subsection{Non-random Fluctuations}

Non-random fluctuations are usually the main cause of departure from "true" time or "true" frequency. 
If, for example, one has the values of the frequency over a period of time and a frequency offset from nominal is observed, one may calculate directly that the phase error will accumulate as a ramp. If the frequency values show some linear orift then the time fluctuations will depart as a quadratic. In almost all oscillators, the above systematics, as they are sometimes called, are the primary cause of time and/or frequency departure. A useful approach to determine the value of the frequency offset is to calculate the simple mean of the set, or for determining the value of the frequency drift by calculating a linear least squares fit to the frequency. A least squares quadratic fit to the phase or to the time derivative is typically not as efficient an estimator of the frequency drift for most oscillators.

\section{2 Random Fluctuations}

After calculating or estimating the systematic or non-random effects of a data set, these may be subtracted from the data leaving the residual random fluctuations. These can usually be best characterized statistically. It is often the case for precision oscillators that these randon fluctuations may be well modeled with power law spectral densities. This topic is discussed later in sections VIII to $X$. We have

$$
S_{y}(f)=h_{\alpha} f^{\alpha}
$$

where $S_{y}(f)$ is the one-sided spectral density of the fractional frequency fluctuations, $f$ is the Fourier frequency at which the density is taken, $h_{\alpha}$ is the intensity coefficient, and $\alpha$ is number modeling the most appropriate power law for the data. It has been shown ${ }^{1,3}$ that in the time domain one can nicely represent a power law spectral density process using a well defined timedomain stability measure, $\sigma_{y}(\tau)$, to be explained in the next section. For example, if one observes from a $\log \sigma_{y}{ }^{2}(\tau)$ versus $\tau$ diagram a particular slope (call it $\mu$ ) over certain regions of sample time, $\tau$, this slope has a correspondence to a power law spectral density or a set of the sane with some amplitude coefficient $h_{\alpha}$. In particular, $\mu=-\alpha-1$ for $-3<\alpha<1$ and $\mu \equiv-2$ for $1 \leq \alpha$. Further a correspondence exists between $h_{\alpha}$ and the coefficient for $\sigma_{y}(\tau)$. These coefficients have been calculated and appear in section XI. The transformations for some of the more common power law spectral densities have been tabulated making it quite easy to transform the frequency stability modeled in the time-domain over to the frequency domain and vice versa. Examples of some power-law spectra that have been simulated by computer are shown in figure 3.1. In descending order these

\section{POWER LAW SPECTRA}

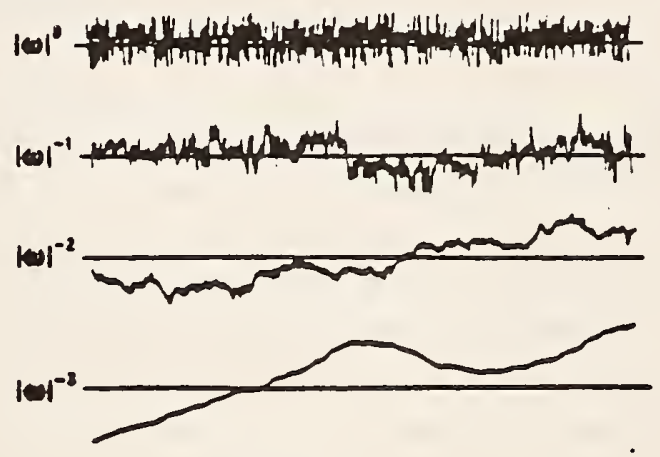

FIGURE 3.1

have been named white noise, flicker noise, random walk, and flicker walk (the $w$ in fig. 3.1 is angular Fourier frequency, $w=2 \pi f$ ).

Once the noise characteristics have been determined, one is often able to deduce whether the oscillators are performing properly or not and whether they are meeting either the design specifications or the manufacturers specifications. For example a cesium beam frequency standard or a rubidium gas cell frequency standard when working properly should exhibit white frequency noise, which is the same as random walk phase (or time) for tau values of the order of a few seconds to severai thousand seconds (see also sec. XI).

\section{ANALYSIS OF TIME DOMAIN DATA}

Suppose now that one is given the time or frequency fluctuations between a pair of precision oscillators measured, for example, by one of the techniques outlined in section $I$, and a stability analysis is desired. Let this comparison be depicted by figure 4.1. The minimum sample time 
is determined by the measurement system. If the time difference or the time fluctuations are available then the frequency or the fractional frequency fluctuations may be calculated from one period of sampling to the next over the data length as indiciated in figure 4.1. Suppose further there are $M$ values of the fractional frequency $y_{1}$. Now there are many ways to analyze these data. Historically, people have typically used the standard deviation equation shown in figure $4.1, \sigma_{\text {std. dev. }}(\tau)$, where $\dot{y}$ is the average fractional frequency over the data set and is subtracted from each value of $y_{1}$ before squaring, summing and dividing by the number of values minus one, $(M-1)$, and taking the square root to get the standard deviation. At NBS, we have studied what happens to the standard deviation when the data set may be characterized by power law spectra which are more dispersive than classical white noise frequency fluctuations. In other words, if the fluctuations are characterized by flicker noise or any other non-white-noise frequency deviations, what happens to the standard deviation for that data set? One can show that the standard deviation is a function of the number of data points in the set; it is also a function of the dead time and of the measurement system bandwidth. For example, using flicker noise frequency modulation as a model, as the number of data points increases, the standard deviation aonotonically increases without limit. Some statistical measures have been developed which do not depend upon the data length and which are readily usabie for characterizing the random fluctuations in precision oscillators. An IEEE subcomittee on frequency stability has recommended what has cone to be known as the "Allan variance" taken fron the set of useful variances developed, and an experimental estimation of the square root of the Allan variance is shown as the bottorn right equation in figure 4.1. This equation is very easy to implement experimentally as one simply need add up the squares of the differences between adjacent values of $y_{i}$, divide by the number of them and by two, and take the square root. One then has the quantity which the IEEE subcomittee has recommended for specification of stability in the time domaindenoted by $\sigma_{y}(\tau)$.

$$
\nabla_{y}(\tau)=\left\langle\frac{1}{2}(\bar{y}(t+\tau)-\bar{y}(t))^{2}\right\rangle^{\frac{1}{2}},
$$

where the brackets "<>" denote infinite time average. In practice this is easily estimated from a fimite data set as follows:

$$
\sigma_{y}(\tau) \cong\left[\frac{1}{2(M-1)} \sum_{i=1}^{\mu-1}\left(y_{i+1}-y_{i}\right)^{2}\right]^{\frac{1}{2}},
$$

where the $y_{i}$ are the discrete frequency averages as illus.trated in figure 4.1 .
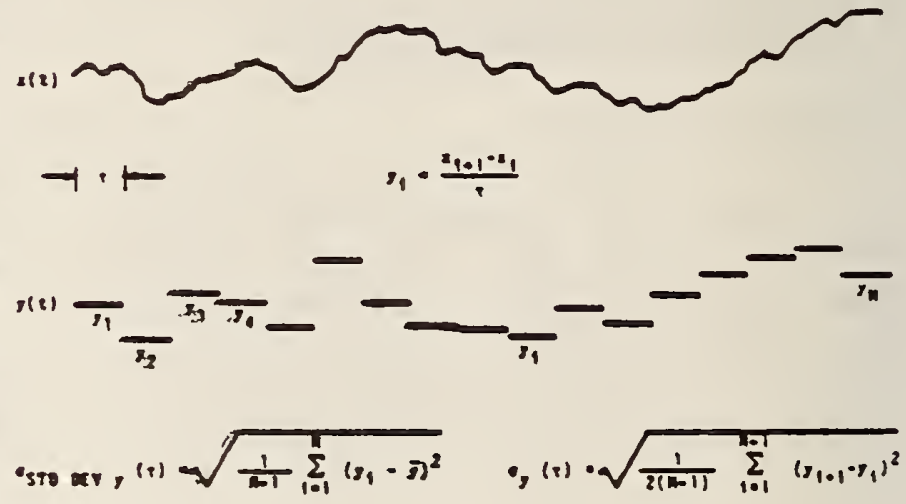

FIGURE 4.I

A simulated plot of the time fluctuations, $x(t)$ between a pair of oscillators and of the corresponding fractional frequencies calculated from the time fluctuations each averaged over a sample time $\tau$. At the bottom are the equations for the standard deviation (left) and for the time-domain measure of frequency stability as recommended by the IEEE subcomittee on frequency stability (right).

One would like to know how $\sigma_{y}(r)$ varies with the sample time, $\tau$. A simple trick that one can use that is very useful if there is no dead time, is to average the previous values for $y_{1}$ and $y_{2}$ and call that a new $y_{1}$ averaged over $2 \tau$, similarly average the previous values for $y_{3}$ and $y_{4}$ and cail that a new $y_{2}$ averaged over $2 \tau$ etc., and finally apply the same equation as before to get $\sigma_{y}(2 \tau)$. One can repeat this process for other desired integer multiples of $\tau$ and from the same data set 
be able to generate values for $\sigma_{y}(m \tau)$ as a function of $m$ from which one may be able to infer a model for the process that is characteristic of this pair of oscillators. If one has dead time in the measurements adjacent pairs cannot be averaged in an unambiguous way to simply increase the sample time. One has to retake the data for each new sample time--often a very time consuming task. This is another instance where dead time can be a problen.

How the classical variance (standard deviation squared) depends on the number of samples is shown in figure 4.2. Plotted is the ratio of the standard deviation squared for $N$ samples to the standard deviation squared for 2 samples; $\left\langle\sigma^{2}(2, \tau)\right\rangle$ is the same as the Allan variance, $\sigma_{y}{ }^{2}(\tau)$. One can see the dependence of the standard deviation upon the number of samples for various kinds of power

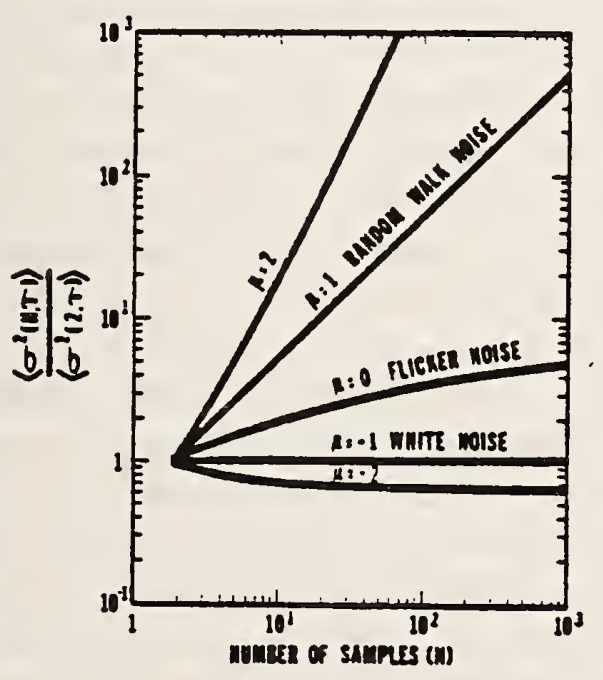

FIGURE 4.2

The ratio of the time average of the standard deviation squared for $N$ samples over the time average of a two sample standard deviation squared as a function of the number of smaples, $N$. The ratio is plotted for various power law spectral densities that commonly occur in precision oscillators. The figure illustrates one reason why the standard deviation is not a convenient measure of frequency stability; i.e. it may be very important to specify how many data points are in a data set if you use the standard deviation.

law spectral densities commonly encountered as reasonable models for many important precision oscillators. Note, $\sigma_{y}{ }^{2}(\tau)$ has the same value as the classical variance for the classical noise case (white noise FM). One main point of figure 4.2 is simply to show that with the increasing data length the standard deviation of the common classical variance is not well behaved for the kinds of noise processes that are very often encountered in most of the precision oscillators of interest.

One may combine eq (1.4) and eq (4.1), which yields an equation for $\sigma_{y}(\tau)$ in terms of the time difference or time deviation measurements.

$\sigma_{y}(\tau)=\left\langle\frac{1}{2 \tau^{2}}(x(t+2 \tau)-2 x(t+\tau)+x(t))^{2}\right\rangle^{\frac{1}{2}}$,

which for $N$ discrete time readings may be estimated as,

$\sigma_{y}(\tau) \cong\left[\frac{1}{2(N-2) \tau^{2}} \sum_{i=1}^{N-2}\left(x_{i+2}-2 x_{i+1}+x_{i}\right)^{2}\right]_{(4,4)}^{\frac{1}{2}}$,

where the $i$ denotes the number of the reading in the set of $N$ and the nominal spacing between readings is $\tau$. If there is no dead time in the data and the original data were taken with the $x$ 's spaced by $\tau_{0}$, we can piek $\tau$ in eq (4.4) to be any integer multiple of $\tau_{0}$, i.e., $\tau=m \tau_{0}$ :

$\sigma_{y}\left(M \tau_{0}\right) \cong\left[\frac{1}{2(N-2 m) m^{2} \tau_{0}^{2}} \sum_{i=1}^{N-2 m}\left(x_{i+2 m}-2 x_{i+m}+x_{i}\right)^{2}\right]^{\frac{1}{2}}$,

Equation (4.5) has some interesting consequences because of the efficient data usage in terms of the confidence of the estimate as will be explained in the next section.

EXAMPLE: Find the Allan variance, $\sigma_{y}{ }^{2}(\tau)$, of the following sequence of fractional frequency fluctuation values $y_{k}$, each value averaged over one "second.

$$
\begin{array}{ll}
y_{1}=4.36 \times 10-5 & y_{5}=4.47 \times 10-5 \\
y_{2}=4.61 \times 10-5 & y_{6}=3.96 \times 10-5 \\
y_{3}=3.19 \times 10.5 & y_{7}=4.10 \times 10-5 \\
y_{4}=4.21 \times 10-5 & y_{8}=3.08 \times 10-5
\end{array}
$$

(assume no dead-time in measurement of averages) 
Since each average of the fractional frequency fluctuation values is for one second, then the first variance calculation will be at $\tau=15$. We are given $M=8$ (eight values); therefore, the number of pairs in sequence is $M-1=7$. We have:

\begin{tabular}{|c|c|c|}
\hline $\begin{array}{l}\text { Dute rolues } \\
y_{k}(x \text { idost) }\end{array}$ & $\begin{array}{l}\text { First alferomeas } \\
\left(y_{k+1}-y_{k}\right)(x 20-5)\end{array}$ & $\begin{array}{l}\text { Firse defforence soupred } \\
\left(y_{t+1}-y_{k}\right)^{2}\left(x 0^{10}\right)\end{array}$ \\
\hline $\begin{array}{l}4.36 \\
4.62 \\
3.19 \\
4.21 \\
4.47 \\
3.98 \\
4.10 \\
3.08\end{array}$ & $\begin{array}{r}-0.25 \\
-1.42 \\
1.02 \\
0.28 \\
-0.51 \\
0.14 \\
-1.02\end{array}$ & $\begin{array}{r}0.05 \\
2.02 \\
1.04 \\
0.07 \\
0.26 \\
0.02 \\
1.04 \\
.51\end{array}$ \\
\hline
\end{tabular}

Therefore,

$$
\sigma_{y}^{2}(15)=\frac{4.51 \times 10-10}{2(7)}=3.2 \times 10-11
$$

and

$\sigma_{y}(\tau)=\left[\sigma_{y}^{2}(1 s)\right]^{\frac{1}{2}}=[3.2 \times 10-11]^{\frac{1}{2}}=5.6 \times 10-6$

Using the same data, one can calculate the variance for $\tau=2 \mathrm{~s}$ by averaging pairs of adjacent values and using these new averages as data values for the same procedure as above. For three second averages $(\tau=3 s)$ take adjacent threesomes and find their averages and proceed in a similiar manner. More data must be acquired for longer averaging times.

One sees that with large numbers of data values, it is nelpful to use a computer or programmable calculator. The confidence of the estimate on $\sigma_{y}(\tau)$ improves nowinally as the square root of the number of data values used. In this example, $M=8$ and the confidence can be expressed as being no better the $2 / \sqrt{8} \times 100 \%=35 \%$. This then is the allowable error in our estimate for the $\tau=1 \mathrm{~s}$ average. The next section shows methods of computing and improving the confidence interval. v. CONFIDENCE OF THE ESTIMATE AND OVERLAPPING SAMPLES $^{4}$

One can imagine taking three phase or time measurements of one oscillator relative to another at equally spaced intervals of time. From this phase data one can obtain two, adjacent values of average frequency. Fron these two frequency measurements, one can calculate a single sample Allan (or two-sample) variance (see fig. 5.1). Of course this variance does not have high precision or confidence since it is based on only one frequency difference.

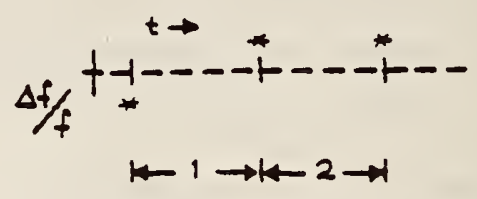

FIGURE 5.1

Statisticians have considered this problem of quantifying the variability of quantities like the Allan Variance. Conceptually, one could imagine repeating the above experiment (of taking the three phase points and calculating the Allan Variance), many times and even calculating the distribution of the values.

For the above eited experiment we know that the results are distributed like the statistician's chi-square distribution with one degree of freedom. That is, we know that for most common oscillators the first difference of the frequency is a normally distributed variable with the typical bell-shaped eurve and zero mean. However, the square of a normally distributed variable is NOT normally distributed. That is easy to see since the square is always positive and the normal curve is completely symetric and negative values are as likely as positive. The resulting distribution is called a chi-square distribution, and it has ONE "degree of freedom" since the distribution was obtained by considering the squares of individual (i.e., one independent sample), nomaily distributed variables.

In contrast, if we took five phase values, then we could calculate four consecutive frequency values, as in figure 5.2. We could then take the 


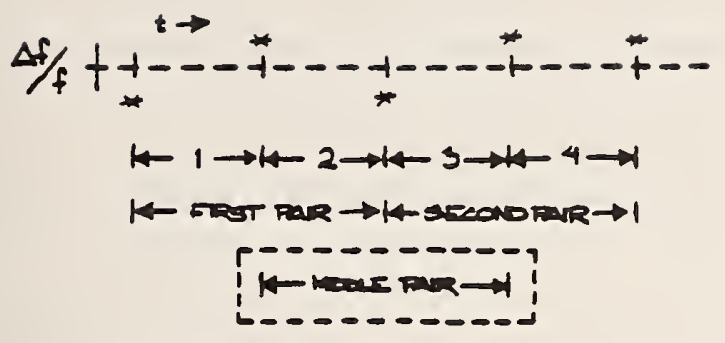

FIGURE 5.2

first pair and caiculate a sample Allan Variance, and we could calculate a second sample Allan Variance from the second pair (i.e., the third and fourth frequency measurements). The average of these two sample Allan Variances provides an improved estimate of the "true" Allan Variance, and we would expect it to have a tighter confidence interval than in the previous example. This could be expressed with the aid of the chi-square distribution with TWO degrees of freedom.

However, there is another option. We could also consider the sample Allan Variance obtained from the second and third frequency measurements. That is the middle sample variance. Now, however, we're in trouble because clearly this last sample Allan Variance is NOT independent of the other two. Indeed, it is made up of parts of each of the other two. This does NOT mean that we can't use it for improving our estimate of the "true" Allan Variance, but it does mean that we can't just assume that the new average of three sample Allan Variances is distributed as chi-square with three degrees of freedom. Indeed, we will encounter chi-square distributions with fractional degrees of freedom. And as one might expect, the number of degrees of freedom will depend upon the underlying noise type, that is, white FM, flicker FM, or whatever.

Before going on with this, it is of value to review some concepts of the chi-square distribution. Sample variances (like sample Allan Variances) are distributed according to the equation:

$$
x^{2}=\frac{(d . f \cdot) \cdot s^{2}}{\sigma^{2}}
$$

where $S^{2}$ is the sample Allan Variance, $X^{2}$ is chi-square, d.f. is the number of degrees of freedom (possibly not an integer), and $\sigma^{2}$ is the "true" Allan Variance we're all interested in knowing--but can only estimate imperfectly. Chi-square is a randon variable and its distribution has been studied extensively. For some reason, chi-square is defined so that d.f., the number of degrees of freedon, appears explicitly in eq (5.1). Still, $x^{2}$ is a (implicit) function of d.f., also.

The probability density for the chi-square distribution is given by the relation

$\rho\left(x^{2}\right)=\frac{1}{2^{d . f \cdot \Gamma\left(\frac{d . f \cdot}{2}\right)}\left(x^{2}\right)^{\frac{d . f .}{2}}-1} e^{\frac{-x^{2}}{2}}$

where $\Gamma\left(\frac{\text { d.f. }}{2}\right)$ is the gamma function, defined by the integral

$$
\Gamma(t)=\int_{0}^{\infty} x^{t-1} e^{-x} d x
$$

Chi-square distributions are useful in determining specified confidence intervals for variances and standard deviations. Here is an example. Suppose we have a sample variance $s^{2}=3.0$ and we know that this variance has 10 degrees of freedom. (Just how we can know the degrees of freedon will be discussed shortly.) Suppose also that we want to know a range around our sample value of $s^{2}=3.0$ which "probably" contains the true value, $\sigma^{2}$. The desired confidence is, say, $90 \%$. That is, $10 \%$ of the time the true value will actually fall outside of the stated bounds. The usual way to proceed is to allocate $5 \%$ to the low end and $5 \%$ to the high end for errors, leaving our $90 \%$ in the middle. This is arbitrary and a specific problem might dictate a different allocation. We now resort to tabies of the chi-square distribution and find that for 10 degrees of freedom the $5 \%$ and $95 \%$ points correspond to:

$$
x^{2}(.05)=3.94
$$

for d.f. $=10$

$x^{2}(.95)=18.3$ 
Thus, with $90 \%$ probability the calculated sample variance, $s^{2}$, satisfies the inequality:

$$
3.94 \leq \frac{(d . f \cdot) \cdot s^{2}}{\sigma^{2}} \leq 18.3
$$

and this inequality can be rearranged in the form

$$
1.64 \leq \sigma^{2} \leq 7.61
$$

or, taking square roots:

$$
1.28 \leq \sigma \leq 2.76
$$

Now someone mignt object to the form of eq (5.7) since it seems to be saying that the true sigma falls within two limits with $90 \%$ probability. Of course, this is either true or not and is not subject to a probabilistic interpretation. Actually eq (5.7) is based on the idea that the true sigma is not known and we estimate it with the square root of a sample variance, $s^{2}$. This sample variance is a random variable and is properly the subject of probability, and its value (which happened to be 3.0 in the example) will conform to eq (5.7) nine times out of ten.

Typically, the sample variance is calculated from a data sample using the relation:

$$
s^{2}=\frac{1}{N-1} \sum_{n=1}^{N}\left(x_{n}-\bar{x}\right)^{2}
$$

where it is implicitly assumed that the $x_{n}$ 's are random and uncorrelated (i.e., white) and where $\bar{x}$ is the sample mean calculated from the same data set. If all of this is true, then $\mathrm{s}^{2}$ is chi-square distributed and has $\mathrm{N}-\mathrm{I}$ degrees of freedom.

Thus, for the case of white $x_{n}$, and a conventional sample variance (i.e., eq (5.8)), the number of degrees of freedom are given by the equation:

$$
\text { d.f. }=N-1
$$

The problem of interest here is to obtain the corresponding equations for Allan Variances using overlapping estimates on various types of noise (i.e., white FM, flicker FM, etc.).

Other authors (Lesage and Audoin, and Yoshimura) have considered the question of the variance of the Allan Variances without regard to the distributions. This is, of course, a closely related problem and use will be made of their results. These authors considered a more restrictive set of overlapping estimates than will be considered here, however.

VI. MAXIMAL USE OF THE OATA AND OETERMINATION OF THE OEGREES OF FREEDOM.

\subsection{Use of Data}

Consider the case of two oscillators being compared in phase and exactly $N$ values of the phase difference are obtained. Assume that the data are taken at equally spaced intervals, $\tau_{0}$. From these $N$ phase values, one can obtain $N-I$ consecutive values of average frequency and from these one can compute $\mathrm{N}-2$ individual, sample Allan Variances (not all independent) for $\tau=\tau_{0}$. These $\mathrm{N}-2$ values can be averaged to obtain an estimate of the Allan Variance at $\tau=\tau_{0}$. The variance of this variance has been calculated by the above eited authors.

Using the same set of data, it is also possible to estimate the Allan Variances for integer multiples of the base sampling interval, $\tau=n \tau_{0}$. Now the possibilities for overlapping sample Allan Variances are even greater. For a phase data set of $N$ points one can obtain exactly $N-2 n$ sample Allan Variances for $\tau=n \tau_{0}$. Of course only a fraction of these are generally independent. Still the use of ALL of the data is well justified (see fig. 6.I).

Consider the case of an experiment extending for several weeks in duration with the aim of getting estimates of the Allan Variance for tau values equal to a week or more. As always the purpose is to estimate reliably the "true" Allan Variance as well as possible--that is, with as tight an uncertainty as possible. Thus one wants 


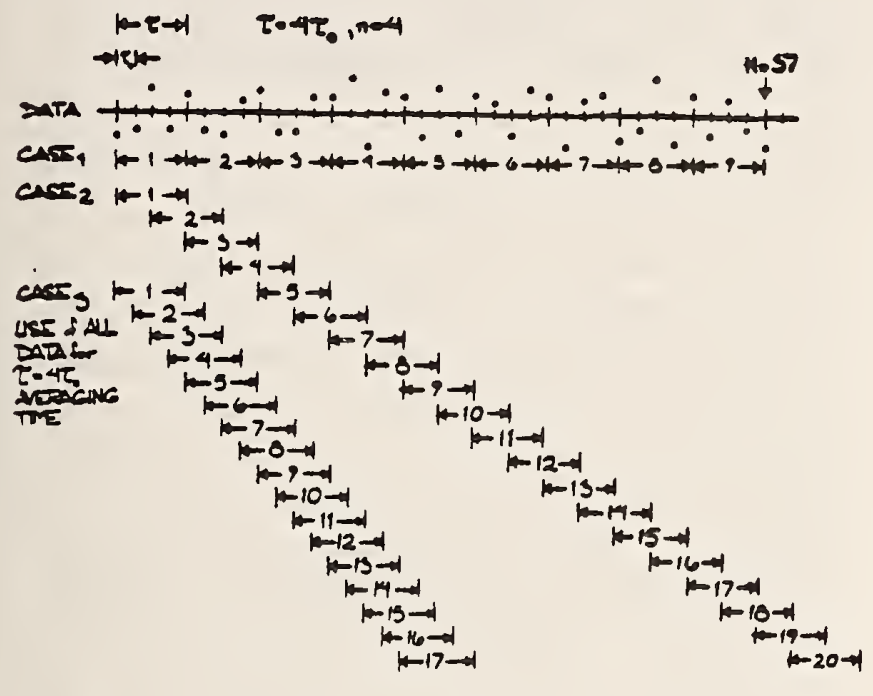

FIGURE 6.1

to use the data as efficiently as possible since obtaining more data can be very expensive. The most efficient use is to average all possible sample Allan Variances of a given tau value that one can compute from the data.

The problem comes in estimating how tight the confidence intervals really are that is, in estimating the number of degrees of freedom. Clearly, if one estimates the confidence intervals pessimistically, then more data is needed to reach a specified tolerance, and that can be expensive. The other error of over-confidence in a questionable value can be even more expensive. Ideally, one has realistic confidence estimates for the most efficient use of the data, which is the intent of this writing.

\section{2 Determining the Degrees of Freedom}

In principle, it should be possible to determine analytically the equations corresponding to eq (5.9) for all cases of interest. Unforiunately the analysis becomes quite complicated. Exact computer algorithms were devised for the cases of white phase noise, white frequency modulation and random walk FM. For the two flicker cases (i.e., flicker $F M$ and $P M$ ) a completely empirical approach was used. Due to the complexity of the computer programs, empirical fits were devised for all five noise types.
The approach used is based on three equations relating to the chi-square distribution:

$$
\begin{aligned}
& \frac{x^{2}}{(d . f .)}=\frac{s^{2}}{\sigma^{2}} \\
& E\left[x^{2}\right]=\text { d.f. } \\
& \operatorname{Var}\left[x^{2}\right]=2(d . f .)
\end{aligned}
$$

where the expression $E\left[X^{2}\right]$ means the "expectation," or average value of $x, \operatorname{Var}\left[x^{2}\right]$ is the variance of $x^{2}$, and $d . f$. is the number of degrees of freedom.

A computer was used to simulate phase data sets of some length, $N$, and then Allan Variances with $\tau=n \tau_{0}$ were calculated for all possibie samples. This "experiment" was repeated at least 1000 times using new simulated data sets of the same spectral type, and always of the same length, $N$. Since the data were simulated on a computer, the "true" Allan Variance, $\sigma^{2}$, was known for many of the noise models and could be substituted into eq (6.1). From the 1000 values of $\mathrm{s}^{2} / \sigma^{2}$, distributions and sample variances were obtained. The "experimental" distributions were compared with theoretical distributions to verify that the observed distributions truely conformed to the chi-square distribution.

The actual calculation of the degrees of freedom were made using the relation:

$$
\text { d. f. }=\frac{2\left(\sigma^{2}\right)^{2}}{\operatorname{Var}\left(s^{2}\right)}
$$

which can be deduced from eqs (6.1), (6.2), and (6.3). The $\operatorname{Var}\left(s^{2}\right)$ was estimated by the sample variance of the 1000 values of the average Allan Variances, each obtained from a phase data set of length $N$.

of course this had to be repeated for various values of $N$ and $n$, as well as for each of the five common noise types: white PM, flicker PM, white FM, flicker FM, and random walk FM. Fortunately, certain limiting values are known and these can be used as checks on the method. For example, when $(N-1) / 2=n$, only one Allan Variance is obtained from each data set and one should get about one degree of freedom for eq (6.4), which was observed 
in fact. Also for $n=1$ the "experimental" conditions correspond to those used by Lesage and Audoin, and by Yoshimura. Indeed, the method also was tested by verifying that it gave results consistent with eq (5.9) when applied to the conventional sample variance. Thus, combining eq (6.4) with the equations for the variance of the Allan Variances from Lesage and Audoin and Yoshimura, one obtains:

$\begin{array}{ll}\text { White PM } & \text { d.f. }=\frac{18(N-2)^{2}}{35 N-88} \text {, for } N \geq 4 \\ \text { Flieker PM } & \text { d.f. }=? \\ \text { White FM } & \text { d.f. }=\frac{2(N-2)^{2}}{3 N-7} \\ \text { Flicker FM } & \text { d.f. }=\frac{2(N-2)^{2}}{2.3 N-4.9} \\ \text { Random Walk FM } & \text { d.f. }=N-2\end{array}$

for $n=1$. Unfortunately, their results are not totally consistent with each other. Where inconsistency arose the value in best agreement with the "experimental" results was chosen.

The empirical equations which were fit to the "experimental" data and the known values are summarized below:

White PM d.f. $\cong \frac{(N+1)(N-2 n)}{2(N-n)}$

Flicker PM d.f. $\cong \exp \left(\ln \frac{N-1}{2 n} \ln \frac{(2 n+1)(N-1)}{4}\right)$

White FM d.f. $\cong\left[\frac{3(N-1)}{2 \pi} \cdot \frac{2(N-2)}{N}\right] \cdot \frac{4 n^{2}}{4 n^{2}+5}$

Flicker FM d.f. $\cong\left\{\begin{array}{l}\frac{2(N-2)}{2.3 N-4.9}, \text { for } n=1 \\ \frac{5 N^{2}}{4 n(N+3 n)}, \text { for } n \geq 2\end{array}\right.$

Randon Walk FM d.f. $\equiv \frac{N-2}{n} \quad \frac{(N-1)^{2}-3 n(N-1)+4 n^{2}}{(N-3)^{2}}$

The figures in Appendix I demonstrate the fit to the "experimental" data.
It is appropriate to give some estimate of just how well these empirical equations approach the "true" values. The equations have approximately (a few percent) the correct assymptotic behavior at $n=I$ and $n=(N-I) / 2$. In between, the values were tested (using the simulation results) over the range of $N=5$ to $N=1025$ for $n=1$ to $n=(N-1) / 2$ changing by octaves. In general, the fit was good to within a few percent. We must acknowledge that distributional problems with the random number generators can cause problems, although there were several known values which should have revealed these problems if they are present. Also for three of the noise types the exact number of degrees of freedom were calculated for many values of $N$ and $n$ and compared with the "Monte Carlo" calculations. The resuits were all very good.

Appendix I presents the data in graphical form. All values are thought to be accurate to within one percent or better for the cases of white PM, white $F M$, and random walk FM. A larger tolerence should be allowed for the flicker cases.

\section{EXAMPLE OF TIME-DOMAIN SIGNAL PROCESSING AND ANALYSIS \\ We will analyze in some detail a commercial} portable clock, Serial No. 102. This cesium was measured against another comnercial cesium whose stability was well documented and verified to be better than the one under test. Plotted in figure 7.1 are the residual time deviations after removing FTS PC VS CSLOH

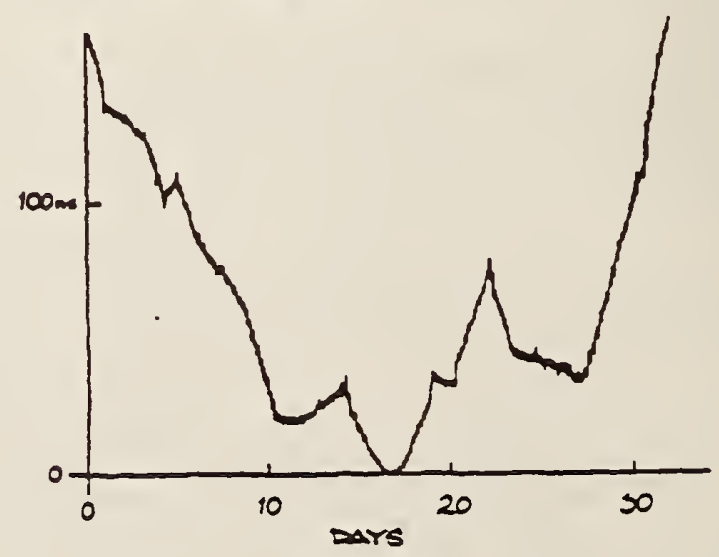

FIGURE 7.1 
a mean frequency of 4.01 parts in $10^{13}$. Applying the methods described in section IV and section $V$, we generated the $\sigma_{y}(\tau)$ diagram shown in figure 7. 2.

FIS FC VS CSLOT

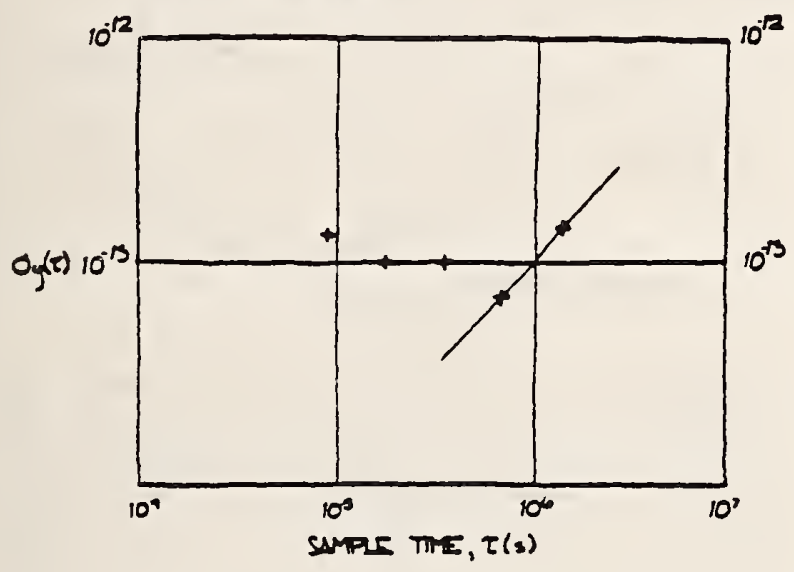

\section{FIGURE 7.2}

One observes that the last two points are proportional to $\tau^{+1}$ and one is suspicious of a significant frequency drift.

If one calculates the drift knowing that $\sigma_{y}(r)$ is equal to the drift times $\frac{r}{\sqrt{2}}$ a drift of $1.22 \times 10^{-14}$ per day is obtained. A linear least squares to the frequency was removed and sections IV and $V$ were applied again. The linear least squares fit showed a drift of $1.23 \times 10-14$ per day, which is in excellent agreement with the previous calculated value obtained from $\sigma_{y}(r)$. Typically, the linear least squares will give a much better estimate of the linear frequency drift than will the estimate from $\sigma_{y}(\tau)$ being propor tional to $\mathrm{r}^{+1}$.

Figure 7.3 gives the plot of the time residwals after removing the linear least squares and figure 7.4 is the corresponding $\sigma_{y}(\tau)$ vs. $\tau$ diagram. From the 33 days of data, we have used the 90\% confidence interval to bracket the stability estimates and one sees a reasonable fit corresponding to white noise frequency modulation at a level of $4.4 \times 10^{-11} \tau^{-\frac{1}{2}}$. This seemed excessive
FTS PC vs cs6or

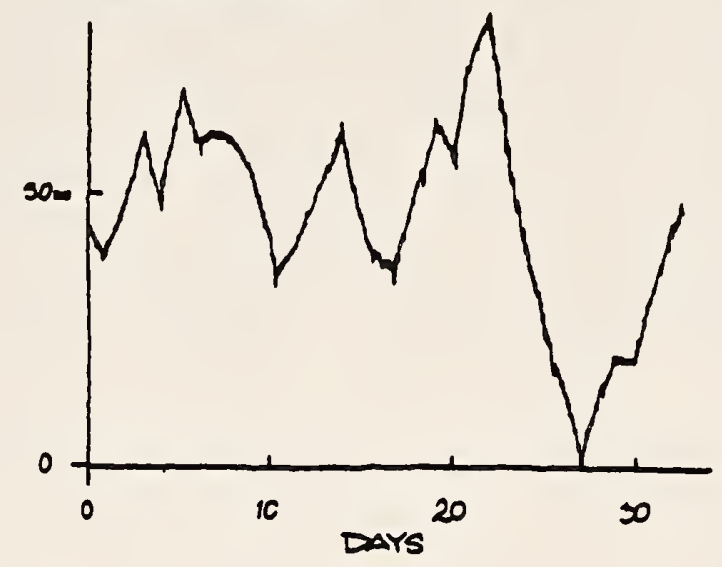

FIGURE 7.3

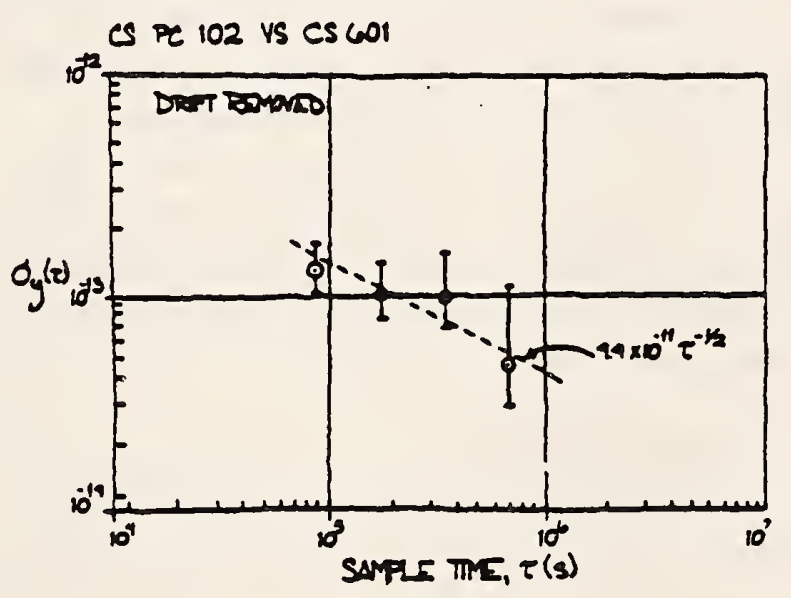

FIGURE 7.4

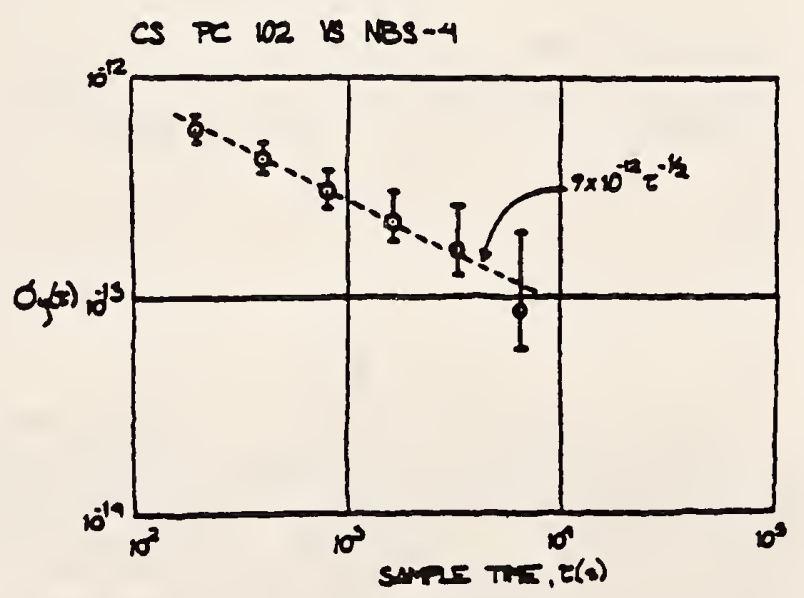

FIGURE 7.5 
in terms of the typical performance of this particular cesium and in as much as we were doing some other testing within the environment, such as working on power supplies and charging and discharging batteries, we did some later tests. Figure 7.5 is a plot of $\sigma_{y}(\tau)$ after the standard had been left alone in a quiet environment and had been allowed to age for about a week. One observes that the white noise frequency modulation level is more than a factor of 4 improved over the previous data. This led us to do some studies on the effects of the power supply on the cesiun frequency as one is charging and discharging batteries, which proved to be significant. One notices in figure 7.4 that the $\sigma_{y}(\tau)$ values plotted are consistent within the error bars with flicker noise frequency modulation. This is more typical of the kind of noise one would expect due to such environmental perturbations as discussed above.

Careful time- and/or frequency-domain analyses can lead to significant insights into problems and their solutions and is highly recommended by the authors. The frequency-domain techniques will be next approached.

\section{SPECTRUM ANALYSIS}

Another method of characterizing the noise in a signal source is by means of spectrum analysis. To understand this approach, let's examine the waveform shown in figure 8.1 .

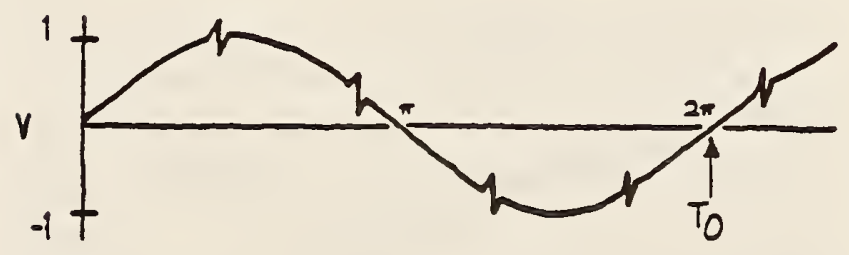

FIGURE 8.1

Here we have a sine wave which is perturbed for short instances by noise. Some loosely refer to these types of noises as "glitches". The waveform has a nominal frequency over one cycle which we'll call " $v_{0} "\left(v_{0}=\frac{1}{\gamma}\right)$. At times, noise causes the instantaneous frequency to differ markedly from the nominal frequency. If a pure sine wave signal of frequency $v_{0}$ is subtracted from this waveform, the renainder is the sum of the noise components. These components are of a variety of frequencies and the sum of their amplitudes is nearly zero except for the intervals during each glitch when their amplitudes momentarily reinforce each other. This is shown graphically in figure 8.2.

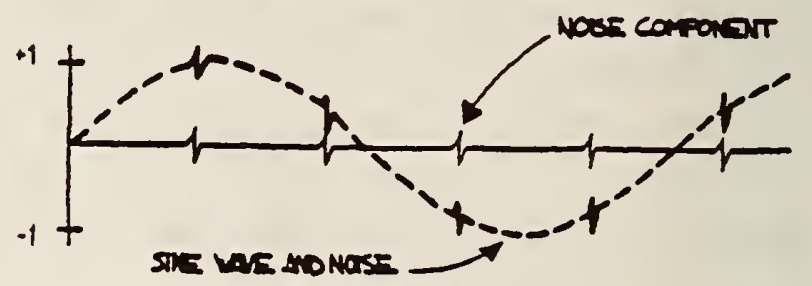

FIGURE 8.2

One can plot a graph showing rms power vs. frequency for a given signal. This kind of plot is called the power spectrum. For the waveform of figure 8.1 the power spectrun will have a high value at $v_{0}$ and will have lower values for the signals produced by the glitches. Closer analysis reveals that there is a recognizable, somewhat constant repetition rate associated with the glitches. In fact, we can deduce that there is a significant anount of power in another signal whose period is the period of the glitches as shown in figure 8.2. Let's call the frequency of the glitches $u_{s}$. Since this is the case, we will observe a noticeable anount of power in the spectrum at $v_{s}$ with an amplitude which is related to the characteristics of the glitches. The power spectrum shown in figure 8.3 has this feature. A predominant $v_{s}$ component has been depicted, but other harmonics also exist.

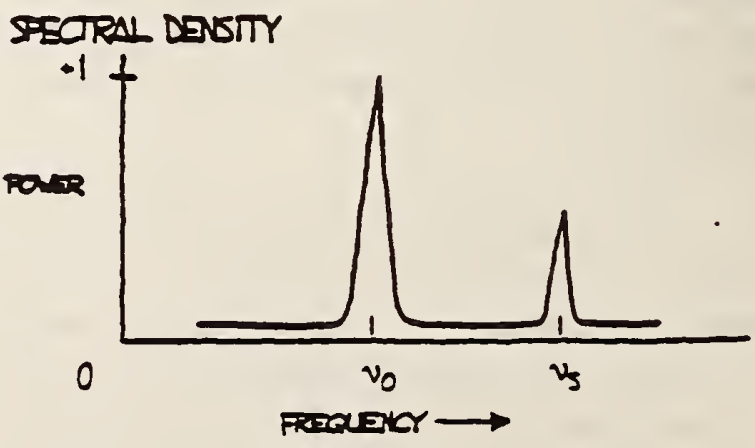

FIGURE 8.3 
Some noise will cause the instantaneous frequency to "jitter" around $v_{0}$, with probability of being higher or lower than $v_{0}$. We thus usually find a "pedestal" associated with $v_{0}$ as shown in figure 8.4 .

\section{SPECTRAL DENSTY}

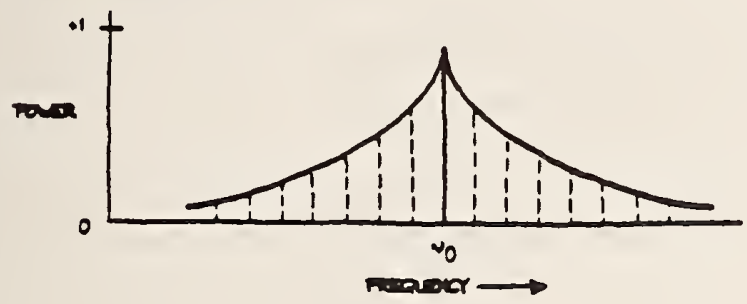

FIGURE 8.4

The process of breaking down a signal into all of its various components of frequency is called Fourier exoansion (see sec. $X$ ). In other words, the addition of all the frequency components, called Fourier frequency components, produces the original signal. The value of a Fourier frequency is the difference between the frequency component and the fundamental frequency. The power spectrum can be normalized to unity such that the total area under the curve equals one. The power spectrum normalized in this way is the power spectral density.

The power spectrum, often called the RF spectrum, of $V(t)$ is very useful in many applications. Unfortunately, if one is given the RF spectrum, it is impossible to detemine whether the power at different Fourier frequencies is a result of amplitude fluctuations " $\varepsilon(t)$ " or phase fluctuations " $\phi(t)$. The RF spectrum can be separ ated into two independent spectra, one being the spectral density of " $\varepsilon(t)$ " often called the AM power spectral density and the other being the spectral density of " $\phi(t)$ ".

For the purposes here, the phase-fluctuation components are the ones of interest. The spectral density of phase fluctuations is denoted by $S_{\phi}(f)$ where " $f$ " is Fourier frequency. For the frequently encountered case where the AM power spectral density is negligibly small and the total modulation of the phase fluctuations is small (mean-square value is much less than one $\operatorname{rad}^{2}$ ), the RF spectrum has approximately the same shape as the phase spectral density. However, a main difference in the representation is that the RF spectrum includes the fundamental signal (carrier), and the phase spectral density does not. Another major difference is that the RF spectrum is a power spectral density and is measured in units of watts/hertz. The phase spectral density involves no "power" measurement of the electrical signal. The units are radians $2 /$ hertz. It is tempting to think of $S_{\phi}(f)$ as a "power" spectral density because in practice it is measured by passing $V(t)$ through a phase detector and measuring the detector's output power spectrum. The measurement technique makes use of the relation that for small deviations ( $\delta \phi \ll 1$ radian),

$$
S_{\phi}(f)=\left(\frac{v_{m s}(f)}{v_{s}}\right)^{2}
$$

where $V_{r m s}(f)$ is the root-mean-square noise voltage per $\sqrt{H Z}$ at a Fourier frequency " $f$ ", and $V_{s}$ is the sensitivity (volts per radian) at the phase quadrature output of a phase detector which is comparing the two oscillators. In the next section, we will look at a scheme for directly measuring $S_{\phi}(f)$.

One question we might ask is, "How do frequency changes relate to phase fluctuations?" After all it's the frequency stability of an oscillator that is a major consideration in many applications. The frequency is equal to a rate of change in the phase of a sine wave. This tells us that fluctuations in an oscillator's output frequency are related to phase fluctuations since we must change the rate of " $\phi(t)$ " to accomplish a shift in " $v(t)$ ", the frequency at time $t$. A rate

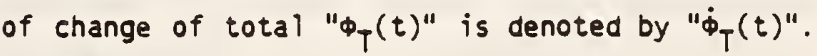
We have then

$$
2 \pi v(t)=\dot{\phi}_{T}(t)
$$

The dot denotes the mathematical operation of differentiation on the function $\Phi_{T}$ with respect to its independent variable t. ${ }^{\star}$ From eq (8.4)

* As an analogy, the same operation relates the position of an object with its velocity. 
and eq (1.1) we get

$$
2 \pi(t)=\dot{\phi}_{T}(t)=2 \pi v_{0}+\dot{\phi}(t)
$$

Rearranging, we have

$$
2 \pi v(t)-2 \pi v_{0}=\dot{\phi}(t)
$$

or

$$
v(t)-v_{0}=\frac{\dot{\phi}(t)}{2 \pi}
$$

The quantity $v(t)$ - $v_{0}$ can be more conveniently denoted as $\delta v(t)$, a change in frequency at time $t$. Equation (8.5) tells us that if. we differentiate the phase fiuctuations $\phi(t)$ and divide by $2 \pi$, we will have calculated the frequency fluctuation $\delta v(t)$. Rather than specifying a frequency fluctuation in terms of shift in frequency, it is useful to denote $\delta v(t)$ with respect to the nominal frequency $v_{0}$. The quantity $\frac{\delta v(t)}{v_{0}}$ is called the fractional frequency fluctuation at time $t$ and is signified by the variable $y(t)$. We have

$$
y(t)=\frac{\delta v(t)}{v_{0}}=\frac{\dot{\phi}(t)}{2 \pi v_{0}}
$$

The fractional frequency fluctuation $y(t)$ is a dimensionless quantity. When talking about frequency stability, its appropriateness becones clearer if we consider the following example. Suppose in two oseillators $\delta v(t)$ is consistently equal to + I Hz and we have sampled this value for many times $t$. Are the two oscillators equal in their ability to produce their desired output frequencies? Not if one oscillator is operating at $10 \mathrm{~Hz}$ and the other at $10 \mathrm{MHz}$. In one case, the average value of the fractional frequency fluetuation is $1 / 10$, and in the second case is $1 / 10,000,000$ or $1 \times 10^{-7}$. The $10 \mathrm{MHz}$ oscillator is then more precise. If frequencies are multiplied or divided using ideal electronics, the fractional stability is not changed.

In the frequency domain, we can measure the spectrum of frequency fluctuations $y(t)$. The

** Some international recommendations replace

"fractional" by "normalized". spectral density of frequency fluctuations is denoted by $S_{y}(f)$ and is obtained by passing the signal from an oscillator through an ideal FM detector and performing spectral analysis on the resultant output voltage. $S_{y}(f)$ has dimensions of (fractional frequency) $)^{2} / \mathrm{Hz}$ or $\mathrm{Hz}^{-1}$. Differentiation of $\phi(t)$ corresponds to multiplication by $\frac{f}{v_{0}}$ in terms of spectral densities. With further calculation, one can derive that

$$
s_{y}(f)=\left(\frac{f}{v_{0}}\right)^{2} S_{\phi}(f)
$$

We will address ourselves primarily to $S_{\phi}(f)$, that is, the spectral density of phase fluctuations. For noise-measurement purposes, $S_{\phi}(f)$ can be measured with a straightforward, easily duplicated equipment set-up. Whether one measures phase or frequency spectral densities is of minor importance since they bear a direct relationship. It is important, however, to make the distinction and to use eq ( 8.7 ) if necessary.

\section{1 The Loose Phase-Locked Loop}

Section I, 1.1, C described a method of measuring phase fluctuations between two phaselocked oscillators. Now we will detail the procedure for measuring $S_{\phi}(f)$.

Suppose we have a noisy oscillator. We wish to measure the oscillator's phase fluctuations relative to nominal phase. One can do this by phaselocking another oscillator (called the reference oscillator) to the test oscillator and mixing the two oscillator signals $90^{\circ}$ out of phase (phase quadrature). This is shown schematically in figure 8.9. The two oscillators are at the same frequency in long term as guaranteed by the phase-lock loop (PLL). A low-pass filter (to filter the R.F. sum component) is used after the mixer since the difference (baseband) signal is the one of interest. By holding the two signals at a relative phase difference of $90^{\circ}$, short-term phase fluctuations between the test and reference oscillators will appear as voltage fluctuations out of the mixer. 


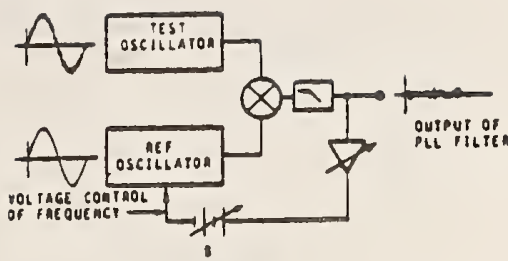

FIGURE 8.9

With a PLL, if we can make the servo time constant very long, then the PLL bandwidth as a filter will be small. This may be done by lowering the gain $A_{v}$ of the loop amplifier. We want to translate the phase modulation spectrum to baseband spectrum so that it is easily measured on a low frequency spectrum analyzer. With a PLL filter, we must keep in mind that the reference oscillator snould be as good or better than the test oscillator. This is because the output of the PLL represents the noise from both oscillators, and if not properly chosen, the reference can have noise masking the noise from the test oscillator. often, the reference and test oscillators are of the same type and have, therefore, approximately the same noise. We can acquire a meaningful measurement by noting that the noise we measure is from two oscillators. Many times a good approximation is to assume that the noise power is twice that which is associated with one oscillator. $S_{\phi}(f)$ is general notation depicting spectral density on a per hertz basis. A PLL filter output necessarily yields noise from two oscillators.

The output of the PLL filter at fourier frequencies above the loop bandwidth is a voltage representing phase fluctuations between reference and test oscillator. It is necessary to make the time-constant of the loop long compared with the inverse of the lowest fourier frequency we wish to measure. That is, $\tau_{c}>\frac{1}{2 \pi f(\text { lowest })}$. This means that if we want to measure $S_{\phi}(f)$ down to $1 \mathrm{~Hz}$, the loop time-constant must be greater than $\frac{1}{2 \pi}$ seconds. One can measure the time-constant by perturbing the loop (momentarily disconnecting the battery is convenient) and noting the time it takes for the control voltage to reach $70 \%$ of its final value. The signal from the mixer can then be inserted into a spectrum analyzer. A preamp may be necessary before the spectrum analyzer.

\footnotetext{
- See Appendix Note \# 3
}

The analyzer determines the mean square volts that pass through the analyzer's bandwidth centered around a pre-chosen Fourier frequency $f$. It is desireable to normalize results to a $1 \mathrm{~Hz}$ bandwidth. Assuming white phase noise (white PM), this can be done by dividing the mean square voltage by the analyzer bandwidth in $\mathrm{Hz}$. One may have to approximate for other noise processes. (The phase noise sideband levels will usually be indicated in ms volts-per-root-Hertz on most analyzers.)

\subsection{Equipment for Frequency Domain Stability Measurements}

(1) Low-noise mixer

This should be a high quality, doublebalanced type, but single-ended types may be used. The oscillators should have well-buffered outputs to be able to isolate the coupling between the two input RF ports of the mixer. Results ** that are too good may be obtained if the two oscillators couple tightly via signal injection through the input ports. We want the PLL to control locking. One should read the specifications in order to prevent exceeding the maximum allowable input power to the mixer. It is best to operate near the maximum for best signal-to-noise out of the IF port of the mixer and, in some cases, it is possible to drive the mixer into saturation without burning out the device.

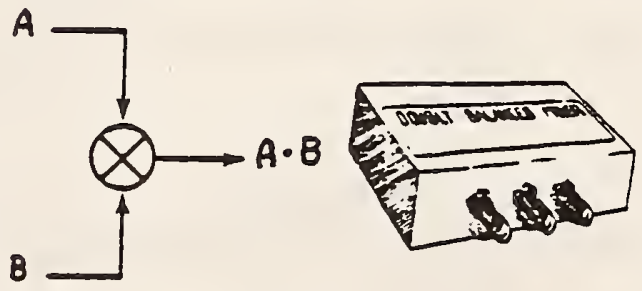

FIGURE 8.10

(2) Lownoise $O C$ amplifier

The amount of gain $A_{v}$ needed in the loop amplifier will depend on the amplitude of the mixer output and the degree of

* See Appendix Note \# 4 
varactor control in the reference oscillator. We may need only a small amount of gain to acquire lock. On the other hand, it may be necessary to add as much as 80 dB of gain. Good low-noise DC amplifiers are available fron a number of sources, and with cascading stages of amplification, each contributing noise, it will be the noise of the first stage which will add most significantly to the noise being measured. If a suitable low-noise first-stage amplifier is not readily available, a schenatic of an amplifier with $40 \mathrm{~d} 8$ of gain is shown in figure 8.11 which will serve nicely for the first stage. Amplifiers with very low equivalent input noise performance are also available from many manufacturers. The response of the amplifier should be flat from $O C$ to the highest Fourier frequency one wishes to measure. The loop time-constant is inversely related to the gain $A_{v}$ and the determination of $A_{v}$ is best made by experimentation knowing that $\tau_{c}<\frac{1}{2 \pi f(\text { lowest })}$.

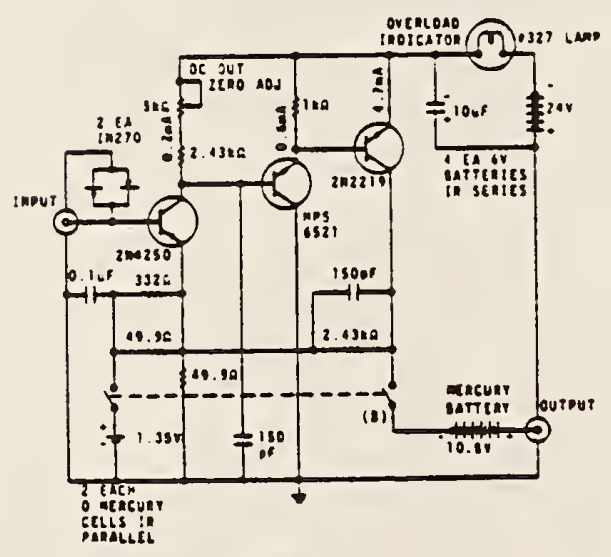

Lo nols MoLIFIER

FIGURE 8.1I

(3) Voltage-controlled reference quartz

oscillator

This oscillator should be a good one with specifications available on its frequency domain stability. The reference should be no worse than the test oscillator. The varactor control should be sufficient to maintain phase-lock of the reference. In general, low quality test oscillators may have varactor control of as much as $I \times 10^{-6}$ fractional frequency change per volt. Some provision should be available on the reference oscillator for tuning the mean frequency over a frequency range that will enable phase-lock. Many factors enter into the choice of the reference oscillator, and often it is convenient to simply use two test oscillators phase-locked together. In this way, one can assume that the noise out of the PLL filter is no worse than $3 \mathrm{~dB}$ greater than the noise from each oscillator. If it is uncertain that both oscillators are contributing approximately equal noise, then one should perform measurements on three oscillators taking two at a time. The noisier-than-average oscillator will reveal itself.

\section{(4) Spectrum analyzer}

The signal analyzer typically should be capable of measuring the noise in $\mathrm{rms}$ volts in a narrow bandwidth from near $1 \mathrm{~Hz}$ to the highest Fourier frequency of interest. This may be $50 \mathrm{kHz}$ for carrier frequencies of $10 \mathrm{MHz}$ or lower. For voltage measuring analyzers, it is typical to use units of "volts per $\sqrt{H z}$ ". The spectrum analyzer and any associated input anolifier will exhibit high-frequency rolloff. The Fourier frequency at which the voltage has dropped by $3 \mathrm{~dB}$ is the measurement system bandwidth ' $h$, or $\omega_{h}=2 \pi f_{h}$. This can be measured directly with a variable signal generator.

Section $x$ describes how analysis can be performed using a discrete fourier transform analyser. Expanding digital technology has made the use of fast-fourier transform analysis affordable and compact. 
Rather than measure the spectral density of phase fluctuations between two oscillators, it is possible to measure the phase fluctuations introduced by a device such as an active filter or amplifier. Only a slight modification of the existing PLL filter equipment set up is needed. The scheme is shown in figure 8.12.

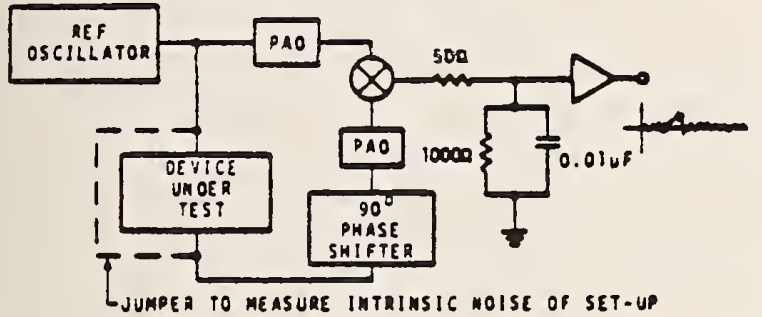

FIGURE 8.12

Figure 8.12 is a differential phase noise measurement set-up. The output of the reference oscillator is split so that part of the signal passes through the device under test. We want the two signals going to the mixer to be $90^{\circ}$ out of phase, thus, phase fluctuations between the two input ports cause voltage fluctuations at the output. The voltage fluctuations then can be measured at various fourier frequencies on a spectrum analyzer.

To estimate the noise inherent in the test set-up, one can in principle bypass the device under test and compensate for any change in amplitude and phase at the mixer. The PLL filter technique must be converted to a differential phase noise technique in order to measure inherent test equipment noise. It is a good practice to measure the system noise before proceeding to measurement of device noise.

A frequency domain measurement set-up is shown schematically in figure 8.13. The component

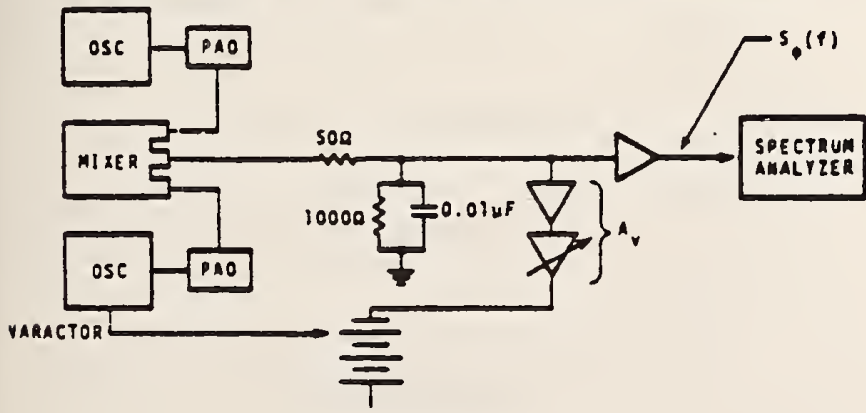

FIGURE 8.13 values for the low-pass filter out of the mixer are suitable for oscillators operating at around $5 \mathrm{MHz}$.

The active gain element $\left(a_{v}\right)$ of the loop is a OC amplifier with flat frequency response. One may replace this element by an integrator to achieve high gain near OC and hence, maintain better lock of the reference oscillator in long term. Otherwise long-term drift between the reference and test oscillators might require manual re-adjustment of the frequency of one or the other oscillator. 5

\subsection{Procedure and Example}

At the input to the spectrum analyzer, the voltage varies as the phase fluctuations in shortterm

$$
S_{\phi}(f)=\left(\frac{V_{\mathrm{rms}}(f)}{V_{s}}\right)^{2}
$$

$V_{s}$ is the phase sensitivity of the mixer in volts per radian. Using the previously described equipment set-up, $V_{s}$ can be measured by disconnecting the feedback loop to the varactor of the reference oscillator. The peak voltage swing is equal to $V_{s}$ in units of volts/rad if the resultant beat note is a sine wave. This may not be the case for state-of-the-art $S_{\phi}(f)$ measurements where one must drive the mixer very hard to achieve low mixer noise levels. Hence, the output will not be a sine wave, and the volts/rad sensitivity must be estimated by the slew-rate (through zero volts) of the resultant square-wave out of the mixer/amplifier.

The value for the measured $S_{\phi}(f)$ in decibels is given by:

$S_{\phi}(f)=20 \log$

$$
\frac{V_{\text {rms }} \text { Voltage at } f}{V_{s} \text { fult-scale } \Phi-d e t e c t o r \text { voltage }}
$$

EXAMPLE: Given a PLL with two oscillators such that, at the mixer output:

$$
v_{s}=1 \mathrm{rolt} / \mathrm{rad}
$$

See Appendix Note \# 6 
$V_{\text {mins }}(45 \mathrm{~Hz})=100 \mathrm{nV}$ per root hertz solve for $S_{\phi}(45 \mathrm{~Hz})$.

$$
\begin{aligned}
S_{\phi}(45 \mathrm{~Hz}) & =\left(\frac{100 \mathrm{nV}\left(\mathrm{Hz}^{-\frac{1}{z}}\right)}{1 \mathrm{~V} / \mathrm{rad}}\right)^{2}=\left(\frac{10-7}{1}\right)^{2} \mathrm{rad}^{2} \mathrm{~Hz}-1 \\
& =10-14 \frac{\mathrm{rad}^{2}}{\mathrm{~Hz}}
\end{aligned}
$$

In decibels,

$$
\begin{aligned}
S_{\phi}(45 \mathrm{~Hz}) & =20 \log \frac{100 \mathrm{nV}}{1 \mathrm{~V}}=20 \log \frac{10-7}{10^{\circ}} \\
& =20(-7)=-140 \text { d8 at } 45 \mathrm{~Hz}
\end{aligned}
$$

In the example, note that the mean frequency of the oscillators in the PLL was not essential to computing $S_{\phi}(f)$. However, in the application of $S_{\phi}(f)$, the mean frequency $\nu_{0}$ is necessary information. Along with an $S_{\phi}(f)$, one should always attach $v_{0}$. In the example above $\nu_{0}=5 \mathrm{MHz}$, so we have

$$
\mathrm{S}_{\phi}(45 \mathrm{~Hz})=10-14 \frac{\mathrm{rad}^{2}}{\mathrm{~Hz}}, v_{0}=5 \mathrm{MHz} \text {. }
$$

From eq (8.7), $S_{y}(f)$ can be computed as

$$
\begin{aligned}
& S_{y}(45 \mathrm{~Hz})=\left(\frac{45}{5 \times 10^{6}}\right)^{2} 10^{-14} \frac{\mathrm{rad}^{2}}{\mathrm{~Hz}} \\
& S_{y}(45 \mathrm{~Hz})=8.1 \times 10^{-25} \mathrm{~Hz}^{-1}, v_{0}=5 \mathrm{MHz} .
\end{aligned}
$$

\section{POWER-LAW NOISE PROCESSES}

Power-law noise processes are models of precision oscillator noise that produce a particular slope on a spectral density plot. We often classify these noise processes into one of five categories. For plots of $S_{\phi}(f)$, they are:

1. Random walk FM (random walk of frequency), $S_{\phi}$ plot goes down as $1 / f^{4}$.

2. Flicker FM (flicker of frequency), $S_{\phi}$ plot goes down as $1 / f^{3}$.

3. White FM (white of frequency), $S_{\phi}$ plot goes down as $1 / f^{2}$.

4. Flicker PM (flicker of phase), $S_{\phi}$ plot goes down as $1 / f$.

5. White PM (white of phase), $S_{\phi}$ plot is flat.
Power law noise processes are characterized by their functional dependence on Fourier frequency. Equation 8.7 relates $S_{\phi}(f)$ to $S_{y}(f)$, the spectral density of frequency fluctuations. Translation of $S_{y}(f)$ to time-domain data $\sigma_{y}(\tau)$ for the five mode 1 noise processes is covered later in section XI.

The spectral density plot of a typical oscillator's output usually is a combination of different power-law noise processes. It is very useful and meaningful to categorize the noise processes. The first job in evaluating a spectral density plot is to determine which type of noise exists for a particular range of Fourier frequencies. It is possible to have all five noise processes being generated from a single oscillator, but, in general, oniy two or three noise processes are dominant. Figure 9.1 is a graph of $S_{\phi}(f)$ showing the five noise processes on a log-log scale. Figure 9.2 shows the spectral density of phase fluctuations for a typical high-quality oscillator.

SPECTRA DEISITY OF PHASE

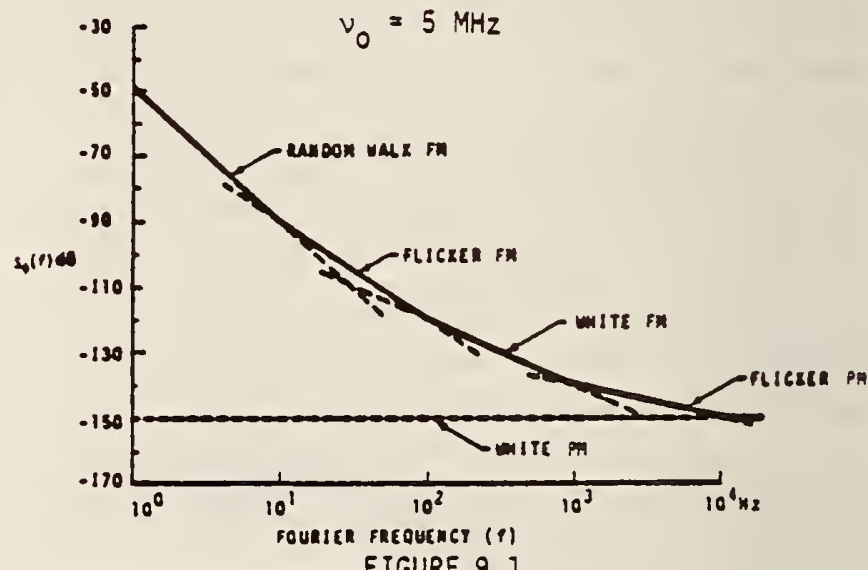

SPECTRAL DEISITY of PMASE

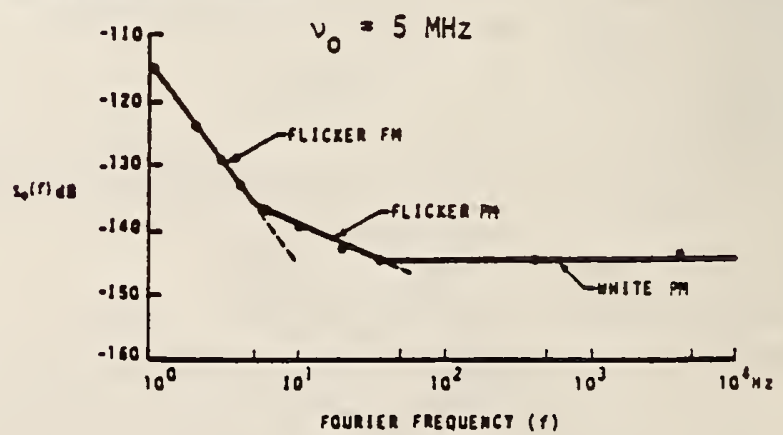

FIGURE 9.2 


\section{x. PITFALLS IN OIGITIZING THE OATA}

The advent and prolific use of digital computers has changed the manner in which processing of analog signals takes place if a computer is used. This section addresses the most common problems in such aralyses.

\subsection{Oiscrete-Continuous Processes}

oigital processing implies that data must be presented to a computer or other processor as an array of numbers whether in a batch or in a time series. If the data are not already in this form (it usually is not when considering frequency stability measurements), then it is necessary to transform to this format by digitizing. Usually, the signal available for analysis is a voltage which varies with frequency or phase difference between two oscillators.

\subsection{Oigitizing the Data}

Oigitizing the data is the process of converting a continuous waveform into discrete numbers. The process is completed in real time using an analog-to-digital converter ( $A D C$ ). Three considerations in the $A D C$ are of importance here:
1. Conversion time
2. Resolution (quantization uncertainty)
3. Linearity

An $A D C$ "looks at" an incoming waveform at equispaced intervals of time $T$. Ideally, the output of the $A D C$ is the waveform (denoted by $y(t)$ ) multiplied by a series of infinitely narrow sampling intervals of unit height as in figure 10.1. We have at $t=T$

$$
y_{I}(t)=y(t) \delta(t-T)=y(T) \delta(t-T)
$$

where $\delta(t-T)$ is a delta function. If $y(t)$ is continuous at $t=n T$ and $n=0, \pm 1, \pm 2, \ldots$, then

$$
\begin{aligned}
y_{\ell}(t) & =\sum_{n=-\infty}^{\infty} y(n T) \delta(t-n T) \\
L & =\text { integer }
\end{aligned}
$$

The delta function respresentation of a sampled waveform eq (10.2) is useful when a subsequent continuous integration is performed using it. 6

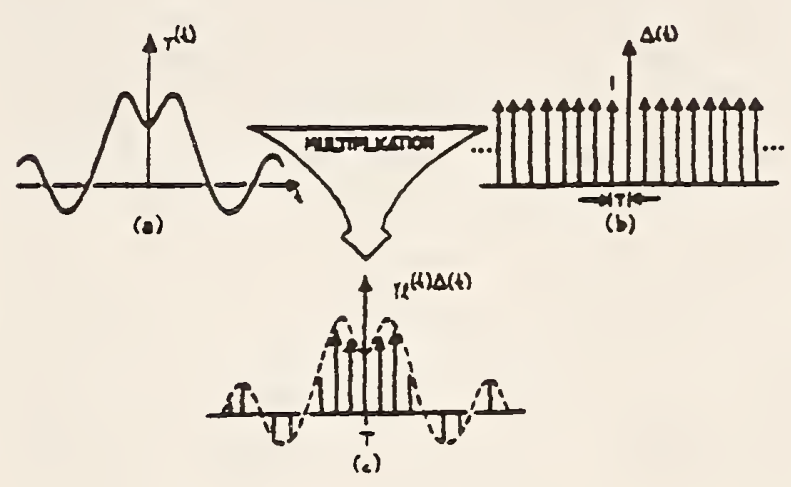

FIGURE 10.1

In $A D C$ 's, the input signal is sampled during an aperture time and held for conversion to a digital number, usually binary. Sampling and processing takes time which is specified as the conversion time. This is the total time required for a complete measurement at one sample to achieve a given level of accuracy. If $y_{L}(t)$ is the ideal discrete-time representation of continuous process $y(t)$, then the $A D C$ output denoted by $y_{L}^{\prime}(t)$ is:

$$
y_{L}^{\prime}=y_{L-d} \pm \varepsilon\left(\frac{d y}{d t}\right)
$$

where " $d$ " is the conversion time and $\varepsilon$. $\frac{d y}{d t}$ the accuracy tolerance at " $d$ " as a function of rate-ofchange in $y(t)$. In general a trade-off exists between $d$ and $\varepsilon$. For example, for a commonly available, high-quality 10 -bit $A O C$, a conversion time of $d=10 \mu s$ yields a maximum error of $3 \%$. Whereas given a $30 \mu s$ conversion time, we can obtain 0. $1 \%$ maximum error.

The error due to conversion time "d" is many times negligible since processing in digital filters and spectrum analysis takes place after the converter. Conversion time delay can be of critical concern, however, where real-time processing at speeds of the order of " $d$ " become important 
such as in digital servo loops where corrections are needed for fast changing errors.

A portion of the conversion-time error is a function of the rate of change $\frac{d y}{d t}$ of the process if the sample-and-hold portion of the $A D C$ relies on the charging of a capacitor during an aperture time. This is true because the charge cycle will have a finite time-constant and because of aperture time uncertainty. For example, if the time-constant is $0.1 \mathrm{~ns}$ (given by say a $0.1 \Omega$ source resistance charging a $0.001 \mu f d$ capacitor), then a $0.1 \%$ nominal error will exist for slope $\frac{\Delta y}{\Delta t}=$ IV/us due to charging. With good design, this error can be reduced. The sampling circuit (before charge) is usually the dominant source of error and logic gate-delay jitter creates an aperture time uncertainty. The jitter typicaliy is between 2-5 ns which means an applied signal slewing at, say, $1 \mathrm{~V} / \mathrm{\mu s}$ produces an uncertainty of 2-5 $\mathrm{mV}$. Since $\varepsilon \cdot \frac{d y}{d t}$ is directly proportional to signal slewing rate, it can be anticipated that nigh-level, high-frequency components of $y(t)$ will have the greatest error in conversion. For typical $A D C^{\prime} s$, less than $0.1 \%$ error can be achieved by nolding $\frac{\Delta y}{\Delta t}$ to less than $0.2 \mathrm{~V} / \mathrm{\mu s}$.

The continuous process $y(t)$ is partitioned into $2^{n}$ discrete ranges for $n$-bit conversion. All analog values within a given range are represented by the same digital code, usually assigned to the nominal midrange value. There is, therefore, an inherent quantization uncertainty of $\pm \frac{1}{2}$ leastsignificant bit (LSB), In addition to other conversion errors. For example, a 10-bit $A D C$ has a total of 1024 discrete ranges with a lowest order bit then representing about $0.1 \%$ of full scale and quantization uncertainty of $\pm 0.05 \%$.

We define the dynamic range of a digital system as the ratio between the maximum allowable value of the process (prior to any overflow condition) and the minimum discernable value. The dynamic range when digitizing the data is set by the quantizing uncertainty, or resolution, and is the ratio of $2^{n}$ to LSB. (If additive noise makes coding ambiguous to the $\frac{1}{2}$ LSB level, then the dynamic range is the ratio of $2^{n}$ to the notse uncertainty, but this is usually not the case.)
For example, the dynamic range of a 10-bit system is $2^{n}=1024$ to $\frac{k}{2}$, or 2048 to 1 . Expressed in dB's, this is

$$
20 \log 2048=66.2 \mathrm{~dB}
$$

if referring to a voltage-to-code converter.

The converter linearity specifies the degree to which the voltage-to-code transfer approximates a straight line. The nonlinearity is the deviation from a straight line drawn between the end points (all zeros to all ones code). It is usually not acceptable to have nonlinearity greater than LSB which means that the sum of the positive errors or the sum of the negative errors of the individual bits must not exceed $\frac{1}{2}$ LSB (Or $\pm \frac{1}{2}$ LSB). The linearity specification used in this context includes all effects such as temperature errors under expected operating temperature extremes and power supply sensitivity errors under expected operating supply variations.

\subsection{Aliasing}

Figure 10.1 illustrates equispaced sampling of continuous process $y(t)$. It is important to have a sufficient number of samples/second to properly describe information in the high frequencies. On the other hand, sampling at too high a rate may unnecessarily increase the processing labor. As we reduce the rate, we see that sample values could represent low or high frequencies in $y(t)$. This property is called aliasing and constitutes a source of error similiar to "imaging" which occurs in analog frequency mixing schemes (i.e., in the multiplication of two different signals).

If the time between samples $(k)$ is $T$ seconds, then the sampling rate is $\frac{1}{T}$ samples per second. Then useful data in $y(t)$ will be from 0 to $\frac{1}{2 T} \mathrm{~Hz}$ and frequencies higher than $\frac{1}{2 T} \mathrm{~Hz}$ will be folded into the lower range from 0 to $\frac{1}{2 T} \mathrm{~Hz}$ and confused with data in this lower range. The cutoff frequency is then given by

$$
f_{s}=\frac{1}{2 T}
$$

and is sometimes called the "Nyquist frequency." 
We can use the convolution theorem to simply illustrate the existence of aliases. This theorem states that multiplication in the time domain corresponds to convolution in the frequency domain, and the time domain and frequency domain representations are Fourier transform pairs. ${ }^{7}$ The Fourier transform of $y(t)$ in figure 10.1(a) is denoted by $Y(f)$; thus:

$$
Y(f)=\int_{-\infty}^{\infty} y(t) e^{-j 2 \pi f t} d t
$$

and

$$
y(t)=\frac{1}{2 \pi} \int_{-\infty}^{\infty} Y(f) e^{j 2 \pi f t} d f
$$

The function $Y(f)$ is depicted in figure 10.2(a). The Fourier transform of $\Delta(t)$ is shown in figure $10.2(b)$ and is given by $\Delta(f)$ where applying the discrete transform yields:

$$
\Delta(f)=\frac{1}{T} \sum_{n=-\infty}^{\infty} \delta\left(f-\frac{n}{T}\right) \text {, }
$$

recalling that

$$
\Delta(t)=\sum_{n=-\infty}^{\infty} \delta(t-n T),
$$

from eq (10.2).
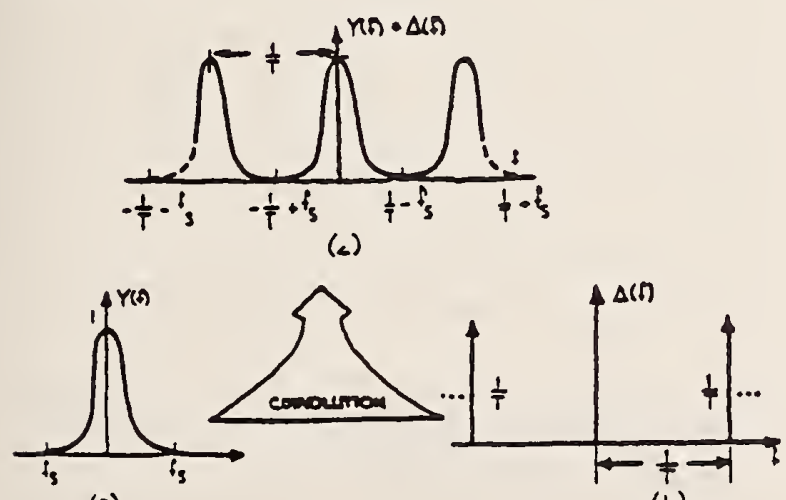

(a)

(b)

FIGURE 10.2
$Y(f)$ convolved with $\Delta f$ is denoted by $Y(f)^{*}$ $\Delta(f)$ and is shown in figure $10.2(c)$. We see that the transform $Y(f)$ is repeated with origins at $f=\stackrel{n}{=}$. Conversely, high frequency data with information around $f=\frac{n}{f}$ will fold into the data around the origin between $-f_{s}$ and $+f_{s}$. In the computation of power spectra, we encounter errors as shown in figure (10.3).

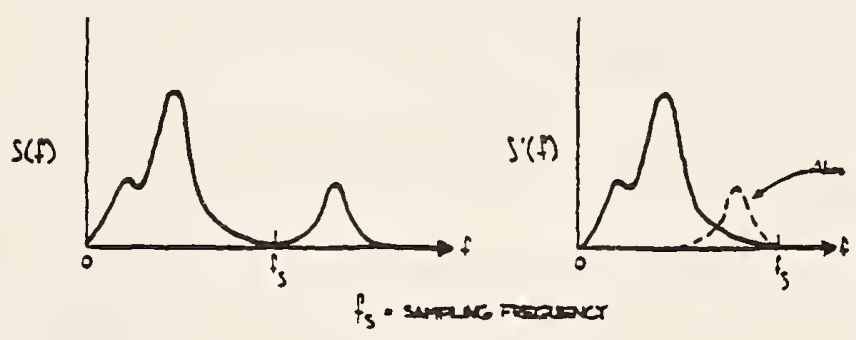

FIGURE 10.3

Aliased power spectra due to folding. (a) True Spectra, (b) Aliased Spectra.

Two pioneers in information theory, Harold Nyquist and Claude Shannon, developed design criteria for discrete-continuous processing systems. Given a specified accuracy, we can convey time-domain process $y(t)$ through a finite bandwidth whose upfer limit $f_{N}$ is the highest significant spectral component of $y(t)$. For discretecontinuous process $y_{k}(t)$, ideally the input signal spectrum should not extend beyond $f_{s}$, or

$$
f_{N} \leq f_{s}
$$

where $f_{s}$ is given by eq (10.4). Equation (10.9) is refered to as the "Shannon limit."

In practice, there is never a case in which there is absolutely no signal or noise component above $f_{N}$. Filters are used before the $A D C$ in order to suppress components above $f_{N}$ which fold into the lower bandwidth of interest. This socalled anti-aliasing filter usually must be quite sophisticated in order to have low ripple in the passband, constant phase delay in the passband, and steep rolloff characteristics. In examining the rolloff requirements of the anti-aliasing filter, we can apply a fundamental filter property that the output spectrum is equal to the input 
spectrum multiplied by the square of the frequency response function; that is,

$$
S(f)[H(f)]^{2}=\underset{\text { out }}{S(f)}
$$

The filter response must be flat to $f_{N}$ and attenuate aliased noise components at $\frac{n}{f} \pm f=$ $2 n f_{s} \pm f$. In digitizing the data, the observed spectra will be the sum of the baseband spectrum (to $f_{N}$ ) and all spectra which are folded into the baseband spectrum

$$
\begin{aligned}
S(f) & =S_{0}(f)+S_{-1}\left(2 f_{s}-f\right)+S_{+1}\left(2 f_{s}+f\right) \\
\text { observed } & +S_{-2}\left(4 f_{s}-f\right) \ldots+S_{j}\left(2 i\left(f_{s}+\frac{1}{j} f\right)\right) \\
& =S_{0}(f)+{ }_{i=-M} S_{i}\left(2 i\left(f_{s}+f\right)\right)
\end{aligned}
$$

where $M$ is an appropriate finite limit.

For a given rejection at an upper frequency, clearly the cutoff frequency $f_{c}$ for the antialiasing filter should be as low as possible to relax the rolloff requirements. Recall that an nth order low-pass filter has frequency response function

$$
H(f)=\frac{1}{1+j\left(\frac{f}{f}\right)^{n}}
$$

and output spectrum

$$
\begin{gathered}
S(f) \\
\text { out }
\end{gathered}=\frac{S(f)}{1+\left(\frac{f}{f}\right)^{2 n}}
$$

and after sampling, we have (applying eq (10.11))

$$
\begin{gathered}
S(f) \\
\text { observed }
\end{gathered}=\frac{S_{0}(f)}{1+\left(\frac{f}{f_{c}}\right)^{2 n}}+\sum_{i=-M}^{M} \frac{s_{i}\left(2 i\left(f_{s}+\frac{1}{j} f\right)\right)}{1+\frac{2 i\left(f_{s}+\frac{1}{f} f\right)^{2 n}}{f_{c}}}
$$

If $f_{c}$ is chosen to be higher than $f_{N}$, then the first term (baseband spectrum) is negligibly affected by the filter, which is our hope. It is the second term (the sum of the folded in spectra) which causes an error.

As an example of the rolloff requirement, consider the measurement of noise process $n(t)$ at $f=400 \mathrm{~Hz}$ in a $1 \mathrm{~Hz}$ bandwidth on a digital spectrum analyzer. Suppose $n(t)$ is white; that is,

$$
\begin{aligned}
& S_{n}(f)=k_{0} \\
& k_{0}=\text { constant }
\end{aligned}
$$

Suppose further that we wish to only measure the noise from $10 \mathrm{~Hz}$ to $1 \mathrm{kHz}$; thus $f_{N}=1 \mathrm{kHz}$. Let us assume a sampling frequency of $f_{s}=2 f_{N}$ or $2 \mathrm{kHz}$. If we impose a $1 \mathrm{~dB}$ error limit in Sobserved and have $60 \mathrm{~dB}$ of dynamic range, then we can tolerate an error limit of $10^{-6}$ due to aliasing effects in this measurement, and the second term in eq (10.14) must be reduced to this level. We can choose $f_{c}=1.5 \mathrm{kHz}$ and obtain

$$
\begin{gathered}
S(f) \\
\text { observed }
\end{gathered}=k_{0}+\sum_{i=-M}^{M} \frac{k_{0}}{1+\frac{2 i\left(f_{s}+\frac{1}{i} f\right)^{2 n}}{f_{c}(10.16)}}
$$

The term in the series which contributes most is at $i=-1$, the nearest fold-in. The denominator must be $10^{6}$ or more to realize the allowable error limit and at $n \geq 8$ this condition is met. The next most contributing term is $i=+1$ at which the error is < $10^{-7}$ for $n=8$, a negligible contribution. The error increases as $f$ increases for a fixed $n$ because the nearest fold-in $(i=-1)$ is coming down in frequency (note fig. $10.2(\mathrm{c})$ ) and power there is filtered less by the anti-aliasing filter. Let us look at the worst case ( $f=1 \mathrm{kHz}$ ) to determine a design criteria for this example. At $f=1 \mathrm{kHz}$, we must have $n \geq 10$.

Thus the requirenent in this example is for a 10-pole low-pass filter ( $60 \mathrm{~dB} /$ octave rolloff).

\subsection{Some History of Spectrum Analysis Leading to the Fast Fourier Transform}

Newton in his Princioia (1687) documented the first mathematical treatment of wave motion al- 
though the concept of harmonics in nature was pointed out by Pythagoras, Kepler, and Galileo. However, it was the work of Joseph Fourier in 1807 which showed that almost any function of a real variable could be represented as the sum of sines and cosines. The theory was rigorously treated in a document in 1822 .

In using Fourier's technique, the periodic nature of a process or signal is analyzed. Fourier analysis assumes we can apply fixed amplitudes, frequencies, and phases to the signal.

In the early 1900's two relatively independent developments took place: (1) radio electronics and electric power haroware were fast growing technologies; and (2) statistical analysis of events or processes which were not periodic became increasingly understood. The radio engineer explored signal and noise properties of a voltage or current into a load by means of the spectrum analyzer and measurement of the power spectrum. On the other hand, statisticians explored deterministic and stochastic properties of a process by means of the variance and self-correlation properties of the process at different times. Wiener (1930) showed that the variance spectrum (i.e., the breakdown of the variance with Fourier frequency) was the Fourier transform of the autocorrelation function of the process. He also theorized that the variance spectrum was the same as the power spectrum normalized to unit area. Tukey (1949) advocated the use of the variance spectrum in the statistical treatment of all processes because ( $I$ ) it is more easily interpreted than correlation-type functions; and (2) it fortuitously is readily measureable by the radio engineer.

The 1950's saw rigorous application of statistics to communication theory. Parallel to this was the rapid advancement of digital computer hardware. Blackman and Tukey (1959) and Welch (1961) elaborated on other useful methods of deriving an estimate for the variance spectrum by taking the ensemble time-average sampled, discrete line spectra. The approach assumes the random process is ergodic. Some digital approaches estimate the variance spectrum using Wiener's theorem if correlation-type functions are useful in the analysis, but in general the time-averaged, sample spectrum is the approach taken since its implementation is direct and straightforward. Most always, ergodicity can be assumed. 8,9

The variance of process $y(t)$ is related to the total power spectrum by

$$
\sigma^{2}[y(t)]=\int_{-\infty}^{\infty} S_{y}(f) d f .
$$

Since

$$
\sigma^{2}[y(t)]=\lim _{T \rightarrow \infty} \frac{1}{2 T} \int_{-T}^{T} y^{2}(t) d t
$$

we see that if $y(t)$ is a voltage or current into a 1-ohm load, then the mean power of $y(t)$ is the integral of $S_{y}(f)$ with respect to frequency over the entire range of frequencies $(-\infty, \infty) . S_{y}(f)$ is, therefore, the power spectrum of process $y(t)$. The power spectrum curve shows how the variance is distributed with frequency and should be expressed in units of watts per unit of frequency, or volts squared per unit of frequency when the load is not considered.

Direct estimation of power spectra has been carried out for many years through the use of analog instruments. These have variously been referred to as sweep spectrum analyzers, harmonic analyzers, filter banks, and wave analyzers. These devices make use of the fact that the spectrum of the output of a linear system (analog filter) is the spectrum of the input multiplied by the square of the systen's frequency response function (real part of the transfer characteristic). Note eq (10.10). If $y(t)$ has spectrum $S_{y}(f)$ feeding a filter with frequency response function $H(f)$, then its output is

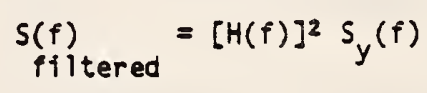

If $H(f)$ is rectangular in shape with width $\Delta f$, then we can measure the contribution to the total power spectrum due to $S_{y}\left(f \pm \frac{\Delta f}{2}\right)$.

The development of the fast fourier transform (FFT) in 1965 made digital methods of spectrum 
estimation increasingly attractive. Today the choice between digital or analog methods depends more on the objectives of the analysis rather than on technical limitations. However, many aspects of digital spectrum analysis are not well known by the casual user in the laboratory while the analog analysis methods and their limitations are understood to a greater extent.

Digital spectrum analysis is realized using the discrete fourier transform (DFT), a modified version of the continuous transform depicted in eqs (10.5) and (10.6). By saapling the input waveform $y(t)$ at discrete intervals of time $t_{2}=$ $\Delta t$ representing the sampled waveform by eq (10.2) and integrating ea (10.5) yields

$$
Y(f)=\sum_{2=-\infty}^{\infty} y(2 t) e^{-j 2 \pi f \ell T}
$$

Equation (10.20) is a Fourier series expansion. Because $f(t)$ is specified as being bandimited, the Fourier transform as calculated by eq (10.20) is as accurate as eq (10.5); however, it cannot extend beyond the Nyquist frequency, eq (10.4).

In practice we cannot compute the Fourier transform to an infinite extent, and we are restricted to some observation time $T$ consisting of $n \Delta t$ intervals. This produces a spectrum which is not continuous in but rather is computed with resolution $\Delta f$ where

$$
\Delta f=\frac{1}{n \Delta t}=\frac{1}{F}
$$

With this change, we get the discrete finite transform

$$
Y(m \Delta f)=\sum_{n=0}^{N-1} y_{\ell}(t) e^{-j 2 \pi m \Delta f n t}
$$

The OFT computes a sampled fourier series, and eq (10.22) assumes that the function $y(t)$ repeats itself with period $T$. $Y(\dot{m} \Delta f)$ is called the "line spectrum." A comparison of the OFT with the continuous fourier transform is shown later in part 10.7 .
The fast Fourier transform (FFT) is an aigorithm which efficiently computes the line spectrum by reducing the number of adds and multiplies involved in eq $(10.22)$. If we choose $T / \Delta t$ to equal a rational power of 2 , then a symnetric matrix can be derived through which $y_{2}(t)$ passes and quickly yields $Y(m \Delta f)$. An N-point transfor mation by the direct method requires a processing time proportional to $\mathrm{N}^{2}$ whereas the FFT requires a time proportional to $\mathrm{N} \log _{2} \mathrm{~N}$. The approximate ratio of FFT to direct computing time is given by

$$
\frac{N \log _{2} N}{N^{2}}=\frac{\log _{2} N}{N}=\frac{Y}{N}
$$

where $N=2^{\gamma}$. For example, if $N=2^{10}$, the FFT requires less than $1 / 100$ of the normal processing time.

We must calculate both the magnitude and phase of a frequency in the line spectrum, i.e., the real and imaginary part at the given frequency. $N$ points in the time domain allow $N / 2$ complex quantities in the frequency domain.

The power spectrum of $y(t)$ is computed by squaring the real and imaginary components, adding the two together and dividing by the total time $T$. We have

$$
S_{y}(m \Delta f)=\frac{R[Y(m \Delta f)]^{2}+I[Y(m \Delta f)]^{2}}{T}
$$

This quantity is the sampled power spectrum and again assumes periodicity in process $y(t)$ with total period T. 10

\subsection{Leakage}

Sampled digital spectrum analysis always involves transforming a finite block of data. Continuous process $y(t)$ is "looked at" for $T$ time through a data window which can functionally be described by

$$
y^{\prime}(t)=w(t) \cdot y(t)
$$

where $w(t)$ is the time domain window. The timediscrete counterpart to eq (10.25) is

$$
y_{2}^{\prime}(t)=w_{2}(t) \cdot y_{2}(t)
$$


and $w_{2}(t)$ is now the sampled version of $w(t)$ derived similarly to eq (10.2). Equation (10.26) is equivalent to convolution in the frequency domain, or

$$
Y^{\prime}(m \Delta f)=W(m \Delta f)^{\star} Y(m \Delta f)
$$

$Y^{\prime}(m \Delta f)$ is called the "modified" line spectrum due to convolution of the original line spectrum with the Fourier transform of the time-domain window function.

Suppose the window function is rectangular, and

$$
\begin{array}{rlrl}
w_{2}(t) & =1, & & \frac{-T}{2} \leq t \leq \frac{T}{2} \\
& =0, & t>\left|\frac{T}{2}\right|^{2}
\end{array}
$$

This window is snown in figure 10.4(a). The Fourier transform of this window is

$$
W(m \Delta f)=T \frac{\sin \pi m \Delta f N T}{\pi m \Delta f N T}
$$

and is shown in figure $10.4(b)$. If $y(t)$ is a sine wave, we convolve the spectrum of the sinusoid, a delta function, with $W(m \Delta f)$.

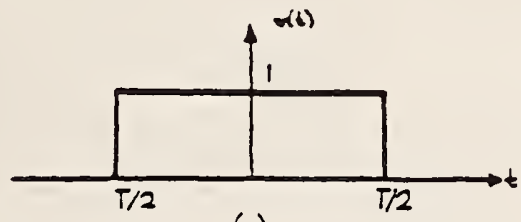

(a)

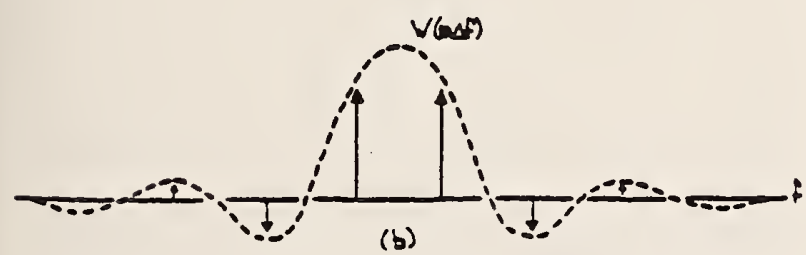

FIGURE 10.4
The transform process (eq 10.22) treats the sample signal as if it were periodically extended. Discontinuities usually occur at the ends of the window function in the extended version of the sampled waveform as in figure 10.5(c). Sample spectra thus represent a periodically extended sampled waveform, complete with discontinuites at its ends, rather than the original waveform.

(a)

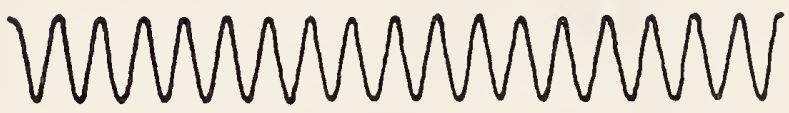

(b)

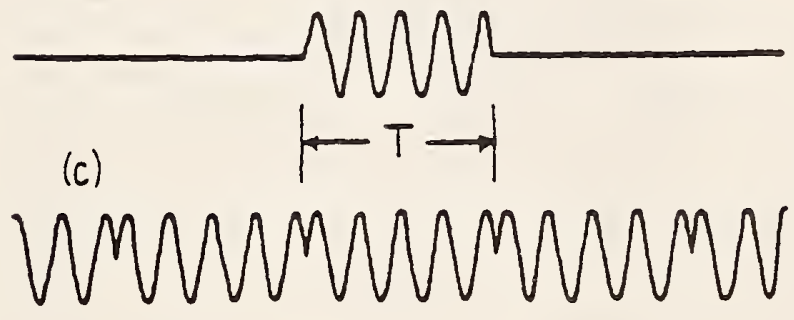

FIGURE 10.5

Spurious components appear near the sinusoid spectrum and this is referred to as "leakage." Leakage results from discontinuites in the periodically extended sample waveform.

Leakage cannot be eliminated entirely, but one can choose an appropriate window function $w(t)$ in order to minimize its effect. This is usually done at the expense of resolution in the frequency domain. An optimum window for most cases is the Hanning window given by:

$$
w(t)=\left[\frac{1}{2}-\frac{1}{2} \cos \left(\frac{2 \pi t}{T}\right)\right]^{a}
$$

for $0 \leq t \leq T$ and " $a$ " designates the number of times the window is implemented. Figure 10.6(a) shows the window function and $10.6(b)$ shows the Hanning line shape in the frequency domain for various numbers of "Hanns." Note that this window 


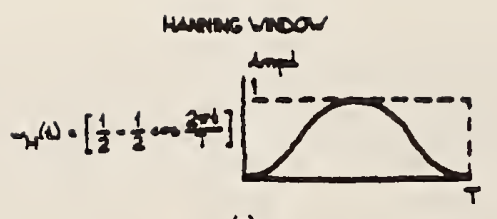

()

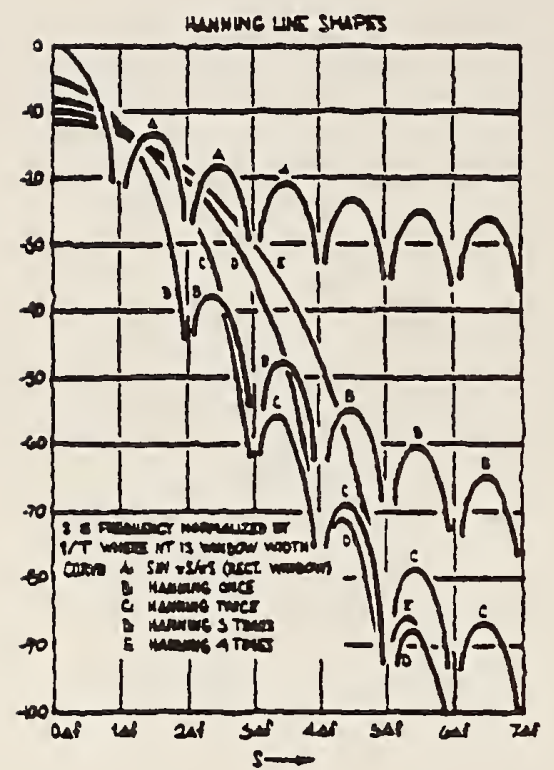

b)

FIGURE 10.6

eliminates discontinuities due to the ends of sample length $T$.

Each time the Hanning window is applied, the sidelobes in the transform are attenuated by $\mathbf{1 2}$ $d B / o c t a v e$, and the main lobe is widened by $2 \Delta f$. The amplitude uncertainty of an arbitrary sine wave input is reduced as we increase the number of Hanns; however, we trade off resolution in frequency.

The effective noise bandwidth indicates the departure of the filter response away from a true rectangularly shaped filtered response (frequency domain). Table 10-I lists equivalent noise bandwidth corrections for up to three applications of the Hanning window. 11

\begin{tabular}{cc} 
Number of Hanns & $\begin{array}{c}\text { Equivalent Noise } \\
\text { Bandwidth }\end{array}$ \\
\hline 1 & $1.5 \Delta f$ \\
2 & $1.92 \Delta f$ \\
3 & $2.31 \Delta f$
\end{tabular}

TABLE 10.I

\section{6 Picket-Fence Effect}

The effect of leakage discussed in the previous section gives rise to a sidelobe type response that can be tailored according to the time-window function through which the analyzed signal passes as a block to be transformed to the frequency domain. Using the Hanning window diminishes the amplitudes of the sidelobes, however, it increases the effective bandwidth of the passband around the center frequency. This is because the effective time-domain window length is shorter than a perfect rectangular window. Directly related to the leakage (or sidelobe) effect is one called the "picket-fence" effect. This is because the sidelobes themselves resemble a frequency response which has geometry much like a picket fence.

The existence of both sidelobe leakage and the resultant picket-fence effect are an artifact of the way in which the FFT analysis is performed. Frequency-domain analysis using analog filters involves a continuous signal in and a continuous signal out. On the other hand, FFT analysis involves a continuous signal in, but the transform to the frequency domain is performed on blocks of data. In order to get discrete frequency information from a block, the assumption is made that the block represents one period of a periodic signal. The picket-fence effect is a direct consequence of this assumption. For example, consider a sinewave signal which is transformed from a time-varying voltage to a frequency-domain representation through an FFT. The block of data to be transformed will be length, $T$, in time. Let's say that the block, $T$, represents only $4 \frac{1}{2}$ cyeles of the input sinewave as in figure 10.5. Artificial sidebands will be created in the transform to the frequency domain, whose frequency spacing equals $\frac{1}{\%}$, or the reciprocal of the block length. This represents a worst-case condition for sidelobe generation and creates a large number of spurious discrete frequency components as shown in figure 10.7(b).

If, on the other hand, one changes the block time, $T$, so that the representation is an integral number of cycles of the input sinewave, then the transform will not contain sidelobe leakage compo- 


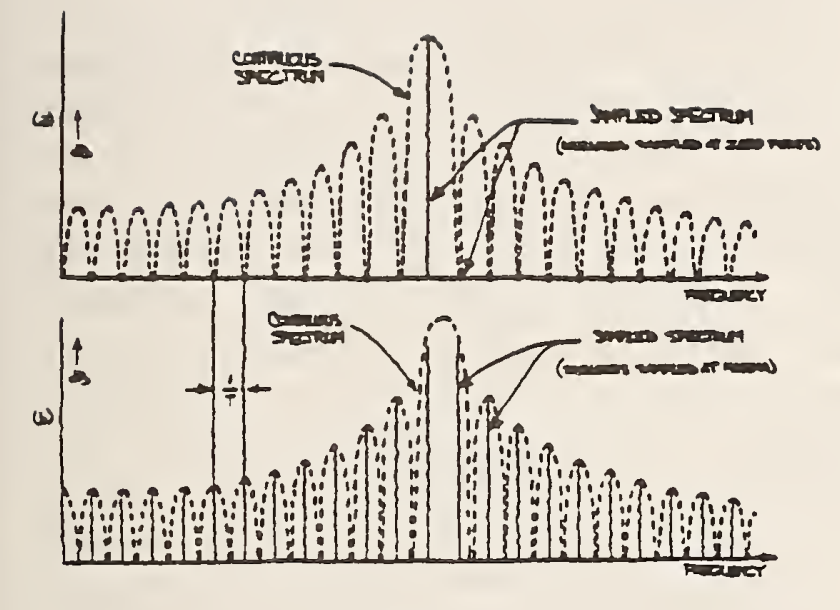

FIGURE 10.7

nents and the artificial sideband frequency components disappear. In practice, when looking at complex input signals, the block time, $T$, is not synchronous with any component of the transformed part of the signal. As a result, discrete frequency components in the frequency domain have associated with them sidebands which come and go depending on the phase of the time window. $T$, relative to the sine components of the incoming signal. The effect is much like looking through a picket fence at the sidebands. 12

An analogy to the sidelobe leakage and picketfence effect is to record the incoming time-varying signal on a tape loop, which has a length of time, $T$. The loop of tape then repeats itself with a period of $T$. This repeating signal is then coupled to a scanning or filter-type spectrum analyzer. The phase discontinuity between the end of one passage of the loop and the beginning of the loop on itself again represents a phase-modulation component, which gives rise to artificial sidebands in the spectrum analysis. A word of caution this is not what actually happens in a FFT analyzer (i.e., there is no recirculating memory). However, the fourier transform treats the incoming block as if this were happening.

\subsection{Time Domain-Frequency Domain Transforms}

A. Integral transform

Figure 10.8 shows the we11-known integral transform, which transforms a continuous timedomain signal extending over all time into a

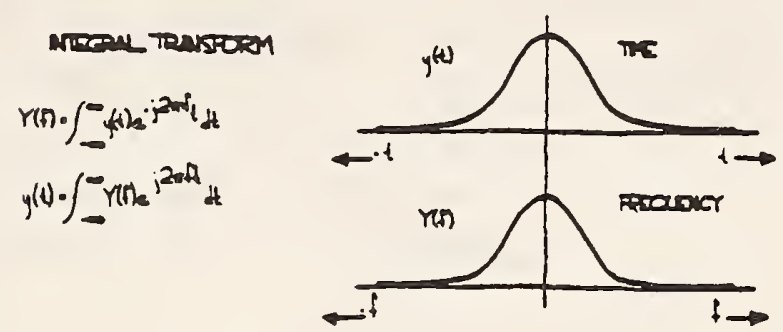

FIGURE 10.8

continuous frequency-domain signal extending over all frequency. This is the ideal transiorm. In practice, however, one deals with finite times and bandwidths. The integral transform then, at best, is an estimate of the transform and is so for only short, well-behaved signals. That is, the signal goes to zero at infinite time and at infinitely high frequency.

\section{B. Fourier series}

The Fourier-series transform assumes periodicity in the time-domain signal for all time. Only one period of the signal (for time $T$ ) is required for this kind of transform. The fourier series treats the incoming signal as periodic with period, $T$, and continuous. The transformed spectrum is then discrete with infinite harmonic components with frequency spacing of $\frac{1}{y}$. This is shown in figure 10.9.

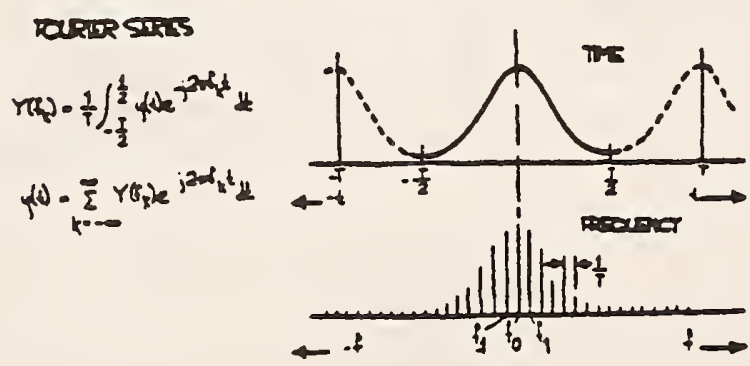

FIGURE 10.9 


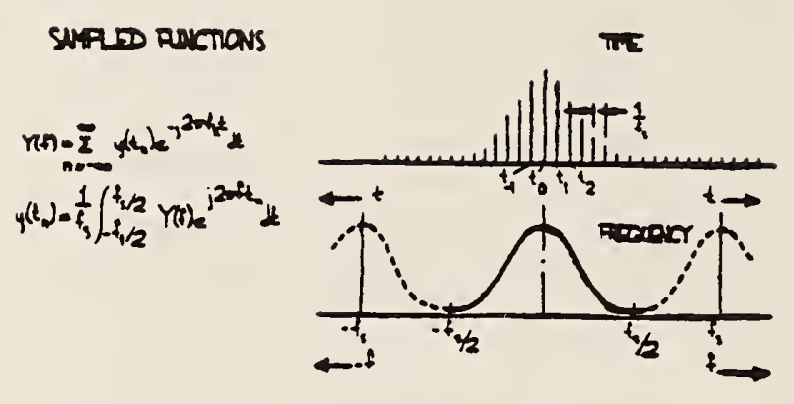

FIGURE 10.10

Figure 10.10 shows the transform from a sam pled time-domain signal to the frequency domain. Note that in the frequency domain, the result is repetitive in frequency. This effect, comanily called aliasing, is discussed earlier in section 10.3. Figure 10.9 and figure 10.10 show the $5 y m-$ metry between the time- and frequency-domain transforms of discrete lines.

\section{Discrete fourier transform}

finally, we have the sampled, periodic (assumed) time-domain signal which is transformed to a discrete and repetitive (aliased) frequencydomain representation. This is shown in figure 10.11

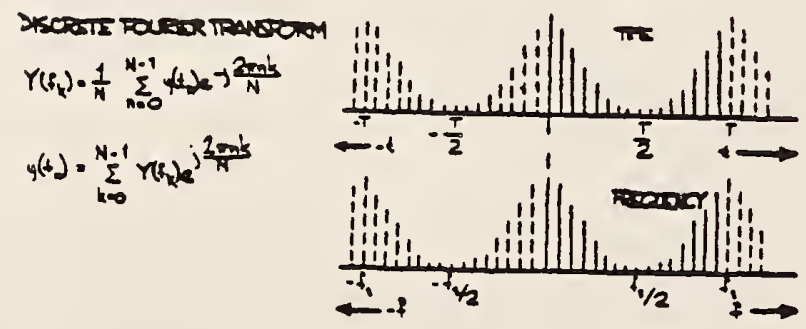

FIGURE 10.11
XI. TRANSLATION FROM FREQUENCY DOMAIN STABILITY MEASUREMENT TO TIME DOMAIN STABILITY MEASUREMENT AND VICE-VERSA.

\section{1 Procedure}

Knowing how to measure $S_{\phi}(f)$ or $S_{y}(f)$ for a pair of oscillators, let us see how to translate the power-law noise process to a plot of $\sigma_{y}{ }^{2}(\tau)$. First, convert the spectrum data to $S_{y}(f)$, the spectral density of frequency fluctuations (see sections III and VIII). There are two quantities which sompletely specify $S_{y}(f)$ for a particular power-law noise process: (1) the slope on a log-log plot for a given range of $f$ and (2) the amplitude. The slope we shall denote by " $\alpha$ "; therefore $f^{\alpha}$ is the straight line (on log-log scale) which relates $s_{y}(f)$ to $f$. The amplitude will be denoted " $h_{\alpha}$ "; it is simply the coefficient of $f$ for a range of $f$. When we examine a plot of spectral density of frequency fluctuations, we are looking at a representation of the addition of all the power-law processes (see sec. IX). We have

$$
s_{y}(f)=\sum_{\alpha=-\infty}^{\infty} n_{\alpha} f^{\alpha}
$$

In section IX, five power-law noise processes were outlined with respect to $S_{\phi}(f)$. These five are the common ones encountered with precision oscillators. Equation (8.7) relates these noise processes to $S_{y}(f)$. One obtains
1. Random Walk FM $(f-2) . ., \alpha=-2$
2. Flicker FM $(f-1) \ldots . \alpha=-I$
3. White FA (1) ... $\alpha=0$
4. Flicker $\phi M$ (f) ... $\alpha=1$
5. White $\phi M$ (f2) ... $\alpha=2$
slope on
$\log -\log$
paper

Table 11.1 is a list of coefficients for translation from $\sigma_{y}{ }^{2}(\tau)$ to $S_{y}(f)$ and from $S_{\phi}(f)$ to 
$\sigma_{y}{ }^{2}(\tau)$. In the table, the left column is the designator for the power-law process. Using the middle column, we can solve for the value of $S_{y}(f)$ by computing the coefficient " $a$ " and using the measured time domain data $\sigma_{y}{ }^{2}(\tau)$. The rightmost column yields a solution for $\sigma_{y}{ }^{2}(\tau)$ given frequency domain data $S_{\phi}(f)$ and a calculation of the appropriate " $b$ " coefficient.

\begin{tabular}{|c|c|c|}
\hline $\begin{aligned} S_{y}(f) & =H_{\alpha} f^{\alpha} \\
\alpha & =\alpha\end{aligned}$ & $\begin{aligned} S_{y}(f) & =a \sigma_{y}^{2}(\tau) \\
a & =a{ }^{2}\end{aligned}$ & $\begin{aligned} \sigma_{y}^{2}(\tau) & =b S_{\phi}(f) \\
b & =\end{aligned}$ \\
\hline (white ${ }^{2}$ phase) & $\frac{(2 \pi)^{2} \tau^{2} f^{2}}{3 f_{h}}$ & $\frac{3 \tau_{h}}{(2 \pi)^{2} \tau^{2} v_{0}^{2}}$ \\
\hline (flicker noise) & $\frac{(2 \pi)^{2} \tau^{2} f}{1.038+3 \ln \left(w_{n} \tau\right)}$ & $\frac{\left[1.038+3 \ln \left(w_{h} \tau\right)\right] f}{(2 \pi)^{2} \tau^{2} v_{0}^{2}}$ \\
\hline $\begin{array}{c}0 \\
\text { (white frequency) }\end{array}$ & $2 \tau$ & $\frac{f^{2}}{2 \tau v_{0}^{2}}$ \\
\hline (flicker frequency) & $\frac{1}{2 \ln (2) \cdot f}$ & $\frac{2 \ln (2) \cdot f^{3}}{v_{0}^{2}}$ \\
\hline (random walk frequency) & $\frac{6}{(2 \pi)^{2} \tau f^{2}}$ & $\frac{(2 \pi)^{2} \tau f^{4}}{6 v_{0}^{2}}$ \\
\hline
\end{tabular}

TABLE 11.1

Conversion table from time domain to frequency domain and from frequency domain to time domain for common kinds of interger power law spectral densities; $f_{h}\left(=w_{h} / 2 \pi\right)$ is the measurement system bandwidth. Measurement reponse should be within $3 \mathrm{~dB}$ from $D . C$. to $f_{h}$ ( $3 \mathrm{~dB}$ down high-frequency cutoff is at $f_{h}$ ).

$$
S_{\phi}(f)=\frac{v_{0}^{2}}{f^{2}} S_{y}(f)
$$

\section{PSCTPAL DESTIY OF PALSE}

?o 1 mate

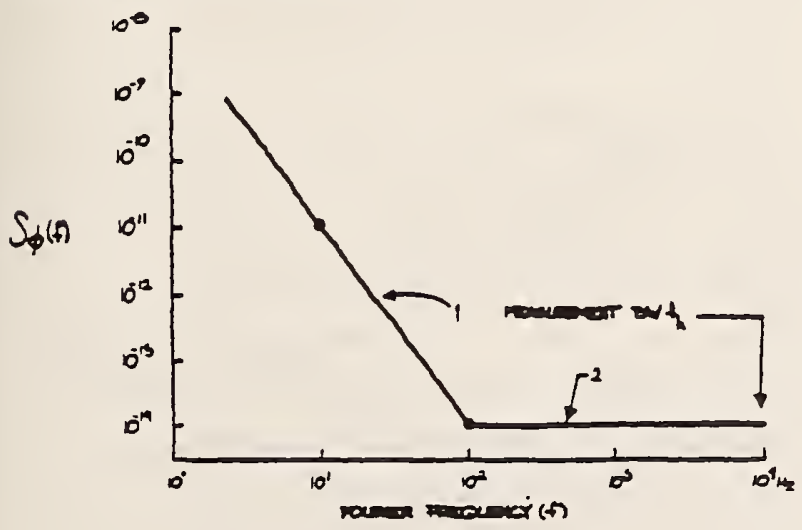

FIGURE 11.1 identify this noise process as flicker FM. The rightmost column of table 11.1 relates $\sigma_{y}^{2}(\tau)$ to $S_{\phi}(f)$. The row designating flicker frequency noise yields:

$$
\sigma_{y}^{2}(\tau)=\frac{2 \ln (2) \cdot f^{3}}{v_{0}^{2}} S_{\phi}(f)
$$

One can pick (arbitrarily) a convenient Fourier frequency $f$ and determine the corresponding values of $S_{\phi}(f)$ given by the plot of figure 11.1. Say, $f=10$, thus $S_{\phi}(10)=10-11$. Solving for $\sigma_{y}^{2}(\tau)$, given $v_{0}=1 \mathrm{MHz}$, we obtain:

$$
\sigma_{y}^{2}(\tau)=1.39 \times 10.20
$$

therefore, $\sigma_{y}(\tau)=1.18 \times 10-10$. For region 2, we have white PM. The relationship between $\sigma_{y}^{2}(\tau)$ and 
$S_{\phi}(f)$ for white $P M$ is:

$$
\sigma_{y}^{2}(\tau)=\frac{3 f_{h}}{(2 \pi)^{2} \tau^{2} v_{0}^{2}} S_{\phi}(f)
$$

Again, we choose a Fourier frequency, say $f=100$, and see that $S_{\phi}(100)=10^{-14}$. Assuming $f_{h}=10^{4}$ $\mathrm{Hz}$, we thus obtain:

$$
\sigma_{y}^{2}(\tau)=7.59 \times 10^{-24} \cdot \frac{1}{\tau^{2}}
$$

therefore,

$$
\sigma_{y}(\tau)=2.76 \times 10^{-12} \frac{1}{\tau} .
$$

The resultant time domain characterization is shown in figure 11.2.

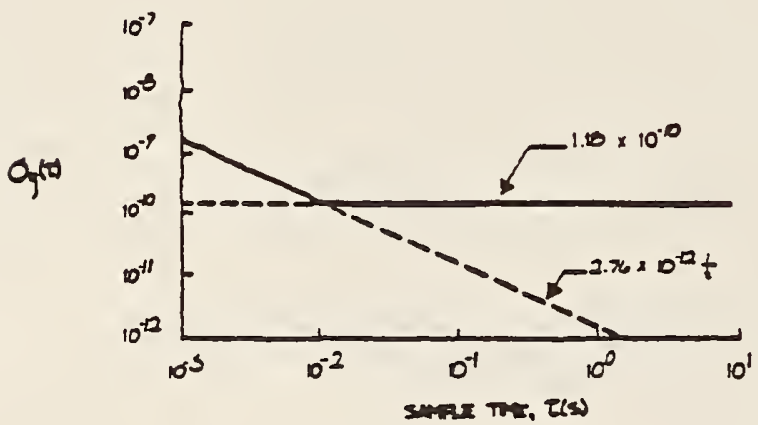

FIGURE 11.2

The translation of $S_{\phi}(f)$ of figure 11 . I yields this $\sigma_{y}(\tau)$ plot.

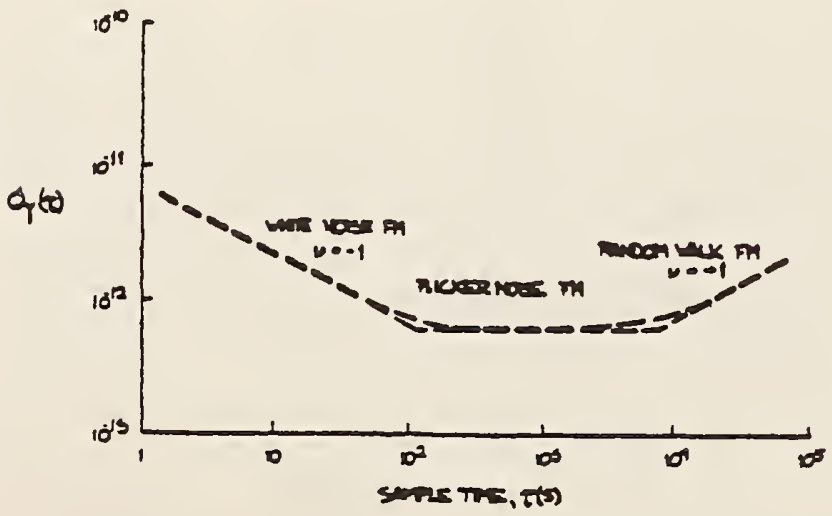

FIGURE 11.3

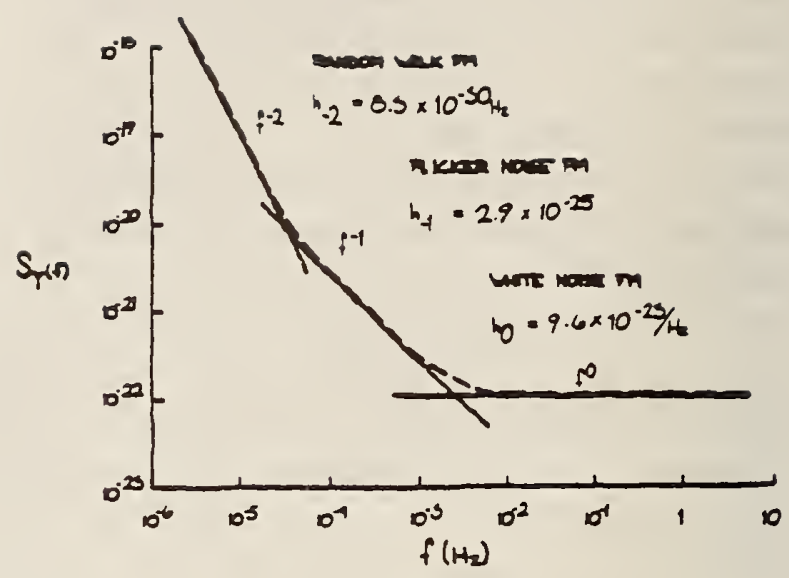

FIGURE 11.4

Figures 11.3 and 11.4 show plots of timedomain stability and a translation to frequency domain. Since table 11.1 has the coefficients which connect both the frequency and time domains, it may be used for translation to and from either domain.

XII. CAUSES OF NOISE PROPERTIES IN A SIGNAL SOURCE

\section{I Power-law Noise Processes}

Section IX pointed out the five commoniy used power-law models of noise. With respect to $S_{\phi}(f)$, one can estimate a staight line slope (on a log-log scale) which corresponds to a particular noise type. This is shown in figure 12.1 (also fig.9.1).

SPECTRAL DEISITY OF PARSE

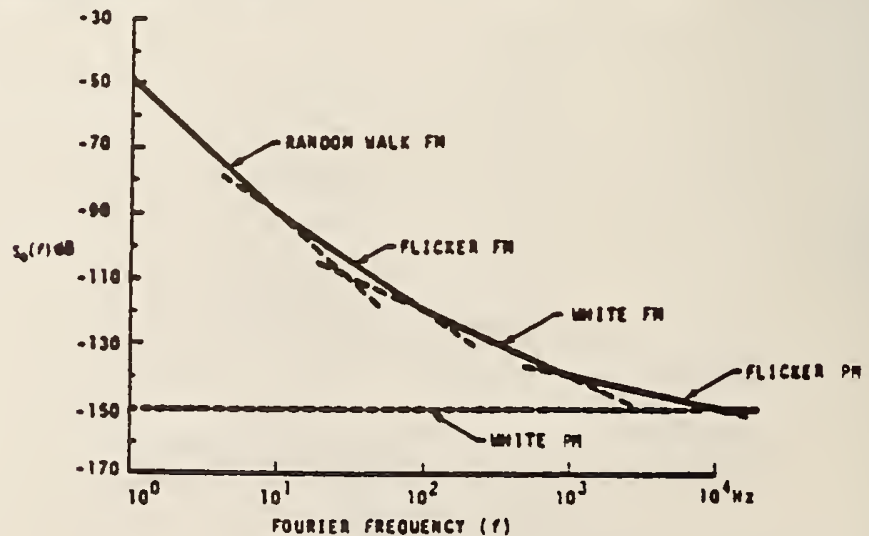

FIGURE 12.1 
We can make the following general remarks about power-law noise processes:

1. Random walk FM $\left(1 / f^{4}\right)$ noise is difficult to measure since it is usualiy very close to the carrier. Random walk FM usualiy relates to the OSCILLATOR'S PHYSICAL ENVIRONMENT. If random waIK FM is a predominant feature of the spectral density plot then MECHANICAL SHOCK, VIBRATION, TEMPERATURE, or other environmental effects may be causing "random" * shifts in the carrier frequency.

2. Flicker FM $\left(1 / f^{3}\right)$ is a noise whose physical cause is usually not fully understood but may typically be related to the PHYSICAL RESONANCE MECHANISM OF AN ACTIVE OSCILLATOR or the DESIGN OR CHOICE OF PARTS USED FOR THE ELECTRONICS, or ENVIRONMENTAL PROPERTIES. Flicker FM is common in high-quality ossillators, but may be masked by white FM $\left(1 / f^{2}\right)$ or flieker PM $(1 / f)$ in lower-quality oscillators.

3. White $F M\left(1 / f^{2}\right)$ noise is a common type found in PASSIVE-RESONATOR FREQUENCY STANDARDS. These contain a slave osciliator, often quartz, which is locked to a resonance feature of another device which behaves much like a nigh-Q filter. Cesium and rubidium standards have white FM noise characteristics.

4. Flicker $P M(1 / f)$ noise may relate to a physical resonance mechanism in an oscillator, but it usually is added by NOISY ELECTRONICS. This type of noise is common, even in the highest quality oscillators, because in order to bring the signal amplitude up to a usable level, amplifiers are used after the signal source. Flicker PM noise may be introduced in these stages. It may also be introduced in a frequency multiplier.
Flicker PM can be reduced with good low-noise amplifier design (e.g., using $r$ ( negative feedback) and hand-selecting transistors and other electronic components.

5. White PM $\left(f^{0}\right)$ noise is broadband phase noise and has little to do with the resonance mechanism. It is probably produced by similar phenomena as flicker PM ( $1 / f$ ) noise. STAGES OF AMPLIFICATION are usually responsible for white $P M$ noise. This noise can be kept at a very low value with good amplifier design, hand-selected components, the addition of narrowband filtering at the output, or increasing, if feasible, the power of the primary frequency source. 13

\section{2 Other types of noise}

A commonly encountered type of noise from a signai source or measurement apparatus is the presence of $60 \mathrm{~Hz}$ A.C. line noise. Shown in figure 12.2 is a constant white $P M$ noise source with $60 \mathrm{~Hz}, 120 \mathrm{~Hz}$ and $180 \mathrm{~Hz}$ components added. This kind of noise is usually caused by $A C$ power getting into the measurement system or the. source under test. In the plot of $S_{\phi}(f)$, one observes discrete line spectra. Although $S_{\phi}(f)$ is a measure of spectral density, one can interpret the line spectra with no loss of generality, although one usually does not refer to spectral densities when characterizing discrete lines. Figure 12.3 is the time domain representation of the same white phase modulation level with $60 \mathrm{~Hz}$ noise. Note that the amplitude of $\sigma_{y}(t)$ varies up and down depending on sampling time. This is because in the time domain the sensitivity to a periodic wave varies directly as the sampling interval. This effect (which is an alias effect) is a very powerful tool for filtering out a periodic wave imposed on a signai source. By sampling in the time domain at integer periods, one is virtualiy insensitive to the periodic (discrete line) term. 


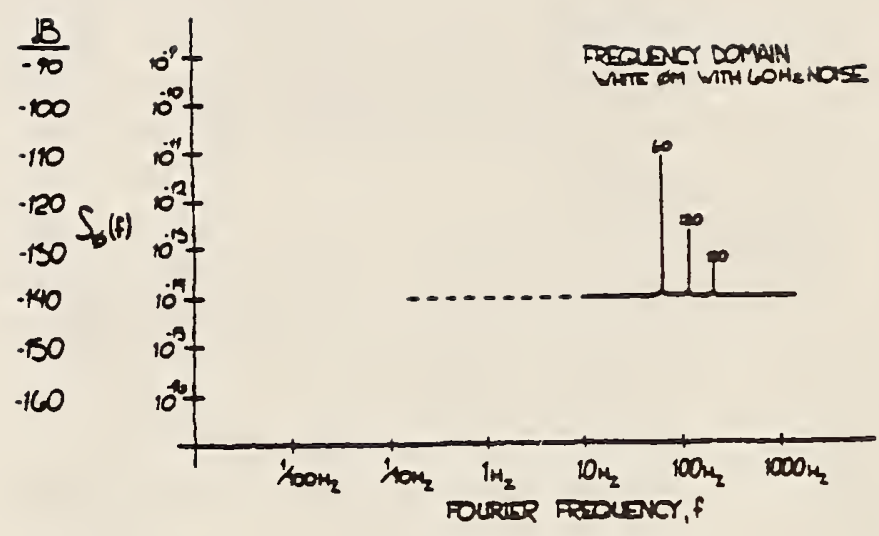

FIGURE 12.2
FM behavior masks the white PM (with the superimposed vibration characteristic) for long averaging times.

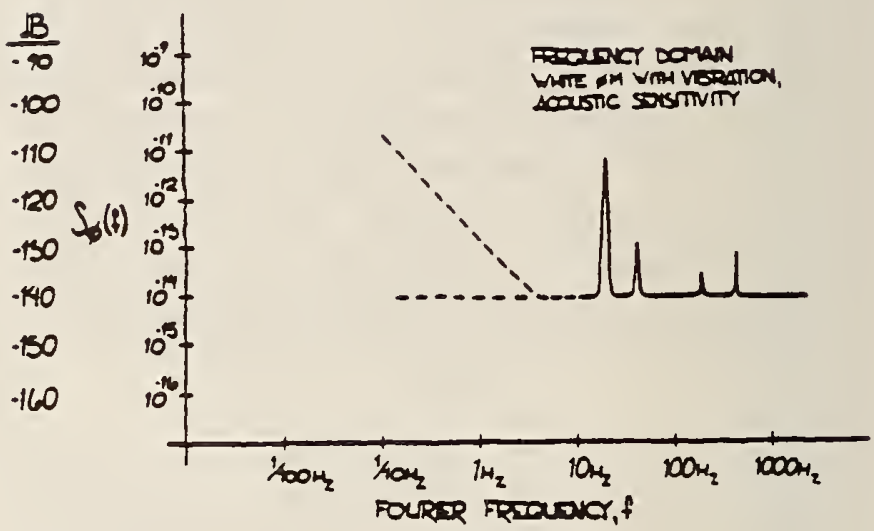

FIGURE 12.4

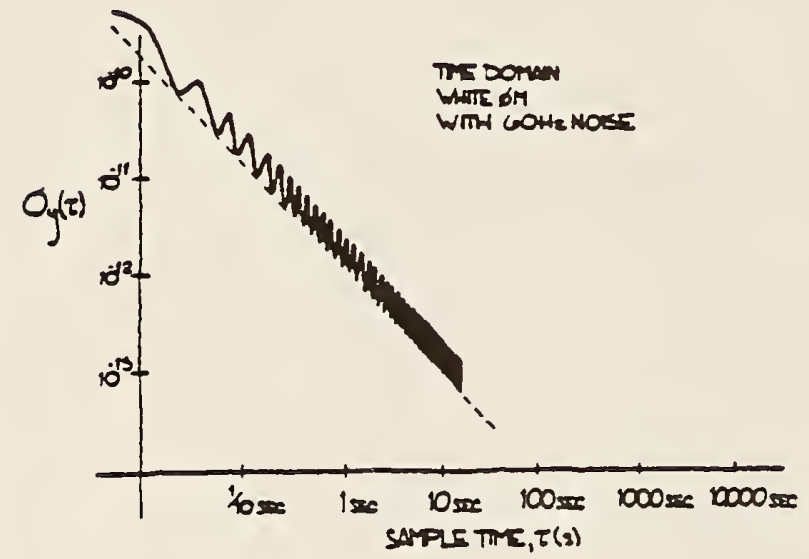

FIGURE 12.3

For example, diurnal variations in data due to day to day temperature, pressure, and other environmental effects can be eliminated by sampling the data once per day. This approach is useful for data with only one periodic term.

Figure 12.4 shows the kind of plot one might see of $S_{\phi}(f)$ with vibration and acoustic sensitivity in the signal source with the device under vibration. Figure 12.5 shows the transiation to the time domain of this effect. Also noted in figure 12.4 is a (typical) flicker FM behavior in the low frequency region. In the translation to time domain (fig. 12.5), the flicker 


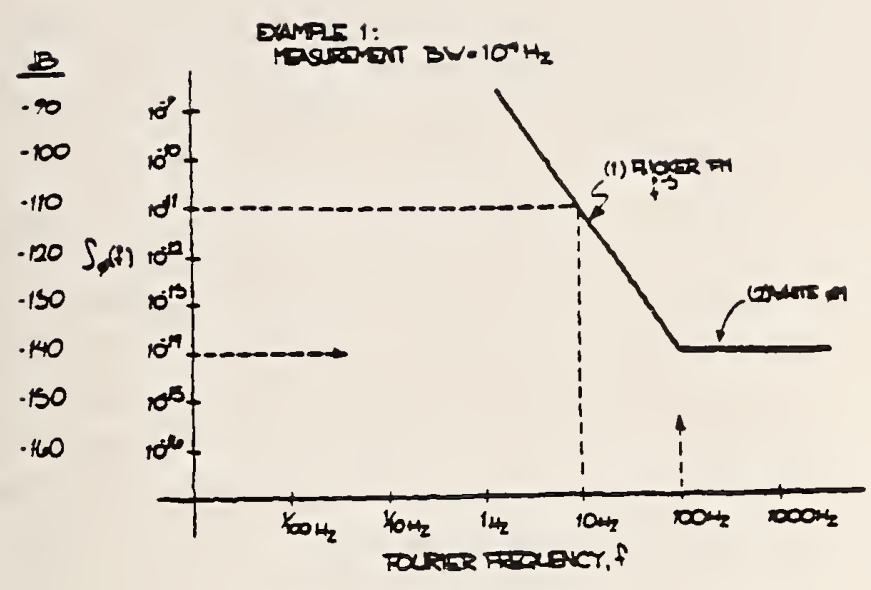

FIGURE $12.6 \mathrm{a}$

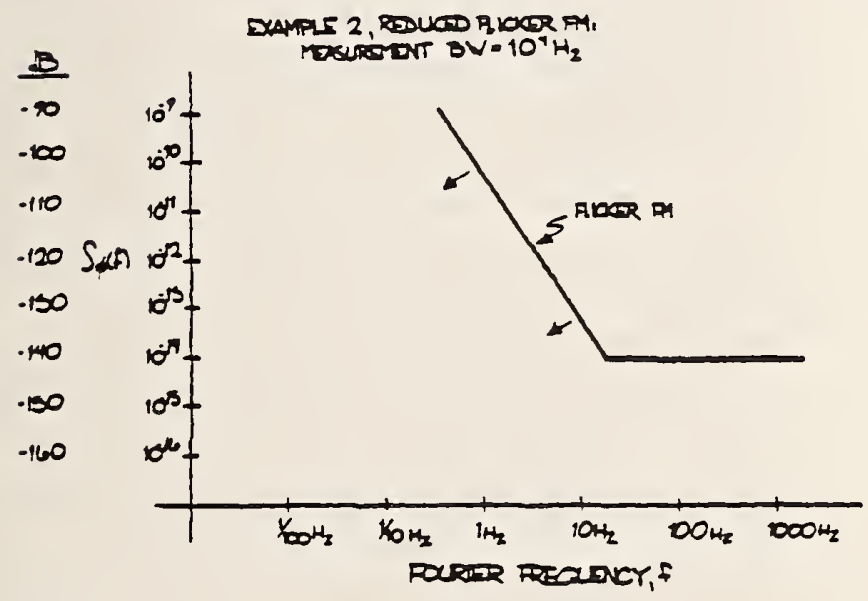

FIGURE $12.6 \mathrm{~b}$

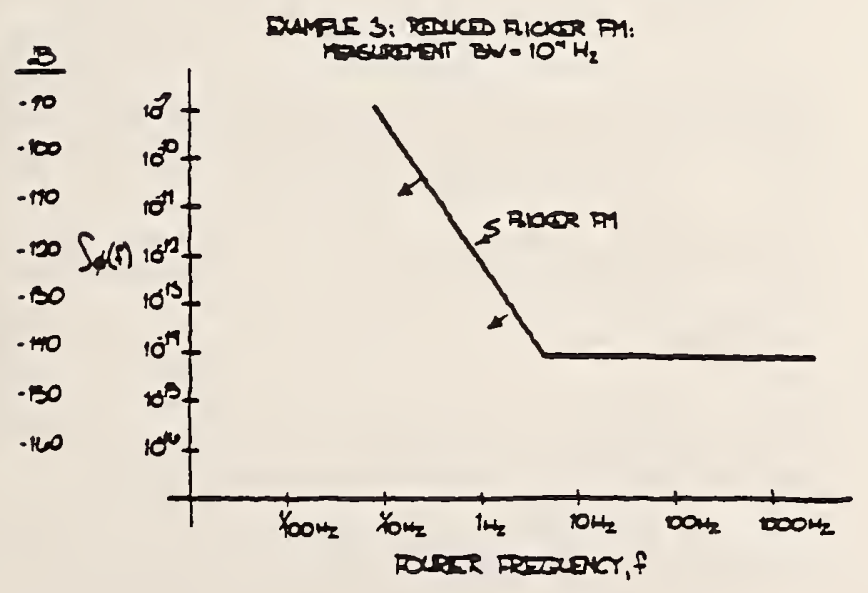

FIGURE $12.6 \mathrm{C}$

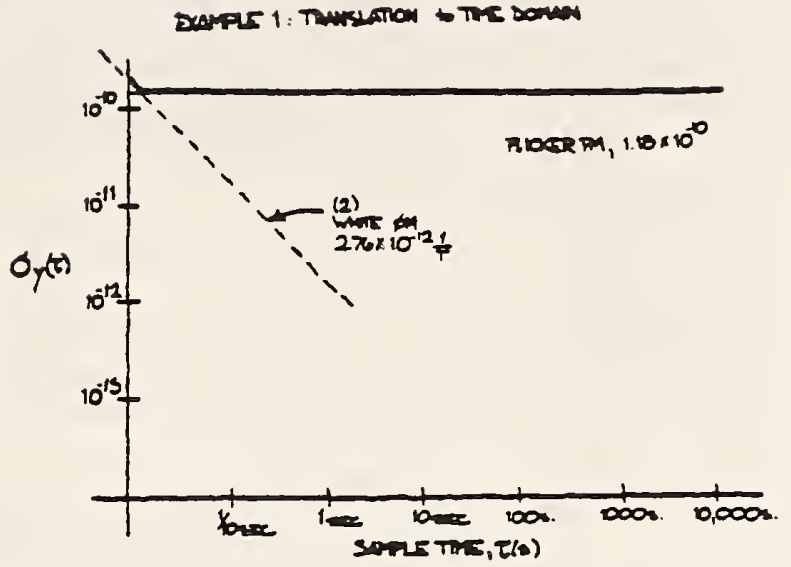

FIGURE $12.7 \mathrm{a}$

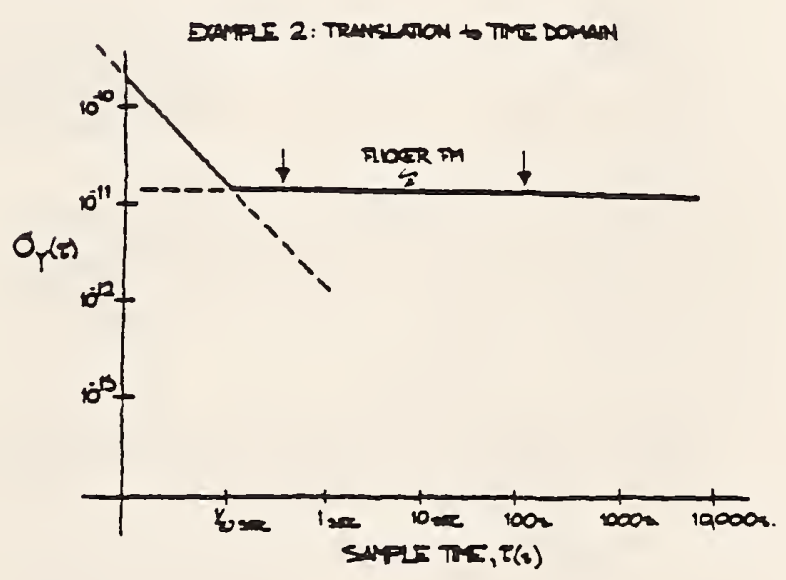

FIGURE $12.7 b$

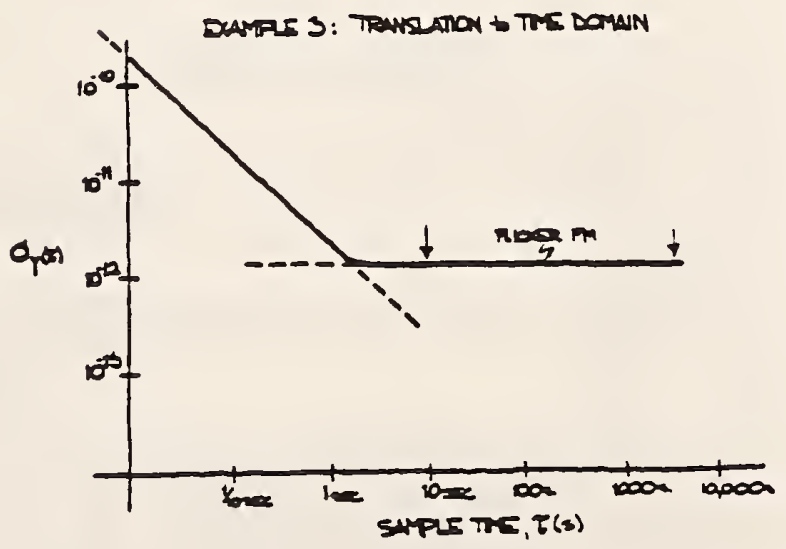

FIGURE $12.7 \mathrm{c}$ 


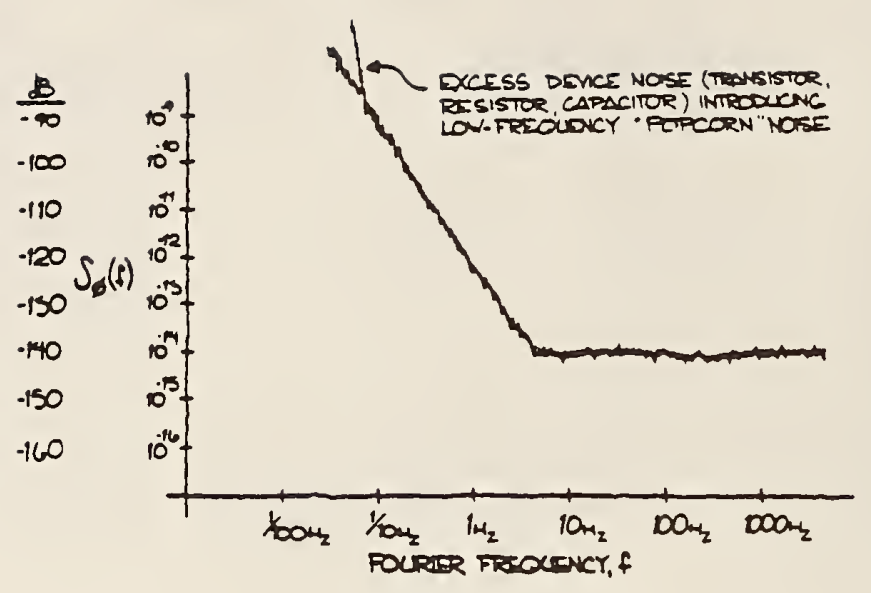

FIGURE 12.8

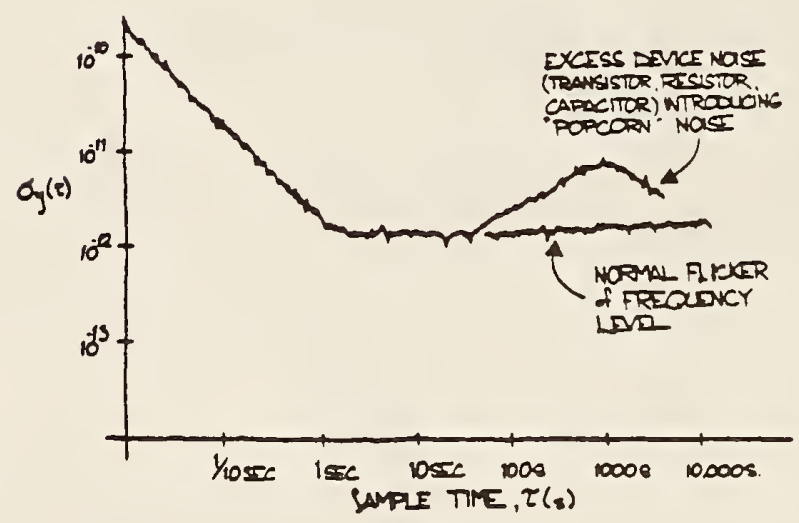

FIGURE 12.9

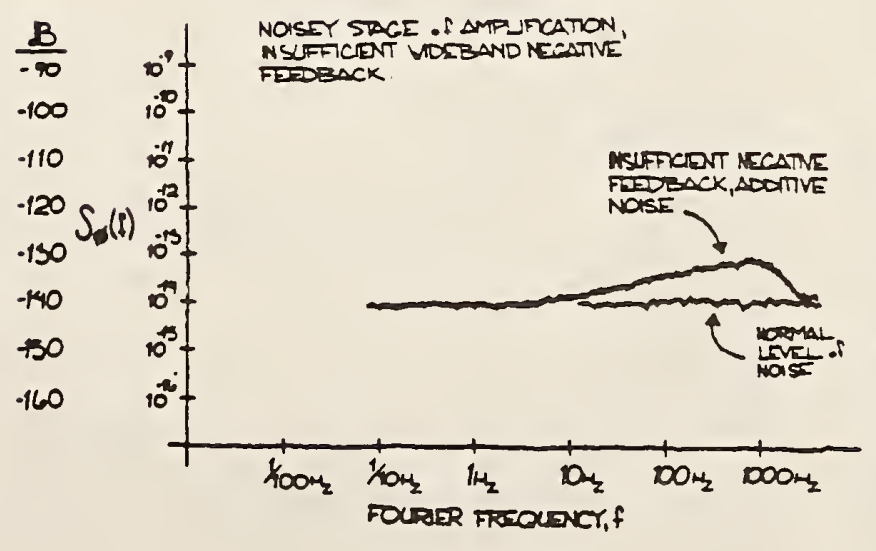

FIGURE 12.10
Excess device noise from transistors, capacitors, resistors, and the like can introduce a low frequency noise which has been referred to as "popcorn" noise because of its sonic qualities. Figure 12.8 shows a plot of $S_{\phi}(f)$ from a signal source having such excess low frequency noise. Figure 12.9 is the time domain representation. The rise in amplitude of $\sigma_{y}$ for long averaging times is particularly aggravating. The solution to this kind of problem if it is introduced by devices is to carefully grade the devices in the assembly and testing process.

Stages of amplification following a signal source many times rely on local degenerate or overall negative feedback schemes in order to minimize the excess noise from active gain elements (such as transistors). This is the recommended design approach. However, phase shift in the negative feedback circuit or poor bandwidth in the gain elements can result in poor high frequency noise behavior. Figure 12.10 shows a kind of result one might see as a gradual rise in $S_{\phi}(f)$ because of insufficient negative feed back at high Fourier frequencies.

Section $X$ discussed aliasing in the frequency domain. Figure 12.11 shows the resultant measurement anomaly due to digital sampling of a poorly bandimited (anti-aliased) white noise source. Noise voltage above the sampling frequency $f_{s}$ is folded into the analysis region of interest. Note also that the stopband ripple characteristics are folded into the high-frequency portion of the passband. For a given sampling frequency, a compromise exists between increasing the high-

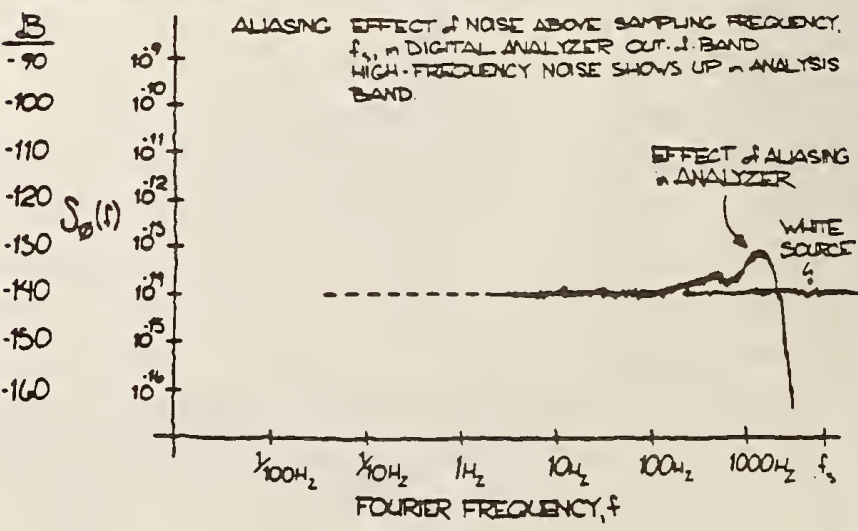

FIGURE 12.11 
frequency extent of the analysis band and improving the anti-aliasing filter's stopband rejection. Section $X$ has an example of the filter requirements for a particular case.

\section{CONCLUSION}

This writing highlights major aspects of time-domain and frequency-domain oscillator signal measurements. The contents are patterned after lectures presented by the authors. The authors have tried to be general in the treatment of topics, and bibliography is attached for readers who would like details about specific items.

\section{References}

1. J. A. Barnes, Andrew R. Chi, Leonard $S$. Cutler, Daniel J. Healey, David B. Leeson, Thomas E. McGunigal, James A. Mullen, Jr., Warren L. Smith, Richard L. Sydnor, Robert F. C. Vessot, and Gernot M. R. Winkler, "Characterization of Frequency Stability," NBS Tech. Note 394 (1970); Proc. IEEE Trans. on Instrum. and Meas. IM-20, 105 (1971).

2. D. W. Allan, "The Measurement of Frequency and Frequency Stability of Precision Oscillators," NBS Tech. Note 669 (1975); Proc. 6th PTTI Planning Meeting, 109 (1975).

3. D. W. Allan, "Statistics of Atomic Frequency Standards," Proc. IEEE 54, 221 (1966).

4. Sections $V$ and $V I$ are from notes to be published by J. A. Barnes.

5. S. R. Stein, "An NBS Phase Noise Measurement System Built for Frequency Domain Measurements Associated with the Global Positioning System, NBSIR 76-846 (1976). See also F. L. Walls and S. R. Stein, "Servo Technique in Oscillators and Measurement Systems," NBS Tech. Note 692 (1976)

6. C. D. McGillen and G. R. Cooper, Continous and Discrete Signal and System Analysis, Holt, Rinehart, and Winston, Inc. (1974).

7. E. 0. Brigham, The Fast Fourier Transform, Prentice-Hail, Inc., New Jersey (1974).

8. Private communication with Norris Nahman, June 1981.

9. G. M. Jenkins and D. G. Watts, Spectral Analysis and Its Application, Holden-Day, San Francisco, 1968.

10. P. F. Panter, Modulation, Noise, and Spectral Analys is, McGraw-Hill, New York, 1965, PD. 137-141.
11. D. Babitch and J. 01iverio, "Phase Noise of Various 0scillators at Very Low Frequencies. Proc. 28th Annual Symp. on Frequency Control, May 1974. U. S. Army Electronics Command, Ft. Monmouth, NJ.

12. N. Thrane, "The Discrete Fourier Transform and FFT Analyzers," Bruel and Kjaer Technical Review, No. 1 (1979).

13. Much of the material presented here is contained in NBS Tech. Note 679, "Frequency Domain Stability Measurements: A Tutorial Introduction," D. A. Howe (1976).

\section{Bibliography}

\section{General References}

1. November or December of even-numbered years, IEEE Transactions on Instrumentation and Measurement (Conference on Precision Electromagnetic Measurements, held every two years).

2. Proceedings of the IEEE. Special issue on frequency stability, vol. 54, February 1966.

3. Proceedings of the IEEE. Special issue on time and frequency, vol. 60, no. 5, May 1972.

4. Proceedings of the IEEE-NASA Symposium on the Definition and Measurement of Short-Term Frequency Stability at Goddard Space Flight Center, Greenbelt, MD, November 23-24, 1964. Prepared by Goddard Space Flight Center (Scientific and Technical Information Division, National Aeronautics and Space Administration, Wastington, DC, 1965). Copies available for $\$ 1.75$ from the US Government Printing Office, Washington, DC 20402.

5. Proceedings of the Annual Symposium on Frequency Control. Prepared by the US Army Electronics Command, Fort Monmouth, NJ. Copies from Electronics Industry Association, 2001 Eye Street, N. W., Washington, DC 20006, starting with 32nd Annual or call (201) 544-4878. Price per copy, $\$ 20.00$.

6. J. A. Barnes, A. R. Chi, L. S. Cutler, et al., "Characterization of Frequency Stability," IEEE Trans. On Intsrum. and Meas. IM-20, no. 2, PP. 105-120 (May 1971). Prepared by the Subcomnittee on Frequency Stability of the Institute of Electrical and Electronics Engineers.

7. Time and Frequency: Theory and Fundamentais, Byron E. Blair, Editor, NBS Monograph 140, May 1974.

8. P. Kartaschoff, Frequency and Time, London, England: Academic Press, 1978.

9. G. M. R. Winkler, Timekeeping and Its Application, Advances in Electonics and Electron Physics, Vol. 44, New York: Academic Press, 1977. 
10. F. M. Gardner, Phase lock Techniques, New York: Wiley \& Sons, 1966.

11. E. A. Gerber, "Precision Frequency Control and Selection, A Bibliography," Proc. 33rd Annual Symposium on Frequency Control, pp. 569-728, 1979. Available from Electronics Industries Association, 2001 Eye St. NW, Wastington, DC 20006.

12. J. Rutman, "Characterization of Phase and Frequency Instabilities in Precision Frequency Sources: Fifteen Years of Progress," Proc. IEEE, vol. 66, no. 9, September 1978, pp. 1048-1075.

\section{Additional Specific References (selected)}

1. D. W. Allan, "Statistics of Atomic Frequency Standards," Proc. IEEE, vol. 54, pp. 221230, February 1966.

2. D. W. Allan, "The Measurement of Frequency and Frequency Stability of Precision Oscillators," NBS Tech. Note 669, July 1975.

3. 3. R. Ashley, C. B. Searles, and F. M. Palka, "The Measurement of Oscillator Noise at Microwave Frequencies," IEEE Trans. on Microwave Theory and Techniques MTT-16, Pp. 753760 , September 1968.

4. E. J. Bagndady, R. D. Lincoln, and B. D. Nelin, "Short-Term Frequency Stability: Theory, Measurement, and Status," Proc. IEEE, vol. 53, pp. 704-722, 2110-2111, 1965.

5. J. A. Barnes, "Models for the Interpretation of Frequency Stability Measurements," NBS Technical Note 683, August 1976.

5. J. A. Barnes, "A Review of Methods of Analyzing Frequency Stability," Proc. 9th Annual Precise Time and Time Interval (PTTI) Applications and Planning Meeting, Nov. 1977, pp. 61-84.

7. J. A. Barnes, "Atomic Timekeeping and the Statistics of Precision Signal Generators," Proc. IEEE, vol. 54, pP. 207-220, February 1966.

8. J. A. Barnes, "Tables of Bias Functions, $8_{1}$ and $B_{2}$, for Variances Based on Finite Samples of Processes with Power Law Spectral Densities," NBS Tech. Note 375, January 1969.

9. J. A. Barnes and D. W. Allan, "A Statistical Model of Flicker Noise," Proc. IEEE, vol. 43, pp. 176-178, February 1966.

10. J. A. Barnes and R. C. Mockler, "The Power Spectrum and its Importance in Precise Frequency Measurements," IRE Trans. on Instrumentation I-9, Pp. 149-155, September 1960.

11. W. R. Bennett, "Methods of Solving Noise Problems," Proc. IRE, May 1956, PP. 609-637.
12. W. R. Bennett, Electrical Noise, New York: MeGraw-Hi11, 1960 .

13. C. Bingham, M. O. Godfrey, and J. W. Tukey, "Modern Techniques of Power Spectrum Estimation," IEEE Trans. AU, vol. 15, PD. 56-66, June 1967.

14. R. B. Blackman and J.W. Tukey, The Measurement of Power Spectra, New York: Dover, 1958.

15. E. O. Brigham and R. E. Morrow, "The Fast Fourier Transform," IEEE Spectrum, vol. 4, no. 12, pp. 63-70, December 1967.

16. J. C. Burgess, "On Digital Spectrum Analysis of Periodic Signals," J. Acoust. Soc. An., vol. 58, no. 3, September 1975, pp. 556-557.

17. R. A. Campbell, "Stability Measurement Techniques in the Frequency Domain," Proc. IEEENASA Symposium on the Definition and Measurement of Short-Term Frequency Stability, NASA SP-80, PP. 231-235, 1965.

18. L. S. Cutler and C. L. Searle, "Some Aspects of the Theory and Measurement of Frequency Fluctuations in Frequency Standards," Proc. IEEE, vol. 54, PD. 136-154, february 1966.

19. W. A. Edson, "Noise in Oscillators," Proc. IRE, vol. 48, pp. 1454-1466, August 1960.

20. C. H. Grauling, Jr. and D. J. Healey, III, "Instrumentation for Measurement of the Short-Term Frequency Stability of Microwave Sources," Proc. IEEE, vol. 54, PP. 249-257, February 1956.

21. D. Halford, "A General Mechanical Model for $|f|^{\alpha}$ Spectral Density Random Noise with Special Reference to Flicker Noise $1 /|f|$," Proc. of the IEEE, vol. 56, no. 3, March 1968, pp. 251-258.

22. H. Hellwig, "Atomic Frequency Standards: A Survey," Proc. IEEE, vol. 63, Pp. 212-229, February 1975.

23. H. Hellwig, "A Review of Precision Oscillators," NBS Tech. Note 662, February 1975.

24. H. Hellwig, "Frequency Standards and Ciocks: A Tutorial Introduction," NBS Tech. Note 616-R, March 1974.

25. Hewlett-Packard, "Precision Frequency Measurements," Application Note 116, July 1969.

26. G. M. Jenkins and D. G. Watts, Spectral Analysis and its Apolications, San Francisco: Holden-Day, 1968.

27. S. L. Johnson, 8. H. Smith, and D. A. Calder, "Noise Spectrum Characteristics of Low-Noise Microwave Tubes and Solid-State Devices," Proc. IEEE, vol. 54, Dp. 258-265, February 1966. 
28. P. Kartaschoff and J. Barnes, "Standard Time and Frequency Generation," Proc. IEEE, vol. 60, pp. 493-501, May 1972.

29. A. L. Lance, W. D. Seal, F. G. Mendoza, and N. W. Hudson, "Automating Phase Noise Measurements in the Frequency Domain," Proc. 31st Annual Symposium on Frequency Control, 1977. pp. $347-358$.

30. D. B. Leeson, "A Simple Model of Feedback Oscillator Noise Spectrum," Proc. IEEE L., vol. 54, pp. 329-330, February 1966.

31. P. Lesage and C. Audoin, "A Time Domain Method for Measurement of the Soectral Density of Frequency fluctuations at Low Fourier Frequencies," Proc. 29th Annual Symposium on Frequency Control, 1975, pp. 394-403.

32. P. Lesage and C. Audoin, "Estimation of the Two-Sample Variance with Limited Number of Data," Proc. 315t Annual Symposium on Frequency Control, 1977, pp. 311-318.

33. P. Lesage and C. Audoin, "Characterization of Frequency Stability: Uncertainty due to Finite Number of Measurements," IEEE Trans on Instrum. and Meas. IM-22, Pp. 157-161, June 1973.

34. W. C. Lindsey and C. M. Chie, "Specification and Measurement of Oscillator Phase Noise Instability," Proc. 31st Annual Symposium on Frequency Control, 1977, pp. 302-310.

35. D. G. Meyer, "A Test Set for Measurement of Phase Noise on High Quality Signal Sources," Proc. IEEE Trans on Instrum. and Meas. IM-19, No. 4, pp. 215-227, November 1970.

36. C. D. Motchenbacher and F. C. Fitchen, Low-Noise Electronic Desian, New York: John Wiley \& Sons, 1973.

37. D. Percival, "Prediction Error Analysis of Atomic Frequency Standards," Proc. 31st Annual Symposium on Frequency Control, 1977 , Pp. 319-326.

38. D. Percival, "A Heuristic Model of Long-Term Clock Behavior," Proc. 30th Annual Symposium on Frequency Control, 1976, PD. 414-419.

39. J. Shoaf, "Specification and Measurement of Frequency Stability," NBS Internal Report 74-396, November 1974

40. J. L. Stewart, "Frequency Modulation Noise in Oscillators," Proc. IRE, vol. 44, pp. 372376, March 1956.

41. R. F. C. Vessot, L. Mueller, and J. Vanier, "The Specification of Oscillator Characteris: tics from Measurements in the Frequency Domain," Proc. IEEE, pp. 199-206, February 1966.
42. F. L. Walls, S. R. Stein, J. E. Gray, and D. J. Giaze, "Design Considerations in State-ofthe-Art Signal Processing and Phase Noise Measurement Systems," Proc. 30th Annual Symposium on Frequency Control, 1976, pp. 269-274.

43. F. Walls and A. Wainwright, "Measurement of the Short-Term Stability of Quartz Crystal Resonators and the Implications for Quartz Oscillator Design and Applications," IEEE Trans. on Instrum. and Meas., pp. 15-20, Maren 1975.

44. G. M. R. Winkler, "A Brief Review of Frequency Stability Measures," Proc. 8th Annual Precise Time and Time Interval (PTI) Applications and Planning Meeting, Nov. 1976, pp. 489-527.

\section{Appendix}

DEGREES OF FREEDOM FOR ALLAN VARIANCE

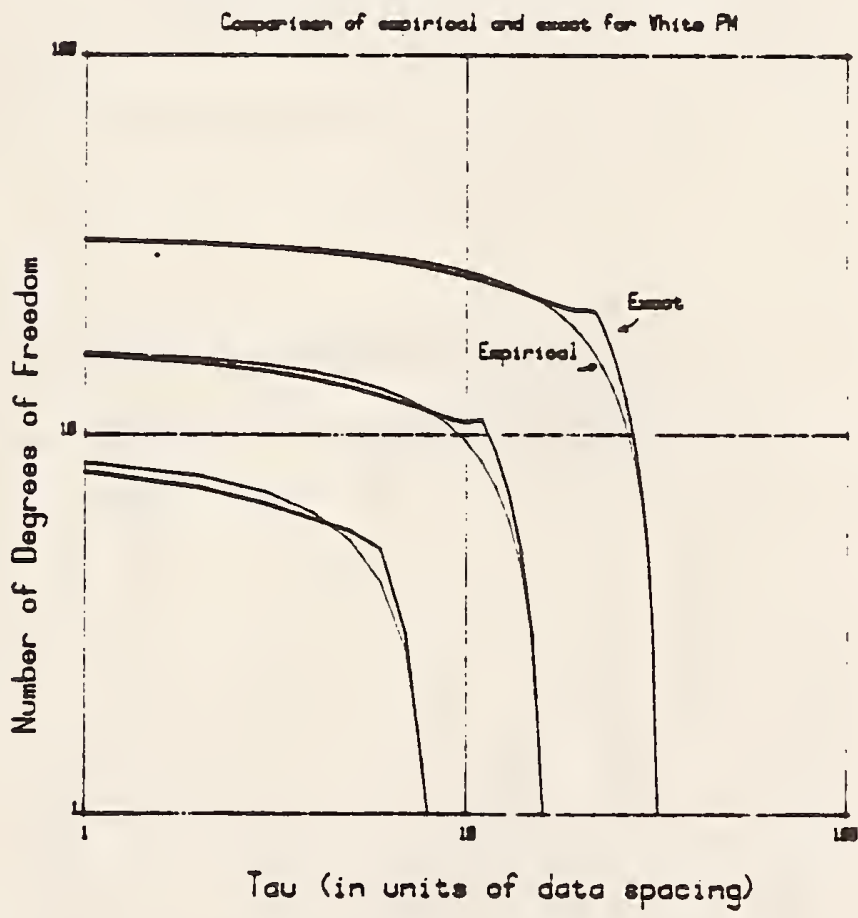



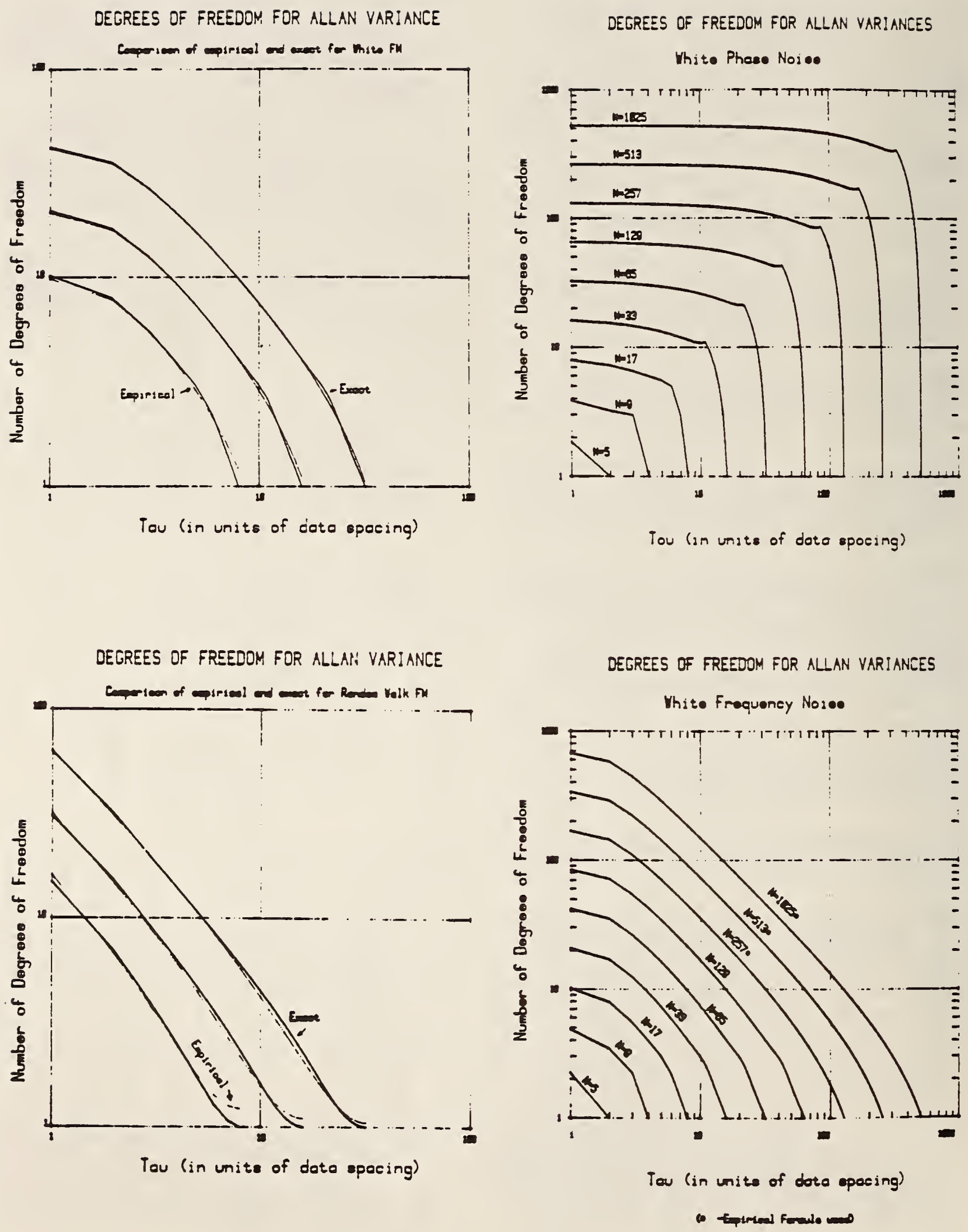
DECREES OF FREEDOM FOR ALLAN VARIANCES

Flackor Phase Modulation

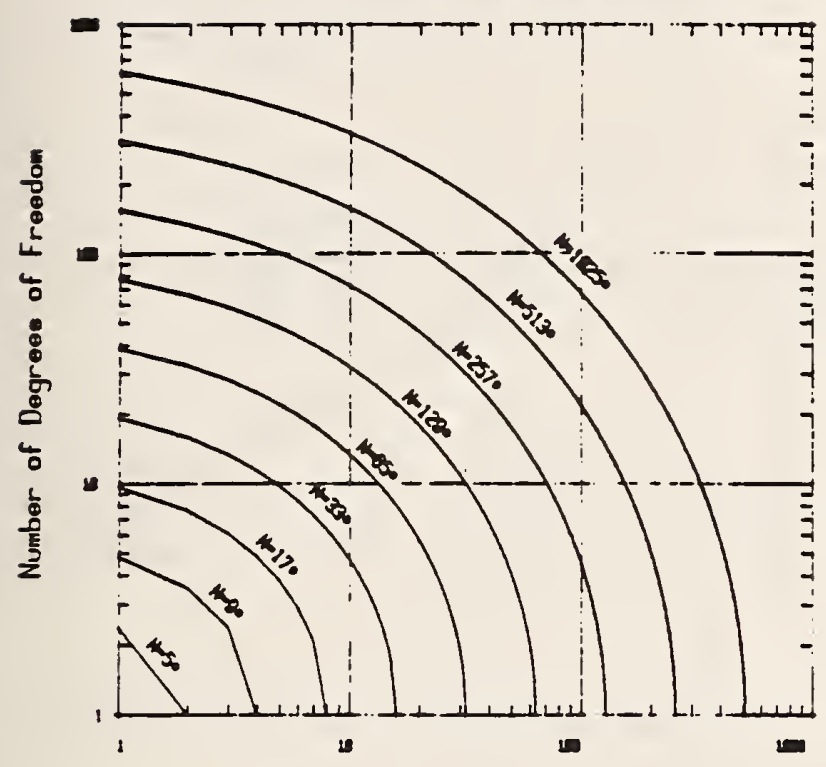

Tou (in unats of doto spocing)

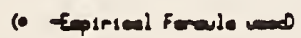

DEGREES OF FREEDOM FOR NLLAN VARIANIEES

Flicker Frequency Modulation

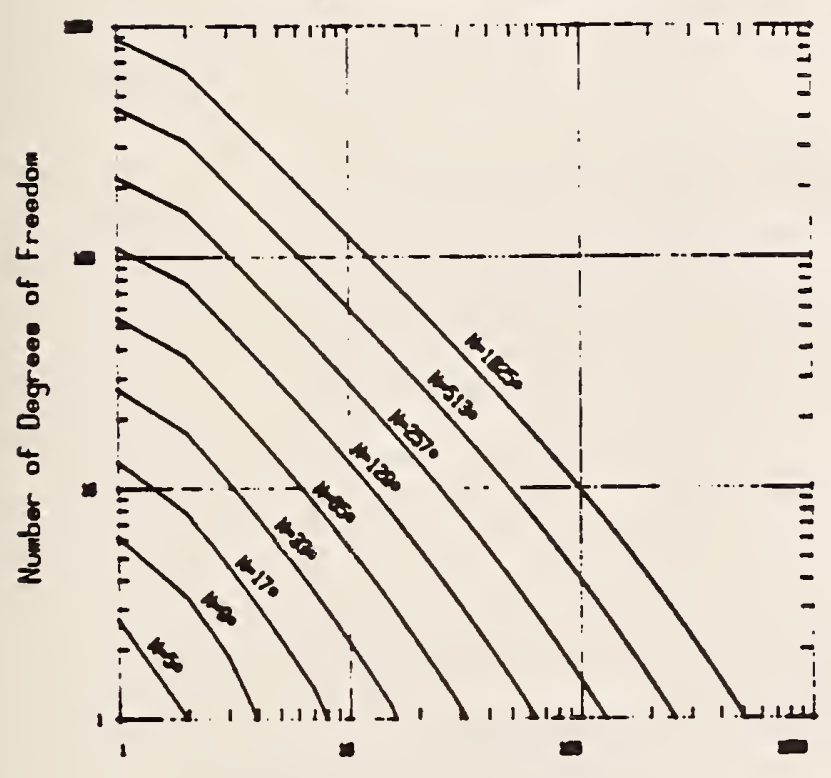

Teu (in unite of deto opoeing)

(1) Eprimad Fonde
OEGREES OF FREEDOM FQR NLLAN VARINICES

Rendon Volk FM

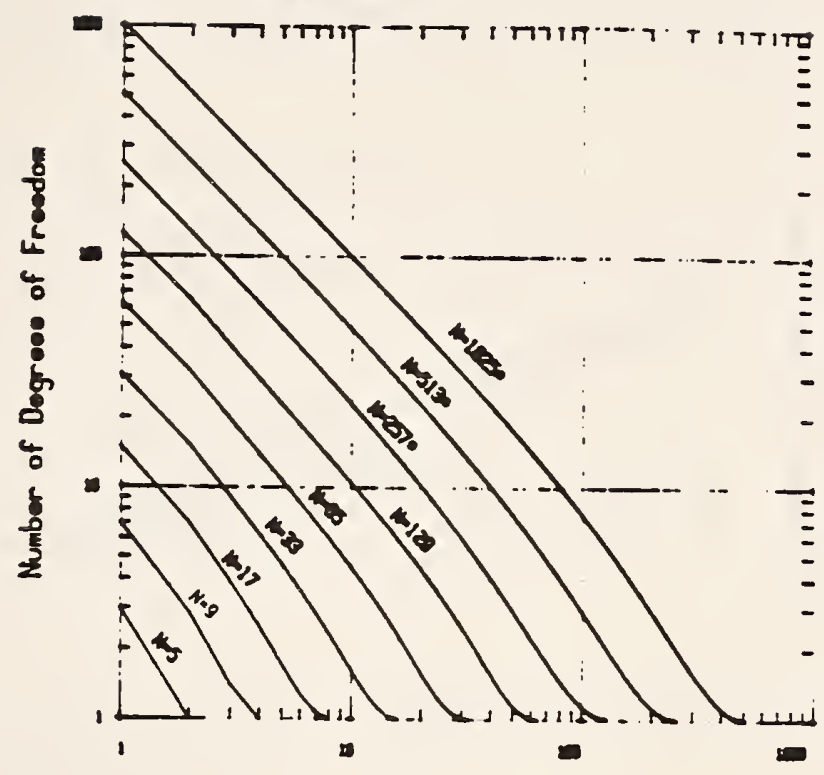

Tou (in unz ts of doto opecing)

6 tpiried fowle 0

CONFIDENCE FACTORS FROM CHI-SJUARE

90\% intorvol.

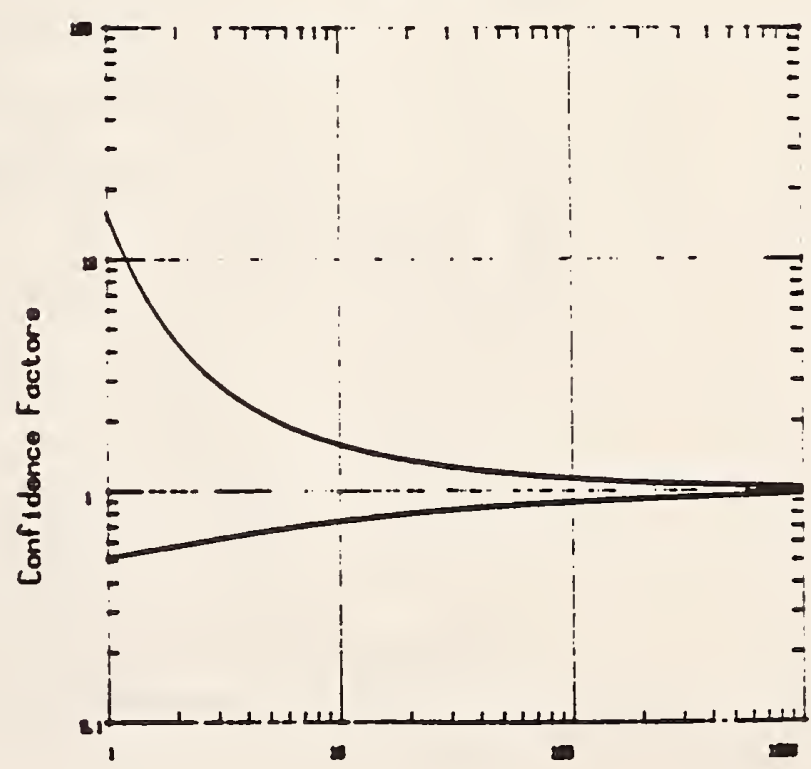

Number of Dograes of Froedom 
LOWER CONFIOENIEE FACIORS

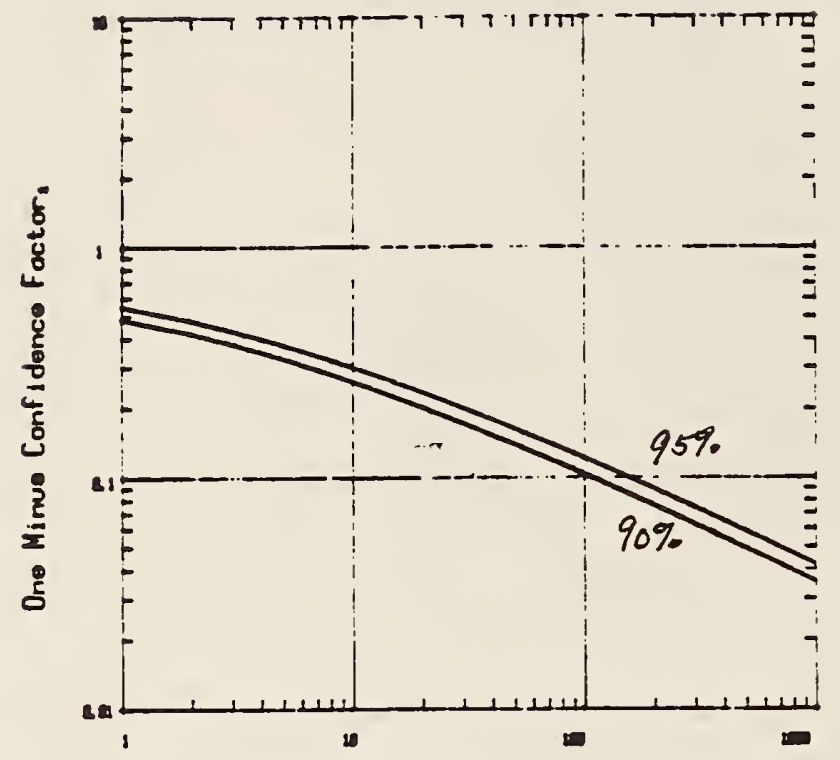

Number of Dogreos of Freodom
UPPER CONFIDENCE FACTORS
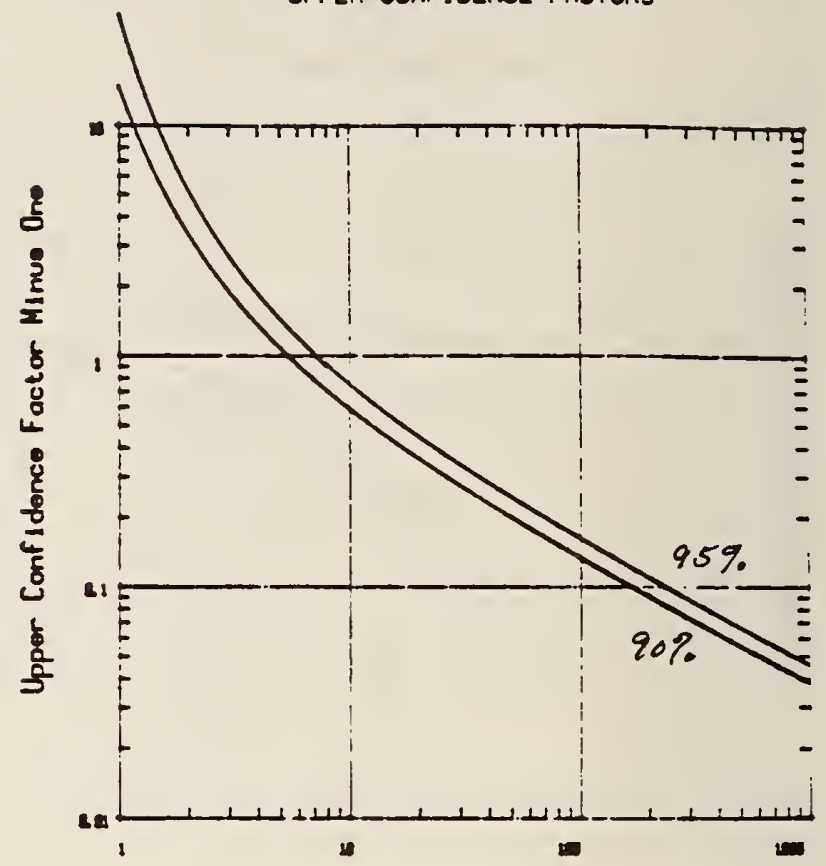

Number of Degrees of Froodom

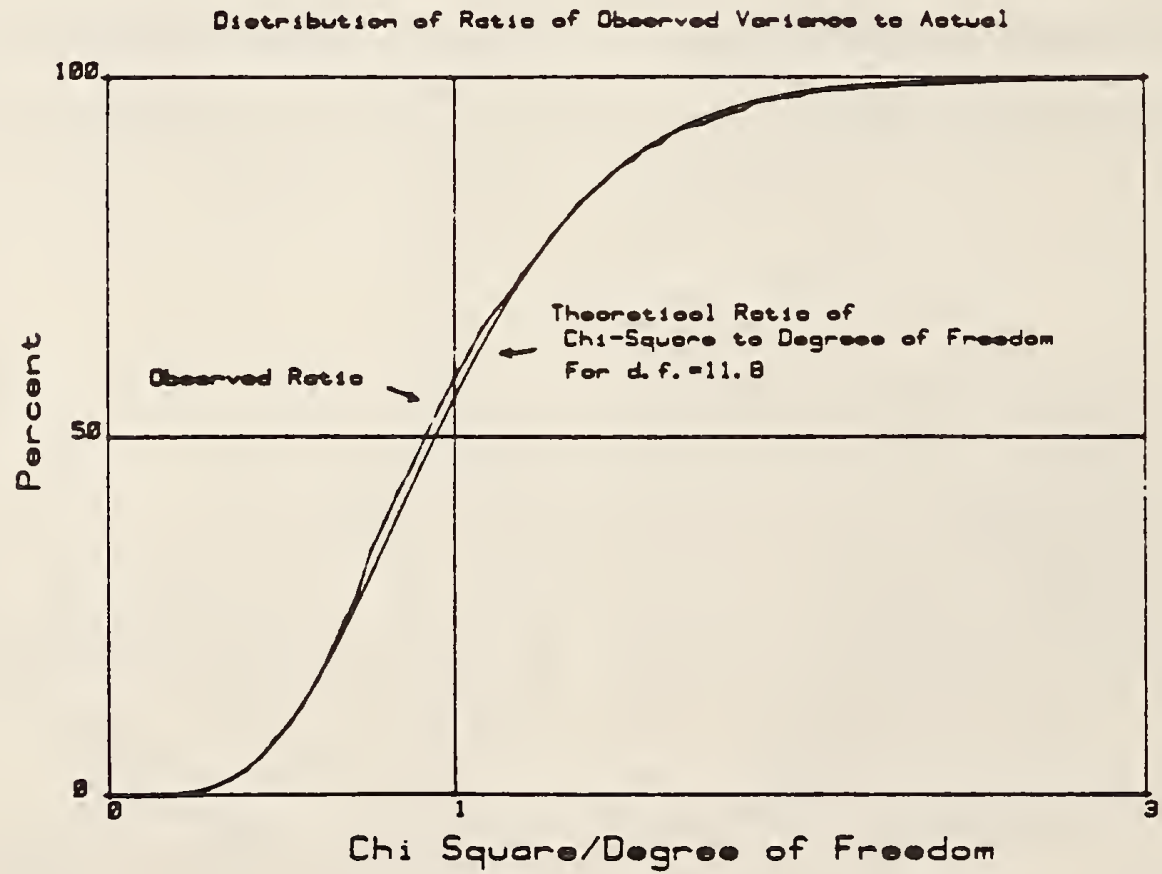


From: Precision Frequency Control, Vol. 2, edited by E.A. Gerber and A. Ballato (Academic Press, New York, 1985), pp. 191-416.

\title{
1 Frequency and Time-Their Measurement and Characterization
}

\author{
Samuel R. Stein \\ Time and Frequency Dirision \\ National Bureau of Standards \\ Boulder, Colorado
}

List of Symbols $\quad 192$

12.1 Concepts, Definitions, and Measures of Stability

12.1.1 Relationship between the Power Spectrum and the Phase Spectrum

12.1.2 The IEEE Recommended Measures of Frequency Stability

12.1.3 The Concepts of the Frequency Domain and the Time Domain

12.1.4 Translation between the Spectral Density of Frequency and the Allan Variance

12.1.5 The Modified Allan Variance

12.1.6 Determination of the Mean Frequency and Frequency Drift of an Oscillator

12.1.7 Confidence of the Estimate and Overlapping Samples

12.1.8 Efficient Use of the Data and Determination of the Degrees of Freedom

12.1.9 Separating the Variances of an Oscillator from the Reterence

12.2 Direct Digital Measurement

12.2.1 Time-Interval Measurements 217

12.2.2 Frequency Measurements 219

122.3 Period Measurements 219

12.3 Sensitivity-Enhancement Methods $\quad 220$

12.3.1 Heterodyne Techniques 220

12.3 .2 Homodyne Techniques 221

12.3.2.1 Discriminator and Delay Line 222

12.3.2.2 Phase-Locked Loop 223

12.3.3 Multiple Conversion Methods 227

12.331 Frequency Synthesis 227

12.33 .2 The Dual-Mixer Time-Difference
Technique

123.3 .3 Frequency Multiplication 231

12.4 Conclusion

PRECISION FREQLENCY CONTROL

ivlurse:

Onildsurs and siandards

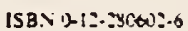




\section{LIST OF SYMBOLS}

\begin{tabular}{|c|c|}
\hline df & Number of degrees of freedom \\
\hline$f$ & Fourier (cycle) frequency \\
\hline$G_{\bullet q}(j \omega)$ & Open-loop transfer function of a phase-locked loop \\
\hline$F(s)$ & Transfer function of the loop filter of a phase-locked loop \\
\hline$H\left(f-f_{0}\right)$ & Transfer function of a filter \\
\hline$K_{\mathrm{d}}$ & Sensitivity of a linear phase detector in volts per radian \\
\hline$P$ & Number of counts accumulated by a time-interval counter \\
\hline$Q$ & $\begin{array}{l}\text { Quality factor of a resonance, equal to the ratio of the stored energy to the } \\
\text { energy lost in one cycle }\end{array}$ \\
\hline$s$ & Independent variable of the Laplace transform \\
\hline$s_{y}^{2}$ & Estimate of the two-sample or Allan variance \\
\hline$S_{h}(f)$ & One-sided power spectral density of the fractional-frequency deviations \\
\hline$S_{0}(f)$ & One-sided power spectral density of the phase deviations \\
\hline$S_{0}^{\mathrm{TS}}(f)$ & Two-sided power spectral density of the phase deviations \\
\hline$S_{0 . \mathrm{e}}^{\text {TS }}(f)$ & Two-sided power spectral density of the phase deviations of the carrier \\
\hline$S_{*}^{\mathrm{TS}}(f)$ & Two-sided power spectral density of the phase deviations of the pedestal \\
\hline$S_{v}^{T s}(f)$ & Two-sided power spectral density of the instantaneous voltage \\
\hline$v_{0}$ & Peak voltage of a signal generator \\
\hline$V_{d}$ & Output voltage of a phase detector \\
\hline$V_{1}$ & $\begin{array}{l}\text { Voltage applied to the tuning element of the voltage-controlled oscillator of a } \\
\text { phase-locked loop }\end{array}$ \\
\hline$V(t)$ & Instantaneous voltage of a signal generator or other device \\
\hline$x(t)$ & $\begin{array}{l}\text { Time deviation required by a signal generator operating at nominal frequency } v_{0} \\
\text { to accumulate phase equal to } \phi(t) \text {. }\end{array}$ \\
\hline$x(f)$ & Fourier transform of $x(t)$ \\
\hline !1t & Instantaneous fractional-frequency offset from nominal \\
\hline$\overline{y_{k}}$ & Mean fractional-frequency offset over the $k$ th interval \\
\hline$x$ & Exponent of $f$ for a power-law spectral density \\
\hline$\Delta r_{0}$ & Frequency uncertainty due to the quantization of measurements \\
\hline 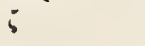 & Damping constant of a phase-locked loop \\
\hline$\mu$ & Exponent of $\tau$ for a power-law Allan variance \\
\hline$v(t)$ & Instantaneous frequency \\
\hline$v_{0}$ & Nominal frequency of a signal generator \\
\hline$\vec{v}\left(t_{1}: t_{2}\right)$ & Mean frequency over the interval $t_{1} \leq t \leq t_{2}$ \\
\hline$v_{\mathrm{H}}$ & Heterodyne frequency or difference frequency between two oscillators \\
\hline$\rho\left(\chi^{2}\right)$ & Probability density of the chi-squared distribution \\
\hline$\sigma ;(. V, T . \tau)$ & $\begin{array}{l}\text { Sample variance of } N \text { fractional-frequency deviations, each averaged over a } \\
\text { period i spaced at intervals } T\end{array}$ \\
\hline$\sigma_{y}^{2}(\tau)$ & The two-sample or Allan variance of the fractional-frequency deviations \\
\hline $\bmod \sigma_{y}^{2}(\tau)$ & Modified Allan variance \\
\hline$\tau$ & A veraging time \\
\hline$\tau_{c}$ & Period of the time base of a counter \\
\hline$\phi(t)$ & Instantaneous phase deviation \\
\hline$x^{2}$ & Chi-squared distribution \\
\hline$\omega$ & Fourier (angular) frequency \\
\hline$\omega_{\mathrm{n}}$ & Natural frequency of a phase-locked loop \\
\hline
\end{tabular}




\subsection{CONCEPTS, DEFINITIONS, AND MEASURES OF STABILITY}

This chapter deals with the measurement of the frequency or time stability of precision oscillators. It is assumed that the average output frequency is determined by a narrow-band circuit so that the signal is very nearly a sine wave. To be specific, it is also assumed that the output is a voltage, which is conventionally (Barnes et al., 1971) represented by the expression

$$
V(t)=\left[V_{0}+\varepsilon(t)\right] \sin \left[2 \pi v_{0} t+\phi(t)\right],
$$

where $V_{0}$ is the nominal peak voltage amplitude, $\varepsilon(t)$ the deviation of amplitude from nominal, $v_{0}$ the nominal fundamental frequency, and $\phi(t)$ the deviation of phase from nominal.

When either specifying or measuring the noise in an oscillator, one must consider the nature of the reference. This may be either a passive circuit such as a narrow-band filter, another similar oscillator, or a set of oscillators, synthesizers, and other signal-generating equipment. A reference with lower noise than the device under test may be available, and in this case the expressions developed in this chapter describe the noise in the oscillator alone. However, a state-of-the-art device will have lower noise than any a vailable reference. In this case all the expressions below refer to the sum of device and reference noise. The most common approach to solving this problem is to compare two or more nearly identical devices. Under most circumstances it is then reasonable to assume that each oscillator contributes half of the measured noise.

The most direct and intuitive method of characterizing the properties of a signal is to determine the two-sided spectrum of $V(t)$, which is denoted $S_{\mathrm{V}}^{\mathrm{TS}}(f)$ (Rutman, 1978). The variable $f$ is called a Fourier frequency and is very closely related to the concept of a modulation frequency. A positive $f$ indicates a frequency above the carrier frequency $v_{0}$, while a negative $f$ indicates a frequency lower than the carrier. Since the noise can in theory modulate the carrier at all possible frequencies, a continuous function is required to describe the modulation of $V(t)$. $S$ is called a spectral density and $S_{\mathrm{V}}^{\mathrm{TS}}(f)$ is the mean-square voltage $\left\langle V^{2}(t)\right\rangle$ in a unit bandwidth centered at $f$. It is proportional to the $f$ power per unit band width delivered by the oscillator to a matched load. The total signal power is proportional to the mean square voltage. which is also called the variance of the signal since the mean value of $V(t)$ is zero. The variance is therefore equal to the two-sided spectral density integrated over all frequencies.

The two-sided spectrum is usually measured by an if spectrum analyzer, a device that functions like a bandpass filter followed by a bolometer, as shown 


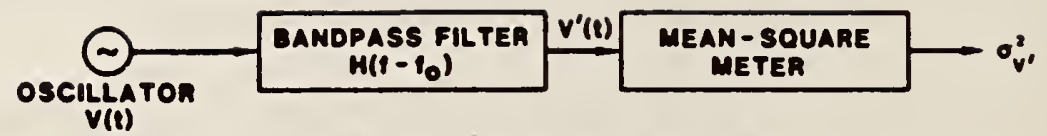

FIG. 12-1 An rf spectrum analyzer. The device produces an output proportional to the mean-square value of the signal passing through a tunable narrow-band filter centered at frequency $\int_{0}$.

in Fig. 12-1. The spectrum of the filtered voltage $V^{\prime}(t)$ is equal to the square of the magnitude of the filter transfer function $H\left(f-f_{0}\right)$ multiplied by the spectrum of the input signal (Cutler and Searle, 1966). The variance of the filtered voltage is obtained from Parseval's theorem:

$$
\sigma_{V}^{2} \cdot\left(f_{0}\right)=\int_{-\infty}^{\infty}\left|H\left(f-f_{0}\right)\right|^{2} S_{\mathrm{v}}^{\mathrm{TS}}(f) d f
$$

If the bandpass filter is sufficiently narrow, so that $S_{\mathrm{v}}^{\text {Ts }}(f)$ changes negligibly over its bandwidth, then Eq. (12-2) may be inverted. With this assumption, the power spectrum is estimated from the measurement using Eq. (12-3):

$$
S_{V}^{\mathrm{TS}}\left(f_{0}\right)=\sigma_{V}^{2} \cdot\left(f_{0}\right) ; B,
$$

where $B=\int_{-}^{\infty}\left|H\left(f^{\prime}-f_{0}\right)\right|^{2} d f^{\prime}$ is the noise bandwidth of the filter and $f_{0}$ its center frequency. Figure 12-2 shows a typical two-sided if spectrum. For many oscillators the spectrum has a Lorentzian shape, that is,

$$
S_{v}^{\mathrm{TS}}(f)=\frac{2\left\langle V^{2}\right\rangle / \pi \Delta f_{3 \mathrm{~dB}}}{1+\left(f\left(\Delta f_{3 \mathrm{~dB}} / 2\right)\right)^{2}} .
$$

The Lorentzian lineshape is completely described by the mean square voltage $\left\langle V^{2}\right\rangle$ and the full width at half maximum $\Delta f_{3 d B}$.

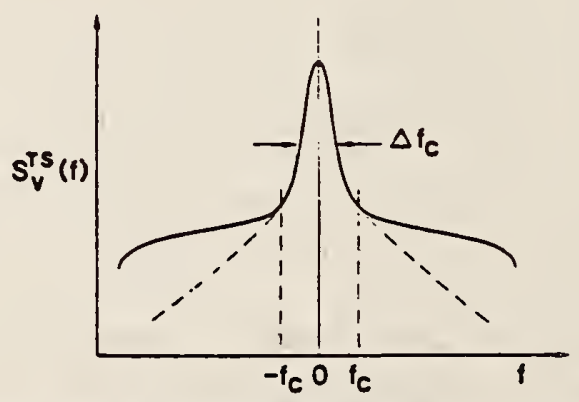

FIG. 12-2 The of spectrum of a signal. It is often useful to divide the spectrum into the carrier and the noise pedestal. The spectral density of the carrier exceeds that of the noise pedestal for Fourier frequencies smaller in magnitude than $f_{e}$. 


\subsubsection{Relationship between the Power Spectrum and the Phase Spectrum}

The power spectrum differs from a delta function $\delta(f)$ due to the presence of the amplitude- and phase-noise terms, $\varepsilon(t)$ and $\phi(t)$, respectively, inciuded in Eq. (12-1). Usually the noise modulation separates into two distinct components, so that one observes a very narrow feature called the carrier above the level of a relatively broad pedestal. The frequency that separates the carrier and pedestal is denoted $f_{c}$. Below this frequency the spectral density of the carrier exceeds that of the pedestal. Assuming that the amplitude noise is negligible compared to the phase noise and that the phase modulation is small, the relationship between the power and phase spectra is given by (Walls and DeMarchi, 1975)

$$
S_{V}^{T S}(f) \cong \frac{V_{0}^{2}}{2} e^{-I\left(S_{c}\right)} \times \begin{cases}\delta(f), & \text { if }-f_{c}<f<f_{c}, \\ S_{\phi}^{T S}(f), & \text { otherwise. }\end{cases}
$$

$S_{\phi}^{\text {TS }}(f)$ is the two-sided spectrum of the phase fluctuations, which divides into a carrier component $S_{\phi . c}^{\text {TS }}(f)$ and a pedestal component $S_{\phi . p}^{\mathrm{TS}}(f) ; I\left(f_{\mathrm{c}}\right)$ is given by

$$
I\left(f_{c}\right)=\int_{f_{c}}^{x} S_{\phi . p}(f) d f \simeq \int_{f_{c}}^{x} S_{\phi}(f) d f
$$

since above $f_{\mathrm{c}}$ the pedestal dominates the noise spectrum. The variance of the carrier is equal to $\left(V_{0}^{2} / 2\right) e^{-l\left(U_{c}\right)}$, with the remaining variance in the pedestal. If $\Delta v_{p}$ is the width of the pedestal and $\Delta v_{c}$ the width of the carrier, then the power density in the carrier is equal to that in the pedestal when $I\left(f_{c}\right)=$ in $\left(\Delta v_{p} / \Delta v_{c}\right)$. For the pedestal, one may use the 3-dB linewidth for $\Delta v_{p}$ provided that $\int_{f_{c}}^{x} S_{\phi}(f) d f<\ln 2$. Otherwise the pedestal width is estimated from $\int_{\Delta v / 2}^{x} S_{\phi}(f) d f=\ln 2$. For the carrier, the linewidth is estimated by calculating $\int_{\Delta v_{c} / 2}^{\infty} S_{\phi . c}(f) d f=\ln 2$.

The foregoing analysis makes it possible to draw certain conclusions concerning detection of the carrier. We use $\int_{p} d f$ to denote the integral over the phase noise pedestal. If $\int_{p} S_{\phi}(f) d f<\ln 2$, then the carrier may be resolved irrespective of detector bandwidth. When $\ln \left(\Delta v_{p} / \Delta v_{c}\right)>\int_{p} S_{\phi}(f) d f>\ln 2$, the carrier may be resolved by restricting the detection bandwidth. But when $\int_{p} S_{\phi}(f) d f>\ln \left(\Delta v_{p} / \Delta v_{c}\right)$, the carrier can no longer be distinguished from the pedestal since its spectral density is smaller.

\subsubsection{The IEEE Recommended Measures of Frequency Stability}

By the mid-1960s the problem of the specification of precision oscillators had become extremely important, but there was very little uniformity among manufacturers, metrologists, and applications engineers in the methods of 
performing measurements or the description of measurement results. This situation was complicated by the difficulty of comparing the various descriptions. A measure of stability is often used to summarize some important feature of the performance of the standard. It may therefore not be possible to translate from one measure to another even though the respective measurement processes are fully described and all relevant parameters are given. This situation resulted in a strong pressure to achieve a higher degree of uniformity.

In order to reduce the difficulty of comparing devices measured in separate laboratories, the IEEE convened a committee to recommend uniform measures of frequency stability. The recommendations made by the committee are based on the rigorous statistical treatment of ideal oscillators that obey a certain model (Barnes et al., 1971). Most importantly, these oscillators are assumed to be elements of a stationary ensemble. A random process is stationary if no translation of the time coordinate changes the probability distribution of the process. That is, if one looks at the ensemble at one instant of time, then the distribution in values for a process within the ensemble is exactly the same as the distribution at any other instant of time. The elements of the ensemble are not constant in time, but as one element changes value other elements of the ensemble assume previous values. Thus, it is not possible to determine the particular time when the measurement uas made.

The stationary noise model has been adopted because many theoretical results, particularly those related to spectral densities, are valid only for this case. It is important for the statistician to exercise considerable care since experimentally one may measure quantities approximately equal to either the instantaneous frequency of the oscillator or the instantaneous phase. But the ideal quantities approximated by these measurements may not both be stationary. The instantaneous angular frequency is conventionally defined as the time derivative of the total oscillator phase. Thus,

$$
\omega(t)=\frac{d}{d t}\left[2 \pi v_{0} t+\phi(t)\right]
$$

and the instantaneous frequency is written

$$
v(t)=v_{0}+\frac{1}{2 \pi} \frac{d \phi}{d t} .
$$

For precision oscillators. the second term on the right-hand side is quite small, and it is useful to define the fractional frequency

$$
y(t)=\frac{v(t)-v_{0}}{v_{0}}=\frac{1}{2 \pi v_{0}} \frac{d \phi}{d t}=\frac{d x}{d t},
$$


where

$$
x(t)=\phi(t) / 2 \pi v_{0}
$$

is the phase expressed in units of time. Alternatively, the phase could be written as the integral of the frequency of the oscillator:

$$
\phi(t)=\phi_{0}+\int_{0}^{t} 2 \pi\left[v(\theta)-v_{0}\right] d \theta .
$$

However, the integral of a stationary process is generally not stationary. Thus, indiscriminate use of Eqs. (12-7) and (12-11) may violate the assumptions of the statistical model. This contradiction is avoided when one accounts for the finite bandwidth of the measurement process. Although a more detailed consideration of the statistics goes beyond the scope of this treatment, it is very important to keep in mind the assumption that lie behind the statistical analysis of oscillators. In order to analyze the behavior of real oscillators, it is necessary to adopt a model of their performance. The model must be consistent with observations of the device being simulated. To make it easier to estimate the device parameters, the models usually include certain predictable features of the oscillator performance, such as a linear frequency drift. A statistical analysis is useful in estimating such parameters to remove their effect from the data. It is just these procedures for estimating the deterministic model parameters that have proved to be the most intractable. A substantial fraction of the total noise power often occurs at Fourier frequencies whose periods are of the same order as the data length or longer. Thus, the process of estimating parameters may bias the noise residuals by reducing the noise power at low Fourier frequencies. A general technique for minimizing this problem in the case of oscillators actually observed in the laboratory is discussed below.

It has been suggested that measurement techniques for frequency and time constitute a hierarchy (Allan and Daams, 1975), with the measurement of the total phase of the oscillator at the peak. Although more difficult to measure with high precision than other quantities, the total phase has this status owing to the fact that all other quantities can be derived from it. Furthermore, missing measurements produce the least deleterious effect on a time series consisting of samples of the total phase. Gaps in the data affect the computation of various time-dependent quantities for times equal to or shorter than the gap length, but have a negligible effect for times much longer than the gap length. The lower levels of the hierarchy consist of the time interval, frequency, and frequency fluctuation. When one measures a quantity somewhere in this hierarchy and wishes to obtain a higher quantity, it is necessary to integrate one or more times. In this case the problem of missing data is quite serious. For example, if frequency is measured and one wants to 
know the time of a clock, one needs to perform the integration in Eq. (12-11). The missing frequency measurements must be bridged by estimating the average frequency over the gap, resulting in a time error that is propagated forever. Thus, it is preferable to always make measurements at a level of the measurement hierarchy equal to or above the level corresponding to the quantity of principle interest. In the past this was rather difficult to do. Measurement systems constructed from simple commercial equipment suffered from dead time, that is, they were inactive for a period after performing a measurement. To make matters worse, methods for measuring time or phase had considerably worse noise performance than methods for measuring frequency. As a result, many powerful statistical techniques were developed to cope with these problems (Barnes, 1969: Allan, 1966). The effect of dead time on the statistical analysis has been determined (Lesage and Audoin, 1979b). Other techniques have been developed to combine short data sets so that the parameters of clocks over long periods of time could be estimated despite missing data (Lesage, 1983). The rationale for these approaches is considerably diminished today. Low-noise techniques for the measurement of oscillator phase have been developed. Now, commercial equipment is capable of measuring the time or the total phase of an oscillator with very high precision. Other equipment exists for measuring the time interval. These devices use the same techniques that were previously employed for the measurement of frequency and are very competitive in performance.

The proliferation of microcomputers and microprocessors has had an equally proiound effect on the field of time and frequency measurement. There has been a dramatic increase in the ability of the metrologist to acquire and process digital data. Many instruments are available with suitable standard interfaces such as IEEE-583 or CAMAC (IEEE, 1975) and IEEE.488 (IEEE, 1978). As a result, there has been a dramatic change in direction away from analog signal processing toward the digital, and this process is accelerating daily. Techniques once used only by national standards laboratories and other major centers of clock development and analysis are now widespread. Consequently, this chapter will focus first on the peak of the measurement hierarchy and the use of digital signal processing. But the analysis is directed toward estimating the traditional measures of frequency stability. Considerable attention will be paid to problems associated with estimating the confidence of these stability measures and obtaining the maximum information from available data.

The IEEE has recommended as its first measure of frequency stability the one-sided spectral density $S_{y}(f)$ of the instantaneous fractional-frequency fluctuations $y(t)$. It is simply related to the spectral density of phase fluctuations since differentiation of the time-dependent functions is equivalent to 
multiplication of their Fourier transforms by $j \omega:$

$$
S_{y}(f)=\left(f / v_{0}\right)^{2} S_{\phi}(f)=(2 \pi f)^{2} S_{x}(f) .
$$

Section 12.1.1 on the relationship between the power spectrum and the phase spectrum described the analog method for the measurement of a spectral density. If a voltage $V$ is the output of the oscillator, then the result of the measurement is proportional to the of power spectral density. But if the voltage were proportional to the frequency or phase of the oscillator, then the result of the measurement would be proportional to the spectral density of the frequency or phase. The most common units of $S_{\phi}(f)$ are radians squared per hertz.

Alternatively, the spectral density can be obtained by digital analysis of the signal. For example, the quantity $S_{x}(f)$ can be calculated from the Fourier transform of $x(t)$. The relevant continuous Fourier-transform pair is defined as follows:

$$
X(f)=\int_{-\infty}^{x} x(t) e^{-j 2 \pi f t} d t
$$

and

$$
x(t)=\frac{1}{2 \pi} \int_{-x}^{x} X(f) e^{j z \pi f t} d f .
$$

However, one does not generally have continuous knowledge of the phase of the oscillator. Since it is relatively easy to measure $x(t)$ at equally spaced time intervals, we assume the existence of the series $x_{l}$, where $x_{l}=x(l \tau)$ for integer values of $l$. The discrete Fourier transform is defined by analogy to the continuous transform (Cochran et al., 1967):

$$
X(f)=\sum_{l=-\infty}^{\infty} x(l) e^{-j 2 \pi f l} .
$$

In practice the time series has finite length $T$ consisting of $N$ intervals of length $\tau$, and it is not possible to compute the infinite sum. Nevertheless, it remains possible to compute a spectrum that is not continuous in $f$ but rather has resolution $\Delta f$, where

$$
\Delta f=1 / T=1 / N \tau \text {. }
$$

The need to sum over all values of the index $l$ is removed by assuming that the function $x(t)$ repeats itself with period $T$. The resulting spectrum contains no information on the spectrum at Fourier frequencies less than $1 / T$. Truncation of the time series also introduces spurious effects due to the turn-on and turnoff transients. These problems can be minimized through the use of a window function. The computed spectrum is actually the square of the magnitude of 
the window function multiplied by the desired spectrum. The use of a window function reduces the variance of the spectrum estimate at the expense of smearing out the spectrum to a small degree. With these changes but no window function, we arrive at the discrete finite transform

$$
X(n \Delta f)=\frac{1}{N} \sum_{k=0}^{N-1} x(k \tau) e^{-j 2 \pi n \Delta s k \tau} .
$$

The spectral density of $x(t)$ is computed from Eq. (12-17) by squaring the real and imaginary components, adding the two together, and dividing by the total time $T$ :

$$
S_{x}(m \Delta f)=\frac{\{R[X(m \Delta f)]\}^{2}+\{I[X(m \Delta f)]\}^{2}}{T} .
$$

The digital method of estimating spectral densities has many advantages over analog signal processing. Most important is the fact that it may be computed from any set of equally spaced samples of a time series. As a result, the technique is compatible with other methods of characterizing the signal, that is, the sampled data can be stored and processed using a variety of algorithms. In addition, each record of length $T$ produces a single estimate of the spectrum for each of the $N$ frequencies $\Delta f, 2 \Delta f, \ldots, N \Delta f$. It is therefore possible to estimate the entire spectrum much more quickly using the digital technique than it would be using analog methods. The fast Fourier transform, a very efficient algorithm for the computation of the discrete finite transform, has opened the way to versatile self-contained, commercial spectrum analysis. It is also very straightforward to compute the spectrum from data acquired by computerized digital data acquisition systems.

A result of the finite sampling rate is that the upper frequency limit of the digital spectrum analysis is $1 / 2 \tau$, called the Nyquist frequency (Jenkins and Watts, 1968). Power in the signal being analyzed that is at frequencies higher than the Nyquist frequency affects the spectrum estimate for lower frequencies. This problem is called aliasing. The out-of-band signal is rejected by only approximately $6 \mathrm{~dB}$ per octave above the Nyquist frequency. Thus, when significant out-of-band signals exist, they must be reduced by analog filtering. One or more low-pass filters are usually sufficient for this purpose.

As its second measure of frequency stability, the IEEE recommended the sample variance $\sigma_{y}^{2}(\tau)$ of the fractional-frequency fluctuations. It is a measure of the variability of the average frequency of an oscillator between two adjacent measurement intervals. The average fractional-frequency deviation $\bar{i}_{k}$ over the time interval from $t_{k}$ to $t_{k}+\tau$ is defined as

$$
\bar{y}_{k}=\frac{1}{\tau} \int_{i_{k}}^{t_{k}+\tau} \nu(t) d t
$$




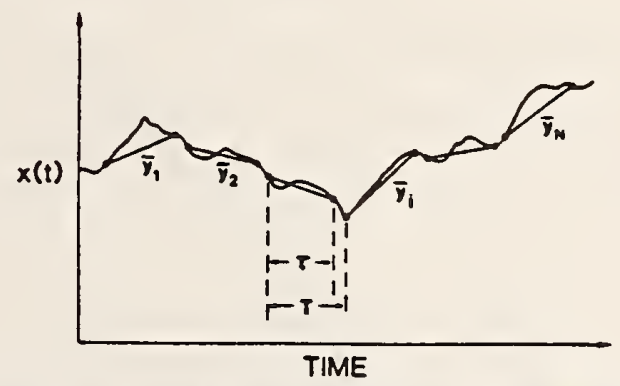

FIG. 12-3 Measurement process for the computation of the sample variance. The phase difference between two oscillators is plotted on the ordinate. The measurement yields a set of frequencies averaged over equal intervals $\tau$ separated by dead time $T-\tau$.

from which it follows that

$$
\bar{y}_{k}=\frac{x\left(t_{k}+\tau\right)-x\left(t_{k}\right)}{\tau} .
$$

The quality $\tau$ is often referred to as the sampling time or the averaging time. Equations (12-19) and (12-20) are not the only way to define mean frequency. but they are the simplest. Other definitions lead to alternative measures of stability that may have desirable properties.

Suppose that one has measured the time or frequency fluctuations between a pair of precision oscillators and a stability analysis is desired. The process is illustrated in Fig. 12-3. These are $N$ values of the fractional frequency $\bar{y}_{i}$. Each one is measured over a time $\tau$, and measurements are repeated after intervals of time $T$. If the measurement repetition time exceeds the averaging time, then there is a dead time equal to $T-\tau$ between each frequency measurement, during which there is no information available.

There are many ways to analyze these data. A fairly general approach is the $N$-sample variance defined by the relation

$$
\left\langle\sigma_{y}^{2}(N, T, \tau)\right\rangle=\left\langle\frac{1}{N-1} \sum_{n=1}^{N}\left(\bar{y}_{n}-\frac{1}{N} \sum_{k=1}^{N} \bar{y}_{k}\right)^{2}\right\rangle,
$$

where the angle brackets denote the infinite time average. Frequently, Eq. (12-21) does not converge as $N \rightarrow x$, since some noise processes in oscillators diverge rapidly at low Fourier frequencies. This implies that the precision with which one estimates the variance does not improve simply as the sample size is increased. For this reason, the two-sample variance with no dead time is preferred. Also called the Allan variance, it converges for all the major noise types observed in precision oscillators. It may be written as

$$
\sigma_{y}^{2}(\tau)=\left\langle\frac{1}{2}\left(\bar{y}_{k-1}-\bar{y}_{k}\right)^{2}\right\rangle \text {. }
$$




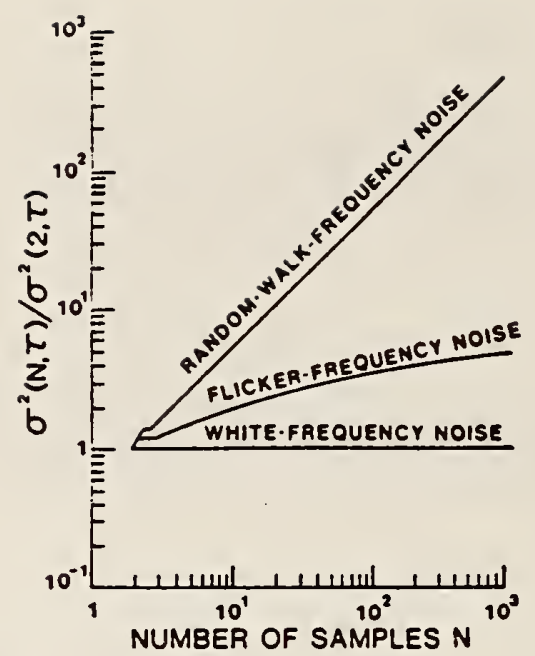

FIG. $12.4 \quad N$-sample variance versus Allan varıance. The I wo-sample variance converges for the important types of noise observed in frequency standards but the ratio of the Iraditional variance 10 the two-sample variance is an increasing funclion of sample size for flicker frequency noise and random-walk frequency noise.

The dependence of the classical variance on the number of samples is shown in Fig. 12-4 for the case of no dead time. The quantity plotted is the ratio of the $N$-sample variance to the Allan variance. Note that $\sigma_{y}^{2}(\tau)$ has the same value as the classical variance for the white-noise frequency modulation. However, the classical variance grows without bound for flicker-frequency and random-walk-frequency noises.

One may combine Eqs. (12-20) and (12-22) to obtain an equation for $\sigma_{y}(\tau)$ in terms of the time-difference or time-deviation measurements:

$$
\sigma_{y}^{2}(\tau)=\left\langle\frac{1}{2} \tau^{-2}[x(t+2 \tau)-2 x(t+\tau)+x(t)]^{2}\right\rangle .
$$

$N$ discrete time readings may be used to estimate the variance

$$
\sigma_{y}^{2}(\tau) \cong \frac{1}{2(x-2) \tau^{2}} \sum_{i=1}^{x-2}\left(x_{i+2}-2 x_{i+1}+x_{i}\right)^{2},
$$

where $i$ denotes the number of the measurement in the set of $N$ and the nominal spacing between measurements is $\tau$. Since it has been assumed that there is no dead time between measurements, one can write $\tau$ in Eq. (12-24) as an integer multiple of $\tau_{0}$. that is, $\tau=m \tau_{0}$, where $\tau_{0}$ is the smallest spacing of the data. In this case

$$
\sigma_{y}^{2}\left(m \tau_{0}\right) \cong \frac{1}{2(N-2 m) m^{2} \tau_{0}^{2}} \sum_{i=1}^{N-2 m}\left(x_{i-2 m}-2 x_{i+m}+x_{i}\right)^{2}
$$




\subsubsection{The Concepts of the Frequency Domain and the Time Domain}

Spectral densities are measures of frequency stability in what is called the frequency domain since they are functions of Fourier frequency. The Allan variance, on the other hand, is an example of a time-domain measure. In a strict mathematical sense, these two descriptions are connected by Fourier transform relationships (Cutler and Searle, 1966). However, for many years the inadequacy of measurement equipment created artificial barriers between these two characterizations of the same noise process. As a result, many specialized techniques have been developed to translate between the various measures of stability (Allan, 1966: Burgoon and Fischer, 1978). The preceding sections have demonstrated how easily both types of stability measures can be computed from the same data provided that the measurement process provides complete information. For example, both $\sigma_{y}^{2}\left(m \tau_{0}\right)$ and $S_{x}(m \Delta f)$ can be computed from evenly spaced samples of $x(t)$. However, incomplete information can result from either measurement dead time or interruptions in the data acquisition process. In these cases translation techniques remain valuable.

Both the spectral density and the Allan Variance are second-moment measures of the time series $x(t)$. However, it is only possible to translate unambiguously from the spectral density to the Allan variance, not the reverse. To calculate the spectral density it is necessary to use the autocorrelation function of the phase. The following discussion on power-law noise processes further demonstrates this dichotomy. As we shall see, the Allan variance for a fixed measurement bandwidth does not distinguish between all of the noise processes that are commonly observed in precision oscillators.

\subsubsection{Translation between the Spectral Density of Frequency and the Allan Variance}

The power-law model is most frequently used for describing oscillator phase noise. It assumes that the spectral density of frequency fluctuations is equal to the sum of terms, each of which varies as an integer power of frequency. Thus, there are two quantities that completely specify $S_{y}(f)$ for a particular power-law noise process: the slope on a log-log plot for a given range of $f$ and the amplitude. The slope is denoted by $x$ and therefore $f^{z}$ is the straight line on a log-log plot that relates $S_{y}(f)$ to $f$. The amplitude is denoted $h_{z}$. When we examine a plot of the spectral density of frequency fluctuations, we represent it by the addition of all the power-law processes (Allan, 1966: Vessot et al., 1966) with the appropriate coefficients:

$$
S_{y}(f)=\sum_{x=-x}^{x} h_{x} f^{x}
$$


TABLE 12-1

Correspondence between Common Power-Law Spectral Densities and the Allan Variance

\begin{tabular}{lcl}
\hline \multicolumn{1}{c}{ Noise type } & \multicolumn{1}{c}{$S, f)$} & \multicolumn{1}{c}{$\sigma_{,}^{2}(\tau)$} \\
\hline White phase & $h_{2} f^{2}$ & $3 f_{1} h_{2} /(2 \pi)^{2} \tau^{2}$ \\
Flicker phase & $h_{1} f$ & $\frac{\left[1.038+3 \ln \left(2 \pi f_{1} \tau\right)\right]}{(2 \pi)^{2}} h_{1} \frac{1}{\tau^{2}}$ \\
White frequency & $h_{0}$ & $\frac{1}{2} h_{0}(1 / \tau)$ \\
Flicker frequency & $h_{-1} f^{-1}$ & $2 \ln (2) h_{-1}$ \\
Random-walk frequency & $h_{-2} f^{-2}$ & $\frac{f(2 \pi)^{2} h_{-2} \tau}{}$
\end{tabular}

- Where necessary for convergence the speetral density has been assumed to be zero for frequencies greater than the cutofi frequency $f_{n}$.

This technique is most valuable when only a few terms in Eq. (12-26) are required to describe the observed noise and each term dominates over several decades of frequency. This situation often prevails. Five power-law noise processes (Allan, 1966: Vessot et al., 1966) are common with precision oscillators:

(1) random-walk frequency modulation

$$
\begin{aligned}
& x=-2 \\
& \alpha=-1 \\
& \alpha=0 \\
& \alpha=1 \\
& \alpha=2
\end{aligned}
$$

(3) White frequency modulation

(4) flicker phase modulation

The spectral density of frequency is an unambiguous description of the oscillator noise. Thus, the spectrum can be used to compute the Allan variance (Barnes et al., 1971):

$$
\sigma_{y}^{2}(\tau)=\frac{2}{\left(\pi v_{0} \tau\right)^{2}} \int_{0}^{x} S_{\phi}(f) \sin ^{4}(\pi f \tau) d f .
$$

However, Eq. (12-27) shows that the Allan variance is very sensitive to the high frequency dependence of the spectral density of phase, thereby necessitating a detailed knowledge of the bandwidth-limiting elements in the measurement setup. The integral has been computed for each of the powerlaw noise processes, and the results are summarized in Table 12-1 (Barnes et al., 1971). For $\alpha$ in the range $-2 \leq \alpha \leq 0$, the Allan variance is proportional to $\tau^{\mu}$, where $\mu=-\alpha-1$. When the log of the Allan variance is plotted as a function of the $\log$ of the averaging time, the graph also consists of straightline segments with integer slopes. However, Table 12-1 also shows that even if

See Appendix Note \#8 
the oscillator is reasonably modeled by power-law spectra, it is not practical to distinguish between white phase noise and ficker phase noise from the dependence of the Allan variance on $\tau$. In both cases $\sigma_{y}^{2} \simeq 1 / \tau^{2}$.

\subsubsection{The Modified Allan Variance}

Table 12-1 also shows that the Allan variance has very different bandwidth dependence for white phase noise and fiicker phase noise. Therefore, these noise types have been distinguished by varying the bandwidth of the measurement system. If $x(t)$ were measured, the noise type could be identified by computing the spectrum. However, both the approach of making measurements as a function of bandwidth and the computation of the spectrum can be avoided by calculating a modified version of the Allan variance. The algorithm for this variance has the effect of changing the bandwidth inversely in proportion to the averaging time (Snyder, 1981; Allan and Barnes, 1981).

Each reading of the time deviation $x_{i}$ has associated with it a measurementsystem bandwidth $f_{b}$. Similarly, we can define a software bandwidth $f_{s}=f_{h} / n$, which is $1 / n$ times narrower than the hardware bandwidth. It can be realized by averaging $n$ adjacent $x_{i}$ 's. Based on this idea it is possible to define a modified Allan variance that allows the reciprocal software bandwidth to change linearly with the sample time $\tau$ :

$$
\bmod \sigma_{y}^{2}(\tau)=\frac{1}{2 \tau^{2}}\left\langle\left[\frac{1}{n} \sum_{i=1}^{n}\left(x_{i+2 n}-2 x_{i+n}+x_{i}\right)\right]^{2}\right\rangle \text {, }
$$

where $\tau=n \tau_{0}$. Equation (12-28) reduces to Eq. (12-23) for $n=1$. One can see that $\bmod \sigma_{y}^{2}(\tau)$ is the second difference of three time values, each of which is a nonoverlapping average of $n$ of the $x_{i}$ 's. As $n$ increases the software bandwidth decreases as $f_{\mathrm{b}} / n$.

For a finite data set of $N$ readings of $x_{i}(i=1$ to $N), \bmod \sigma_{y}^{2}(\tau)$ can be estimated from the expression

$$
\bmod \sigma_{y}^{2}(\tau) \cong \frac{1}{2 \tau^{2} n^{2}(N-3 n+1)} \sum_{j=1}^{N-3 n+1} \sum_{i=j}^{n+j-1}\left(x_{i+2 n}-2 x_{i+n}+x_{i}\right)^{2},
$$

which is easy to program but takes more time to compute than the corresponding equation (12-24) for $\sigma_{y}^{2}(\tau)$.

Table 12-2 gives the relationship between the time-domain measure $\bmod \sigma_{y}^{2}(\tau)$ and its power-law spectral counterpart. In the right-hand column are the asymptotic values of the ratio of the modified Allan variance to the Allan variance. It is clear from the table that $\bmod \sigma_{y}^{2}(\tau)$ is very useful for white

- See Appendix Note * 9 
TABLE $12 \cdot 2$

Correspondence beiween Cummun Power-Law Spectral Densities and the Modified Allan Variance

\begin{tabular}{llll}
\hline \multicolumn{1}{c}{ Noise type } & \multicolumn{1}{c}{$S_{\lambda}(S)$} & \multicolumn{1}{c}{$\bmod \sigma_{1}^{2}(\tau)$} & $\bmod \sigma_{2}^{2} ; \sigma^{2}$ \\
\hline White phase & $h_{2} f^{2}$ & $h_{2} \frac{3 f_{1}}{n(2 \pi)^{2}} \frac{1}{\tau^{2}}$ & $n$ \\
Flicker phase & $h_{1} f$ & $h_{1} \frac{\left[1.038+3 \ln \left(2 \pi f_{1} \tau\right)\right]}{(2 \pi)^{2}} \frac{1}{\tau^{2}}$ & 1 \\
White frequency & $h_{0}$ & $h_{0} \cdot 4 \tau$ & 0.5 \\
Flicker frequency & $h_{-1} f^{-1}$ & $h_{-1}(0.936)$ & 0.674 \\
Random-walk frequency & $h_{-2} f^{-2}$ & $h_{-2}(5.42)$ & 0.824 \\
\hline
\end{tabular}

- Where necessary the spectral density has been assumed to be zero for frequencies greater than the cutoff frequency $f$. The constant $n$ ir the number of adjacent phase values that are averaged to produce the bandwidth reduction. The values in the last iwo columns are for the asymptotic limit $n \rightarrow x$. In practice, $n$ only needs to be 10 or larger before the asymptotic limit is approached within a few percent. When $n=1$ the ratio in the last column is 1 in all cases.

phase modulation and flicker phase modulation, but for $x \leq 1$ the conventional Allan variance gives both an easier-to-interpret and an easier-tocalculate measure of stability.

It is interesting to make a graph of $z$ versus $\mu$ for both the ordinary Allan variance and the modified Allan variance, such as the one shown in Fig. 12-5.

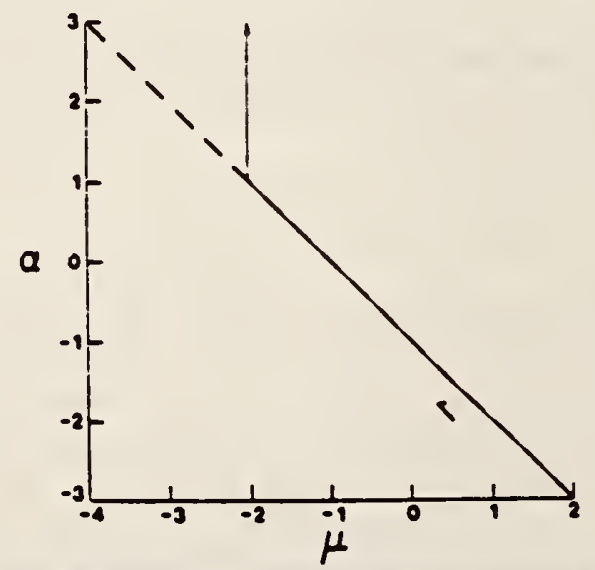

FIG. 12.5 Relationship between a power-law spectral density whose slope on a log-log plot is $x$ and the corresponding sample variance whose slope on a $\log -\log$ plot is $\mu$. The solid line describes the behavior of the Allan variance, while the dashed line shows the advantage of the modified Allan variance for white phase noise and flicker phase noise. 
This graph allows one to determine power-law spectra for noninteger as well as integer values of $\alpha$. In the asymtotic limit the equation relating $\mu$ and $\alpha$ for the modified Allan variance is

$$
\alpha=-\mu-1 \text { for }-3<x<3 .
$$

\subsubsection{Determination of the Mean Frequency and Frequency Drift of an Oscillator}

Before the techniques of the previous four sections can be meaningfully applied to practical measurements, it is necessary to separate the deterministic and random components of the time deviation $x(t)$. Suppose, for example, that an oscillator has significant drift, such as might be the case for a quartz crystal oscillator. With no additional signal processing, the Allan variance would be proportional to $\tau^{2}$. The variance of the Allan variance would be very small, further demonstrating that deterministic behavior has been improperly described in statistical terms and the oscillator's predictability is much better than the Allan variance indicated. Unfortunately, it is difficult to estimate the oscillator's deterministic behavior without introducing a bias in the noise at Fourier frequencies comparable to the inverse of the record length. In practice, it has been sufficient to consider two deterministic terms in $x(t)$ :

$$
x(t)=x_{0}+\left(\Delta v_{i}^{\prime} v_{0}\right) t+\frac{1}{2} D t^{2}+x_{1}(t)
$$

The first term on the right-hand side is the synchronization error. The second term is due to imperfect knowledge of the mean frequency and is sometimes called syntonization error. The quadratic term, which results from frequency drift, is the most difficult problem for the statistical analysis because the Allan variance is insensitive to both synchronization and syntonization errors.

For white noise, the optimum estimate of the process is the mean. Therefore, a general statistical procedure that can be followed is to filter the data until the residuals are white (Allan et al., 1974; Barnes and Allan, 1966). For example, at short times the frequency fluctuations of atomic clocks are usually white. Taking the first difference of Eq. (12-31), we find that

$$
\bar{y}(t)=\frac{\Delta v}{v_{0}}+D t+\frac{x_{1}(t+\tau)-x_{1}(t)}{\tau},
$$

and a linear least square fit to the frequency data yields the optimum estimate of $\Delta v$. However, the drift in atomic clocks is generally so small that the value obtained for $D$ will not be statistically significant when $\tau$ is small enough to 
satisfy the assumption of white frequency noise. Thus, we are led to consider the first difference of the frequency,

$$
\frac{\bar{y}(t+\tau)-\bar{y}(t)}{\tau}=D+\frac{x_{1}(t+2 \tau)-2 x_{1}(t+\tau)+x_{1}(t)}{\tau^{2}} .
$$

Many atomic clocks are dominated by random walk of frequency noise for long averaging times. Thus, the first difference of the frequency data (the second difference of the phase data) is white, and the optimum estimate of the drift is just the simple mean. If instead, a linear least square fit were removed from the frequency data in this region of $\tau$, then the random-walk residuals would be biased, and it is likely that an optimistic estimate of $\sigma_{y}(\tau)$ would be obtained.

The optimum procedure would be different if the dominant noise type were flicker of frequency, rather than random walk. But there is no simple prescription that can be followed to estimate the drift in that case. Fortunately, a maximum likelihood estimate of the prameters for some typical cases has shown that the mean second difference of phase is still a good estimator of frequency drift in the sense that it introduces negligible bias in the Allan variance. Thus, in practice there is a simple prescription for computing the Allan variance in the presence of significant drift. Starting with the phase data, one forms the second difference and uses the simple average to estimate the mean. The value of $\tau$ chosen for creating these second differences must be long enough so that the predominant noise process is random-walk frequency modulation. After subtracting this estimate, the second-difference data is integrated twice to recover phase data with drift removed, and further analysis, including the computation of the Allan variance, may proceed. Figures 12-6 through 12-10 illustrate the estimation of drift. The quadratic dependence of the phase data in Fig. 12-6 nearly obscures the noise. The first difference of this data produces the nearly linear frequency dependence shown in Fig. 12-7, and the second difference produces the residuals shown in Fig. 12-8, which appear to be nearly white. Rigorous statistical analysis of this data indicates that the first difference of the frequency is indeed white with $90 \%$ confidence. Next, the mean frequency difference is subtracted. Then the residuals of Fig. 12-8 are integrated twice, and the result is the estimate of the phase deviation with drift removed shown in Fig. 12-9. Fig. 12-10 illustrates the Allan variance of this data calculated by three techniques. The squares were computed from the data of Fig. 12-6, while the open circles were computed following the recommended procedure for estimating the drift. The validity of the approach is illustrated by the black dots, which are the result of a statistically optimum parameter estimation procedure. 


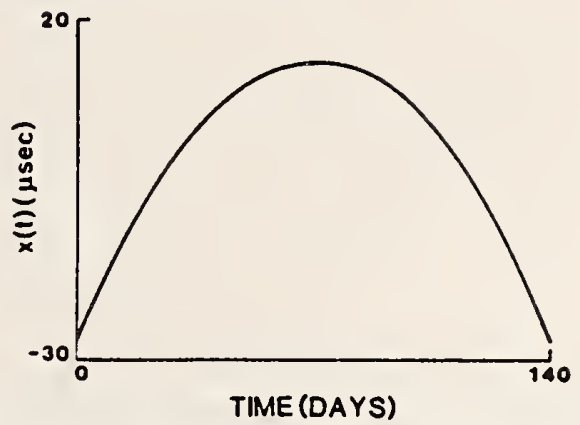

FIG. 12-6 Measured phase difference between a frequency standard and a reference during a 140-day experiment. The nearly quadratic form of the data effectively obscures the noise.

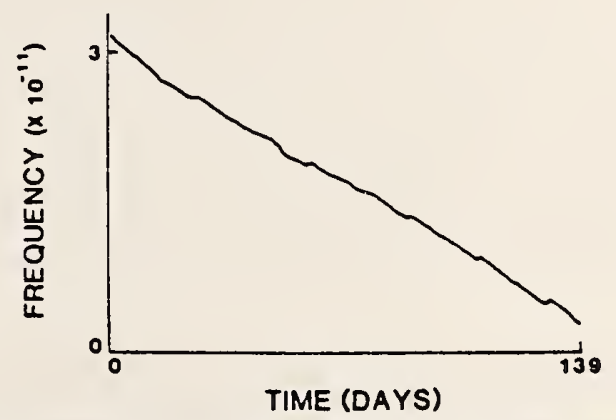

FIG. 12-7 One-day frequency averages obtained by taking the first differences of the data in Fig. 12-6. The ordinate is the fractional difference of the daily frequency from a nominal value. The nearly linear change in frequency with time is apparent, although the random deviations are visible.

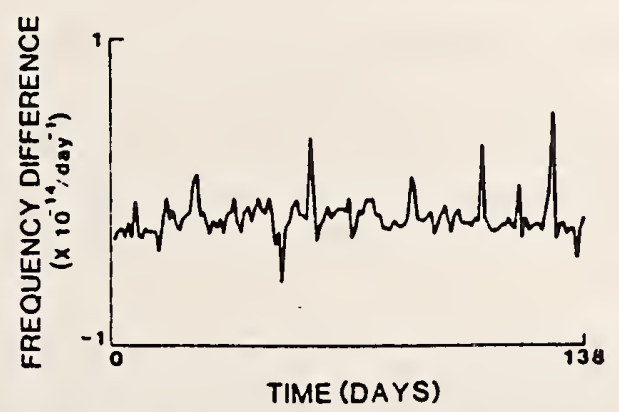

FIG. 12.8 Second difference of the data in Fig. 12-6. The second difference operation has removed the nonrandom behavior and the residuals appear to be nearly white. 


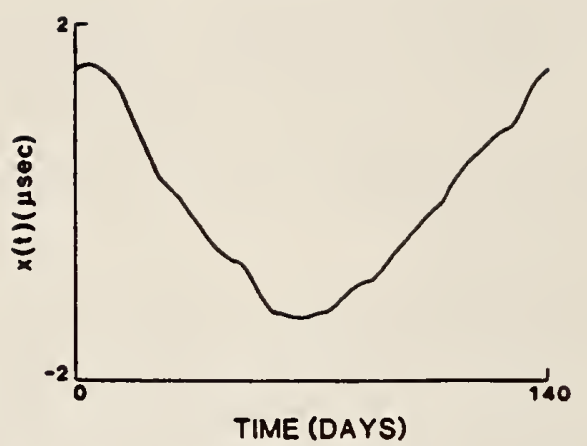

FIG. 12.9 Phase variations of the frequency standard due to the residuals. obtained by performing two integrations on the data of Fig. 12-8. The ordinate scale is expanded approximately 10 times compared to Fig. 12-6.

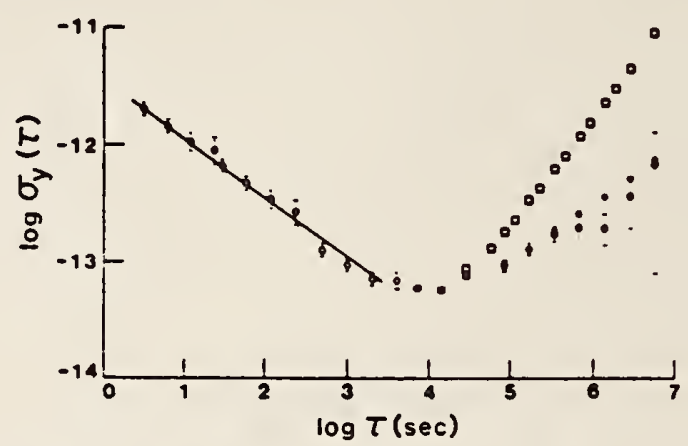

FIG. 12-10 Logarithm of the square root of the Allan variance as a function of the logarithm of the averaging time for three different computation methods. The squares were computed from the data of Fig. 12-6 and show the effect of the drift. The open circles were computed from the data of Fig. 12-9. The closed circles were computed using an optimumparameter estimation procedure.

\subsubsection{Confidence of the Estimate and Overlapping Samples}

Consider three phase or time measurements of one oscillator relative to another at equally spaced intervals of time. From this phase data one can obtain two adjacent values of average frequency and one can calculate a single sample Allan variance (see Fig. 12-11). Of course, this estimate does not have high precision or confidence, since it is based on only one frequency difference.

For most commonly encountered oscillators, the first difference of the frequency is a normally distributed variable with zero mean. However, the square of a normally distributed variable is not normally distributed. This is so because the square is always positive and the normal distribution is 


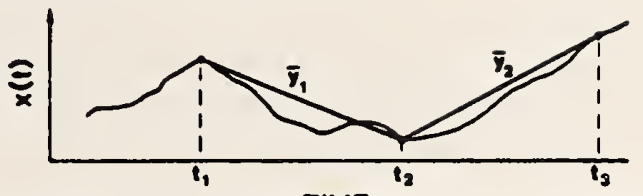

TIME

FIG. 12.11 Calculation of two average frequencies $\bar{y}_{1}$ and $\bar{y}_{2}$ by measuring the phase of an oscillator $x(t)$ at times $t_{1}, t_{2}$, and $t_{3}$.

completely symmetric, with negative values being as likely as positive ones. The resulting distribution is called a chi-squared distribution, and it has one "degree of freedom" since the distribution was obtained by considering the squares of individual (i.e., one independent sample), normally distributed variables (Jenkins and Watts, 1968).

In contrast, from five phase values four consecutive frequency values can be calculated, as shown in Fig. 12-12. It is possible to take the first pair and calculate a sample Allan variance. A second sample Allan variance can be calculated from the second pair (i.e., the third and fourth frequency measurements). The a verage of these two sample Allan variances provides an improved estimate of the true Allan variance, and one would expect it to have a tighter confidence interval than in the previous example. This could be expressed with the aid of the chi-squared distribution with two degrees of freedom.

However, there is another option. One could also consider the sample Allan variance obtained from the second and third frequency measurements, that is, the middle sample variance. This last sample Allan variance is not independent of the other two, since it is made up of parts of each of the others. But this does not mean that it cannot be used to improve the estimate of the true Allan variance. It does mean that the new average of three sample Allan variances is not distributed as chi squared with three degrees of freedom. The

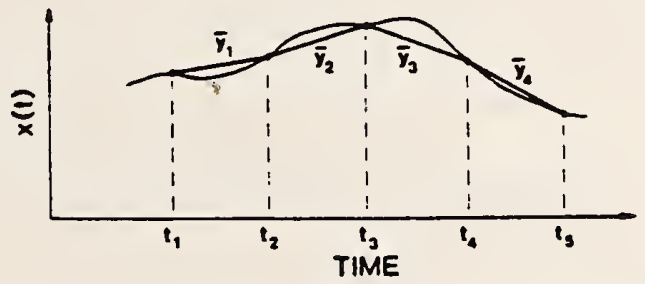

FIG. 12.12 Calculation of four frequency values $\bar{y}_{1}, \bar{y}_{2}, \bar{y}_{3}$, and $\bar{y}_{6}$ from five phase measurements at times $t_{1}, t_{2}, t_{3}, t_{4}$, and $t_{3}$. The sample variance formed from $\bar{y}_{1}$ and $\bar{y}_{2}$ and the one formed from $\bar{y}_{3}$ and $\bar{y}_{4}$ are independent. The sample variance formed from $\bar{y}_{2}$ and $\bar{y}_{3}$ is not independent of the other two but does contain some additional information useful in estimating the true sample variance. 
number of degrees of freedom depends on the underlying noise type, that is, white frequency, flicker frequency, etc., and may have a fractional value.

Sample Allan variances are distributed as chi square according to the equation

$$
\chi^{2}=(\mathrm{df}) s_{y}^{2} / \sigma_{y}^{2},
$$

where $s_{y}^{2}$ is the sample Allan variance, df the number of degrees of freedom (possibly not an integer), and $\sigma_{y}^{2}$ the true Allan variance, which we are interested in knowing but can only estimate imperfectly.

The probability density for the chi-squared distribution is given by the relation (Jenkins and Watts, 1968)

$$
\rho\left(\chi^{2}\right)=\frac{1}{2^{\mathrm{d} / 2} \Gamma(\mathrm{df} / 2)}\left(\chi^{2}\right)^{\mathrm{dr} / 2-1} e^{-z^{2 / 2}},
$$

where $\Gamma(\mathrm{df} ; 2)$ is the gamma function, defined by the integral

$$
\Gamma(t)=\int_{0}^{\infty} x^{1-1} e^{-x} d x
$$

A typical distribution is shown in Fig. (12-13).

Chi-squared distributions are useful in determining confidence intervals for variances and standard deviations, as shown in the following example. Suppose one has a sample variance $s^{2}=3.0$ and it is known that this variance has 10 degrees of freedom. The object is to calculate a range around the sample value of $s_{y}^{2}=3.0$ that probably contains the true value $\sigma_{y}^{2}$. The desired confidence is, say, $90 \%$. That is, $10 \%$ of the time the true value will actually fall outside of the stated bounds. The usual way to proceed is to allocate $5 \%$ to the low end and $5 \%$ to the high end for errors, leaving $90 \%$ in the middle. This is arbitrary and a specific problem might dictate a different

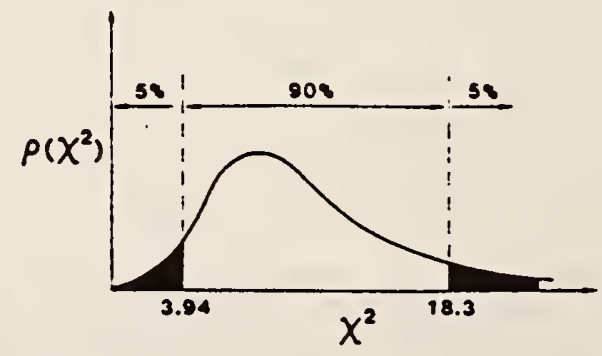

FIG. 12.13 Approximate form of a typical chi-squared distribution. For 10 degrees of freedom $5 \%$ of the area under the curve corresponds to values of $\chi^{2}$ less than 3.94 , and an additional $5 \%$ corresponds to values of $\chi^{2}$ greater than 18.3 . 
allocation. By referring to tables of the chi-squared distribution, one finds that for 10 degrees of freedom ( $\mathrm{df}=10$ ) the $5 \%$ and $95 \%$ points correspond to

$$
\chi^{2}(0.05)=3.94, \quad \chi^{2}(0.95)=18.3 .
$$

Thus, with $90 \%$ probability the calculated sample variance $s_{y}^{2}=3$ satisfies the inequality

$$
3.94<(\mathrm{df}) s_{y}^{2} / \sigma_{y}^{2}<18.3,
$$

and this inequality can be rearranged in the form

$$
1.64<\sigma_{y}^{2}<7.61 \text {. }
$$

The estimate $s_{y}^{2}=3$ is a point estimate. The estimate $1.64<\sigma_{y}^{2}<7.61$ is an interval estimate and should be interpreted to mean that $90 \%$ of the time the interval calculated in this manner will contain the true $\sigma_{y}^{2}$.

\subsubsection{Efficient Use of the Data and Determination of the Degrees of Freedom}

Typically, the sample variance is calculated from a data set using the relation

$$
s^{2} \cong \frac{1}{N-1} \sum_{n=1}^{N}\left(z_{n}-\bar{z}\right)^{2},
$$

where it is implicitly assumed that the $z_{n}$ 's are random and uncorrelated (i.e., white) and where $\bar{z}$ is the sample mean calculated from the same data set. If all of this is true, then $s^{2}$ is chi-squared distributed and has $N-1$ degrees of freedom.

Consider the case of two oscillators being compared in phase with $N$ values of the phase difference obtained at equally spaced intervals $\tau_{0}$. From these $N$ phase values one obtains $N-1$ consecutive values of average frequency, and from these one can compute $N-2$ individual sample Allan variances (not all independent) for $\tau=\tau_{0}$. These $N-2$ values can be averaged to obtain an estimate of the Allan variance at $\tau=\tau_{0}$.

The variance of this Allan variance has been calculated (Lesage and Audoin, 1973: Yoshimura, 1978). This approach is less versatile than the method of the previous section since it yields only symmetric error limits. However, it is simple and easy to use. Let $\Delta(N)$ be the relative difference between the sample Allan variance and the true value. Thus,

$$
s_{y}^{2}=[1+\Delta(N)] \sigma_{y}^{2}(\tau) .
$$


TABLE 12-3

Variance of the Relative Difference between the Sample Allan Variance and the True Value $\left(C_{\mathbf{z}} / N\right)^{\bullet}$

\begin{tabular}{lrr}
\hline \multicolumn{1}{c}{ Noise type } & \multicolumn{1}{c}{$C_{\text {. }}$} \\
\hline White phase & 2 & 3.88 \\
Flicker phase & 1 & 3.88 \\
White frequency & 0 & 2.99 \\
Flicker frequency & -1 & 2.31 \\
Random-walk frequency & -2 & 2.25 \\
\hline
\end{tabular}

- $N$ is the number of phase measurements. The result is accurate to better than $10^{\circ} ;$ for $N$ larger than 10 .

The quantity $\Delta(N)$ has mean zero. For $N$ larger than 10 , the variance of $\Delta$ is approximately

$$
\sigma^{2}(\Delta)=C_{2} / N .
$$

Table 12-3 gives the constant $C_{a}$ for the five major noise types.

Using the same set of data it is also possible to estimate the Allan variances for integer multiples of the base sampling interval $\tau=m \tau_{0}$. Now the possibilities for overlapping sample Allan variances are even greater. For a data set of $N$ phase points, one can obtain a maximum of exactly $N-2 m$ sample Allan variances for $\tau=m \tau_{0}$. Of course only $(N-1) 2 m$ of these are generally independent. Still, the use of all of the data is well justified since the confidence of the estimate is always improved by so doing. Consider the case of an experiment extending for several weeks in duration with the aim of getting estimates of the Allan variance for $\tau$ values equal to a week or more. As always, the purpose is to estimate the "true" Allan variance as well as possible, that is, with as tight an uncertainty as possible. Thus, one wants to use the data as efficiently as possible. The most efficient use is to average all possible sample Allan variances of a given $\tau$ value that one can compute from the data. This procedure is illustrated in Fig. 12-14.

In order to calculate confidence intervals for a sample variance, it is necessary to know the number of degrees of freedom. This has been done by both analytical and Monte Carlo techniques, and empirical equations have been found that are accurate to $1 \%$ for white phase, white frequency, and random-walk frequency modulation. The tolerance is somewhat larger for flicker frequency and phase modulation (Howe et al., 1981). The empirical equations for the degrees of freedom are given in Table 12-4. Table 12-5 gives the degrees of freedom for selected values of $N$, the total number of phase values, and $m$, the number of intervals averaged. Figure 12-15 illustrates the number of degrees of freedom for all noise processes as a function of $\tau$ for the case of 101 total phase measurements. 


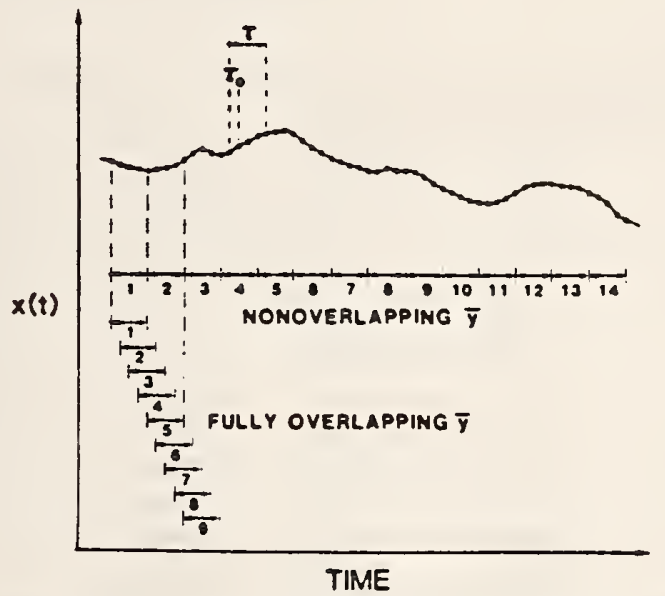

FIG. 12-14 Illustration of the case of $\tau=4 \tau_{0}$, for which the ratio of the number of fully overlapping to nonoverlapping estimates of the variance is more than 8 for the 57 phase points shown. When the averaging time for the computation of mean frequencies $?$ exceeds the sampling time $\tau_{0}$, the number of fully overlapping mean frequencies is far larger than the number of nonoverlapping frequencies. In general. for large $N$ approximately $2 m$ times as many estimates of the sample variances can be computed using the fully overlapping technique.

TABLE $12-4$

Number of Degrees of Freedom for Calculation of the Confidence of the Estimate of a Sample Allan Variance

\begin{tabular}{|c|c|}
\hline Noise type & $d f$ \\
\hline White phase & $\frac{(N+1)(N-2 m)}{2(N-m)}$ \\
\hline Flicker phase & $\exp \left[\ln \left(\frac{N-1}{2 n}\right) \ln \left(\frac{(2 m+1)(N-1)}{4}\right)\right]$ \\
\hline White frequeacy & {$\left[\frac{3(. V-1)}{2 m}-\frac{2(N-2)}{N}\right] \frac{4 m^{2}}{4 m^{2}+5}$} \\
\hline Flicker frequency & $\frac{2(N-2)}{2.3 N-4.9}$ for $m=1$ \\
\hline \multirow{3}{*}{ Random-walk frequency } & $\frac{5 N^{2}}{4 m 1 N+3 m 1}$ \\
\hline & $N-2(N-1)^{2}-3 m(N-1)+4 m^{2}$ \\
\hline & $m \quad(. N-3)^{2}$ \\
\hline
\end{tabular}

- For $:=m s_{0}$ from.$V$ phase points spaced $i_{0}$ apart. 
TABLE 12.5

Number of Degrees of Freedom for Calculation of the Confidence of the Estimate of a Sample Allan Variance for the Major Noise Types*

\begin{tabular}{rrrrrrr}
\hline \multicolumn{1}{c}{$\boldsymbol{N}$} & $\boldsymbol{m}$ & $\begin{array}{c}\text { White } \\
\text { phase }\end{array}$ & $\begin{array}{c}\text { Flicker } \\
\text { phase }\end{array}$ & $\begin{array}{c}\text { White } \\
\text { frequency }\end{array}$ & $\begin{array}{c}\text { Flicker } \\
\text { frequency }\end{array}$ & $\begin{array}{c}\text { Random-walk } \\
\text { frequency }\end{array}$ \\
\hline 9 & 1 & 3.665 & 4.835 & 4.900 & 6.202 & 7.000 \\
& 2 & 3.237 & 3.537 & 3.448 & 3.375 & 2.866 \\
129 & 4 & 1.000 & 1.000 & 1.000 & 1.000 & 0.999 \\
& 1 & 65.579 & 79.015 & 84.889 & 110.548 & 127.000 \\
& 2 & 64.819 & 66.284 & 71.642 & 77.041 & 62.524 \\
& 4 & 63.304 & 52.586 & 42.695 & 36.881 & 29.822 \\
& 8 & 60.310 & 37.306 & 21.608 & 16.994 & 13.567 \\
& 16 & 54.509 & 22.347 & 9.982 & 7.345 & 5.631 \\
& 32 & 44.761 & 9.986 & 4.026 & 2.889 & 2.047 \\
& 64 & 1.000 & 1.000 & 1.000 & 1.000 & 1.000 \\
& 1 & 526.373 & 625.071 & 682.222 & 889.675 & 1023.000 \\
& 2 & 525.615 & 543.863 & 583.622 & 636.896 & 510.502 \\
& 4 & 524.088 & 459.041 & 354.322 & 316.605 & 253.755 \\
& 8 & 521.038 & 366.113 & 186.363 & 156.492 & 125.398 \\
& 16 & 514.952 & 269.849 & 93.547 & 76.495 & 61.241 \\
& 32 & 502.839 & 179.680 & 45.947 & 36.610 & 29.210 \\
& 64 & 478.886 & 104.743 & 21.997 & 16.861 & 13.288 \\
& 128 & 432.509 & 50.487 & 10.003 & 7.281 & 5.516 \\
& 256 & 354.914 & 17.429 & 4.003 & 2.861 & 2.005 \\
& 512 & 1.000 & 1.000 & 1.000 & 1.000 & 1.005 \\
\hline
\end{tabular}

- $N$ is the number of equally spaced phase points that are taken $m$ at a time to form the averaging time.

\subsubsection{Separating the Variances of the Oscillator and the Reference}

A measured variance contains noise contributions from both the oscillator under test and the reference. The individual contributions are easily separated if it is known a priori that the reference is much less noisy than the device under test or equal to it in performance. Otherwise, the individual contributions can be estimated by comparing three devices (Barnes, 1966). The three possible joint variances are denoted by $\sigma_{i j}^{2}, \sigma_{j k}^{2}$, and $\sigma_{i k}^{2}$, while the individual device variances are $\sigma_{i}^{2}, \sigma_{j}^{2}$, and $\sigma_{k}^{2}$. The joint variances are composed of the sum of the individual contributions under the assumption that the oscillators are independent:

$$
\begin{aligned}
& \sigma_{i j}^{2}=\sigma_{i}^{2}+\sigma_{j}^{2}, \\
& \sigma_{j k}^{2}=\sigma_{j}^{2}+\sigma_{k}^{2}, \\
& \sigma_{i k}^{2}=\sigma_{i}^{2}+\sigma_{k}^{2} .
\end{aligned}
$$




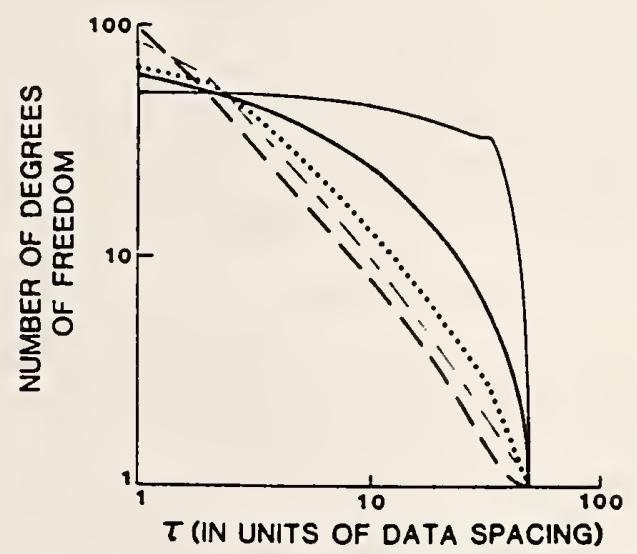

FIG. 12-15 Number of degrees of freedom as a function of averaging time for the case of 101 phase measurements: The heavy broken line is for random-walk frequency noise, the light broken line is for flicker frequency noise, the dotted line is for white frequency noise, the heavy solid line is for flicker phase noise, and the light solid line is for white phase noise.

An expression for each individual variance is obtained by adding two joint variances and subtracting the third:

$$
\begin{aligned}
& \sigma_{i}^{2}=\frac{1}{2}\left(\sigma_{i j}^{2}+\sigma_{i k}^{2}-\sigma_{j k}^{2}\right), \\
& \sigma_{j}^{2}=\frac{1}{2}\left(\sigma_{j k}^{2}+\sigma_{i j}^{2}-\sigma_{i k}^{2}\right), \\
& \sigma_{k}^{2}=\frac{1}{2}\left(\sigma_{j k}^{2}+\sigma_{i k}^{2}-\sigma_{i j}^{2}\right),
\end{aligned}
$$

This method works best if the three devices are comparable in performance. Caution must be exercised since Eqs. (12-44) may give a negative sample Allan variance despite the fact that the true Allan variance is positive definite. This is possible because the confidence interval of the estimate is sufficiently large to include negative variances. Such a result is an indication that the confidence intervals of the sample Allan variances are too large and that more data is required.

\subsection{DIRECT DIGITAL MEASUREMENT}

\subsubsection{Time-Interval Measurements}

A common technique for measuring the phase difference between oscillators having nearly equal nominal frequencies is the use of direct timeinterval measurements. In this section and those that follow, the symbols $v_{10}$ and $v_{20}$ are used to indicate the nominal values of $v_{1}$ and $v_{2}$, respectively. In the simplest form of this technique, a time-interval counter is started on some 


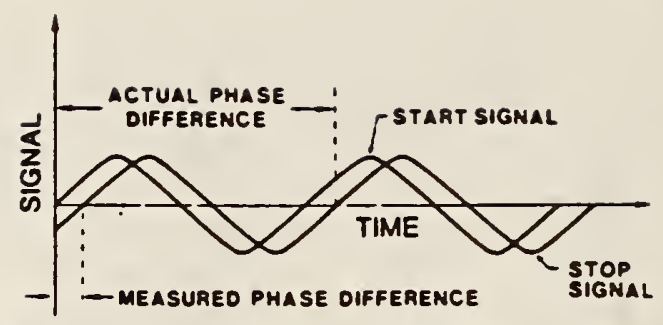

FIG. 12-16 The phase difference measured by a time-interval counter is the phase difference between the start signal and the stop signal modulo the period of the stop signal.

arbitrarily selected positive-going zero crossing of the signal from one oscillator (started on $v_{10}$ at time $t_{1}$ ) and stopped in the next positive-going zero crossing of the second oscillator (stopped on $v_{20}$ at time $t_{2}$ ). The measured time difference is

$$
x_{2}\left(t_{1}\right)-x_{1}\left(t_{1}\right) \cong-P \tau_{c}\left[1+\left(v_{20}-v_{10}\right) / v_{10}\right],
$$

where $P$ is the reading of the time-interval counter and $\tau_{c}$ the period of its time base (Allan et al., 1974). The units of the time difference is seconds of oscillator number 1. Equation (12-45) demonstrates an important characteristic of both time- and phase-difference measurements. Because of distortion the phase of an oscillator is generally not well known except at zero crossings. Thus, the quantity usually measured is $x_{2}\left(t_{2}\right)-x_{1}\left(t_{1}\right)$. However, all analysis techniques require the phase difference at the same time, and the translation requires a correction that takes into account the difference in frequency between the two oscillators. This correction is the reason for the second term in the brackets on the right-hand side of Eq. (12-45).

The simple scheme described above measures a maximum accumulated phase difference of one cycle of the signal. When the phase difference exceeds one cycle the counter reading is periodic, as shown in Fig. 12-16. This ambiguity can be reduced by dividing the signals from each oscillator before the time-interval measurement. The complete system is shown in Fig. 12-17. The effect of the divider is to increase the time interval before an ambiguity

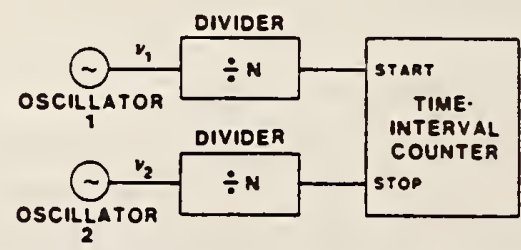

FIG. 12-17 Schematic diagram of the dividers used in conjunction with a time-interval counter to increase the maximum measurable phase difference to $N$ cycles of the stop signal. 
occurs to $N / v_{10}$, where $N$ is the divisor. Such measurement systems are used at many standards laboratories for the long-term measurement of atomic clocks, whose output is usually divided down to 1 pulse/sec. Since timeinterval counters with resolution better than $0.1 \mathrm{nsec}$ are available, this measurement scheme is suitable for long-term performance monitoring, yielding frequency-measurement precision of $10^{-1+}$ for 1 -day averages.

\subsubsection{Frequency Measurements}

Average frequency is measured most directly using a frequency counter. Used this way, the counter determines the number of whole cycles $M$ occurring during a time interval $\tau$ given by the counter's time base. Thus

$$
\bar{v}(0 ; \tau)=(M+\Delta . M) / \tau \simeq M / \tau,
$$

where $\bar{v}\left(t_{1}: t_{2}\right)$ denotes the average frequency over the interval from $t_{1}$ to $t_{2}$ and $\Delta . M$, the fractional cycle, is not measured by the counter. The starting time is arbitrarily called $t=0$. Thus, the quantization error is given by

$$
\Delta v_{Q^{\prime}}\langle v\rangle<1 / M \text {. }
$$

\subsubsection{Period Measurements}

For low frequencies, the number of cycles counted may be small and the quantization error can be very large. By measuring the period instead of the frequency, it is possible to decrease the error without increasing the duration of the measurement. A period counter measures the duration of $M$ whole cycles of the signal as $N$ cycles of the time base $\tau_{c}$. The fraction of a cycle $\Delta N$ is not measured. Thus, we have

$$
M=\bar{v}\left(0: M / v_{0}\right)(N+\Delta N) \tau_{c},
$$

and therefore

$$
\bar{v}\left(0 ; M / v_{0}\right) \cong M / N \tau_{c}
$$

and the quantization error is

$$
\Delta v_{\mathrm{Q}} /\langle v\rangle<1 / N
$$

Frequency measurements are almost never used to characterize precision oscillators, but period measurements are very common. A straightforward extension of this method eliminates the bias potentially introduced by the quantization error and permits the measurement of accumulated phase. The counter must be capable of being read without halting the counting process. 


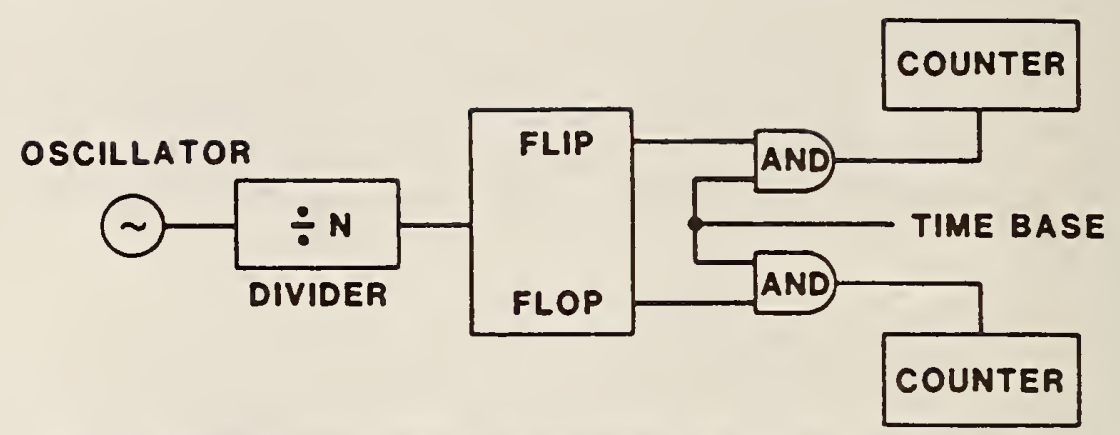

FIG. 12.18 Two-counter system to eliminate dead time in period measurements. The two counters alternately count the number of cycles of the time base in $N$ periods of the oscillator under test.

Alternatively, a second counter may be used to begin counting the same time base when the first counter stops. The second approach is illustrated in Fig. 12-18. This type of measurement system is sometimes called a chronograph.

\subsection{SENSITIVITY-ENHA.NCEMENT METHODS}

\subsubsection{Heterodyne Techniques}

It is possible for oscillators to be very stable, and values of $\sigma_{y}(\tau)$ can be as small as $10^{-16}$ in some state-of-the-art standards. Thus, one often needs measuring techniques capable of resolving very small fluctuations in $y(t)$. One of the most common techniques is the heterodune or beat-frequency technique. In this method the signal from the oscillator under test is mixed with a reference signal of almost the same frequency so that one is left with a lower average frequency for analysis without reducing the frequency (or phase) fluctuations themselves.

In principle, it is possible to analyze the most general measurement case, where no restrictions are placed on the average frequency or phase difference between the two oscillators under test. Equation 12-1 can be inverted as

$$
2 \pi v_{0} t+\phi(t)=\arcsin \left[V(t) / v_{0}\right]
$$

and used to obtain the series $\phi(m \tau)$ by sampling the voltage at regular time intervals. This direct technique is not used, because it requires unobtainable mixer performance characteristics. The high-level if signals that are required for low-noise phase measurements produce significant harmonic distortion, so that the output of the phase detector deviates significantly from a sine wave. Furthermore, the distortions are generally sensitive to level and environmental perturbations. However, the phase relationships among the various harmonics are very stable, so it is possible to use the repetition of one 
point on the waveform in order to count cycles. The positive-going zero crossings are normally chosen in order to provide immunity from changes in both the amplitude and symmetry of the waveform.

Consider two signals whose frequency difference is much less than the frequency of either oscillator:

$$
V_{1}(t)=V_{10} \sin \left[2 \pi v_{10} t+\phi_{1}(t)+\phi_{10}\right]
$$

and

$$
V_{2}(t)=V_{20} \sin \left[2 \pi v_{20} t+\phi_{2}(t)+\phi_{20}\right],
$$

where $\left|v_{10}-v_{20}\right| \ll v_{10}$ and the constants $\phi_{10}$ and $\phi_{20}$ represent the nominal phases of the two signals.

Suppose that the two signals are mixed in a linear product detector and filtered so that the signal at the sum frequency $v_{10}+v_{20}$ is highly attenuated. The result is

$$
V(t) \cong V_{0} \cos \left[2 \pi\left(v_{10}-v_{20}\right) t+\phi_{10}-\phi_{20}+\phi_{1}(t)-\phi_{2}(t)\right],
$$

which may be characterized by any of the measurement techniques discussed in Section 12.2. The amplitude $V_{0}$ of the mixer output is a function of the mixer design, the input amplitudes, and the output termination (Walls et al., 1976). Using the definition (12-10), we find that for the heterodyned signal

$$
x_{H}(t)=\left(1 / 2 \pi v_{H}\right) \Delta \phi(t),
$$

where

$$
v_{\mathrm{H}}=\left|v_{10}-v_{20}\right|
$$

and

$$
\Delta \phi(t)=\phi_{1}(t)-\phi_{2}(t)
$$

Equation (12-54) may be rewritten as

$$
x_{\mathrm{H}}(t)=\left(v_{0} / v_{\mathrm{H}}\right) x(t)
$$

from which we conclude that a given phase change corresponds to a larger time deviation for the heterodyne signal than for the original signal. As a result, the quantization error for the period measurement technique is reduced by the factor $v_{\mathrm{H}} / v_{\mathrm{O}}$.

\subsubsection{Homodyne Techniques}

The limit of the heterodyne method, called homodyne, occurs when $v_{10}=v_{20}$. In this case the output of the phase detector is given by

$$
V(t) \cong V_{0} \cos \left[\phi_{10}-\phi_{20}+\phi_{1}(t)-\phi_{2}(t)\right] \text {. }
$$

* See Appendix Note \# 6 
The analysis of phase noise is accomplished by arranging that $\phi_{10}-\phi_{20}=\pi / 2$, which can be achieved with a phase shifter. Then,

$$
V(t) \cong-V_{0} \sin \left[\phi_{1}(t)-\phi_{2}(t)\right] \cong V_{0}\left[\phi_{2}(t)-\phi_{1}(t)\right] .
$$

There are various methods by which one can control the signal $V_{2}(t)$ so that $v_{10}=v_{20}$ without producing significant correlation between $\phi_{2}(t)$ and $\phi_{1}(t)$. When any one of these methods is used, it is possible to use $V(t)$ as a measure of $\phi(t)$. Two methods, delay lines and phase-locked loops (Gardner, 1966), are described below.

\subsubsection{DISCRIMINATOR AND DELAY LINE}

The circuit of a discriminator or delay-line system for measuring phase noise is illustrated in Fig. 12-19. The delayed signal is given by

$$
V_{2}(t)=V_{1}\left(t-t_{d}\right)=V_{20} \sin \left[2 \pi v_{10}\left(t-t_{d}\right)+\phi_{1}\left(t-t_{d}\right)+\phi_{10}+\phi_{s}\right] .
$$

When the phase shifter is set for quadrature, $\phi_{s}-2 \pi v_{10} t_{d}=\pi ; 2$ and

$$
V_{2}(t)=V_{20} \sin \left[2 \pi v_{10} t+\phi_{1}\left(t-t_{d}\right)+\phi_{10}+\pi_{t}^{\prime 2}\right] \text {. }
$$

The output of the phase detector is given by

$$
V(t)=V_{0}\left[\phi_{1}\left(t-t_{d}\right)-\phi_{1}(t)\right] .
$$

Substituting Eq. (12-62) into Eq. (12-20), we obtain

$$
i\left(t-t_{\mathrm{d}}: t\right)=-V(t) i 2 \pi v_{0} V_{0} t_{d}
$$

and we see that the delay-line method can be used to produce samples of ix $\left(m \tau_{0}\right)$ by varying the delay time. However, the technique is used more frequently with a fixed delay by restricting its application to the region of $\tau$ much greater than the delay time, so that $i\left(t-t_{\mathrm{d}}: t\right)$ is a good approximation for the instantaneous frequency. Under this assumption spectrum analysis of

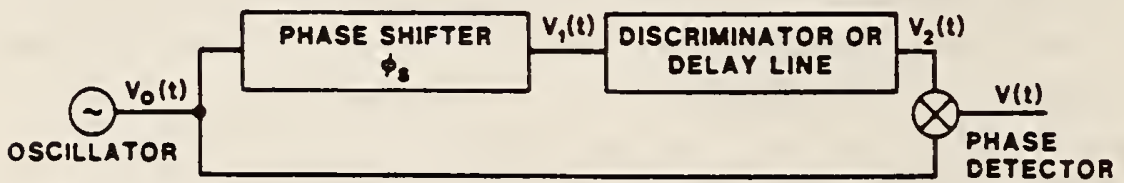

FIG. 12-19 A delay-line phase-noise-measurement system. When the phase shifter is adjusted so that $V_{2}^{\prime}(t)$ is in phase quadrature with $V_{0}(t)$, the output of the phase detector is approximately equal to the instantaneous frequency deviation of the oscillator. The spectral density of the source may be estimated for Fourier frequencies small compared to the inverse of the delay time. 
the signal from the mixer can be used to estimate the spectral density of the frequency fluctuations:

$$
S_{y}(f) \cong \frac{1}{\left(2 \pi v_{0} t_{\mathrm{d}}\right)^{2}} S_{V, v_{0}}(f) \quad \text { for } f \ll 1 / \pi i_{\mathrm{d}} .
$$

Frequency discriminators are applied in an analogous fashion. A resonant circuit is often used to provide discrimination since it produces a phase shift proportional to the frequency deviation from the resonant frequency. For example, the phase shift on reflection from a resonance with loaded quality factor $Q$ is

$$
\phi=\arctan (2 Q y) \cong 2 Q \mathrm{y},
$$

provided that the frequency deviation is small compared to the bandwidth of the resonance and the applied signal is nearly at the center frequency of the discriminator. This can be accomplished either manually or with a frequencylocked loop. The design of such a loop is similar to the phase-locked loop of the next section. Once again, one can spectrum analyze the signal from the mixer to obtain

$$
S_{y}(f)=\frac{1}{(2 Q)^{2}} S_{V, V_{0}}(f) \quad \text { for } f \ll v_{0} / Q
$$

The noise floor for measurements made with either a delay line or discriminator normally results from white voltage noise in the analysis circuitry and is independent of the Fourier frequency. We denote the noise floor $S_{v i v_{0}}$ (minimum) and find the noise floor for frequency or phase measurements by

$$
\left.S_{\phi}(\text { noise limit })=\frac{v_{0}^{2}}{f^{2}} S_{y} \text { (noise limit }\right)=\frac{v_{0}^{2}}{f^{2}(2 Q)^{2}} S_{V / V_{0}}(\text { minimum }) .
$$

Consequently, the discriminator or delay-line technique is limited in sensitivity since the output voltage is proportional to the frequency deviations. Greater sensitivity is possible using two oscillators in a phase-locked loop. The noise in the reference is an important consideration, even though the reference is passive in the case of a discriminator or a delay line. If the oscillator has sufficiently low noise, then the circuits described measure the variations of the discriminator center frequency or the delay variations in the delay line.

\subsubsection{PHASE-LOCKED LOOP}

The block diagram for the most general phase-locked loop that will be considered here is shown in Fig. 12-20. The noise voltage summed into the loop is a schematic way of representing $\phi_{n}(t)$, the open-loop phase noise of the 


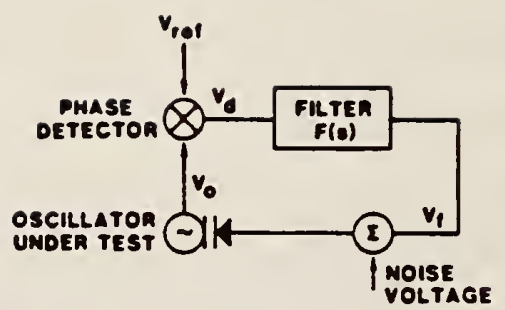

FIG. 12-20 Block diagram of a phase-locked loop. The order of the loop is determined by the filter transfer function. For convenience, noise in the oscillator under test is introduced at the summing junction.

oscillator under test. Phase noise in the reference oscillator is denoted by $\phi_{\text {reer }}(t)$.

The purpose of using a phase-locked loop is simply to guarantee that the two oscillators are, on the average, in phase quadrature. When the oscillators are near quadrature, the voltage output of the phase detector is proportional to the difference in phase between the two output signals.

Analysis of the phase-locked loop yields the result

$$
\phi_{0}(s)=\phi_{n}(s)\left[\frac{1}{1+G_{e q}(s)}\right]+\phi_{\text {rer }}(s)\left[\frac{G_{e q}(s)}{1+G_{\text {eq }}(s)}\right],
$$

where $G_{\text {eq }}(s)$ is the open-loop transfer function defined by

$$
G_{e q}(s)=\frac{K_{0} K_{d} F(s)}{s}
$$

and $\phi_{n}(s)$ and $\phi_{\text {ref }}(s)$ are the Laplace transforms of the corresponding timevarying quantities. We can also calculate the voltage output of the phase detector,

$$
V_{\mathrm{d}}(s)=\frac{K_{\mathrm{d}}\left[\phi_{\mathrm{ref}}(s)-\phi_{\mathrm{n}}(s)\right]}{1+G_{\mathrm{eq}}(s)},
$$

as well as the feedback voltage to the varactor,

$$
V_{\mathrm{f}}(s)=F(s) V_{\mathrm{d}}(s)=\frac{s G_{e q}(s)}{G_{e q}(s)}\left[\phi_{\mathrm{rer}}(s)-\phi_{\mathrm{D}}(s)\right]
$$

Assuming that the phase noise of the two oscillators is not correlated,

$$
\begin{aligned}
& S_{V_{d}}(\omega)=\frac{K_{j}^{2}}{\| 1+\left.G_{e q}(j \omega)\right|^{2}}\left[S_{\phi_{\text {rer }}}(\omega)+S_{\phi_{a}}(\omega)\right] . \\
& S_{V_{r}}(\omega)=\frac{\omega^{2} G_{e q}(j \omega)^{2}}{\| 1+\left.G_{e q}(j \omega)\right|^{2}}\left[S_{\phi_{\text {rer }}}(\omega)+S_{\phi_{\mathbf{a}}}(\omega)\right] .
\end{aligned}
$$




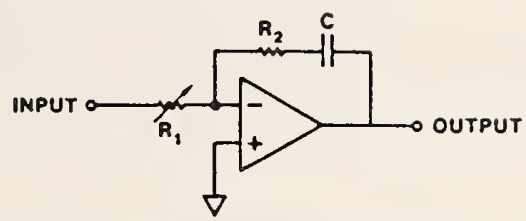

FIG. 12-21 Circuit diagram of the most common loop filter for a second-order phaselocked loop. Resistor $R_{2}$ is required for stable operation. Capacitor $C$ provides the lowfrequency gain needed to reduce the phase errors of the first-order loop.

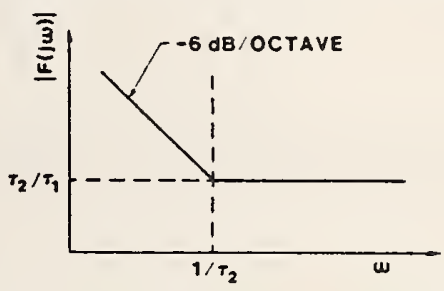

FIG. 12-22 Bode plot for the loop filter of Fig. 12-21.

Thus, if we know the behavior of $G_{\text {eq }}(j \omega)$, then we can relate the measured spectrum of the voltage at the output of the phase detector or at the varactor tuner to the sum of the spectral densities of the phase noise of the two oscillators.

The loop filter is often chosen to be a pure gain. The resulting first-order loop has a significant drawback: the two oscillators are offset from quadrature by a phase shift proportional to their open-loop frequency difference. In order to maintain system calibration, the operator must remove the frequency offset from time to time. This problem can be eliminated by using a second-order loop. Figure 12-21 illustrates one loop filter that can be used to achieve the desired frequency response. The transfer function of this filter is

$$
F(s)=\left(1+s \tau_{2}\right) / s \tau_{1},
$$

where $\tau_{2}=R_{2} C$ and $\tau_{1}=R_{1} C$. Figure 12-22 shows the Bode plot of the frequency-response function of this filter. Substitution of Eq. (12-74) into Eq. (12-69) yields the open-loop frequency-response function

$$
G_{e q}(j \omega)=-\frac{\omega_{n}^{2}+2 j \zeta \omega_{n} \omega}{\omega^{2}}
$$

where

$$
\omega_{\mathrm{n}}=\left[K_{0} K_{\mathrm{d}} / \tau_{1}\right]^{1: 2}
$$

and

$$
\zeta=\frac{1}{2} \tau_{2} \omega_{n} .
$$




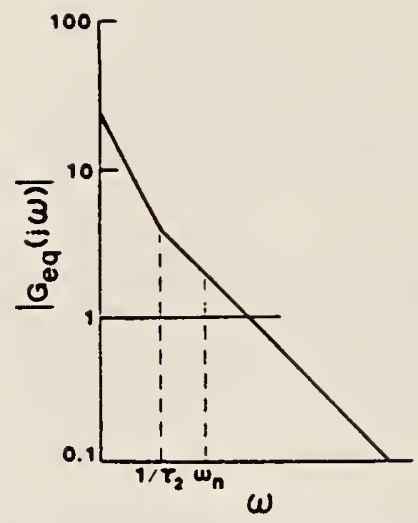

FIG. 12-23 Bode plot of the open-loop frequency-response function for a phase-locked loop having the loop filter of Fig. 12-21. Parameters were chosen to illustrate a stable condition.

The first requirement to be satisfied by the loop parameters is that the closed loop be stable. Since the transfer function $G_{e q}(s)$ has no poles or zeros for $s>0$, a sufficient requirement for the phase-locked loop to be stable is that the slope of the Bode plot of $\left|G_{\text {eq }}(j \omega)\right|$ be less steep than $-12 \mathrm{~dB} /$ octave at the point where $\left|G_{\text {eq }}(j \omega)\right|=1$. The Bode plot of $\left|G_{\text {eq }}(j \omega)\right|$ is shown in Fig. 12-23 for a case where the loop operation is stable.

It is desirable for the loop to be nearly critically damped, that is, $\zeta=1$. At critical damping the natural frequency of the loop is related to $\tau_{2}$ by

$$
\omega_{n,:=1}=2 / \tau_{2} \text {. }
$$

Under the same conditions the unity gain frequency is

$$
\omega_{1, \zeta=1}=4.12 ; \tau_{2} \text {. }
$$

The second requirement to be satisfied by the phase-locked loop is related to the accuracy with which spectral-density measurements can be made. Substitution of Eq. (12-75) into Eq. (12-72) yields

$$
S_{v_{\mathrm{d}}}(\omega)=\frac{K_{\mathrm{d}}^{2} \omega^{4}}{\left(\omega^{2}-\omega_{\mathrm{n}}^{2}\right)^{2}+4 \sigma_{\varphi}^{-2} \omega^{2} \omega_{\mathrm{n}}^{2}}\left[S_{\phi_{\mathrm{rer}}}(\omega)+S_{\phi_{\mathrm{n}}}(\omega)\right] .
$$

Since the proportionality factor has a high pass response, it is possible to use an essentially constant calibration to relate $S_{V_{d}}(\omega)$ and $S_{\phi}(\omega)$. For example, if we require that

$$
S_{i_{\mathrm{d}}}(\omega) \cong K_{d}^{2}\left[S_{\varphi_{\text {rer }}}(\omega)+S_{\phi_{\mathrm{n}}}(\omega)\right]
$$

with no more than $10 \%$ error for all Fourier frequencies greater than $2 \pi \mathrm{rad}$ isec, then for the critically damped loop the requirement on $\tau_{2}$ is $\tau_{2}>1.4 \mathrm{sec}$. 
The third requirement on loop performance is that the frequency offset between the two oscillators produce negligible phase shift of the oscillators from quadrature. In the ideal loop the phase error for a frequency error $\Delta v$ introduced at time $t=0$ is

$$
\phi_{\text {error }}=2 \pi \Delta v t e^{-\omega_{n} t} .
$$

However, in the actual circuit there is a finite phase error due to the limited loop gain of the amplifier of Fig. 12-21. Nevertheless, the phase error is reduced by $10^{5}$ compared to its value for a first-order loop. Typically, the error is less than the residual phase error due to the voltage offset at the mixer output and should be much less than $1^{\circ}$.

The feedback loop reduces the sensitivity of the system for measurements of the phase spectral density for Fourier frequencies less than the unity-gain frequency of the phase-locked loop. One way to avoid this problem is to utilize the feedback voltage $V_{f}$. Substituting Eq. (12-75) into Eq. (12-73), we find that

$$
S_{V_{f}}(\omega)=\frac{2 \pi v_{0}^{2}\left(\omega_{\mathrm{n}}^{4}-4 \omega_{\zeta}^{-2} \omega_{n}^{2} \omega^{2}\right)}{\left(\omega^{2}-\omega_{n}^{2}\right)^{2}+4 \omega_{\zeta}^{-2} \omega_{n}^{2} \omega^{2}}\left[S_{y_{\mathrm{ref}}}(\omega)+S_{y_{\mathrm{n}}}(\omega)\right]
$$

For this case, the proportionality factor has a low pass response and a constant calibration factor may be used to relate $S_{V_{f}}(\omega)$ to $S_{y}(\omega)$.

\subsubsection{Multiple Conversion Methods}

Quite often the beat frequency between the signal under test and the laboratory reference is unsuitable or inconvenient for frequency-stability measurements. The frequency may be too high for the available counters or the heterodyne factor may be too small to yield the required noise enhancement. Under these circumstances a second mixing stage in series with the first can be used to produce the desired beat frequency. On the other hand, the direct beat frequency between two oscillators may be too small. For example, the frequencies of commercial cesium-beam frequency standards are usually so close together that the beat frequency between two devices would be near l cycle day, making it impossible to observe the stability at shorter times. This limitation can be overcome by the use of two parallel mixing stages.

\subsubsection{FREQUENCY SYNTHESIS}

A commercial frequency synthesizer is usually the most convenient way to produce arbitrary reference frequencies for stability measurements. A mixing stage preceding the synthesizer can be used both to bring the signal into the 


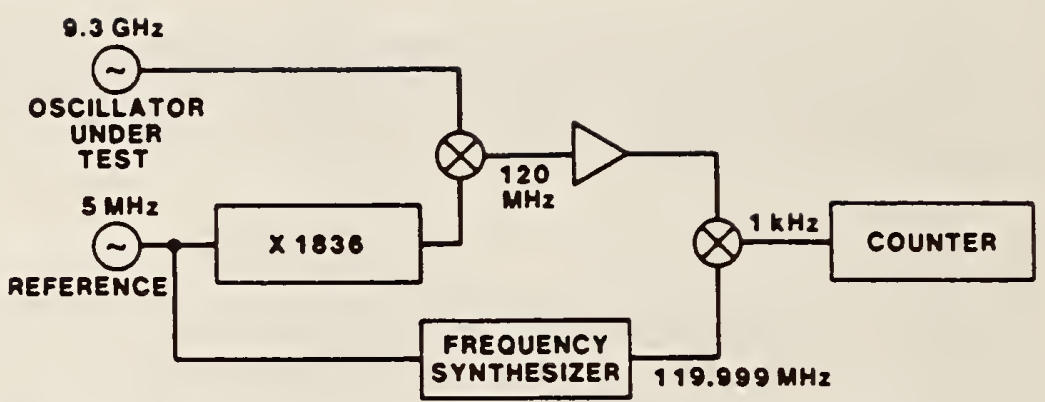

FIG. 12-24 Use of frequency synthesis to measure oscillators whose frequency differs significantly from the available low-noise reference. It may be necessary to use a frequency multiplier to bring the signal into the range of the available synthesizer or to overcome the synthesizer's phase noise.

appropriate range and to enhance the oscillator noise compared to the shortterm phase noise of the synthesizer. Figure 12-24 demonstrates both aspects of the technique.

The initial mixing stage from the microwave frequency to the $r f$ results in a substantial heterodyne factor, 77.5 for the example chosen. The output of the first conversion stage lies $u$ ithin the range of low-noise commercial frequency synthesizers, which makes it possible to obtain a fixed, low beat frequency over a wide range of input frequencies. The initial mixing stage also reduces the frequency synthesizer's contribution to the measurement-system noise. Figure 12-25 shows the typical phase excursions of a high-quality commercial synthesizer operated near $5 \mathrm{MHz}$.

Under some circumstances a frequency divider may be used to provide the signal for the second mixing stage, as shown in Fig. 12-26. This technique has the disadvantage of requiring a custom divider but results in much lower measurement noise than the direct use of a synthesizer with a single heterodyne stage.

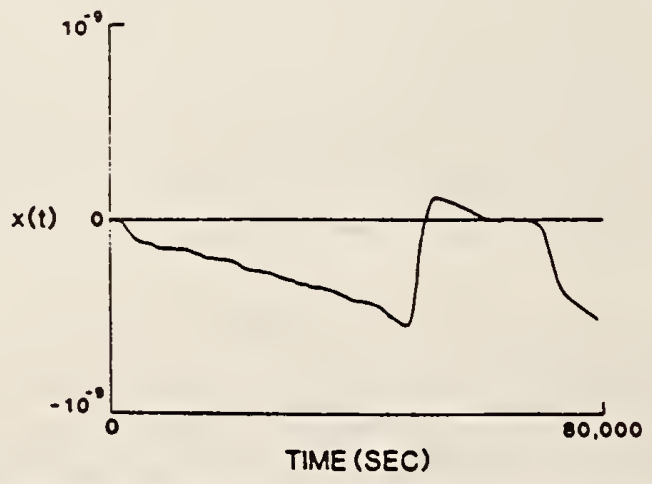

FIG. 12-25 Typical phase excursions of a commercial frequency synthesizer. 


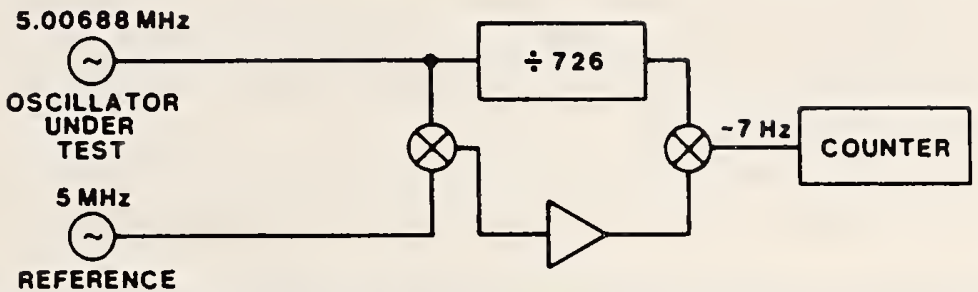

FIG. 12-26 Use of a simple divider as a substitute for a commercial frequency synthesizer in a heterodyne measurement system. Better noise performance can result from the initial mixing stage.

\subsubsection{THE DUAL-MIXER TIME-DIFFERENCE TECHNIQUE}

There is no best answer to the question of how to make frequency-stability measurements. However, by combining versatility with low-noise performance, the dual-mixer time-difference technique (Cutler and Searle, 1966: Allan and Daams, 1975) shown in Fig. 12-27 comes close to the ideal. The original motivation for this method was to use a transfer oscillator and two mixers in parallel to permit short-term frequency-stability measurements between oscillators that have an inconveniently small frequency difference. The transfer oscillator is most easily realized with a frequency synthesizer locked to one of the oscillators, designated oscillator 1 in Fig. 12-27. By convention the frequency of the synthesizer is set low compared to the oscillator under test, so we write the frequency of the synthesizer as

$$
v_{s}=v_{1}(1-1 / R) \text {. }
$$

The constant $R$ is equal to the heterodyne factor, which can be seen by calculating the beat frequency between oscillator 1 and the synthesizer:

$$
v_{B 1}=v_{1}-v_{s}=v_{1} / R \text {. }
$$

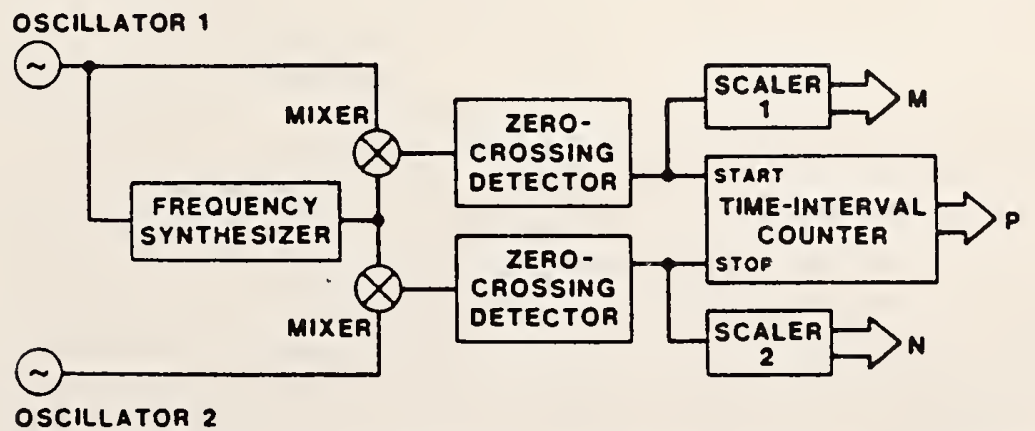

FIG. 12.27 A dual-mixer measurement system. The scalars measure the number of whole cycles of elapsed phase, while the time-interval counter measures the fractional cycle. 
The combination of oscillator, frequency synthesizer, and mixer functions as a divider and scaler 1 functions as the system clock, recording elapsed time in units of cycles of oscillator 1 .

The signals from oscillators 1 and 2 are represented according to Eq. (12-52) with $\phi_{10}=\phi_{20}=0$, and the signal from the synthesizer is written

$$
V_{s}(t)=V_{s 0} \cos \left[2 \pi v_{s 0} t+\phi_{s}(t)\right] \text {. }
$$

The phase of the synthesizer retards nearly linearly in time compared to the phase of oscillators 1 and 2 . At time $t_{M}$ the synthesizer reaches phase quadrature with oscillator 1 and the beat signal crosses zero (in the positive direction), producing a pulse from the zero-crossing detector and starting the time-interval counter. At a later time $t_{N}$ the continued sweep of the synthesizer has brought it into quadrature with oscillator 2 , and a pulse is produced that stops the time-interval counter. The phase difference between the oscillators can be written in terms of the three counter readings:

$$
\phi_{2}\left(t_{M}\right)-\phi_{1}\left(t_{M}\right)=2(N-M) \pi-2 \pi\left[\bar{v}_{B 2}\left(t_{M}: t_{N}\right)\right] \tau_{c} P,
$$

where $N$ is the reading of scaler $2, M$ the reading of scaler $1, P$ the reading of the time-interval counter, and $\tau_{c}$ the period of its time base (Stein et al., 1983). Comparison with Eq. (12-45) for direct time-interval measurements reveals that the role of the scalers is to accumulate the coarse phase difference between the oscillators, while the time-interval counter provides fine-grain resolution of the fractional cycle. This process is illustrated in Fig. 12-28. The advantage of the technique over direct time-interval measurements is that the noise performance is improved by the large heterodyne factor, allowing time resolution of $0.1 \mathrm{psec}$ to be obtained. The synthesizer degrades the noise performance very little since it contributes to the noise only over the interval $\tau_{\mathrm{c}} P$.

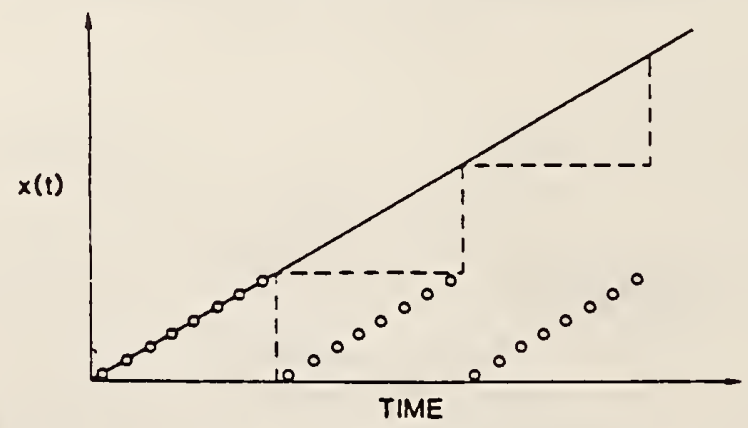

FIG. 12-28 Total elapsed phase measured by the dual-mixer system of Fig. 12-27 (solid linel. This phase measurement consists of two components: the number of full cycles that have elapsed is the step function plotted as a dashed line: the fractional cycle is the saw-tooth function plonted as open circles. 
The average beat frequency $\bar{v}_{B 2}\left(t_{M} ; t_{N}\right)$ cannot be known exactly, but it may be estimated with sufficient precision if it changes slowly compared to the interval between measurements. If the primed and unprimed variables represent two independent measurements, then

$$
\bar{v}_{\mathrm{B2}}\left(t_{\mathrm{M}}: t_{\mathrm{N}}\right) \cong\left(N^{\prime}-N\right) /\left[R\left(M^{\prime}-M\right) / v_{10}+\tau_{\mathrm{c}}\left(P^{\prime}-P\right)\right] .
$$

\subsubsection{FREQUENCY MULTIPLICATION}

A frequency multiplier produces $n$ full cycles of the output signal for each cycle of the input signal, where $n$ is an integer determined by the design of the device. Such a device is also a phase multiplier, that is, the total phase accumulation of the output signal is $n$ times as great as the phase accumulation of the input signal:

$$
\Phi_{\text {out }}=2 \pi v_{\text {out }} t+\phi_{\text {our }}(t)=2 \pi\left(n v_{\text {in }}\right) t+n \phi_{\text {in }}(t) .
$$

It follows that the spectral density of the output signal is enhanced by a factor of $n^{2}$ compared to the input signal,

$$
S_{\text {कovi }}(f)=n^{2} S_{\phi_{\text {in }}}(f),
$$

making it easier to perform the necessary noise measurements. Similarly. it is also easier to make Allan-variance measurements. If the oscillator under test and the reference are both multiplied by the same factor, the beat frequency will be $n$ times larger than with no multiplication but the heterodyne factor will be the same. The zero crossings that must be detected by the counter have $n$ times higher slope and more easily overcome the voltage noise in the counter trigger circuits. The ability to measure frequency stability is only enhanced if the multipliers have extremely low phase noise themselves. This is the case for many modern multipliers that are triggered by the zero crossings of the input signal. As a result, the use of multipliers can reduce the performance requirements on the phase detector and the following low-noise amplifiers.

\subsection{CONCLUSION}

The IEEE recommendations have achieved the goal of introducing substantial uniformity in the specification of oscillator performance. The Allan variance and the one-sided power spectral density of phase have proved sufficient to evaluate oscillators for all common applications. In a few cases more specialized measures are helpful in relating performance to the specific application. For example, the rms time-prediction error is helpful in judging a clock's ability to keep time over long intervals (Allan and Hellwig, 1978). 
However, the specialized performance measures are generally calculable in terms of the IEEE recommended measures.

Significant progress has been made during the last 15 years in measurement techniques and data processing. These advances have obscured the dividing line between the frequency domain and the time domain. Today the spectral density and the variance are most often computed from the identical input data set, the equally spaced time series of the phase deviations. The choice of a specific measurement setup can be made mostly on a cost versus performance basis. Perhaps the biggest advance in commercially available equipment is the introduction of heterodyne measurement techniques for time-domain (counter-based) measurements. As a result, the noise performance of these systems has improved dramatically.

One recommendation that should be made is to perform measurements as high up in the measurement hierarchy as possible. Direct measurement of the phase deviation is most desirable. This approach places the largest share of the burden on the measurement equipment, minimizes long-term errors, and maximizes data processing flexibility. 


\section{Chapter 12}

Ablowich. D.. Jr. (1966). Some observations concerning frequency and time standardization Proc. 21st Insirum. Soc. Am. Conf. 21. 20

Akimochkin. I K.. Denisov. V. P.. and Cherenkova. E. M (1973). Long-term frequency comparator Meas. Tech (Engl. Transl.) 16. 83-85

Aldridge. D. (1975). $5 \mathrm{MHz}$ digital frequency meter. Eleciron (82). 61. 63-64.66. 68

Aldridge. D. (1978). $5 \mathrm{MHz}$ digital frequency measunng instrument. Elekiron. An: 10.15-18. In German

Allan. D W. (1966). Statistics of atomic frequency standards Proc. IEEE \$4. 221-230

Allan. D (1975). "The Measurement of Frequency and Frequency Siability of Precision Oscillators." NBS Technical Note 669. National Bureau of Standards. Boulder. Colorade Allan. D. W.. and Bames. J A. (1981). A modified "Allan vanance" with increased oscilldior characterization ability Proc 35 ih Annu Freq Conirol S.mp.. Pp $4^{\top 7} 0-4^{\top} 3$ 
Allan. D. W., and Daams. H. (1975). Picosecond time difference measurement system. Proc. 29th Annu. Freq. Control Symp., pp. 404-411.

Allan. D. W., and Hellwig. H. (1978). Time deviation and the prediction error for clock specification, characterization and application. Proc. Position Location and Narigation Simp., pp. 29-36.

Allan. D. W.. Gray. J. E.. and Machlan. H. E. (1972). The National Bureau of Standards atomic time scales: generation. dissemination, stability, and accuracy. IEEE Trans. Instrum. Meas. IM-21. 388-391.

Allan. D. W., Gray. J. E., and Machlan. H. E. (1974). The National Bureau of Standards atomic time scales: generation. stability. accuracy and accessibility. In "Time and Frequency: Theory and Fundamedeals" (B. E. Blair, ed.). NBS Monogr. 140. pp. 205-231.

Allan, D. W.. Hellwig. H.. and Glaze. D. J. (1975). AD accuracy algorithm for an atomic time scale. Metrologia 11. 133-138.

Andres. 1. (1977). Straight talk on frequedcy counters. Communications 54. 56-60.

Andriyanov, A. V., Glebovich, G. V., Krylov, V. V.. Korsakov, S. Ya., and Ponomarev, D. M. (1980). Automated measurements in the time domain and a method of increasing itheir sccuracy I:mer. Tekh. 23, 42-44. In Russian.

Anonymous (1967). Definition, realisation and use of time and frequency. Collog. Dig. IEE. Paper no. 11.87.

Anonymous (1975a). Electromagnetic radiation in frequency measurements. Electron. Aust. 37. 3i. 33.

Anonymous (1975b). Analogue frequency meter (rev. counter). Eleklor (GB) 1. 737.

Anonymous (1975c). L'niversal frequency reference. Elekıor $(G B)$ 1. 715.

Anonymous (1975d). A programmable counter type frequency meter. Electron. Microelectron. Ind. (213), 45-49. In French.

Anonymous (19?6a). High accuracy timing devices. Elektron. Elektrotech. 31. 9-11. In Dutch.

Anon!mous (1976b). Time and frequency: two important quantities in modern measuremeats. Tecnol. Elettr. (12), 66-69. In Italian.

Anonymous (1977a). $50 \mathrm{MHz}$ counter timer. CSIO Commun. (India) 4. 34.

Anonymous (1977b). A universal counting and time measuring device. Elekron Int. (5), 167-169. In German.

Anunymous $11977 \mathrm{c}$ ). Precision timebase for frequency counter. Elekior (GB) 3. 22-25.

Anonymous (1977d). Which counter? Automation 12.21, 23, 25.

Anonymous (1978a). Time measurement where are we? Mes. Regul. Autom. 43. 19-23. In French.

Anonymous (19:8b). A universal counter on one chip. Elektronikschau \$4. 48. In German.

Anonymous (1978c). Yaesu Musen YC-500S frequency councer. Electron. Aust. 40. 103.

Anunymous (19;9a). A system of measuremed and display of frequency for a AM FM receiver. Eleciron. .tppl. Ind. 271. 55-56. In French.

Anonymous (19:9b). A frequency counter for radio receivers. Elektronikschau 55. 56-57. In German.

Anonymous (1979\%: A special IC revolutionizes the design of universal councers. Elekron. Prex. 14. 1\&-18. In German.

Anonymous (1979d). Microprocessor and LSI: two festures of a universal $100 \mathrm{MHz}$ counter. Eleciron .tppl. Ind. 270. 23-26. In French.

thonymous (1979e). Time frequency measurements in radar equipment-which technique? Onde Elecir. 59. 47-54. In French

Antyufeev. V. I.. Breslavets, V. P., Fedulov, V. M.. and Fertik. N. S. (1980). Application of digital tiltrution for messuring instubility Rudiolekhnika (Kharkor) 45. 43-49. In Russian.

Ars. M $(19 ; 8 d)$. Frequency counter trends: $\mu$-processors facilitate improvements. JEE. $J$ Eleciron. Eng. 136. 30-31.

Ara. M. (1978b). L'niversal counters are be:oming intelligent. JEE. J. E!ectrun. Eng 136. 32-34. 
Arnoldt. M (1974). A frequency counter with time-multuple indication. 1. Funk-Tech. 20. 721-724. In German.

Arnoldt, M. (1977). Frequency counter for ultra short wave radio receivers. Funk-Tech. 32. 237-242. 244-245. In German.

Amoldt. M. (1979). A frequency counter for VHF receivers with frequency control loops. Fundschau 51, 1371-1374. In German.

Artyukh. Yu. N., Arnit. M. A., Gotlib, G. I., and Zagurskii, V. Ya. (1978). Instrument for measuring time intervals to an accuracy of $2 \mathrm{nsec}$. Instrum. Exp. Tech. (Engl. Transl.) 20, 1039-1041.

Ashley. J. R., Searles, C. B.. and Palka, F. M. (1968). The measurement of oscillator noise at microwave frequencies. IEEE Trans. Microwate Theory Tech. MTT-16. 753-760.

Auchterlonie, L. J., and Bryant, D. L. (1978). A direct method of measuring small frequency shifts using two open resonators at millimetre wavelengths. Int. J. Electron. 45, 11 3-122.

Ausejo. R. (1976). Frequency and time meters. Mundo Electron. 50, 59-66. In Spanish.

Azoubib. J.. Granveaud, M., and Guinot, B. (1977). Estimation of the scale unit duration of time scales. Metrologia 13, 87-93.

Baev, A. V., Rozkina, M. S., and Torbenkov, G. B. (1973). Capacitive method for measuring frequency. Meas. Tech. (Engl. Transl.) 16. 1034-1036.

Bahadur. H., and Parshad, R. (1977). Measurement of time. J. Inst. Electron Telecommun. Eng. (.Vex. Delhi) 17. 103-108

Baidakov, I. G.. Pushkin, S. B., and Titov, V.P. (1973). Securing unanimity of time and frequency measurement in the USSR at the highest section of the reference system. Meas. Tech. (Engl. Transl.) 16. 60-63.

Baird. K. M.. Hanes, G. R., Evenson, K. M., Jennings, D. A., and Petersen, F. R. (1979). Extension of absolute-frequency measurements to the visible: frequencies of ten hyperfine components of iodine. Opt. Lett. 4, 263-264.

Balph, T. (1976). A $200 \mathrm{MHz}$ autoranging frequency counter. Electron. Equip. New's. April. p. 17.

Barber. R. E. (1971). Short-term frequency stability of precision oscillators and frequency generators. Bell Syst. Tech. J. 50, 881-915.

Barnaba. J. F. (1972). USAF time and frequency calibration program. Proc. 26th Annu. Freq. Control Simp.. pp. 260-263.

Barnes. J. A. (1966). Atomic timekeeping and the statistics of precision signal generators. Proc. IEEE \$4, 207-219.

Barnes. J. A. (1969). Tables of bias functions $B_{1}$ and $B_{2}$ for variances based on finite samples of processes with power lau spectral densities. NBS Tech. Note (L'S.) 375.

Barnes. J. A. (1972). Problems in the definition and measurement of frequency stability. Proc. 26th .Annu. Freq. Control S. 1 mp.. p. 20.

Barnes. J. A. (1976a). A simulation of the fluctuations of International Atomic Time. ABS Tech. Viote (L:S.) 609, 20 pp.

Barnes. J. A. (1976b). Models for the interpretation of frequency stability measurements. NBS Tech. Nole ( $($ '.S.) 689,39 pp.

Barnes. J. A.. and Allan, D. W. (1966). A statistical model of ficker noise. Proc. IEEE 54 . $176-178$.

Barnes. J. A.. and Winkler, G. M. R. (1972). The standard of time and frequency in the LSA. Proc. 26ih Annu. Freq. Control Symp., pp. 269-278.

Barnes, J. A.. Chi, A. R.. Cutler. L. S., Healey, D. J.. Leeson. D. B.. McGunigal. T. E.. Mullen. J. A., Jr.. Smith, W. L., Sydnor. R. L., Vessot. R. C. F., and Winkler. G. M. R. (1971). Characterization of frequency stability. IEEE Trans. Instrum. Weas. I.M1-20, $105-120$.

Barrier, B.. and Humbert. J.P. (1973). Numerical frequency meters trom A to Z. Int. Electron. 28. 33-39. In French.

Bastelberker. J. (1968). Normal frequencies, their investigation and measurement. FernmeldeInve'nie'ur 22. 23. In Cerman. 
Bisugh. R. A. (1971). Frequency modulation analysis with the Hadamard variance. Proc. 25ih .tnnu. Freq. Conirol Symp.. pp. 222-225.

Baugh. R. A. (1972). Low noise frequency multiplication. Proc. 26th Annu. Freq. Control Symp.. pp. 50-54.

Bava. E.. De Marchi. A., and Godone. A. (1977). A narrow output linewidth multiplier chain for precision frequency measurements in the $1 \mathrm{THz}$ region. Proc. 3/st Annu. Freq. Control S.

Bay. Z.. and Luther. G. G. (1968). Locking a laser frequency to the time standard. Appl. Phys. Lelt. 13. 303-304.

Becker. G. (1976). The second as standard unit of time. Lmsch. Wiss. Tech. 76. 706-707. In German.

Becker. G., and Hubner. U. (1978). The generation of time scales. Radio Sci. 14. 593-603.

Beckman. D. (1974). How accurate is your timer-counter? Electron. Equip. New's 15. 32-33.

Begley. W. W., and Shapiro. A. H. (1970). Precision timing systems. I. Standard scales. Instrum. Control Syst. 43. 87-89.

Behnke, H. (1973). Standard frequency comparison by error multiplication. Radio Fernsehen Elekiron. 22.642-643. In German.

Beisner. H. M. (1980). Clock error with a Wiener predictor and by numerical calculation, IEEE Trans. Instrum. Meas. I.M1-29. $105-113$.

Belenov, E. M.. and Uskov, A. V. (1979). Measurement of laser frequencies using superconducting point contacts. Sot. J. Quantum Electron. (Engl. Transl.) 9, 1519-1522.

Belham. N. D. N. (1980). How accurate is a digital frequency meter? Radio Commun. 56. 368-371.

Belomestnov. G. I., Dmitrienko, A. D., Emakov, A. S., and Korzh, V. F. (1976). Automatic system for acquiring. recording. and processing information by computer for secondary time-and-frequency standards. Meas. Tech. (Engl. Transl.) 19, 1572-1574.

Belotserkovskii. D. Yu., Il'in. V. G., and Stepanova. I. V. (1976). International cooperation in the field of exact time and frequency measurements. Meas. Tech. (Engl. Transl.) 18. 517-519.

Berger. F. (1975). Time metrology and quartz application. I. Time metrology. Ret: Gen. Electr. 84. 907-918. In French.

Bezdrukov. S. M., and Preobrizhenskii. N. I. (1979). Increased accuracy in audio-frequency measurements with the $\mathrm{S} 4-12$ and $\mathrm{S}+-53$ spectrum analyzers. Instrum. Exp. Tech. (Engl. Transl.) 21. 714-716.

Bichler. H.. and Fenk. J. (1981a). Simple universal 0 to $30 \mathrm{MHz}$ frequency counter with SDA 5680A. Siemens Components (Engl. Transl.) 16. 21-24.

Bichler, H... and Fenk. J. (1981b). The IC unit. SDA 5680A. universal frequency counter covering $100 \mathrm{kHz}$ to $30 \mathrm{MHz}$. Elektron Int. 3-4, 89-92. In German.

Blachman. N. M. (1976). The effect of noise upon polar-display instantaneous frequency measurement. IEEE Trans. Insirum. Meas. IM1-25. 214-21.

Blair. B. E. (1974). Time and frequency: theory and fundamentals. NBS .Wonogr. (C:S.) $1+0$, $460 \mathrm{pp}$.

Blaney. T. G.. Cross, N. R.. Knight, D. J. E.. Edwards. G. J., and Pearce. P. R. (1980). Frequency measurement at $4.25 \mathrm{THz}(70.5 \mu \mathrm{m})$ using Josephson harmonic mixer and phase-lock techniques. J. Phi's. D 13, 1365-1370.

Bodea. M.. and Dunilis. T. (1979). Autoranging. digital time-interval meter. Bull. Inst. Politeh. Gheorghe Gheorghiu-Dej Bucuresti Ser. Electroteh. 40. 57-63. In Rumanian.

Body, 1.. and Szemok. 1. (1974). General problems of frequency measurement. Weres futom. 22. 422-428. In Hungartian.

Bologlu. A. 11975). Automutic 4.5-GHz counter provides I-Hz resolution. Hew/et1-Puckard J. 27. $14-18$. 
Bologlu. A. (1980). A new method of measuring the frequency of microwave signals, using microprocessors. Nachr. Elektron. 34, 149-152. In German.

Bologlu. A.. and Barber. V. A. (1978). Microprocessor-controlled harmonic heterodyne microwave counter also measures amplitudes. Hewlets-Packard J. 29. 2-4, 6-7, 9-12, 14-16.

Bordt, A. (1973a). Universal digital counter for frequencies up to $12 \mathrm{MHz}$. I. Radio Fernsehen Elektron. 22. 365-368. In German.

Bordt. A. (1973b). Universal digital counter for frequencies up to $12 \mathrm{MHz}$. II. Radio Fernsehen Elektron. 22. 402-406. In German.

Borisochkin, V. V. (1972). Checking the frequency of highly stable generators. Meas. Tech. (Engl. Transl.) 15. 460-463.

Bosnjakovic. P. (1976). A new solution of the precision line frequency measurement. Automatika 17. 194-196. In Serbo-Croatian.

Bowman. J. A. (1973). Short and long term stability measurements using automatic data recording system. Proc. 27th Annu. Freq. Control Symp.. pp. 440-445.

Bowman, M. J., and Whitehead, D. G. (1977). A picosecond timing system. IEEE Trans. Instrum. Meas. IM-26, 153-157.

Brandenberger, H., Hadorn, F., Halford, D., and Shoaf, J. H. (1971). High quality quartz crystal oscillators: frequency domain and time domain stability. Proc. 25th Annu. Freq. Control Simp.. pp. 226-230.

Brown. D. E. (1957). Frequency measurement of quartz crystal oscillators. Marconi Instrum. 11. 12-15.

Budreau, A. J., Slobodnik, A. J., Jr., and Carr, P. H. (1982). Fast frequency hopping achieved with SAW synthesizers. Microwave J. 25, 71-73, 76-80.

Burgoon, R., and Fischer. M. C. (1978). Conversion between time and frequency domain of intersection points of slopes of various noise processes. Proc. 32nd Annu. Freq. Control Symp..pp. 514-519.

Buchuev, F. I., and Ivakin. V. M. (1979). Electronic converter of the unit time dimension. Meas. Tech. (Engl. Transl.) 21. 788-789.

Buxton. A. J. (1976). Digital frequency meter. Pract. Electron. 12, 376-382.

Buzek. O.. Cermak. J., Smid, J., and Medellin. H. (1980a). A time base controlled by the OMA $50 \mathrm{kHz}$ transmitter. Sdelovaci Tech. 28, 362-365. In Czech.

Buzek, O., Cermak. J., and Tolman, J. (1980b). Accurate time and frequency. Slaboproudy Obz. 41, 263-266. In Czech.

Cantero, J. A., Pollan. T., and Barquillas, J. (1978). Digital frequency meter uses variable measuring and automatic scale conversion. Mundo Electron. 71, 34-35. In Spanish.

Cardwell, W. T.. Jr. (1978). Frequency counter in a probe. Radio Electron Eng. 49, 67, 72-73. $100-101,103$

Charriere. J.-J. (1977). A digital chronometer. I. Rev. Polyrech. (6), 585. 587. In French.

Chebotayev, V. P.. Goldort, V. G., Klementyev, V. M.. Nikitin, M. V., Timchenko, B.A., and Ziskharyash. V. F. (1982). Development of an optical time scale. Appl. Phys. B 29, 63-65.

Coates, P. B. (1968). Fast measurement of short time intervals. J. Sci. Instrum. 1. 1123-1127.

Cochran, W. T.. Cooley. J. W., Favin. D. L.. Helms, H. D.. Kaenel, R. A., Lang, W. W., Maling. G. C.. Jr.. Nelson. D. E., Rader, C. M.. and Welch. P. D. (1967). What is the fast Fourier transform? IEEE Trans. Audio Electroacoust. AV-15. 45-55.

Colburn. J., and Owen. B. (1979). $600 \mathrm{MHz}$ portable frequency counter. Radio Electron. Eng. $\mathbf{5 0 .}$ $39-43.90$.

Cole. H. M. (1975). Time domain measurements. Electron, Electro-Optic Inf. Countermeas. 1. $38-42.55$.

Conway. A., and Urdaneta. N. (1978a). Considerations in selecting a frequency counter. Communications 15, 22-23. 
Conway. A., and Urdaneta, N. (1978b). Selecting a frequency counter. Teleph. Eng. Management 82. 77-78.

Cordwell. C.R. (1968). "Frequency Measurements." Vacation school on electrical measurement practice. Institution of Electrical Engineers. London. 13 pp.

Cordwell. C. R. (1969). Frequency measurements. Electron. Radio Tech. 3, 95-103.

Cosmelli. C.. and Frasca. S. (1979). Fast new technique for measuring the resonance frequencies of high $Q$ systems. Ret. Sci. Instrum. 50. 1650-1651.

Cutler, L. S.. and Searle. C. L. (1966). Some aspects of the theory and measurement of frequency fluctuations in frequency standards. Proc. IEEE 54. 136-154.

Czarnecki. A. (1980). Test set for the comparison of standard frequencies, type FZ-530. Pr. Inst. Tele-Radiorech. (83). 27-32. In Polish.

Danilevich. V. V.. Chernyavskii. A. F., and Yakushev. A. K. (1977a). Selectro-type time interval analyzer with a measurement range of $10^{-10}$ to $10^{-2} \mathrm{sec}$. Instrum. Exp. Tech. (Engl. Transl.) 20. 326-327.

Danilevich. V. V., Kvachenok, V. G., Priimak. M. A., Chernyavskii, A. F., and Yakushev. A. K. (1977b). Signal generator for adjusting and calibrating time measurement systems. Instrum. Exp. Tech. (Engl. Transl.) 20, 600.

Danzeisen. K. (1977). Remote frequency measurement using VHF-UHF receivers. Funk-Tech. 32. 196-197. In German.

Davis. D. D. (1975). Calibrating crystal oscillators with TV color-reference signals. Electronics 48. 107-112.

De Costanzo. L. (1979). Radio receiver frequency counter clock. Electron. Ind. 5. 16-17.

De Jong. G., and Kaarls, R. (1980). An automated time-keeping system. IEEE Trans. Instrum. .Meas. 1M-29. 230-233.

Denbnovetskii, S. V.. and Kovtun, A. K. (1970). Time measurements. Digital time-interval meters. I:mer. Tekh. 10. 29-31. In Russian.

Denbnovetskii. S. V.. and Shkuro, A. N. (1975). Principles of eliminating ambiguity in digital time-interval meters (review). Instrum. Exp. Tech. (Engl. Transl.) 18. 1659-1670.

De Prins. J. (1974). Timing systems. In "ELF-VLF Radio Wave Propagation Spatind. Norway," pp. 385-398. Reidel. Dordrecht. The Netherlands.

De Prins. J. (1977). Aspects of time and frequency measurement. Rer. HF 10, 123-134. In French.

De Prins. J., and Cornelissen. G. (1974). Critique and discussion about frequency stability definitions with respect to various kinds of noises. CPE.M 74 Dig., pp. 54-56.

Dietzel. R. (1975). Passive resonators with noise feeding in frequency-analogue measuring systems. Mess., Steuern, Regeln 18. 389-393. In German.

Dombrovskii, A. S.. Zaitsev, V. N.. and Pashev, G. P. (1974). Apparatus for time and frequency measurement. .Meas. Tech. (Engl. Transl.) 17. 919-921.

Domnin. Y.S.. Kosheljaevsky. N. B.. Tatarenkov. V. M.. and Shumjatsky. P. S. (1980a). Precise frequency measurements in submillimeter and infrared region. IEEE Trans. Instrum. Meas. IM-29. 264-26:

Dumnin. Yu. S.. Kosheljaevsky. N. B.. Tatarenkov. V. M., and Shumjatsky. P. S. (1980b). Precise frequency measurements in submillimeter and infrared region [lasers]. CPE.M Dig.. p. 85.

Doring. F. W... and Hollenberg. K. (1981). Eight-channel time measurement system with 2 ns resolution. J. Phy's. E 14. 638-640.

Doronina. O. M., and Petukh. A. M. (1980). Interpolation method of increasing measurement accuracy for low frequencies. Attometriya (5), 89-92. In Russian.

Drake. J. M.. and Rodriguez-Izquierdo. G. (1978). Analogue setup for fast-response frequency measuremen:. Elecuron. Eng. 50, 41. 43.

Drake. J M.. Rodriguez-Izquierdo. G. (1980). Fast analogue measurement of low frequencies. Ret. Telegr. Electron. 69, 1359-1360. In Spanish. 
Egidi. C. (1969). Frequency;time metrology at the I.E.N.G.F. (Instituto Elettrotechnico Nazionale Galileo Ferraris). RGE. Rer. Gen. Electr. 78. 275-282. In French.

Egidi. C., and Leschiutta. S. (1970). The true meaning of the word " time." Alta Freq. 39. 689-693.

Egidi, C., and Leschiutta. S. (1971). The metrology of time. Rir. Nuoto Cimento 1. 496-518. In Italian.

El-Ghoroury. H. S.. and Gupta. S. C. (1976). Algorithmic measurement of digital instantaneous frequency. IEEE Trans. Commun. COM-24. 1115-1122.

Elkin. G. A., and Purtov. V. 1. (1976). Automatic system for comparing the frequency of a [quantum] store with the transition frequency of cesium atoms. Meas. Tech. (Engl. Transl.) 19. $1456-1460$.

Ely. R. D. (1978). Frequency measurements by frequency meter or timer counter? Electron. Poner 24. 45-46.

Englert. R. (1978). Circuit for measuring very low frequencies. Elektronik (.Munich) 27.67-68. In German.

Essen. L. (1967). Atomic units and scales of time. IEE Collog. Definition. Realisation and lise of Time and Frequency, Dig., Paper No. 1.

Essen, L. (1968). Time scales. Metrologia 4. 161-165.

Essen. L. (1981). Leap seconds [transfer from astronomical to atomic time]. Wireless World 87. $39-40$.

Evenson. K. M.. Wells. J. S.. Petersen. F. R., Danielson. B. L.. Day. G. W.. Barger. R. L.. and Hall. J. L. (1972). Laser frequency and wavelength measurement and the speed of light. Abstr. Ann. Meeting Opi. Soc. Am., p. 22.

Faulkner. N. D.. and Vilar. E. (1982). Sub-harmonic sampling for the accurate measurement of frequency stability of microwave oscillators. CPE.M Dig.. pp. M,10-11.

Ferrari, H. 1. (1976). Frequency meter and digital counter. Rer. Telegr. Electron. 64, 234-238. 241-242. In Spanish.

Fichtenbaum, M. L. (1976). Counter inverts period to measure low frequency. Electronics 49. $100-101$.

Filin. N. A., and Trofimov. A. V. (1976). Time counter. Instrum. Exp. Tech. (Engl. Transl.) 19. 1582.

Filous, L. (1979). Analogue measurement of frequency. Sdeloraci Tech. 27, 325-328. In Czech.

Firth. G. F.. and Pratt. D. M. (1976). A digital frequency counter and timer (direct read-out at $150 \mathrm{MHz}$ ). Radio Commun. 52. 182-189.

Fischer. M. C. (1978). Frequency stability mea surement procedures. Onde Electr. 58. 291-299. In French.

Fischer. M. C. (1981). Methods for the measurement of the degree of stability in a frequency spectrum. Nachr. Elektron. 35. 381-389. In German.

Fleys. M. (1978). Microwave frequency counters-which conversion technique to choose?.Mess. Regul. Autom. 43, 31-38. In French.

Freeman. R. L. (1977). The science of time and its inverse. Telecommun. J. (Engl. Transl.) 44. 66-7?.

Frenkel. L. (1969). Methods for the measurement of frequency and stability of laser generators of submillimeter oscillations. Electron Technol. 2. 47-52.

Fukuyo. H. (1975). Time and frequency stundards. J. Soc. Instrum. Conirol Eng. 14. 521-528. In Japanese.

Fullagar. D. (1976). Frequency counter. Aen Electron. 9. 28

Gabry. A.. Faucheron. G.. Dubouis. B., and Petit. P. (19;i). Distant comparison of stable irequency standards by means of the transmission of a beat note between the carrier of a TV broadcast signal and a frequency synthesized from the frequency standards. Proc. 3/st Annu. Freq. Control S!mp.. pp. 499-502. 
Gardner, F. M. (1966). "Phaselock Techniques." Wiley. New York.

Godone, A., De Marchi. A.. and Bava, E. (1979). An improved multiplier chain for precise frequency measurements up to $20 \mathrm{THz}$. Proc. 33rd Annu. Freq. Conirol Symp.. pp. 498-503.

Gonzalez., R. L., and Olivero, M. A. (1980). A digital frequency meter. Ret. Telegr. Electron. 68. 67-72. In Spanish.

Granveaud. M. (1980). Contribution of the computation algorithm to the instability of atomic time scales. CPEM Dig., pp. 7-8.

Granveaud. M., and Azoubib. J. (1974). The problem of ageing in the derivation of TAI [International Atomic Time]. CPEM Dig., pp. 279-280.

Granveaud. M., and Guinot. B. (1978). The role of primary standards in establishing the International Atomic Time TAI. CPEM Dig.. pp. 39-41.

Grauling. C. H.. Jr., and Healey. D. J., III (I966). Instrumentation for measurement of the shortterm frequency stability of microwave sources. Proc. IEEE 54, 249-257.

Gray, J. E. (1976). Clock synchronization and comparison: problems, techniques and hardware. NBS Tech. Note (U.S.) 691, 9 pp.

Greivulis, Ya. P.. and Blumbergs. E. A. (1976). Frequency meter. Izv. Vyssh. Vchebn. Zaved. Priborosir. 19. 13-15. In Russian.

Grenchik. T. J., and Fang. B. T. (I977). Time determination for spacecraft users of the Navstar Global Positioning System (GPS). Proc. 31st Annu. Freq. Control Symp., pp. 1-3.

Groslambert, J. M. (1971). Automatic measurement system of oscillator frequency stability. Eurocon Dig. (IEEE), p. I.

Groslambert, J., Olivier, M., and Uebersfeld. J. (1972). Automatic plotting systems for frequency stability curves and discrete spectral density of frequency standards. IEEE Trans. Instrum. Meas. IM-21, 405-409.

Groslambert, J., Olivier, M., and Uebersfeld, J. ([974a). Spectral and short term stability measurements. IEEE Trans. Instrum. Meas. IM-23, 518-521.

Groslambert. J., Olivier. M., and Uebersfeld. J. (1974b). Spectral and short term stability measurements. CPEM Dig.. pp. 60-61.

Guignard. A.. and Berney, J. C. (1967). A new series of electronic instruments for time measurements and standard-time distribution. Mes., Regul., Autom. 32. 100. In French.

Guinot. B. (1967). Mean atomic time-scales. Bull. Astron. 2. 449-464. In French.

Guinot. B. (1971). Characteristics of a scale of time. Alta Freq. 40.684-686.

Guinot. B. (1974). Long term stability and accuracy of time scales. CPE.M Dig.. pp. 333-335.

Guinot. B. (1980). Problems of the generation, quality, and availability of the International Atomic Time Scale. CPE.M Dig.. pp. 5-6.

Guinot. B., and Granveaud, M. (1972). Atomic time scales. IEEE Trans. Instrum. Meas. IM-21. $396-400$.

Guinot. B., Granveaud. M., and Azoubib. J. (1981). Stability and accuracy of the international atomic time. J. Insi. Electron. Telecommun. Eng. (vew Delhi) 27, 524-527.

Gutnikov, V. S.. Nedashkovskii. A. I., and Palkin. V. S. (1977). A specialised digital frequency meter used for operation with measuring frequency convertors. Prib. Sist. $C_{p r}$. 5, 22-24. In Russian.

Hafner. E. (1969). Frequency and time. Proc. Conf. Vien Horizons in Measurements-Trends in Theory and Application (JEEE). pp. 58-68

Halford. D. Wainwright. A. E., and Barnes. J. A. (1968). Flicker noise of phase in RF amplifiers and frequency multipliers: characterization. cause and cure. Proc. 2?nd Annu. Freq. Control S!mp..pp. 340-341.

Halford. D.. Shoaf, J. H.. and Risley. A. S. (1973). Spectral density analysis: frequency domain measurements of frequency stability. Proc. 27 th Annu. Freq. Control Symp.. pp. 421-431 
Hamanaka, A. (1978). New frequency counter series features high reliability. JEE, J. Electron. Eng. (142), 50-54.

Harry, E. (1978). Counter, scope team up for precise timing measurements. Electronics 51 , 126-127.

Healey, D. J., III (1972). Flicker of frequency and phase, and white frequency and phase fluctuations in frequency sources. Proc. 26th Annu. Freq. Control Symp., pp. 29-42.

Healey. D. J., III (1974). $L(f)$ measurements on UHF sources comprising VHF crystal controlled oscillator followed by a frequency multiplier. Proc. 28 th Annu. Freq. Control Symp., pp. 190-202.

Hellwig. H. (1981). Reliability, serviceability and performance of frequency standards. J. Inst. Electron. Telecommun. Eng. (New Delhi) 27, 362-365.

Hellwig, H., and Wineland, D. J. (1978). Time, frequency and physical measurement. Phys. Today' 31, 23-30.

Hocker. L. O.. Small. J. G., and Javan, A. (1969). Extension of absolute frequency measurements to the $84 \mu$ range. Phy.s. Lett. A 20, 321-322.

Hodgkinson. G. (1977). Counters and timers, once lab luxuries, now everyday instruments Electron 13, 16. 19

Hoene, J., and Holland-Nell. U. (1970). Time measuring equipment for the delayed representation of signals with high definition. Nachrichtentechnik 20,405-411. In German

Hoffman, K. (1982). Time synchronisation via OTS-2. CPEM Dig., pp. M:16-17.

Howe, D. A., Allan, D. W., and Barnes, J. A. (1981). Properties of signal sources and measurement methods. Proc. 35th Annu. Freq. Control Symp., pp. Al-A47.

Hubner. U. (1977). Models and predictions for the realization of time scales. Proc. 3/st Annu. Freq. Control Symp., pp. 327-334

Hudson, G. E., and Barnes. J. A. (1968). Clock error statistics as a renewal process. Proc. 22nd Annu. Freq. Consrol Symp., pp. 384-418.

Hutchinson. F. (1976). Frequency measurement and timer-counters. Electron. Equip. New's (March), 22-23.

IEEE (1975). "IEEE Standard Modular Instrumentation and Digital Interface System (CAMAC)-1EEE Standard 583-1975." IEEE, New York.

IEEE (1978). "IEEE Standard Digital Interface for Programmable InstrumentationANSI IEEE Standard 488-1978." IEEE, New York.

Iijima. S.. and Fujiwara. K. (1968). Precise measurement of radio time signals by means of pulsed oscillator. Tokyo Astron. Obs. Rep. 14. 273-285.

Il'in. V. G., and Lukin. A. E. (1973). Phase fluctuations in a heterodyne multistage frequency multiplier [for standard frequency comparison]. .Meas. Tech. (Engl. Transl.) 15. 1659-1661.

Il'in. V. G.. Sazhin. V. V.. Bolotinikov, M. V., and Elkin, G. A. (1974). High-precision time and frequency measurements in the national economy and scientific investigations. Meas. Tech. (Engl. Transl.) 17, 1033-1937.

Imshenetskii. V. V.. and Martyniv. M. S. (1974). Extending the frequency limits of an electronic counting frequency meter. Otbor Peredacha Inf. 39, 89-92. In Russian.

Ioannides. G. (1974). The $50 \mathrm{MHz}$ universal counter UZ56 UZ56s. Grundig Tech. Inf. 21. 307-310. In German.

Ivanov. N. I., and Kravshenko, V.F. (1973). Short-term frequency instability of an oscillator. lar. Vissh. Cichebn. Zated. Radioelektron. 15, 1391-1393. In Russian.

Ivanov. V. V., and Stepanov. B. M. (1971). Measurement of small duration intervals. Ismer. Tekh. 14. 25-27. In Russian.

Izokh. V. V.. and Tabachnik. E. 1. (1977). Mean-square and information-theory estimates of time-interval measurement accuracy in the presence of noise. Meas. Tech. (Engl. Transl.) 20. $343-347$. 
Jackisch. C.. Schubert, H. J., and Hartmann. H. L. (1980). Measurement of time fluctuations in synchronous communication networks. CPEM Dig., pp. 34-39.

Jayapal, R. (1977). Measure power frequency with a conventional counter. Electron. Eng. 49, 17.

Jenkins, G. M.. and Watts. D. G. (1968). "Spectral Analysis and Its Applications." Holden-Day, San Francisco.

Jennings. D. A., Petersen, F. R., and Evenson, K. M. (1975). Extension of absolute frequency measurements to $148 \mathrm{THz}$ : frequencies of the 2.0 and $3.5 \mu \mathrm{m}$ Xe laser. Appl. Phys. Lett. 26. 510-511.

Jennings. D.A., Petersen, F. R., and Evenson. K. M. (1979). Frequency measurement of the 260$\mathrm{THz}(1.15 \mu \mathrm{m}) \mathrm{He}-\mathrm{Ne}$ laser. Opt. Lett. 4. 129-130.

Jimenez, J. J., and Petersen, F. R. (1977). Recent progress in laser frequency synthesis. Infrared Phys. 17, 541-546.

Job. A. (1969). Short term stability of sinusoidal generators. Onde Electr. 49, 528-538. In French.

Jones, C. H. (1979). A test equipment for the measurement of phase and frequency instability in VHF and SHF sources. Radio Eleciron. Eng. 49. 187-196.

Jutzi, K. H. (1973). Frequency measurement by signal sampling. Frequenz 27, 309-311. In German.

Kahnt, D. (1977). ASMW time and frequency signals and their applications. Radio Fernsehen Elekiron. 26, 112-113. In German.

Kalau, M. (1980). The GDR atomic time scale. CPEM Dig., pp. 11-14.

Kamas, G., and Howe, S. L. (1979). Time and frequency user's manual. NBS Spec. Publ.(U.S.) 559, 248 pp.

Kamyshan. V. V., and Valitov, R. A. (1969). Heterodyne method of frequency measurement in the millimeter range. Instrum. Exp. Tech. (Engl. Transl.) (4), 925-928.

Karpov, N. R. (1980). Vernier method of measuring time intervals. Meas. Tech. (Engl. Transl.) 23. 817-822.

Kartaschoff. P. (1973). Terms and methods to describe frequency stability. Tech. Mitt. PTT 51 , 520-529. In German.

Kartaschoff, P. (1977). Frequency control and timing requirements for communications systems. Proc. 3lst Annu. Freq. Control Symp., pp. 478-483.

Kazi, A. M., and Perini, G. J. (1971). Program execution time measurement. IB.M Tech. Disclosure Bull. 14, 428.

Kendall, W. B., and Parsons, P. L. (1968). A precision frequency measurement technique for a moving signal with a low signal to noise ratio. Conf. Precision Electromagn. Meas (IEEE), pp. 54-55.

Kesner, D. (1973). Take the guesswork out of phase-locked loop design. ED.V 18, 54.

Kiehne. F. (1967). FEGR-A multi-purpose $500 \mathrm{kHz}$ counter. Nex's Rhode and Schwar: 7. 15-16.

Kimel"blat. V. 1. (1978). Evaluation of measuring accuracy of middle frequency and bandwidth for radio-receiving apparatus. Meas. Tech. (Engl. Transl.) 21, 541-543.

Kirchner, D. (1983). Precision timekeeping at the Observatory Lustbuehel. Graz. Austria. Proc. 37 th Annu. Freq. Control Symp.. pp. 67-77.

Kirianaki. N. V.. Kazantsev. E. M.. and Mosievich. P. M. (1977). Multirange fast automatic digital frequency meter. Izr. Vyssh. L'chebn. Zaved. Priborostr. 20. 9-14. In Russian.

Klimov, A. I.. and Meleshko. E. A. (1977). Monitoring unit for time-interval analyzers of nanosecond range. Instrum. Exp. Tech. (Engl. Transl.) 20. 1058-1060.

Knight. D. J. E., Edwards, G. J., and Blaney. T. G. (1977). Developments in laser frequency measurement towards realising the metre from a unified frequency and length standard. European Conf. Precise Electr. Meas. (IEE). p. 40.

Kobayashi, M., Osawa. H., Morinaga. N., and Namekawa. T. (1973). Output signal-to-noise ratios in mean frequency measurement system. Tech. Rep. Osaka Lnit. 23, 515-526. 
Kobayashi, M.. Osawa. H., Morinaga, N., and Namekawa. T. (1976). Mean frequency and mean power measurement using correlation detection. Trans. Soc. Instrum. Control Eng. 12. 613-618. In Japanese.

Kocaurek, R. (1968). Frequency comparison receiver EF 151 k. New's Rohde and Schwar: 8, 23-24.

Koch, E. (1979). An all-waveband receiver with a digital frequency display and a quartz clock. Funkschau 51, 236-239. In German.

Koga, I. (1970). Precision measurement of crystal frequency by means of "center line method." Proc. 24th Annu. Freq. Control Symp..pp. 168-171.

Kogan, L. R. (1970). Oscillator stability requirements in radio interferometers. Radiotekh. Elektron. 15. 1292-1294. In Russian.

Kolbasin, A. I., and Pavlenko, Yu. F. (1978). Transfer of the dimension of a unit deviation of frequency from the special state standard to prototype means of measurement. Meas. Tech. (Engl. Transt.) 21. 806-809.

Koppe. H. (1979). Frequency measurement using a microcomputer-a program and an instruction program. Elektron. Prax. 14. 30, 35-36. 39. In German.

Kotel'nikov, A. V., Rubailo. G. T., and Uvarov, N. E. (1975). An instrument for measuring the ratio of time intervals. Telecommun. Radio Eng. (Engl. Transl.). Part / 29, 60-61.

Kourigin. E. I., Chernyshev, V. S., and Shumkin. Yu. V. (1979). High-speed device for time interval measurement. Instrum. Exp. Tech. (Engl. Transl.) 22, 484-486.

Kramer, G. (1970). An electronic time intervalometer with high resolution. Nachrichtentech. $Z$. 23. 433-436. In German.

Krause, W. H. U. (1968). Accurate time and frequency measurement. Frequenz 22, 178-182. In German.

Kuchynka, K., and Preuss, A. (1981). Electronic counters facilitate time measurement. Siemens Power Eng. 3, 179-182.

Kul'gavchuk, V. M. (1975). Frequency meters using devices with S-shaped volt-ampere characteristics. Instrum. Exp. Tech. (Engl. Transl.) 18. 514-516.

Kuznetsov, V.P., and Reznikov, A. L. (1974). Adjacent time intervals measured by two frequency meters. Meas. Tech. (Engl. Transl.) 17, 474-475.

Laker, K. R., and Slobodnik. A. J., Jr. (1975). "Impact of SAW Filters on RF. Pulse Frequency Measurement by Double Detection." Report AFCRL-PSRP-621. Air Force Cambridge Research Laboratories. Hanscom Air Force Base. Massachusetts. 194 pp. Available from the National Technical Information Service, Springfield, Virginia.

Lance, A. L., Seal, W. D.. Mendoza, F. G.. and Hudson, N. H. (1977). Automating phase noise measurements in the frequency domain. Proc. 31st Annu. Freq. Control Stmp., pp. 347-358.

Leah. P. (1977). Frequency counter timer. Pract. Electron. 14. 22-28.

Leikin, A. Ya., and Orlov. E. Z. (1973). Equipment for comparing frequencies of highly-stable generators. Meas. Tech. (Engl. Transl.) 16, 1469-1470.

Leikin. A. Ya.. Tomashenko. I. V., and Fertik. N. S. (1974). Application of a rubidium discriminator for the measurement of frequency fluctuations. $l=r$. Vissh. Licheth. Zated. Radtofi:. 17. 85i-854. In Russian.

Lemesev. S. G.. Rachev. B. T.. Strugach. V. G.. and Shub. S. M. (1975). Statistical method for measunng a recurrent time interval using a random pulse signal. $l z t$. Vyssh. Uchebn. Zared. Priborostr. 18. 16-21. In Russian.

Lesage. P. (1982). Characterization of frequency stability: bias due to the addition of time interval measurements. CPEM Dig.. pp. M 8-9.

Lesage. P. (1983). Characterization of frequency stability: bias due to the juxtaposition of umeinterval measurements. IEEE Trans. Instrum. Meas. 1.M-32. 204-207. 
Lesage. P.. and Audoin. C. (1973). Characterization of frequency stability: uncertainty due to the finite number of measurements. IEEE Trans. Instrum. Meas. I.M-22. 157-161, with corrections from IEEE Trans. Instrum. Meas. IM-25. 270.

Lesage. P.. and Audoin. C. (1975a). A time domain method for measurement of the spectral density of frequency fluctuations at low Fourier frequencies. Proc. 29th Annu. Freq. Control S.mp., pp. 394-403.

Lesage. P., and Audoin. C. (1975b). Comments on "Characterization of frequency stability: uncertainty due to the finite number of measurements." IEEE Trans. Instrum. Meas. I.M-24. 86.

Lesage. P., and Audoin. C. (1977). Estimation of the two-sample variance with a limited number of data [signal generator frequency stability]. Proc. 3/st Annu. Freq. Control Symp., pp. $311-318$.

Lesage, P., and Audoin. C. (1979a). Characterization and measurement of time and frequency stability. Radio Sci. 14, 521-539.

Lesage. P., and Audoin C. (1979b). Effect of dead time on the estimation of the two-sample variance. IEEE Trans. Instrum. Meas. IM-28, 6-10.

Lo Faso, G. (1968a). Digital frequency meter and chronometer in RTL micrologic. Int. Elektron. Rundsch. 22, 63-66. In German.

Lo Faso. G. (1968b). RTL Micrologic in a digital frequency and time counter. Int. Elektron. Rundsch. 22. 47-50. In German.

Lomas. G. (1974). Signal-frequency meter. Wireless World 80, 429-434.

Loose. P. (1979). Modern electronic-based timing systems. Electron. Eng. 51. 81. 83-84.

Lubentsov, V. F. (1973). The errors of the absolute method for measuring difference-signal frequency. Telecommun. Radio Eng. (Engl. Transl.), Parl 2 28. 96-99.

Lubentsov, V. F., and Ashitkov. I. N. (1968). Device for measuring the frequencies and errors of crystal oscillators. Meas. Tech. (Engl. Transl.) (7), 906-911.

Lukas, M. (1968). An intermediate frequency measuring equipment with an unusual frequency indication. Slaboproudy $O b=29.323-326$. In Czech.

MacLeod. K. J. (1973). A portable high-resolution counter for low-frequency measurements. Hev:letr-Packard J. 25, 10-15.

Maletz. W. (1974). $100 \mathrm{MHz}$ universal counter FET 100. New's Rohde \& Schwar: 14. 5-7.

Markowitz. W. (1968). Time and frequency measurement. atomic and astronomical. Proc. 5th Annu. IS A Test Meas. Simp.. Instrument Society of America. Pittsburgh. Pennsylvania. p. 6.

Murtin. D. (1971). Frequency stability measurements by computıng counter system. HexteltPackard J. 23. 9-14.

Martin. D. (1974). Measure time interval precisely. Electron. Des. 22. 162-167.

Marun. J. D. (1972). Digital methods of frequency measurements: a comparison. Rudio Electron. Eng. 42. 285-294.

Maton. G. W. (1978). Thoughts on frequency meters and counter-timers. Electron (134), 24, 27.

Maxwell. B. J. (1980). Perception of errors in instruments and applications. 1. Time, our most accurate medsurement. Autom. Control (Viex Zealand) 10, 27-29.

McCarthy. E. P. (1979). A digital instantaneous frequency meter. IEEE Trans. Instrum. Meas. I.M-28, 224-226.

McCaskill. T. B., and Buisson, J. A. (1975). NTS-1 (TIMATION 111) [satellite] quartz and rubidium oscillator frequency stability results. Proc. 29th Annu. Freq. Controi Symp., pp. $425-428$.

McCaskill. T.. White. J.. Stebbins. S.. and Buisson. J. (1978). NTS-2 cesium frequency stability results. Proc. 3.nd Annu. Freq. Control Symp.. pp. $560-566$.

McCellin. G. (1978). Prescaler and LSI chip form 135-.MHz counter. Electronics $51,97,99$.

Meer. P. (1977). Four function calculator can measure time. Electron. Eng. 49. 35. 
Meisa. J. L.. and Schultz. D. G. (1969). "Linear Control Systems." McGraw-Hill. New York.

Mende. F. F., and Charkin, V. A. (1973). Superhigh frequency measurement with electroniccounter frequency meters. Meas. Tech. (Engl. Transl.) 16, 1360-1362.

Millham. E. H. (1974). High-accuracy time meter. IBM Tech. Disclosure Bull. 17. 1143-1144.

Mints. M. Ya.. and Chinkov. V. N. (1980). Accuracy of time interval measurement by pulse counting. Meas. Tech. (Engl. Transl.) 23, 603-606.

Mitsenko. I. D., and Borisov, S. K. (1975). Phase-pulse discriminator for measurement of nanosecond time intervals. Instrum. Exp. Tech. (Engl. Transl.) 18, 144-145.

Mohos. Z. (1973). Time interval measurement by averaging. Meres Autom. 21. 268-272. In Hungarian.

Mroz. T. J. (1977). Time-interval meter reads digitally to $99.9 \mathrm{~ms}$ on a DVM IC. Electron. Des. 25. 108. 110.

Muchiauri. A. A. (1973). A digital method of frequency measurement for harmonic signals. Meas. Tech. (Engl. Transl.) 16. 1710-1712.

Muhlenbroich. H. P. (1980). The mode of operation of time interval measurement. Elektron. $J$. 15. 32-34. In German.

Mungall, A. G. (1971). Atomic time scales. Metrologia 7, 146-153.

Murashov. B. P., and Tsvetkov. Yu. P. (1976). Instrument for checking quartz-crystal oscillators with standard frequency signals in the long-wave and very-long-wave ranges. Meas. Tech. (Engl. Transl.) 19, 1012-1015.

Muzychuk. O. V., and Shepelevich. L. G. (1974). On the determination of short time signal frequency instability. Izi. Vyssh. Uchebn. Zaved. Radiofiz. 17, 855-863. In Russian.

Nakadan. Y., and Koga. Y.(1977). Measurements of frequency stability of precision oscillators. Oyo Butsuri 46, 572-579. In Japanese.

Navarro-Fernandez. A. (1974). Adapting a frequency meter into a digital clock. Mundo Electron. (34), 92. In Spanish.

Niki. S. (1975). Automatic frequency counter uses TRAHET technique to count $1032 \mathrm{GHz}$ with $-20 \mathrm{dBm}$ sensitivity. JEE. J. Electron. Eng. (107), 20-23.

Offermann, R. W., Schultz. S. E., and Trimble, C. R. (1975). Active probes improve precision of time interval measurements. Hew'lett-Packard J. 27, 11-16.

Ohman. B. (1978). New task for the telecommunication authority-national measurement standards for time and frequency. Tele 84. 14-24. In Swedish.

Orte. A. (1976). Comparison of various fundamental methods of defining time. with special reference to astronomical a pplication. Narigation 24. 72-84.

Osakabe. M.. Sasao, K., and Kato. E. (1973). Field strength and frequency measuring instruments. Anritsu Tech. Bull. (29), 52-56. In Japanese.

Pacheu. Y. (1979). Analogue frequency meter. Electron. Appl. Ind. (262S), 15-16. In French.

Page. R. (1979). Time and frequency counting techniques. Electron. Eng. 51, 47-48, 51, 53, 57-58.

Palamaryuk. G. O., and Kholkin. I. I. (1967). Digital smoothing frequency meter for variablesign pulse signals. Meas. Tech. (Engl. Transl.) (7), 845-847.

Palii. G. N.. and Pushkin. S. B. (1980). Improvement of time and frequency stabilization. Meas. Tech. (Engl. Transl.) 23. 115-118.

Parkinson. B. A. R. (1970). Precise time and frequency measurement. II. Electron. Equip. News 12, 60-65.

Pashev. G. P.. and Parfenov, G. A. (1977). Analysis of the errors of statistical devices measuring repeated time intervals. Meas. Tech. (Engl. Transl.) 20, 347-349.

Patankar. A. R.. Nambiar. K. P. P.. and Sitharaman. M. R. (1974). An automatic electronic frequency comparator. J. Inst. Electron. Telecommun. Eng. (Vew Delhi) 20, 405-407.

Patykov, V. G.. Podlesnyi, S. A., and Chmykh. M. K. (1976). Digital frequency meter with high noise immunity. Instrum. Exp. Tech. (Engl. Transl.) 19, 1581. 
Paul. F. (1973). Reciprocal frequency meters. Electron. Microelectron. Ind. (168), 55-59. In French.

Peltz, I. G. (1971). Highly stable audio generator for frequency measurements on clock crystals. Elektronik (Munich) 20, 345-348. In German.

Percival, D. B. (1978a). The U.S. Naval Observatory clock time scales. IEEE Trans. Instrum. Meas. IM-27, 376-385.

Percival, D. B. (1978b). The U.S. Naval Observatory clock time scale. CPEM Dig., Conf. Precision Electromagn. Meas., pp. 42-44.

Percival. D. B. (1982). Characterization of time and frequency instabilities: prediction error variance. CPE.M Dig.. pp. N/7-9.

Percival, D. B., and Winkler. G. M. R. (1975). Timekeeping and the reliability problem. Proc. 29th Annu. Freq. Control Symp., pp. 412-416.

Peres. A. (1980). Measurement of time by quantum clocks. Am. J. Phys. 48. 552-557.

Perkinson. R. E. (1970). Time frequency technology in system development. Proc. 24th Annu. Freq. Control Symp., pp. 315-321.

Petersen, A. P. (1980). A new apparatus for the precise determination of time and frequency on the LF band using transmitter DCF 77. CPEM Dig.. pp. 17-19.

Petrov, A. F., Zhukov, E. T., Kosharnovskii, G. V., Breslavets, V. P., and Fedulov, V. M. (1977). Frequency comparator based on a delay line. Meas. Tech. (Engl. Transl.) 20, 1456-1458.

Pokorny. L. (1976). Modern frequency counters. Radio Elektron. Schau (Austria) 52. 20-24. In German.

Pokorny, L. (1977). Hewlwtt-Packard 5328A universal counter with DVM options. Elektroniskschau (Austria) 53, 56.

Potschke. H. (1976). Time measurement. Nachr. Elektron. 30, 107-108. In German.

Powers, R. S., and Snyder. W. F. (1969). Radio-frequency measurements in the NBS Institute for Basic Standards NBS Tech. Note (L'.S.) 373, pp. 37-42.

Pritchard. N. B. (1979). A frequency counter for a $144 . \mathrm{MHz}$ transmitter. Radio Commun. 55, 410-415.

Prusakov, V. K., and Kilna. A. A. (1974). About digital harmonic signal frequency measuring accuracy. Liet. TSR Mokslu Akad. Darb., Ser. B (80), 141-148. In Russian.

Putkovich. K. (1972). Automated timekeeping. IEEE Trans. Instrum. Meas. IM-21, 401-405.

Ramamurthy, T. V., and Kumar, M. K. (1979). A measurement technique for low frequencies. $J$. Phys. E. 12, 187-188.

Reeder, R. (1977). The not so simple counter. Electron. Equip. New's (November), 9-10.

Reider. G. A. (1980). Standard deviation of averaged digital time interval measurement [error values]. Rev. Sci. Instrum 51. 1423-1424.

Reid. J. H. (1972). The measurement of time. J. R. Astron. Soc. Can. 66, 135-148.

Reinhardt. V.. and Lavanceau. J. (1974). A comparison of the cesium and hydrogen hyperfine frequencies by means of Loran C and portable clocks. Proc. 28 ih Annu. Freq. Control Symp.. pp. 379-383.

Reussner, J., and Weiss. H.-J. (1973). Standard frequency receiver for calibrating frequency counters. Radio Fernsehen Elektron. 22. 638-640. In German.

Ribenyi. A. (1967). Digital frequency measuring instruments. .Meres .Automat. 15. 436-439. In Hungarian.

Rikhter. V., and Fogler. G. (1977). Influence of stresses due to discontinuous jamming on the accuracy of digital frequency measuring devices. Prib. Sist. Cpr. (1). 21-23. In Russian.

Risley, A. S. (1976). The national measurement system for time and frequency. NBS Spec. Publ. (U.S.) $445-1.64$ pp.

Rivamonte. J. M. (1972). U.S. Army calibration program. Proc. 26th Annu. Freq. Control Symp., pp. 258-259.

Roth, R. (1977). Alignment generator AS5 with quartz controlled frequency indicator. Grundig Tech. Inf. 24, 209-216. In German. 
Rozwadouski. M. (1971). Modern frequency stabilisation methods. Przegl. Telekomun 44. 335-338. In Polish.

Ruderfer. M. (1979). One-way doppler effects in atomic timekeeping. Speculations Sci. Technol. 2. 387-404.

Runyon. S. (1977). Focus on electronic counters. Electron. Des. 25, 54-63.

Rusconi, L., and Sedmak. G. (1977). Estimates of the limiting standard deviation in the absolute timing of periodic sources. Astrophys. Space Sci. 46, 301-308.

Rutman, J. (1972a). Comment on "Characterization of frequency stability." IEEE Trans. Instrum. Meas. IMI-21. 85.

Rutman. J. (1972b). Short term frequency instability of signal generators. Onde Electr. 52. 480-487. In French.

Rutman, J. (1974a). Characterization of frequency stability: a transfer function approach and its application to measurements via filtering of phase noise. IEEE Trans. Instrum. Meas. IM-23. $40-48$.

Rutman. J. (1974b). Relations between spectral purity and frequency stability. Proc. 28th Annu. Freq. Control Symp.. pp. 160-165.

Rutman, J. (1977). Oscillator specifications: a review of classical and new ideas. Proc. 3/st Annu. Freq. Control Symp.. pp. 291-301.

Rutman, J. (1978). Characterization of phase and frequency instabilities in precision frequency sources: fifteen years of progress. Proc. IEEE 66, 1048-1075.

Rutman. J.. and Sauvage. G. (1974a). Measurement of frequency stability in time and frequency domains via filtering of phase noise. CPEM Dig.. pp. 57-59.

Rutman. J.. and Sauvage. G. (1974b). Measurement of frequency stability in time and frequency domains via filtering of phase noise. IEEE Trans. Instrum. Meas. 1M-23. 515-518.

Rzewvski, M. (1976). Frequency deviation measurements by synchronized sampling method. Przegl. Telekomun. 49. 7-9. In Polish.

Sakai, T. (1977). Frequency counters become sophisticated. JEE. J. Electron. Eng. (121). 20-23.

Saunders, W. K. (1972). Short term frequency stability in coherent radar applications. Proc. 26th Annu. Freq. Control Symp., pp. 21-28.

Schneider, R. J. (1971). Time and frequency standardization. Signal 25, 42-45.

Schommartz. G. (1979). On the measurement dynamics of digital frequency meters. Mess. Steuern. Regeln 22. 201-267. In German.

Schreiber. H. (1978). Active test head for a frequency counter. Funk-Tech. 33. 241-242. In German.

Secaze. G. (1975). Measurement of time-standards and their marketing. Electron. Microelectron. Ind. (208), 71-75. In French.

Senftleben. H. (1977). A digital universal counter. Radio Fernsehen Elektron. 26. 4\$1-445. In German.

Shoaf. J. H.. Halford. D. and Risley. A. S. (1973). Frequency stability specification and measurement: high frequency and microwave signals. NBS Tech. Note (U'S.) $632,65 \mathrm{pp}$.

Simpson. L. (1977). A low-cost $200 \mathrm{MHz}$ digital frequency meter. Electron. Aust. 38. 30-35. 37

Simpson. L. (1978). L'pgraded $200 \mathrm{MHz}$ digital frequency meter. II. Construction details. Electron. Aust. 40, 60-63

Small. J. C.. Monchalin. J. P., Kelly. M. J.. Keilmann. F.. Senchez. A.. Singh. S. K. Kurnit. N. A.. and Javan. A. (1972). Precision absolute frequency and wavelength measurements in the infrared: a revieu of activities at MIT. Proc. 26th Annu. Freq. Control Symp.. pp. 248-249.

Smith. H. M. (1967). Astronomical time scales. Colloq. Definition. Realisation and (se of Time and Frequency, IEE Colloq. Dig.. Paper No. ?.

Snyder. J. J. (1981). An ultra-high resolution frequency meter. Proc. 35th Annu. Freq. Control Simp.. pp. 464-469.

Sopel nikov: M. D.. and Fedorov. Yu. A. (1980). Investigation of errors in synchronizing time scales over wide-band communication channels. Meas. Tech. (Engl. Transl.) 23, 118-120 
Sorden. J. L. (1974). A new generation in frequency and time measurements. Hewlett-Packard J. 25. $2-8$.

Spapens. W. (1972). Accurate time measurements with a counter and an oscilloscope. Philips Test Meas. Not. (2), 4-8.

Speake. B. (1979). The accurate setting of microwave frequencies. Commun. Int. 6. 19, 21.

Stecher, R. (1973). Electronic time interval measurement with nanosecond resolution. Nachrichtentech. Elektron. 23, 413-4I6. In German.

Steele. J. McA. (1967). Frequency measurement using standard frequency emissions. Conf. Freq. Generation and Control Radio Syst. IEE Conference Publication No. 31.

Steer, G. (1973). A new UHF frequency measuring device. Fernmelde-Praxis 50. 571-600. In German.

Stein, S., Glaze, D., Levine, J., Gray, J., Hilliard, D., Howe, D., and Erb, L. A. (1983). Automated high-accuracy phase measurement system. IEEE Trans. Instrum. Meas. I.M-32. 227-231.

Steiner, C. I. (1969). Electronic methods of time measurement. Elektroniker 8. 3-16. In German.

Stoyko, A. (1967). Ephemeris time according to the revised lunar theory of Brown. Bull. Astron. 2. 411-416. In French.

Szata, R. (1968). New method of oscilloscopic frequency measurement. Pomiary, Autom., Kontrola 14, 200-301. In Polish.

Szepan, R. (1978). Determining accuracy of receiver-controlled frequency standards. New's Rohde \& Schwar: 18, 23-26.

Taylor, D. (1968). Time measurement. Instrum. Rer. 15, 173-I 76.

Taylor, G. W. (1977). Time-a precious commodity. Electron. Power 23, 61-64.

Tolman, J. (1970). Accurate frequency and time measurement at the Institute of Radio Engineering and Electronics. Slaboproudy' Obz. 31, 286-290. In Czech.

Tooley, M. (1979). In-line crystal calibrator. Pract. Wireless 55, 49-50, 55.

Triplet. J. M. (I967). Ephemeris time determination by lunar photography. Bull. Astron. 2, 465-527. In French.

Trkal, V. (1968). A new definition of the second on a quantum basis. Cesk. Cas. Fyz. A. 18, 641-669. In Czechoslavakian.

Turrini. A. (1974). Heterodyne frequency meter No. 35IC E.I.P. Antenna $46,62-68$. In Italian.

Tyrsa, V. E., and Dyunyashev, V. V. (1981). Accuracy of frequency measurement based on the pulsed coincidence principle. Meas. Tech. (Engl. Transl.) 24, 308-312.

Underhill, M. J., Sarhadi, M.. and Aitchinson, C. S. (I978). Fast-sampling frequency meter. Electron. Lett. 14, 366-367.

Upton. R. (1977). An objective comparison of analog and digital methods of realtime frequency analysis. Bruel Kjaer Tech. Rev. (Denmark) 1. 18-26.

Vakman, D. Ye. (1979). The evaluation of frequency stability. Telecommun. Radio Eng. (Eng!. Transl.), Part 2 34. 49-53.

Vakman. D. Ye. (1979). Measuring the frequency of an analytical signal. Radio Eng. Electron. Phy.s. (Engl. Transl.) 24. 63-69.

Valaskova. E. (1971). Measuring the frequency of damped oscillations. Sdeloraci Tech. 19. 174-175. In Czech.

Valverde. R. M. (1968). A digital instantaneous frequency-meter. Automa:. Strum. 16, 216-221. In Italian.

Van Blerkom. R., Freeman. D. G.. and Crutchfield, R. C.. Jr. (1968). Frequency measurement techniques. IEEE Trans. Instrum. Meas. IM-17. 133-145.

Van Den Brock, H. G. J. (1977). Two frequency counters for routine measurements. Polytech Tijdschr. Elektrotech. Elektron. 32, 517-518. In Dutch.

Van den Steen. L. (1979). A low frequency meter with instantaneous response. IEEE Trans. Biomed. Eng. B.ME-26, 137-140. 
Van Straaten, J. (1979). A one-chip LSI frequency meter in the $f^{2} L$ lechnology for FM and AM receivers. IEEE Trans. Consum. Eleciron. CE-25. 134-143.

Vessot, R., Mueller. L., and Vanier, J. (1966). The specification of oscillator characteristics from measurements made in the frequency domain. Proc. IEEE S4. 199-206.

Viles, R. S. (1972). Swept frequency techniques for accurate r.f. measurements. Marconi Instrum. 13. 88-92.

Voss, R. F. (1979). I// noise. Proc. 33rd Annu. Freq. Control Symp.. pp. 40-46.

Wainwright. A. E.. Walls, F. L., and MCCaa. W. D. (1974). Direct measurement of the inherent frequency stability of quartz crystal resonators. Proc. 28 th Annu. Freq. Control Symp.. pp. 177-180.

Walls. F. L.. and DeMarchi. A. (1975). RF spectrum of a signal after frequency multiplication: measurement and comparison with a simple calculation. IEEE Trans. Insirum. Meas. IM-24, 310-317.

Walls. F. L., and Stein. S. R. (1976). Servo techniques in oscillators and measurement systems. NBS Tech. Note (L:S.)69?.

Walls, F. L., Stein, S. R., Gray. J. A., and Glaze, D. J. (1976). Design considerations in state-ofthe-art signal processing and phase noise measurement systems. Proc. 30th Annu. Freg. Conirol Symp.. pp. 269-274.

Ward. R. B. (1982). VHF/UHF frequency measurement using the surface acoustic wave delay difference device. Appl. Phys. Lett. 40. 1010-1012.

Weiss. C. O., and Godone. A. (1982). Extension of frequency measurements with Schottky diodes to the $4 \mathrm{THz}$ range. Appl. Phys. B 27, 167-168.

Weiss. R. (1976). Time-signal and standard-frequency receiver for the DCF 77 with reserve. 1. Funkschau 48, 964-968. In German.

Wessel. R. (1973). A digital frequency meter. Elektromeister Disch. Elektrohandwerk 48. 1066-1068. In German.

Westlund, R. C. (1978). Very short time interval measurements. New Electron. 11, 13-14. 18.

Wheeler. K. G. (1976). An optimum solution for counting and timing. Niex Electron. 9. 24. 28. 32.

Whittaker, A. E., and Westbury. P. R. (1976). Measuring low frequencies. New Electron. 9. 36.

Wiley. R. G. (1977). A direct time-domain measure of frequency stability: the modified Allan variance. IEEE Trans. Instrum. Meas. IM-26, 38-41.

Woschni. E. G. (1975). Errors in high-precision frequency measurements due to interactive effects. Wiss. Z. Tech. Hochsch. Karl-Marx-Stadt 17. 633-639. In German.

Woschni. E. G. (1976). On the optimum difference frequency in high accuracy frequency measurements of industrial parameters. Mess.. Stevern. Regeln Automatisierungsprax. 19. 334-335. In German.

Woschni. E. G. (1978). Application of microprocessors to the calculation of ertor correction for the syncbronisation effect in highly accurate frequency measurements. Wiss. Z. Tech. Hochsch. Karl-Mar.x-Stadt 20, 665-668. In German.

Wresien. M. (1976). Instantaneous frequency measuring circuit. Pomiary. Autom. Kontrola 22. 90-91. In Polish.

Yasuda. Y. (1974). Accuracy of estimating clockface and mean rate of the frequency standird by: normal regression theory. J. Radio Res. Lab. (Japan) 21. 39-60.

Yoshimura. K. (1972). The generation of an accurate and uniform time seale with calibrations and prediction. NBS Tech. Note (l:S.) 626.59 pp.

Yoshimura. K. (1978). Characterization of frequency stability: uncertainty due to the autocorrelation of the frequency fluctuations. IEEE Trans. Insirum. Meas. IN-27. 1-7.

Yoshimura. K. (1980). Calculation of unbıased clock-variances in uncalibrated atomic time scale algorithms. Metrologia 16. 133-139.

See Appendix Note \# 10 
Yoshimurs. K.. Harada. K.. and Kobayashi. M. (1971). Measurement of short-lerm frequency stability of frequency standards. Ret. Radio Res. Labs. 17. 437-443. In Ja panese.

Yuval. I. (1982). Frequency comparator uses synchronous detection. Electronics \$5. 160.

Zander. H. (1977). Mea'surement of low frequencies with short duration. Elektronik (Munich) 26. 63-64. In German.

Zelentosov, V. I. Sulakshin. A. S., and Fedorov. N. P. (1976). Meter for measuring the carrier frequency of single ultrahigh-frequency pulses. Insirum. Exp. Tech. (Engl. Tronsl.) 19. 4:6-4?7.

Zhilin. N. S. and Subbotin. L. S. (1997). Devices for reproducing reference time intervals. Meos. Tech. (Engl. Transl.) 20. 995-999

Zhuk. A V.. Kzzanisev. E. M.. Kitidankı. N. K.. and Mosievich. P. M. 119:71. Computer investigation of the metrological characteristics of frequency meters with digutal positive feesback and measures to improve them. Orbor Peredacho Inf. S0. 110-115. In Russian 


\title{
Time and Frequency (Time-Domain) Characterization, Estimation, and Prediction of Precision Clocks and Oscillators
}

\author{
Invited Paper
}

DAVID W. ALLAN

\begin{abstract}
A tutorial review of some time-domain methods of characterizing the performance of precision clocks and oscillators is presented. Characterizing both the systematic and random deviations is considered. The Allan variance and the modified Allan variance are defined, and methods of utilizing them are presented along $w$ ith ranges and areas of applicability. The standard deviation is contrasted and shoun not to be. in general, a good measure for precision clocks and oscillators. Once a proper characterization model has been developed, then optimum estimation and prediclion techniques can be employed. Some important cases are illustrated. As precision clocks and oscillalors become increasingly important in society, communication of their characteristics and specifications among the vendors. manufaclurers, design engineers. managers, and metrologists of this equipment becomes increasingl! imporlant.
\end{abstract}

\section{INTRODUCTION}

66 W H.t THEN.” asked St. Augustine, “is time? If no one asks me. I know what it is. If I wish to explain it to him who asks me, I do not know." Though Einstein and others have taught us a lot since St. Augustine, there are still many unanswered questions. In particular. can time be measured? It seems that it cannot; what is measured is the time difference betw'een two clocks. The time of an event with reference to a particular clock can be measured. If time cannot be measured, is it physical, an abstraction, or is it an artifact?

We conceptualize some of the law's of physics with time as the independent variable. We attempt to approximate our conceptualized ideal time by inverting these laws so that time is the dependent variable. The fact is that time as we now generate it is dependent upon defined origins, a defined resonance in the cesium atom. interrogating electronics, induced biases. timescale algorithms. and random perurbations from the ideal. Hence, at a significant level, time-as man generates it by the best means available to him-is an artifact. Corollaries to this are that every clock disagrees with every other clock essentially always, and no clock keeps ideal or "true" time in an abstract sense except as we may choose to define it. Frequency or time interval, on the other hand. is fundamental to nature: hence the definition of the second can approach

Manusirnpt resured .May 11. 1987: ravised June 15. 1987.

The duthor is with the Time and Frequency Division. National Bureau of Stundards. 325 Broadwas. Boulder. CO 80303.

IEEE Log Number $8716 \$ 61$ the ideal-down to some accuracy limit. Noise in nature is also fundamental. Characterizing the random variations of a clock opens the door to optimum estimation of environmental influences and to the design of optimum combining algorithms for the generation of uniform time and for providing a stable and accurate frequency reference.

Let us define $V(t)$ as the sine-wave voltage output of a precision oscillator:

$$
V(t)=V_{0} \sin \Phi(t)
$$

where $\Phi(t)$ is the abstract but actual total time-dependent accumulated phase stanting from some arbitrary origin $\Phi(t$ $=0$ ) $=0$. We assume that the amplitude fluctuations are negligible around $V_{0}$. Cases exist in which this assumption is not valid, but we will not treat those in the context of this paper. This lack of treatment has no impact on the development or the conclusions in this paper. Since infinite bandwidth measurement equipment is not available to us, we cannot measure instantaneous frequency; therefore $\nu(t)=(1 / 2 \pi) d \Phi / d t$ is not measurable. We can rewrite this equation with $\nu_{0}$ being a constant nominal frequency and place all of the deviations in a residual phase $\phi(t)$ :

$$
V(t)=V_{0} \sin \left(2 \pi \nu_{0} t+\phi(t)\right) .
$$

We then define a quantity $y(t)=\left(\nu(t)-\nu_{0}\right) / \nu_{0}$, which is dimensionless and which is the fractional or normalized frequency deviation of $\nu(t)$ from its nominal value. Integrating $y(t)$ yields the time deviation $x(t)$, which has the dimensions of time

$$
x(t)=\int_{0}^{t} y\left(t^{\prime}\right) d t^{\prime} .
$$

From this, the time deviation of a clock can be written as a function of the phase deviation:

$$
x(t)=\frac{\phi(t)}{2 \pi \nu_{0}} .
$$

\section{Systematic Models for Clocks and Oscillators}

The next question one may ask is why does a clock deviate from the ideal? We conceptualize two categories 


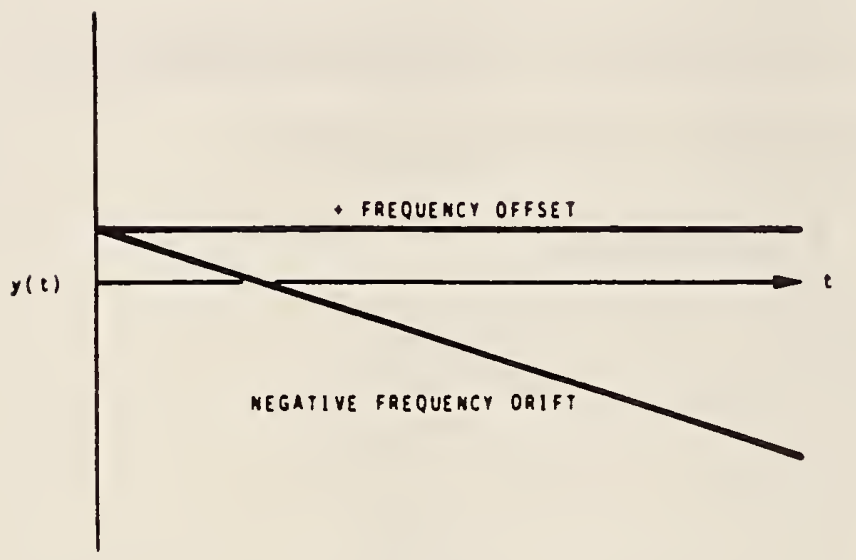

(a)

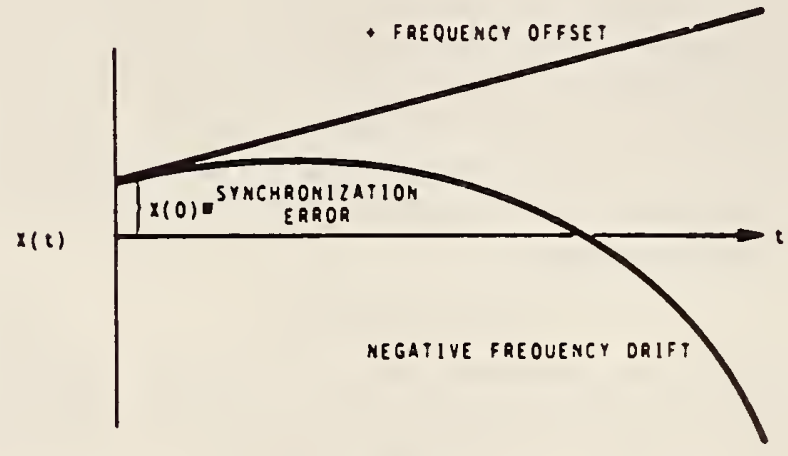

(b)

Fig. 1. Frequency $y(t)$ and time $x(t)$ deviations due to frequency offset and to frequency drift in clock. (a) Fractional frequency ertor versus time. (b) Time ertor versus time.

of reasons, the first being systematics such as frequency drift $(D)$. frequency offset $\left(y_{0}\right)$, and time offset $\left(x_{0}\right)$. In addition. there are systematic deviations that are often environmentally induced. The second category is the random deviations $\epsilon(t)$. which are usually not thought to be deterministic. In general, we may write

$$
x(t)=x_{0}+y_{0} t+1 / 2 D t^{2}+\epsilon(t) \text {. }
$$

Though generally useful, the model in (5) does not apply in all cases: e.g.. some oscillators have significant frequency-modulation sidebands, and in others the frequency drift $D$ is not constant. In some clocks and oscillators. e.g.. cesium-beam standards, setting $D=0$ is usually a better model.

Note that the quadratic $D$ term occurs because $x(t)$ is the integral of $y(t)$, the fractional frequency. and is often the predominant cause of time deviation. In Fig. I we have simulated two systematic-error cases: a clock with frequency offset. and a clock with negative frequency drift. Figs. 2-6 summarize some of the imporant systematic influences on precision clocks and oscillators. In addition to Figs. 1-6, imporant systematic deviations may include modulation sidebands, e.g.. $60 \mathrm{~Hz}, 120 \mathrm{~Hz}$, daily, and annual dependences, which can be manifestations of environmental effects such as deviations induced by vibrations, shock, radiation, humidity, and temperature.

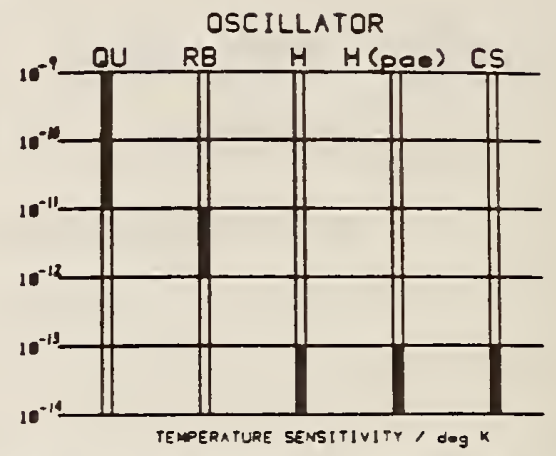

Fig. 2. Nominal values for temperature coefficient for frequency standards: $\mathrm{QU}=$ quanz crystal, $\mathrm{RB}=$ nubidium gas cell, $\mathrm{H}=$ active hydrogen maser, $\mathrm{H}$ (pas) = passive hydrogen maser, and $\mathrm{CS}=$ cesium beam.

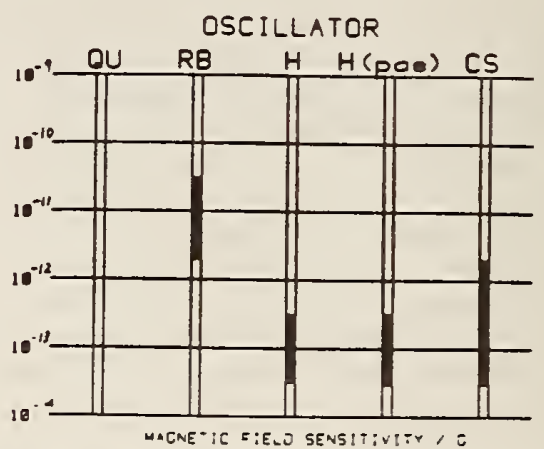

Fig. 3. Nominal values for magnetic field sensitivity for frequency standards: $\mathrm{QU}=$ quarzz crystal. $\mathrm{RB}=$ rubidium gas cell, $\mathrm{H}=$ active hydrogen maser, $\mathrm{H}($ pas $)=$ passive hydrogen maser, and $\mathrm{CS}=$ cesium beam.

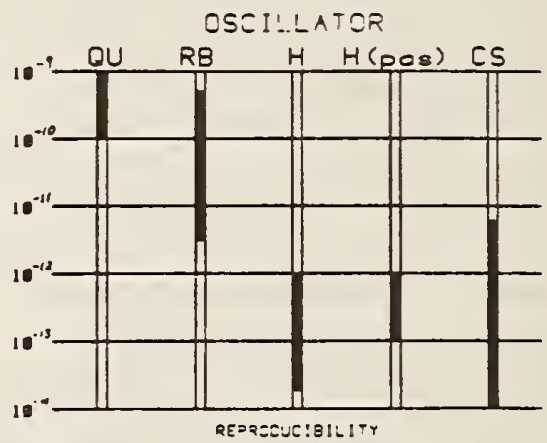

Fig. 4. Nominal capability of frequency standard to reproduce same frequency after period of time for standards: $Q U=$ quarz crystal. $R B=$ rubidium gas cell, $\mathrm{H}=$ active hydrogen maser, $\mathrm{H}$ (pas) = passive hydrogen maser. and CS = cesium beam.

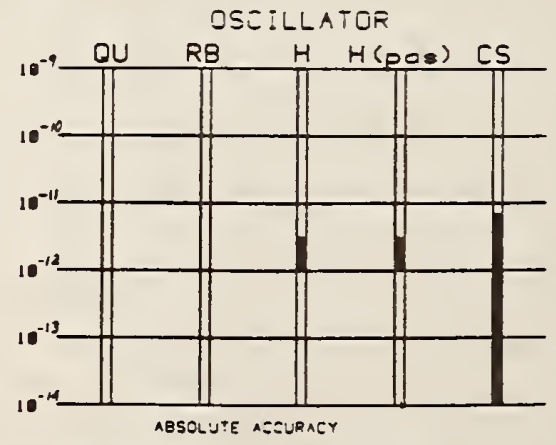

Fig. 5. Nominal capability for frequency standard to produce frequency determined by fundamental constants of nature for standards: $Q U=$ quanz crystal, $\mathrm{RB}=$ rubidium gas cell, $\mathrm{H}=$ active hydrogen maser. $H($ pas $)=$ passive hydrogen maser, and $C S=$ cesium beam. 
TABLE I

APPLICABle OSCILLATORS AND RANGe OF APPLICABILITY

\begin{tabular}{|c|c|c|c|c|c|}
\hline $\begin{array}{l}\text { Typical Noise Types } \\
\alpha \quad \text { Name }\end{array}$ & Cs & H-Active & H-Passive & Qu & $R b$ \\
\hline $\begin{aligned} 2 & \text { white-noise PM } \\
1 & \text { flicker-noise PM } \\
0 & \text { white-noise FM } \\
-1 & \text { flicker-noise FM } \\
-2 & \text { random-walk FM }\end{aligned}$ & $\begin{array}{l}\geq 10 \mathrm{~s} \\
\geq \text { days } \\
\geq \text { weeks }\end{array}$ & $\begin{array}{l}\leq 100 \mathrm{~s} \\
100 \mathrm{~s} s \tau \geq 10^{4} \mathrm{~s} \\
\geq 10^{4} \mathrm{~s} \\
\geq \text { weeks }\end{array}$ & $\begin{array}{l}\geq 1 \mathrm{~s} \\
\geq \text { days } \\
\geq \text { weeks }\end{array}$ & $\begin{array}{l}\leq 1 \mathrm{~ms} \\
\leq 1 \mathrm{~s} \\
\geq 1 \mathrm{~s} \\
\geq \mathrm{h}\end{array}$ & $\begin{array}{l}\geq 1 \mathrm{~s} \\
\geq 10^{4} \\
\geq \text { days }\end{array}$ \\
\hline
\end{tabular}

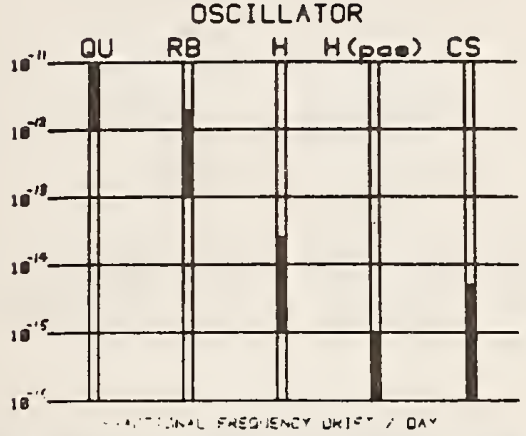

Fig. 6. Nominal values (ignonng sign) for frequency drift for frequency standards: $\mathrm{QU}=$ quarz crystal, $\mathrm{RB}=$ rubidium gas cell, $\mathrm{H}=$ active hydrogen maser, $\mathbf{H}$ (pas) = passive hydrogen maser, and $\mathrm{CS}=$ cesium beam.

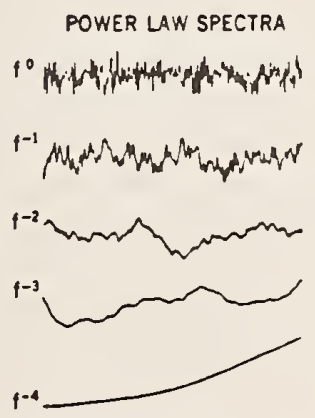

Fig. 7. Simulated random processes commonly occurring in output signal of atomic clocks. Power law spectra $S(f)$ are proportional to $\omega$ to some * exponent, where $f$ is Fourier frequency $\left((w=2 \pi f)\right.$ and $S_{y}(f)=$ $\left.w^{2} S_{y}(f)\right)$

\section{Random Models for Clocks and Oscillators}

The random-frequency deviations of precision clocks and oscillators can often be characterized by power-law spectra $S_{y}(f) \sim f^{\alpha}$, where $f$ is the Fourier frequency and $\alpha$ typically takes on integer values, i.e., $-2,-1,0,1,2$ [1]-[4]. Fig. 7 shows noise samples corresponding to these different power law spectra, and Table I shows the nominal range of applicability of these power-law models.

\section{Time-Domain Signal Characterization}

Given a discrete set of time deviations $x_{i}$ taken in sequence for the measurable time difference between a pair of clocks or between a clock and some primary reference, and given that the nominal spacing between adjacent time difference measurements is $\tau_{0}$ (see Fig. 8 for an example), then the average fractional frequency for the $i$ th measurement interval is

$$
\bar{y}_{i}^{+0}=\frac{x_{i+1}-x_{i}}{\tau_{0}}
$$

where $-\tau_{0}$ over $y_{i}$ denotes the average over an interval $\tau_{0}$.

We can thus construct a set of discrete frequency values from such a time-difference data set. If the standard deviation is calculated for this set of values, one can show that for some kinds of power-law spectra encountered in precision oscillators the standard deviation is divergent [1], [2], [5], i.e., it does not converge to a well-defined value and is a function of data length. Hence the standard deviation is seldom useful and can be misleading in characterizing clocks. An IEEE subcommittee has recommended $S_{y}(f)$ in the frequency domain and a measure $\sigma_{y}^{2}(\tau)$ in the time domain [1]. $S_{y}(f)$ is the one-sided spectral density of $y$ as a function of Fourier frequency $f$. The latter is often called the Allan variance or two-sample variance. The convergence of $\sigma_{y}(\tau)$ has been verified [1][4] for the power law spectra of interest in precision clocks and oscillators. The measure $\sigma_{y}^{2}(\tau)$ is defined as [1]

$$
\sigma_{y}^{2}(\tau)=\frac{1}{2}\left\langle\left(\Delta \bar{y}^{\tau}\right)^{2}\right\rangle
$$

where $\Delta \bar{y}^{r}$ is the difference between adjacent fractional frequency measurements, each sampled over an interval $\tau$, and the brackets $\langle$ > indicate an infinite time average or expectation value. A pictorial description is shown in Fig. 9 for a finite data set. A data set of the order of 100 points is more than adequate for convergence of $\sigma_{y}(\tau)$, though of course the confidence of the estimate will typically improve as the data length increases [6].

Given a discrete set of stored evenly spaced data, the value of $\tau$ can be varied in the software [7]. If $\tau_{0}$ is the minimum data spacing for the original stored data set $\bar{y}_{i}^{\tau_{0}}$, then one can change the sampling time to $\tau=n \tau_{0}$ by averaging $n$ adjacent values of $\bar{y}_{i}^{- \text {-ro }}$ to obtain a new fractional $* *$ frequency estimate $\bar{y}_{i}^{\tau}$, with sample time $\tau$ as input to (7). Note this is different from averaging adjacent values of $x$. Hence in a very convenient way one can calculate $\sigma_{y}(\tau)$ as a function of $\tau$, which will be shown to be very useful. For a finite data set of $M$ values of $\bar{y}_{i}^{70},(7)$ for general $\tau$ becomes (see Fig. 8 for an example computation of $\sigma_{y}(\tau$ 

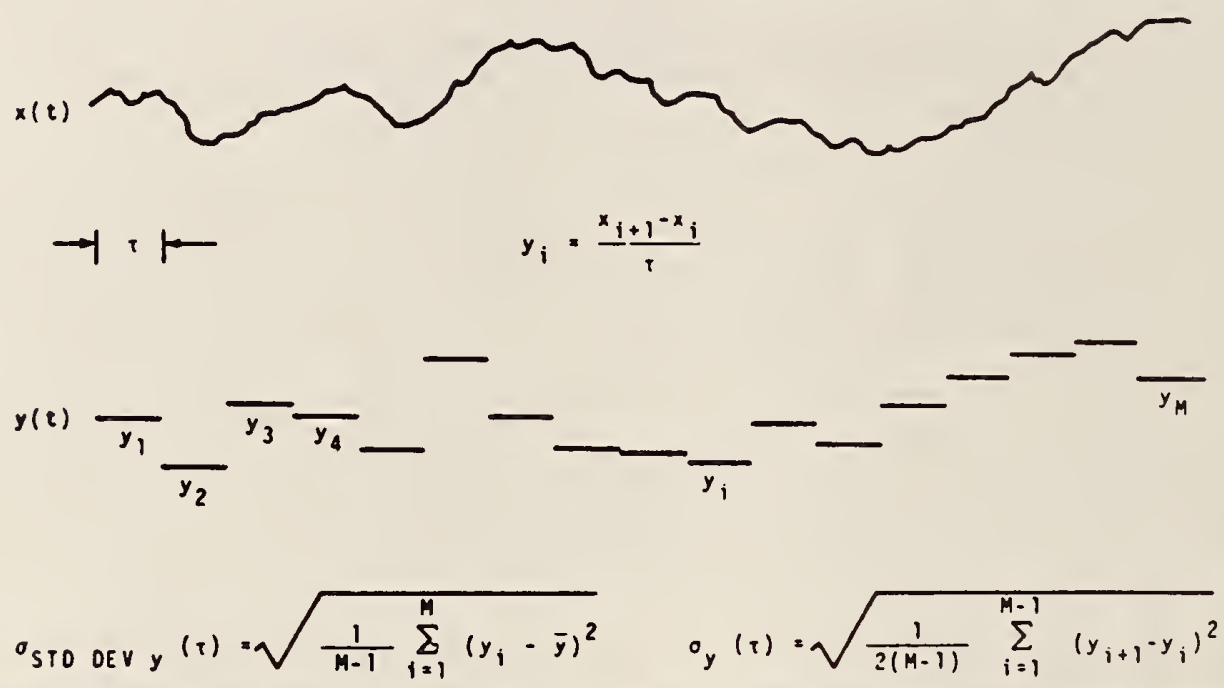

Fig. 8. Simulated time deviation plot $x(t)$ with indicated sample time $\tau$ over which each adjacent fractional frequency $y$, is measured. Equations are for standard deviation and for estimate of $\sigma_{y}(\tau)$ for finite data set of $M$ frequency measurements. Often standard deviation diverges as data length increases when measuring long-term frequency stability of precision oscillators, whereas $\sigma_{y}(\tau)$ converges.

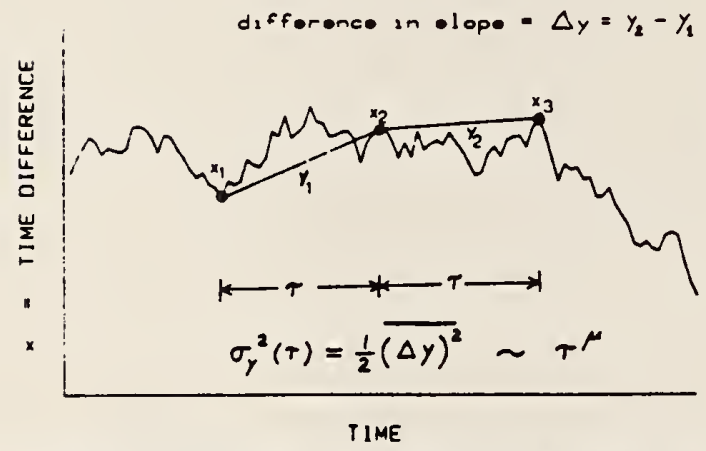

Fig. 9. Pictorial of computation of Allan variance. Simulated time variations plotted are random walk. At set sample time $\tau_{1} \Delta y=\left(x_{3}-2 r_{2}-\right.$ $\left.x_{1}\right) / \tau$ is computed. With time of measurement of $x_{3} \tau$ ahead of $x_{2}$ and that of $x_{2} T$ ahead of $x_{1}$, all possible values of $\Delta y$ are computed. Each $\Delta y$ is squared and average squared value determined, $\overline{(\Delta y)^{2}} ;$ taking $1 / 2$ of this yields two-sample or Allan variance for that value of $\tau$. Value of $r$ can then be changed either in hardware or software to determine Allan variance for another value of $T$.

$\left.=\tau_{0}\right)$. i.e., $n=1$ )

$\sigma_{y}^{2}(\tau)=\frac{1}{2(M-2 n+1)} \sum_{k=1}^{M-2 n+1}\left(\bar{y}_{k+n}^{\tau}-\bar{y}_{k}^{T}\right)^{2}$

where $\bar{y}_{k+n}^{r}$ and $\bar{y}_{k}^{r}$ are still adjacent fractional frequencies (i.e., no dead time exists between the measurements), each averaged over $\tau=n \tau_{0}$, and

$$
\bar{y}_{k}^{r}=\frac{1}{n} \sum_{i=k}^{k+n-1} \bar{y}_{i}^{r_{0}}=\frac{x_{k-n}-x_{k}}{\tau} .
$$

Altemately, one may write (see Fig. 9 for an example)

$$
\begin{aligned}
\sigma_{i}^{2}(\tau)= & \frac{1}{2 \tau^{2}(M-2 n+1)} \\
& \sum_{i=1}^{M-2 n+1}\left(x_{i+2 n}-2 x_{i+n}+x_{i}\right)^{2}
\end{aligned}
$$

where $x_{1}$ is taken from the set of $M+1=N$ discrete time deviation measurements between a pair of clocks or oscillators, $i=1$ to $M+1$ :

$$
x_{k+1}=\tau_{0} \sum_{i=1}^{k} \bar{y}_{i}^{\tau_{0}}+x_{1} \text {. }
$$

Equation (8) is obtained from a first difference on frequency, and (10) from the second difference on the time; they are mathematically identical, yielding the option of using frequency or time (phase) data.

For power-law spectra the following proportionality applies: $\sigma_{y}^{2}(\tau)-\tau^{\mu}$, where $\mu$ is typically constant for a particular value of $\alpha$. A simple and elegant relationship exists between the spectral density exponent $\alpha$ (in the relationship $\left.S_{y}(f)-f^{\alpha}\right)$ and $\mu$, i.e., $\mu=-\alpha-1(-3$ $<\alpha \leq 1)$ and $\mu=-2(\alpha \geq 1)$ [8]. For example, for a significant range of $\tau$ values. $\sigma_{y}(\tau) \sim \tau^{\mu / 2}$ is propurtional to $\tau^{-(1 / 2)}$ for cesium, rubidium, and passive hydrogen maser frequency standards. Therefore $\mu$ has the value of -1 , and hence $\alpha$ has the value of 0 (white-noise frequency modulation). This is the classical noise exhibited by an important set of atomic clocks for $\tau$ 's beyond a few seconds. In this case, $\sigma_{y}\left(\tau_{0}\right)$ is equal to the standard deviation. Fortunately, for most cases with precision clocks and oscillators where $\tau \geq 1 \mathrm{~s}$, the simple relationship $\mu=-\alpha-1$ is applicable. It is convenient to plot $\log \sigma_{y}(\tau)$ versus $\log \tau$ to estimate the value of $\mu$ and to let $n=2^{l}, l=0,1,2, \cdots\left(\tau=n \tau_{0}\right)$.

An ambiguity exists at $\mu=-2$; one cannot conveniently tell whether the noise process is flicker-noise phase modulation (PM), $\alpha=+1$, or white-noise PM, $\alpha=+2$. This ambiguity can be resolved by,realizing that for these cases $\sigma_{y}(\tau)$ depends on the measurement bandwidth [2], [3]. One can construct a variable software bandwidth $f_{s}$ by realizing the following [9]. [10]. In any measurement system a hardware bandwidth $f_{h}$ exists through which we 
TABLE $11^{4}$

\begin{tabular}{|c|c|c|c|c|}
\hline$\alpha$ & $\begin{array}{c}\text { pical Noise Types } \\
\text { Name }\end{array}$ & $\sigma_{y}^{2}(\tau)=$ & $\begin{array}{c}\text { Classical } \\
\text { Standard } \\
\text { Deviation of } x\end{array}$ & $\begin{array}{c}\text { Classical } \\
\text { Standard } \\
\text { Deviation of } y\end{array}$ \\
\hline 2 & white-noise PM & $a_{2} \tau^{-\infty}$ & $\tau \cdot \sigma_{y}(\tau) / \sqrt{3}$ (constant) & $\sigma_{y}(\tau) \sqrt{2(N+1) / 3 N}$ \\
\hline 1 & flicker-noise PM & $a_{1} 7^{-2}$ & $\sim \tau \cdot \sigma_{y}(\tau) \sqrt{\ln M / \ln 2}$ & $\sim \sigma_{y}(\tau) \sqrt{2(N+1 / 3 N}$ \\
\hline $\begin{array}{r}0 \\
-1 \\
-2\end{array}$ & $\begin{array}{l}\text { white-noise FM } \\
\text { flicker-noise FM } \\
\text { random-walk FM }\end{array}$ & $\begin{array}{l}a_{0} \tau^{-1} \\
a_{-1} \tau^{0} \\
a_{-2} T^{2}\end{array}$ & $\begin{array}{l}\tau_{0} \cdot \sigma_{y}\left(\tau_{0}\right) \sqrt{(M+1) / 6} \\
\text { undefined } \\
\text { undefined }\end{array}$ & $\begin{array}{l}\sigma_{y}\left(\tau_{0}\right) \\
\sigma_{y}(\tau) \sqrt{N \ln N /(2(N-1) \ln 2)} \\
\sigma_{y}(\tau) \sqrt{N / 2}\end{array}$ \\
\hline
\end{tabular}

- Note $\tau$ is a general averaging time and $\tau_{0}$ is the initial averaging time ( $\tau=n \tau_{0}$, where $n$ is an integer). Also note that the last four entries in the fourth column and the last two entries in the fifth column go to infinity as $M$ or $N$ go to infinity. $M$ is the initial number of frequency difference measurements and $N$ the number of phase or time difference measurements $N=M+1$. If the spectral density is given by $S,(f)$ $=h_{a} f^{\alpha}$, then

$$
\begin{aligned}
& a_{2}=\left(\frac{1}{2 \pi}\right)^{2} 3 f_{h} \tau_{0} h_{2}{ }^{b} \\
& a_{1}=\left(\frac{1}{2 \pi}\right)^{2}\left(1.038+3 \log _{e}\left(2 \pi f_{h} \tau\right) h_{1}\right) \\
& a_{0}=\left(\frac{1}{2}\right) h_{0} \\
& a_{-1}=2 \log _{e}(2) h_{-1} \\
& a_{-2}=\frac{1}{6}(2 \pi)^{2} h_{-2} .
\end{aligned}
$$

${ }^{b}$ Note this equality assumes use of modified $\sigma_{y}^{2}(\tau)=\bar{\sigma}_{y}^{2}(\tau)$.

measure the phase difference or the time difference between a pair of oscillators or clocks and we define $\tau_{h}=$ $1 / f_{h}$. In other words, $\tau_{h}$ is the sample time period through which the time or phase date are observed or averaged. Averaging $n$ time or phase readings increases the sample time window to $n \tau_{h}=\tau_{s}$. Let $\tau_{s}=1 / f_{s}$; then $f_{s}=f_{h} / n$, i.e., the software bandwidth is narrowed to $f_{s}$. In other words, $f_{s}=f_{h} / n$ decreases as we average more values; i.e., increase $n\left(\tau=n \tau_{0}\right)$. One can therefore construct a second difference composed of time deviations so-averaged and then define a modified $\sigma_{y}^{2}(\tau)=\bar{\sigma}_{y}^{2}(\tau)$ that will remove the ambiguity through bandwidth variation:

$$
\begin{aligned}
\bar{\sigma}_{y}^{2}(\tau)= & \frac{1}{2 \tau^{2} n^{2}(N-3 n+1)} \\
& \sum_{j=1}^{N-3 n+1}\left(\sum_{i=j}^{n+j-1}\left(x_{i+2 n}-2 x_{i+n}+x_{i}\right)\right)^{2}
\end{aligned}
$$

where $N=M+1$, the number of time-deviation measurements available from the data set. Now if $\bar{\sigma}_{y}^{2}(\tau)-$ $\tau^{\mu^{\prime}}$, then $\mu^{\prime}=-\alpha-1(1 \leq \alpha \leq 3)$ [10], [11]. Thus $\bar{\sigma}_{y}(\tau)$ is typically employed as a subroutine to remove the ambiguity if $\sigma_{y}(\tau)-\tau^{-1}$. This is because the $\mu^{\prime}=-\alpha$ -1 relationship is valid as an asymptotic limit for large $n$ and $\alpha<1$ and is not valid in general; however, the re is evidence that $\bar{\sigma}_{y}^{2}(\tau)$ may be a better measure [12]. Specifically, for $\alpha=2$ and $1, \mu^{\prime} / 2$ equals $-3 / 2$ and -1 , respectively, providing a clean differentiation between white-noise PM and flicker-noise PM.

If three or more independent oscillators or clocks are available along with time (phase) or frequency measure- ments between them, then it is possible to estimate a variance for each oscillator or clock. Often there is a reference to which the rest are periodically measured at a sampling rate $1 / \tau_{0}$. If at each measurement the time or frequency differences betweer the clocks are measured at nominally the same time, then the time difference or frequency difference can usually be estimated or calculated between every possible pair in the set of oscillators or clocks. Given a series of measurements, variances $s_{i j}^{2}$ can be calculated on the time or frequency data between all pairs. It has been shown [13] that the individual clock variances can be estimated using the following equations:

$$
\sigma_{i}^{2} \doteq \frac{1}{m-2}\left(\sum_{j=1}^{m} s_{i j}^{2}-B\right)
$$

where

$$
B=\frac{1}{m-1} \sum_{i<j}^{m} s_{i j}^{2}
$$

$m$ is the number of clocks available in the set, and $s_{i i}^{2}=$ 0 . If the variance measures used are $\sigma_{y}^{2}(\tau)$ or $\bar{\sigma}_{y}^{2}(\tau)$, then (13) can be used to estimate the individual variances as a function of $\tau$.

Table II illustrates why one should not use the standard deviation to characterize clocks. For the different kinds of noise processes we list the standard deviation of the time deviations and of the fractional frequency deviations as a function of $\sigma_{y}(\tau)$. The divergent nature of the standard deviation is apparent. Even for classical white-noise FM the standard deviation of the time diverges as the square root of the data length, i.e., the number of samples $N$ [2]. 
TABLE III

\begin{tabular}{cccc}
\hline \hline$\alpha$ & $\begin{array}{c}\text { Typical Noise Types } \\
\text { Name }\end{array}$ & \multicolumn{1}{c}{$\begin{array}{c}\text { Optimum } \\
\text { Prediction } \\
x\left(\tau_{p}\right) \mathrm{rms}^{\mu}\end{array}$} & $\begin{array}{c}\text { Time Error: } \\
\text { Asymptotic Form }\end{array}$ \\
\hline 2 & whice-noise PM & $\tau_{p} \cdot \sigma_{y}\left(\tau_{p}\right) / \sqrt{3}$ & constant \\
1 & Alicker-noise PM & $\tau_{p} \cdot \sigma_{y}\left(\tau_{p}\right) \sqrt{\ln \tau_{p} / 2 \ln \tau_{0}}$ & $\sqrt{\ln \tau_{p}}$ \\
0 & white-noise FM & $\tau_{p} \cdot \sigma_{y}\left(\tau_{p}\right)$ & $\tau_{p}^{1 / 2}$ \\
-1 & Alicker-noise FM & $\tau_{p} \cdot \sigma_{y}\left(\tau_{p}\right) / \sqrt{\ln 2}$ & $\tau_{p}$ \\
-2 & random-walk FM & $\tau_{p} \cdot \sigma_{y}\left(\tau_{p}\right)$ & $\tau_{p}^{3 / 2}$ \\
\hline
\end{tabular}

${ }^{\top}{ }^{\prime}$, is the prediction interval.

\section{Time and Frequency Estimation and Prediction}

Using $\sigma_{y}(\tau), \bar{\sigma}_{y}(\tau), S_{y}(f)$, or $S_{\phi}(f)$, one can characterize typical power law processes. Once characterized, this opens the opportunity for determining optimum estimates of values by employing the statistical theorem that the optimum estimate of a white-noise process is the simple mean.

For example, consider the very common and very important case of white-noise FM typically found on the signals from cesium standards, rubidium standards, and passive hydrogen masers. The optimum estimate of the frequency is the simple mean frequency, which is equivalent to $\left(x_{N}-x_{1}\right) / M \tau_{0}$. It is still all too common within our discipline to see our colleagues erroneously determining the frequency for these kinds of oscillators by calculating the slope from a linear least-squares fit to the time deviations and quoting the standard deviation around that fit as a measure of the clock performance. There are three problems in proceeding this way. First, the frequency estimate is not optimum in a mean-square-error sense. It is equivalent to throwing away about 20 percent of the data and thereby increasing the cost in the case of a calibration. Second, the standard deviation diverges as the square root of the data length. Third, the standard deviation is significantly dependent on the filter form, e.g., linear least squares, as well as the clock deviations. On the other hand, such a filter is sometimes useful for assessing outliers. The optimum "end-point" method outlined earlier has the risk that if either of the points is abnormal, (i.e., the model fails), the result will of course be adversely effected. Therefore such a filter is useful to assess whether there are outliers-paying special attention to the end points. Also, if the measurement noise exceeds the combined noise in the clocks, then the end points will be adversely affected. The key message is that the end-point method for estimating frequency is only optimum if the noise is pure white $F M$, which is easy to determine from a $\log \sigma_{y}(\tau)$ versus $\log \tau$ plot.

There are other useful, and maybe not so obvious, optimum estimators appropriate for time-difference data sets.

1) Given white-noise PM, the best time estimate is the simple mean of the time deviations; the frequency estimate then is the slope from a linear least-squares fit to the time deviations, and the frequency drift $D$ is determined from a quadratic least-squares fit to the time deviations per (1).
2) Given white-noise FM, the optimum estimate of the time is the last value; the optimum-frequency estimate is outlined in the previous paragraph, and the optimum-frequency-drift estimate is derived from a linear least-squares fit to the frequency.

3) Given random-walk FM, the current optimum time estimate is the last value plus the last slope (clock rate) times the time since the last value; the optimum-frequency estimate is obtained from the last slope of the time deviations; and the optimum-frequency-drift estimate is calculated from the mean second difference of the time deviations. Caution needs to be exercised here, for typically there will be higher frequency component noise in a real data stream, such as white-noise FM, along with random-walk FM, and this can significantly contaminate the drift estimate from a mean second difference. If random-walk FM is the predominant longterm power-law process, which is often the case, then the effect of high-frequency noise can be reduced by calculating the second difference from the first, middle, and end-time deviation points of the data set.

The flicker-noise cases are significantly more complicated, though filters can be designed to approximate optimum estimation [14]-[16]. As the data length increases without limit, time is not defined for flicker-noise PM, and frequency is not defined for flicker-noise FM. This has some philosophical implications for the definitions of time and frequency, unless some low-frequency cutoff limits exist. If significant frequency drift exists in the data, it should be optimally subtracted from the data or it will bias the long-term values of $\sigma_{y}(\tau)$ :

$$
\sigma_{y}(\tau)=\frac{D \tau}{\sqrt{2}} .
$$

Once the power-law spectra are deduced for a pair of oscillators or clocks, then one can also develop an optimum predictor. Table III gives both the optimum prediction uncertainty values for the various relevant pure power-law spectra as well as their asymptotic forms. Special forecasting techniques must be used for optimal prediction when combinations of these processes are present [17]. To illustrate how these concepts relate to real devices, Fig. 10 shows a $\sigma_{y}(\tau)$ diagram for some interesting 


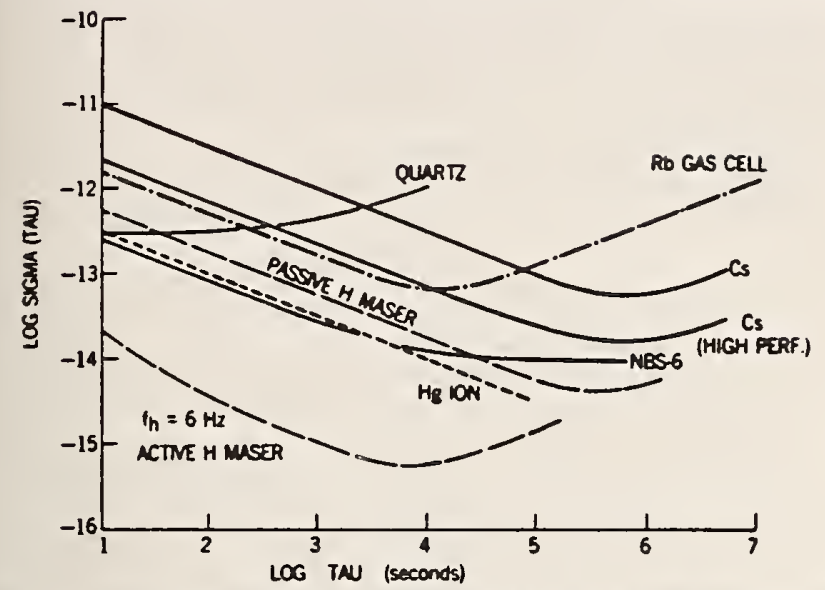

Fig. 10. Square root of Allan variance for variety of state-of-the-an precision oscillators including NBS-6, NBS primary frequency standand.

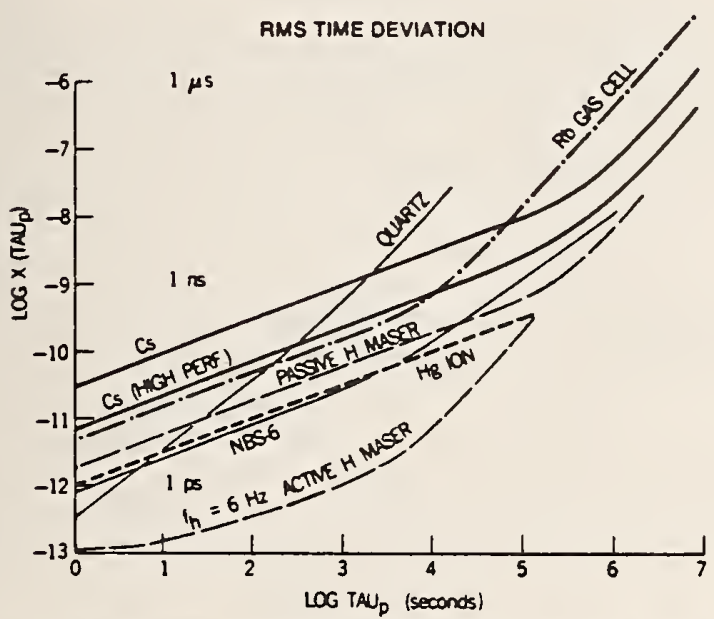

Fig. 11. From frequency stability characterization shown in Fig. 10, optimum prediction algorithms to minimize time error can be obtained. Based on optimum prediction procedures rms time prediction error for prediction interval $\tau_{p}$ can be calculated for each oscillator shown in Fig. 10 and corresponding values are piotted in Fig. 11

state-of-the-art oscillators, and Fig. 11 shows the rms time prediction errors for the same set of oscillators.

\section{Conclusion}

In conclusion, it is clear that classical statistics do not allow characterization of common kinds of random signal variations found in precision oscillators. The two-sample Allan variance provides a valuable and convergent measure of the power-law spectral-density models useful in characterizing random deviations for most oscillators and clocks. Once characterized, we can calculate optimum time and frequency estimates as well as predicted values. Characterizing the random variations also provides nearoptimum estimation of systematic effects, which often cause the predominant time and frequency deviations. For example, if we wanted to optimally determine the static temperature dependence with the temperature set at two different values, we would stabilize the oscillator at one temperature and measure the frequency against a reference for a time $\tau_{m}$, corresponding to the $\tau$ for the nominal minimum $\sigma_{y}(\tau)$ value. We would then change the temperature to the other value and repeat the measurement with the same criteria and note the $\Delta \bar{y}^{r_{m}}$ between the two optimally determined frequency values. If these two steps are repeated several times, an arbitrarily good precision for the temperature coefficient is achieved if it is linear. The uncerainty is approximately given by $\sigma_{y}\left(\tau_{m}\right) / \sqrt{P}$, where $P$ is the number of $\Delta \bar{y}^{r_{m}}$ values obtained from switching back and forth. Knowing the characteristics of both the random and the systematic deviations of precision clocks and oscillators clearly is useful to the designer, the manufacturer, the planner, and the user as well as the vendor of these devices.

The aforementioned procedures usually work well if the clocks or oscillators are in a reasonable environment. If the environment is adverse, other procedures and analysis methods may have to be employed. As a general rule it is often useful to analyze the data in the frequency domain as well as the time domain. The frequency domain is especially useful if there are bright lines, i.e., sidebands to the carrier frequency. The effect of a modulation sideband $f_{m}$ on $\sigma_{y}(\tau)$ can be calculated, and is given by [18]

$$
\sigma_{y}(\tau)=\frac{x_{p p}}{\tau} \sin ^{2}\left(\pi f_{m} \tau\right)
$$

where $x_{p p}$ is equal to the peak-to-peak or twice the amplitude of the time-deviation modulation.

If one is trying to estimate the power-law spectral behavior between a pair of oscillators or clocks using $\sigma_{y}(\tau)$, it is apparent from (15) that if significant modulation sidebands are present on the signal, these can seriously contaminate that estimate. However, if a $\sigma_{y}(\tau)$ plot displays a character as given by (15), then the amplitude and frequency of that modulation sideband can be estimated from this time-domain analysis technique. In practice, this approach is often used, but these modulation sidebands can be more efficiently estimated in the frequency domain. If the measurement sampling rate $1 / \tau_{0}$ is set equal to $f_{m}$, then the modulation sideband is aliased away and has no effect on $\sigma_{y}(\tau)$.

The best rule in all analysis is to use common sense. Very often the most revealing information may be in a plot of the raw time (phase) difference or frequency-difference residuals after some trend has been removed. Such a plot is usually the first thing to look at when characterizing clocks and oscillators. Caution here is also important as a pure random walk on the time residuals (white FM) may be visually interpreted as having frequency steps. This is especially true for flicker FM as often seen in quarz-crystal oscillators. Following the time-residual plot with a $\sigma_{y}(\tau)$ analysis often answers the question as to whether or not such steps are statistically significant.

\section{ACKNOWLEDGMENT}

Because the paper is mainly a review, the author is indebted to a large number of people-many of whom are reflected in the references. Specifically, sincere appreciation is expressed to Dr. James A. Bames, Mr. Dick D. 
Davis, Dr. John Vig, Dr. Donald B. Sullivan, Dr. Fred L. Walls, and Dr. Marc A. Weiss for their extremely helpful comments and suggestions.

\section{REFERENCES}

[I] J. A. Bames et al., "Characterization of frequency stability," in IEEE Trans. Instrum. Meas., vol. IM-20, p. 105, NBS Tech. Note 394, 1971.

[2] D. W. Allan, "Statistics of atomic frequency standards," in Proc. IEEE, vol. 54, 1966, p. 221.

[3] R. Vessot, L. Mueller, and J. Vanier, "The specification of oscillator characteristics from measurements made in the frequency domain," in Proc. IEEE Special Issue on Frequency Stability, vol. 54, Feb. 1966, pp. 199-207.

[4] P. Lesage and C. Audoin, "Characterization and measurement of time and frequency stability." Radio Sci., vol. 14. pp. 521-539, July/Aug. 1979.

[5] J. A. Bames, "Atomic timekeeping and the statistics of precision signal generators," in Proc. IEEE Special /ssue on Frequency Stability, vol. 54, Feb. 1966, pp. 207-220.

[6] D. A. Howe, D. W. Allan, and J. A. Bames, "Properties of signal sources and measurement methods," in Proc. 35th Ann. Symp. Frequency Control (SFC), May 1981, pp. 669. Al-A47.

[7] D. W. Allan. "The measurement of frequency and frequency stability of precision oscillator," Nat. Bur. Standards, Boulder, CO, NBS Tech. Note 669. May 1975.

[8] M. J. Lighthill, "Introduction to Fourier analysis and generalized functions," in Cambridge Monographs on Mechanics and Applied Mathematics, G. K. Batchelor and J. W. Miles, Eds. London. England: Cambridge Univ. Press, 1964.

[9] J. J. Snyder, "An ultra-high resolution frequency meter," in Proc. 35 th Ann. Frequency Control Symp., USAERADCOM, Ft. Monmouth, NJ, May 1981, pp. 464-469.
[10] D. W. Allan and J. A. Barnes, "A modified 'Allan variance' with increased oscillator characterization ability," in Proc. $35 \mathrm{th}$ Ann. Frequency Control Symp., USAERADCOM, Ft. Monmouth, NJ, May 1981, pp. 470-475.

[11] P. Lesage and T. Ayi, "Characterization of frequency stability: Analysis of the modified Allan variance and properties of its estimate," IEEE Trans. Instrum. Meas., vol. IM-33, no. 4, pp. 332-336, Dec. 1984.

[12] L. G. Bemier, "Theoretical analysis of the modified Allan variance," in Proc. 4lst Ann. Frequency Control Symp., USAERADCOM, Fi. Monmouth, NJ, May 1987.

[13] J. A. Bames, Notes from NBS 2nd Sem. Atomic Time Scale Algorithms, June 1982, Time and Frequency Division, Nat. Bur. Standards, Boulder, CO 80303 .

[14] J. A. Barnes and S. Jarvis, Jr., "Efficient numerical and analog modeling of flicker noise processes," Nat. Bur. Standards, Boulder, $\mathrm{CO}$, NBS Tech. Note 604, June 1971.

[15] J. A. Bames, "The measurement of linear frequency drift in oscillators," in Proc. 15th Ann. Precise Time and Time Interval (PTTI) Applications and Planning Meeting, Naval Res. Lab., Washington, DC. Dec. 1983, pp. 551-582.

[16] D. W. Allan et al., "Performance, modeling, and simulation of some cesium beam clocks." in Proc. 27 th Ann. Symp. Frequency Control, 1972, p. 309, AD 771042.

[17] D. W. Allan and H. Hellwig. "Time deviation and the prediction error for clock specification, characterization, and application," in Proc. Position Location and Navigation Symp. (PLANS), 1978, p. 29.

[18] S. R. Stein, private communication.

David W. Allan for a photograph and biography, please see page 571 of this Transactions. 
From: Proceedings of the 42nd Annual Symposium on Frequency Control, 1988.

EXTENDING THE RANGE AND ACCURACY OF PHASE NOISE MEASUREMIENTS

F.L. Walls, A.J.D. Clements, C.M. Felton, M.A. Lombardi, and M.D. Vanek

Time and Frequencv Division

National Institute of Standards and Technology

Boulder, Colorado 80303

Summary

This paper describes recent progress in extending high accuracy measurements of phase nolse in oscillators and other devices for carrier frequencies from the rf to the millimeter region and Fourier frequencies up to $10 \%$ of the carrier (or a maximum of about $1 \mathrm{GHz}$ ). A brief survey of traditional precision techniques for measuring phase noise is included as a basis for comparing their relative performance and limitations. The single oscillator techniques, although conceptually simple, require a set of 5 to 10 references to adequately measure the phase noise from $1 \mathrm{~Hz}$ to $1 \mathrm{GHz}$ from the carrier. The two oscillator technique yields excellent noise floors if, for oscillator measurements, one has a comparable or better oscillator for the reference and, for other devices, either pairs of devices or a reference oscillator with comparable or better noise. We have developed several new calibration techniques which, when combined with previous two oscillator techniques, permits one to calibrate all factors affecting the measurements of phase noise of oscillator pairs to an accuracy which typically exceeds $1 \mathrm{~dB}$ and in favorable cases can approach 0.4 $d B$. In order to illustrate this expanded two oscillator approach, measurements at $5 \mathrm{MHz}$ and $10 \mathrm{GHz}$ are described in detail. At $5 \mathrm{MHz}$ we achieved accuracies of about $\pm 0.6 \mathrm{~dB}$ for phase noise measurements from $20 \mathrm{~Hz}$ to $100 \mathrm{kHz}$ from the carrier At $10 \mathrm{GHz}$ we achieved an accuracy of $\pm 0.6 \mathrm{~dB}$ for phase noise measurements a few $\mathrm{kHz}$ from the carrier degrading to about $\pm 1.5 \mathrm{~dB}, 1 \mathrm{GHz}$ from the carrier.

\section{Introduction}

This paper describes recent progress at the National Bureau of Standards (NBS) in extending high accuracy measurements of phase noise in oscillators,

amplifiers, frequency synthesizers, and passive components at carrier frequencies from the rf to the millimeter region and Fourier frequencies up to 108 of the carrier (or a maximum of about $1 \mathrm{GHz}$ ). An examination of existing techniques for precision phase noise measurements of oscillators [1-11] showed that present approaches which don't require a second "reference" oscillator have good resolution or noise floor for Fourier frequencies extending over only 1 or 2 decades.[7-10] Consequently, these approaches require a set of 5 to 10 references, either delay lines or high $Q$ factor cavities, in order to adequately measure the phase noise from $1 \mathrm{~Hz}$ to $1 \mathrm{GHz}$ from the carrier. Using the cavity approach would require a entire set of reference cavities for each carrier frequency measured. Similar considerations also apply to phase noise measurements of the other devices unless one can measure pairs of devices.

The limitations of the single oscillator techniques led us to adopt a two oscillator method for all measurements. This approach yields good resolution or noise floor from essentially dc to the bandwidth of the mixer if, for oscillator measurements, one has a comparable or better oscillator for the reference

Contribution of the U.S. Government, not subject to copyright. and, for other devices, either pairs of devices or a reference oscillator with comparable or better noise

The major limitations in the accuracy of the two oscillator method (which also apply to the single oscillator methods) are the calibration of: the mixer phase-to-voltage conversion factor, the amplifier gain versus Fourier frequency, and the accuracy of the spectrum analyzer. We have developed several new calibration techniques which, when combined with previous techniques, allow us to address each of these limitations. The net result is the development of a complete measurement concept that permits one to calibrate all factors affecting the measurements of phase noise of oscillator pairs to an accuracy which typically exceeds $1 \mathrm{~dB}$ and in favorable cases can approach $0.4 \mathrm{~dB}$. For other types of devices the limitations are similar if the noise of the reference oscillator can be neglected. The ultimate accuracy that can be easily achieved with this approach is now limited by the accuracy of the attenuators in available spectrum analyzers.

Measurements at $5 \mathrm{MHz}$ and $10 \mathrm{GHz}$ are described in detail, in order to illustrate this expanded two oscillator approach. Specifically we measure the mixer phase-to-voltage conversion factor multiplied by amplifier gain on all channels versus Fourier frequency using a new ultra-wideband phase modulator. We then measure the absolute mixer conversion factor multiplied by the amplifier gain at one Fourier frequency, the effect of the phase-lock loop on the measured noise voltage, and spectral density function of the spectrum analyzers. These measurements are used to normalize the relative gains of the noise measurements on the various channels. At $5 \mathrm{MHz}$ we achieved accuracies of about $\pm 0.6 \mathrm{~dB}$ for phase noise measurements from $20 \mathrm{~Hz}$ to $100 \mathrm{kHz}$ from the carrier. At $10 \mathrm{GHz}$ we achieved an accuracy of $\pm 0.6 \mathrm{~dB}$ for phase noise measurements a few $\mathrm{kHz}$ to $500 \mathrm{MHz}$ from the carrier. The accuracy degrades to about $\pm 1.5 \mathrm{~dB}$, $1 \mathrm{GHz}$ from the carrier.

\section{Model of a Noisy Signal}

The output of an oscillator can be expressed as

$$
V(t)=\left[V_{0}+\epsilon(t)\right] \sin \left(2 \pi \nu_{0} t+\phi(t)\right),
$$

where $v_{0}$ is the nominal peak output voltage, and $\nu_{0}$ is the nominal frequency of the oscillator. The time variations of amplitude have been incorporated into $\epsilon(t)$ and the time variations of the instantaneous frequency, $\nu(t)$, have been incorporated into $\phi(t)$. The instantaneous frequency is

$$
\nu(t)=\nu_{0}+\frac{d[\phi(t)]}{2 \pi d t} .
$$

The fractional frequency deviation is defined as

$$
y(t)=\frac{\nu(t)-\nu_{0}}{\nu_{0}}=\frac{d[\phi(t)]}{2 \pi \nu_{0} d t} .
$$


Power spectral analysis of the output signal $V(t)$ combines the power in the carrier $\nu_{0}$ with the power in $\epsilon(t)$ and $\phi(t)$ and therefore is not a good method to characterize $\epsilon(t)$ or $\phi(t)$.

Since in many precision sources understanding the variations in $\phi(t)$ or $y(t)$ is of primary importance, we will confine the following discussion to frequency-domain measures of $y(t)$, neglecting $\epsilon(t)$ except in cases where it sets limits on the measurement of $y(t)$. The amplitude fluctuations, $\epsilon(t)$, can be reduced using limiters whereas $\phi(t)$ can be reduced in some cases by the use of narrow band filters.

Spectral (Fourier) analysis of $y(t)$ is often expressed in terms of $S_{\phi}(f)$, the spectral density of phase fluctuations in units of radians squared per $\mathrm{Hz}$ bandwidth at Fourler frequency $f$ from the carrier $v_{0}$. Alternately $S_{y}(f)$, the spectral density of fractional frequency fluctuations in a $1 \mathrm{~Hz}$ bandwidth at Fourier frequency $f$ from the carrier $\nu_{0}$ can be used [1]. These are related as

$$
S_{\phi}(f)=\frac{\nu_{0}^{2}}{f^{2}} \quad S_{y}(f) \quad \mathrm{rad}^{2} / \mathrm{Hz} \quad 0<f<\infty .
$$

$S_{\phi}(f)$ can be intuitively understood as,

$$
S_{\phi}(f)=\frac{\Delta \phi^{2}(f)}{B W}
$$

where $\Delta \phi(f)$ is the rms phase deviation measured at Fourler frequency $f$ from the carrier in a bandwidth BW. It should be noted that these are single-sided spectral density measures containing the phase or frequency fluctuations from both sides of the carrier. The wean squared phase modulation in a measurement bandwidth, $B W$, is given by

$$
\Delta \phi^{2}(f)=\int_{-B W / 2}^{+B W / 2} s_{\phi}(f) d f .
$$

Other measures sometimes encountered are $\varphi(f)$, $\mathrm{dBC} / \mathrm{Hz}$, and $S_{\Delta \nu}(f)$. These are related by $[1-3]$

$$
\begin{aligned}
& \mathrm{S}_{\Delta \nu}(\mathrm{f})=\nu_{0}^{2} \mathrm{~S}_{\mathrm{y}} \text { (f) } \mathrm{Hz}^{2} / \mathrm{Hz} \\
& S_{\phi}(f)=\mathscr{L}\left(\nu_{0}-f\right)+\mathscr{L}\left(\nu_{0}+f\right) \equiv 2 \mathscr{L}(\mathrm{f})
\end{aligned}
$$

$$
\mathrm{dBC} / \mathrm{Hz}=10 \log \mathscr{L}(\mathrm{f})
$$

$\psi(f)$ and $\mathrm{dBC} / \mathrm{Hz}$ are single sideband measures of phase noise. These are the revised definitions of $L(f)$ and $\mathrm{dBC}$ as per the most recent recommendation of the Standards Coordinating Committee for Time and Frequency of the IEEE [3]. With these revised definitions $\mathcal{L}(f)$ and $\mathrm{dBC} / \mathrm{Hz}$ are now defined for arbitrarily large phase modulation, whereas previously they were restricted to small angle modulation. In some situations, especially where $f$ becomes a sizeable fraction of $\nu_{0}$, the phase noise spectrum is asymetric about the carrier. In these cases one should specify whether the upper sideband noise $\varphi\left(\nu_{0}+f\right)$ or the lower sideband noise $\psi\left(\nu_{0}-f\right)$ is being referenced. These distinctions are becoming

See Appendix Note \# 6 increasingly important as users require the specification of phase noise near the carrier where the phase excursions are large compared to 1 radian, or at Fourier frequencies which exceed the bandwidth of typical circuit elements.

The above measures provide the most powerful (and detailed) analysis for evaluating types and levels of fundamental noise and spectral density structure in precision oscillators and signal handing equipment as it allows one to examine individual fourfer components of residual phase (or frequency) modulation.

\section{A. Two Oscillator Method}

Fig. 1 shows the block diagram for a typical scheme used to measure the phase noise of a precision source using a double balanced mixer and a reference source. Fig. 2 illustrates a simllar technique for measuring only the phase noise added in multipliers, dividers, amplifiers, and passive components. It is very important that the substitution oscillator be at the same drive level, impedance, and at the equivalent electrical length from the mixer as the signal coming from the reference osclllator. This is dramatically illustrated in Fig. 3, discussed below. The output voltage of the mixer as a function of phase deviation, $\Delta \phi$, between the two inputs to the mixer is nominally given by

$$
\mathrm{V}_{\mathrm{out}}=\mathrm{K} \cos \Delta \phi
$$

Near quadrature ( $90^{\circ}$ phase difference) this can be approximated by

$v_{\text {out }}=k_{d} \delta \phi$, where $\delta \phi \equiv\left[\Delta \phi-\frac{2 \pi-1}{2} \pi\right]<0.1$

where $\mathrm{n}$ is the integer to make $\delta \phi-0$. The voltage to phase conversion ratio sensitivity, $K_{d}$, is dependent on the frequency, the drive level, and impedance of both input signals, and the IF termination of the mixer [4]. The combined spectral density of phase nolse of both input signals, the noise of the mixer and the amplitude noise from the If amplifiers is

Measurement of $S_{\varphi}(f)$ Between Two Oscillators

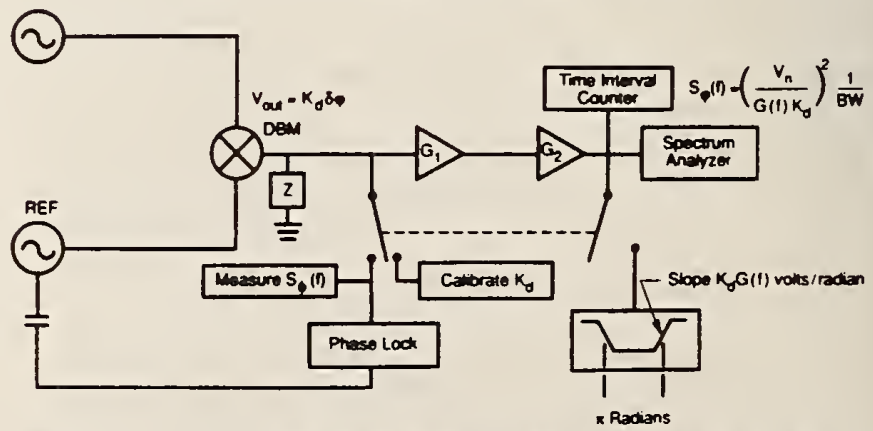

Fig. 1. Precision phase measurement system using a spectrum analyzer. Calibration requires a recording device to measure the slope at the zero crossing. The accuracy is better than $0.4 \mathrm{~dB}$ from dc to $0.1 \nu_{\text {o }}$ Fourier frequency offset from the carrler $\nu_{0}$. Carrier frequencles from a few $\mathrm{Hz}$ to $10^{11} \mathrm{~Hz}$ can be accommodated with this type of measurement system [4]. 
given by

$$
S_{b}(f)=\left(\frac{V_{n}(f)}{G(f) K_{d}}\right)^{2} \quad \frac{1}{B W} .
$$

Measurement of $S_{\varphi}(f)$ for Two Amplifiers

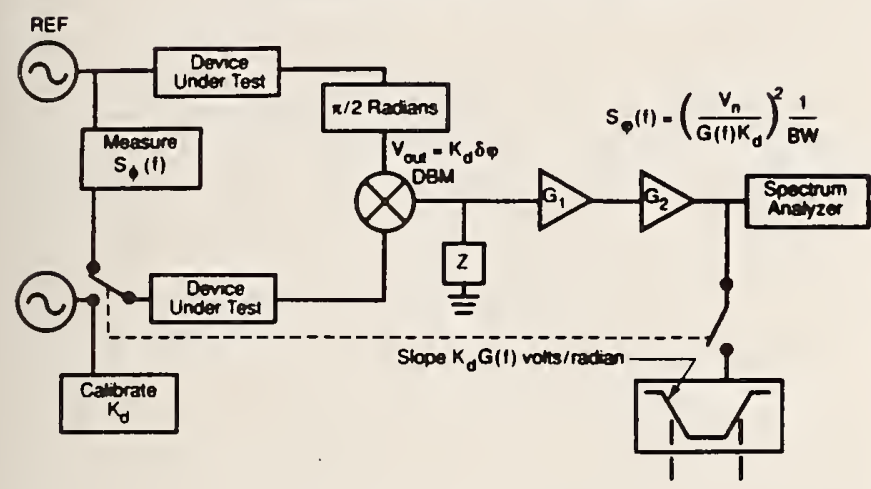

Fig. 2. Precision phase measurement system featuring self calibration to $0.4 \mathrm{~dB}$ accuracy from dc to $0.1 \nu_{0}$ Fourier frequency offset from carrier. This system is suitable for measuring signal handling equipment, multipliers, dividers, frequency synthesizers, as well as passive components [4].

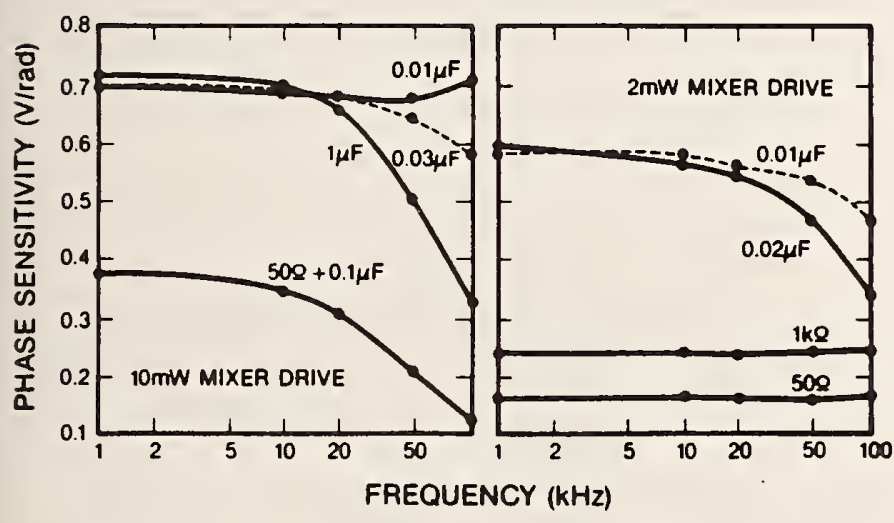

Fig. 3. Double-balanced mixer phase sensitivity at 5 $\mathrm{MHz}$ as a function of Fourier frequency for various output terminations. The curves on the left were obtained with $10 \mathrm{~mW}$ drive while those on the right were obtained with $2 \mathrm{~mW}$ drive. The data demonstrate a clear choice between constant, but low sensitivity or much higher, but frequency dependent sensitivity [4].

where $V_{n}(f)$ is the RMS noise voltage at Fourier frequency from the carrier measured after IF gain $G(f)$ in a noise bandwidth $B W$. Obviously BW must be small compared to $f$. This is very important where $S_{\phi}(f)$ is changing rapidly with $f, e . g ., S_{\phi}(f)$ often varies as $f^{-3}$ near the carrier. In Fig. 1 , the output of the second amplifier following the mixer contains contributions from the phase noise of the oscillators, the noise of the mixers, and the post amplifiers for Fourier frequencies much larger than the phase-lock loop bandwidth. In Fig. 2, the phase noise of the oscillator cancels out to a high degree (often more than $20 \mathrm{~dB}$ ). Termination of the mixer IF port with $50 \Omega$ maximizes the IF bandwidth, however. termination with reactive loads can reduce the mixer noise by $-6 \mathrm{~dB}$, and increase $K_{d}$ by 3 to $6 \mathrm{~dB}$ as shown in Fig. $3[4]$. Accurate determination of $k_{d}$ can be achieved by measuring the slope of the zero crossing in volts/radian with an oscilloscope or other recording device when the two oscillators are beating slowly. For some applications the digitizer in the spectrum analyzer can be used to measure both the beat period and the slope in $V / s$ at the zero crossing. The time axis is easily calibrated since one beat period equals $2 \pi$ radians. The slope in volts/radian is then calculated with a typical accuracy of $0.2 \mathrm{~dB}$. Estimates of $\mathrm{K}_{d}$ obtained from measurements of the peak to peak output voltage induced can introduce errors as large as $6 \mathrm{~dB}$ in $S_{\text {, }}(f)$ even if the amplitude of the other harmonics is measured unless the phase relationship is also taken into account [4]. $S_{f}(f)$ can be made independent of the accuracy of the spectrum analyzer voltage reference by comparing the level of an externally If signal (a pure tone is best), on the spectrum analyzer used to measure $V_{n}$ with the level recorded on the device used to measure $K_{d}$.

The noise bandwidth of the spectrum analyzer also needs to be verified. This calibration procedure is sufficient for small Fourier frequencies but looses precision and accuracy due to the problems illustrated in Fig. 3 and the variations of amplifier gain with Fourier frequency. If measurements need to be made at Fourier frequencies near or below the phase-lock-loop bandwidth, a probe signal can be infected inside the phase-lock-loop and the attenuation measured versus Fourier frequency.

Some care is necessary to assure that the spectrum analyzer is not saturated by spurious signals such as the power line frequency and its multiples.

Sometimes aliasing in the spectrum analyzer is a problem. If narrow spectral features are to be measured it is usually recommended that a flat top window function (in the spectrum analyzer) be used. In the region where the measured noise is changing rapidly with Fourler frequency, the noise bandwidth should be much smaller than the measurement frequency. The approximate level of the noise floor of the measurement system should be measured in order to verify that it does not significantly bias the measurements or, if necessary, to subtract its effect from the results.

Typical best performance for various measurement techniques is shown in Fig. 4. The two oscillator approach exceeds the performance of almost all available oscillators from below $0.1 \mathrm{MHz}$ to over 100 $\mathrm{GHz}$ and is generally the technique of first choice because of lts versatility and simplicity. Figs. 10 - 12 give some examples. Phase noise measurements on pairs of signal sources can be made with an absolute accuracy better than $1 \mathrm{~dB}$ using the above calibration procedure. Such accuracy is not always attainable when the phase noise of the source exceeds that of the added noise of the components under test (see Fig. 2). The use of specialized high level mixers with multiple diodes per leg increases the phase to voltage conversion sensitivity, $K_{d}$ and therefore reduces the contribution of IF amplifier noise [5] as shown in Fig. 4. Phase noise measurements can generally be made at Fourier frequencies from approximately dc to $1 / 2$ the source frequency. 
major difficulty is designing a mixer terminations to remove the source frequency from the output signal, which would generally saturate the low noise amplifiers following the mixer, without degrading the signal-to-noise ratio. As mentioned earlier the phase noise spectrum is quite likely asymetric when f exceeds the bandwidth of the tuned circuits in the device under test. For example one expects that the phase noise at $1 / 2 \nu_{0}$ is different than the phase noise at $3 / 2 v_{0}$.

\section{Comparison of Noise Floor for Different Techniques}

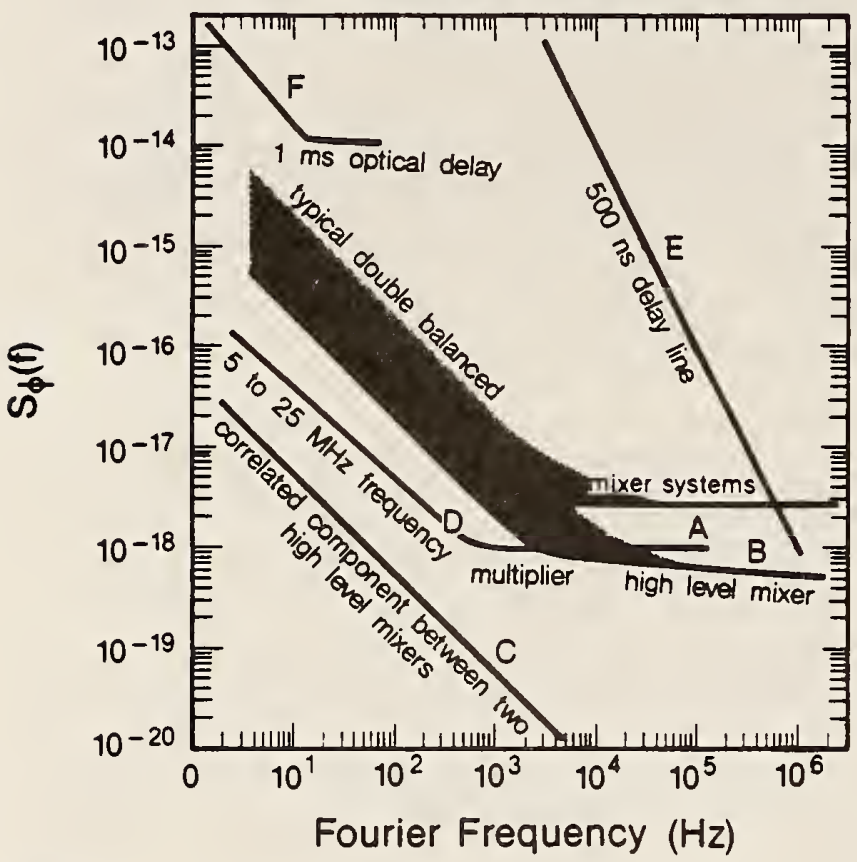

Fig. 4.

Curve $A$. The noise floor $S_{\phi}(f)$ (resolution) of typical double balanced mixer systems (e.g. Fig. 1 and Fig. 2) at carrier frequencies from $0.1 \mathrm{MHz}$ to $26 \mathrm{GHz}$. Similar performance possible to $100 \mathrm{GHz}$ [5].

Curve B. The noise floor, $S_{\phi}(f)$, for a high level mixer [5].

Curve $C$. The correlated component of $S_{\phi}(f)$ between two channels using high level mixers [5].

Curve $D$. The equivalent noise floor $S_{*}(f)$ of a 5 to $25 \mathrm{MHz}$ frequency multiplier.

Curve E. Approximate phase noise floor of Fig. 8 using a 500 ns delay line.

Curve F. Approximate phase noise floor of Fig. 8 where a 1 ms delay has been achieved by encoding the signal on an optical carrier and transmitted it across a long optical fiber to a detector.

Most double balanced mixers have a substantial nonIinearity that can be exploited to make phase comparison between the reference source and odd multiples of the reference frequency. Some mixers even feature internal even harmonic generation. The measurement block diagram looks identical to that of in Fig. 1, except that the source under test is at an odd (even) harmonic of the reference source. This method is relatively efficient (as long as the harmonics fall within the bandwidth of the mixer) for multiples up to $x 5$ although multiples as high as 25 have been used. The noise floor is approximately degraded by the amount of reduction in the phase sensitivity of the mixer. The phase noise of the reference source is also higher at the multiplied frequency as shown in Section III.F below.

\section{B. Enhanced Performance Using Corfelation}

\section{Techniques}

The resolution of the many systems can be greatly enhanced (typically $20 \mathrm{~dB}$ ) by using correlation techniques to separate the phase noise due to the device under test from the noise in the mixer and IF amplifier $[5,11]$.

For purposes of illustration, consider the scheme shown in Fig. 5. At the output of each double balanced mixer there is a signal which is proportional to the phase difference, $\Delta \phi$, between the two oscillators and a noise term, $V_{n}$, due to contributions from the mixer and amplifier. The voltages at the input of each bandpass filter are

$$
\begin{aligned}
& \mathrm{V}_{1} \text { (BP filter input) }=\mathrm{G}_{1} \Delta \phi(t)+\mathrm{C}_{1} \mathrm{~V}_{\mathrm{N}_{1}}(t) \\
& \mathrm{V}_{2}(B P \text { filter input })=\mathrm{G}_{2} \Delta \phi(t)+\mathrm{C}_{2} \mathrm{~V}_{\mathrm{N}_{2}}(t) .
\end{aligned}
$$

where $V_{H_{1}}(t)$ and $V_{N_{2}}(t)$ are substantially uncorrelated and $C_{1}$ and $C_{2}$ are constants. Each bandpass filter produces a narrow band noise function around its center frequency $f$ :

$$
\begin{aligned}
& V_{1} \text { (BP filter output) }=\underset{\phi(t)]}{G_{1}\left[S_{\phi}(f)\right]^{*} B_{1} * \cos [2 \pi f t+} \\
& +C_{1}\left[S_{V X_{1}}(f)\right]^{t} B_{1}+\cos \left[2 \pi f t+n_{1}(t)\right] \\
& V_{2} \text { (BP filter output) }=G_{2}\left[S_{4}(f)\right]^{t} B_{2} * \cos [2 \pi f t+ \\
& \psi(t)] \\
& +C_{2}\left[S_{\nabla_{2}}(f)\right]^{t} B_{2}+\cos \left[2 \pi f t+n_{2}(t)\right]
\end{aligned}
$$

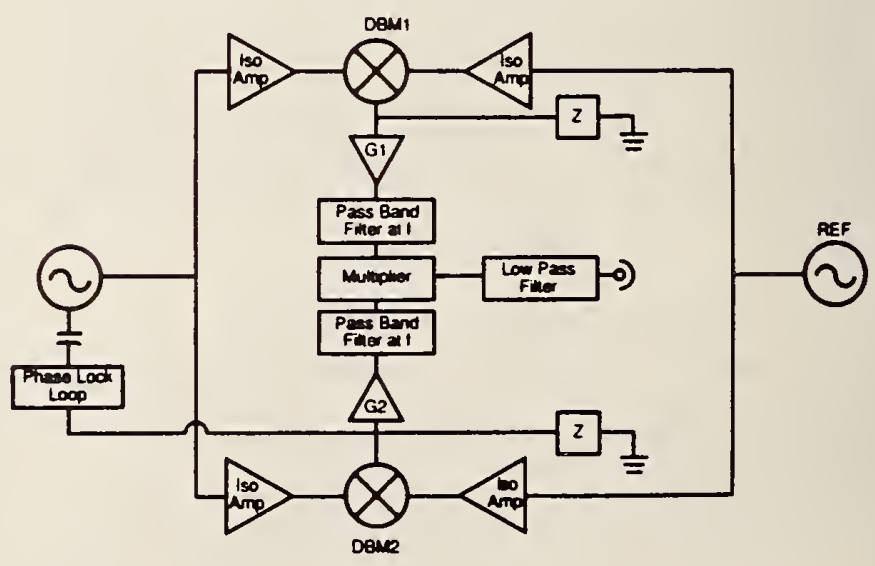

Fig. 5. Correlation phase noise measurement system.

where $B_{1}$ and $B_{2}$ are the equivalent noise bandwidths of filters 1 and 2 respectively. Both channels are bandpass filtered in order to help eliminate aliasing and dynamic range problems. The phases $b(t), n_{1}(t)$ and $n_{2}(t)$ take on all values between 0 and $2 \pi$ with equal likelihood. They vary slowly compared to $1 / f$ and are substantially uncorrelated. When these two voltages are multiplied together and low pass filtered, only one term has finite average value. 
$V_{o u t}^{2}=G_{1} G_{2} S_{\phi}(f) B_{1} h B_{2}+D_{1}\left\langle\cos \left[\psi(t)-n_{1}(t)\right]\right\rangle$

$$
+D_{2}\left\langle\cos \left[\phi(t)-n_{2}(t)\right]\right\rangle+D_{3}\left\langle\cos \left[n_{1}(t)-n_{2}(t)\right]\right\rangle \text {. }
$$

so that $S_{\phi}(f)$ is given by

$$
S_{4}(f)=\frac{(2) V_{n}^{2}(f)}{G_{1} G_{2} \sqrt{B_{1} B_{2}}} .
$$

For times long compared to $\mathrm{B}_{1}^{-{ }^{-}} \mathrm{B}_{2}^{-t}$ the noise terms $D_{1}, D_{2}$ and $D_{3}$ tend towards zero as $J_{t}$. Limits in the reduction of these terms are usually associated with harmonics of $60 \mathrm{~Hz}$ pickup, dc offset drifts, and nonlinearities in the multiplier. Also if the isolation amplifiers have input current noise, they will pump current through the source resistance. The resulting noise voltage will appear coherently on both channels and cannot be distinguished from real phase noise between the two oscillators. One half of the noise power appears in amplitude and one half in phase modulation.

Obviously the simple single frequency correlator used in this example can be replaced by a fast digital system which simultaneously computes the correlated phase noise for a large band of Fourier frequencies. Typical results show a reduction in noise floor of order $20 \mathrm{~dB}$ over the noise floor of a single channel (See Fig. 4). The great power of this technique is that it can be applied at any carrier frequency where are available double balanced mixers. The primary limitations come from the bandwidth and nonlinearities in the cross correlator $[5,11]$.

\section{C Reference Phase Modulation Method}

Another method of determining $S_{\phi}(f)$ uses phase modulation of the reference oscillator by a known amount. The ratio of the reference phase modulation to the rest of the spectrum then can be used for a relative calibration. This approach can save an enormous amount of time for measurements which are repeated a great many times. An adaptation of this approach is utilized in the new NBS phase noise system described in section IV below.

\section{D. Frequency Discriminator Methods}

It is sometimes convenient to use a high-Q resonance directly as a frequency discriminator as shown in Fig. 6. The oscillator can be tuned $1 / 2$ linewidth $\left(\nu_{0} / 2 Q\right)$ away from line center yielding a detected amplitude signal of the form

$$
V_{\text {out }}=G(f) k_{d} Q d y(f)[V+\epsilon(t)]
$$

This approach mixes frequency fluctuations between the oscillator and reference resonance with the amplitude noise of the transmitted signal. By using amplitude control (e.g. by processing to normalize the data), one can reduce the effect of amplitude noise. [5] The measured nolse at the detector is then related to the phase fluctuation of the reference resonance by

$$
S_{b}(f)=\left(\frac{\nu_{0} V_{H}(f)}{f Q k_{d} G(f)}\right)^{2} \frac{1}{B W} \text { for } f<\frac{\nu_{0}}{2 Q} .
$$

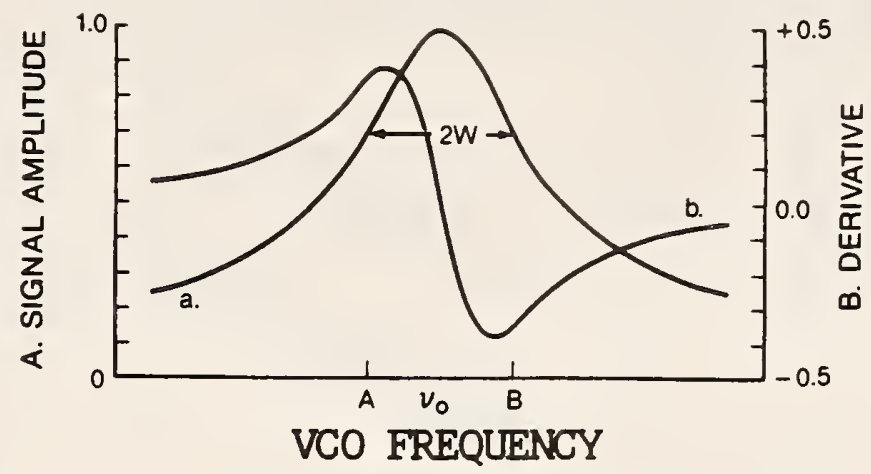

Fig. 6. High-Q resonance used as a frequency discriminator. Note that the peak response is displaced from the center of the resonance by about the half bandwidth.

This approach normally has the limitations that $f$ must be small compared to the linewidth of the cavity, and the effect of residual amplitude noise is difficult to remove; however, no reference source is needed. The calibration factors $G(f) K d Q$ can be measured even for Fourier frequencies larger than $\nu_{0} / 2 Q$ by stepping the source frequency an amount dy (which is small compared to $1 / 2 Q$ ) and measuring the output voltage versus the modulation frequency, $f$.

Differential techniques $c a n$ be used to measure the inherent frequency (phase) fluctuations of two high-Q resonators as shown in Fig. 7 [7]. The output voltage is of the form $V_{\text {out }}=2 Q_{d} G(f)$ dy(f). The phase noise spectrum of the resonators is then obtained using Eq. 4.

$$
S_{\phi}(f)=\left(\frac{\nu_{0} V_{H}(f)}{2 Q f K_{d} G(f)}\right)^{2} \frac{1}{B W} \quad f<\frac{\nu_{0}}{2 Q}
$$

Measurement of the Inherent Phase Noise in High-Q Resonators

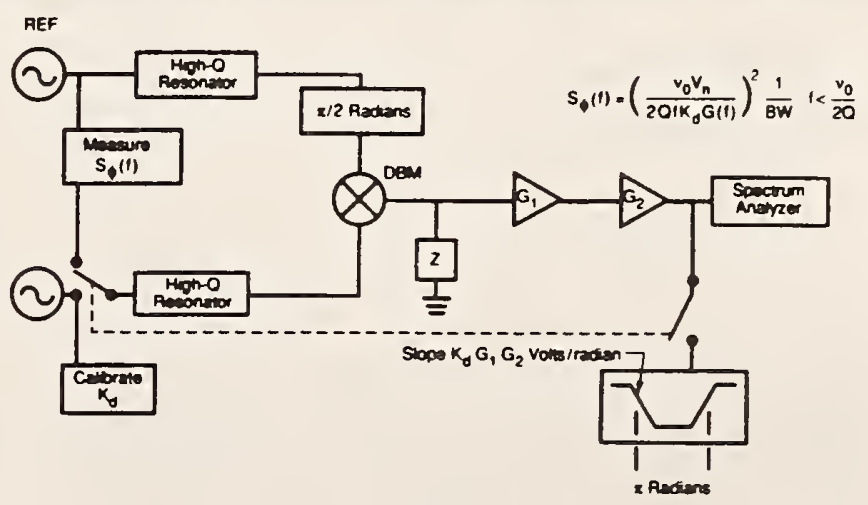

Fig. 7. Differential frequency discriminator using a pair of high-Q resonators. In this approach the phase noise of the source tends to cancel out. 
The phase nolse in the source can cancel out by 20 to $40 \mathrm{~dB}$ depending on the similarity of resonant frequencles $Q^{\prime} s$ and the transmission properties of the two resonators. This approach was first used to demonstrate that the inherent frequency stability of precision quartz resonators exceeds the performance of most quartz crystal controlled oscillators [7].

If only one resonance is used, the output includes the phase fluctuations of both the source and the resonator. The calibration is accomplished by stepping the frequency of the source and measuring the output voltage, 1.e., $\Delta V=G(f) K^{1}(f) \Delta \nu_{1}$. From this measurement the phase spectrum can be calculated as

$$
S_{*}(f)=\left(\frac{V_{N}(f)}{f K^{1}(f)}\right)^{2}\left(\frac{1}{B W}\right) \text {. }
$$

Fig. 8A shows one method of implementing this approach at $X$-band. The cavity has a loaded quality factor of order 25,000. Fig. 8B shows the measured frequency discriminator curve. Note that $K^{1}(f)$ is constant for $f<\nu /(2 Q)$ and decreases at values of $f$ larger than the half bandwidth of the resonance as

$$
K^{1}(f)-\frac{1}{1+\left(\frac{2 Q f^{2}}{v_{0}}\right)}
$$

The frequency dependence shown in eq. (19) can be accurately determined by measuring $\Delta V$ when stepping the source an amount $\Delta \nu$, which is small compared to $\nu_{0} / Q$, versus the modulation frequency, $f$. This approach has poor resolution near the carrier and limited high frequency response. Therefore the measurement of phase from close to the carrier out to 108 of the carrier could require a large set of cavities with different $Q$ factors. In order to achieve the required $Q$ factor for close in measurements it may even be necessary to use cryogenic techniques.

\section{E. Delay Line Method}

Another different approach uses a delay line to make a pseudo reference which is retarded relative to the incoming signal $[7-10]$ as shown in Fig. 9.

The mixer output is of the form

$$
V_{\text {out }}=2 \pi t_{d} K_{d} \nu_{0} d y \text {. }
$$

and the input phase noise is given by

$S_{f}(f)=\left(\frac{V_{B}(f)}{2 \pi f r_{d} G(f) K_{d}}\right)^{2} \frac{1}{B W}, \quad f<\frac{1}{\tau_{d}}$

This approach is often used at microwave frequencles when only one oscillator is avallable. In this technique the ability to resolve phase noise close to the carrier depends on the delay time. For example, If $\mathrm{f}=1 \mathrm{~Hz}$ and $r_{\mathrm{d}}=500 \mathrm{~ns}$, then, $\left(2 \pi \mathrm{fr} r_{\mathrm{d}}\right)^{2}-10^{-11}$. The nolse floor is $110 \mathrm{~dB}$ higher at $f=1 \mathrm{~Hz}$ than that of the two oscillator method, decreasing as $1 / f^{2}$. Recent advances make it possible to encode the
If signal on an optical signal which then can be transmitted down an optical fiber to achieve delays up to the order of $10^{-3} \mathrm{~s}$ with an increase in the noise floor to approximately $-140 \mathrm{~dB}$ relative to 1 $\mathrm{rad}^{2} / \mathrm{Hz}$. The noise floor can be reduced by -20 to 40 $\mathrm{dB}$ using the correlation techniques described above [11]. Note that the range of Fourler frequencies is usually limited to less than $-1 / r_{d}$. This technique normally has good resolution over it to 2 decodes in Fourler frequency. Therefore, measurements of phase nolse from close to the carrier out to 108 of the carrier require a large set of different delay lines and hardware including optical delay lines, associated Lasers, modulators, and detectors.

Measurement of Phase Nolse using a High-Q Cavity
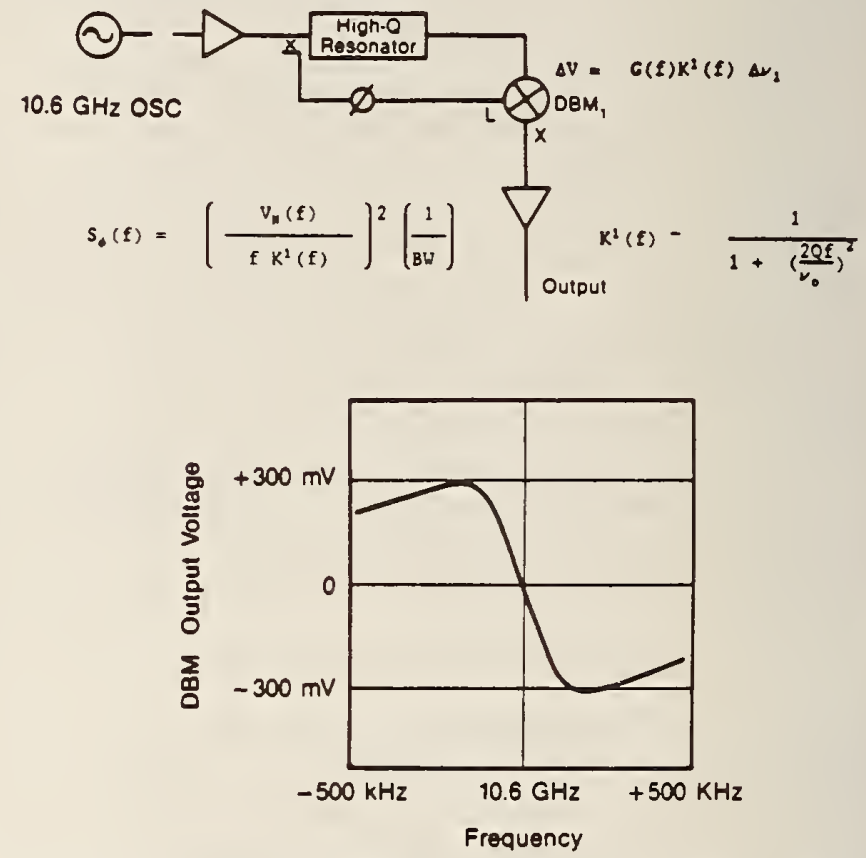

Fig. 8. A. block diagram of a high $Q$ resonator used as a frequency discriminator. B. Frequency discriminator curve for the scheme shown in $A$ used at $\mathrm{X}$-band with a cavity having a loaded quality factor of approximately 25,000 .

Measurement of $S_{\phi}(\ell)$ Using a Delay Line

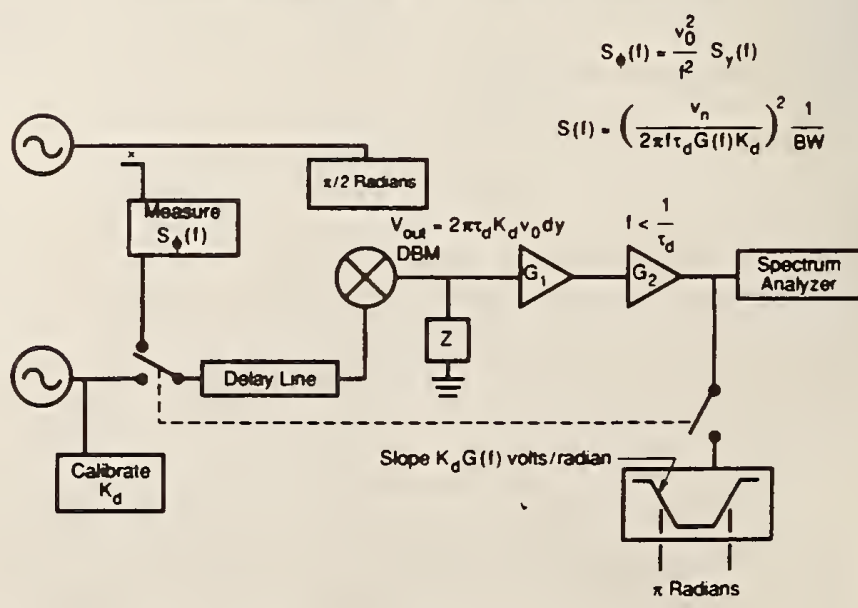

Fig. 9. Delay line frequency discriminator. 
efther increase or decrease the phase sensitivity of the mixer system. Fig. 4 shows the noise of a

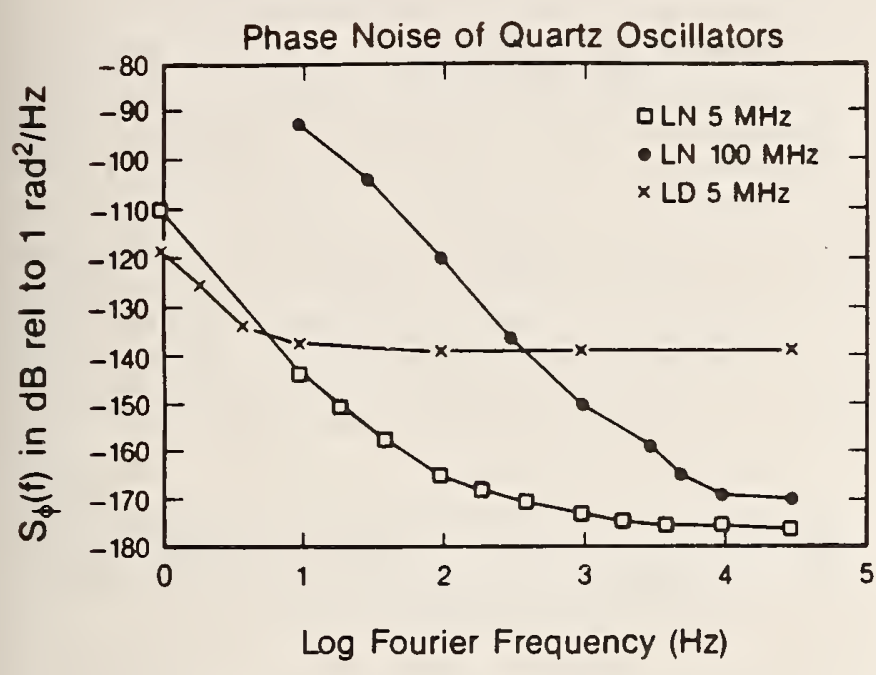

Fig. 10. Phase noise performance of selected quartz oscillators. The $\mathrm{LN} 5 \mathrm{MHz}$ oscillator is driven at a high level to reduce the wideband noise while the LD $5 \mathrm{MHz}$ oscillator is driven at a lower level to obtain low phase noise close to the carrier.

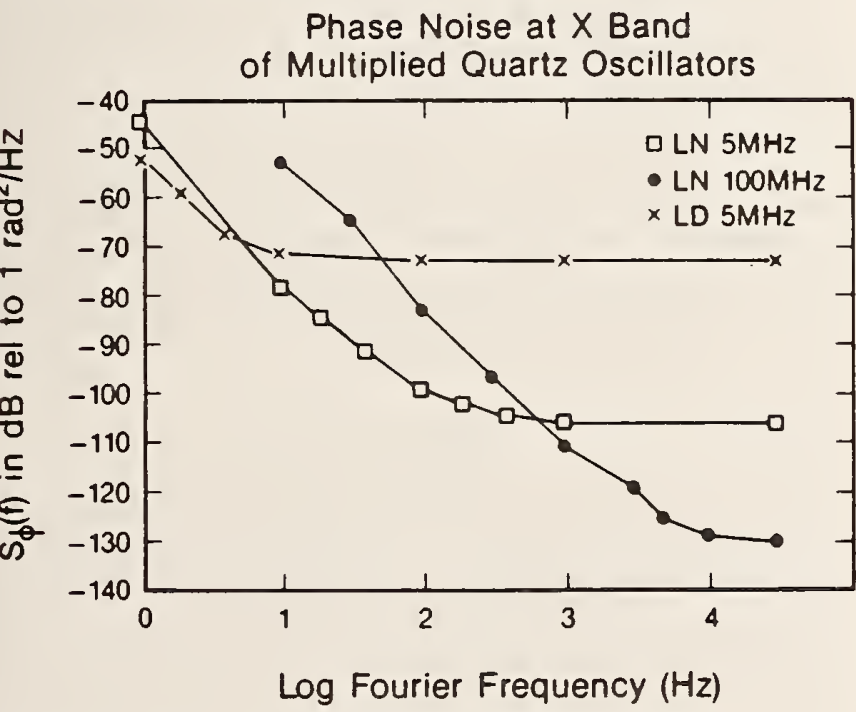

Fig. 11. Phase noise of the oscillators of Fig. 10 if multiplied to $X$-band in a perfect frequency multiplier.

\section{F. Multiplication/Division}

The use of perfect frequency multipliers (or dividers) between the signal source and the double balanced mixer increases (decreases) the phase noise level [12] as

$$
S_{\phi} \nu_{1}(f)=\left(\frac{\nu_{2}}{\nu_{1}}\right)^{2} S_{\phi} \nu_{1}(f)
$$

where $\nu_{1}$ is the initial carrier frequency and $\nu_{2}$ is the final carrier frequency. This can be used to

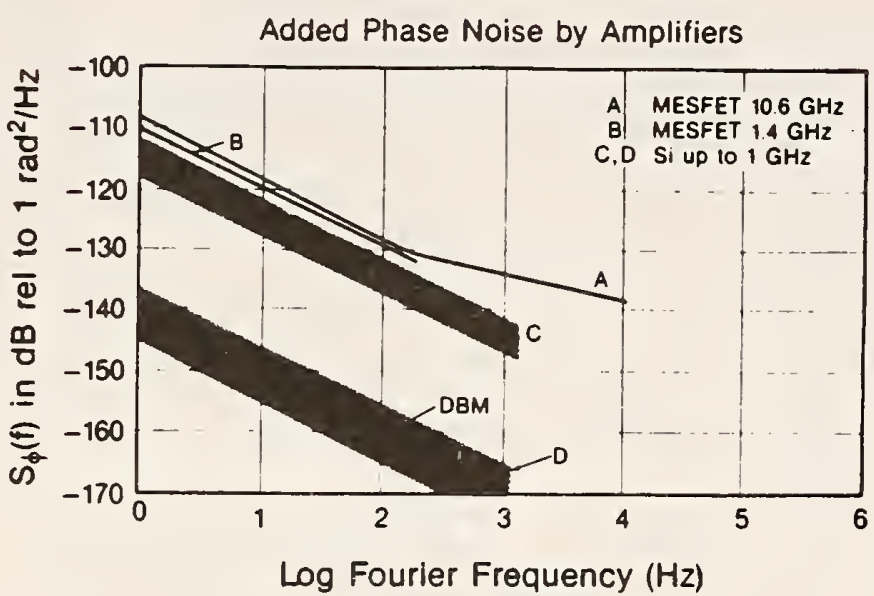

Fig. 12. Curves $A$ and $B$ show the phase noise added by selected GaAs MESFET amplifiers. Curve $C$ shows the phase noise added by a typical common emitter silicon bipolar transistor with a "good" rf bypass on the emitter lead. Curve D shows the typical performance of the same amplifier with a small unbypassed impedance (approximately $1 /$ transconductance) in the emitter lead. The added phase noise is generally independent of frequency over a very large range.

specialized 5 to $25 \mathrm{MHz}$ multiplier referred to the 5 $\mathrm{MHz}$ input. A potential problem with the use of the multiplier approach comes from exceeding the linear range of the mixer. Once the phase excursion, $\Delta \phi$, exceeds about 0.1 radian, non-linearities start to become important and at $\Delta \phi-1$ radian, the measurement is no longer valid [12]. An additional practical problem is that low noise multipliers are usually narrowband devices. Each significantly new frequency generally requires a new set of frequency multipliers.

\section{A. The New NBS Phase Noise Measurement Systems}

The new NBS phase nolse measurement systems are a combination of the traditional two oscillator approach shown in Figs. 1 and 2 plus the reference phase modulation technique mentioned in section IIIC. The complete block diagram is sketched in Fig. 13. This approach yields the widest possible bandwidth and the lowest phase noise of a single channel system. It does, however, require the use of two sources for oscillator measurements. From hardware considerations we generally use 3 different phase noise measurement systems. Test set A accepts carrier frequencies from 5 to $1300 \mathrm{MHz}$ and can measure the phase noise from $1 \mathrm{~Hz}$ to about 108 of the carrier or a maximum of $100 \mathrm{MHz}$. Test set $B$ accepts carrier frequencies from $1 \mathrm{GHz}$ to $26 \mathrm{GHz}$ and can measure the phase noise from $0.01 \mathrm{~Hz}$ to about $500 \mathrm{MHz}$ from the carrier. Test set $C$ accepts carrier frequencies from about 2 to $26 \mathrm{GHz}$ and can measure the phase noise from $0.01 \mathrm{~Hz}$ to $1 \mathrm{GHz}$ from the carrier. Test set $D$ accepts carrier frequencies from 33 to $50 \mathrm{GHz}$ in WR22 waveguide and can measure the phase noise from $0.01 \mathrm{~Hz}$ to about $1.3 \mathrm{GHz}$ from the carrier.

The construction of the phase modulator between the reference source and the mixer will be described in 
detail elsewhere [12]. The low pass filter section is used in order not to saturate the amplifiers with the carrier feedthrough signal from the mixer. One dc amplifier is used for phase noise measurements from dc to $100 \mathrm{kHz}$ from the carrier. One ac amplifier is used for phase noise measurements from $50 \mathrm{kHz}$ to $32 \mathrm{MHz}$. This range is well matched for one of our spectrum analyzers. The wideband ac amplifier has a bandwidth of $50 \mathrm{kHz}$ to $1.3 \mathrm{GHz}$ and is used when the desired measurement bandwidth exceeds $32 \mathrm{MHz}$.

The wide bandwidth spectrum analyzer also provides a convenient way to observe the gross features of the output phase noise and to identify any major spurious outputs if present. In order to obtain the most accurate measurement of the phase noise it is, however, necessary to measure the amplitude of the first IF signal at about $21 \mathrm{MHz}$ in order to avoid the variations in gains of the $\log$ amplifiers with various environmental factors.

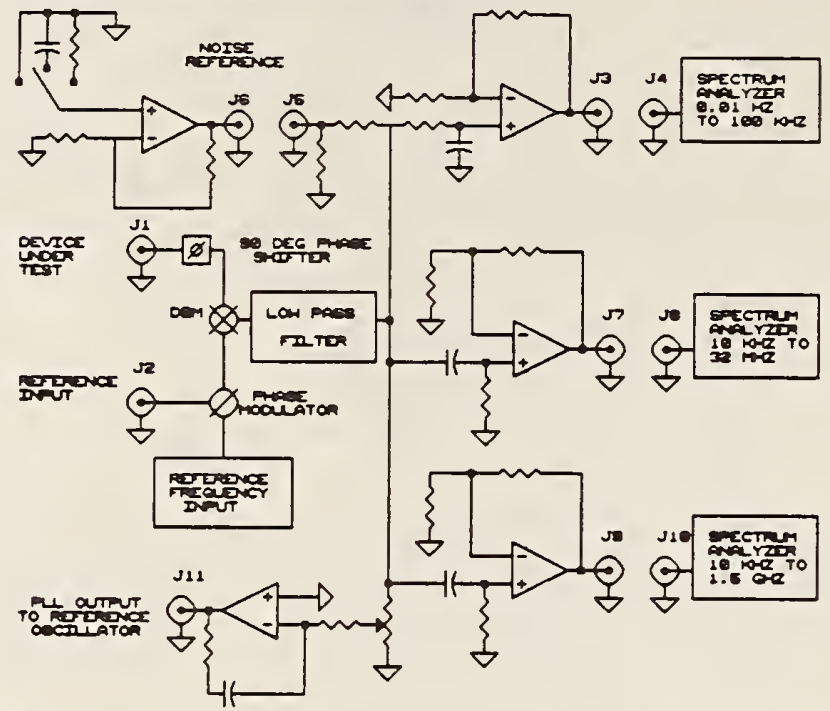

Fig. 13. Generalized block diagram of the new NBS phase noise measurement systems. The phase noise of carrier frequencies from $1 \mathrm{MHz}$ to $100 \mathrm{GHz}$ can be measured by varying the components in the phase shifters and mixers. Dedicated measurement systems covering $5 \mathrm{MHz}$ to $50 \mathrm{GHz}$ are described in the text.

\section{B. Measurement Sequence}

1) The output power of the two sources to be measured is typically set to between +5 and $+13 \mathrm{dBm}$ at the mixer. This takes into account the insertion loss of the phase modulator. If the oscillators don't posses sufficient internal isolation to prevent unwanted frequency pulling, isolators (or isolation amplifiers) are generally inserted between the sources and the mixer.

2) The absolute sensitivity of the nixer and the dc amplifier for converting small changes in phase to voltage changes is determined in a way similar to the traditional method, namely by allowing the two oscillators to slowly beat. The output of the dc amplifier is recorded by the digitizer in the FFT connected to the dc amplifier in order to accurately determine the period of the beat. In test sets $C$ and $D$ an additional $50 \mathrm{MHz}$ digitizer is used to average and record the beat frequency. The time scale of the digitizer is then expanded to approximately 10 of the beat period and pretrigerred at about $-2 \mathrm{~V}$ in order to accurately determine the slope of the output in volts per radian. This calculation, shown in eq. (23) below, is typically accurate \pm 2 or $0.2 \mathrm{~dB}$.

$$
\mathrm{K}=\text { Voles/Second })(\operatorname{Period} /(2 \pi)
$$

3) The two sources to be measured are phase locked together with sufficient bandwidth that the phase excursions at the mixer are less than 0.1 radian. The necessary phase lock gain is calculated using an estimate for the noise of the oscillators and the tuning rate for the reference oscillator. This is then verified by noting the peak to peak excursions of the dc amplifier and using the measured conversion sensitivity measured in step 2 above. If the peak phase excursions are in excess of 0.1 radian, then the phase lock loop bandwidth is increased (if possible) in order to satisfy this condition.

4) The modulator is driven by a reference frequency (typically at +7 to $+10 \mathrm{dBm}$ ) which steps through the Fourier frequencies of interest and the detected RMS voltage recorded on the appropriate spectrum analyzer. This approach accurately yields the relative gains of each amplifier and its respective spectrum analyzer since it automatically accounts for the effect of the phase lock loop and residual frequency pulling as well as the termination of the mixer and the variations in gain of the various amplifiers with Fourler frequency. The amplitude of the phase modulation on the carrier is constant in amplitude to better than $\pm 1.5 \mathrm{~dB}$ (typically $\pm 0.2 \mathrm{~dB}$ for $f<500 \mathrm{kHz}$ ) for reference frequencies $d c$ to about 10 of the carrier frequency or a maximum of 1 $\mathrm{GHz}$. Initial measurements of the prototype modulator are shown in Fig. 14.

This measurement is then combined with the measurement of the absolute mixer sensitivity multiplied by the gain of the dc amplifier described in step 2 above. The absolute gain of all the amplifiers shown in Fig. 13 can generally be determined to an accuracy of $\pm 0.3 \mathrm{~dB}$ ( $1.5 \mathrm{~dB}$ for Fourier frequencies from $500 \mathrm{MHz}$ to $1 \mathrm{GHz}$ ) over the Fourler frequencies of interest.

5) Next the spectral density function of the FFT is verified. The level of the noise determined by the FFT for the input of the noise source amplifier sequentialiy shorted to ground, connected to ground through a $100 \mathrm{k}$ metal film resister, and connected to ground through $200 \mathrm{pF}$, is then recorded. From these data one can determine the inherent noise voltage and noise current of the noise source amplifier plus the FFI as well as the noise of the resistor to about $\pm 0.25 \mathrm{~dB}$ which is the stated accuracy of the FFT. This primary calibration of the FFT can be carried out from about $20 \mathrm{~Hz}$ to over 50 $\mathrm{kHz}$. Above $50 \mathrm{kHz}$, the noise gain of the amplifier we used contributes a significant amount of noise. With sone compensation the noise is flat to within $\pm 0.2 \mathrm{~dB}$ from $20 \mathrm{~Hz}$ to $100 \mathrm{kHz}$. Next the noise source is switched into the output of the mixer and the relative noise spectrum of all the spectrum analyzers is calibrated by knowing their relative gains. This procedure verifies the voltage references and noise bandwidths of the various spectrum analyzers.

6) The noise voltage is recorded on the three spectrum analyzers over the Fourier frequencies of interest, generally the same one used in step 4 
above. The measured noise voltages are scaled using the measured gains and the spectral density of phase noise calculated. The overlapping ranges of the various spectrum analyzers allows one the opportunity to compare the measurements on the three spectrum analyzers. Typically one can obtain $S_{\phi}(f)$ of the oscillator pair to an accuracy of about $\pm 0.6 \mathrm{~dB}$ at Fourier frequency above $100 \mathrm{~Hz}$. The agreement with repeat measurements is often of order $\pm 0.2 \mathrm{~dB}$ as shown in Fig. 15.

7) The noise floor of the system is determined by driving both sides of the measurement system with one oscillator having a similar power and impedance level as that used in these measurements. If the noise floor is within $13 \mathrm{~dB}$ of that measured in step 7 above, corrections are made to the measurement data to remove the bias generated by the noise floor.

Measurements of the amplitude accuracy of the phase aodulation side bands generated by the prototype phase modulators are sumarized in Fig. 15. The same modulator was used for carrier frequencles from 5 to $300 \mathrm{MHz}$. The error in the phase modulation amplitude is less than $0.5 \mathrm{~dB}$ for modulation frequencies from dc to 10 of the carrier. The $10 \mathrm{GHz}$ modulator also maintains an accuracy of better than $0.5 \mathrm{~dB}$ out to $500 \mathrm{MHz}$ from the carrier. At $1 \mathrm{GHz}$ the modulation amplitude is $1.5 \mathrm{~dB} h 1 \mathrm{gh}$. Once this is measured it can be taken into account in the calibration procedure. The $45 \mathrm{GHz}$ modulator results shown in Fig. 15 should be attainable over the entire WR22 wavequide bandwidth.

The performance that can be obtained with this measurement technique is illustrated by actual phase noise data on oscillator pairs shown in Figs. 15 and 16. Typical accuracies are $\pm 0.6 \mathrm{~dB}$ with a noise floor at about - $175 \mathrm{~dB}$ relative to $1 \mathrm{radian}^{2} / \mathrm{Hz}$. The corrections applied to the raw data at $10 \mathrm{GHz}$ are shown in Fig. 17. At low frequencles the effect of the phase-locked loop is apparent while at the higher frequencies the roll-off of the amplifiers are important. These effects have been emphasized here in order to examine the ability of the calibration process to correct for instrumentation gain variations.

\section{v. Conclusion}

We have analyzed several traditional approaches to making phase measurements and found that they all lacked some element necessary for making phase noise measurements from essentially dc out to 108 of the carrier frequency with good phase noise floors and an accuracy of order $1 \mathrm{~dB}$. By combining several of the techniques and adding a phase nodulator which is exceptionally flat from dc to about 10 of the carrier frequency, we have been able to achieve excellent phase noise floors, bandwidths of at least 10 of the carrier, and accuracies of order $\pm 0.6 \mathrm{~dB}$.

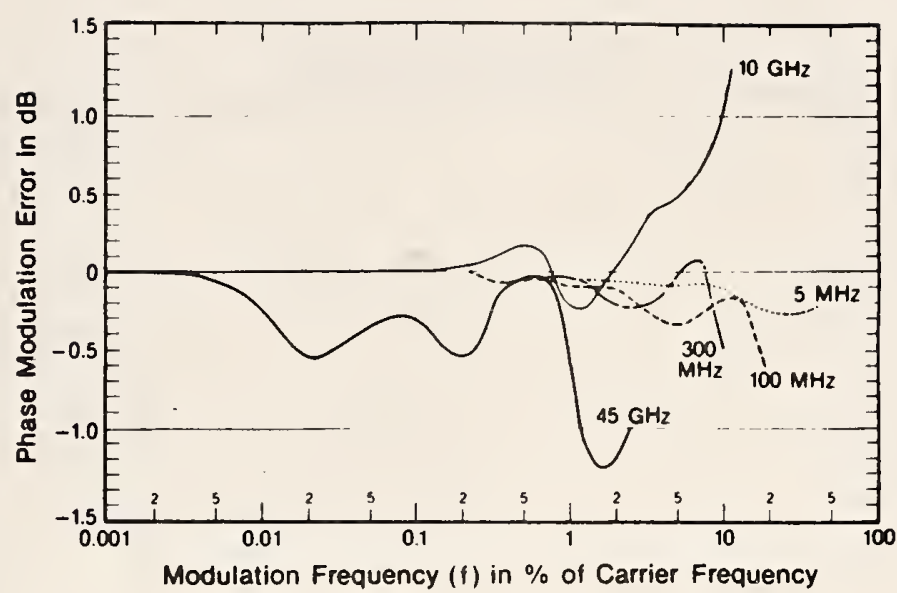

Fig. 14 Measurement of the amplitude error of modulation signal versus Fourier frequencies $f$, for these prototype phase modulators. Curves labeled 5 100 , and $300 \mathrm{MHz}$ were obtained with the modulator used in 5 to $1,300 \mathrm{kHz}$ test set. The curve labeled $10 \mathrm{GHz}$ was obtained with the modulator for the 2 to $26 \mathrm{GHz}$ test set. The curve labeled $45 \mathrm{GHz}$ was obtained with the WR22 test set.

Two Oscillator Calibration Test @ $5 \mathrm{MHz}$

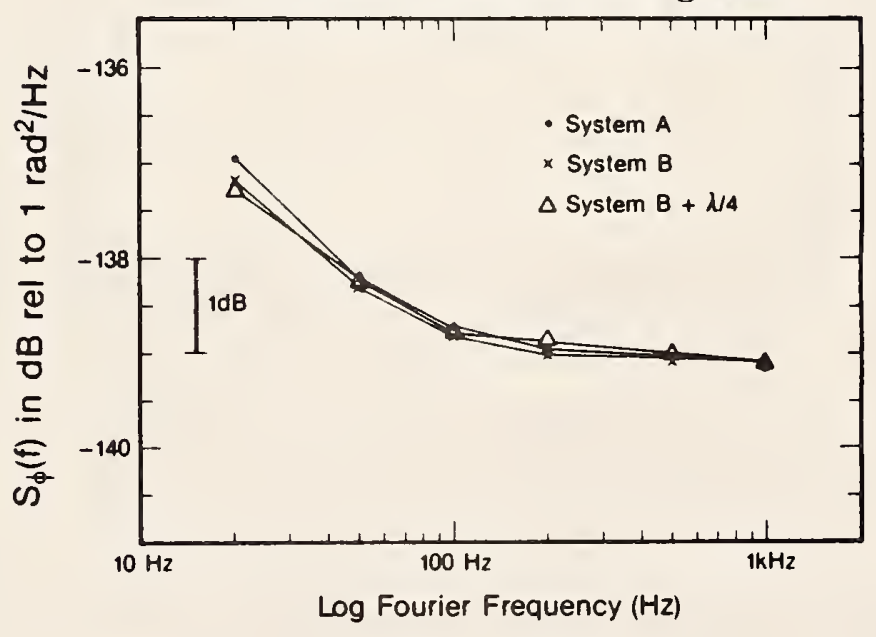

Fig. 15 Demonstration of calibration accuracy for two oscillator concept. The curve labeled System A shows the measured phase noise of a pair of $5 \mathrm{MHz}$ oscillators using the test set shown in Fig. 13. The curve labeled System $B$ shows the measured phase noise of the same pair of $5 \mathrm{MHz}$ oscillators using a totally separate measurement system with the oscillators held in phase quadrature with the measurement test set of A. The curve labeled System $B+\lambda / 4$ shows the phase noise of the same pair of oscillators using test set $B$ with and extra cable length of $\lambda / 4$ inserted into each signal path. The agreement between the three curves is in the worse case $\pm 0.15 \mathrm{~dB}$. 
Phase Noise at $\times$ Band

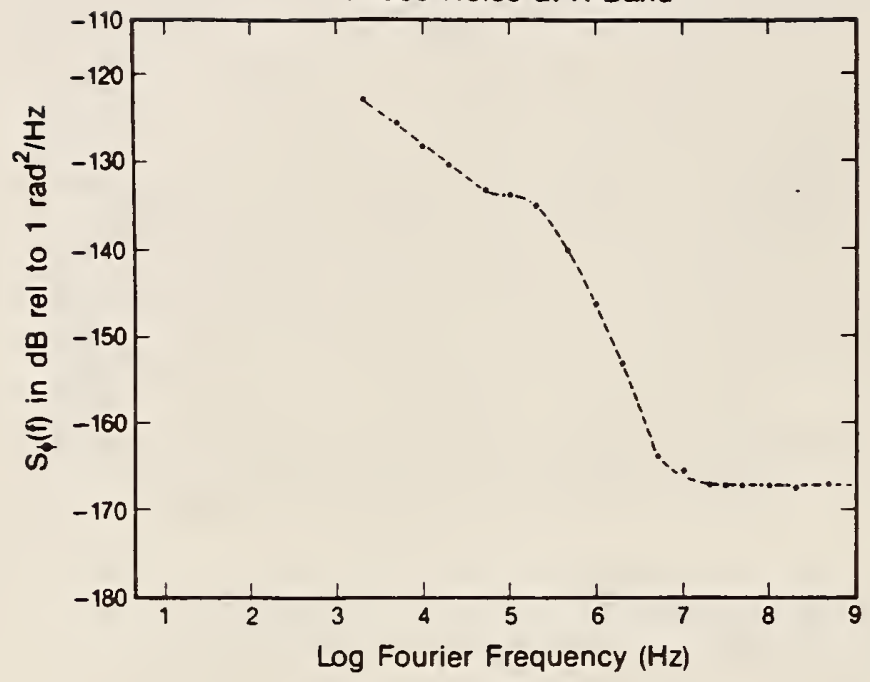

Fig. 16 Phase noise measurement on a pair of 10.6 GHz sources using the new NBS measurement technique.

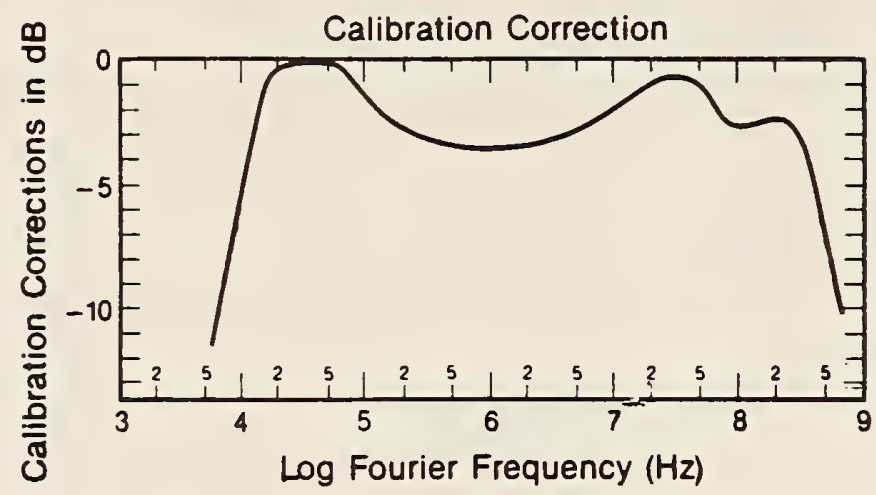

Fig. 17 Correction factor applied to the measurement data made to obtain the results of Fig. 16 .

\section{Acknowledgements}

The authors are grateful to many colleagues, especially David W. Allan, James C. Bergquist, Andrea DeMarch1, David J. Glaze, James E. Gray, David A. Howe, John P. Lowe, Samuel R. Stein and Charles Stone for many fruitful discussions on this topic and the Calibration Coordination Group for the funding to improve the accuracy and bandwidth of phase noise metrology.

\section{References}

1. J. A. Barnes, A. R. Chi, L. S. Cutler, D. J. Healey, D. B. Leeson, T. E. McGunigal, J. A. Mullen, Jr., W. L. Smith R. L. Sydnor, R. F. C. Vessot, G. M. Winkler, Characterization of Frequency Stability, Proc. IEEE Trans. on I \& M 20, 105-120 (1971).

2. J. H. Shoaf, D. Halford, and A. S. Risley, Frequency Stability Specifications and Measurement, NBS Technical Note 632, (1973). Document available from US Government printing office. Order SD at \#C13.46:632.
3. D.W. Allan, H. Hellwig, P. Kartaschoff, J. Vanier, J. Vig, G.M.R. Winkler, and N.F. Yannoni, Standard Terminology for Fundamental Frequency and Time Metrology, to be published in the Proc. of the 42nd Symposium on Frequency Control, Baltimore, MD, June $1-4,1988$.

4. F. L. Walls, and S. R. Stein, Accurate Measurements of Spectral Density of Phase Noise in Devices, Proc. of 31st SFC, 335-343, (1977).

(National Technical Information Service, Sills Bullding, 5825 Port Royal Road, Springfield, VA 22161).

5. F. L. Walls, S. R. Stein, J. E. Gray, and D. J. Glaze, Design Considerations in State-of-the-Art Signal Processing and Phase Noise Measurement Systems, Proc. 30th Ann. SFC, 269-274 (1976). (National Technical Information Service, Sills Bullding, 5285 Port Royal Road, Springfield, VA 22161).

6. R. L. Barger, M. S. Soren, and J. L. Hall, Frequency Stabilization of a cw Dye Laser, Appl. Phys. Lett. 22, 573 (1973).

7. F. L. Walls and A. E. Wainwright, Measurement of the Short-Term Stability of Quartz Crystal Resonators and the Implications for Crystal Oscillator Design and Applications, IEEE Trans. on I \& M 24, 15-20 (1975).

8. A. S. Risley, J. H. Shoaf, and J. R. Ashley, Frequency Stablilzation of $X$-Band Sources for Use in Frequency Synthesis into the Infrared, IEEE Trans. on I \& $\mathrm{M}, 23,187-195$ (1974).

9. J. R. Ashley, T. A. Barley, and G. J. Rast, The Measurement of Nolse in Microwave Transmitters, IEEE Trans. on Microwave Theory and Techniques, Special Issue on Low Noise Technology, (1977).

10. A. L. Lance, W. D. Seal, F. G. Mendoza, and N. W. Hudson, Automating Phase Nolse Measurements in the Frequency Domain, Proc. 31 st Ann. Symp. on Freq. Control, 347-358 (1977).

11. A. L. Lance and W. D. Seal, Phase Noise and AM Noise Measurements in the Frequency Domain at Millimeter Wave Frequencles, from Infrared and Millimeter Haves, Ken Button Ed., Academic Press, NY 1985 .

12. F. L. Walls and A. DeMarch1, RF Spectrum of a Signal After Frequency Multiplication Measurement and Comparison with a Simple Calculation, IEEE Trans, on I\& $M$ 24, 210-217 (1975).

13. F.L. Walls, A New Phase Modulator for Wideband Phase Nolse Measurement Systems, to be submitted to IEEE Transactions on Ultrasonics, Ferroelectrics and Frequency Control. 
David Allan, Naclonal Bureau of Standards, Boulder, CO 80303 Helmue Hellwig. Naelonal Bureau of Seandards, Galthersburg. MD 20899

Perer Kartaschoff, Swiss PTT, R\&D, CH 3000 Bern 29, Swlezerland Jacques Vanier. National Research Council, Oetawa, Canada KIA OR6 John Vig, U.S. Army Electronics Technology and Devices Laboratory, Fore Monmoueh, NS 07703

Gernor M.R. Winkler, U.S. Naval Observarory, Washingeon, DC 20390

Nicholas F. Yannoni, Rome Alr Development Center, Hanscom AFB, Bedford, MA 01731

\section{Incroducten}

Techniques to characterize and to measure the frequency and phase instabllieles in frequency and time devices and in recelved radio signals are of fundamental imporeance to all manufacturers and users of frequency and eime eechnology.

In 1964, a subcomitece on frequency stability was formed within the Institute of Electrical and Eleceronics Engineers (IEEE) Standards Comlecee 14 and, later (In 1966), In the Technical Commitcee on Frequency and Time within the Soclecy of Instrumentation and Measuremene (SIM), to prepare an IEEE scandard on frequency seab111ey. In 1969, this subcomilecee completed a document proposing definitions for weasures on frequency and phase stabilities (Barnes, et al., 1971). These recommended measures of instabilities in frequency generators have gained general acceptance among frequency and time users throughoue the world.

In this paper, measures in the eime and in the frequency domains aro reviewed. The particular cholce as to which domain is used depends on the application. However, the users are reminded that conversions using mathomatical formulacions (see Appendix I) from one domain to the other can present problems.

Most of the major manufacturers now speclfy lnstabilfty charactaristics of their standards in ceras of these recommended measures. This paper thus defines and formalizes the general practice of more than a decade.

\section{Measures of Erequency and Phase Instablificy}

Frequency and phase instabilicies shall be measured in cerms of the inseaneaneous, normalized frequency depareure $y(k)$ from the nominal frequency $\nu$, and/or by phase depareure $\phi_{(}(=)$, In radians, from the nominal piase $2 \pi \nu, e$ as follows:

$$
\begin{aligned}
& y(t)=\frac{1}{2 \pi \nu_{0}} \frac{d \phi(t)}{d t}=\frac{\dot{d}(t)}{2 \pi \nu_{0}} \\
& x(t)=\frac{\phi(t)}{2 \pi \nu_{0}}
\end{aligned}
$$

where $x(\xi)$ is the phase departure expressed in units of i.ime.

\section{Characterizacion of Erequency and Phase insiabilizies}

\section{a. Eequency Domalo:}

In the frequency domain, frequency and phase inscabllisy is defined by any of the following onesided spectral densleles (che Fourier frequency ranges from 0 to 0 ):

- Sec Appendix Note \# 17

$$
\begin{aligned}
& S_{y}(f) \text { of } y(t) \\
& S_{1}(f) \text { of } \phi(t) \\
& S_{\phi}^{*}(f) \text { of } \phi(t) \\
& S_{x}(f) \text { of } x(t) .
\end{aligned}
$$

These speceral densicles are relaced by the equa elons:

$$
\begin{aligned}
& s_{y}(f)=\frac{f^{2}}{v_{0}^{2}} s_{\phi}(f) \\
& s_{\phi}(f)=(2 \pi f)^{2} s_{\phi}(f) \\
& S_{x}(f)=\frac{1}{\left(2 \pi \nu_{0}\right)^{2}} S_{\phi}(f) .
\end{aligned}
$$

A device or signal shall be characterized by a plot of spectral densley vs. Fourler frequency or by tabulating discrete values or by equivalene weans such as a scacement of power law(s) (Appendix I).

According to the conventonal defintelon (Kartaschoff. 1978) of $\varphi(f)$ (pronounced "script (11"), $\varphi(f)$ is the raclo of the power in one sideband due to phase modulacton by nolse (for a $1 \mathrm{~Hz}$ banduldth) to the cotal signal power (carrier plus sidebands), that is,

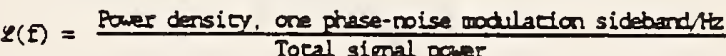 Toeal sigral pourer}

The convenelonal definielon of $\varphi(E)$ is related co $S,(E)$ by

$$
\varphi(E)=4 S_{1}(E)
$$

only if the wean squared phase deviation, $\left\langle\phi^{2}(f)\right\rangle=$ the Incegral of $S$, (f) from $f$ to $\infty$, is much swaller than one radian. In other words, ehis relacionship is valid only for Fourler frequencles f far enough from the carrier frequency and is always violaced near the cartier.

Slnce $S,(f)$ is the quanticy that is generally weasured in frequency standards metrology, and $\varphi(f)$ has become the prevalling weasure of phase noise among manufacturers and users of frequency standards, $L^{\prime}(f)$ is redefined as

$$
\varphi(f)=4 S_{0}(\varepsilon) \text {. }
$$

This redefinteion is incended to avold erroneous use of $\varphi(f)$ in sftuztions whera the saall angle approximation is not valid. In oeher words, $S_{0}(f)$ 
is the preferred aeasure, since, unamblguously, it always can be neasured.

\section{b. TIme-Domaln:}

In the time domaln, frequency instabllity shall be deflned by the ewo-sample deviation $\sigma_{y}(r)$ whlch is the square root of the two-sample varlance $\sigma_{y}^{2}(r)$. This varlance, $\sigma_{y}^{2}(r)$, has no dead-tiae between the frequency samples and is also called the Allan varlance. For the sampling time $r$, we write:

$$
\sigma_{y}^{2}(r)=\frac{1}{2}\left\langle\left(\bar{y}_{k+1}-\bar{y}_{k}\right)^{2}\right\rangle
$$

where

$$
\bar{y}_{k}=\frac{1}{r} \int_{c_{k}}^{t_{k}+r} y(t) d t=\frac{x_{k+1} \cdot x_{k}}{r} .
$$

The symbol <> denotes an Infinlte time average. In practice, the requirement of Infintee time average is never fulfilled; the use of the foregoIng terms shall be peralcted for finice time averages. The $x_{1}$ and $x_{1}$ are time residual measureaents made $\xi_{k}$ and $\xi_{k+1}=\tau_{t}+r, k=1,2,3, \ldots$. and $1 / r$ is the nominal flxed sampling rate which glves zero dead time between frequency measurements. "Residual" Implies the known systomatlc effects have been removed.

If dead tiae exlsts between the frequency departure measurements and this is ignored in the computation of $\sigma_{y}(r)$, resulelng instability values will be blased (except for white frequency nolse). Some of the blases have been studied and some correction tables published (Barnes, 1969; Lesage, 1983; Barnes and Allan, 1988). Therefore, the term $\sigma_{y}(r)$ shall not be used to describe such blased measurements. Racher, if biased instabillity measures are made, the Information in the roferences should be used to report an unblased estimate.

If the Initial sampling rate is specifled as $1 / r_{0}$, then 1t has been shown that, in general, we may obtaln a wore efflclent estimate of $\sigma_{y}(r)$ using what is called "overlapping estinates." This estloate is obtalned by computing

$$
\sigma_{y}{ }^{2}(r)=\frac{1}{2(N-2 \pi) r^{2}} \sum_{1=1}^{N-2 m}\left(x_{1+2 m}-2 x_{i+\infty}+x_{1}\right)^{2}
$$

where $N$ is the number of original time resldual measurements spaced by $r_{0}(N=M+1$, where $M$ is che number of orlglnal frequency measuremenes of sample elme $\left.r_{0}\right)$ and $r=m r_{0}$.

From the above equation, we see that $\sigma_{y}^{2}(r)$ acts like a second-difference operator on the time deviation residuals--providing a sicionary measure of the stochastic behavior even for nonstationary processes. Addlelonal variances, which may be used to describe frequency instabllitles, ara dofined in Appendix II.

\section{c. Cleck-Time Prediction}

The vartation of the tise difference betreen a real clock and an ideal uniform elme scale, also known as elae Incerval error, TIE, observed over a elme Interral stazing a: time $t_{0}$ and ending at $t_{0}+t$ shall be defined as:

$$
\operatorname{IIE}(t)=x\left(t_{0}+t\right) \cdot x\left(t_{0}\right)=\int_{t_{0}}^{t_{0}+t} y\left(t^{\prime}\right) d t^{\prime} .
$$

For falrly slaple models, regression analysis can provide efficient estimates of the IIE (Draper and Silth, 1966; CCIR, 1986). In general, there are many estimators posstble for any statistical quan. eley. Ideally, we would like an efflelent and unblased estimator. Using the tlue domain measure $\sigma_{y}^{2}(r)$ defined in (b). the following estimate of the standard deviation (RYS) of TIE and Its assoclated systematic departure due to a IInear frequency drift (or les uncertalney) can be used to predict a probable tlae lnterval error of a clock synchronized at $t=t_{0}=0$ and left free running thereafter:

RYSTIE ${ }_{\text {est }}=t\left[\frac{a^{2}}{4} t^{2}+\sigma_{y_{0}}^{2}+\sigma_{y}^{2}(r=t)+\left(\frac{x\left(t_{0}\right)}{t}\right)^{2}\right]^{4}$,

where "a" is the normallzed IInear frequency drife per unle of time (aging) or the uncertalnty in the drif: astimate, $O$, the two-sample deviation of the initlal frequehey adjuscment, $\sigma_{y}(r)$ the twosample deviation describling the random frequency Instabllity of the clock at $t=r$, and $x\left(t_{0}\right)$ is the Inltial synchronlzation uncertalnty. The thled cerm in the brackets provides an opeloum and unblased estimate (under the condition of an optinum (RYS) prediction method) in the cases of whlte nolse $\mathrm{M}$ and/or randon walk $\mathrm{F}$. The third term is too optiolstic, by about a factor of 1.4, for flicke? nolse FM, and too pesstmis=1c, by about a factor of 3, for whlte nolse PY.

This estimate is a useful and falrly slople approxtmation. In general, a more complete error analysis becomes difilcult; if carried out, such an analysis needs to include the methods of time prediction, the uncertalnties of the clock parameters, using the confidence lioles of measurements deflned below, the detalled clock nolse models, systematic effects, etc.

\section{Confidence Limits of Measuremencs}

An estimaze for $\sigma,(r)$ can be made from a finite daca set with $M$ measurements of $y_{2}$ as follows:

$\sigma_{y}(r)=\left|\frac{1}{2(M-1)} \sum_{j=1}^{M-1}\left(\bar{y}_{j+1}-\bar{y}_{j}\right)^{2}\right|^{h}$

or, If the dasa are time readings $x_{j}$ :

$o_{y}(r)=\left|\frac{1}{2 r^{2}(y-1)} \sum_{j=1}^{M-1}\left(x_{j+2}-2 x_{j+1}+x_{j}\right)^{2}\right|^{h}$

The 68 pezcent confidence interval (or error baz), Ia for Gausilan nolse of a parifcular value $\sigma_{y}(p)$ obsalned from a finfice number of samples can be estimazed as follows:

$$
I_{3}=\sigma_{y}(\varphi) \kappa_{a} Y^{-i}
$$

where:

$$
\begin{aligned}
& M \quad= \text { total number of daza points used in the } \\
& \text { estimase, } \\
& a= \text { an laceger as defined in Appendix I, } \\
& x_{2}=x_{1}=0.99 .
\end{aligned}
$$


$\pi_{0}=0.87$
$\kappa_{-1}=0.77$

$\kappa_{-2}=0.75$

As an example of the Gausian aodel with $M=100, a=-1$ (Ellcker frequency nolse) and $\sigma_{y}(r=1$ second $)=10^{-12}$. we may wrize:

$$
I_{0}=\sigma_{y}(r) \cdot(0.77) \cdot(100)^{-t}=\sigma_{y}(r) \cdot(0.077)
$$

which gives:

$$
a_{y}(r=1 \text { second })=(1 \pm 0.08) \times 10^{-12} \text {. }
$$

If $M$ is seall, then the plus and alnus confidence Intervals becowe asymerile and the $*_{a}$ coefflelents are not valld; however, these confidence Intervals can be calculaced (Lesage and Audoin, 1973).

If "overlapping" estimaces are used, as ouclined above, then the confidence Interval of the estimace can be shown to be less than of equal to $I_{\text {a }}$ as glven above (Howe, Allan, Barnes, 1981).

\section{Recomendaclons for Charactertzing or Reporting} Heasuremencs of Frequency and Phase Instabllifles

a. Nonrandom phenomena should be recognlzed, for example:

- any observed eime dependency of the seaelstical measures should be staced;

- the method of modeling systematlc behavior should be speclfled (for example, an estimate of the linear Erequency drlfe was obtalned from the coefficlenes of a linear lease-squares regression co M Erequency measurements, each wich a specleled averagling or sample time $r$ and measuremenc bandwidth $E_{b}$ ):

- the environmental sensleivities should be staced (for example, the dependence of frequency and/or phase on remperature, magnetic field, barometric pressure, vibraclon, etc.);

b. Relevant measurement or speclflcation paramecars should be given:

- the method of measurements:

- The characteristics of the reference signal:

- the nominal signal Erequency $\nu_{0}$ :

- the measurement system bandwidth $E_{b}$ and the corresponding low pass filter response:

- The tocal measurement $t$ ime and the number of measuremencs $M$;

- the calculation rechnlques (For example, detalls of the window function when estimating power spectral densteles from ine domain daca, of the assumptions about effects of dead-time when estimacing the two-sample deviation $\sigma_{y}(r)$ ):

- the confidence of the escimaze (or error bar) and les stacistical probabllity (e.g. "chree-sigma"):
If a passlve eleaent, such as a crystal flicer, is being mesured in contrast to - frequency and/or elme generacor.

\section{References}

Barnes, J.A., Tables of blas functons, $B_{1}$ and $B_{2}$. for varlances based on finlee samples of processes with power law spectral densitles, NBS, Hashingeon, DC, Tech. Note 375, (Jan. 1969).

Barnes, J.A. and Allan, D.H., "Varlances Based on Dara with Dead IIme Between the Measurements: Theory and Tables, NBS Tech Note 1318 (1988).

Barnes, J.A., Chl, A.R., Cueler, L.S., Healey, D.J., Leeson, D.B., McGunlgal, T.E., Mullen, J.A., Smleh,W.L., Sydnor, R., Vessor, R.F. and Winklez, G.M.R., Characterlzation of frequency stabllity, IEEE Trans. Instr. and Meas., Vol. IY-20, 105-120, (May 1971)

CCIR Report 898, Dubrovnlk, (1986).

Draper, N.R. and Smleh, H., Applled Regression Analys ls, John Ulley and Sons, (1966).

Howe, D. A., Allan, D. W., and Barnes, J.A., Propertles of slgnal sources and measurement mechods. Proc. 35th Annual Sympostun on Frequency Control, 669717. (May 1981).

Kartaschoff, P., Frequency and IIme, Academic Press, New York (1978).

Lesage, P., and Audoin, C. Characterization of frequency stability: uncertalnty due to the finlte number of mesurements. IEEE Trans. Instr. and Meas., Vol. IM-22, 157-161, (June 1973).

Lesage, P., Cheracterlzarion of Frequency Seabll1ey: Blas due to the juxtaposition of elme Interval measureqents. IEEE Trans. on Instr. and Meas, IK32. 204-207. (1983).

Seein, S. R, Frequency and Time: The lo measurement and characterlzation. Preciston Frequency Concrol. Vol. 2. Academlc Press, New York, 191-232, (1985).

\section{APPENDIX I}

\section{Power-Law Spectral Denst-les}

Powez-law spectral densleles aze often exploved as reasonable and accurace models of the randon fluctuarions in precision osclllacors. In practice, these random fluctuacions can often be represenced by tie sum of five Independent nolse processes, and hence:

$$
S_{y}(E)= \begin{cases}\sum_{a=-2}^{+2} h_{\alpha} E^{a} & \text { for } 0<E<E_{h} \\ 0 & \text { for } \varepsilon \geq E_{h}\end{cases}
$$

where $h_{\alpha}$ 's are constanes, $a$ 's are Ineegers, and $F_{y}$ is the hlgh Erequency cut-off of a low pass fllcer. HIgh Erequency divergence is eliminaced by che restrictions on $f$ in this equation. The 1dentiflcation and characrerlzazion of the flve nolse processes are given in Table 1, and shown in Fig. 1 . 


\section{Comversten Between Frequency and Time Domato}

The operation of the counter, averaging the frequency for a elae $r$, aay be thoughe of as a flleering operaclon. The transfer funcelon, $H(f)$, of thls equivalent fllter is then the Fourler eransform of the Iapulse response of the flleer. The eloe domaln frequency instablliey is then given by

$$
\sigma^{2}(Y, T, r)=\int_{0}^{\infty} S_{Y}(E)|H(E)|^{2} d E \text {, }
$$

where $S_{y}(f)$ is the spectral density of frequency fluceuselons. $1 / T$ is the aesureaent rate ( $T-r$ is the dasd elme between measurements). In the case of the twosample variance $|H(f)|^{2}$ is $2(\sin x+f) /(\pi r f)^{2}$. The twosample varlance can ehus be compued froa

$$
\sigma_{y}{ }^{2}(r)=2 \int_{0}^{f_{h}} s_{y}(\varepsilon) \frac{\sin ^{4} \pi r f}{(\pi r f)^{2}} d f .
$$

Specifically, for the power las model given, the tiae domain measure also follows a power law.

TABLE 1 - The functional characteristics of the independent nolse processes

\begin{tabular}{|c|c|c|c|c|c|}
\hline \multirow{4}{*}{$\begin{array}{l}\text { Description of } \\
\text { Nolse Process }\end{array}$} & \multicolumn{5}{|c|}{ Slope characteriselcs of $\log \mathrm{log}$ plot } \\
\hline & \multicolumn{2}{|c|}{ Frequency doesin } & \multicolumn{3}{|c|}{ Ine-domain } \\
\hline & $\begin{array}{c}S_{y}(f) \text { of } \\
S_{f}(f)\end{array}$ & $\begin{array}{l}S_{1}(f) \text { or } \\
S_{x}(f)\end{array}$ & $\sigma_{Y}^{2}(p)$ & $\sigma_{y}(r)$ & $\operatorname{Mod} . \sigma_{y}(r)$ \\
\hline & $a$ & $\boldsymbol{B}$ & $\mu$ & $\mu / 2$ & $\mu^{\circ}$ \\
\hline Randow Walk Freq Modularion & -2 & -4 & 1 & $1 / 2$ & 1 \\
\hline Flicker Frequency Modulation & -1 & -3 & 0 & 0 & 0 \\
\hline White Frequency Modulation & 0 & -2 & -1 & $-1 / 2$ & -1 \\
\hline Fllcker Phase Modulation & 1 & -1 & -2 & -1 & -2 \\
\hline White Phase Modulacion & 2 & 0 & -2 & -1 & -3 \\
\hline
\end{tabular}
used in modeling frequency instabllicy of osclllators

$$
\begin{aligned}
& S_{Y}(f)=\frac{(2 \pi f)^{2}}{\left(2 \pi \nu_{0}\right)^{2}} S_{\rho}(f)=h_{0} f=\quad \sigma_{g}^{2}(f)-|r|^{\mu} \\
& S_{0}(f)=v_{0}^{2} h_{e} f^{-2}=\nu_{0}^{2} h_{e} E^{g} \quad(\beta=\alpha-2) \quad \sigma_{y}(\varphi)-|+|^{\mu / 2} \\
& S_{z}(f)=\frac{1}{4 \pi^{2}} h_{e} f^{-2}=\frac{1}{4 \pi^{2}} h_{e} f^{0} \quad \operatorname{Mod} \sigma_{y}(r)-|r|^{\prime}
\end{aligned}
$$

IABLE ? - Translation of frequency instabll1ty measures from spectal densities in frequency domain $c 0$ varlances in elme domain and vice versa (For $2 \pi f_{b}$, $\gg$ )

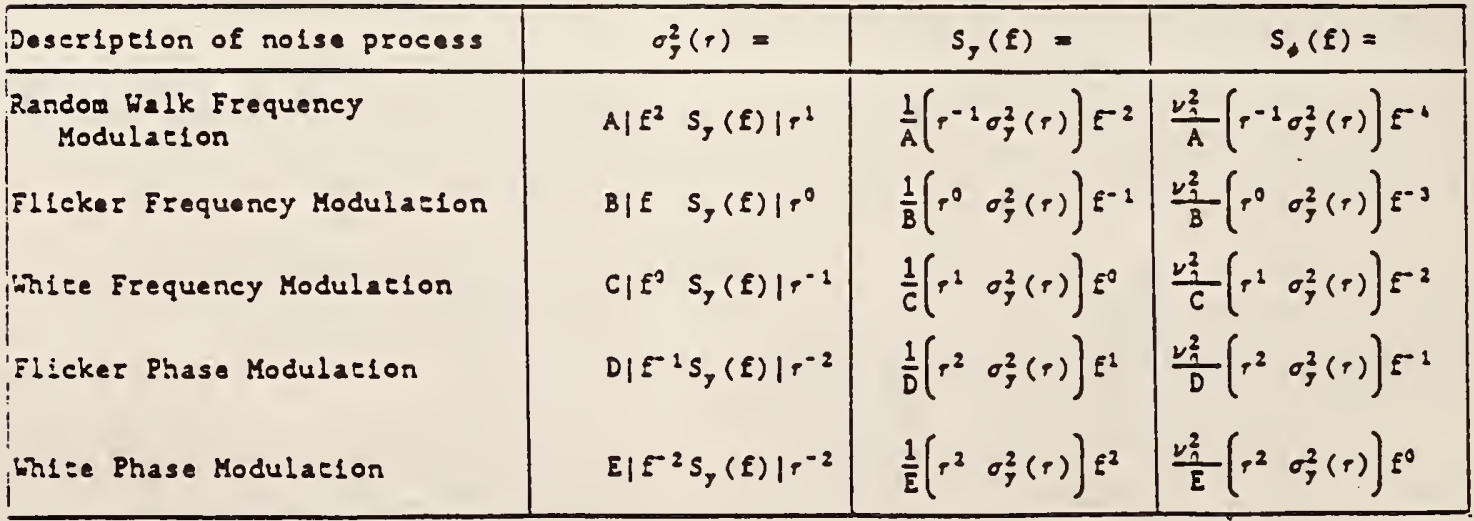

$$
\begin{array}{ll}
A=\frac{4 \pi^{2}}{6} & C=1 / 2 \\
B=2 \log _{0} 2 & D=\frac{1.038+3 \log _{0}\left(2 \pi E_{b} r\right)}{4 \pi^{2}}
\end{array} \quad E=\frac{3 E_{i}}{4 \pi^{2}}
$$




$$
\begin{aligned}
\sigma_{y}^{2}(r) & =h_{-2} \frac{(2 \pi)^{2}}{6}++h_{-1} 2 \log _{\theta} 2+h_{0} \frac{1}{2 r} \\
& +h_{1} \frac{1.038+3 \log _{g}\left(2 \pi f_{h} r\right)}{(2 \pi)^{2} r^{2}}+h_{2} \frac{3 f_{h}}{(2 \pi)^{2} r^{2}} .
\end{aligned}
$$

This implictely assumes that the randon driving aechanisa for each tera is Independent of the others. In addition, there is the iaplicit assumption that the mechanlsa is valld over all Fourier frequencles, which may not always be true.

The values of $h_{a}$ are characterlstic models of oscillator frequency noise. For integer values (as often seens to be the case for reasonable models), $\mu=-\alpha-1$,

for $-3 \leq a \leq 1$, and $\mu=-2$ for $a \geq 1$, where $\sigma_{y}^{2}(r)-r^{4}$.

Table 2 gives the coefficlents of the translation among the frequency stabilley measures from eime domain to frequency domain and from frequency domain to time domaln.

The slope characteristics of the five independent nolse processes are plotted in the frequency and time domalns In Flg. I (log-10g scale).

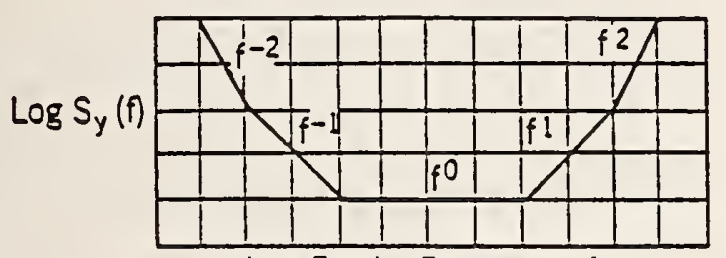

Log Fourier Frequency, $f$

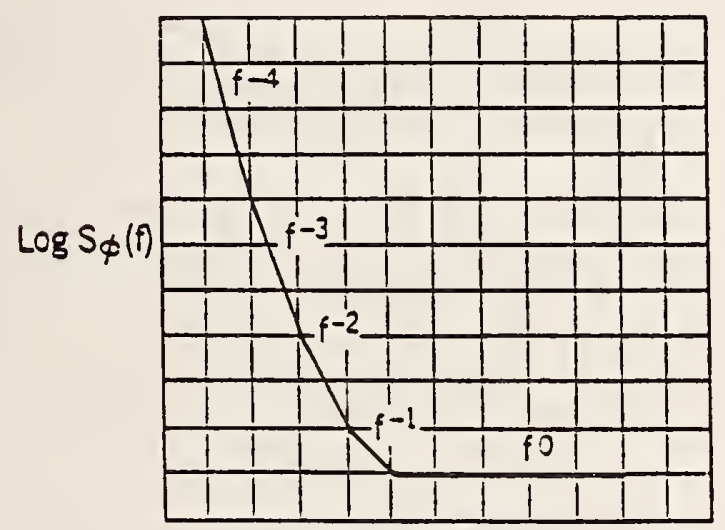

Log Fourier Frequency, $f$

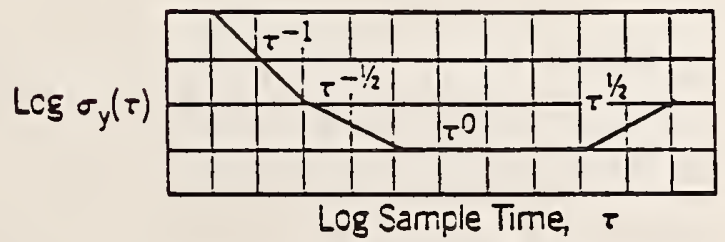

Slope characteristics of the five independent noise frocesses.

ELCtRE 1
APRSNDIX II

ADOITJONAL VARIANCES THAT MAY BE USBD TO DESCRTBE, BREUPAC INSTABILITIES IN THE TIYE DOMATN

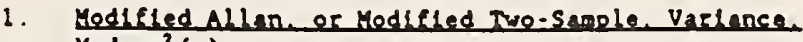 Mod $g_{1}^{2}(r)$}

Instead of the use of $\sigma_{y}^{2}(r)$, a "Modified Variance" Mod $\sigma_{y}^{2}(r)$ may be used to characterize frequency instabll1tles (Stein, 1985: Allan, 1987). It has the property of ylelding different dependence on $r$ for white phase nolse and flicker phase nolse. The dependence for Mod $o_{y}(r)$ is,$-3 / 2$ and $r^{-1}$ respectivaly. Mod $\sigma_{y}^{2}(r)$ is defined as:

$\operatorname{Mod} \sigma_{y}^{2}(r)=\frac{1}{2 r^{2} m^{2}(N-3 m+1)} \sum_{j=1}^{N-3 m+1}\left(\sum_{i=j}^{m+j-1}\left(x_{1+2 m^{-2}}-2 x_{1+m}+x_{1}\right)\right)^{2}$

Where $N$ is the original number of eime measurements spaced by $r$ and $r=m$, the sample time of cholce $(N=M+1)$. A device or signal shall be characterized by a plot of $\sigma_{y}(r)$ or $\sigma_{y}^{2}(r)$ or $\operatorname{Mod} \sigma_{y}(r)$ or $\operatorname{Mod} \sigma_{y}^{2}(r)$ vs. sampling time $r$. or by tabulating discrete values or by equivalene geans such as a stacemene of power laws (Appendix I).

\section{Qcher Variances}

Several other varlances have been introduced by workers in this field. In particular, before the introduction of the cso-sample varlance, it was standard practice to use the sample varlance, $s^{2}$, defined as

$$
s^{2}=\int_{0}^{f_{h}} S_{y}(E)\left(\frac{\sin \pi f_{r}}{\pi f_{r}}\right)^{2} d f
$$

In practice it may be obeained from a set of measurements of the frequency of the oscillator as

$$
s^{2}=\frac{1}{N} \sum_{i=1}^{N}\left(y_{i}-\bar{y}\right)^{2} .
$$

The sample varlance diverges for some eypes of nolse and, therefore, is not generally useful.

Other variances based on the seructure funceion approach can also be defined (Lindsey and Chl, 1976). For example, these are the Hadamard variance, the threesample variance and the high pass variance (Rutzan 1978). They are occasionally used in researcin and sclentifle works for specific purposes, such as differentating between different types of roise and for dealing with systematics and sidebands in the spectrum.

\section{APPENDTE III}

\section{Blbliosraphy}

Allan. D. W., Statistics of atomic frequency siandards, $P=O C$. IEEE, Vol. 54,221-230, (Feb 1960).

Allan, D. W., et al., Perforaance, modeling, and simuLasion of some cesium bean clocks. Proc. $27 \mathrm{ch}$ Anrual Symosium on Frequency Control, 334-346. (1973).

Allan, D. W., The measurement of frequency and frequency stablltey of preclsion oscillacors. Proc. 6eh Annual Precise Ilme and Ifme Incerval Planning Mee=1ng. 109-142. (Dec 1974). 
Allen, D. Y., and Daens, H., Plcosecond elne difference eesurenent systen. Proc. of the $29 \mathrm{eh}$ Annusl Syuposiun on Frequency Concrol, 404-411, (May 1979).

Allan, D. H., and Hellvig, H. W., Tlae Deviaclon and IIne Prediction Error for Clock Specificarion, Characterlzation, and Applicafion, Proc. IEEz Position Locaclon and Navigation Symposium, 29-36, (1978).

Allan, D. H., and Barnes, J. A., A modifled "Allan Varlance" with Increased osclllator characterliza. tion abllity. Proc. 35th Annual Symposiun on Frequency Control, 470-476. (May 1981).

Allan, D. H., Time and Frequency (Ilae Domain) Characterlzation, Estination and Prediction of Precl. slon Clocks and Osclllators. IEEE Trans. UFFC Vol. 34. 647-654 (Nov 1987).

Ackinson, W. K., Fey, L., and Newran, J., Spectrun analysis of extremely low-frequency varlactons of quartz osclllators. Proc. IEEE, Vol. S1, 379-380, (Feb 1963).

Bablech, D., and Ollver10, J.. Phase nolse of varlous oscillators at very low Fourler frequencles. Proc. 28 eh Annual Symposium on Frequency Conerol, 150159. (1974).

Barnes, J. A., Models for the Interpretation of frequency stabllity measurements. NBS Tech. Note 683. (Aug 1976).

Barnes, J. A., Jones, R. H., Iryon, P. V., and Allan, D.W.. Nolse models for acomic clocks. Proc. 14th Annual Precise Time and Ilme Incerval Planning Meeting, 295-307, (Dec 1982).

Baugh, R. A., Frequency modulation analysis with the Hadamard varlance. Proc. 25eh Annual Symposiun on Frequency control, 222-225. (1971).

Bernler, L.C., IInear Prediction of the Non-Stationary Clock Error Funcelon, Proc. 2nd European Frequency and Iize Forun, Neuchatel (1988).

Blackman, R. B., and Iukey, J. M. , The measurement of power spectea, Dover Publicalion. Inc.. New York, NY, (1959).

B1a1r, B. E., IIme and frequency: Theory and fundamentals. NBS Monograph No. 140, US Govermente Prineing office, Washingeon, DC 20402, (May 1974).

Bolleau, E., and Plcinbono, B.. Statistical seudy of phese fluctuetions and oscillator ssabllley. IEEE Trans. Instr. and Meas., Vol. 25, 66-75, (Mar 1976).

Brandenbe:ge:, H., Hadorn, F., Halford, D., and Shoaf, 3. H., High qualley quarez cryseal osclllacors: frequency-domaln and elme-domain seabllicy. Proc. 25 th Annual Symposium on Frequency Control, 226. 230. (1971).

Ch1. A. R., The mechanles of eranslarlon of frequency stab1llisy measures between frequency and time. domain measurements. Proc. 9th Annual Precise Time and I'ze Interval Planning Meeting. (Dec 1977).

Cueler, L. S., and Searle, C. L., Sore aspects of the cheory and measurement of frequency fluctuations in frequency standards, Proc. IEEE, Vo1. \$4, 136-154, (Feb 1966).
De Prins. J., and Comelissen, G., Anelyse specrale discrece. Eurocon (Lausanne, Swlezerland), (Oct 1971).

De Prins, J., Descorner, G., Gorsk1, M., and Tanine, J., Frequency-doealn Interprecation of osclllacor phase stabllicy. IEEE Irans. Inser. and Meas., Vol. IM18, 251-261, (Dec 1969).

Flscher, M. C., Frequency seablliey measurement procedures. Proc. 8th Annual Preclse IIne and Ilae Interval Plannlng Meeting. 575-618, (Dec 1976).

Greenhall, C.A., Inlelalizing a Fllcker Nolse Generator, IEEE Trans. Inser, and Meas., Vol. MM-35, 222-224 (June 1986).

Groslambert, J., Oliver, M., and Uebersfeld, J., Spectral and short-term stab111ty measurements. IEEE Irans. Inser. and Meas., Vol. IM-23, 518-521. (Dec 1974).

Halford, D.. A general mechanlcal model for $\mid$ f densley nolse with special reference to flicker nolse $1 /|f|$. Proc. I IEE Vol. 56, 251-258, (Mar 1968).

IEEE, Spectal issue on tine and frequency. Proc. IEEE, Vol. 60. (May 1972).

IEEE-NASA, Proceedings of Symposlum on shore-retm frequency scablllcy. NASA Eublicaclon SP 80. (1964).

Jones, R. H., and Tryon, P. V., Estimating elme from acomlc clocks. NBS Journal of Research, Vol. 88 . November 17-24, (Jan-Feb 1983).

Kartaschoff, P., Computer Simulation of the Conventional Clock Mode1, IEEE Irans. Inser, and Meas., Vol. IM28. 193-197 (Sepe 1979).

Kroupa, V. F., Frequency scabll1cy: Fundamentals and Measurement. IEEE Press. IEEE Selected Reprine Serles. Prepared under the sponsorship of the IEEE Inserumentacion and Measurement Soclecy, 77-80, (1984).

Lesage P., and Audoln, C., Correction to: Character1zation of frequency stabllity: Uncertalney due to flnite number of measurexents. IEEE Trans. Instr. and Meas., Vol. IK-23, 103, (Mar 1974).

Lesage, P., and Audoln, F., A eime domaln method for measuremene of the spectral densiey of frequency Eluctuations at low Fourler frequencles. Proc. 29 th Annual Symposlue on Frequency Concrol, 394403. (May 1975).

Lesage, P., and Audoln, C., Corzection to: Character1zacion of frequency seabllity: Uncersalney due to the flalte number of measurements. IEEE Trans. Inser. and Meas., Vol. IY-25, 270, (Sepe 1976).

Lesage, P., and Ay1, T., Characterlzarion of frequency seabliley: Analysis of modifled Allan variance and propereles of les estigate. IEEE Trans. on Instr. and Meas., Vol. IM-33, 332-336, (Dec 1934).

IIndsey, W. C., and Chle, C. M. , Theory of oscillator Insiabllicy based upon siructure funcelons. Proc. IEES, Vol. 64, 1662-1666, (Dec 1976).

Mandelbrot, B., Some nolses with $1 /$ f specrrum; a bridge becween direce currene and white nolse. IEEE Irans. Inf. Theory, Vol. IT-13. 289-298, (ApF 1967). 
Meyer. D. C., A test set for the accurate measurements of phase nolse on hlgh-qualley 3 gnal sources. IEEE Trans. Inser. and Meas.. Vol. IM-19, 215-227. (Nov 1970).

Percival, D. B.. A heuristic model of long-tere atomlc clock behavior. Proc. 30th Annual Syroposiun on Erequency Control. (June 1976).

Ricc1, D. H., and Peregrino, L., Phase nolse weasureaent using a high resolution counter with on-ilne data processing. Proc. 30th Annual Symposium on Erequency Conerol. (June 1976).

Rutman, J., Coment on characterlzation of frequency stablilty. IEEE Trans. Inser. and Meas.. Vol. IM21. 85. (Feb 1972).

Rutman, J., Characterization of frequency stabillty: A eransfer function approsch and les appllcarlon to measurements via filtering of phase noise. IEEE Irans. Instr. and Meas., Vol. IX-23, 40-48, (Mar 1974).

Rueman, J., Characterlzarion of phase and frequency Instablities in precision frequency sources: Fifteen years of progress. Proc. IEEE, Vo1. 66. 1048-1075. (Sepe 1978).

Rutman, J., and Sauvage, G., Measuremene of frequency stability in the eime and frequency domains via filtering of phase nolse. IEEE Trans. Instr. and Meas., Vo1. IY -23, 515-518, (Dec 1974).

Rurman, J., and Uebersfeld, J., A model for flicker frequency nolse of osclilacors. Proc. IEEE, Vol. 60. 233-235, (Feb 1972).

Sauvage, G., and Rueman, J., Analyse spectrale du brule de frequence des oscillateurs par la variance de Hadamard. Ann. des Telecom., Vol. 28, 304-314, (July-Aug 1973).

Vanter, J., and Teru, M., Time domain measuremene of frequency stabllity: A cutorial approach, Proc. loth Arnual Precise Time and Time Interval Planning Meeting, 247-291, (1978).

Vessor, R., Frequency and Iime Seandards. Mechods of Experimental Physics, Acaderlc Press, Ner York, 198-227, (1976).

Vessor, R., Mueller, L., and Vanier, J., The speciflcarion of oscillator characteristics from measurements made in the frequency domain. Proc. IEEE, Vo1. 54, 199-207 (Feb 1966).

Von Neumann, J., Kent, R. H., Bellinson, H. R., and Ha:c, B. I. The mean square successive difference, Arn. Math. Stat., Vol. 12, 153-162, (1941).

Walls, F.L., and Allan, D.H., Measurements of Erequency Stability, Proc. IEEE, Special Issue on Radio Measurements and Standards, Vol. 74, No. 1, pp. 162168 ( $\mathrm{J}$ an 1986).

Walls, F. L., Seein, S. R., Gray, J. E., and Glaze, D. J., Design considerations in scace-of-the-art sigmal processing and phase nolse measurement systems. Proc. 30ch Annual Symposium on Erequency Conerol, (June 1976).

Winkler, G. M. R., A brief reviow of frequency stabllley neasures. Proc. $8 \mathrm{eh}$ Annual Precise $\mathrm{I}$ ime and Time Incerval Planning Meeting, 489-528, U.S. Naval Research Laboratory, HashIngton, D.C., (Dec 1976).
Hinkler, G. M. R., Hall, R. G., and Percival, D. B., The U.S. Naval Observatory elock that reference and the performance of a sapple of atonle clocks. Metro$\log 12$, Vol. 6. 126-134, (Oet 1970).

Yoshiqura, K., Characterizarion of frequency stabll1ty: Uncertainty due to the autocorrelation of the frequency fluctuations. IRER Irans. Instr. and Meas., IH-27. 1-7. (Mar 1978).

The authors are nexbers of the Technleal Comlttee TC-3, TIae and Frequency, of the IEEE Instrumentation and Measurement Soclety. This paper is part of this Comaltree's effort to develop an IEEE standard. To this end, the IEEE authorlzed a Standerds Coordinaring Counitee, SCC 21, and the developane of the IEEE standard under PAR-P-1139. SCC 21 is formed out of the IEM, UFTC and ITT Socleties: the authors are also members of SCC 21. 


\section{Characterization of Frequency Stability}

JAMES A. BARNES, SENIOR MEMBER, IEEE, ANDREW R. CHI, SENIOR MEMBER, IEEE, LEONARD S. CUTLER, MEMBER, IEEE, DANIEL J. HEALEY, MEMBER, IEEE, DAVID B. LEESON, SENIOR MEMBER, IEEE, THOMAS E. MCGUNIGAL, MEMBER, IEEE, JAMES A. MULLEN, JR., SENIOR MEMBER, IEEE, WARREN L. SMITH, SENIOR MEMBER, IEEE, RICHARD L. SYDNOR, MEMBER, IEEE, ROBERT F. C. VESSOT, AND GERNOT M. R. WINKLER, MEMBER, IEEE

Abstract-Consider a signal generator whose instantaneous output voltage $V(t)$ may be written as

$$
V(t)=\left[V_{0}+\epsilon(t)\right] \sin \left[2 \pi \nu_{0} t+\varphi(t)\right]
$$

where $V_{0}$ and $y_{0}$ are the nominal amplitude and frequency, respectively, of the output. Provided that $e(t)$ and $\dot{\varphi}(l)=(d \varphi /(d t)$ are sufficiently small for all time $t$, one may define the fractional instantaneous frequency deviation from nominal by the relation

$$
y(t) \equiv \frac{\dot{\varphi}(t)}{2 \pi \nu_{0}}
$$

A proposed definition for the measure of frequency stability is the spectral density $S_{y}(f)$ of the function $y(t)$ where the spectrum is considered to be one sided on a per hertz basis.

An alternative definition for the measure of stability is the infinite time average of the sample variance of two adjacent averages of $y(t)$; that is, if

$$
\bar{y}_{k}=\frac{1}{\tau} \int_{1,}^{t+1} y(t) d t
$$

where $r$ is the averaging period, $t_{4+1}=t_{k}+T, k=0,1,2 \cdots, t_{0}$ is arbitrary, and $T$ is the time interval between the beginnings of two successive measurements of arerage frequency; then the second measure of stability is

$$
\sigma_{v}^{2}(\tau) \equiv\left\langle\frac{\left(\bar{y}_{k+1}-\bar{y}_{k}\right)^{2}}{2}\right\rangle
$$

where $\langle$ > denotes infinite time average and where $T=T$.

In practice, data records are of finite length and the infinite

Manuscript received December 1, 1970. The authors of this paper are members of the Subcommittee on Frequency Stability of the Technical Committee on Frequency and Time of the IEEE Group on Instrumentation and Measurement.

J. A. Barnes is with the Time and Frequency Division, Institute for Basic Standards, NBS, Boulder, Colo. 80302.

A. R. Chi is with the Head Timing Systems Section, NASA Goddard Space Flight Center, Greenbelt, Md. 20771.

L. S. Cutler is with the Physical Research Laboratory, HewlettPackard Company, Palo Alto, Calif. 94304.

D. J. Healey is with the Aerospace Division, Westinghouse Electric Corporation, Baltimore, Md. 21203.

D. B. Leeson is with California Microwave, Inc., Sunnyvale, Calif. 94086.

T. E. McGunigal is with the RF and Quantum Techoiques Section, NASA Goddard Space Flight Center, Greenbelt, Md. 21715.

J. A. Mullen is with the Research Division, Raytheon Company, Waltham, Maes. 02154.

W. L. Smith is with Bell Telephone Laboratories, Allentown, Pa. 18108 .

R. L. Sydnor is with the Telecommunications Department, Jet Propulsion Laboratory, Pasadena, Calif. 91103.

G. M. R. Winkler is with the Time Service Division, U. S. Naval Observatory, Washington, D. C. 20390

R. F. C. Vessot is with the Smitheonian Astrophysical Ob. servatory, Cambridge, Mass. 02138.

time averages implied in the definitions are normally not available; thus estimates for the two measures must be used. Entimates of $S_{y}(f)$ would be obtained from suitable averages either in the time domain or the frequency domain. An obrious estimate for $\sigma_{y}^{2}(\tau)$ is

$$
\sigma_{y}^{2}(\tau) \approx \frac{1}{m} \sum_{k=1}^{m} \frac{\left(\bar{y}_{k+1}-\bar{y}_{k}\right)^{2}}{2} .
$$

Parameters of the measuring system and estimating procedure are of critical importance in the specification of frequency stability. In practice, one should experimentally establish confidence limits for an estimate of frequency stability by repeated trials.

\section{Glossary of Srmbols}

$$
\begin{gathered}
B_{1}(N, r, \mu), \\
B_{2}(r, \mu)
\end{gathered}
$$

$C_{a}$

$c_{0}, c_{1}$

$c(t)$

$D_{x}^{2}(\tau)$

$f \equiv \omega / 2 \pi$

f.

$f$

$g(t)$

$h_{\mathrm{s}}$

$i, j, k, m, n$

$M$

$N$

$n(t)$

$R_{\boldsymbol{v}}(\tau)$

$r$
Bias function for variances based on finite samples of a process with a power-law spectral density. (See [13].)

A real constant defined by (70).

Real constants.

A real, deterministic function of time.

Expected value of the squared second difference of $x(t)$ with lag time $\tau$. See (80).

Fourier frequency variable.

High-frequency cutoff of an idealized infinitely sharp cutoff low-pass filter.

Low-frequency cutoff of an idealized infinitely sharp cutoff, highpass filter.

A real function of time.

Positive real coefficient of $f^{*}$ in a power series expansion of the spectral density of the function $y(t)$.

Integers, often a dummy index of summation.

Positive integer giving the number of cycles averaged.

Positive integer giving the number of data points used in obtaining a sample variance.

A nondeterministic function of time. Autocovariance function of $y(t)$. See (58).

Positive real number defined by $r \equiv T / \tau$. 
An intermediate term used in deriving (23). The definition of $S$ is given by (64).

One-sided (power) spectral density on a per hertz basis of the pure real function $g(t)$. The dimensions of $S_{v}(f)$ are the dimensions of $g^{2}(t) / f$. A definition for the measure of frequency stability. One-sided (power) spectral density of $y(t)$ on a per hertz basis. The dimensions of $S_{y}(f)$ are $\mathrm{Hz}_{z}^{-1}$.

Time interval between the beginnings of two successive measurements of average frequency.

Time variable.

An arbitrary fixed instant of time. The time coordinate of the beginning of the $k$ th messurement of average frequency. By definition, $t_{\mathrm{b}+1}=t_{\mathrm{b}}+T, k=0,1,2 \cdots$.

Dummy variable of integration; $u \equiv \pi f r$.

Instantaneous output voltage of signal generator. See (2).

Nominal peak amplitude of signal generator output. See (2).

Instantaneous voltage of reference sigral. Seo (40).

Peak amplitude of reference signal. See (40).

Voltage output of ideal product detector.

Low-pass filtered output of product detector.

Real function of time related to the phase of the sigaal $V(t)$ by $x(t) \equiv$ $[\varphi(t)] /\left(2 \pi v_{0}\right)$.

\section{A predicted value for $x(t)$}

Fractional frequency offset of $V(t)$ from the nominal frequency. See (7). Average fractional frequency offset during the kth measurement interval. See (9).

The sample average of $N$ successive values of $y_{k}$. See (76).

Nondeterministic (noise) function with (power) spectral density given by (25).

Exponent of $f$ for a power-law spectral density.

Positive real constant.

The Kronecker $\delta$ function defined by $\delta_{\Delta}(r-1) \equiv \begin{cases}1, & \text { if } r=1 \\ 0, & \text { otherwise. }\end{cases}$

Amplitude fluctuations of signal. See (2). $\mu$

$\nu(t)$

$\nu_{0}$

$\kappa(t)$

$\sigma_{v}^{2}(N, T, \tau)$

$\left\langle\sigma_{v}^{2}(N, T, \tau)\right\rangle$

$\sigma_{y}^{2}(\tau)$

$\sigma_{x}^{2}(\tau)$

$\tau$

$\Phi(t)$

$\varphi(t)$

$\psi_{s}^{2}(T, \tau)$

$\omega \equiv 2 \pi f$
Exponent of $\tau$. See (29).

Instantaneous frequency of $V(t)$. Defined by

$$
\nu(t) \equiv \frac{1}{2 \pi} \frac{d}{d t} \Phi(t) .
$$

Nominal (constant) frequency of $V(t)$.

The Fourier transform of $n(t)$.

Sample variance of $N$ averages of $y(t)$, each of duration $\tau$, and spaced every $T$ units of time. See (10).

Average value of the sample variance $\sigma_{y}^{2}(N, T, \tau)$.

A second choice of the definition for the measure of frequency stability. Defined by $\sigma_{v}^{2}(\tau) \equiv\left\langle\sigma_{v}^{2}(N=2\right.$, $T=\tau, \tau)\rangle$.

Time stability measure defined by $\sigma_{x}^{2}(\tau) \equiv \tau^{2} \sigma_{y}^{2}(\tau)$.

Duration of averaging period of $y(t)$ to obtain $\bar{y}_{k}$. See (9).

Instantaneous phase of $V(t)$. Defined by $\Phi(t) \equiv 2 \pi \nu_{0} t+\varphi(t)$.

Instantaneous phase fluctuations about the ideal phase $2 \pi \nu_{0} t$. See (2). Mean-square time error for Doppler radar. See (82).

Angular Fourier frequency variable.

\section{INTRODUCTION}

$\prod$

$\mathrm{HE}$ measurement of frequency and fluctuations in frequency has received such great attention for so many years that it is surprising that the concept of frequency stability does not have a universally accepted definition. At least part of the reason has been that some uses are most readily described in the frequency domain and other uses in the time domain, as well as in combinations of the two. This situation is further complicated by the fact that only recently have noise models been presented that both adequately describe performance and allow a translation between the time and frequency domains. Indeed, only recently has it been recognized that there can be a wide discrepancy between commonly used time domain measures themselves. Following the NASA-IEEE Symposium on ShortTerm Stability in 1964 and the Special Issue on Frequency Stability in the Procesdings of THE IEEE, February 1966, it now seems reasonable to propose a definition of frequency stability. The present paper is presented as technical background for an eventual IEEE standard definition.

This paper attempts to present (as concisely as practical) adequate, self-consistent definitions of frequency stability. Since more than one definition of frequency stability is presented, an important part of this paper 
(perhaps the most important part) deals with translations among the suggested definitions of frequency stability. The applicability of these definitions to the more common noise models is demonstrated.

Consistent with an attempt to be concise, the references cited have been selected on the basis of being of most value to the reader rather than on the basis of being exhaustive. An exhaustive reference list covering the subject of frequency stability would itself be a voluminous publication.

Almost any signal generator is influenced to some extent by its environment. Thus observed frequency instabilities may be traced, for example, to changes in ambient temperature, supply voltages, magnetic field, barometric pressure, humidity, physical vibration, or even output loading, to mention the more obvious. While these environmental influences may be extremely important for many applications, the definition of frequency stability presented here is independent of these causal factors. In effect, we cannot hope to present an exhaustive list of environmental factors and a prescription for handling each even though, in some cases, these environmental factors may be by far the most important. Given a particular signal generator in a particular environment, one can obtain its frequency stability with the measures presented below, but one should not then expect an accurate prediction of frequency stability in a new environment.

It is natural to expect any definition of stability to involve various statistical considerations auch as stationarity, ergodicity, average, variance, spectral density, etc. There often exist fundamental difficulties in rigorous attempts to bring these concepts into the laboratory. It is worth considering, specifically, the concept of stationarity since it is a concept at the root of many statistical discussions.

A random process is matbematically defined as stationary if every translation of the time coordinate maps the ensemble onto itself. As a necessary condition, if one looks at the ensemble at one instant of time $t$, the distribution in values within the ensemble is exactly the same 8 s at any other instant of time $t^{\prime}$. This is not to imply that the elements of the ensemble are constant in time, but, as one element changes value with time, other elements of the ensemble assume the previous values. Looking at it in snother way, by observing the ensemble at some instant of time, one can deduce no information as to when the particular instant was chosen. This same sort of invariance of the joint distribution holds for any set of times $t_{1}, t_{2}, \cdots, t_{n}$ and its translation $t_{1}+\tau, t_{2}+\tau, \cdots, t_{n}+\tau$.

It is apparent that any ensemble that has a finite past as well as a finite future cannot be stationary, and this neatly excludes the real world and anything of practical interest. The concept of stationarity does violence to concepts of causality since we implicitly feel that current performance (i.e., the applicability of sta- tionary statistics) cannot be logically dependent upon future events (i.e., if the process is terminated some time in the distant future). Also, the verification of stationarity would involve hypothetical measurements that are not experimentally feasible, and therefore the concept of stationarity is not directly relevant to experimentation.

Actually the utility of statistics is in the formation of idealized models that reasonably describe significant observables of real systems. One may, for example, consider a hypotbetical ensemble of noises with certain properties (such as stationarity) as a model for a particular real device. If a model is to be acceptsble, it should have at least two properties: first, the model should be tractable; that is, one should be able to easily arrive at estimates for the elements of the models; and second, the model should be consistent with observables derived from the real device that it is simulating.

Notice that one does not need to know that the device was selected from a stationary ensemble, but only that the observables derived from the device are consistent with, say, elements of a hypotbetically stationary ensemble. Notice also that the actual model used may depend upon how clever the experimenter-theorist is in generating models.

It is worth noting, however, that while some texts on statistics give "tests for stationarity," these tests are almost always inadequate. Typically, these tests determine only if there is a substantial fraction of the noise power in Fourier frequencies whose periods are of the same order as the dats length or longer. While this may be very important, it is not logically essential to the concept of stationarity. If a nonstationary model actually becomes common, it will almost surely be because it is useful or convenient and not because the process is "actually nonstationary." Indeed, the phrase "actually nonstationary" appears to bave no meaning in an operational sense. In short, stationarity (or nonstationarity) is a property of models, not a property of data [1].

Fortunately, many statistical models exist that adequately describe most present-day signal generators; many of these models are considered below. It is obvious that one cannot guarantee that all signal generators are adequately described by these models, but the authors do feel they are adequate for the description of most signal generators presently encountered.

\section{Statement of the Problem}

To be useful, a measure of frequency stability must allow one to predict performance of signal generators used in a wide variety of situations as well as allow one to make meaningful relative comparisons among signal generators. One must be able to predict performance in devices that may most easily be described either in the time domain, or in the frequency domain, or in a combination of the two. This prediction of performance may involve actual distribution functions, and thus 
second moment measures (such as power spectra and variances) are not totally adequate.

Two common types of equipment used to evaluate the performance of a frequency source are (analog) spectrum analyzers (frequency domain) and digital electronic counters (time domain). On occasion the digital counter data are converted to power spectra by computers. One must realize that any piece of equipment simultaneously has certain aspects most easily described in the time domain and other aspects most easily described in the frequency domain. For example, an electronic counter has a high-frequency limitation, an experimental spectra are determined with finite time averages.

Research has established that ordinary oscillators demonstrate noise, which appears to be a superposition of causally generated signals and random nondeterministic noises. The random noises include thermal noise, shot noise, noises of undetermined origin (such as flicker noise), and integrals of these noises.

One might well expect that for the more general cases one would need to use a nonstationary model (not stationary even in the wide sense, i.e., the covariance sense). Nonstationarity would, however, introduce significant dif ficulties in the passage between the frequency and time domains. It is interesting to note that, so far, experimenters have seldom found a nonstationary (covariance) model useful in describing actual oscillators.

In what follows, an attempt has been made to separate general statements that hold for any noise or perturbation from the statements that apply only to specific models. It is important that these distinctions be kept in mind.

\section{Background and Definitions}

To discuss the concept of frequency stability immediately implies that frequency can change with time and thus one is not considering Fourier frequencies (at least at this point). The conventional definition of instantaneous (angular) frequency is the time rate of change of phase; that is

$$
2 \pi \nu(t) \equiv \frac{d \Phi(t)}{d l} \equiv \Phi(t)
$$

where $\Phi(t)$ is the instantaneous phase of the oscillator. This paper uses the convention that time-dependent frequencies of oscillators are denoted by $\nu(t)$ (cycle frequency, hertz), and Fourier frequencies are denoted by $\omega$ (angular frequency) or $f$ (cycle frequency, hertz) where $\omega \equiv 2 \pi f$. In order for (1) to have meaning, the phase $\Phi(t)$ must be a well-defined function. This restriction immediately eliminates some "nonsinusoidal" signals such as a pure random uncorrelated ("white") noise. For most real signal generators, the concept of phase is reasonably amenable to an operational definition and this restriction is not serious.

Of great importance to this paper is the concept of spectral density, $S_{\imath}(f)$. The notation $S_{v}(f)$ is to repre- sent the one-sided spectral density of the (pure real, function $g(t)$ on a per hertz basis; that is, the total "power" or mean-square value of $g(t)$ is given by

$$
\int_{0}^{\infty} S_{\theta}(f) d f
$$

Since the spectral density is such an important concept to what follows, it is worthwhile to present some important references on spectrum estimation. There are many references on the estimation of spectra from data records, but worthy of special note are [2]-[5].

\section{Definition of Measures of Frequency Stability (SECOND-Moment TYPe)}

\section{A. General}

Consider a signal generator whose instantaneous output voltage $V(t)$ may be written as

$$
V(t)=\left[V_{0}+\epsilon(t)\right] \sin \left[2 \pi \nu_{0} t+\varphi(t)\right]
$$

where $V_{0}$ and $\nu_{0}$ are the nominal amplitude and frequency, respectively, of the output and it is assumed that

$$
\left|\frac{\epsilon(t)}{V_{0}}\right| \ll 1
$$

and

$$
\left|\frac{\dot{\varphi}(t)}{2 \pi \nu_{0}}\right| \ll 1
$$

for substantially all time $t$. Making use of (1) and (2) one sees that

$$
\Phi(t)=2 \pi \nu_{0} l+\varphi(t)
$$

and

$$
\nu(t)=\nu_{0}+\frac{1}{2 \pi} \dot{\varphi}(t)
$$

Equations (3) and (4) are essential in order that $\psi(t)$ may be defined conveniently and unambiguously (sec measurement section).

Since (4) must be ralid even to speak of an instantaneous frequency, there is no real need to distinguish stability measures from instability measures. That is. any fractional frequency stability measure will be far from unity, and the chance of confusion is slight. It is true that in a very strict sense people usually measure instability and speak of stability. Bccause the chances of confusion are so slight, the authors have chosen to continue in the custom of measuring "instability" and speaking of stability (a number always much less than unity).

Of significant interest to many people is the radio frequency (RF) spectral density $S_{V}(f)$. This is of direct concern in spectroscopy and radar. However, this is not a good primary measure of frequency stability for two reasons. First, fluctuations in the amplitude $\epsilon(t)$ contribute directly to $S_{v}(f)$; and second, for many cases when 
$\epsilon(t)$ is insignificant, the RF spectrum $S_{\nabla}(f)$ is not uniquely related to the frequency fluctuations [6].

\section{B. General:First Definition of the Measure of Frequency Stability-Frequency Domain}

By definition, let

$$
y(t) \equiv \frac{\dot{\varphi}(t)}{2 \pi \nu_{0}}
$$

where $\varphi(t)$ and $v_{0}$ are as in (2). Thus $y(t)$ is the instantaneous fractional frequency deviation from the nominal frequency $\nu_{0}$. A proposed definition of frequency stability is the spectral density $S_{y}(f)$ of the instantaneous fractional frequency fluctuations $y(t)$. The function $S_{y}(f)$ has the dimensions of $\mathrm{Hz}^{-1}$.

One can show [7] that if $S_{\phi}(f)$ is the spectral density of the phase fluctuations, then

$$
\begin{aligned}
S_{y}(f) & =\left(\frac{1}{2 \pi \nu_{0}}\right)^{2} S_{:}(f) \\
& =\left(\frac{1}{\nu_{0}}\right)^{2} f^{2} S_{0}(f) .
\end{aligned}
$$

Thus a knowledge of the spectral density of the phase fluctuations $S_{\varphi}(f)$ allows a knowledge of the spectral density of the frequency fluctuations $S_{y}(f)$, the first definition of frequency stability. Of course, $S_{y}(f)$ cannot be perfectly measured-this is the case for any physical quantity; useful estimates of $S_{y}(f)$ are, however, easily obtainable.

\section{General: Second Definition of the Measure of Fre- quency Stability-Time Domain}

The second definition is based on the sample variance of the fractional frequency fluctuations. In order to present this measure of frequency stability, define $\bar{y}_{k}$ by the relation

$$
\bar{y}_{k} \equiv \frac{1}{\tau} \int_{10}^{t_{1}+r} y(t) d t=\frac{\varphi\left(t_{k}+\tau\right)-\varphi\left(t_{k}\right)}{2 \pi \nu_{0} \tau}
$$

where $t_{b+1}=t_{k}+T, k=0,1,2, \cdots, T$ is the repetition interval for measurements of duration $r$, and $l_{0}$ is arbitrary. Conventional frequency counters measure the number of cycles in a period $r$; that is, they messure $\nu_{0} r\left(1+\bar{y}_{k}\right)$. When $r$ is $1 \mathrm{~s}$ they count the number of $\nu_{0}\left(1+\bar{y}_{k}\right)$. The second measure of frequency stability, then, is defined in analogy to the sample variance by the relation

$$
\left\langle\sigma_{v}^{2}(N, T, \tau)\right\rangle \equiv\left\langle\frac{1}{N-1} \sum_{n=1}^{N}\left(\bar{y}_{n}-\frac{1}{N} \sum_{k=1}^{N} \bar{y}_{k}\right)^{2}\right\rangle
$$

where $\langle g\rangle$ denotes the infinite time average of $g$. This measure of frequency stability is dimensionless.

In many situations it would be wrong to assume that (10) converges to a meaningful limit as $N \rightarrow \infty$. First, of course, one cannot practically let $N$ approach infinity and, second, it is known that some actual noise processes contain substantial fractions of the total noise power in the Fourier frequency range below one cycle per year. In order to improve comparability of data, it is important to specify particular $N$ and $T$. For the preferred definition we recommend choosing $N=2$ and $T=\tau$ (i.e., no dead time between measurements). Writing $\left\langle\sigma_{v}^{2}(N=2, T=\tau, \tau)\right\rangle$ as $\sigma_{v}^{2}(\tau)$, the Allan variance [8], the proposed measure of frequency stability in the time domain may be written as

$$
\sigma_{y}^{2}(\tau)=\left\langle\frac{\left(\bar{y}_{k+1}-\bar{y}_{k}\right)^{2}}{2}\right\rangle
$$

for $T=\tau$.

Of course, the experimental estimate of $\sigma_{y}^{2}(\tau)$ must be obtained from finite samples of data, and one can never obtain perfect confidence in the estimate; the true time average is not realizable in a real situation. One estimates $\sigma_{y}^{2}(\tau)$ from a finite number (say, $m$ ) of values of $\sigma_{y}^{2}(2, \tau, \tau)$ and averages to obtain an estimste of $\sigma_{v}^{2}(\tau)$. Appendix I shows that the ensemble average of $\sigma_{y}^{2}(2, \tau, \tau)$ is convergent (i.e., as $m \rightarrow \infty$ ) even for noise processes that do not have convergent $\left\langle\sigma_{y}^{2}(N, \tau, \tau)\right\rangle$ as $N \rightarrow \infty$. Therefore, $\sigma_{y}^{2}(\tau)$ has greater utility as an idealization than does $\left\langle\sigma_{v}^{2}(\infty, \tau, \tau)\right\rangle$ even though both involve assumptions of infinite averages. In effect, increasing $N$ causes $\sigma_{y}^{2}(N, T, \tau)$ to become more sensitive to the low-frequency components of $S_{y}(f)$. In practice, one must distinguish between an experimental estimate of a quantity (s\&y, of $\sigma_{y}^{2}(\tau)$ ) and its idealized value. It is reasonable to believe that extensions to the concept of statistical ("quality") control [9] may prove useful here. One should, of course, specify the actual number $m$ of independent samples used for an estimate of $\sigma_{y}^{2}(r)$.

In summary, therefore, $S,(f)$ is the proposed measure of (instantaneous) frequency stability in the (Fourier) frequency domain and $\sigma_{y}^{2}(\tau)$ is the proposed measure of frequency stability in the time domain.

\section{Distributions}

It is natural that people first become involved with second moment measures of statistical quantities and only later with actual distributions. This is certainly true with frequency stability. While one can specify the argument of a distribution function to be, say $\left(\bar{y}_{k+1}-\bar{y}_{k}\right)$, it makes sense to postpone such a specfication until a real use has materialized for a particular distribution function. This paper does not attempt to specify a preferred distribution function for frequency fluctuations.

\section{E. Treatment of Systematic Variations}

1) General: The definition of frequency stability $\sigma_{y}^{2}(r)$ in the time domain is useful for many situations. However, some oscillators, for example, exhibit an aging or almost linear drift of frequency with time. For some applications, this trend may be calculated and should be removed [8] before estimating $\sigma_{v}^{2}(\tau)$.

In general, a systematic trend is perfectly deterministic (i.e., predictable) while the noise is nondeterministic. Consider a function $g(t)$, which may be written in the form 


$$
g(t)=c(t)+n(t)
$$

where $c(t)$ is some deterministic function of time and $n(t)$, the noise, is a nondeterministic function of time. We will define $c(t)$ to be the systematic trend to the function $g(t)$. A problem of significance bere is to determine when and in what sense $c(t)$ is measurable.

2) Specific Case-Linear Drift: As an example, if we consider a typical quartz crystal oscillator whose fractional frequency deviation is $y(t)$, we may let

$$
g(t)=\frac{d}{d t} y(t)
$$

With these conditions, $c(t)$ is the drift rate of the oscillator (e.g., $10^{-10} /$ day) and $n(t)$ is related to the frequency "noise" of the oscillator by a time derivative. One sees that the time average of $g(t)$ becomes

$$
\frac{1}{T} \int_{1.0}^{t_{0}+T} g(t) d t=c_{1}+\frac{1}{T} \int_{t_{0}}^{t_{0}+T} n(t) d t
$$

where $c(t)=c_{1}$ is assumed to be the constant drift rate of the oscillator. In order for $c_{1}$ to be an observable, it is natural to expect the average of the noise term to vanish, that is, converge to zero.

It is instructive to assume [8], [10] that in addition to a linear drift, the oscillator is perturbed by a flicker noise, i.e.,

$$
S_{y}(f)= \begin{cases}h_{-1} f^{-1}, & 0<f \leq f_{A} \\ 0, & f>f_{A}\end{cases}
$$

where $h_{-1}$ is a constant (see Section V-A-2) and thus,

$$
S_{n}(f)= \begin{cases}(2 \pi)^{2} h_{-1} f, & 0 \leq f \leq f_{A} \\ 0, & f>f_{h}\end{cases}
$$

for the oscillator we are considering. With these assumptions, it is seen that

$$
\lim _{T \rightarrow \infty} \frac{1}{T} \int_{t_{0}}^{t_{0}+r} n(t) d t=\kappa(0)=0
$$

and that

$$
\lim _{T \rightarrow \infty}\left\{\text { variance }\left[\frac{1}{T} \int_{t_{t}}^{t_{t}+T} n(t) d t\right]\right\}=0
$$

where $\alpha(f)$ is the fourier transform of $n(t)$. Since $S_{n}(0)$ $=0, \kappa(0)$ must also vanish both in probability and in mean square. Thus, not only does $n(t)$ average to zero, but one may obtain arbitrarily good confidence on the result by longer averages.

Having shown that one can reliably estimate the drift rate $c_{1}$ of this (common) oscillator, it is instructive to attempt to fit a straight line to the frequency aging. That is, let

$$
g(t)=y(t)
$$

and thus

$$
g(t)=c_{0}+c_{1}\left(t-t_{0}\right)+n^{\prime}(t)
$$

where $c_{0}$ is the frequency intercept at $t=t_{0}$ and $c_{1}$ is the drift rate previously determined. A problem arises bere because

$$
S_{*} \cdot(f)=S_{v}(f)
$$

and

$$
\lim _{T \rightarrow \infty}\left\{\text { variance }\left[\frac{1}{T} \int_{t^{t}}^{t_{t}+T} n^{\prime}(t) d t\right]\right\}=\infty
$$

for the noise model we have assumed. This follows from the fact that the (infinite $N$ ) variance of a flicker noise process is infinite [7], [8], [10]. Thus, co cannot be measured with any realistic precision, at least, in an absolute sense.

We may interpret these results as follows. After experimenting with the oscillator for a period of time one can fit an empirical equation to $y(t)$ of the form

$$
y(t)=c_{0}+t c_{1}+n^{\prime}(t)
$$

where $n^{\prime}(t)$ is nondeterministic. At some later time it is possible to reevaluate the coefficients $c_{0}$ and $c_{1}$. According to what has been said, the drift rate $c_{1}$ should be reproducible to within the confidence estimates of the experiment regardless of when it is reevaluated. For $c_{0}$, however, this is not true. In fact, the more one attempts to evaluate $c_{0}$, the larger the fluctuations are in the result.

Depending on the spectral density of the noise term, it may be possible to predict future measurements of $c_{0}$ and to place realistic confidence limits on the prediction [11]. For the case considered here, however, these confidence limits tend to infinity when the prediction interval is increased. Thus, in a certain sense, $c_{0}$ is "measurable" but it is not in statistical control (to use the language of the quality control engineer [9]).

\section{Translations Among Frequency Stability Measures}

\section{A. Frequency Domain to Time Domain}

1) General: It is of value to define $r=T / \tau$; that is, $r$ is the ratio of the time interval between successive measurements to the duration of the averaging period. Cutler has shown (see Appendix I) that

$$
\begin{aligned}
& \left\langle\sigma_{v}^{2}(N, T, \tau)\right\rangle \\
& \quad=\frac{N}{(N-1)} \int_{0}^{\infty} d f S_{\nu}(f) \frac{\left[\sin ^{2}(\pi f \tau)\right]}{(\pi f \tau)^{2}}\left\{1-\frac{\sin ^{2}(\pi r f N \tau)}{N^{2} \sin ^{2}(\pi \tau f \tau)}\right\} .
\end{aligned}
$$

Equation (23) in principle allows one to calculate the time-domain stability $\left\langle\sigma_{y}^{2}(N, T, \tau)\right\rangle$ from the frequencydomain stability $S_{y}(f)$.

2) Specific Model: A model that has been found useful [8], [10]-[13] consists of a set of five independent noise processes $z_{n}(t), n=-2,-1,0,1,2$, such that

* See Appendix Note \#19 


$$
y(t)=z_{-2}(l)+z_{-1}(t)+z_{0}(t)+z_{1}(t)+z_{3}(l)
$$

and the spectral density of $z_{n}$ is given by

$$
S .(f)= \begin{cases}h_{n} f^{n}, & 0 \leq f \leq f_{h} \\ 0, & f>f_{h}, n=-2,-1,0,1,2,\end{cases}
$$

where the $h_{n}$ are constants. Thus, $S_{y}(f)$ becomes

$$
S_{y}(f)=h_{-2} f^{-2}+h_{-1} f^{-1}+h_{0}+h_{1} f+h_{2} f^{2},
$$

for $0 \leq f \leq f_{A}$ and $S_{y}(f)$ is assumed to be negligible beyond this range. In effect, each $z_{n}$ contributes to both $S_{y}(f)$ and $\left\langle\sigma_{v}^{2}(N, T, \tau)\right\rangle$ independently of the other $z_{n}$. The contributions of the $z_{n}$ to $\left\langle\sigma_{y}^{2}(N, T, \tau)\right\rangle$ are tabulated in Appendix II.

Any electronic device has a finite bandwidth and this certainly applies to frequency-measuring equipment also. For fractional frequency fluctuations $y(t)$ whose spectral density varies as

$$
S_{v}(f) \sim f^{\alpha}, \quad \alpha \geq-1
$$

for the higher Fourier components, one sees (from Appendix I) that $\left\langle\sigma_{y}^{2}(N, T, \tau)\right\rangle$ may depend on the exact shape of the frequency cutoff. This is true because 8 substantial fraction of the noise "power" may be in these higher Fourier components. As a simplifying assumption, this paper assumes a sharp cutoff in noise "power" at the frequency $f_{A}$ for the noise models. It is apparent from the tables of Appendix II that the time domain measure of frequency stability may depend on $f_{A}$ in a very important way, and, in some practical cases, the actual shape of the frequency cutoff may be very important [7]. On the other hand, there are many practical measurements where the value of $f_{A}$ has little or no effect. Good practice, however, dictates that the system noise bandwidth $f_{h}$ should be specified with any results.

In actual practice, the model of (24)-(26) seems to fit almost all real frequency sources. Typically, only two or three of the $h$-coefficients are actually significant for a real device and the others can be neglected. Because of its applicability, this model is used in much of what follows. Since the $z_{n}$ are assumed to be independent noises, it is normally sufficient to compute the effects for a general $z_{n}$ and recognize that the superposition can be accomplished by simple additions for their contributions to $S_{y}(f)$ or $\left\langle\sigma_{y}^{2}(N, T, \tau)\right\rangle$.

\section{B. Time Domain to Frequency Domain}

1) General: For general $\left\langle\sigma_{y}^{2}(N, T, \tau)\right\rangle$ no simple prescription is available for translation into the frequency domain. For this reason, one might prefer $S_{y}(f)$ as a general measure of frequency stability. This is especially true for theoretical work.

2) Specific Model: Equations (24)-(26) form a realistic model that fits the random nondeterministic noises found on most signal generators. Obviously, if this is a good model, then the tables in Appendix II may be used (in reverse) to translate into the frequency domain.
Allan [8] and Vessot [12] showed that if

$$
S_{y}(f)= \begin{cases}h_{a} f^{a}, & 0 \leq f \leq f_{A} \\ 0, & f>f_{h}\end{cases}
$$

where $\alpha$ is a constant, then

$$
\left\langle\sigma_{v}^{2}(N, T, \tau)\right\rangle \sim|\tau|^{*}, \quad 2 \pi \tau f_{\star} \gg 1
$$

for $N$ and $r=T / \tau$ held constant. The constant $\mu$ is related to $\alpha$ by the mapping shown ${ }^{1}$ in Fig. 1. If (28) and (29) hold over a reasonable range for a signal generator, then (28) can be substituted into (23) and evaluated to determine the constant $h_{\mathrm{a}}$ from measurements of $\left\langle\sigma_{v}^{2}(N, T, \tau)\right\rangle$. It should be noted that the model of (28) and (29) may be easily extended to a superposition of similar noises as in (26).

\section{Translations Among the Time-Domain Mearures}

1) General: Since $\left\langle\sigma_{y}^{2}(N, T, \tau)\right\rangle$ is a function of $N, T$, and $\tau$ (for some types of noise $f_{A}$ is also important), it is very desirable to be able to translate among different. sets of $N, T$, and $\tau$ ( $f_{h}$ held constant). This is, however. not possible in general.

2) Specific Model: It is useful to restrict consideration to a case described by (28) and (29). Superpositions of independent noises with different power-law types of spectral densities (i.e., different $\alpha$ ) can also bo treated by this technique, e.g., (26). One may define two "bias functions," $B_{1}$ and $B_{2}$ by the relations [13]

$$
B_{1}(N, \tau, \mu) \equiv \frac{\left\langle\sigma_{y}^{2}(N, T, \tau)\right\rangle}{\left\langle\sigma_{v}^{2}(2, T, \tau)\right\rangle}
$$

and

$$
B_{2}(\tau, \mu) \equiv \frac{\left\langle\sigma_{v}^{2}(2, T, \tau)\right\rangle}{\left\langle\sigma_{y}^{2}(2, \tau, \tau)\right\rangle}
$$

where $r \equiv T / \tau$ and $\mu$ is related to $\alpha$ by the mapping of Fig. 1. In words, $B_{1}$ is the ratio of the average variance for $N$ samples to the average variance for two samples (everything else held constant), while $B_{2}$ is the ratio of the average variance with dead time between measurements $(r \neq 1)$ to that of no dead time $(r=1$ and with $N=2$ and $\tau$ held constant). These functions are tabulated in [13]. Figs. 2 and 3 show a computer plot of $B_{1}(N, r=1, \mu)$ and $B_{2}(r, \mu)$.

Suppose one has an experimental estimate of $\left\langle\sigma_{y}^{2}\left(N_{1}\right.\right.$, $\left.\left.T_{1}, \tau_{1}\right)\right\rangle$ and its spectral type is known, i.e., (28) and (29) form a good model and $\mu$ is known. Suppose also that one wishes to know the variance at some other set of measurement parameters $N_{2}, T_{2}, \tau_{2}$. An unbiased estimate of $\left\langle\sigma_{v}^{2}\left(N_{2}, T_{2}, \tau_{2}\right)\right\rangle$ may be calculated by

\footnotetext{
2 It should be noted that in Allan [8], the exponent a corresponds to the spectrum of phase fluctuations while variances are taken over average frequency fluctuations. In the present paper, $\alpha$ is identical to the exponent $\alpha+2$ in [8].
} 


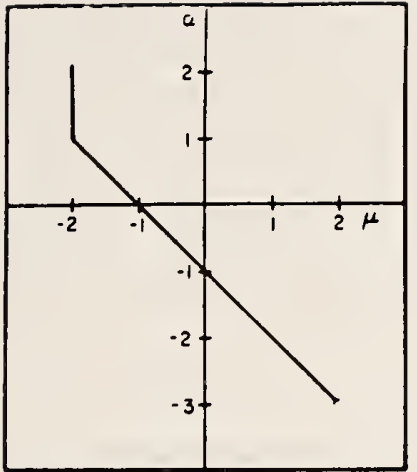

Fig. 1. $\mu-a$ mapping.

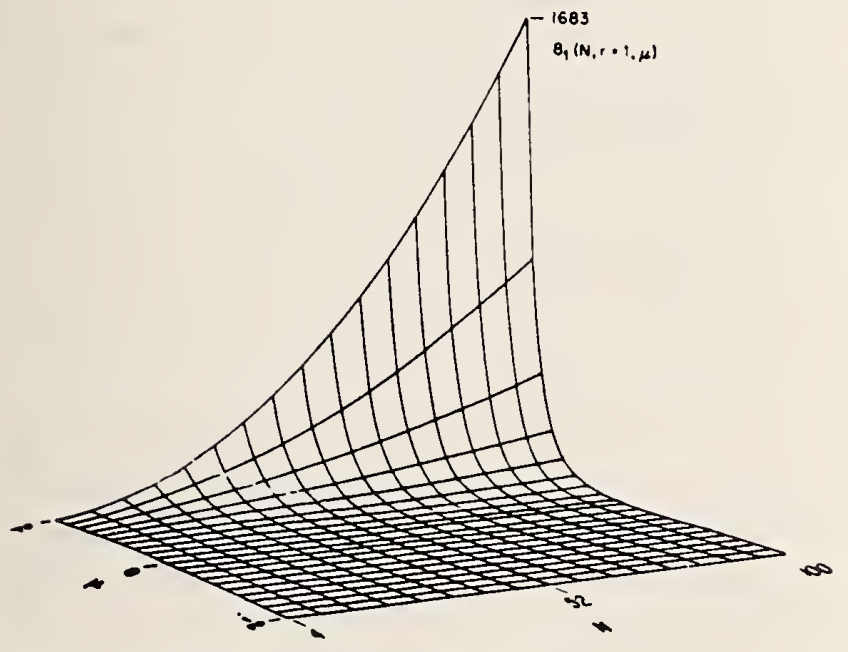

Fig. 2. Function $B_{1}(N, r=1, \mu)$.

$\left\langle\sigma_{y}^{2}\left(N_{2}, T_{2}, \tau_{2}\right)\right\rangle=\left(\frac{\tau_{2}}{\tau_{1}}\right)^{\mu}$

$$
\cdot\left[\frac{B_{1}\left(N_{2}, r_{2}, \mu\right) B_{2}\left(r_{2}, \mu\right)}{B_{1}\left(N_{1}, r_{1}, \mu\right) B_{2}\left(r_{1}, \mu\right)}\right]\left\langle\sigma_{v}^{2}\left(N_{1}, T_{1}, r_{1}\right)\right\rangle,
$$

where $r_{1}=T_{1} / \tau_{1}$ and $r_{2}=T_{2} / \tau_{2}$.

3) General: While it is true that the concept of the bias functions $B_{1}$ and $B_{2}$ could be extended to other processes besides those with the power-law types of spectral densities, this generalization has not been done. Indeed, spectra of the form given in (28) [or superpositions of such spectra as in (26)] seem to be the most common types of nondeterministic noises encountered in signal generators and associated equipment. For other types of fluctuations (such as causally generated perturbations), translations must be handled on an individual basis.

\section{Applications of Stability Measures}

Obviously, if one of the stability measures is exactly the important parameter in the use of a signal generator, the stability measure's application is trivial. Some nontrivial applications arise when one is interested in a dif-

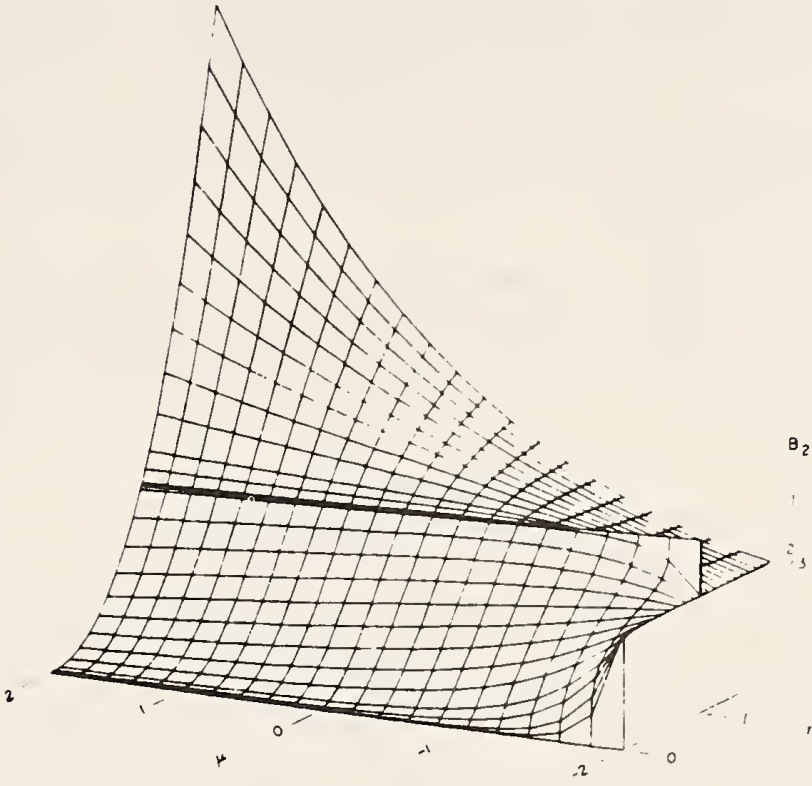

Fig. 3. Bias function $B_{2}(r, \mu)$.

ferent parameter, such as in the use of an oscillator in Doppler radar measurements or in clocks.

\section{A. Doppler Radar}

1) General: From its transmitted signal, a Doppler radar receives from a moving target a frequency-shifted return signal in the presence of other large signals. These large signals can include clutter (ground return) and transmitter leakage into the receiver (spillover). Instabilities of radar signals result in noise energy on the clutter return, on spillover, and on local oscillators in the equipment.

The limitations of subclutter visibility (SCV) rejections due to the radar signals themselves are related to the RF power spectral density $S_{V}(f)$. The quantity typically referred to is the carrier-to-noise ratio and can be mathematically approximated by the quantity

$$
\frac{S_{v}(f)}{\int_{0}^{\infty} S_{v}\left(f^{\prime}\right) d f^{\prime}} .
$$

The effects of coherence of target return and other radar parameters are amply considered in the literature [14]-[17].

2) Special Case: Because FM effects generally predominate over AM effects, this carrier-to-noise ratio is approximately given by [6]

$$
\frac{S_{V}(f)}{\int_{0}^{\infty} S_{V}\left(f^{\prime}\right) d f^{\prime}} \approx \frac{1}{2} S_{\varphi}\left(\left|f-\nu_{0}\right|\right) .
$$

for many signal sources provided $\left|f-v_{0}\right|$ is sufficiently greater than zero. (The factor of $\frac{1}{2}$ arises from the fact that $S_{\varphi}(f)$ is a one-sided spectrum.) Thus, if $f-v_{1}$ is 
in frequency separation from the carricr, the carrier-tonoise ratio at that point is approximately

$$
\frac{1}{2} s_{0}\left(\left|f-v_{0}\right|\right)=\frac{1}{2}\left(\frac{\nu_{0}}{f-\nu_{0}}\right)^{2} S_{\nu}\left(\left|f-\nu_{0}\right|\right) .
$$

\section{B. Clock Errors}

1) General: A clock is a device that counts the cycles of a periodic phenomenon. Thus, the reading error $x(t)$ of a clock run from the signal given by (2) is

$$
x(t)=\frac{\varphi(t)}{2 \pi v_{0}}
$$

and the dimensions of $x(t)$ are seconds.

If this clock is a sccondary standard, then one could have available some past history of $x(t)$, the time error relative to the standard clock. It often occurs that one is intercsted in predicting the clock error $x(t)$ for some future date, say $t_{0}+\tau$, where $t_{0}$ is the present date. Obviously, this is a problem in pure prediction and can be handled by conrentional methods [3].

2) Special Case: Although one could handle the prediction of clock errors by the rigorous methods of prediction theory, it is more common to use simpler prediction methods [10], [11]. In particular, one often predicts a clock error for the future by adding to the present error a correction that is derived from the current rate of gain (or loss) of time. That is, the predicted error $\hat{x}\left(t_{0}+\tau\right)$ is related to the past history of $x(t)$ by

$$
\hat{x}\left(l_{10}+\tau\right)=x\left(t_{10}\right)+T\left[\frac{x\left(l_{0}\right)-x\left(l_{0}-T\right)}{T}\right] .
$$

It is typical to let $T=\tau$.

Thus, the mean-square error of prediction for $T=\tau$ becomes

$$
\begin{aligned}
\left\langle\left[ x\left(t_{0}+\tau\right)\right.\right. & \left.\left.-\hat{x}\left(t_{0}+\tau\right)\right]^{2}\right\rangle \\
= & \left\langle\left[x\left(t_{0}+\tau\right)-2 x\left(t_{0}\right)+x\left(t_{0}-\tau\right)\right]^{2}\right\rangle,
\end{aligned}
$$

which, with the aid of (11), can be written in the form

$$
\left\langle\left[x\left(t_{0}+\tau\right)-\hat{x}\left(t_{0}+\tau\right)\right]^{2}\right\rangle=2 \tau^{2} \sigma_{v}^{2}(\tau) .
$$

One can define a time stability measure $\sigma_{x}^{2}(\tau)$ by

$$
\sigma_{x}^{2}(\tau) \equiv \tau^{2} \sigma_{y}^{2}(\tau)
$$

Clearly, however, the actual errors of prediction of clock readings are dependent on the prediction algorithm used and the utility of such a definition as $\sigma_{x}^{2}(\tau)$ is not great. Caution should be used in employing this definition.

\section{Measuremext Techniques for Frequency Stability}

\section{A. Heterodyne Techniques (General)}

It is possible for oscillators to be very stable and values of $\sigma_{\nu}(\tau)$ can be as small as $10^{-14}$ in some state-ofthe-art equipment. Thus, one often needs measuring techniques capable of resolving very small fluctuations in $y(t)$. One of the most common techniques is a heterodync or beat frequency technique. In this method, the signal from the oscillator to be tested is mixed with a reference signal of almost the same frequency as the test oscillator in order that one is left with a lower average frequency for analysis without reducing the frequency (or phase) fluctuations themselves. Following Vessot et al. [18], consider an ideal reference oscillator whose output signal is

$$
V_{r}(t)=V_{\text {or }} \sin 2 \pi \nu_{0} t
$$

and a second oscillator whose output voltage $V(t)$ is given by $(2): V(t)=\left[V_{0}+\epsilon(t)\right] \sin \left[2 \pi \nu_{0} t+\varphi(t)\right]$. Let these two signals be mixed in a product detector; that is, the output of the product detector $v(t)$ is equal to the product $\gamma V(t) \times V_{,}(t)$, where $\gamma$ is a constant (see Fig. 4).

Let $v(t)$, in turn, be processed by a sharp low-pass filter with cutoff frequency $f_{h}^{\prime}$ such that

$$
0<f_{h}<f_{h}^{\prime}<\nu_{n} \text {. }
$$

One may write

$$
\begin{aligned}
& \gamma V^{\prime}(t) \cdot V_{r}(t) \\
& \quad=\gamma V_{0 r}\left(V_{0}+\epsilon\right)\left[\sin 2 \pi \nu_{0} t\right]\left[\sin \left(2 \pi \nu_{0} t+\varphi\right)\right] \\
& \quad=v(t)=\gamma \frac{\left(V_{n} V_{n}\right)}{2}\left(1+\frac{\epsilon}{V_{0}}\right)\left[\cos \varphi-\cos \left(4 \pi \nu_{0} t+\varphi\right)\right]
\end{aligned}
$$

Assume that $\cos [\varphi(t)]$ has essentially no power in Fourier frequencies $f$ in the region $f \geq f_{h}^{\prime}$. The effect of the low-pass filter then is to remove the second term on the extreme right of (42); that is

$$
v^{\prime}(l)=\gamma \frac{V_{n} V_{n}}{2}\left(1+\frac{\epsilon}{V_{0}}\right) \cos \varphi(t) .
$$

This separation of terms by the filter is correct only if $\left|\left[\dot{\varphi}(t) / 2 \pi \nu_{0}\right]\right| \ll 1$ for all $t(4)$.

The following two cases are of interest.

Case I: The relative phase of the oscillators is adjusted so that $|\varphi(t)| \ll 1$ (in-phase condition) during the period of measurement. Under these conditions

$$
v^{\prime}(t) \approx \frac{\gamma}{2} V_{0,} V_{0}+\frac{\gamma}{2} V_{0 r} \epsilon(t)
$$

since $\cos \psi(t) \approx 1$. That is to say one detects the amplitude noise $\epsilon(t)$ of the signal.

Case II: The relative phase of the oscillators is adjusted to be in approximate quadrature; that is

$$
\varphi^{\prime}(l)=\varphi(t)+\frac{\pi}{2}
$$

where $\mid \varphi^{\prime}(t) ! \ll 1$. Under these conditions,

$$
\cos \varphi(t)=\sin \varphi^{\prime}(t) \approx \varphi^{\prime}(t)
$$

and

$$
l^{\prime}(t)=\frac{\gamma}{2} V_{0 r} V_{0 \varphi^{\prime}}(t)+\frac{\gamma}{2} V_{0} \varphi^{\prime}(t) \epsilon(t)
$$




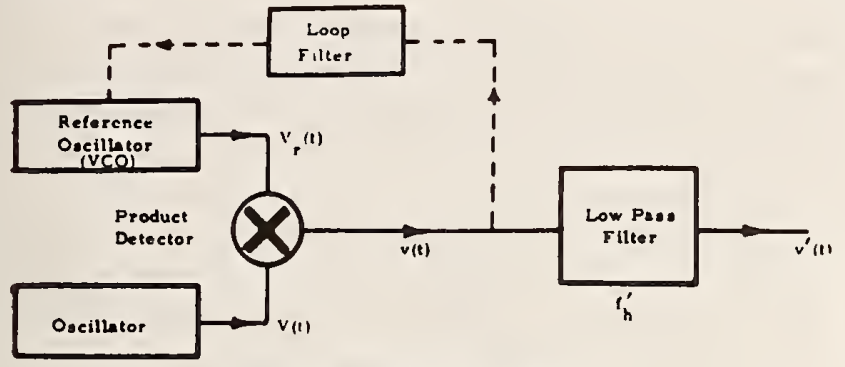

Fig. 4. Heterodyne scheme.

If it is true that $\left|\left[e(t) / V_{0}\right]\right| \ll 1$ for all $t(3)$, then (47) becomes

$$
v^{\prime}(t) \approx \frac{\gamma}{2} V_{O r} V_{O \phi^{\prime}}(t)
$$

that is, $v^{\prime}(t)$ is proportional to the phase fluctuations. Thus, in order to observe $\varphi^{\prime}(t)$ by this method, (3) and (1) must be valid. For different average phase values, mixtures of amplitude and phase noise are observed.

In order to maintain the two signals in quadrature for long observational periods, the reference oscillator can be a voltage-controlled oscillator (VCO) and one may feed back the phase error voltage as defined in (48) to control the frequency of the VCO [19]. In this condition of the phase-locked oscillator, the voltage $v^{\prime}(t)$ is the analog of the phase fluctuations for Fourier frequencies above the loop cutoff frequency of the locked loop. For Fourier frequencies below the loop cutoff frequency of the loop, $v^{\prime}(t)$ is the analog of frequency fluctuations. In practice, one should measure the complete servo-loop response.

\section{B. Period Measurement}

Assume one has an oscillator whose voltage output may be represented by (2). If $\left|\left[e(t) / V_{0}\right]\right| \ll 1$ for all $t$ and the total phase

$$
\Phi(t)=2 \pi v_{0} t+\varphi(t)
$$

is a monotonic function of time (that is, $\left.\| \dot{\varphi}(t) / 2 \pi \nu_{0}\right] \mid \leq 1$ ), then the time $t$ between successive positive going zero crossings of $V(t)$ is related to the average frequency during the interval $\tau$. Specifically

$$
\frac{1}{\tau}=v_{0}\left(1+\bar{y}_{n}\right) \text {. }
$$

If one lets $r$ be the time between a positive going zero crossing of $V(t)$ and the $M$ th successive positive going sero crossing, then

$$
\frac{M}{\tau}=v_{0}\left(1+\eta_{n}\right) .
$$

If the variations $\Delta r$ of the period are small compared to the average period $\tau_{0}$, Cutler and Searle [7] have shown that one may make a reasonable approximation to $\left\langle\sigma_{v}^{2}\left(N, T, \tau_{0}\right)\right\rangle$ using period measurements.

\section{Period Measurement With Heterodyning}

Suppose that $\varphi(t)$ is a monotonic function of time. The output of the filter of Section VII-A (43) becomes

$$
v^{\prime}(t) \approx \gamma \frac{V_{0,} V_{0}}{2} \cos \varphi(t)
$$

if $\left|\left[\epsilon(t) / V_{0}\right]\right| \ll 1$. Then one may measure the period $r$ of two successive positive zero crossings of $v^{\prime}(t)$. Thus

$$
\frac{1}{r}=\nu_{0}\left|\bar{y}_{0}\right|
$$

and for the $M$ th positive crossover

$$
\frac{M}{T}=v_{0}\left|\bar{y}_{n}\right| .
$$

The magnitude bars appear because $\cos \varphi(t)$ is an even function of $\varphi(t)$. It is impossible to determine by this method alone whether $\varphi$ is increasing with time or decreasing with time. Since $\bar{y}_{n}$ may be very small $\left(\sim 10^{-11}\right.$ or $10^{-12}$ for very good oscillators), $\tau$ may be quite long and thus measurable with a good relative precision.

If the phase $p(t)$ is not monotonic, the true $\dot{y}_{n}$ may be near zero but one could still have many zeros of $\cos \varphi(t)$ and thus (52) and (53) would not be valid.

\section{Frequency Counters}

Assume the phase (either $\Phi$ or $\varphi$ ) is a montonic function of time. If one counts the number $M$ of positive going zero crossings in a period of time $\tau$, then the sverage frequency of the signal is $M / \tau$. If we assume that the signal is $V(t)$ as defined in (2), then

$$
\frac{M}{T}=v_{0}\left(1+\bar{y}_{n}\right) .
$$

If we sssume that the signal is $v^{\prime}(t)$ as defined in (48), then

$$
\frac{M}{T}=\nu_{0}\left|\bar{y}_{m}\right| .
$$

Again, one measures only positive frequencies.

\section{E. Frequency Discriminators}

A frequency discriminator is a device that converts frequency fluctuations into an analog voltage by means of a dispersive element. For example, by slightly detuning a resonant circuit from the signal $V(\ell)$ the frequency fluctuations $(1 / 2 \pi)] \dot{\varphi}(t)$ are converted to amplitude fluctuations of the output signal. Provided the input amplitude fluctuations $[\epsilon(t)] / V_{0}$ are insignificant, the output amplitude fluctuations can be a good measure of the frequency fuctuations. Obviously, more sophisticated frequency discriminators exist (e.g., the cesium beam). 
From the analog voltage one may use analog spectrum analyzers to determine $S_{v}(f)$, the frequency stability. By converting to digital data, other analyses are possible on a computer.

\section{F. Common Hazards}

1) Errors Caused by Signal-Processing Equipment: The intent of most frequency stability measurements is to evaluate the source and not the measuring equipment. Thus, one must know the performance of the measuring system. Of obvious importance are such aspects of the measuring equipment as noise level, dynamic range, resolution (dead time), and frequency range.

It has been pointed out that the noise bandwidth $f_{b}$ is very essential for the mathematical convergence of certain expressions. Insofar as one wants to measure the signal source, one must know that the measuring system is not limiting the frequency response. At the very least, one must recognize that the frequency limit of the measuring system may be a very important, implicit parameter for either $\sigma_{y}^{2}(t)$ or $S_{y}(f)$. Indeed, one must account for any deviations of the measuring system form ideality such as a "nonflat" frequency response of the spectrum analyzer itself.

Almost any electronic circuit that processes a signal will, to some extent, convert amplitude fluctuations at the input terminals into phase fluctuations at the output. Thus, AM noise at the input will cause a time-varying phase (or FM noise) at the output. This can impose important constraints on limiters and automatic gain control (AGC) circuits when good frequency stability is needed. Similarly, this imposes constraints on equipment used for frequency stability measurements.

2) Analog Spectrum Analyzers (Frequency Domain): Typical analog spectrum analyzers are very similar in design to radio receivers of the superheterodyne type, and thus certain design features are quite similar. For example, image rejection (related to predetection bandwidth) is very important. Similarly, the actual shape of the analyzer's frequency window is important since this affects spectral resolution. As with receivers, dynamic range can be critical for the analysis of weak signals in the presence of substantial power in relatively narrow bandwidths (e.g., $60 \mathrm{~Hz}$ ).

The slewing rate of the analyzer must be consistent with the analyzer's frequency window and the post-detection bandwidth. If one has a frequency window of $1 \mathrm{~Hz}$, one cannot reliably estimate the intensity of a bright line unless the slewing rate is much slower than $1 \mathrm{~Hz} / \mathrm{s}$. Additional post-detection filtering will further reduce the maximum usable slewing rate.

S) Spectral Density Estimation from Time Domain Data: It is beyond the scope of this paper to present a comprehensive list of hazards for spectral density estimation; one should consult the literature [2]-[5]. There are a few points, however, which are worthy of special notice: a) data aliasing (similar to predetection bandwidth problems); b) spectral resolution; and c) confidence of the estimate.

4) Variances of Frequency Fluctuations $\sigma_{v}^{2}(\tau)$ : It is not uncommon to have discrete frequency modulation of a source such as that associated with the power supply frequencies. The existence of discrete frequencies in $S,(f)$ can cause $\sigma_{v}^{2}(\tau)$ to be a very rapidly changing function of $\tau$. An interesting situation results when $\tau$ is an exact multiple of the period of the modulation frequency (e.g., one makes $\tau=1 \mathrm{~s}$ and there exists $60-\mathrm{Hz}$ frequency modulation on the signal). In this situation, $\sigma_{v}^{2}(\tau=1 \mathrm{~s})$ can be very optimistic relative to values with slightly different values of $\tau$.

One also must be concerned with the convergence properties of $\sigma_{v}^{2}(\tau)$ since not all noise processes will have finite limits to the estimates of $\sigma_{\gamma}^{2}(\tau)$ (see Appendix I). One must be as critically aware of any "dead time" in the measurement process as of the system bandwidth.

5) Signal Source and Loading: In measuring frequency stability one should specify the exact location in the circuit from which the signal is obtained and the nature of the load used. It is obvious that the transfer characteristics of the device being specified will depend on the load and that the measured frequency stability might be affected. If the load itself is not constant during the measurements, one expects large effects on frequency stability.

6) Confidence of the Estimate: As with any measurement in science, one wants to know the confidence to assign to numerical results. Thus, when one measures $S_{y}(f)$ or $\sigma_{y}^{2}(\tau)$, it is important to know the accuracies of these estimstes.

a) The Allan Variance: It is apparent that a single sample variance $\sigma_{v}^{2}(4, \tau, \tau)$ does not have good confidence, but, by averaging many independent samples, one can improve the accuracy of the estimate greatly. There is a key point in this statement, "independent samples." For this argument to be true, it is important that one sample variance be independent of the next. Since $\sigma_{y}^{2}(2, \tau, \tau)$ is related to the first difference of the frequency (11), it is sufficient that the noise perturbing $y(t)$ have "independent increments," i.e., that $y(t)$ be a random walk. In other words, it is sufficient that $S_{y}(f) \sim f^{-2}$ for low frequencies. One can show that for noise processes that are more divergent at low frequencies than $f^{-2}$, it is difficult (or impossible) to gain good confidence on estimates of $\sigma_{y}^{2}(\tau)$. For noise processes that are less divergent than $f^{-2}$, no problem exists.

It is worth noting that if we were interested in $\sigma_{y}^{2}(N=\infty, \tau, \tau)$, then the limit noise would become $S_{y}(f) \sim f^{0}$ instead of $f^{-2}$ as it is for $\sigma_{y}^{2}(2, \tau, \tau)$. Since most real signal generators possess low-frequency divergent noises, $\left\langle\sigma_{v}^{2}(2, \tau, \tau)\right\rangle$ is more useful than $\sigma_{v}^{2}(N=\infty, \tau, \tau)$.

Although the sample variances $\sigma_{*}^{2}(2, \tau, \tau)$ will not be normally distributed, the variance of the average of $m$ 
independent (nonoverlapping) samples of $\sigma_{v}^{2}(2, \tau, \tau)$ (i.e., the variance of the Allan variance) will decrease as $1 / m$ provided the conditions on low-frequency divergence are met. For sufficiently large $m$, the distribution of the $m$ sample averages of $\sigma_{n}^{2}(2, \tau, \tau)$ will tend toward normal (central limit theorem). It is thus possible to estimate confidence intervals based on the normal distribution.

As always, one may be interested in $\tau$ values approaching the limits of available data. Clearly, when one is interested in $\tau$ values of the order of a year, one is severely limited in the size of $m$, the number of samples of $\sigma_{y}^{2}(2, \tau, \tau)$. Unfortunately, there seems to be no substitute for many samples and one extends $\tau$ at the expense of confidence in the results. "Truth in packaging" dictates that the sample sire $m$ be stated with the results.

b) Spectral Density: As before, one is referred to the literature for discussions of spectrum estimation [2]-[5]. It is worth pointing out, however, that for $S,(f)$ there are basically two different types of averaging that can be employed: sample averaging of independent estimates of $S_{v}(f)$, and frequency averaging where the resolution bandwidth is made much greater than the reciprocal data length.

\section{Conclusions}

A good measure of frequency stability is the spectral density $S_{y}(f)$ of fractional frequency fluctuations $y(t)$. An alternative is the expected variance of $N$ sample averages of $y(t)$ taken over a duration $\tau$. With the beginning of successive sample periods spaced every $T$ units of time, the variance is denoted by $\sigma_{s}^{2}(N, T, \tau)$. The atability measure, then, is the expected value of many messurements of $\sigma_{v}^{2}(N, T, \tau)$ with $N=2$ and $T=\tau$; that is, $\sigma_{n}^{2}(\tau)$. For all real experiments one has a finite bandwidth. In general, the time domain measure of frequency stability $\sigma_{y}^{2}(\tau)$ is dependent on the noise bandwidth of the system. Thus, there are four important parameters to the time domain measure of frequency stability.

$N$ Number of sample averages $(N=2$ for preferred measure).

$T$ Repetition time for successive sample averages ( $T=\tau$ for preferred measure).

T Duration of each sample average.

f. System noise bandwidth.

Translations among the various stability measures for common noise types are possible, but there are significant reasons for choosing $N=2$ and $T=\tau$ for the preferred measure of frequency stability in the time domain. This measure, the Allan variance, $(N=2)$ has been referenced by [12], [20]-[22] and more.

Although $S_{y}(f)$ appears to be a function of the single variable $f$, actual experimental estimation procedures for the spectral density involve a great many parameters. Indeed, its experimental estimation can be at least as involved as the estimation of $\sigma_{y}^{2}(\tau)$.

\section{APPENDIX I}

We want to derive (23) in the text. Starting from (10, we have

$$
\begin{aligned}
& \left\langle\sigma_{v}^{2}(N, T, \tau)\right\rangle \\
& \equiv\left\langle\frac{1}{N-1} \sum_{n=1}^{N}\left(\bar{y}_{n}-\frac{1}{N} \sum_{k=1}^{N} \bar{y}_{k}\right)^{2}\right\rangle \\
& =\frac{1}{N-1}\left\{\sum_{n=1}^{N}\left\langle\bar{y}_{n}^{2}\right\rangle-\frac{1}{N} \sum_{i=1}^{N} \sum_{i=1}^{N}\left(\bar{y}_{i} \bar{y}_{i}\right)\right\} \\
& =\frac{1}{(N-1) \tau^{2}}\left\{\sum_{n=1}^{N} \int_{t_{0}}^{t_{0}+r} d l^{\prime \prime} \int_{t_{0}}^{t_{+}+r} d l^{\prime}\left\langle y\left(\ell^{\prime}\right) y\left(t^{\prime \prime}\right)\right\rangle\right.
\end{aligned}
$$

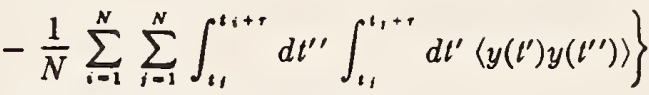

where (9) has been used. Now

$$
\left\langle y\left(t^{\prime}\right) y\left(t^{\prime \prime}\right)\right\rangle=R_{v}\left(t^{\prime}-t^{\prime \prime}\right)
$$

where $R_{y}(r)$ is the autocorrelation function of $y(t)$ and is the Fourier transform of $S_{y}(f)$, the power spectral density of $y(t)$. Equation (57) is true provided that $y(t)$ is stationary (at least in the wide or covariance sense), and that the average exists. If we assume the power spectral density of $y(t), S_{y}(f)$ has low and high frequency cutoffs $f_{l}$ and $f_{h}$ (if necessary) so that

$$
\int_{0}^{\infty} S_{v}(f) d f
$$

exists, then if $y$ is a random variable, the average does exist and we may safely assume stationarity.

In practice, the high-frequency cutoff $f_{A}$ is always present either in the device being measured or in the messuring equipment itself. When the high-frequency cutoff is necessary for convergence of integrals of $S_{y}(f)$ (or is too low in frequency), the stability measure will depend on $f_{A}$. The latter case can occur when the measuring equipment is too narrow-band. In fact, a useful type of spectral analysis may be done by varying $f_{n}$ purposefully [18].

The low-frequency cutoff $f_{l}$ may be taken to be much smaller than the reciprocal of the longest time of interest. The results of calculations as well as measurements will be meaningful if they are independent of $f_{l} \& 5 f_{l}$ approsches zero. The range of exponents in power law spectral densities for which this is true will be discussed and are given in Fig. 1.

To continue, the derivation requires the Fourier transform relationships between the autocorrelation function and the power spectral density

$$
\begin{aligned}
& S_{\nu}(f)=4 \int_{0}^{\infty} R_{\nu}(\tau) \cos 2 \pi f \tau d \tau \\
& R_{\nu}(\tau)=\int_{0}^{\infty} S_{\nu}(f) \cos 2 \pi f \tau d f .
\end{aligned}
$$

Using (58) and (57) in (56) gives 


$$
\begin{aligned}
& \left\langle\sigma_{y}^{2}(N, T, \tau)\right\rangle=\frac{1}{(N-1) \tau^{2}}\left\{\sum_{n=1}^{N} \int_{0}^{\infty} d f S_{y}(f) \int_{1 .}^{1,++r} d t^{\prime \prime}\right. \\
& \text { - } \int_{1 .}^{t .++} d t^{\prime} \cos 2 \pi f\left(t^{\prime}-t^{\prime \prime}\right)-\frac{1}{N} \sum_{i=1}^{N} \sum_{i=1}^{N} \int_{0}^{\infty} d f S_{y}(f) \\
& \left.\int_{1,}^{t_{i++}} d t^{\prime \prime} \int_{t_{i}}^{t_{1++}} d t^{\prime} \cos 2 \pi f\left(t^{\prime}-t^{\prime \prime}\right)\right\} \\
& =\frac{1}{(N-1) \tau^{2}}\left\{\sum_{n=1}^{N}\left[\int_{0}^{\infty} d f S_{v}(f) \frac{\sin ^{2} \pi f \tau}{(\pi f)^{2}}\right]\right. \\
& -\frac{1}{N} \sum_{i=1}^{N} \sum_{i=1}^{N}\left[\int_{0}^{\infty} d f \frac{S_{v}(f)}{(2 \pi f)^{2}}(2 \cos 2 \pi f T(j-i)\right. \\
& -\cos 2 \pi f[T(j-i)+\tau]-\cos 2 \pi f[T(j-i)-\tau])]\} \text {. }
\end{aligned}
$$

(The interchanges in order of integration are permissible here since the integrals are uniformly convergent with the given restrictions on $S_{\nu}(f)$.) The first summation in the curly brackets is independent of the summation index $n$ and thus gives just

$$
N \int_{0}^{\infty} d f S_{\nu}(f) \frac{\sin ^{2} \pi f \tau}{(\pi f)^{2}}
$$

The kernel in the second term in the curly brackets may be further simplified

$$
\begin{aligned}
& 2 \cos 2 \pi f T(j-i)-\cos 2 \pi f(T(j-i)+\tau) \\
& \quad-\cos 2 \pi f(T(j-i)-\tau)=4 \sin ^{2} \pi f \tau \cos 2 \pi f T(j-i) .
\end{aligned}
$$

The second term is then

$-\frac{1}{N}\left(\int_{0}^{\infty} d f \frac{S_{u}(f)}{(\pi f)^{2}} \sin ^{2} \pi f \tau \sum_{i=1}^{N} \sum_{i=1}^{N} \cos 2 \pi f T(j-i)\right)$.

(The interchange of summation and integration is justified.) We must now do the double sum. Let

$$
\begin{aligned}
& j-i=k \\
& 2 \pi f T=x .
\end{aligned}
$$

Changing summation indices from $i$ and $j$ to $i$ and $k$ gives for the sum

$$
S \equiv \sum_{i=1}^{N} \sum_{i=1}^{N} \cos x(j-i)=\sum_{i=1}^{N} \sum_{k=1-i}^{N-i} \cos k x .
$$

The region of summation over the discrete variables $i$ and $k$ is shown in Fig. 5 for $N=4$.

The summand is independent of $i$ so that one may interchange the order of summation and sum over $i$ first. The summand is even in $k$ and the contributions for $k<0$ are equal to those for $k>0$, and so we may pull out the term for $k=0$ separately and write

$$
\begin{aligned}
S & =2\left(\sum_{k=1}^{N-1} \cos k x \sum_{i=1}^{N-k} 1\right)+\sum_{i+1}^{N} 1 \\
& =2\left(\sum_{k=1}^{N-1}(N-k) \cos k x\right)+N .
\end{aligned}
$$

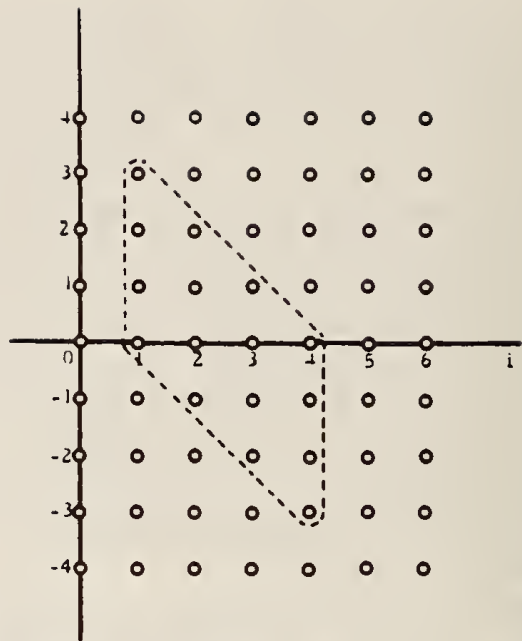

Fig. 5. Region of summation for $i$ and $k$ for $N=4$.

This may be written as

$$
S=N+2 \operatorname{Re}\left[N-\frac{1}{i} \frac{d}{d x}\right] \sum_{k=1}^{N-1} e^{i k x}
$$

where $\operatorname{Re}[U]$ means the real part of $U$ and $d / d x$ is the differential operator. The series is a simple geometric series and may be summed easily, giving

$$
\begin{aligned}
S & =N+2 \operatorname{Re}\left\{\left[N-\frac{1}{i} \frac{d}{d x}\right] \frac{e^{i x}-e^{i N s}}{1-e^{i x}}\right\} \\
& =N+2 \operatorname{Re}\left\{\frac{1-e^{i N s}-N\left(1-e^{i s}\right)}{4 \sin ^{2} x / 2}\right\} \\
& =\frac{\sin ^{2} N x / 2}{\sin ^{2} x / 2} .
\end{aligned}
$$

Combining everything we get, after some rearrangement, $\left\langle\sigma_{y}^{2}(N, T, \tau)\right\rangle$

$$
=\frac{N}{N-1} \int_{0}^{\infty} d f S_{y}(f) \frac{\sin ^{2} \pi f \tau}{(\pi f \tau)^{2}}\left[1-\frac{\sin ^{2} \pi f N_{\tau}}{N^{2} \sin ^{2} \pi \tau f \tau}\right]
$$

where $r=T / \tau$. This is the result given in (23).

We can determine a number of things very easily from this equation. First let us change variables. Let $\pi \tau=u$, then

$$
\begin{aligned}
& \left\langle\sigma_{\nu}^{2}(N, T, \tau)\right\rangle \\
& \quad=\frac{N}{(N-1) \pi \tau} \int_{0}^{\infty} d u S_{\nu}\left(\frac{u}{\pi \tau}\right) \frac{\sin ^{2} u}{u^{2}}\left\{1-\frac{\sin ^{2} N r u}{N^{2} \sin ^{2} r u}\right\} .
\end{aligned}
$$

The kernel behaves like $u^{2}$ as $u \rightarrow 0$ and like $u^{-2}$ as $u \rightarrow \infty$. Therefore $\left\langle\sigma_{y}^{2}(N, T, \tau)\right\rangle$ is convergent for power law spectral densities, $S_{y}(f)=h_{\alpha} f^{a}$, without any low- or highfrequency cutoffs for $-3<\alpha,<1$. Using (69) for power law spectral densities we find

$$
\begin{aligned}
\left\langle\sigma_{y}^{2}(N, T, \tau)\right\rangle & =\tau^{-a-1} h_{a} C_{a,} & & -3<\alpha<1 \\
& =\tau^{\mu} h_{a} C_{a}, & \mu & \equiv-\alpha-1
\end{aligned}
$$


and

$C_{a} \equiv \frac{N}{(. V-1) \pi^{a+1}} \int_{0}^{\infty} d u u^{a} \frac{\sin ^{2} u}{u^{2}}\left\{1-\frac{\sin ^{2} \lambda r u}{N^{2} \sin ^{2} r u}\right\}$.

This is the basis for the plot in Fig. 1 in the text of $\mu$ versus $\alpha$. For $\alpha \geq 1$ we must include the high-frequency cutoff $f_{b}$.

For $N=2$ and $r=1$ the results are particularly simple. We have

$$
\left\langle\sigma_{v}^{2}(2, \tau, \tau)\right\rangle=\tau^{-\alpha-1} h_{a} \frac{2}{\pi^{a+1}} \int_{0}^{\infty} d u u^{\alpha-2} \sin ^{4} u
$$

for power law spectral densities. For $N=2$ and general $r$ we get

$$
\begin{aligned}
\left\langle\sigma_{v}^{2}(2, T, \tau)\right) & \\
= & \frac{1}{2 \pi \tau} \int_{0}^{\infty} d u S_{v}\left(\frac{u}{\pi \tau}\right) \\
& \frac{1-\cos 2 u-\cos 2 r u+\frac{\cos 2 u(\tau+1)}{2}+\frac{\cos 2 u(r-1)}{2}}{u^{2}} \\
= & \frac{2}{\pi \tau} \int_{0}^{\infty} d u S_{v}\left(\frac{u}{\pi \tau}\right) \frac{\sin ^{2} u \sin ^{2} r u}{u^{2}} .
\end{aligned}
$$

The first form in (72) is particularly simple and is also useful for $r=1$ in place of (71).

Let us discuss the case for $\alpha \geq 1$ in a little more detail. As mentioned above we must include the high-frequency cutoff $f_{A}$ for convergence. The general behavior can be seen most easily from (68). After placing the factor $\tau^{-2}$ outside the integral and combining the factor $f^{-2}$ with $S_{y}(f)$ we find that the remaining part of the kernel consists of some constants and some oscillatory terms. If $2 \pi f_{h} \gg 1$ it is apparent that the rapidly oscillating terms contribute very little to the integral. Most of the contribution comes from the integral over the constant term causing the major portion of the $r$ dependence to be the $\tau^{-2}$ factor outside the integral. This is the reason for the vertical slope at $\mu=-2$ in the $\mu$ versus $\alpha$ plot in Fig. 1 in the text.

One other point deserves some mention. The constant term of the kernel discussed in the preceding paragraph is different for $r=1$ from the value for $r \neq 1$. This is readily seen from (72) for $N=2$; for $r=1$ the constant term is $3 / 2$ while for $r \neq 1$ it is 1 . This is the reason for $\delta_{k}(r-1)$, which appears in some of the results of Appendix II. In practice, $\delta_{k}(r-1)$ does not have zero width but is smeared out over a width of approximately $\left(2 \pi f_{\Lambda} \tau\right)^{-1}$. If there must be dead time $r \neq 1$, it is wise to choose $(r-1) \gg\left(2 \pi f_{A} \tau\right)^{-1}$ or $(r-1) \ll\left(2 \pi f_{A} \tau\right)^{-1}$ but with $2 \pi f_{\lambda} r \gg 1$. In the latter case, one may assume $r \approx 1$.

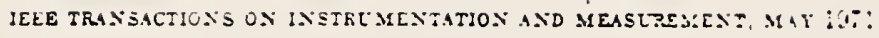

\section{APPEXDLX 11}

Let $y(t)$ be a sample function of a random noise process with a spectral density $S_{y}(f)$. The function $y(t)$ is assumed to be pure real and $S_{y}(f)$ is a one-sided spectrai density relative to a cycle frequency (i.e., the dimensions of $S_{y}(f)$ are that of $y^{2}$ per hertz). (For additional information see Appendix I, [7], [8], [18].)

Let $x(t)$ be defined by the equation

$$
\dot{x}(t) \equiv \frac{d x}{d t} \equiv y(t) .
$$

Define the following. $t_{0}$ is arbitrary instant of time and

$$
\begin{aligned}
t_{n+1} & \equiv t_{n}+T, \quad n=0,1,2, \cdots, \\
\bar{y}_{n} & \equiv \frac{1}{\tau} \int_{1 .}^{1 .+\cdot} y(t) d t=\frac{x\left(t_{n}+\tau\right)-x\left(t_{n}\right)}{\tau} \\
\langle\bar{y}\rangle_{N} & \equiv \frac{1}{N} \sum_{n=1}^{N} \bar{y}_{n,}
\end{aligned}
$$

and let $f_{A}$ be a high-frequency cutoff (infinitely sharp) with $2 \pi f_{h} \tau \gg 1$.

Definition:

$$
\left\langle\sigma_{v}^{2}(N, T, \tau)\right\rangle \equiv\left\langle\frac{1}{N-1} \sum_{n=1}^{N}\left(\bar{y}_{n}-\langle\bar{y}\rangle_{s}\right)^{2}\right\rangle .
$$

Special Case:

$$
\left\langle\sigma_{\nu}^{2}(2, T, \tau)\right\rangle=\left\langle\frac{\left(\bar{y}_{2}-\bar{y}_{1}\right)^{2}}{2}\right\rangle .
$$

Special Case:

$$
\begin{aligned}
\sigma_{v}^{2}(\tau) & \equiv\left\langle\sigma_{v}^{2}(2, \tau, \tau)\right\rangle \\
& =\left\langle\frac{\left[x\left(l_{n}+2 \tau\right)-2 x\left(l_{n}+\tau\right)+x\left(l_{n}\right)\right]^{2}}{2 \tau^{2}}\right\rangle .
\end{aligned}
$$

Definition:

$$
D_{s}^{2}(\tau) \equiv\left\langle\left[x\left(t_{0}+2 \tau\right)-2 x\left(t_{0}+\tau\right)+x\left(t_{0}\right)\right]^{2}\right\rangle .
$$

Consequence of Definitions:

$$
D_{x}^{2}(\tau)=2 \tau^{2} \sigma_{v}^{2}(\tau) \equiv 2 \sigma_{x}^{2}(\tau) .
$$

Definition:

$$
\begin{aligned}
\psi_{z}^{2}(T, \tau) \equiv\left\langle\left[ x\left(t_{0}+T+\tau\right)\right.\right. & -x\left(t_{0}+T\right) \\
& \left.\left.-x\left(t_{0}+\tau\right)+x\left(t_{0}\right)\right]^{2}\right\rangle .
\end{aligned}
$$

Consequence of Definitions:

$$
\psi_{z}^{2}(T, \tau)=2 \tau^{2}\left\langle\sigma_{v}^{2}(2, T, \tau)\right\rangle .
$$

Special Case:

$$
\psi_{s}^{2}(\tau, \tau)=D_{s}^{2}(\tau)
$$

Random Walk $y$

$$
\begin{array}{cc}
S_{y}(f)=\frac{h_{-2}}{f^{2}} & \left(S_{x}(f)=\frac{h_{-2}}{(2 \pi)^{2} f^{4}}\right) \\
r \equiv \frac{T}{\tau}, & 0 \leq f \leq f_{A} .
\end{array}
$$




$$
\begin{array}{ll}
\multicolumn{1}{c}{\text { Quantity }} & \text { Relation } \\
\left\langle\sigma_{v}^{2}(N, T, \tau)\right\rangle & h_{-2} \cdot \frac{(2 \pi)^{2}|\tau|}{12}[r(N+1)-1], \quad r \geq \\
\left\langle\sigma_{v}^{2}(N, \tau, \tau)\right) & h_{-2} \cdot \frac{(2 \pi)^{2}|\tau|}{12} \cdot N, \quad r=1 \\
\sigma_{v}^{2}(\tau) & h_{-2} \cdot \frac{(2 \pi)^{2}|\tau|}{6}, \quad N=2, r=1 \\
D_{x}^{2}(\tau)=2 \sigma_{x}^{2}(\tau) & h_{-2} \cdot \frac{2(2 \pi)^{2}|r|^{3}}{6} \\
\psi_{x}^{2}(T, \tau) & h_{-2} \cdot \frac{(2 \pi)^{2}|\tau|^{3}}{6}(3 r-1), \quad r \geq 1 \\
& h_{-2} \cdot \frac{(2 \pi)^{2}|T|^{3}}{6}\left(\frac{3}{r}-1\right), \quad r \leq 1 .
\end{array}
$$

Flicker $y$

$$
\begin{aligned}
& S_{\nu}(f)=\frac{h_{-1}}{f} \quad\left(S_{x}(f)=\frac{h_{-1}}{(2 \pi)^{2} f^{3}}\right) \\
& r=T / \tau, \quad 0 \leq f \leq f_{h} . \\
& \text { Quantity } \\
& \text { Relation } \\
& \left\langle\sigma_{\nu}^{2}(N, T, \tau)\right\rangle \quad h_{-1} \cdot \frac{1}{N(N-1)} \sum_{n=1}^{N}(N-n) \\
& \cdot\left[-2(n r)^{2} \ln (n r)+(n r+1)^{2} \ln (n r+1)\right. \\
& +(n r-1)^{2} \ln [n r-1 \mid] \\
& \left\langle\sigma_{v}^{2}(N, \tau, \tau)\right\rangle \quad h_{-1} \cdot \frac{N \ln N}{N-1}, \quad(r=1) \\
& \sigma_{v}^{2}(r) \quad h_{-1} \cdot 2 \ln 2,(N=2, r=1) \\
& D_{z}^{2}(\tau)=2 \sigma_{z}^{2}(\tau) \quad h_{-1} \cdot 4 \tau^{2} \ln 2 \\
& \psi_{r}^{2}(I, \tau) \quad h_{-1} \cdot \tau^{2}\left(-2 r^{2} \ln r+(r+1)^{2} \ln (r+1)\right. \\
& \left.+(r-1)^{2} \ln |r-1|\right] \\
& \sim h_{-1} \cdot 2 \tau^{2}(2+\ln r), \quad r \gg 1 \\
& \sim h_{-1} \cdot 2 T^{2}(2-\ln r), \quad r \ll 1 .
\end{aligned}
$$

White $y$ (Random Walk $x$ )

$$
\begin{array}{rlrl}
S_{y}(f) & =h_{0} \quad & S_{x}(f)=\frac{h_{0}}{(2 \pi)^{2} f^{2}} \\
r & =T / \tau, \quad 0 \leq f \leq f_{h} .
\end{array}
$$

\section{Quantity}

$$
\begin{aligned}
& \left(\sigma_{v}^{2}(N, T, \tau)\right\rangle \quad \frac{h_{n}}{2} \cdot|\tau|^{-1}, \quad r \geq 1 \\
& h_{0} \cdot \frac{1}{6} r(N+1)|r|^{-1}, \quad N r \leq 1 \\
& \left(\sigma_{v}^{2}(N, \tau, \tau)\right\rangle \quad \frac{h_{n}}{2} \cdot|\tau|^{-1}, \quad r=1 \\
& \sigma_{v}^{2}(\tau) \quad \frac{h_{n}}{2} \cdot|\tau|^{-1}, \quad x=2, r=1 \\
& D_{s}^{2}(\tau)=2 \sigma_{r}^{2}(\tau) \quad h_{n} \cdot|\tau|
\end{aligned}
$$$$
\text { Relation }
$$

$\psi_{s}^{2}(T, \tau) \quad h_{0} \cdot|\tau|, \quad r \geq 1$

$$
h_{0} \cdot T, \quad r \leq 1 \text {. }
$$

Flicker $x$

$$
S_{v}(f)=h_{1}|f|\left(S_{x}(f)=\frac{h_{1}}{(2 \pi)^{2} f}\right)
$$

$r=T / \tau, \quad 2 \pi f_{h} \tau \gg 1, \quad 2 \pi f_{h} T \gg 1, \quad 0 \leq f \leq f_{h}$.

Quantity

\section{Relation}

$\left\langle\sigma_{v}^{2}(N, T, \tau)\right) \quad h_{1} \cdot \frac{2}{(2 \pi \tau)^{2}}\left\{2+\ln \left(2 \pi f_{h} \tau\right)\right.$

$$
\begin{aligned}
& +\frac{1}{N(N-1)} \sum_{n=1}^{N-1}(. V-n) \\
& \left.\cdot \ln \left[\frac{n^{2} r^{2}}{n^{2} r^{2}-1}\right]\right\}, \quad r \gg 1
\end{aligned}
$$

$(101) * *$

$$
\begin{array}{rr}
\left\langle\sigma_{h}^{2}(N, \tau, \tau)\right\rangle \quad & h_{1} \cdot \frac{2(N+1)}{N \tau^{2}(2 \pi)^{2}}\left[2+\ln \left(2 \pi f_{h} \tau\right)-\frac{\ln N}{N^{2}-1}\right], \\
r=1 \quad(102) * * \\
\sigma_{v}^{2}(\tau) \quad & h_{1} \cdot \frac{1}{\tau^{2}(2 \pi)^{2}}\left\{3\left[2+\ln \left(2 \pi f_{h} \tau\right)\right]-\ln 2\right\},
\end{array}
$$

$$
Y=2, r=1 \quad(103) * *
$$

$D_{x}^{2}(\tau)=2 \sigma_{x}^{2}(\tau) \frac{h_{1}}{(2 \pi)^{2}} \cdot 2\left\{3\left[2+\ln \left(2 \pi f_{h} \tau\right)\right]-\ln 2\right\} \quad(104) * *$

$\psi_{x}^{2}(T, \tau) \quad h_{1} \cdot \frac{4}{(2 \pi)^{2}}\left[2+\ln \left(2 \pi f_{h} \tau\right)\right], \quad r \gg 1$

$$
h_{1} \cdot \frac{2}{(2 \pi)^{2}}\left\{3\left[2+\ln \left(2 \pi f_{h} \tau\right)\right]-\ln 2\right\} \text {, }
$$$$
\tau=1 \quad(105) * *
$$

$h_{1} \cdot \frac{4}{(2 \pi)^{2}}\left[2+\ln \left(2 \pi f_{h} T\right)\right], \quad r \ll 1$.

White $x$

$$
\begin{gathered}
S_{v}(f)=h_{2} f^{2} \quad\left(S_{x}(f)=\frac{h_{2}}{(2 \pi)^{2}}\right) \\
r=T / \tau ; \delta_{k}(r-1)= \begin{cases}1, & \text { if } r=1 \\
0, & \text { otherwise }\end{cases}
\end{gathered}
$$

$2 \pi f_{h} \tau \gg 1, \quad 0 \leq f \leq f_{h}$.

Quantity

\section{Relation}

$\left\langle\sigma_{v}^{2}(N, T, \tau)\right\rangle \quad h_{2} \cdot \frac{N+\delta_{k}(\tau-1)}{N(2 \pi)^{2}} \cdot \frac{2 f_{h}}{\tau^{2}}$

$$
\left(\sigma_{v}^{2}(N, \tau, \tau)\right) \quad h_{2} \cdot \frac{V+1}{V(2 \pi)^{2}} \cdot \frac{2 f_{h}}{\tau^{2}}, \quad r=1
$$

$\sigma_{y}^{2}(r) \quad h_{2} \cdot \frac{3 f_{\Lambda}}{(2 \pi)^{2} \tau^{2}}, \quad N=2, r=1$

$D_{x}^{2}(\tau)=2 \sigma_{x}^{2}(\tau) \quad h_{2} \cdot \frac{6 f_{h}}{(2 \pi)^{2}}$

$\psi_{x}^{2}(T, \tau) \quad \dot{h}_{2} \cdot\left[2+\delta_{k}(r-1)\right] \frac{2 f_{k}}{(2 \pi)^{2}}$ 


\section{ACENOTLEDGMENT}

The author are particularly indebted to D. W. Allan, Dr. D. Halford, Dr. S. Jarris, and Dr. J. J. Filliben of the National Bureau of Standards. The suthors are also indebted to Mrs. Carol Wright for preparing the many revised copies of this paper.

\section{REFERENCES}

[1] E. T. Jaypes, "Information theory and atatistical mechanics," Phys. Rev., vol. 108, rec. 15, Oct. 1957, pp. 171-190.

[2] C. Bingham, M. D. Godfrey, and J. W. Tukey, "Modern techniques of power spectrum estimation," IEEE Trars. Audio Electrocoust., vol. AU-15, June 1967, pp. 56-66.

[3] R. B. Blackman, Linear Data Smoothing and Prediction in Theory and Practice. Reading, Mass.: Addison-Wesley, 1965.

[1] R. B. Blackman and J. W. Tukey, The Measurement of Power Spectra. New lork: Dover, 1958.

[5] E. O. Brigham and R. E. Mortor, "The fast Fourier transiorm," IEEE Spectrum, vol. 4, Dec. 1967, pp. 63-70.

(6] E. J. Baghdady, R. D. Lincoln, and B. D. Nelin. "Shortterm frequency stability: theor", measurement, and status," Proc. IEEE-NASA Symp. on Short-Term Frequency Stability (NASA SP-80), Nor. 1964, pp. 65-87; also in Proc. IEEE, vol. 53. July 1965, pp. 704-722 and Proc. IEEE (Corresp.), vol 53, Dec. 1965. pp. 2110-2111.

(i) L. Cutler and C. Searle, "Some aspects of the theory and measurement of frequency fluctuations in frequency standards." Proc. IEEE, vol. 54, Feb. 1966, pp. 136-154.

[8] D. F. Allan "Statistics of atomic frequency standards," Proc. IEEE, vol. 54, Feb. 1966 , pp. 221-230.

19] N. A. Sherart, Economic Control of Qualiey of Manufactured Product. Princeton, N. J.: Van Nostrand, 193!, p. 146.
[10] J. A. Bames, "Atomic timeleeping and the otatistics of precirion signal generator," Proc. IEEE, vol. 54, Feb. 1866, pp. $207-220$.

[11] J. A. Barnes and D. W. Allan, "An approach to the prediction of coordinated universal time," Frequency, vol. 5, Nov.-Dec. 196\%, pp. 15-20.

[12] R. F. C. Vessot el al. "An intercompgrison of bydrogen and cesum frequency otanderds," IEEE Trans. Instrum. Mees., vol. IM-15, Dec. 1966, pp. 165-176.

[18] J. A. Barnes, "Tables of bias functions, $B_{2}$ and $B_{8}$ for variances based on finite samples of processes with power law spectral densities," NBS, Wanbington, D. C., Tech. Note 375, Jan. 1969.

[14] D. B. Leeson and G. F. Johnson, "Short-term stability for a Doppler radar: requirements, measurements, and techniques," Proc. IEEE, vol. 54, Feb. 1866, pp. 244-248.

[15] W. K. Saunders in Rodar Handbook, M. I. Stolnik, Ed. New York: McGraw Hill, 1970, cb. 16.

[16] R. S. Raven, "Requirements on master oscillators for coberent rader," Proc. IEEE, vol. 54, Feb. 1966, pp. 23i-243

[1i] D. B. Leeson, "A simple model of feedback oscillator Doise spectrum," Proc. IEEE (Lett.), vol. 34, Feb. 1966, pp. 329330

[18] R. F. C. Tessot, L. Mueller, and J. Vanier, "The opecification of oscillator characteristics from mersurements made in the frequency domsin," Proc. IEEE, vol. 54, Feb. 1966. pp. $199-20 \%$.

[19] Floy'd M. Gardner, Phasclock Techniques. Nex Iork: Wiler. 1966.

[20] C. Menoud, J. Racipe and P. Kartaschof, "Atomic hrdrogen maser work at LI.R.H.. Neuchatel. Sritze:land." Proc. 21st Ann. Symp. Frequency Control, Apr. 196:. pp. 543-567

[21] A. G. Mungall, D. Morris, H. Daams, and R. Boiley. "A tomic hrdrogen maser development at the National Research Council of Cansda." Metrologia, vol. 4. July 1965, pp. 8i-94

(22] R. F. C. Vessot, "Atomic hydrogen masers. an introduction and progress report," Hewlelt-Packard J., vol. 20, Oct. 1968, pp. $15-20$.

\author{
Reprinted by permission from \\ IEEE TR + NS ACTIONS OY \\ INSTRLME.TTATION AND MEASLREMENT \\ Vol 1M-20, No. 2, Mar 19; \\ Copyrigbt (c) 1971, by the Institute of Electrical and Electronics Engineers, Inc \\ PRINTED IN THE L'.S.A
}


Reprinted, with permission, from Report 580 of the International Radio Consultative Committee (C.C.I.R.), pp. 142-150, 1986.

CHARACTERIZATION OF FREQUENCY AND PHASE NOISE

(Study Programme 3B/7)
*

(1974-1978-1986)

\section{Introduction}

Techniques to characterize and to measure the frequency and phase instabilities in frequency generators and received radio signals are of fundamental importance to users of frequency and time standards.

In 1964 a subcommittee on frequency stability was formed, within the Institute of Electrical and Electronic Engineers (IEEE) Standards Committee 14 and later (in 1966) in the Technical Committee on Frequency and Time within the Society of Instrumentation and Measurement (SIM), to prepare an IEEE standard on frequency stability. In 1969, this subcommittee completed a document proposing definitions for measures on frequency and phase stabilities. These recommended measures of stabilities in frequency generators have gained general acceptance among frequency and time users throughout the world. Some of the major manufacturers now specify stability characteristics of their standards in terms of these recommended measures.

Models of the instabilities may include both stationary and non-stationary random processes as well as systematic processes. Concerning the apparently random processes, considerable progress has been made [IEEE-NASA, 1964: IEEE, 1972] in characterizing these processes with reasonable statistical models. In contrast, the presence of systematic changes of frequencies such as drifts should not be modelled statistically, but should be described in some reasonable analytic way as measured with respect to an adequate reference standard, e.g., linear regression to determine a model for linear frequency drift. The separation between systematic and random parts however is not always easy or obvious. The systematic effects generally become predominant in the long term, and thus it is extremely important to specify them in order to giye a full characterization of a signal's stability. This Report presents some methods of characterizing the random processes and some important types of systematic processes.

Since then, additional significant work has been accomplished. For example, Baugh [1971] illustrated the properties of the Hadamard variance - a time-domain method of estimating discrete frequency modulation sidebands - particularly appropriate for Fourier frequencies less than about $10 \mathrm{~Hz}$; a mathematical analysis of this technique has been made by Sauvage and Rutman [1973]: Rutman [1972] has suggested some altemative time-domain measures while still giving general support to the subcommittee's recommendations; De Prins ef al. [1969] and De Prins and Cornelissen, [1971] have proposed alternatives for the measure of frequency stability in the frequency domain with specific emphasis on sample averages of discrete spectra. A National Bureau of Standards Monograph devotes Chapter 8 to the "Statistics of time and frequency data analysis" [Blair, 1974]. This chapter contains some measurement methods, and applications of both frequency-domain and time-domain measures of frequency/phase instabilities. It also describes methods of conversion among various time-domain measures of frequency stability, as well as conversion relationships from frequency-domain measures to timedomain measures and vice versa. The effect of a finite number of measurements on the accuracy with which the two-sample variance is determined has been specified [Lesage and Audoin, 1973, 1974 and 1976; Yoshimura, 1978]. Box-Jenkins-type models have been applied for the interpretation of frequency stability measurements [Barnes, 1976; Percival, 1976] and reviewed by Winkler [1976].

Lindsey and Chie [1976] have generalized the r.m.s. fractional frequency deviation and the two-sample variance in the sense of providing a larger class of time-domain oscillator stability measures. They have developed measures which characterize the random time-domain phase stability and the frequency stability of an oscillator's signal by the use of Kolmogorov structure functions. These measures are connected to the frequency-domain stability measure $S_{y}(\Omega$ via the Mellin transform. In this theory, polynominal type drifts are included and some theoretical convergence problems due to power-law type spectra are alleviated. They also show the close relationship of these measures to the r.m.s. fractional deviation [Cutler and Searle, 1966] and to the two-sample variance [Allan, 1966]. And finally, they show that other members from the set of stability measures developed are important in specifying performance and writing system specifications for applications such as radar, communica. tions, and tracking system engineering work.

Other forms of limited sample variances have been discussed [Baugh, 1971; Lesage and Audoin, 1975; Boileau and Picinbono, 1976] and a review of the classical and new approaches has been published [Rutman, 1978]. 
Frequency and phase instabilities may be characterized by random processes that can be represented statistically in either the Fourier frequency domain or in the time domain [Blackman and Tukey, 1959]. The instantaneous, normalized frequency departure $y(t)$ from the nominal frequency $v_{0}$ is related to the instantaneousphase fluctuation $\varphi(t)$ about the nominal phase $2 \pi v_{0} t$ by:

$$
\begin{gathered}
y(t)=\frac{1}{2 \pi v_{0}} \frac{\mathrm{d} \varphi(t)}{\mathrm{d} t}=\frac{\dot{\varphi}(t)}{2 \pi v_{0}} \\
x(t)=\frac{\varphi(t)}{2 \pi v_{0}}
\end{gathered}
$$

where $x(t)$ is the phase variation expressed in units of time.

\section{Fourier frequency domain}

In the Fourier frequency domain, frequency stability may be defined by several one-sided (the Fourier frequency ranges from 0 to $\infty$ ) spectral densities such as:

$$
S_{y}(f) \text { of } y(t), S_{\varphi}(f) \text { of } \varphi(t), S_{\dot{\varphi}}(f) \text { of } \dot{\varphi}(t), S_{x}(f) \text { of } x(t), \text { etc. }
$$

These spectral densities are related by the equations:

$$
\begin{gathered}
S_{y}(f)=\frac{\rho^{2}}{v_{0}^{2}} S_{\varphi}(f) \\
S_{\varphi}(f)=4 \pi^{2} f^{2} S_{\varphi}(f) \\
S_{x}(f)=\frac{1}{\left(2 \pi v_{0}\right)^{2}} S_{\varphi}(f)
\end{gathered}
$$

Power-law spectral densities are often employed as reasonable models of the random nluctuations in precision oscillators. In practice, it has been recognized that these random fluctuations are the sum of five independent noise processes and hence:

$$
S_{y}(f)=\left\{\begin{array}{cl}
\sum_{a=-2}^{+2} h_{a} f^{a} & \text { for } 0<f<f_{h} \\
0 & \text { for } f>f_{h}
\end{array}\right.
$$

where $h_{a}$ 's are constants, $\alpha$ 's are integers, and $f_{h}$ is the high frequency cut-off of a low pass filter. Equations (2), (3) and (4) are correct and consistent for stationary noises including phase noise. High frequency divergence is eliminated by the restrictions on $f$ in equation (5). The identification and characterization of the five noise processes are given in Table 1, and shown in Fig. 1. In practice, only two or three noise processes are sufficient to describe the random frequency fluctuations in a specific oscillator; the others may be neglected.

\section{Time-domain}

Random frequency instability in the time-domain may be defined by several sample variances. The recommended measure is the two-sample standard deviation which is the square root of the two-sample zero dead-time variance $\sigma_{y}{ }^{2}(\tau)$ [von Neumann et al., 1941; Allan, 1966; Barnes et al., 1971] defined as:

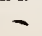

where

$$
\sigma_{y}^{2}(\tau)=\left\langle\frac{\left(\bar{y}_{k+1}-\bar{y}_{k}\right)^{2}}{2}\right\rangle
$$

$$
\bar{y}_{k}=\frac{1}{\tau} \int_{t_{k}}^{t_{k}+\tau} y(t) \mathrm{d} t=\frac{x_{k+1}-x_{k}}{\tau} \text { and } t_{k+1}=t_{k}+\tau \text { (adjacent samples) }
$$

$<>$ denotes an infinite time average. The $x_{k}$ and $x_{k+1}$ are time residual measurements made at $t_{k}$ and $t_{k+1}=t_{k}+\tau, k=0,1,2, \ldots$, and $1 / \tau$ is the fixed sampling rate which gives zero dead time between frequency measurements. By "residual" it is understood that the known systematic effects have been removed. 
If the initial sampling rate is specified as $1 / \tau_{0}$, then it has been shown [Howe et al., 1981] that in general one may obtain a more efficient estimate of $\sigma_{y}(\tau)$ using what is called "overlapping estimates". This estimate is obtained by computing equation (7).

$$
\sigma_{y}^{2}(\tau)=\frac{1}{2(N-2 m) \tau^{2}} \sum_{i=1}^{N-2 m}\left(x_{i+2 m}-2 x_{i+m}+x_{i}\right)^{2}
$$

where $N$ is the number of original time departure measurements spaced by $\tau_{0},(N=M+1$, where $M$ is the number of original frequency measurements of sample time, $\tau_{0}$ ) and $\tau=m \tau_{0}$. The corresponding confidence intervals [Howe et al., 1981], discussed in $\$ 6$, are smaller than those obtained by using equation (12), and the estimate is still unbiased.

If dead time exists between the frequency departure measurements and this is ignored in computing equation (6), it has been shown that the resulting stability values (which are no longer the Allan variances), will be biased (except for the white frequency noise) as the frequency measurements are regrouped to estimate the stability for $m \tau_{0}(m>1)$. This bias has been studied and some tables for its correction published [Barnes, 1969; Lesage, 1983].

A plot of $\sigma_{y}(\tau)$ versus $\tau$ for a frequency standard typically shows a behaviour consisting of elements as shown in Fig. 1. The first part, with $\sigma_{y}(\tau) \sim \tau^{-1 / 2}$ (white frequency noise) and/or $\sigma_{y}(\tau) \sim \tau^{-1}$ (white or flicker phase noise) reflects the fundamental noise properties of the standard. In the case where $\sigma_{y}(\tau) \sim \tau^{-1}$, it is not practical to decide whether the oscillator is perturbed by white phase noise or by flicker phase noise. Alternative techniques are suggested below. This is a limitation of the usefulness of $\sigma_{y}(\tau)$ when one wishes to study the nature of the existing noise sources in the oscillator. A frequency-domain analysis is typically more adequate for Fourier frequencies greater than about $1 \mathrm{~Hz}$. This $\tau^{-1}$ and/or $\tau^{-1 / 2}$ law continues with increasing averaging time until the so-called flicker "floor" is reached, where $\sigma_{y}(\tau)$ is independent of the averaging time $\tau$. This behaviour is found in almost all frequency standards; it depends on the particular frequency standard and is not fully understood in its physical basis. Examples of probable causes for the flicker "floor" are power supply voltage fluctuations, magnetic field fluctuations, changes in components of the standard, and microwave power changes. Finally the curve shows a deterioration of the stability with increasing averaging time. This occurs typically at times ranging from hours to days, depending on the particular kind of standard.

A "modified Allan variance", MOD $\sigma_{y}^{2}(\tau)$, has been developed [Allan and Barnes, 1981] which has the property of yielding different dependences on $\tau$ for white phase noise and flicker phase noise. The dependences for $M O D \sigma_{y}(\tau)$ are $\tau^{-3 / 2}$ and $\tau^{-1}$ respectively. The relationships between $\sigma_{y}(\tau)$ and $M O D \sigma_{y}(\tau)$ are also explained in [Allan and Barnes, 1981; IEEE 1983, Lesage and Ayi, 1984]. MOD $\sigma_{y}(\tau)$ is estimated using the following equation:

$$
M O D \sigma_{y}^{2}(\tau)=\frac{1}{2 \tau^{2} m^{2}(N-3 m+1)} \sum_{j=1}^{N-3 m+1}\left[\sum_{i=j}^{m+j-1}\left(x_{i+2 m}-2 x_{i+m}+x_{i}\right)\right]^{2}
$$

where $N$ is the original number of time measurements spaced by $\tau_{0}$, and $\tau=m \tau_{0}$ the sample time of choice. Properties and confidence of the estimate are discussed in Lesage and Ayi [1984]. Jones and Tryon [1983] and Barnes et al. [1982] have developed maximum likelihood methods of estimating $\sigma_{y}(\tau)$ for the specific models of white frequency noise and random walk frequency noise, which has been shown to be a good model for observation times longer than a few seconds for caesium beam standards.

\section{Conversion between frequency and time domains}

In general, if the spectral density of the normalized frequency fuctuations $S_{y}(f)$ is known, the two-sample variance can be computed [Barnes et al., 1971; Rutman, 1972]:

$$
\sigma_{y}^{2}(\tau)=2 \int_{0}^{f_{h}} S_{y}(f) \frac{\sin ^{4} \pi \tau f}{(\pi \tau f)^{2}} d f
$$



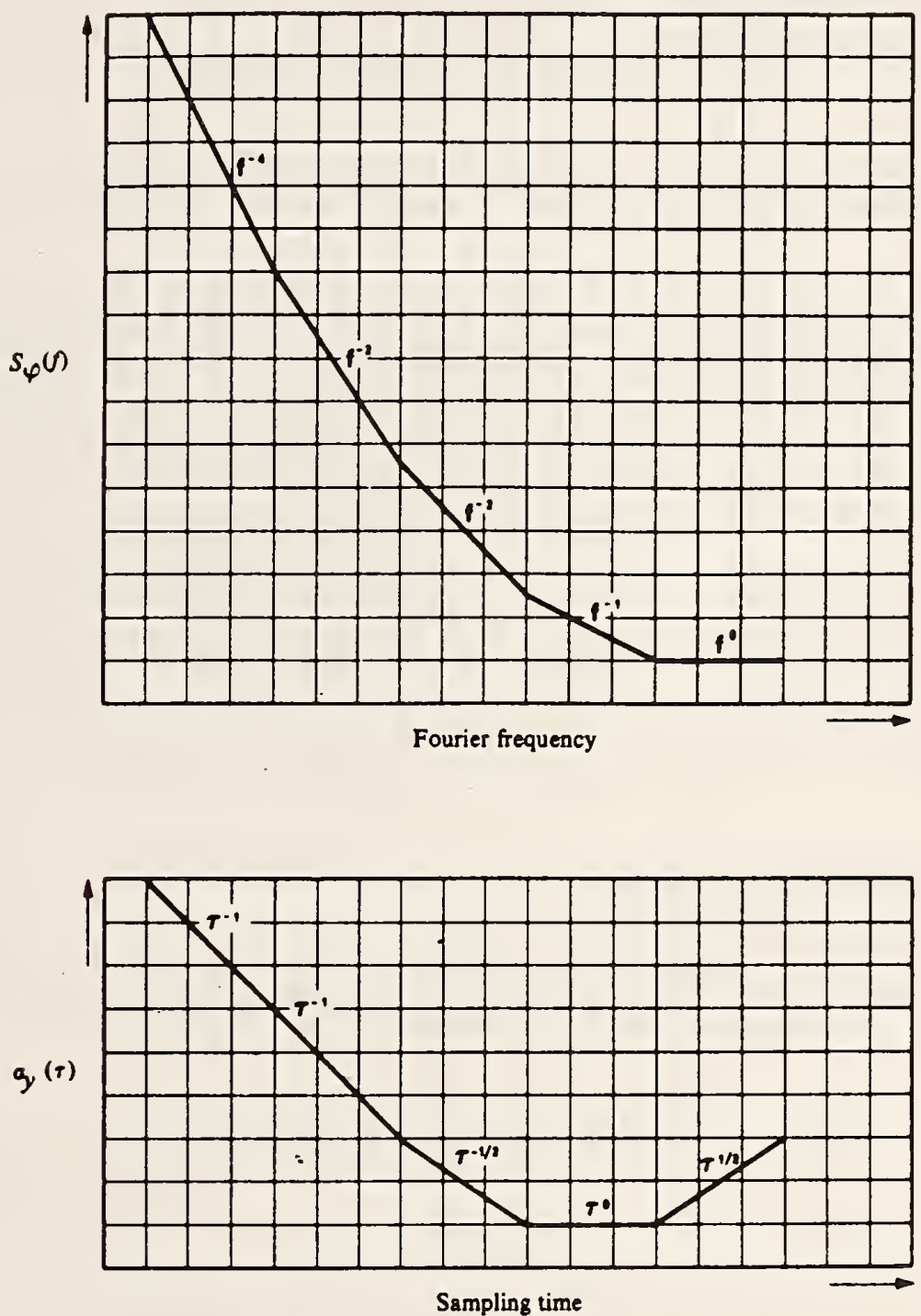

FIGURE 1 - Slope characteristics of the five independent noise processes (log scale)

Specifically, for the power law model given by equation (5), the time-domain measure also follows the power law as derived by Cutler from equations (5) and (9).

$$
\sigma_{y}^{2}(\tau)=h_{-2} \frac{(2 \pi)^{2}}{6} \tau+h_{-1} 2 \log _{e} 2+h_{0} \frac{1}{2 \tau}+h_{1} \frac{1.038+3 \log _{e}\left(2 \pi f_{h} \tau\right)}{(2 \pi)^{2} \tau^{2}}+h_{2} \frac{3 f_{h}}{(2 \pi)^{2} \tau^{2}}
$$

Note. - The factor 1.038 in the fourth term of equation (10) is different from the value given in most previous publications. 
The values of $h_{a}$ are characteristics of oscillator frequency noise. One may note for integer values (as often seems to be the case) that $\mu=-\alpha-1$, for $-3 \leqslant \alpha \leqslant 1$, and $\mu \approx-2$ for $\alpha \geq 1$ where $\sigma_{y}^{2}(\tau) \sim \tau^{\mu}$.

These conversions have been verified experimentally [Brandenberger et al., 1971] and by computation [Chi, 1977]. Table II gives the coefficients of the translation among the frequency stability measures from time domain to frequency domain and from frequency domain to time domain.

The slope characteristics of the five independent noise processes are plotted in the frequency and time domains in Fig. 1 (log log scale).

\section{Messurement techniques}

The spectral density of phase fluctuations $S_{\Phi}(f)$ may be approximately measured using a phase-locked loop and a low frequency wave analyzer [Meyer, 1970; Walls et al., 1976]. A double-balanced mixer is used as the phase detector in a lightly coupled phase lock loop. The measuring system uses available state-of-the-art electronic components; also a very high quality oscillator is used as the reference. For very low Fourier frequencies (well below $1 \mathrm{~Hz}$ ), digital techniques have been used [Atkinson et al., 1963; De Prins et al., 1969; Babitch and Oliverio, 1974]. New methods of measuring time (phase) and frequency stabilities have been introduced with picosecond time precision [Allan and Daams, 1975], and of measuring the Fourier frequencies of phase noise with $30 \mathrm{~dB}$ more sensitivity than the previous state of the art [Walls et al., 1976].

Several measurement systems using frequency counters have been used to determine time-domain stability with or without measurement dead time [Allan, 1974; Allan and Daams, 1975]. A system without any counter has - also been developed [Rutman, 1974: Rutman and Sauvage, 1974]. Frequency measurements without dead time can be made by sampling time intervals instead of measuring frequency directly. Problems encountered when dead time exists between adjacent frequency measurements have also been discussed and solutions recommended [Blair, 1974; Allan and Daams, 1975; Ricci and Peregrino, 1976]. Discrete spectra have been measured by Groslambert et al. [1974].

\section{Confidence limits of time domain measurements}

A method of data acquisition is to measure time variations $x_{j}$ at intervals $\tau_{0}$. Then $\sigma_{y}(\tau)$ can be estimated for any $\tau=n \tau_{0}$ ( $n$ is any positive integer) since one may use those $x_{j}$ values for which $j$ is equal to $n k$. An estimate for $\sigma_{y}(\tau)$ can be made from a data set with $M$ measurements of $\bar{y}_{j}$ as follows:

$$
\hat{\sigma}_{y}\left(n \tau_{0}\right)=\hat{\sigma}_{y}(\tau) \simeq\left|\frac{1}{2(M-1)} \sum_{j=1}^{M-1}\left(\bar{y}_{j+1}-y_{j}\right)^{2}\right|^{1 / 2}
$$

or equivalent

$$
\hat{\sigma}_{y}(\tau) \cong\left|\frac{1}{2 \tau^{2}(M-1)} \sum_{j=1}^{M-1}\left(x_{j+2}-2 x_{j+1}+x_{j}\right)^{2}\right|^{1 / 2}
$$

Thus, one can ascertain the dependence of $\sigma_{y}(\tau)$ as a function of $\tau$ from a single data set in a very simple way. For a given data set, $M$ of course decreases as $n$ increases.

To estimate the confidence interval or error bar for a Gaussian type of noise of a particular value $\sigma_{y}(\tau)$ obtained from a finite number of samples [Lesage and Audoin, 1973] have shown that:

$$
\text { Confidence Interval } I_{\alpha} \approx \sigma_{y}(\tau) \cdot \kappa_{\alpha} \cdot M^{-1 / 2} \text { for } M>10
$$

where:

$M:$ total number of data points used in the estimate,

$\alpha$ : as defined in the previous section,

$\kappa_{2}=\kappa_{1}=0.99$,

$x_{0}=0.87$,

$\kappa_{-1}=0.77$,

$\kappa_{-2}=0.75$. 
As an example of the Gaussian model with $M=100, \alpha=-1$ (flicker frequency noise) and $\sigma_{y}(\tau=1$ second $)=10^{-12}$, one may write:

$$
l_{a}=\sigma_{y}(\tau) \cdot \kappa_{a} \cdot M^{-1 / 2}=\sigma_{y}(\tau) \cdot(0.77) \cdot(100)^{-1 / 2}=\sigma_{y}(\tau) \cdot(0.077)
$$

which gives:

$$
\sigma_{y}(\tau=1 \text { second })=(1 \pm 0.08) \times 10^{-12}
$$

A modified estimation procedure including dead-time between pairs of measurements has also been developed [Yoshimura, 1978], showing the influence of frequency fluctuations auto-correlation.

\section{Conclusion}

The statistical methods for describing frequency and phase instability and the corresponding power law spectral density model described are sufficient for describing oscillator instability on the short term. Equation (9) shows that the spectral density can be unambiguously transformed into the time-domain measure. The converse is not true in all cases but is true for the power law spectra often used to model precision oscillators.

Non-random variations are not covered by the model described. These can be either periodic or monotonic. Periodic variations are to be analyzed by means of known methods of harmonic analysis. Monotonic variations are described by linear or higher order drift terms.

TABLE I - The functional characteristics of five independent noise processes

\begin{tabular}{|c|c|c|c|c|}
\hline \multirow{4}{*}{ Description of noise process } & \multicolumn{4}{|c|}{ Slope characteristics of $\log \log$ plot } \\
\hline & \multicolumn{2}{|c|}{ Frequency-domaine } & \multicolumn{2}{|c|}{ Time-domaine } \\
\hline & $S_{y}(\Omega)$ & $S_{\varphi}(\Omega)$ or $S_{x}(\Omega)$ & $\sigma^{2}(\tau)$ & $\sigma(\tau)$ \\
\hline & $\alpha$ & $\beta=\alpha-2$ & $\mu$ & $\mu / 2$ \\
\hline Random walk frequency : & -2 & -4 & 1 & Hh \\
\hline Flicker frequency & -1 & -3 & 0 & 0 \\
\hline White frequency & 0 & -2 & -1 & $-1 / 2$ \\
\hline Flicker phase & 1 & -1 & -2 & -1 \\
\hline White phase & 2 & 0 & -2 & -1 \\
\hline
\end{tabular}
for frequency instability of oscillators

$$
\begin{aligned}
& S_{y}(f)=h_{a} f^{a} \\
& S_{Q}(f)=v_{0}^{2} h_{a} f^{a-2}=v_{0}^{2} h_{a} f^{\beta} \quad(\beta=\alpha-2) \\
& \sigma^{2}(\tau) \sim|\tau|^{\mu} \\
& S_{x}(f)=\frac{1}{4 \pi^{2}} h_{a} f^{a-2}=\frac{1}{4 \pi^{2}} h_{a} f^{\beta} \\
& \sigma(\tau) \sim|\tau|^{\mu \Omega}
\end{aligned}
$$


TABLE II - Translation of frequency stability measures from spectral densities in frequency domain 10 variance in time domain and vice versa $\left(\right.$ for $\left.2 \pi f_{h} \tau>1\right)$

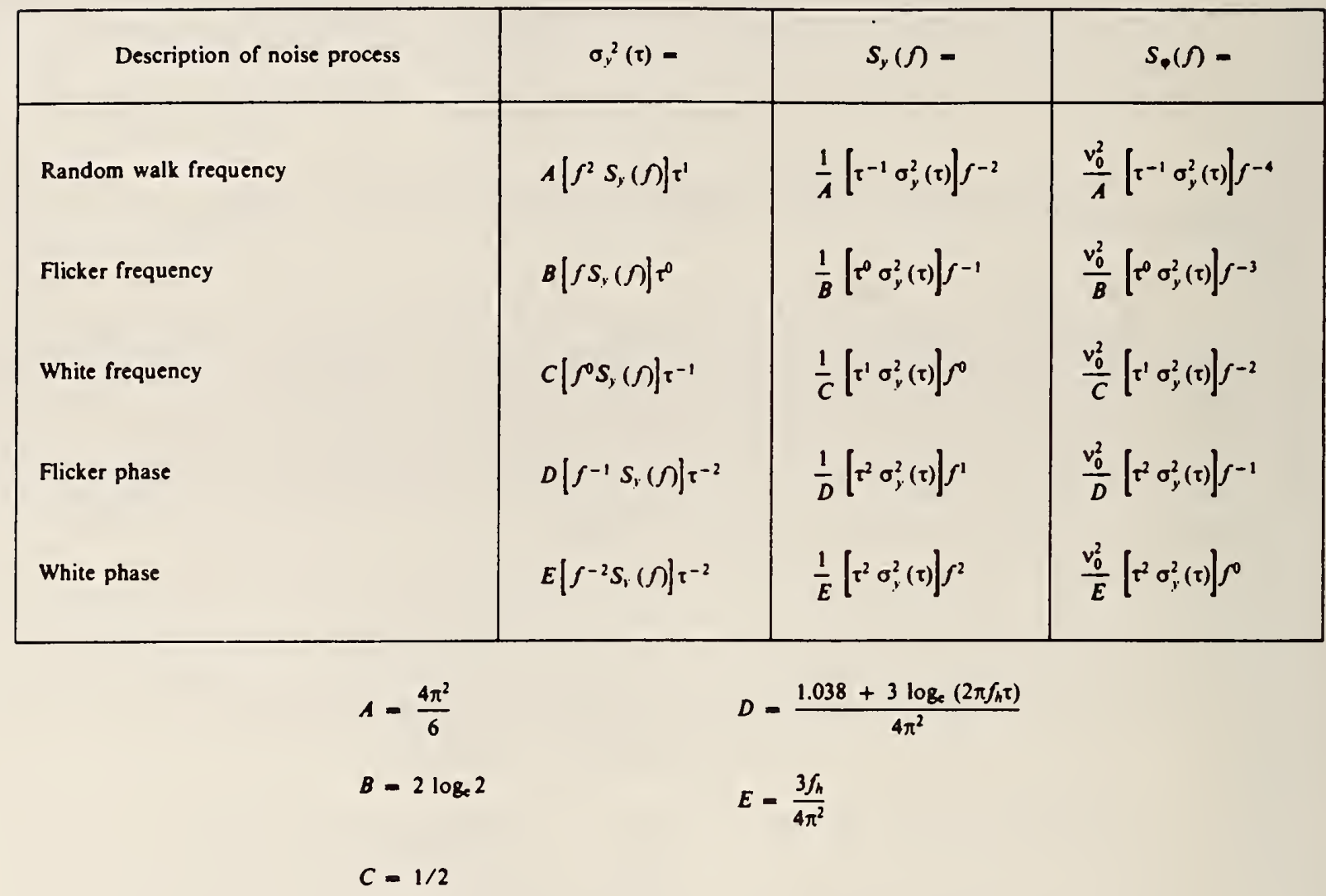

\section{REFERENCES}

ALLAN, D. W. [February, 1966] Statistics of atomic frequency standards. Proc. IEEE, Vol. 54, 221-230.

ALLAN, D. W. [December, 1974] The measurement of frequency and frequency stability of precision oscillators. Proc. 6th Annual Precise Time and Time Interval (PTTI) Planning Meeting NASA/DOD (US Naval Research Laboratory, Washington, DC), 109-142.

ALLAN, D. W. and BARNES, J. A. [May, 1981] A modified "Allan Variance" with increased oscillator characterization ability. Proc. 35th Annual Symposium on Frequency Control. Philadelphia, PA, USA (US Army Electronics Command, Ft. Monmouth, NJ 07703), 470-476 (Electronic Industries Association, Washington, DC 20006, USA).

ALLAN, D. W. and DAAMS, H. [May, 1975] Picosecond time difference measurement system. Proc. of the 29th Annual Symposium on Frequency Control, 404-411.

ATKINSON, W. K., FEY, L. and NEWMAN, J. [February, 1963] Spectrum analysis of extremely low-frequency variations of quartz oscillators. Proc. IEEE, Vol. 51, $2,379$.

BABITCH, D. and OLIVERIO, J. [1974] Phase noise of various oscillators at very low Fourier frequencies. Proc. of the 28th Annual Symposium on Frequency Control, 150-159.

BARNES, J. A. [January, 1969] Tables of bias functions, $B_{1}$ and $B_{2}$, for variances based on finite samples of processes" with power law spectral densities. Tech. Note No. 375, National Bureau of Standards, Washington, DC, USA.

BARNES, J. A. [August, 1976] Models for the interpretation of frequency stability measurements. NBS Technical Note 683.

BARNES, J. A., CHI, A. R., CUTLER, L. S., HEALEY, D. J., LEESON, D. B., McGUNIGAL, T. E., MULLEN, J. A., SMITH, W. L., SYDNOR, R., VESSOT, R. F. and WINKLER, G. M. R. [May, 1971] Characterization of frequency stability. IEEE Trans. Instr. Meas., Vol. IM-20, 105-120.

BARNES, J. A., JONES, R. H., TRYON, P. V. and ALLAN, D. W. [December, 1982] Noise models for atomic clocks. Proc. 14th Annual Precise Time and Time Interval (PTTl) Applications and Planning Meeting, Washington, DC, USA, 295-307.

BAUGH, R. A. [1971] Frequency modulation analysis with the Hadamard variance. Proc. 25th Annual Symposium on Frequency Control, 222-225.

BLACKMAN, R. B. and TUKEY, J. M. [1959] The measurement of power spectra. (Dover Publication, Inc., New York, N.Y.). 
BLAIR, B. E. (Ed.) [May, 1974] Time and frequency: theory and fundamentals. NBS Monograph No. 140. (US Government Printing Office, Washington, D.C. 20402).

BOILEAU, E. and PICINBONO, B. [March, 1976] Statistical study of phase fluctuations and oscillator stability. IEEE Trans. Instr. Meas., Vol. 25, 1, 66-75.

BRANDENBERGER, H., HADORN, F., HALFORD, D. and SHOAF, J. H. [1971] High quality quartz crystal oscillators: frequency-domain and time-domain stability. Proc. 25th Annual Symposium on Frequency Control, 226-230.

CHI, A. R. [December, 1977] The mechanics of translation of frequency stability measures between frequency and time-domain measurements. Proc. 9th Annual Precise Time and Time Interval (PTTI) Applications and Planning Meeting, Greenbelt, MD, USA.

CUTLER, L. S. and SEARLE, C. L. [February, 1966] Some aspects of the theory and measurement of frequency fluctuations in frequency standards. Proc. IEEE, Vol. 54, 136-154.

DE PRINS, J. and CORNELISSEN, G. [October, 1971] Analyse spectrale discrète. Eurocon (Lausanne, Switzerland).

DE PRINS, J., DESCORNET, G., GORSKI, M. and TAMINE, J. [December, 1969] Frequency-domain interpretation of oscillator phase stability. IEEE Trans. Instr. and Meas., Vol. IM-18, 251-261.

GROSLAMBERT, J., OLIVIER, M. and UEBERSFELD, J. [December, 1974] Spectral and short-term stability measurements. IEEE Trans. Instr. Meas., Vol. IM-23 4, 518-521.

HOWE, D. A., ALlAN, D. W. and BARNES, J. A. [May, 1981] Properties of signal sources and measurement. Proc. 35th Annual Symposium on Frequency Control, Philadelphia, PA, USA (US Army Electronics Command, Ft. Monmouth, NJ 07703), 669-717 (Electronics Industries Association, Washington, DC 20006, USA).

IEEE [May, 1972] Special issue on time and frequency. Proc. IEEE, Vol. 60, 5.

IEEE [1983] Frequency Stability: Fundamentals and Measurement, 77-80. Ed. V. F. Kroupa. IEEE Press Books.

IEEE-NASA [1964] Proceedings of Symposium on short-term frequency stability. NASA Publication SP 80.

JONES, R. H. and TRYON, P. V. [January-February, 1983] Estimating time from atomic clocks. NBS Res., Vol. 88, 1, $17-24$.

LESAGE, P. [1983] Characterization of frequency stability: Bias due to the juxtaposition of time interval measurements. IEEE Trans. Instr. Meas., Vol. IM-32, 1, 204-207.

LESAGE, P. and AUDOIN, F. [June, 1973] Characterization of frequency stability: uncertainty due to the finite number of measurements. IEEE Trans. Instr. Meas., Vol. IM-22, 1, 103.

LESAGE, P. and AUDOIN, C. [March, 1974] Correction to: Characterization of frequency stability: uncertainty due to finite number of measurements. IEEE Trans. Instr. Meas., Vol. IM-23, 1, 103.

LESAGE, P. and AUDOIN, F. [May, 1975] A time domain method for measurement of the spectral density of frequency fluctuations at low Fourier frequencies. Proc. of the 29th Annual Symposium on Frequency Control, 394-403.

LESAGE, P. and AUDOIN, C. [September, 1976] Correction to: Characterization of frequency stability: uncertainty due to the finite number of measurements. IEEE Trans. Instr. Meas., Vol. IM-25, 3.

LESAGE, P. and AYI, T. [December, 1984] Characterization of frequency stability: analysis of modified Allan variance and properties of its estimate. IEEE Trans. Instr. Meas., Vol. IM-33, 4, 332-336.

LINDSEY, W.C. and CHIE, C.M. [Decẹmber, 1976] Theory of oscillator instability based upon structure functions. Proc. IEEE, Vol. 64, 1662-1666.

MEYER, D. G. [November, 1970] A test set for the accurate measurements of phase noise on high-quality signal sources. IEEE Trans. Insir. Meas., Vol. IM-19, 215-227.

PERCIVAL, D. B. [June, 1976] A heuristic model of long-term atomic clock behavior. Proc. of the 30th Annual Symposium on Frequency Control.

RICCI, D. W. and PEREGRINO, L. [June, 1976] Phase noise measurement using a high resolution counter with on-line data processing. Proc. of the 30th Annual Symposium on Frequency Control.

RUTMAN, J. [February, 1972] Comment on characterization of frequency stability. IEEE Trans. Instr. Meas., Vol. IM-21, 2, 85.

RUTMAN, J. [March, 1974] Characterization of frequency stability: a transfer function approach and its application to measurements via filtering of phase noise. IEEE Trans. Instr. Meas., Vol. IM-23, 1, 40-48.

RUTMAN, J. [September, 1978] Characterization of phase and frequency instabilities in precision frequency sources: fifteen years of progress. Proc. IEEE, Vol. 66, 9, 1048-1075.

RUTMAN, J. and SAUVAGE, G. [December, 1974] Measurement of frequency stability in the time and frequency domains via filtering of phase noise. IEEE Trans. Instr. Meas., Vol. IM-23, 4, 515, 518.

SAUVAGE, G. and RUTMAN, J. [July-August, 1973] Analyse spectrale du bruit de fréquence des oscillateurs par la variance de Hadamard. Ann. des Télécom., Vol. 28, 7-8, 304-314.

VON NEUMANN, J., KENT, R. H., BELLINSON, H. R. and HART, B. 1. [1941] The mean square successive difference. Ann. Math. Stat., 12, 153-162.

WALLS, F. L., STEIN, S. R., GRAY, J.E. and GLAZE, D. J. [June, 1976] Design considerations in state-of-the-art signal processing and phase noise measurement systems. Proc. of the 30th Annual Symposium on Frequency Control.

WINKLER, G. M.R. [December, 1976] A brief review of frequency stability measures. Proc. 8th Annual Precise Time and Time Interval (PTTI) Applications and Planning Meeting (US Naval Research Laboratory, Washington, DC), 489-528.

YOSHIMURA, K. [March, 1978] Characterization of frequency stability: Uncertainty due to the autocorrelation of the frequency fluctuations. IEEE Trans. Instr. Meas., IM-27 1, 1-7. 


\section{BIBLIOGRAPHY}

ALLAN, D. W., et al. [1973] Performance, modelling, and simulation of some caesium beam clocks. Proc. 27th Annual Symposium on Frequency Control, 334-346.

FISCHER, M.C. [December, 1976] Frequency stability measurement procedures. Proc. 8th Annual Precise Time and Time Interval (PTTl) Applications and Planning Meeting (US Naval Research Laboratory, Washington, DC), 575-618.

HALFORD, D. [March, 1968] A general mechanical model for $|f|^{\circ}$ spectral density noise with special reference to nicker noise $1 /|f|$. Proc. IEEE (Corres.), Vol. 56, 3, 251-258.

MANDELBROT, B. [April, 1967] Some noises with 1/f spectrum, a bridge between direct current and white noise. IEEE Trans. Inf. Theory, Vol. IT-13, 2, 289-298.

RUTMAN, J. and UEBERSFELD, J. [February, 1972] A model for flicker frequency noise of oscillators. Proc. IEEE, Vol. 60, 2, 233-235.

VANIER, J. and TETU, M. [1978] Time domain measurement of frequency stability: a tutorial approach. Proc. 10th Annual Precise Time and Time Interval (PTTI) Applications and Planning Meeting, Washington, DC, USA, 247-291.

VESSOT, R. [1976] Frequency and Time Standards. Chap. 5.4 Methods of Experimental Physics, Academic Press, $198-227$.

VESSOT, R., MUELLER, L. and VANIER, J. [February, 1966] The specification of oscillator characteristics from measurements made in the frequency domain. Proc. IEEE, Vol. 54, 2, 199-207.

WINKLER, G. M. R., HALL, R. G. and PERCIVAL, D. B. [October, 1970] The US Naval Observatory clock time reference and the performance of a sample of atomic clocks. Metrologia, Vol. 6, 4, 126-134. 
Reprinted, with permission, from P. Lesage and C. Audoin in Radio Science, Vol. 14, No. 4, pp. 521-539, 1979, Copyright ${ }^{\odot}$ by the American Geophysical Union.

Characterization and measurement of time and frequency stability

\author{
P. Lesage and C. Audoin
}

Laboratoire de l'Horloge Atomique, Equipe de Recherche du CNRS, Université Paris-Sud, Orsay, France

(Received February 1, 1979.)

\begin{abstract}
The roles which spectral density of fractional frequency fluctuations, two-sample variance, and power spectra play in different parts of the electromagnetic spectrum are introduced. Their relationship is discussed. Data acquisition in the frequency and the time domain is considered, and examples are given throughout the spectrum. Recently proposed methods for the characterization of a single high-quality frequency source are briefly described. Possible difficulties and limitations in the interpretation of measurement results are specified, mostly in the presence of a dead time between measurements. The link between past developments in the field, such as two-sample variance and spectral analysis from time domain measurement, and recentiy introduced structure functions is emphasized.
\end{abstract}

\section{INTRODUCTION}

Progress in the characterization of time and frequency stability has been initiated owing to the work of the various authors of papers delivered at the IEEE-NASA Symposium on Short Term Frequency Stability [1964] and of articles published in a special issue of the Proceedings of the IEEE [1966]. Presently widespread definitions of frequency stability have been given by Barnes et al. [1971]. Many of the most important articles on the subject of time and frequency have been gathered in the NBS Monograph 140 [1974]. Since that time, many papers have been published which outline different aspects of the field. Owing to the extent of the subject, they will be only partly reviewed here. We will emphasize recently proposed principles of measurements and recent developments in the time domain characterization. of frequency stability. The subject of time prediction and modeling as well as its use for estimation of the spectrum of frequency fluctuations [Percival, 1978] are beyond the scope of this paper. Recent reviews which outline several different aspects of the field of time and frequency characterization have been published [Barnes, 1976; Winkler, 1976; Barnes, 1977; Rutman, 1978; Kartaschoff, 1978].

\section{DEFINITIONS: MODEL OF FREQUENCY FLUCTUATIONS}

The instantaneous output voltage of a frequency generator can be written as

Copyright 1979 by the American Geophysical Uajoa.

$$
\nu(t)=\left[V_{0}+\Delta V(t)\right] \cos \left[2 \pi v_{0} t+\varphi(t)\right]
$$

where $V_{0}$ and $\nu_{0}$ are constants which represent the nominal amplitude and frequency, respectively. $\Delta V(t)$ and $\varphi(t)$ denote time-dependent voltage and phase variations.

Fractional amplitude fluctuations are defined by

$$
\varepsilon(t)=\Delta V(t) / V_{0}
$$

A power spectral density of fractional amplitude fluctuations $S_{e}(f)$ can be introduced if amplitude fluctuations are random and stationary in the wide sense. Usually, for high-quality frequency sources, one has

$$
|\varepsilon(t)| \ll 1
$$

and amplitude fluctuations are neglected. However, it is known that amplitude fluctuations can be converted into phase fluctuations in electronic circuits used for frequency metrology [Barillet and Audoin, 1976; Bava et al., 1977a] and that they may perturb measurement of phase fluctuations [Brendel et al., 1977]. It is then likely that amplitude fluctuations will become the subject of more detailed analysis in the future.

According to the conventional definition of instantaneous frequency we have

$$
\nu(t)=\nu_{0}+(1 / 2 \pi) \dot{\varphi}(t)
$$

In a stable frequency generator the condition

$$
|\dot{\varphi}(t)| / 2 \pi v_{0} \ll 1
$$

is generally satisfied.

We will use the following notations [Barnes et 
al., 1971]:

$$
x(t)=\frac{\varphi(t)}{2 \pi v_{0}} \text { and } y(t)=\frac{\dot{\varphi}(t)}{2 \pi v_{0}}
$$

where $x(t)$ and $y(t)$ are the fractional phase and frequency fluctuations, respectively. The quantity $x(t)$ represents the fluctuation in the time defined by the generator considered as a clock.

At first, we will make the following assumptions:

1. The quantities $x(t)$ and $y(t)$ are random functions of time with zero mean values, which implies that systematic trends are removed [Barnes et al., 1971]. They might be due to ageing or to imperfect decoupling from environmental changes such as temperature, pressure, acceleration, or voltage. Characterization of drifts will be considered in section 8.

2. The statistical properties of the stable frequency generators are described by a model which is stationary of order 2. This point has been fully discussed in the literature [Barnes et al., 1971; Boileau and Picinbono, 1976; Barnes, 1976]. This assumption allows one to derive useful results and to define simple data processing for the characterization of frequency stability.

Actual experimental practice shows that, besides long-term frequency drifts, the frequency of a high-quality frequency source can be perturbed by a superposition of independent noise processes, which can be adequately represented by random fluctuations having the following one-sided power spectral density of fractional frequency fluctuations:

$$
S_{y}(f)=\sum_{a=-2}^{2} h_{a} f^{a}
$$

$S_{y}(f)$ is depicted in Figure 1. Its dimensions are $\mathrm{Hz}^{-1}$. Lower values of a may be present in the spectral density of frequency fluctuations. They have not been clearly identified yet because of experimental difficulties related to very long term data acquisition and to control of experimental conditions for long times. Moreover, the related noise processes may be difficult to distinguish from systematic drifts.

Finite duration of measurements introduces a low-frequency cutoff which prevents one from obtaining information at Fourier frequencies smaller

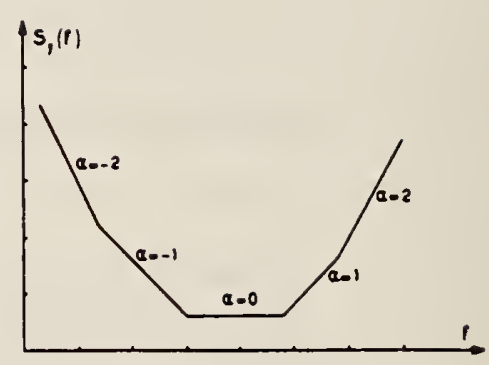

Fig. 1. Asymptotic log-log plot os $S_{y}(\cap)$ for commonly encountered noise processes.

than $1 / \theta$, approximately, where $\theta$ is the total duration of the measurement [Cutler and Searle, 1966]. Alternatively, this made it possible to invoke physical arguments to remove some possible mathematical difficulties related to the divergence of $S_{y}(f)$ as $f \rightarrow 0$ for $\alpha<0$.

Furthermore, high pass filtering is always present in the measuring instruments or in the frequency generator to be characterized. It insures convergence conditions at the higher-frequency side of the power spectra for $\alpha>0$.

The spectral density of fractional phase fluctuations is also often considered. From (6), one can write, at least formally,

$$
S_{x}(n)=\left(1 / 4 \pi^{2} f^{2}\right) S_{y}(n)
$$

The dimensions of $S_{x}(f)$ are $\mathrm{s}^{2} \mathrm{~Hz}^{-1}$. Similarly, the spectral density of phase fluctuations $\varphi(t)$ is such as

$$
S_{\varphi}(f)=\left(2 \pi v_{0}\right)^{2} S_{s}(f)
$$

It is expressed in (rad) ${ }^{2} \mathrm{~Hz}^{-1}$.

The quantity $\mathscr{L}(f)$ [Halford et al., 1973] is sometimes considered to characterize phase fluctuations. If phase fluctuations at frequencies $>f$ are small compared with 1 rad, one has

$$
\mathscr{L}(\Omega)=\frac{1}{2} S_{i}(\Omega)
$$

where $S_{\varphi}(f)$ is the spectral density of phase fluctuations of the frequency generator considered. The definition of $\mathscr{L}(f)$ implies a connection with the radio frequency spectrum, and its use is not recommended.

Since the class of noise processes for which $y(t)$ is stationary is broader than that for which the 
TABLE 1. Designation of noise processes

\begin{tabular}{clc}
\hline a & \multicolumn{1}{c}{ Designation } & Class of Stationarity \\
\hline 2 & white noise of phase & $\begin{array}{c}\text { stationary phase } \\
\text { fluctuations } \\
\text { stationary first-order } \\
\text { phase increments } \\
\text { stationary first-order } \\
\text { phase increments } \\
\text { stationary second-order } \\
\text { phase increments } \\
\text { stationary second-order } \\
\text { phase increments }\end{array}$ \\
\hline-1 flicker noise of frequency & random walk of frequency &
\end{tabular}

phase is stationary [Boileau and Picinbono, 1976], $S_{y}(f)$ should preferably be used in mathematical analysis. However, it is true that many experimental setups transduce $\varphi(t)$ into voltage fluctuations and allow one to experimentally determine an estimate of its power spectral density $S_{\varphi}(f)$.

Table 1 shows the designations of the noise processes considered. It also indicates the class of stationarity to which they pertain, as will be justified later on.

$S_{y}(f)$ is one of the recommended definitions of frequency stability [Barnes et al., 1971]. It gives the widest information on frequency deviations $y(t)$ within the limits stated previously.

\section{NOISE PROCESSES IN FREQUENCY GENERATORS}

The white phase noise $(\alpha=2)$ predominates for $f$ large enough. It is the result of the additive thermal (for the lower part of the electromagnetic spectrum, including microwaves) or quantum (for optical frequencies) noise which is unavoidably. superimposed on the signal generated in the oscillator [Cutler and Searle, 1966]. It leads to a one-sided spectral density $S_{y}(f)$ of the form $F k T \gamma^{2} / \nu_{0}^{2} P$ or $F h v_{0} f^{2} / \nu_{0}^{2} P$, depending on the frequency range, where $k$ is Boltzmann's constant, $h$ is Planck's constant, $T$ is the absolute temperature, $F$ is the noise figure of the components under consideration, and $P$ is the power delivered by atoms.

The flicker phase noise $(\alpha=1)$ is generated mainly in transistors, where this noise modulates the current [Halford et al., 1968; Healey, 1972]. The theory of this noise is not yet very well understood. Diffusion processes across junctions of semiconductor devices may produce this noise.

White noise of frequency $(\alpha=0)$ is present in oscillators. It is the result of the noise perturbation in the generation of the oscillation which is due to white noise within the bandwidth of the frequency-determining element of the oscillator [Blaquière, 1953a, b]. It is often masked by other types of noise but has been observed in lasers [Siegman and Arrathoon, 1968] and more recently in masers [Vessot et al., 1977]. The one-sided spectral density of fractional frequency fluctuations is then $k T / P Q^{2}$ of $h v_{0} / P Q^{2}$ depending on the frequency range, as stated above. $Q$ is the quality factor of the frequency-determining element.

White noise of frequency is typical of passive frequency standards such as cesium beam tube and rubidium cell devices as well as stabilized lasers. It is related to the shot noise in the detection of the resonance to which an oscillator is slaved [Cutler and Searle, 1966].

Flicker noise of frequency and the random walk frequency noise for which $\alpha=-1$ and -2 , respectively, are sources of limitation in the long-term frequency stability of frequency sources. They are observed in active devices as well as passive ones. For instance, flicker and random walk frequency noises have been observed in quartz crystal resonators [Wainwright et al., 1974] and rubidium masers [Vanier et al., 1977]. The origin is not well understood yet. It might be connected, in the first case, with fluctuations in the phonon energy density [Musha, 1975].

Figures 3 and 5 show, for the purpose of illustration, $S_{y}(f)$ for a hydrogen maser for $10^{-3} \leqslant f \leqslant$ $3 \mathrm{~Hz}$ [Vessot et al., 1977] and for an iodinestabilized He-Ne laser for $10^{-2} \leqslant f \leqslant 100 \mathrm{~Hz}$ [Cérez et al., 1978]. In both cases, $S_{y}(f)$ is derived from the results of time domain frequency measurements

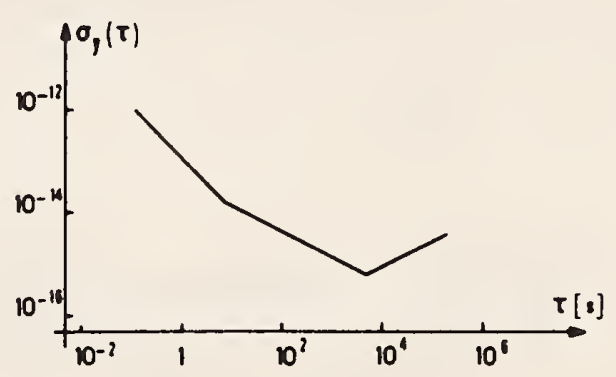

Fig. 2. Frequency stability, characterized by the root mean square of the two-sample variance of fractional frequency fluctuation, of hydrogen maser. The part of the graph with a slope of $-\frac{1}{2}$ is typical of white noise of frequency. 


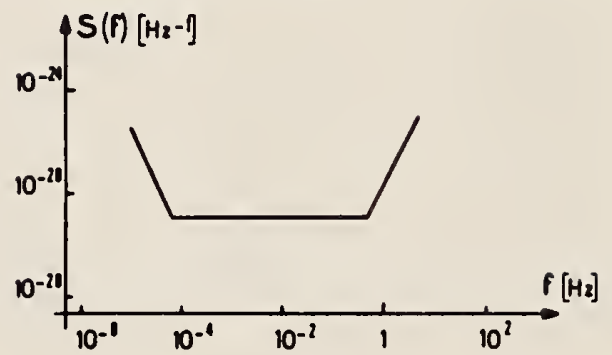

Fig. 3. Spectral density of fractional frequency fluctuations of a hydrogen maser. The parts of the graph with slopes 0 and 2 coincide with theoretical expectations.

(see section 6.4), as shown in Figures 2 and 4 but is very close to theoretical limits specified above.

It is worth pointing out here that in systems where a frequency source is frequency slaved to a frequency reference or phase locked to another frequency generator the different kinds of noise involved are filtered in the system [Cutler and Searle, 1966; C. Audoin, unpublished manuscript, 1976]. In these cases, at the output of the system, one can find noise contributions pertaining to the model (7) but appearing on the Fourier frequency scale in an order different than that shown in Figure 1. This is depicted in Figures 6 and 7 for the case of a cesium beam frequency standard consisting of a good quartz crystal oscillator which is frequency controlled by a cesium beam tube resonator.

The model for the frequency fluctuations is more useful if the noise processes can be assumed to be gaussian ones (in particular, momenta of all orders can then be expressed with the help of momenta of second order). The deviation of the frequency being the result of a number of elementary perturbations, this assumption seems a reasonable one. Furthermore, the normal distribution of

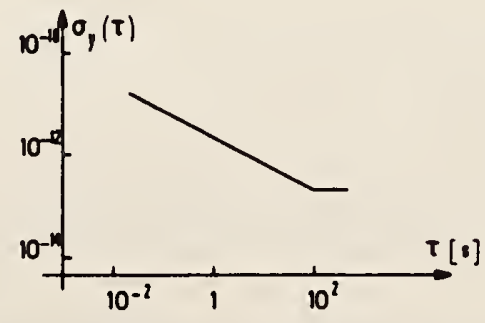

Fig. 4. Frequency stability, characterized by the root mean square of the two-sample variance of fractional frequency nuctuations, of a He-Ne iodine-stabilized laser.

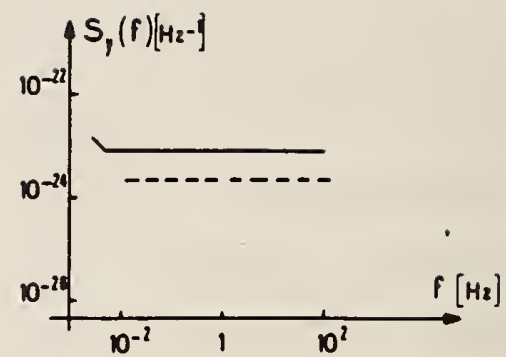

Fig. 5. Spectral density of fractional frequency fluctuations of a He-Ne iodine-stabilized laser. The solid line represents the spectral density of fractional frequency fluctuations corresponding to experimental results, and the dotted line represents the expected value of $S_{y}(f)$.

$\bar{y}$, the mean value of frequency fluctuations averaged over time interval $\tau$ as defined in (16), has been experimentally checked for $\alpha=2,1,0$, and -1 [Lesage and Audoin, 1973, 1977]. This is shown in Figure 8 for white noise of frequency, for instance.

\section{MEASUREMENTS IN THE FREQUENCY DOMAIN}

Measurement of power spectral density of frequency and phase fluctuations can be performed in the frequency domain for Fourier frequencies greater than a few $10^{-3} \mathrm{~Hz}$ owing to the availability of good low-frequency spectrum analyzers.

\subsection{Use of a frequency discriminator}

Frequency discriminators are of current use to characterize radio frequency and microwave generators. A resonant device such as a tuned circuit or a microwave cavity acts as a transducer which

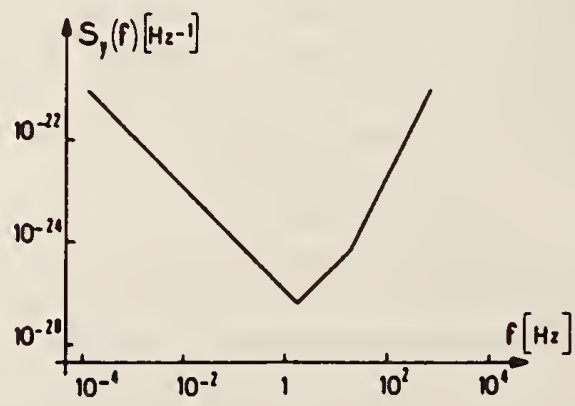

Fig. 6. Spectral density of fractional frequency fluctuations of a good quartz crystal oscillator.

* See Appendix Note \# 6 


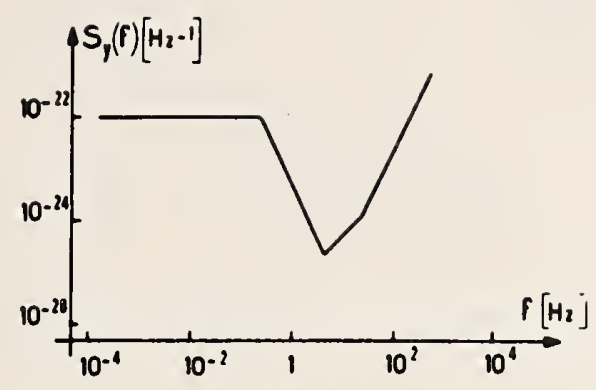

Fis. 7. Spectral density of fractional frequency fluctuations of the same quartz crystal oscillator as in Figure 6 but frequency controlled by a $\mathrm{Cs}$ beam tube resonance.

transforms frequency to voltage fluctuations. This method can be applied to optical frequency sources too, as shown in Figures 9 and 10 . Here the source is, for instance, a CW dye laser, and the frequency selective device is a Fabry-Perot etalon. The second light pass allows one to compensate for the effects of amplitude fluctuations and to adjust to a null the mean value of the output voltage. The slope of this frequency discriminator equals $1 \mathrm{~V} \mathrm{MHz}^{-1}$, typically, with a good Fabry-Perot etalon in the visible.

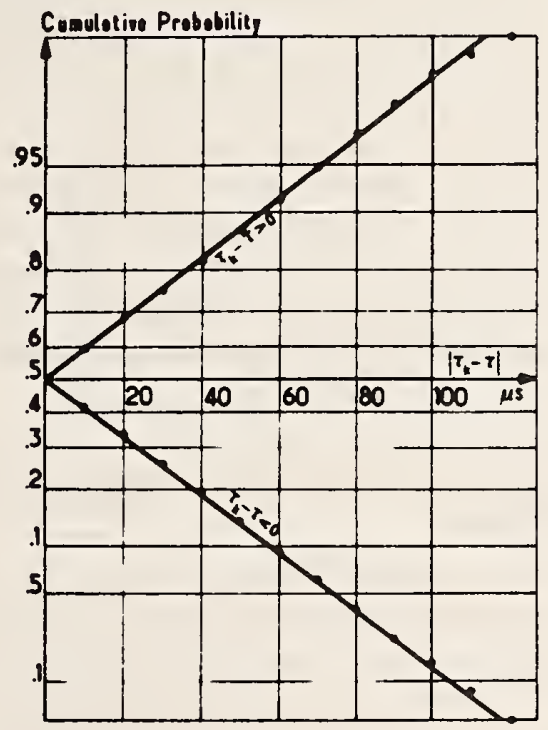

Fig. 8. Distribution of counting time results for white frequency noise (cesium beam frequency standards, $\tau=10 \mathrm{~s}$ ) in Galtonian coordinates. Circles represent the cumulative probability corresponding to $\left|\tau_{k}-\tau\right|$ with $\tau=\left\langle\tau_{k}\right\rangle$. Solid lines correspond to the normal distribution of the same width.

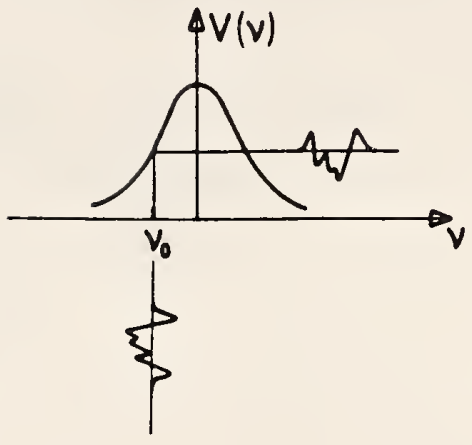

Fig. 9. Principle of frequency to voltage transfer in a frequency discriminator.

\subsection{Use of a phase detector}

This technique is well suited for the study of frequency sources in the radio frequency domain $0.2 \mathrm{MHz}<v_{0}<500 \mathrm{MHz}$, in a range where very low noise balanced-diode mixers which utilize Schottky barrier diodes are available. This technique has mainly been promoted by the National Bureau of Standards [Shoof, 1971; Walls and Stein, 1977].

Figure 11 shows the principle of the determination of the phase fluctuations in frequency multipliers, for instance. The two frequency multipliers are driven by the same source. A phase shifter is adjusted in order to satisfy the quadrature condition. One then has

$$
v(t)=D\left[\varphi_{1}(t)-\varphi_{2}(t)\right]
$$

where $D$ is a constant and $\varphi_{1}$ and $\varphi_{2}$ are the phase fluctuations introduced in the devices under test. It is assumed that the mixer is properly used to allow a balance of the phase and amplitude fluctuations of the frequency source.

This technique is often used to characterize phase fluctuations of two separate frequency sources of the same frequency. The quadrature condition is

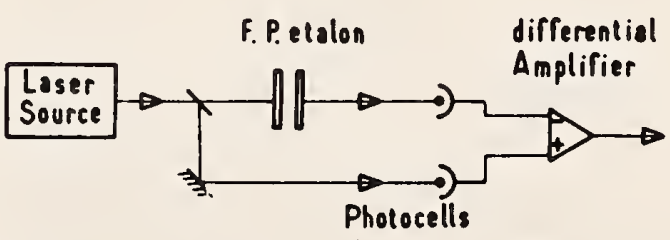

Fig. 10. Principle of frequency noise analysis of a dye laser.

* See Appendix Note \# 25 


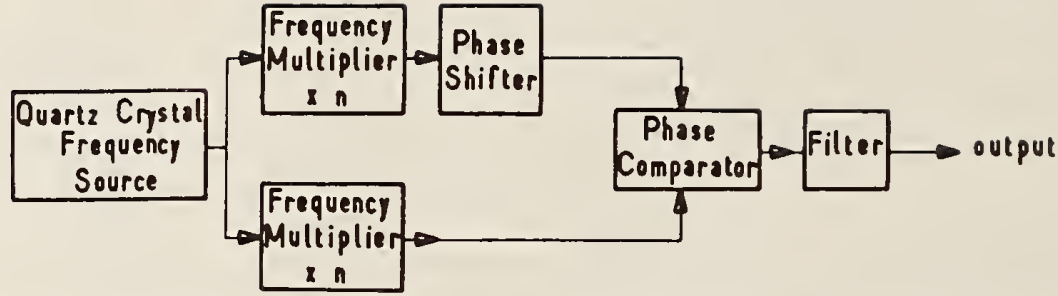

Fig. 11. Principle of measurement of phase noise with a high-quality balanced mixer used as a phase comparator.

insured by phase locking the reference oscillator, number 2 of Figure 12, to the oscillator under test. Fluctuations of the output voltage $u(t)$ at frequencies $f$ larger than the frequency cutoff of the phase loop are proportional to phase fluctuations of oscillator number 1 . On the contrary, components of $u(t)$ at frequencies smaller than the above frequency cutoff are representative of frequency fluctuations of oscillator number 1 .

The requirement of having a reference oscillator of the same quality as the oscillator to be tested may be inconvenient. It has recently been shown that the phase noise of a single oscillator can be measured by using the mixer technique, but with a delay line [Lance et al., 1977]. Figure 13 shows a schematic of the setup. The signal from the frequency source is split into two channels. The reference channel includes a phase shifter for the purpose of adjustment. It feeds one of the mixer inputs. The other channel delays the signal before it is applied to the second mixer input. It can be seen that the power spectra density of the mixer output is proportional to $\left(2 \pi f \tau_{d}\right)^{2} S_{\varphi}(f)$, where $\tau_{d}$ is the delay. The sensitivity of this technique is then reduced for low Fourier frequencies. However,

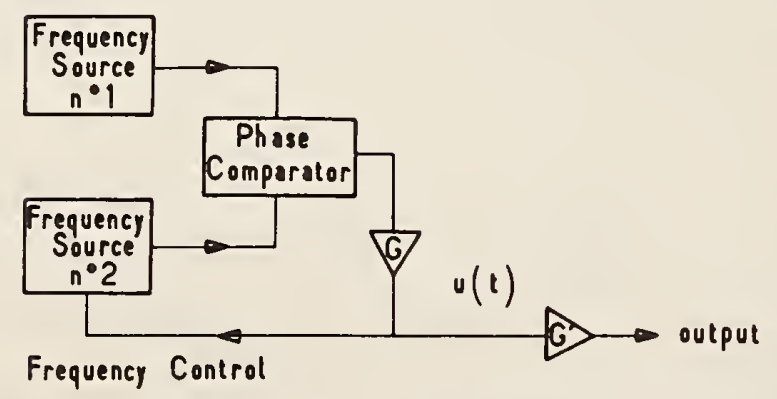

Fig. 12. Principle of phase noise measurement of oscillators. A phase lock loop insures the phase quadrature of the two phase-compared signals. some signal to noise enhancement can be achieved in a more elaborate configuration with two differential delay line systems in which cross-spectrum analysis is performed on the signal output from the two delay line systems [Lance et al., 1978].

Another method has been proposed to determine the power spectrum of fractional frequency fluctuations of a single high-quality frequency source [Groslambert, 1977]. It is shown in Figure 14. Two auxiliary oscillators, which do not need to be of the same quality as the oscillator under test, are used. They are phase locked to the frequency generator to be characterized. The control voltages $v_{1}(t)$ and $v_{2}(t)$ are appropriately filtered in order to obtain, at their outputs, a voltage $v_{1}^{\prime}(t)=K_{1}\left(\varphi_{1}\right.$ $\left.-\dot{\varphi}_{0}\right)$ and $v_{2}^{\prime}(t)=K_{2}\left(\dot{\varphi}_{2}-\dot{\varphi}_{0}\right)$, respectively, where $K_{1}$ and $K_{2}$ are constants and the subscripts 0,1 , and 2 refer to the oscillator under test, oscillator number 1 , and oscillator number 2 , respectively. It can be shown that the cross-correlation function of $v_{1}^{\prime}$ and $v_{2}^{\prime}$ is proportional to the autocorrelation function of the frequency fluctuations of the oscillator under test. Its spectral density of fractional frequency fluctuations can then be obtained via Fourier transform.

\subsection{Precision of measurement in the frequency domain}

A spectrum analyzer includes a filter of bandwidth $\Delta f$, centered at frequency $f$, a nonlinear device which measures the power in the filtered signal, and a low pass filter which integrates the output signal for the time $T$. The integration time $T$ is not infinite, and the filter bandwidth $\Delta f$ is not extremely narrow. Only an estimate $\hat{S}(f, \Delta f, T)$ of $S(f)$ can then be obtained. Well-known results show that the precision $p$ in the measurement of the power spectral density of a gaussian process is given by 


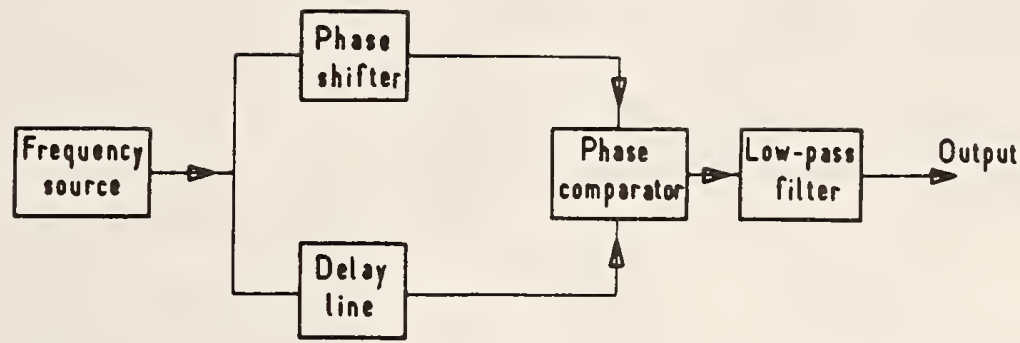

Fig. 13. Principle of phase noise measurement of a single-frequency source with a delay line.

$$
p \simeq(T \cdot \Delta v)^{-1 / 2}
$$

No attempt has been made, to our knowledge, to specify $p$ more accurately for the different noise processes which can be encountered in frequency metrology of stable sources.

\section{MEASUREMENTS IN THE TIME DOMAIN}

Time and frequency counting techniques are well known [Cutler and Searle, 1966]. They are the easiest to implement to provide information on the low-frequency content $(f \leqslant 1 \mathrm{~Hz})$ of the power spectra of fractional frequency fluctuations.

\subsection{The beat frequency method}

A beat note at frequency $\nu_{1}$ is obtained from two frequency sources under test, with frequencies $\nu_{0}$ and $\nu_{0}^{\prime}$, respectively, such that $\nu_{0} \simeq \nu_{0}^{\prime}$. If $\Delta \nu_{0}$

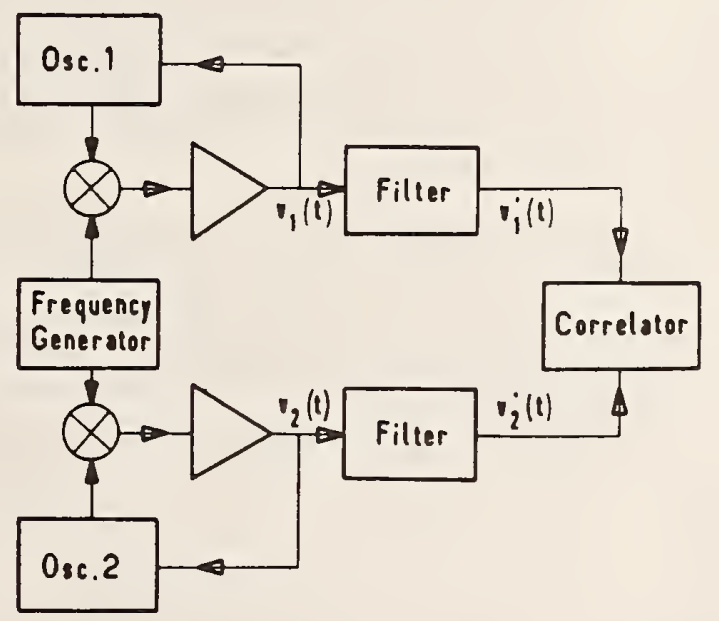

Fig. 14. Principle of phase noise measurement of a single highquality frequency source with a correlator. and $\Delta v_{0}^{\prime}$ denote the frequency fluctuations of the two sources and $\Delta \nu_{1}$ the frequency fluctuations of the beat note, one has

$$
y_{1}=\frac{\Delta \nu_{1}}{v_{1}}=\frac{v_{0}}{v_{1}}\left|\frac{\Delta v_{0}}{v_{0}}-\frac{\Delta v_{0}^{\prime}}{v_{0}^{\prime}}\right|
$$

The fractional frequency fluctuations of the beat note are then proportional to those of the frequencies $\nu_{0}$ and $\nu_{0}^{\prime}$ but multiplied by the factor $\nu_{0} / \nu_{1}$ which is much larger than unity.

With stable generators at frequencies lower than approximately $100 \mathrm{GHz}$ the frequency fluctuations are small enough that the beat note can be at low frequency. The counter is then used as a period meter, and a high precision in the measurement is achievable.

Optical frequency standards show larger frequency fluctuations in absolute value. For instance, a laser stabilized at $500 \mathrm{THz}(\lambda=0.6 \mu \mathrm{m})$ with a fractional frequency stability of $1 \times 10^{-13}$ exhibits frequency fluctuations of $50 \mathrm{~Hz}$. They can be easily measured if the beat note is at $50 \mathrm{MHz}$, say, when the counter is used as a frequency meter. In the case of iodine-stabilized He-Ne lasers the beat note is easily obtained by locking the two lasers to different hyperfine components of the considered iodine transition. Otherwise, the frequency offset technique is used [Barger and Hall, 1969].

\subsection{The time difference method}

The time difference method [Allan and Daams, 1975] must be used with time standards which deliver pulses as time scale marks. Distant time comparison and synchronization by TV pulses, light pulses, Loran-C pulses, for instance, pertain to this category. It provides information on the relative phases of the two clocks under test.

Time interval measurements being very precise

* See Appendix Note \# 26 


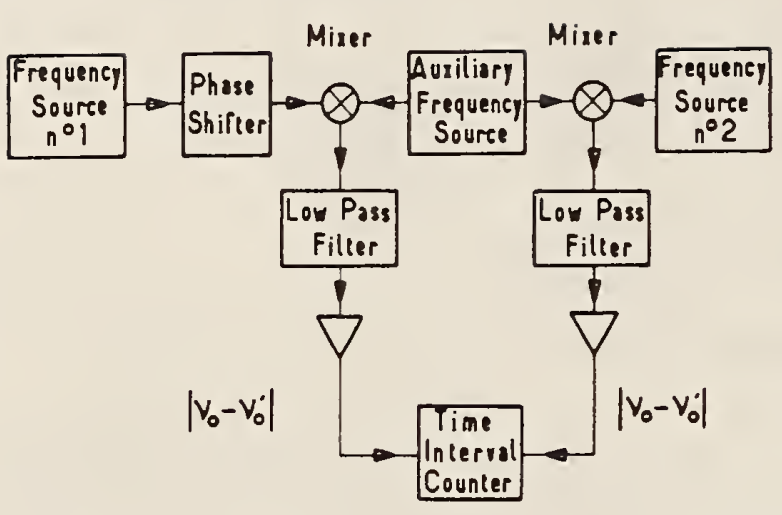

Fig. 15. Principle of the time difference method.

(a precision of 10-100 ns is typical, depending on the class of the counter), they are also used with c.w. frequency generators, as shown in Figure 15. This method is then well suited to the case in which the frequency sources under test have the same nominal frequency $v_{0}$, e.g., atomic frequency standards. An auxiliary frequency source, such as a frequency synthesizer, with frequency $v_{0}^{\prime}$ allows one to obtain, at the output of the mixers, two beat notes at the desired frequency $\nu_{1}=\mid v_{0}-$ $v_{0}^{\prime} \mid$. After amplification the zero crossing of one of the beat notes starts the time interval counter, and the zero crossing of the other beat note stops it. One has

$$
x_{1}=\left(v_{0} / \nu_{1}\right)\left(x_{0}-x_{0}^{\prime}\right)
$$

where $x_{1}$ is the fractional phase fluctuation of the beat note and $x_{0}$ and $x_{0}^{\prime}$ that of the two frequency standards. For instance, with $v_{0}=5 \mathrm{MHz}, v_{1}=$ $0.5 \mathrm{~Hz}$, and a precision in the time interval measurement of $0.1 \mu \mathrm{s}$ a precision of $10^{-14} \mathrm{~s}$ at the nominal frequency $\nu_{0}$ is achieved.

\section{CHARACTERIZATION OF FREQUENCY STABILITY IN THE TIME DOMAIN}

\subsection{Significance of experimental data}

It is well established that measurement in the time domain with an electronic counter samples phase increments and gives $\Delta_{\tau} \varphi\left(t_{k}\right)$ defined as

$$
\Delta_{1} \varphi\left(t_{k}\right)=\varphi\left(t_{k}+\tau\right)-\varphi\left(t_{k}\right)
$$

The phase increment $\Delta_{\tau} \varphi\left(t_{k}\right)$ is related to $\bar{y}_{k}$, the average over time interval $\left[t_{k}, t_{k}+\tau\right]$ of fractional frequency fluctuations. We have

$$
\overline{y_{k}}=\frac{1}{\tau} \int_{t_{k}}^{t_{k}+\varphi} y\left(t^{\prime}\right) d t^{\prime}=\frac{1}{2 \pi \nu_{1} \tau} \Delta_{i} \varphi\left(t_{k}\right)
$$

where $v_{1}$ is the mean frequency of the processed signal.

Samples of $\overline{y_{k}}$ can be combined in many different ways. Some of those which have been considered will be reviewed here. On the other hand, the number of samples is finite, and the question arises as to the related uncertainty in the characterization of frequency stability and of the best use of the data.

\subsection{N-sample variance}

The sequence of measurement is as shown in Figure 16. The mean duration of each measurement is $\tau$, and $T$ is the time interval between the beginnings of them.

In statistical estimation it is common to consider sample variance [Papoulis, 1965]. The $N$-sample variance of $y_{k}$ is defined as

$\sigma_{y}^{2}(N, T, \tau)=\frac{1}{N-1} \sum_{k=0}^{N-1}\left(\overline{y_{k}}-\frac{1}{N} \sum_{k=0}^{N-1} \overline{y_{k}}\right)^{2}$

where the factor $N /(N-1)$ removes bias in the estimation.

The dependence of the expectation value of the $N$-sample variance on the number $N$ of samples, the sample time $\tau$, and the power spectral density has been considered by Allan [1966]. We will only consider special cases in the following.

It can be shown that computation of the average of the $N$-sample variance introduces a filtering of the power spectral density $S_{y}(f)$ [Barnes et al., 1971]. We have

$$
\left\langle\sigma_{y}^{2}(N, T, \tau)\right\rangle=\int_{0}^{\infty} S_{y}(f)|H(f)|^{2} d f
$$

$H(f)$ is the transfer function of a linear filter which has the following expression:

$$
|H(f)|^{2}=\frac{N}{N-1}\left[\frac{\sin \pi f \tau}{\pi f \tau}\right]^{2}\left\{1-\left[\frac{\sin \pi f N T}{N \sin \pi f T}\right]^{2}\right\}
$$

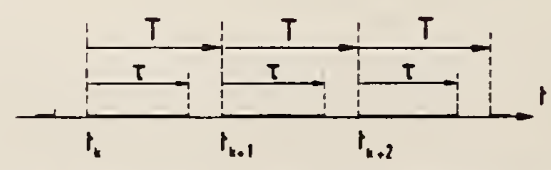

Fig. 16. Sequence of time domain measurement. 
For $f$ such that

$$
\pi f T \ll 1
$$

we have

$$
|H(f)|^{2}=\frac{N(N+1)}{3}(\pi f T)^{2}
$$

Equation (21) shows that for finite $N$ the integral in (18) will converge at the lower limit for $\alpha=$ -1 and $\alpha=-2$, as well as for $\alpha=2,1$, and 0 . One sees that very low frequency components of $S_{y}(f)$ are best eliminated for small values of $N$.

\subsection{Variance of time-averaged frequency} fluctuations

When $N$ goes to infinity, $\left\langle\sigma_{y}^{2}(\infty, \tau, \tau)\right\rangle$ becomes $\sigma^{2}\left(y_{k}\right)$, the variance of time-averaged frequency fluctuations or of the first difference of phase fluctuations [Cutler and Searle, 1966] as given by

$$
\sigma^{2}\left(\overline{y_{k}}\right)=\left\langle\left(\overline{y_{k}}\right)^{2}\right\rangle
$$

where angle brackets denote mathematical expectation.

In the presence of a single-pole low-pass filter with cutoff frequency $f_{c}$ we have the following relation between $\sigma_{y}^{2}\left(y_{k}\right)$ and $S_{y}(f)$ :

$\sigma^{2}\left(\overline{y_{k}}\right)=\int_{0}^{\infty} S_{y}(f) \frac{1}{1+\left(f^{2} / f_{c}^{2}\right)}\left|H_{1}(f)\right|^{2} d f$

with

$$
\left|H_{1}(f)\right|^{2}=\left[\frac{\sin \pi f \tau}{\pi f \tau}\right]^{2}
$$

Equation (24) shows that $\sigma^{2}\left(\bar{y}_{k}\right)$ converges for $\alpha$ $=2,1,0$ but diverges for flicker noise of frequency $(\alpha=-1)$ and random walk of frequency $(\alpha=-2)$. The variance $\sigma^{2}\left(y_{k}\right)$ is no longer used to characterize frequency stability. However, it is useful to relate the RF power spectral density to $S_{y}(f)$ [Rutman, 1974a].

\subsection{Two-sample variance}

For the special case $N=2$, (17) gives

$$
\left\langle\sigma_{y}^{2}(2, T, \tau)\right\rangle=\frac{1}{2}\left\langle\left(\tilde{y}_{k+1}-\bar{y}_{k}\right)^{2}\right\rangle
$$

and we have

$\left\langle\sigma_{y}^{2}(2, T, \tau)\right\rangle=\int_{0}^{\infty} S_{y}(f) \frac{1}{1+\left(f / f_{c}\right)^{2}}\left|H_{2}(f)\right|^{2} d f$

with

$$
\left|H_{2}(f)\right|^{2}=2\left[\frac{\sin \pi f \tau}{\pi f \tau}\right]^{2}(\sin \pi f T)^{2}
$$

For small $f$, such that $\pi f T \ll 1,\left|H_{2}(f)\right|^{2}$ varies as $f^{2}$. The integral in (26) is thus defined for flicker noise of frequency $(\alpha=-1)$ and random walk of frequency $(\alpha=-2)$, as well as for $\alpha=2,1$, and 0 . It is easy to show that the quantity $\bar{y}_{k+1}-$ $\bar{y}_{k}$ ) represents a second-order difference of phase fluctuations. It follows that second-order phase increments are stationary for $\alpha=-1$ and -2 (as specified in Table 1).

6.4.1. Two-sample variance without dead time. The two-sample variance (Allan variance) without dead time, for $T=\tau$, is now generally accepted as the measure of frequency stability in the time domain. One sets

$$
\sigma_{y}^{2}(\tau)=\left\langle\sigma_{y}^{2}(2, \tau, \tau)\right\rangle
$$

Table 2 gives asymptotic expressions of $\sigma_{y}^{2}(\tau)$ in the cases $2 \pi f_{c} \tau \gg 1$ and $2 \pi f_{c} \tau \ll 1$. Expressions of $\sigma_{y}^{2}(\tau)$ in the presence of a sharp high-frequency cutoff $f_{h}$ have been given by Barnes et al. [1971] for the case $2 \pi f_{h} \tau \gg 1$.

One sees in Table 2 that $\sigma_{y}^{2}(\tau)$ has a characteristic $\tau$ dependence for each type of noise considered,

TABLE 2. Asymptotic expressions of the two-sample variance for the noise processes considered

\begin{tabular}{ccc}
\hline & \multicolumn{2}{c}{$\sigma_{y}^{2}(\tau)$} \\
\cline { 2 - 3 }$S_{y}(f)$ & $2 \pi f_{c} \tau>1$ & $2 \pi f_{c} \tau \ll 1$ \\
$h_{1} f$ & $\frac{3 h_{2} f_{c}}{8 \pi \pi^{2}}$ & $\frac{h_{2} f_{c}^{2}}{2 \tau}$ \\
$h_{0}$ & $\frac{3 h_{1} \ln \left(2 \pi f_{c} \tau\right)}{4 \pi^{2} \tau^{2}}$ & $2 h_{1} f_{c}^{2} \ln 2$ \\
$h_{-1} f^{-1}$ & $\frac{h_{0}}{2 \tau}$ & $\frac{2}{3} \pi^{2} h_{0} f_{c}^{2} \tau$ \\
$h_{-2} f^{-2}$ & $\frac{2 h_{-1}^{2} \ln 2}{3} h_{-2}{ }^{2}$ & $2 \pi^{2} h_{-1} f_{c}^{2} \tau^{2}$ \\
\end{tabular}

* See Appendix Note \# 27 
such as $\sigma_{y}^{2}(\tau)=k / \tau^{\mu}$. This is specified as follows for $2 \pi f_{c} \tau \gg 1$ :

\begin{tabular}{|c|c|}
\hline & $\boldsymbol{\mu}$ \\
\hline 2 & 2 \\
\hline 1 & 2 \\
\hline 0 & 1 \\
\hline-1 & 0 \\
\hline-2 & -1 \\
\hline
\end{tabular}

For $\alpha=-1$ the $\sigma_{y}(\tau)$ graph is a horizontal line, which justifies the designation 'flicker floor' for that part of the graph.

Usually, under experimental conditions the relation $2 \pi f_{c} \tau \gg 1$ is satisfied. The noise processes which perturb the oscillation can then be identified from a $\sigma_{y}(\tau)$ graph if it is assumed that the aforementioned model of frequency fluctuations is valid. Table 2 shows in which cases $\sigma_{y}^{2}(\tau)$ depends on the frequency cutoff. The latter must then be specified.

The case $2 \pi f_{c} \tau \ll 1$ is useful for the analysis of the effect of frequency of phase servocontrol loops where the frequency fluctuations of the frequency reference are low-pass filtered. Bias functions have been given to relate (1) the two-sample variance with and without dead time and (2) the two-sample variance to the $N$-sample variance [Barnes et al., 1971].

6.4.2. Two-sample variance with dead time. * General expressions for the $N$-sample variance with dead time have been given [Barnes et al., 1971] for useful values of $\alpha$ if the condition $2 \pi f_{h} \tau>$ 1 is satisfied. The case of the two-sample variance with dead time has not been emphasized enough yet. Table 3 compares the two-sample variance with and without dead time when the condition $2 \pi f_{c} \tau$ $>1$ is fulfilled. The condition of negligible dead

TABLE 3. Comparison of the two-sample variance with and without dead time for $2 \pi f_{c} \tau>1$

\begin{tabular}{ccc}
\hline & \multicolumn{2}{c}{$\sigma_{y}^{2}(2, T, \tau)$} \\
\cline { 2 - 3 }$S_{y}(f)$ & $2 \pi f_{c}(T-\tau)<1$ & $2 \pi f_{c}(T-\tau)>1$ \\
$h_{2} f^{2}$ & $\frac{3 h_{2} f_{c}}{8 \pi \tau^{2}}$ & $\frac{h_{2} f_{c}}{4 \pi \tau^{2}}$ \\
& $\frac{3 h_{1} \ln \left(2 \pi f_{c} \tau\right)}{4 \pi^{2} \tau^{2}}$ & $\frac{h_{1} \ln \left(2 \pi f_{c} \tau\right)}{2 \pi^{2} \tau^{2}}$ \\
$h_{1} f$ & $\frac{2 h_{0} / 2 \tau}{h_{0} \ln 2}$ & $h_{-1} \ln (T / \tau) ; T>\tau$ \\
$h_{-1} f^{-1}$ & $\frac{2 \pi^{2}}{3} h_{-2} \tau$ & $\pi^{2} h_{-2} T$ \\
$h_{-2} f^{-2}$ & 3 &
\end{tabular}

* See Appendix Note \# 28

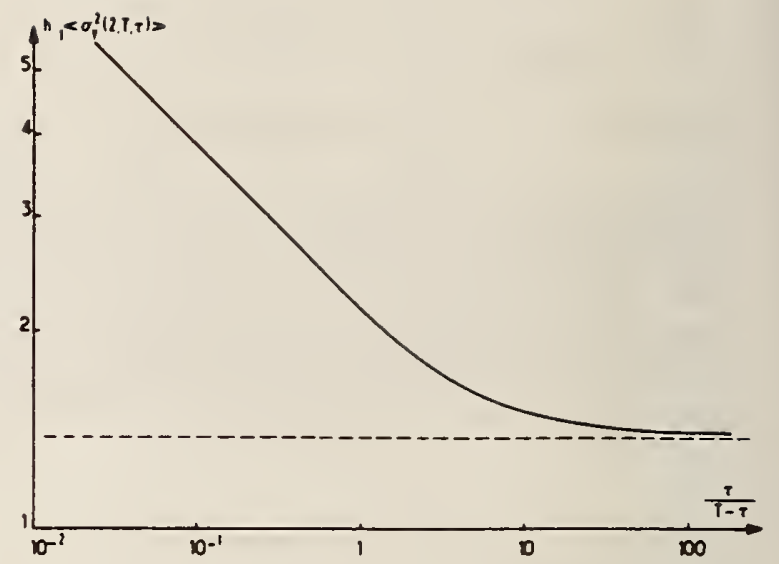

Fig. 17. The solid line represents the variation of $h_{-1}\left\langle\sigma_{y}^{2}(2\right.$, $T, \tau)\rangle$ versus $\tau /(T-\tau)$ for the flicker noise of frequency. The dotted line represents the asymptotic value for $(T-T)$ $<\tau$.

time is then $2 \pi f_{c}(T-\tau) \ll 1$. Results for $2 \pi f_{c} \tau$ $\ll 1$ are also available (P. Lesage and C. Audoin, private communication, 1978). Table 3 shows that in the presence of -dead time, i.e., $2 \pi f_{c}(T-\tau)$ $>1$, the expression for the two-sample variance is noticeably modified for $\alpha=-1$ and -2 .

The case of the flicker noise of frequency is particularly interesting. Figure 17 shows the variation of the two-sample variance with dead time as a function of $\tau /(T-\tau)$. The flicker floor does not exist anymore if the value of this ratio is modified when the sampling time $\tau$ is changed. The identification of the noise process which perturbs the oscillation might then be wrong if the effect of dead time is not taken into account.

\subsection{Precision in the estimation of the two-sample variance}

Measurements are always of finite duration, and therefore the number of available values of $y_{k}$ is finite. We are then faced with the problem of the precision in the estimation of the time domain frequency stability measurement. This is an important one because successive characterizations of the frequency stability of a given device allow one to get information on the stationarity of the processes involved in the perturbation of its frequency but within the limits of the precision of the characterization. Precision in the estimation of the frequency stability of individual oscillators of a set of $p$ frequency generatörs $(p>2)$ [Gray and Allan,

$\overline{* * \text { See Appendix Note \# } 29}$ 
1974] critically depends on the precision of the $p(p-1) / 2$ frequency comparisons which can be performed by arranging oscillators in pairs. Furthermore, the uncertainty in the determination of $\sigma_{y}(\tau)$ translates directly into the uncertainty in determining the $h_{\alpha}$ coefficients if the frequency generator is perturbed by noise processes modeled by (7).

The precision in the estimation of time domain measurements of frequency stability has been considered by several authors [Tausworthe, 1972; Lesage and Audoin, 1973; Yoshimura, 1978]. It has been determined for most of the experimental situations which can be encountered in the twosample variance characterization of frequency stability, with or without dead time (P. Lesage and C. Audoin, private communication, 1978).

Calculation of the expectation value of the twosample variance according to (25) requires an infinite number of data. But, in practice, only $m$ counting results are available, and one calculates the estimated average of the two-sample variance as follows:

$\partial_{y}^{2}(2, T, \tau, m)=\frac{1}{2(m-1)} \sum_{k=1}^{m-1}\left(\bar{y}_{k+1}-\overline{y_{k}}\right)^{2}$

One can easily show that the expectation value of $\partial_{y}^{2}(2, T, \tau, m)$ equals the averaged two-sample variance with dead time. Thus the finite number of measurements does not introduce bias in the estimation of the two-sample variance.

The estimated averaged two-sample variance (EATSV) being a random function of $m$, we need to characterize the uncertainty" in the estimation. We thus introduce the variance of the EATSV, according to the common understanding of variance. We set

$$
\begin{aligned}
& \sigma^{2}\left[\partial_{y}^{2}(2, T, \tau, m)\right] \\
&=\left\langle\left[\partial_{y}^{2}(2, T, \tau, m)-\left\langle\sigma_{y}^{2}(2, T, \tau)\right\rangle\right]^{2}\right\rangle
\end{aligned}
$$

With the expression (29) of the EATSV we get $\sigma^{2}\left[\partial_{y}^{2}(2, T, \tau, m)\right]$

$$
=\left[\frac{1}{2(m-1)}\right]^{2}\left\langle\sum_{i=1}^{m-1} \sum_{j=1}^{m-1} \beta_{i} \beta_{j}\right\rangle
$$

with

$$
\beta_{l}=\left(\bar{y}_{1+1}-\bar{y}_{1}\right)^{2}-2\left(\sigma_{y}^{2}(2, T, \tau)\right\rangle
$$

The classical law of large numbers [Papoulis, 1965] which states that the true variance of a sum of $(m-1)$ uncorrelated random variables decreases as $1 /(m-1)$, even for small values of $(m-1)$, does not apply here. We are considering the quantities $\beta$, which are correlated because two adjacent differences $\left(\bar{y}_{1+1}-\bar{y}_{1}\right)$ and $\left(\bar{y}_{1+2}-\bar{y}_{1+1}\right)$ are obviously not independent.

Equation (31) can also be written as

$$
\begin{aligned}
\sigma^{2}\left[\hat{\partial}_{y}^{2}(2, T, \tau, m)\right] & =\frac{1}{m-1}\left[\frac{\Gamma_{0}}{4}\right. \\
+ & \left.\frac{1}{2(m-1)} \sum_{k=1}^{m-2}(m-1-k) \Gamma_{k}\right]
\end{aligned}
$$

with

$$
\Gamma_{k}=\left\langle\beta, \beta_{1-k}\right\rangle
$$

$\Gamma_{k}$, which does not depend on $m$, represents the autocorrelation coefficient of $\beta_{1}$ and $\beta_{i-k}$. Since the same data are used in two adjacent pairs, the autocorrelation coefficient $\Gamma_{1}$, and possibly others, differs from zero. Equation (33) then shows that the $1 /(m-1)$ dependence also occurs for the random variables considered, but asymptotically for large enough values of $(m-1)$.

The variance of the EATSV can be related to $S_{y}(f)$ if it is assumed that the quantities $y_{1}$ are normally distributed. This is a reasonable assumption, as shown in section 2 .

It is useful to introduce $\Delta(m)$, the fractional deviation of $\hat{\partial}_{y}^{2}(2, T \tau, m)$ defined as

$$
\Delta(m)=\frac{\partial_{y}^{2}(2, T, \tau, m)-\left\langle\sigma_{y}^{2}(2, T, \tau)\right\rangle}{\left\langle\sigma_{y}^{2}(2, T, \tau)\right\rangle}
$$

The standard deviation $\sigma[\Delta(m)]$ defines the precision in the estimation of the two-sample variance. Expressions for $\sigma[\Delta(m)]$ which are valid for $m$ $>2$ have been established for all possible values of $2 \pi f_{c} \tau$ and $2 \pi f_{c}(T-\tau)$ but will not be given here.

In practice, the time domain frequency stability of a frequency source is characterized by the standard deviation $\left[\hat{\sigma}_{y}^{2}(2, T, \tau, m)\right]^{1 / 2}$. We therefore consider $\delta$ defined as

$\delta=\frac{\left[\partial_{y}^{2}(2, T, \tau, m)\right]^{1 / 2}-\left\langle\sigma_{y}^{2}(2, T, \tau)\right\rangle^{1 / 2}}{-\left(\sigma_{y}^{2}(2, T, \tau)\right\rangle^{1 / 2}}$

$\sigma(\delta)$ specifies the precision in the estimation of the time domain frequency stability measurement 


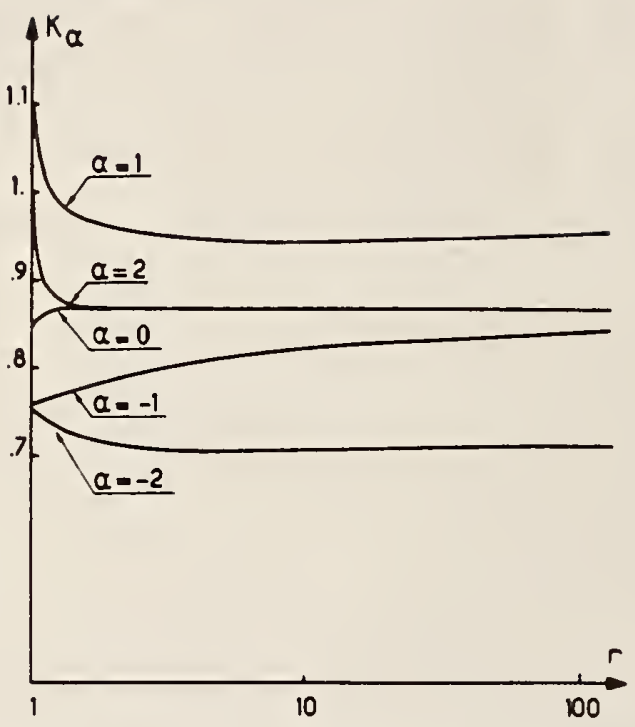

Fis. 18. Variation of $K_{0}$ as a function of $r=T / T$ for the commonly encountered noise processes and for $2 \pi f_{c} T=10$.

from a limited number of data and allows one to draw error bars on a frequency stability graph.

For $2 \pi f_{c} \tau \gg 1$ and $m>1$ we have

$$
\sigma(\delta)=K_{0} m^{-1 / 2}
$$

The values of $K_{\mathrm{a}}$ are given as follows subject to the condition that the dead time is negligible, i.e., $2 \pi f_{c}(T-\tau)<1$ :

\begin{tabular}{rrr}
$\alpha$ & & $K_{0}$ \\
\cline { 1 - 1 } & 0.99 \\
1 & & 0.99 \\
0 & & 0.87 \\
-1 & & 0.77 \\
-2 & & 0.75
\end{tabular}

In the presence of dead time the values of $K_{\mathrm{a}}$ depend on the noise process considered as well as the values of $2 \pi f_{c} \tau$ and $r=T / \tau$. Figure 18 , valid for $2 \pi f_{c} \tau=10$ shows that the dependence of $K_{\alpha}$ with dead time is especially pronounced in the vicinity of $r=1$ for $\alpha=1$ and 2 .

\section{CHARACTERIZATION OF FREQUENCY STABILITY VIA FILTERING OF PHASE OR FREQUENCY NOISE}

Equations (27) and (28) show that the definition of the time domain measurement of frequency stability $\sigma_{y}^{2}(\tau)$ involves a filtering of $S_{y}(f)$ in a linear filter. Figure 19 shows the impulse response of this filter, which represents the sequence of measure-

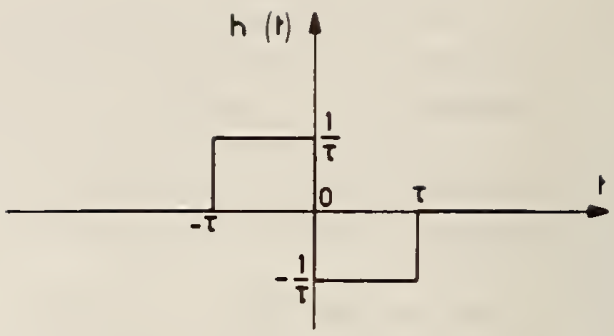

Fig. 19. Impulse response of a linear filter which represents computation of two-sample variance.

ment for $T=\tau$, and Figure 20 depicts the related transfer function. One can also consider the effect of filtering a voltage proportional to $y(t)$ or $x(t)$ in a physically realizable analog filter.

A high pass filter of cutoff frequency $1 / \pi$ t has been considered [Rutman, 1974b; Rutman and Sauvage, 1974]. Its input receives a voltage proportional to $x(t)$. It is provided by a mixer used as a phase comparator. The rms value of the filtered signal is measured. When the frequency cutoff is changed, this rms value shows the same $\mu$ versus $\alpha$ dependence as shown in section 6.4.1. More interesting is a bandpass filter centered at the variable frequency $f=1 / 2 \tau$ but with a fixed value of its quality factor. It allows one to distinguish white and flicker noise of phase, as it gives $\mu=$ 3 for $\alpha=2$; the $\mu$ versus $\alpha$ dependence being otherwise unchanged for $\alpha=1,0,-1$, and -2 .

Similarly, a frequency discriminator, giving an output proportional to $y(t)$, followed by two cascaded resistance-capacitance (RC) filters and a.rms voltmeter allows one to obtain a useful approximation of the two-sample variance. The filters insure low-pass and high-pass filtering with $\mathrm{RC}=$ $\tau / 2$ [Wiley, 1977].

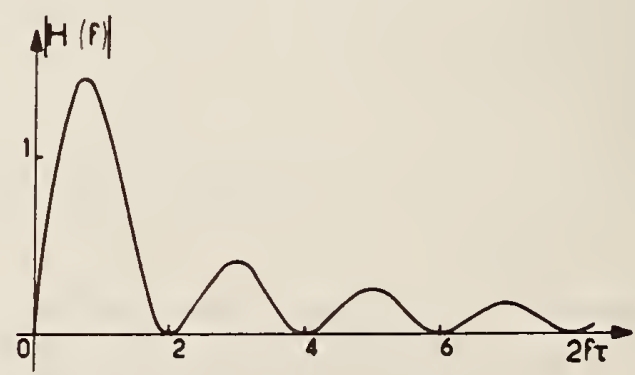

Fig. 20. Transfer function, of the linear filter with impulse response shown in Figure 19. 


\section{SPECTRAL ANALYSIS INFERRED FROM TIME DOMAIN MEASUREMENTS}

The methods of time domain characterization of frequency stability reviewed above allow one to identify noise process if they are described by

$$
S_{y}(f)=\sum_{a=-2}^{2} h_{a} f^{\alpha}
$$

This may not be the case. Furthermore, it is of interest to determine the power spectral density of fractional frequency fluctuations for Fourier frequencies lower than $1 \mathrm{~Hz}$. In this region, time domain measurements are the most convenient, and the question arises as to their best use for spectral analysis.

\subsection{Selective numerical filtering}

Equations (24) and (27) show that calculation of the variance of the second difference of phase fluctuations (the two-sample variance) involves a more selective filtering than calculation of the variance of the first difference of phase fluctuations. One can then consider higher-order differences [Barnes, 1966; Lesage and Audoin, 1975a, b]. The $n$ th-order difference of phase fluctuations is denoted as " $\Delta_{T, \tau} \varphi\left(t_{k}\right)$, where $\tau$ and $T$ have the same meaning as in section 6.1. and 6.2. This $n$th difference is defined by the following recursive equation:

${ }^{n} \Delta_{T, \tau} \varphi\left(l_{k}\right)={ }^{(n-1)} \Delta_{T, 7} \varphi\left(l_{k}+T\right)-{ }^{(n-1)} \Delta_{T, \tau} \varphi\left(l_{k}\right)$

which introduces binomial coeffícients $C_{n-1}^{i}$. We have

$$
\begin{array}{r}
{ }^{n} \Delta_{T . \tau} \varphi\left(t_{k}\right)=\sum_{i=0}^{n-1}(-1)^{\prime} C_{n-1}^{\prime}\left\{\varphi\left[l_{k}+(n-1-i) T+\tau\right]\right. \\
\left.-\varphi\left[l_{k}+(n-1-i) T\right]\right\}
\end{array}
$$

The transfer function $H_{n}(f, T, \tau)$ of the linear filter which represents the calculation of the variance of the $n$th difference of phase fluctuations is given by

$\left|H_{n}(f, T, \tau)\right|=\frac{2^{n-1}}{\pi f \tau}(\sin \pi f T)^{n-1} \sin \pi f \tau$

It should be pointed out that for $f \tau \ll 1$, one has

$$
\left|H_{n}(f, T, \tau)\right|=(2 \pi f T)^{n-1} \quad f \tau \ll 1
$$

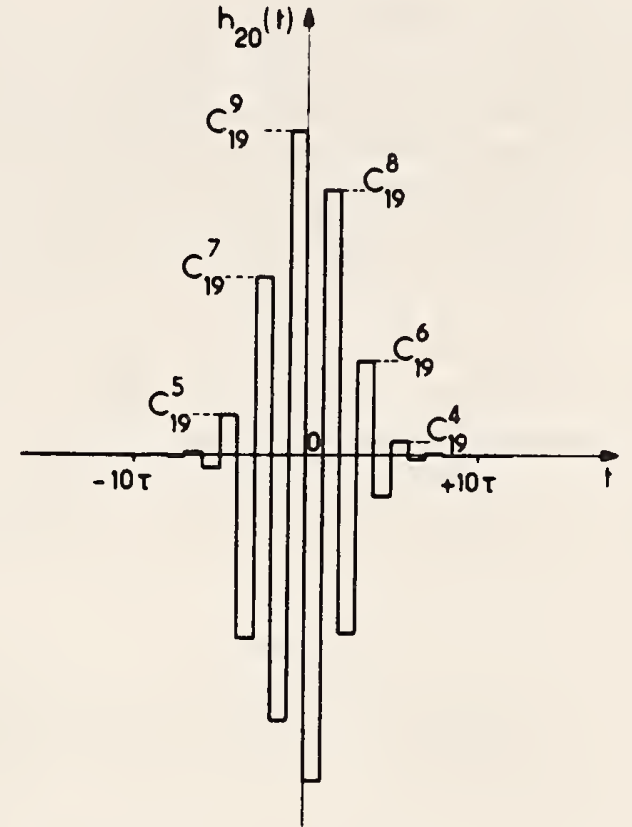

Fig. 21. Impulse response of a linear filter which represents computation of the variance of the 20th difference of phase fluctuations. $C$, represents binomial coefficients.

Figure 21 shows the impulse response of the linear filter which represents the calculation of the variance of the 20th difference of phase fluctuations, and Figure 22 shows the related transfer function. A selective filtering is then involved around frequency $1 / 2 \tau$.

Such a variance is also known as a modified Hadamard variance [Baugh, 1971]. The spurious responses at frequencies $(2 l+1) / 2 \tau$, where $l$ is an integer, can be eliminated by a proper weighting

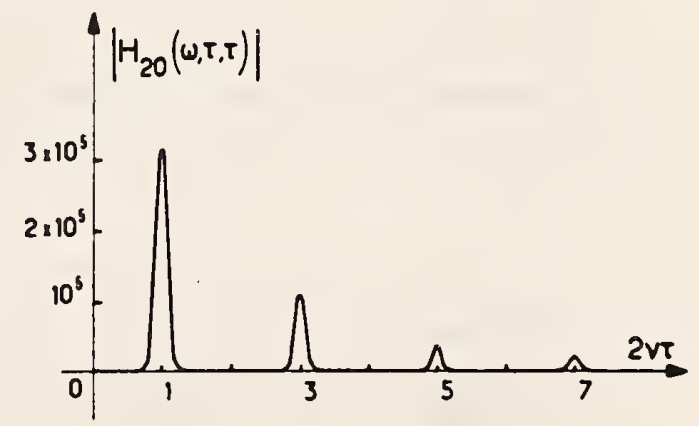

Fig. 22. Transfer function of the linear filter considered in Figure 21. 
of measurement results or by filtering with the help of an analog filter [Groslambert, 1976].

Such a technique of linear filtering has been used to show that good quartz crystal oscillators exhibit flicker noise of frequency for Fourier frequencies as low as $10^{-3} \mathrm{~Hz}$ [Lesage and Audoin, 1975b]. Furthermore, it is well suited to the design of automated measurement setups [Peregrino and Ricci, 1976; Groslambert, 1977].

The best use of experimental time domain data for selective filtering has been considered by Boileau [1976].

\subsection{High-pass filtering}

If frequency fluctuations $y(t)$ are filtered in an ideal high-pass filter with transfer function $G_{0}\left(f_{1}\right.$, $f$ such that

$$
G_{0}\left(f_{1}, f\right)= \begin{cases}0 & f<f_{1} \\ 1 & f>f_{1}\end{cases}
$$

its output $z(t)$ is such that

$$
\sigma_{z}^{2}=\int_{0}^{\infty} G_{0}\left(f, f_{1}\right) S_{y}(f) d f=\int_{f_{1}}^{\infty} S_{y}(\rho) d f
$$

Equation (44) shows that the derivative of $\sigma_{z}^{2}$ is $-S_{y}(f)$, and spectral analysis, and therefore characterization of frequency stability, are possible, in principle, by high-pass filtering.

Possible realization of the high-pass filter by techniques of digital data processing have been specified, such as the method of finite-time variance and the method of finite-time frequency control. Processing of finite-time data is aimed to properly deal with the nonintegrable singularity of the power spectral density at $v=0$ [Boileau, 1975; Boileau and Picinbono, 1976]. The method is well suited to the analysis of drifts or slow frequency changes. Practical use of this method has not been reported yet.

\subsection{Use of the sample spectral density}

It has been shown in section 8.1. that spectral analysis from the Hadamard variance or its modified forms requires a series of measurements at time interval $\tau$ in order to specify the spectral density at frequency $1 / 2 \tau$. Another point of view has been considered [Boileau and Lecourtier, 1977]. From a set of measurements of $y_{k}$, sampled at frequency $1 / \tau$, it allows one to obtain an estimation of the spectral density for discrete values of the Fourier frequency.

\section{STRUCTURE FUNCTIONS OF OSCILLATOR FRACTIONAL PHASE AND FREQUENCY FLUCTUATIONS}

Interest in the variance of $n$ th-order difference of phase fluctuations was recognized early in the field of time keeping (see for instance, Barnes [1966]). This can be easily understood from (43), which shows that an efficient filtering of low. frequency components of frequency fluctuations is then introduced. It allows one to deal properly with frequency drifts, which will now be considered, and poles of $S_{y}(f)$ of order $2(n-1)$ at the origin. It is equivalent to saying that the $n$th difference of phase fluctuations allows one to consider random processes with stationary $n$ th-order phase increments.

This question has been formalized by Lindsey and Chie [1976, 1977], who introduce structure functions of oscillator phase fluctuations. The $n$th order structure function of phase fluctuations is nothing else but the variance of the $n$th difference of phase fluctuations, as considered in section 8. Then, by definition, the $n$ th-order structure function of fractional phase fluctuations is given by

$$
D_{x}^{(n)}(\tau)=E\left\{\left[{ }^{n} \Delta_{\tau .+} x\left(t_{k}\right)\right]^{2}\right\}
$$

where $E\{\cdot\}$ means expectation value. The fractional phase (or the clock reading) at time $t_{k}$ is $x\left(t_{k}\right)$. We assume $T=\tau$.

Let us consider an oscillator, the phase $\varphi^{\prime}(t)$ of which is of the following form except for an additive constant:

$$
\varphi^{\prime}(t)=\sum_{k=2}^{t} \Omega_{k-1} \frac{t^{k}}{k !}+\varphi(t)
$$

where $\Omega_{k}$ is a random variable modeling the $k$ thorder frequency drift and $\varphi(t)$ represents random phase fluctuations. We then have

$$
x^{\prime}(t)=\sum_{k=2}^{t} d_{k-1} \frac{t^{k}}{k !}+x(t)
$$

and

$$
y^{\prime}(t)=\sum_{k=1}^{1-1} d_{k} \frac{t^{k}}{k !}+y(t)
$$

where $d_{k}=\Omega_{k} / 2 \pi v_{0}$ is the normalized drift coeffi- 
cient and $x(t)$ and $y(t)$ have the same meaning as in the preceding sections. The notations $x^{\prime}$ and $y^{\prime}$ refer to an oscillator with drift.

It can then be shown that we have

$$
\begin{aligned}
D_{x}^{(n)}(\tau)= & \tau^{2 n} E\left[d_{n-1}^{2}\right] \\
& +2^{2 n} \int_{0}^{\infty} S_{y}(f) \frac{\sin ^{2 n}(\pi f \tau)}{(2 \pi f)^{2}} \mathrm{df} \quad l=n
\end{aligned}
$$

and

$$
D_{x}^{(n)}(\tau)=2^{2 n} \int_{0}^{\infty} S_{y}(f) \frac{\sin ^{2 n}(\pi f \tau)}{(2 \pi f)^{2}} d f \quad l>n
$$

If one applies (49) to the case of an oscillator without drift, one can easily show that the following equations are satisfied:

$$
\sigma^{2}\left(\overline{y_{k}}\right)=\left(1 / \tau^{2}\right) D_{x}^{(1)}(\tau)
$$

and

$$
\sigma_{y}^{2}(\tau)=\left(1 / 2 \tau^{2}\right) D_{x}^{(2)}(\tau)
$$

This is indeed not surprising because apart from more or less complicated mathematical formalism the definitions of the considered variances and structure functions are closely related, as has been emphasized here.

Relations between sample variance and structure functions have been given by Lindsey and Chie [1976], whereas the relation between structure functions and several different approaches of frequency stability characterization has been analyzed by Rutman [1977, 1978] .

For the generally accepted noise model defined by (7) the $\tau$ dependence of higher-order structure functions is the same as the two-sample variance, as shown in Table 4 [Lindsey and Chie, 1978b]. As stated above, structure functions of order $n$ allow one to consider spectral densities which vary as $f^{\alpha}$ at the origin with $\alpha \geq-2(n-1)$. For instance, for $n=3$ it is possible to characterize frequency fluctuations of an oscillator with a power spectral density of fractional frequency fluctuations given by $S_{y}(f)=\Sigma_{\alpha=-4}^{2} h_{\alpha} f^{\alpha}$. This oscillator exhibits stationary third-order increments of phase fluctuations.

In the presence of a frequency drift described by a polynomial of degree $l-1$, structure functions of degree $n<l$ are meaningless: their computation yields a time-dependent result. For $n=l$ the $l$ th structure function shows a long-term $\tau$ dependence proportional to $\tau^{2 l}$. This dependence disappears for $n>l$. Although a power spectral density of the form $f^{-(21-1)}$ would also give the structure functions a variation of the form $\tau^{2 l}$, this variation does not depend on $n$, provided that the function is meaningful. It is then possible, at least in principle, to identify frequency drifts and to specify their order. This is illustrated in Figure 23 according to Lindsey and Chie [1978b]. However, there are not yet experimental proofs that such a characterization is achievable in practice.

\section{PO'NER SPECTRAL DENSITY OF STABLE FREQUENCY SOURCES}

The power emitted by a source of time-dependent voltage $v(t)$ given by $(1)$ is $S_{v}(v) d v$ in the frequency range $[v, v+d v]$, where $S_{v}(v)$ is the power spectral density of the source. The dimensions of $S_{v}(v)$ are $V^{2} \mathrm{~Hz}^{-1}$. The main interest of power spectral density, in frequency metrology, is related to highorder frequency multiplication. We will only introduce the subject by giving the relations between $S_{v}(v)$ and $S_{\varphi}(f)$ and stating present problems in the field.

TABLE 4. Structure functions of orders $1,2,3$, and 4 for fractional phase fluctuations of commonly encountered noise processes for $2 \pi f_{A} \tau>1$

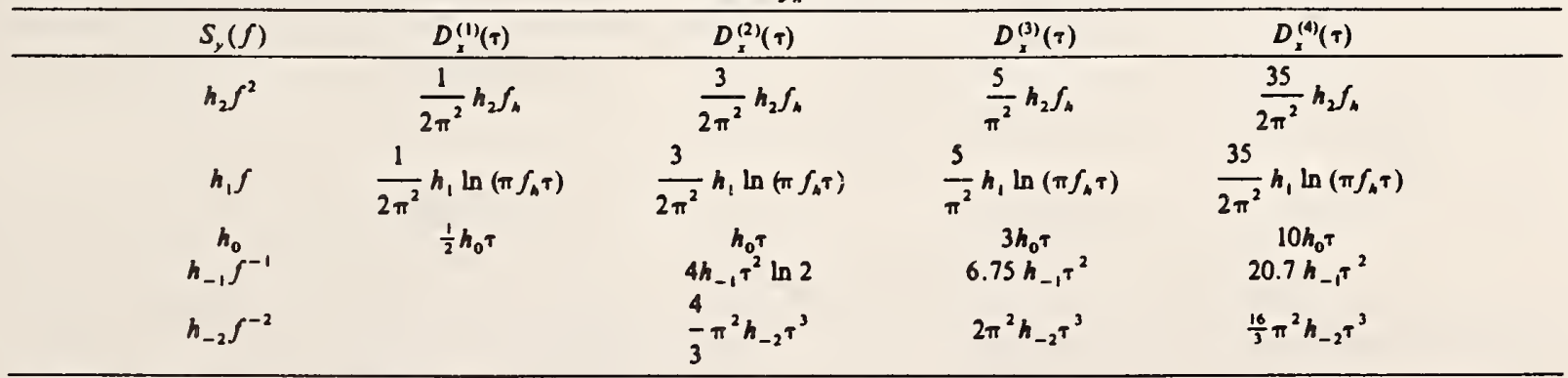

From Lindsey and Chie [1978b] 


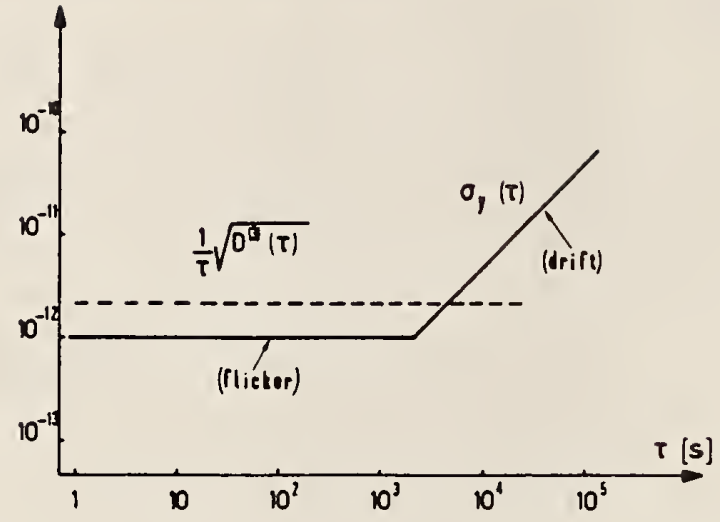

Fig. 23. (Solid line) Two-sample variance of an oscillator showing a linear frequency drift of $10^{-10}$ per day and nicker noise of frequency given by $S_{y}(f)=7.2 \times 10^{-25} f^{-1}$. (Dotted line) The third difference of fractional phase fluctuation is independent of the drift (according to Lindsey and Chie [1978b].

Negligible amplitude noise and gaussian phase fluctuations being assumed, it is well known that the autocorrelation function of $v(t)$ is $R_{v}(\tau)$ given by

$R_{v}(\tau)=\frac{V_{0}^{2}}{2} \cos 2 \pi v_{0} \tau \exp \left[-\frac{1}{2}\left(2 \pi v_{0}\right)^{2} \sigma^{2}\left(\bar{y}_{k}\right)\right]$

As $\sigma^{2}\left(\overline{y_{k}}\right)$ is only defined for stationary phase fluctuations and for phase fluctuations with stationary first increments, the same is true for $R_{v}(\tau)$ and therefore $S_{\nu}(f)$.

\subsection{White noise of frequency}

This is the simplest to deal with. If the frequency of the source is perturbed by a broadband white noise of frequency, one has $S_{y}(f)=h_{0}$ and $\sigma^{2}\left(\overline{y_{k}}\right)$ $=\left(h_{0} / 2 \tau\right)$. Whence

$$
R_{v}(\tau)=\frac{V_{0}^{2}}{2} \cos 2 \pi \nu_{0} \tau \exp \left(-\frac{|\tau|}{\tau_{c}}\right)
$$

where $\tau_{c}$ is the coherence time of the signal. We have

$$
\tau_{c}=\left(\pi^{2} v_{0}^{2} h_{0}\right)^{-1}
$$

The one-sided power spectral density is then represented by a Lorentzian given by

$$
S_{\nu}(v)=V_{0}^{2} \frac{2 \pi \Delta v}{(2 \pi \Delta v)^{2}+\left[2 \pi\left(\nu-v_{0}\right)\right]^{2}}
$$

where $\Delta v$ is the half width at half maximum of the power spectra defined as

$$
2 \Delta v=\pi v_{0}^{2} h_{0}
$$

We obviously have $2 \pi \Delta v \cdot \tau_{c}=1$. If oscillators are considered, one has $h_{0}=k T / P Q^{2}$ in the radiof requency and microwave domain and $h_{0}=$ $h \nu_{0} / P Q^{2}$ in the optical frequency domain, where $P$ is the power delivered by the oscillator and $Q$ the quality factor of the frequency-determining element. Table 5 gives theoretical values of coherence time and linewidth of good oscillators. It is only intended to illustrate a comparison, often made, of the spectral purity of oscillators. It must be pointed out that, in practice, other noise processes exist which modify these results. Even any meaning of $\tau_{c}$ and $\Delta v$ is removed if $R_{v}(\tau)$ is not defined.

Multiplication of the frequency by $n$ multiplies $\Delta v$ and divides $\tau_{c}$ by the factor $n^{2}$.

\subsection{White noise of phase}

Presently available good quartz oscillators are affected by white noise of phase. It is easy to show from the definition (16) of $y_{k}$ that the following equation is satisfied:

$$
\frac{1}{2}\left[2 \pi v_{0} \tau \sigma\left(\overline{y_{k}}\right)\right]^{2}=R_{\varphi}(0)-R_{\varphi}(\tau)
$$

where $R_{\varphi}(\tau)$ denotes the autocorrelation function of the stationary phase fluctuations $\varphi(t)$.

The expression of the one-sided $S_{v}(v)$ then follows [Rutman, 1974a; Lindsey and Chie, 1978a]:

$$
\begin{aligned}
S_{v}(v)= & \frac{V_{0}^{2}}{2} e^{-R_{0}(0)}\left[\delta\left(v-v_{0}\right)\right. \\
& \left.+S_{\varphi}\left(v-v_{0}\right)+\frac{1}{2} S_{\varphi}(v) * S_{\varphi}(v)+\cdots\right]
\end{aligned}
$$

where the asterisk denotes convolution and the bracket contains an infinite set of multiple-convolution products of $S_{\varphi}(v)$ by itself. Such an equation is not easily tractable. It is the reason why the

TABLE 5. Theoretical values of correlation time and power spectrum linewidth for various oscillators

\begin{tabular}{lrlll}
\hline Oscillator & $\nu_{0}, \mathrm{~Hz}$ & \multicolumn{1}{c}{$h_{0}, \mathrm{~Hz}^{-1}$} & $T_{c}, \mathrm{~s}$ & $2 \Delta \nu, \mathrm{Hz}$ \\
\hline S-MHz & & & & \\
quartz xtal & $5 \times 10^{6}$ & $4 \times 10^{-27}$ & $10^{12}$ & $3 \times 10^{-13}$ \\
H maser & $1.4 \times 10^{9}$, & $4 \times 10^{-27}$ & $10^{7}$ & $3 \times 10^{-1}$ \\
He-Ne laser & $5 \times 10^{14}$ & $3 \times 10^{-20}$ & $10^{-2}$ & 30 \\
\hline
\end{tabular}


approximation of small-phase fluctuations is often made. If $R_{\varphi}(0)=\overline{\varphi^{2}} \ll 1$, one has

$$
S_{v}(v)=\frac{v_{0}^{2}}{2} e^{\overline{-\phi^{2}}}\left[\delta\left(v-v_{0}\right)+S_{\varphi}\left(v-v_{0}\right)\right]
$$

In this approximation the power spectrum consists of a carrier at frequency $v_{0}$ around which the spectrum of the phase fluctuations is translated.

If the frequency of the signal is multiplied by $n$, the mean squared frequency fluctuation becomes $n^{2} \overline{\varphi^{2}}$. If $n^{2} \overline{\varphi^{2}}<1$, the power spectral density is then given by

$S_{\nu}(v)=\frac{V_{0}^{2}}{2} e^{-n \overline{\phi^{2}}}\left[\delta\left(v-n v_{0}\right)+n^{2} S_{\varphi}\left(v-v_{0}\right)\right]$

The power in the carrier decreases, and the power in the pedestal increases. The relative powers in the carrier $P_{c}$ and in the pedestal $P_{p}$ are then given by

$$
\begin{gathered}
P_{c}=e^{-\overline{\phi^{2}}} \\
P_{p}=1-e^{-\overline{\phi^{2}}}
\end{gathered}
$$

respectively, where $\phi^{2}$ represents the mean squared value of phase fluctuations at the signal frequency considered. It has been proved that (62) and (63) are valid, even if the condition $\overline{\phi^{2}} \ll 1$ is not satisfied (F. Clerc, private communication, 1977).

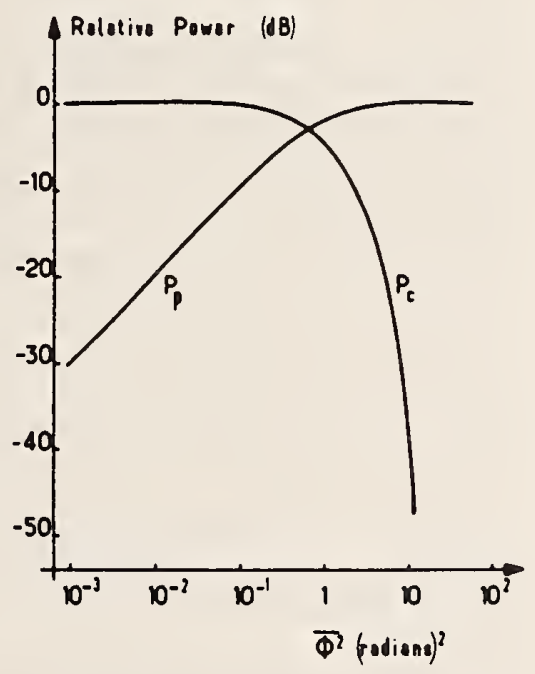

Fig. 24. Variation of the relative power in the carrier $P_{c}$ and the pedestal $P$, as a function of $\phi^{2}$, the mean squared phase fluctuations.
Figure 24 shows the variation of $P_{c}$ and $P_{p}$ as a function of $\overline{\phi^{2}}$. One easily understands that the carrier may disappear if the multiplicative factor is high enough. This has been theoretically and experimentally investigated by Walls and de Marchi [1975], Bava et al. [1977b], and Godone et al. [1978]. A signal has been synthesized at $761 \mathrm{GHz}$, starting from a $5-\mathrm{MHz}$ quartz oscillator, which verifies theoretical conclusions.

\subsection{Other noise processes}

Much work remains to be done to analyze properly the effect of noise such as the flicker noise of frequency or the random walk of frequency which contributes power very close to the carrier. The very interesting semiempirical approach by Halford [1971] has not yet been justified either theoretically or experimentally in a convincing manner.

\section{CONCLUSION}

Widely used theoretical and experimental methods for the characterization of frequency stability in the time and frequency domain have been outlined. Recently used or proposed experimental methods have been reviewed. The effect of dead time on the interpretation of time domain measurements, as well as on their precision has been emphasized. Recently introduced structure functions have been considered as well as their interest for the elimination of frequency drifts. The problems in the relation between the radiofrequency power spectral density and the power spectral density of phase fluctuations have been briefly summarized.

\section{REFERENCES}

Allan, D. W. (1966), Statistics of atomic frequency standards, Proc. IEEE, S4(2), 221-230.

Allan, D. W., and H. Daams (1975), Picosecond time difference measurement system, Proceedings of the $29 \mathrm{th}$ Annual Frequency Control Symposium, pp. 404-411, Electronic Industries Association, Washington, D.C.

Barillet, R., and C. Audoin (1976), Electronic circuits showing a level-independent phase shift for hydrogen-maser work applications, IEEE Trans. Instrum. Meas., IM-25(14), 465468.

Barger, R. L., and J. L. Hall (1969), Pressure shift and broadening of methane line at $3.39 \mu \mathrm{m}$ studied by laser saturated molecular absorption, Phys. Rev. Lett., 22(1), 4-8.

Barnes, J. A. (1966), Atomic time-keeping and the statistics of precision signal generators, Proc. IEEE, 54(2), 207-220.

Barnes, J. A. (1976), Models for the interpretation of frequency 
stability measurements, Nat. Bur. Stand. Tech. Note, 683.

Barnes, J. A. (1977), A review of methods of analyzing frequency stability, Proceedings of the 9 h Annual Precise Time and Time Interval Applications and Planning Meeting, pp. 61-84, Technical Information and Administrative Support Division, Goddard Space Flight Center, Greenbelt, Md.

Barnes, J. A., A. R. Chi, L. S. Cutler, D. J. Healey, D. B. Leeson, T. E. McGunigal, J. A. Mullen, W. L. Smith, R. L. Sydnor, R. F. C. Vessot, and G. M. R. Winkler (1971), Characterization of frequency stability, IEEE Trans. Instrum. Meas., IM-20(2), 105-120.

Baugh, R. A. (1971), Frequency modulation analysis with the Hadamard variance, Proceedings of the 25th Annual Frequency Control Symposium, pp. 222-225, National Techoical Information Service, Springfield, Va.

Bava, E., G. P. Bava, A. de Marchi, and A. Godone (1977a), Measurement of static AM-PM conversion in frequency multipliers, IEEE Trans. Insirum. Meas., IM-26(1), 33-38.

Bava, E., A. de Marchi, and A. Godone (1977b), Spectral analysis of synthesized signals in the $\mathrm{mm}$ wavelength region, IEEE Trans. Instrum. Meas., IM-26(2), 128-132.

Blaquière, A. (1953a), Effet du bruir de fond sur la fréquence des auto-oscillateurs à lampes: Précision ultime des borloges radioélectriques, Ann. Radioelec. 8(31), 36-80.

Blaquière, A. (1953b), Spectre de puissance d'un oscillateur non-linéaire perturbé par le bruit, Ann. Radioelec., 8(32), 153-179.

Boileau, E. (1975), Elimination optimale des dérives lentes dans les mesures de fluctuation, Ann. Télécommun., 30(5-6), 163166.

Boileau, E. (1976), Optimisation de certains traitements numériques avec une forme quadratique, Ann. Télécommun., 31 (56), 179-189.

Boileau, E., and Y. Lecourtier (1977), Description statistique de la stabilité des oscillateurs, Proceedings of the Conference on Measurement in Telecommunications, Pp. 231-236, Centre National d'Etudes des Télécommunications, Lannion, France.

Boileau, E., and B. Picinbono (1976), Statistical study of phase fluctuations and oscillator stability, IEEE Trans. Instrum. Meas., $I M-25(1), 66-75$.

Brendel, R., G. Marianneau, and J. Uebersfeld (1977), Phase and amplitude modulation effects in a phase detector using an incorrectly balanced mixer, IEEE Trans. Instrum. Meas., IM-26(2), 98-102.

Cérez, P., S. J. Bennell, and C. Audoin (1978), Stabilité de fréquence du laser hélium-néon comportant une cuve d'iode à parois chaudes, C. R. Acad. Sci., 286B, 53-56.

Cutler, L. S., and C. L. Searle (1966), Some aspects of the theory and measurement of frequency fluctuations in frequency standards, Proc. IEEE, 54 (2), 136-154.

Godone, A., C. O. Weiss, and G. Kramer (1978), FM noise measurements on an optically pumped FIR laser, IEEE J. Quantum Electron, $Q E-14(5), 339-342$.

Gray, J. E., and D. W. Allan (1974), A method for estimating the frequency stability of an individual oscillator, Proceedings of the 28th Annual Frequency Control Symposium, pp. 243246, Electronic Industries Association, Washington, D.C.

Groslambert, J. (1976), A new spectrum analyzer using both analog and digital filtering via Hadamard variance, IEEE Trans. Instrum. Meas., IM-25(4), 480-483.

Groslambert, J. (1977), Mesure des instabilités de fréquence des oscillateurs dans le domaine spectral et dans le domaine temporel, Proceedings of the Conference on Measurements in Telecommunications, pp. 274-279, Centre National d'Etudes des Télécommunications, Lannion, France.

Halford, D. (1971), Infrared-microwave frequency synthesis design: Some relevant conceptual noise aspects, Proceedings of the Frequency Standard and Metrology Seminar, pp. 431466, Université Laval, Quebec, Ontario.

Halford, D., A. E. Wainwright, and J. A. Barnes (1968), Flicker noise of phase in R.F. amplifiers and frequency multipliers: Characterization, cause and cure, Proceedings of the 22nd Annual Frequency Control Symposium, pp. 340-341, National Technical Information Service, Springfield, Va.

Halford, D., J. H. Shoaf, and A. S. Risley (1973), Spectral density analysis: Frequency domain specification and measurement of signal stability. Proceedings of the 27th Annual Frequency Control Symposium, pp. 421-430, Electronic Industries Association, Washington, D.C.

Healey, D. 3. (1972), Flicker of frequency and phase and white frequency and phase fluctuations in frequency sources, Proceedings of the 26th Annual Frequency Control Symposium, pp. 29-42, Electronic Industries Association, Washington, D.C.

IEEE-NASA (1964), Symposium on Short-Term Frequency Stability, 317 pp., Goddard Space Flight Center, Greenbelt, Md.

Kartaschoff, P. (1978), Frequency and Time, 260 pp., Academic, New York.

Lance, A. L., W. D. Seal, F. G. Mendoza, and N. W. Hudson (1977), Automated phase noise measurements, Microwave J., 20(6), 87-103.

Lance, A. L., W. D. Seal, F. G. Mendoza, and N. W. Hudson (1978), Phase noise measurements using cross-spectrum analysis, Digest of the Conference on Precision Electromagnetic Measurements-Ottawa, pp. 94-96, IEEE Catalogue $78 \mathrm{Cb}$ 1320-1 IM

Lesage, P., and C. Audoin (1973), Characterization of frequency stability: Uncertainty due to the finite number of measurements, IEEE Trans. Instrum. Meas., IM-22(2), 157-161.

Lesage, P., and C. Audoin (1975a), A time domain method for measurement of the spectral deasity of frequency fluctus. tions at low Fourier frequencies. Proceedings of the $29 \mathrm{rh}$ Annual Frequency Control Symposium, pp. 394-403, Electronic Industries Association, Washington, D.C.

Lesage, P., and C. Audoin (1975b), Instabilité de fréquence des oscillateurs: Mesure dans le domaine fréquence par une méthode temporelle, L'onde Elec., 55(2), 82-89.

Lesage, P., and C. Audoin (1977), Estimation of the two-sample variance with a limited number of data, Proceedings of the 31st Annual Frequency Control Symposium, pp. 311-318, Electronic Industries Association, Washington, D.C.

Lindsey, W. C., and C. M. Chie (1976), Theory of oscillator instability based upon structure functions, Proc. IEEE, 64(12), 1652-1666.

Lindsey, W. C., and C. M. Chie (1977a), Specification and measurement of oscillator phase noise instability, Proceedings of the 31st Annual Frequency Control Symposium, pp. 302-310, Electronic Industries Association, Washington, D.C.

Lindsey, W. C., and C. M. Chie (1977b), Structure functions of oscillator fractional phase and frequency fluctuations, $I E E E$ Trans. Instrum. Meas., 'IM-26(4), 420-421. 
Lindsey, W. C., and C. M. Chie (1978a), Frequency multiplication effects on oscillator instability, IEEE Trans. Instrum. Meas., $I M-27(1), 26-28$.

Lindsey, W. C., and C. M. Chie (1978b), Identification of power-law type oscillator phase noise spectra from measurements, IEEE Trans. Instrum. Meas., IM-27(1), 46-53.

Musha, T. (1975), $1 / f$ resonant frequency fluctuation of a quartz crystal, Proceedings of the $29 \mathrm{~h}$ Annual Frequency Control Symposium, Pp. 308-310, Electronic Industries Association, Washington, D.C.

National Bureau of Standards (1974), Time and Frequency: Theory and Fundamentals, Monogr. 140, 459 pp., Washington, D.C.

Papoulis, A. (1965), Probability Random and Stochastic Processes, 583 pp., McGraw-Hill, New York.

Percival, D. B. (1978), Estimation of the spectral density of fractional frequency deviates, Proceedings of the 32nd Annual Frequency Control Symposium, pp. 542-548, Electronic Industries Association, Washington, D.C.

Peregrino, L., and D. W. Ricci (1976), Phase noise measurement using a high resolution counter with on-line data processing, Proceedings of the 30h A nnual Frequency Control Symposium, pp. 309-317, Electronic Industries Association, Washington, D.C.

IEEE (1966), Special Issue on Frequency Stability, Proc. IEEE, $54(2)$.

Rutman, J. (1974a), Relations between spectral purity and frequency stability, Proceedings of the 28th A nnual Frequency Control Symposium, pp. 160-165, Electronic Industries Associstion, Washington, D.C.

Rutman, J. (1974b), Characterization of frequency stability: A transfer function approach and its application to measurements via filtering of phase noise, IEEE Trans. Instrum. Meas., IM-23(1), 40-48.

Rutman, J. (1977), Oscillator specifications: A review of classical and new ideas, Proceedings of the 3lst Annual Frequency Control Symposium, pp. 291-301, Electronic Industries Association, Washington, D.C.

Rutman, J. (1978), Characterization of phase and frequency instabilities in precision frequency sources: Fifteen years of progress, Proc. IEEE, 66(9), 1048-1075.

Rutman, J., and G. Sauvage (1974), Measurement of frequency stability in time and frequency domains via filtering of phase. noise, IEEE Trans. Instrum. Meas., IM-23(4), 515-518.

Siegman, A. E., and R. Arrathoon (1968), Observation of quantum phase noise in laser oscillator, Phys. Rev. Lett., 20(17), 901-902.

Shoaf, J. H. (1971), Specification and measurement of frequency stability, NBS Rep. 9794, U.S. Government Printing Office, Washington, D.C.

Tausworthe, R. C. (1972), Convergence of oscillator spectral estimators for counted-frequency measurements, IEEE Trans. Commun., COM-20(2), 214-217.

Vanier, J., M. Tétu, and R. Brousseau (1977), Frequency and time domain stability of the $\mathrm{Rb}^{\text {" }}$ maser and related oscillators: A progress report, Proceedings of the 31st Annual Frequency Control Symposium, pp. 344-346, Electronic Industries Association, Washington, D.C.

Vessot, R. F. C., M. W. Levine, and E. M. Mattison (1977), Comparison of theoretical and observed maser stability limitations due to thermal noise and the prospect of improvement by low temperature operation, Proceedings of the 9 th Annual Precise Time and Time Interval Applications and Planning Meeting, pp. 549-566, Technical Information and Administrative Support Division, Goddard Space Flight Center, Greenbelt, Md.

Wainwright, A. E., F. L. Walls, and W. D. McCoa (1974), Direct measurements of the inherent frequency stability of quartz crystal resonators, Proceedings of the $28 \mathrm{th}$ Annual Frequency Control Symposium, pp. 177-180, Electronic Industries Association, Washington, D.C.

Walls, F. L., and A. de Marchi (1975), RF spectrum of a signal after frequency multiplication: Measurement and comparison with a simple calculation, IEEE Trans. Instrum. Meas., IM-24(3), 210-217.

Walls, F. L., and S. R. Stein (1977), Accurate measurements of spectral density of phase noise in devices, Proceedings of the 31st A nnual Frequency Control Symposium, pp. 335-343, Electronic Industries Association, Washington, D.C.

Wiley, R. G. (1977), A direct time-domain measure of frequency stability: The modified Allan variance, IEEE Trans. Instrum. Meas., IM-26(1), 38-41.

Winkler, G. M. R. (1976), A brief review of frequency stability measures, Proceedings of the 8th Annual Precise Time and Time Interval Applications and Planning Meeting, pp. 489527, Technical Information and Administrative Support Division, Goddard Space Flight Center, Greenbelt, Md.

Yoshimura, K. (1978), Characterization of frequency stability: Uncertainty due to the sutocorrelation of the frequency fluctuations, IEEE Trans. Insirum. Meas., IM-27(1), 1-7. 
Copyright 1984 Academic Press. Reprinted, with permission, from Infrared and Millimeter Waves, Vol. 11, pp. 239-289, 1984.

INFRARED AND MILLIMETER WAVES. VOL. II

\section{CHAPTER 7}

\section{Phase Noise and AM Noise Measurements in the Frequency Domain}

\begin{tabular}{|c|c|c|}
\hline $\begin{array}{l}\text { One } \\
\text { Red }\end{array}$ & $\begin{array}{l}\text { Operations and Support Group } \\
\text { Space Park } \\
\text { ndo Beach. California }\end{array}$ & \\
\hline I. & INTRODUCTION & \\
\hline II. & FUNDAMENTAL CONCEPTS & \\
\hline & A. Noise Sidebands & \\
\hline & B. Speciral Density & \\
\hline & C. Spectral Densities of Phase Fluctuations in the & \\
\hline & Frequency Domain & \\
\hline & D. Modulation Theory and Spectral Density Relationships & \\
\hline & E. Noise Processes & \\
\hline & F. Integrated Phase Noise & \\
\hline & G. AM Noise in the Frequency Domain & \\
\hline & Phase-Noise Measurements Using the & \\
\hline & TWO-OSCILLATOR TECHNIQUe & \\
\hline & A. Two Notsy Oscillators & \\
\hline & B. Automated Phase-Noise Measurements Using the & \\
\hline & Two-Oscillator Technique & \\
\hline & C. Calibration and Measurements Using the & \\
\hline & Two-Oscillator Svstem & \\
\hline & Single-Oscillator Phase-Noise Measurement & \\
\hline & SYSTEMS AND TECHNIQUES & 26 \\
\hline & A. The Delay Line as an FM Discriminator & 268 \\
\hline & $\begin{array}{l}\text { B. Calibration and Measurements Using the Delay Line } \\
\text { as an FM Discriminator }\end{array}$ & \\
\hline & C. Dual Delay-Line Discriminator & \\
\hline & D. Millimeter-Wave Phase-Noise Measurements & \\
\hline & REFERENCES & \\
\hline
\end{tabular}

\section{Introduction}

Frequency sources contain noise that appears to be a superposition of causally generated signals and random, nondeterministic noises. The random noises include thermal noise, shot noise, and noises of undetermined origin (such as flicker noise). The end result is time-dependent phase and amplitude fluctuations. Measurements of these fluctuations characterize the frequency source in terms of amplitude modulation (AM) and phase modulation (PM) noise (frequency stability). 
The term frequency stability encompasses the concepts of random noise, intended and incidental modulation, and any other fluctuations of the output frequency of a device. In general, frequency stability is the degree to which an oscillating source produces the same frequency value throughout a specified period of time. It is implicit in this general definition of frequency stability that the stability of a given frequency decreases if anything except a perfect sine function is the signal wave shape.

Phase noise is the term most widely used to describe the characteristic randomness of frequency stability. The term spectral purity refers to the ratio of signal power to phase-noise sideband power. Measurements of phase noise and AM noise are performed in the frequency domain using a spectrum analyzer that provides a frequency window following the detector (doublebalanced mixer). Frequency stability can also be measured in the time domain with a gated counter that provides a time window following the detector.

Long-term stability is usually expressed in terms of parts per million per hour, day, week, month, or year. This stability represents phenomena caused by the aging process of circuit elements and of the material used in the frequency-determining element. Short-term stability relates to frequency changes of less than a few seconds duration about the nominal frequency.

Automated measurement systems have been developed for measuring the combined phase noise of two signal sources (the two-oscillator technique) and a single signal source (the single-oscillator technique), as reported by Lance et al. (1977) and Seal and Lance (1981). When two source signals are applied in quadrature to a phase-sensitive detector (double-balanced mixer), the voltage fluctuations analogous to phase fluctuations are measured at the detector output. The single-oscillator measurement system is usually designed using a frequency cavity or a delay line as an FM discriminator. Voltage fluctuations analogous to frequency fluctuations are measured at the detector output.

The integrated phase noise can be calculated for any selected range of Fourier frequencies. A representation of fluctuations in the frequency domain is called spectral density graph. This graph is the distribution of power variance versus frequency.

\section{Fundamental Concepts}

In this presentation we shall attempt to conform to the definitions. symbols, and terminology set forth by Barnes et al. (1970). The Greek letter $v$ represents frequency for carrier-related measures. Modulationrelated frequencies are designated $f$. If the carrier is considered as $\mathrm{dc}$, the frequencies measured with respect to the carrier are referred to as baseband, offset from the carrier, modulation, noise, or Fourier frequencies. 


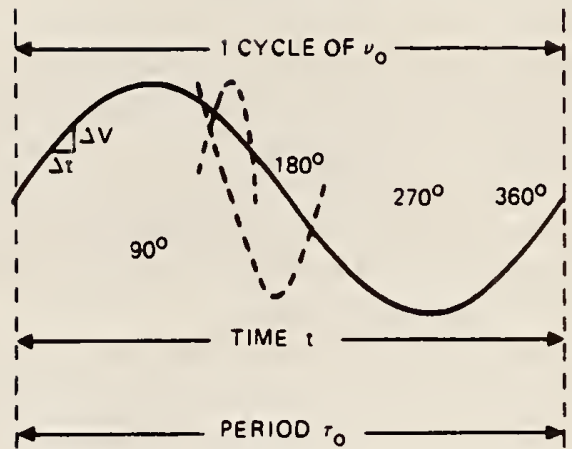

(a)

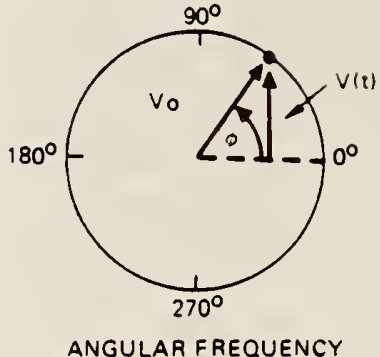

(b)

FIG. I Sine wave characteristics: (a) voltage $V$ changes with time $\tau$ as (b) amplitude changes with phase angle $\phi$.

A sine wave generator produces a voltage that changes in time $t$ as the amplitude $V$ changes with the phase angle $\phi$, shown in Fig. 1. Phase is measured from a zero crossing, as illustrated by plotting the phase angle as the radius vector rotates at a constant angular rate determined by the frequency. The ideal (perfect) sine-wave-related parameters are as follows: $v_{0}$, average (nominal) frequency of the signal; $v(t)$, instantaneous frequency of a signal

$$
v(t)=\frac{1}{2 \pi} \frac{d \phi}{d t}(t)
$$

$V_{0}$, nominal peak amplitude of a signal source output; $\tau$, period of an oscillaion $\left(1 / v_{0}\right) ; \Omega$, signal (carrier) angular frequency (rate of change of phase with time) in radians

$$
\Omega=2 \pi v_{0}
$$

$\Omega t$, instantaneous angular frequency; $V(t)$, instantaneous output voltage of a signal. For the ideal sine wave signal of Fig. 1, in volts,

$$
V(t)=V_{0} \sin \left(2 \pi v_{0} t\right) \text {. }
$$

The basic relationship between phase $\phi$, frequency $v_{0}$, and time interval $\tau$ of the ideal sine wave is given in radians by the following:

$$
\phi=2 \pi v_{0} \tau,
$$

where $\phi(t)$ is the instantaneous phase of the signal voltage, $V(t)$, defined for the ideal sine wave in radians as

$$
\phi(t)=2 \pi v_{0} t .
$$


The instantaneous phase $\phi(t)$ of $V(t)$ for the noisy signal is

$$
\phi(t)=2 \pi v_{0} t+\phi(t),
$$

where $\phi(t)$ is the instantaneous phase fluctuation about the ideal phase $2 \pi v_{0} \tau$ of Eq. (4).

The simplified illustration in Fig. 1 shows the sine-wave signal perturbed for a short instant by noise. In the perturbed area, the $\Delta v$ and $\Delta t$ relationships correspond to other frequencies, as shown by the dashed-line waveforms. In this sense, frequency variations (phase noise) occur for a given instant within the cycle.

The instantaneous output voltage $V(t)$ of a signal generator or oscillator is now

$$
V(t)=\left[V_{0}+\varepsilon(t)\right] \sin \left[2 \pi v_{0} t+\phi(t)\right]
$$

where $V_{0}$ and $v_{0}$ are the nominal amplitude and frequency, respectively, and $\varepsilon(t)$ and $\phi(t)$ are the instantaneous amplitude and phase fluctuations of the signal.

It is assumed in Eq. (7) that

$$
\varepsilon(t) / V_{0} \ll 1 \text { and } \frac{\dot{\phi}(t)}{v_{0}} \ll 1 \quad \text { for all } \quad(t), \dot{\phi}(t)=d \phi / d t
$$

Equation (7) can also be expressed as

$$
V(T)=\left[V_{0}+\delta \varepsilon(t)\right] \sin \left[2 \pi v_{0} t+\phi_{0}+\delta \phi(t)\right],
$$

where $\phi_{0}$ is a constant, $\delta$ is the fluctuations operator, and $\delta \varepsilon(t)$ and $\delta \phi(t)$ represent the fluctuations of signal amplitude and phase, respectively.

Frequency fluctuations $\delta v$ are related to phase fluctuations $\delta \phi$, in hertz, by

$$
\delta v=\frac{\delta \Omega}{2 \pi}=\frac{1}{2 \pi} \frac{d(\delta \phi)}{d t},
$$

i.e., radian frequency deviation is equal to the rate of change of phase deviation (the first-time derivative of the instantaneous phase deviation).

The fluctuations of time interval $\delta \tau$ are related to fluctuations of phase $\delta \phi$, in radians, by

$$
\delta \phi=\left(2 \pi v_{0}\right) \delta \tau .
$$

In the following, $y$ is defined as the fractional frequency fluctuation or fractional frequency deviation. It is the dimensionless value of $\delta v$ normalized to the a verage (nominal) signal frequency $v_{0}$,

$$
y=\delta v / v_{0}
$$


where $y(t)$ is the instantaneous fractional frequency deviation from the nominal frequency $v_{0}$.

\section{A. NoISE SIDEBandS}

Noise sidebands can be thought of as arising from a composite of low. frequency signals. Each of these signals modulate the carrier-producing components in both sidebands separated by the modulation frequency, as illustrated in Fig. 2. The signal is represented by a pair of symmetrical sidebands (pure AM) and a pair of antisymmetrical sidebands (pure FM).

The basis of measurement is that when noise modulation indices are small, correlation noise can be neglected. Two signals are uncorrelated if their phase and amplitudes have different time distributions so that they do not cancel in a phase detector. The separation of the AM and FM components are illustrated as a modulation phenomenon in Fig. 3. Amplitude fluctuations can be measured with a simple detector such as a crystal. Phase or frequency fluctuations can be detected with a discriminator. Frequency modulation (FM) noise or rms frequency deviation can also be measured with an amplitude (AM) detection system after the FM variations are converted to $\mathrm{AM}$ variations, as shown in Fig. 3a. The FM-AM conversion is obtained by applying two signals in phase quadrature $\left(90^{\circ}\right)$ at the inputs to a balanced mixer (detector). This is illustrated in Fig. 3 by the $90^{\circ}$ phase advances of the carrier.

\section{B. Spectral Density}

Stability in the frequency domain is commonly specified in terms of spectral densities. There are several different, but closely related, spectral densities that are relevant to the specification and measurement of stability of the frequency, phase, period, amplitude, and power of signals. Concise, tutorial

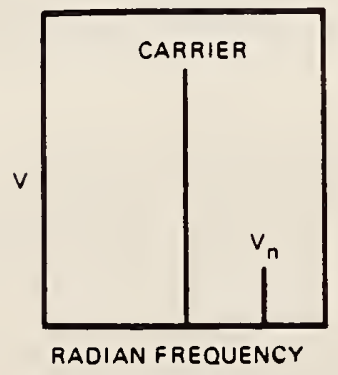

(a)

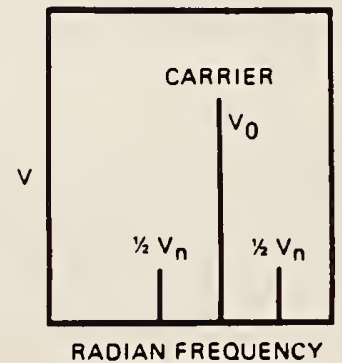

(b)

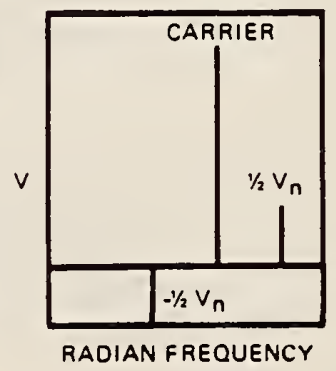

(c)

FIG. 2 (a) Carrier and single upper sideband signals; (b) symmetrical sidebands (pure $A M) ;(c)$ an antisymmetrical pair of sidebands (pure FM). 


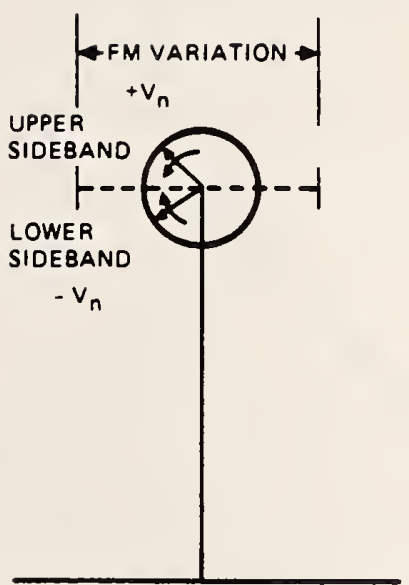

(a)

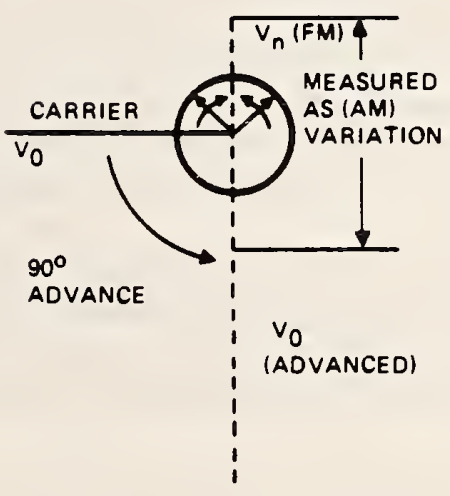

(c)

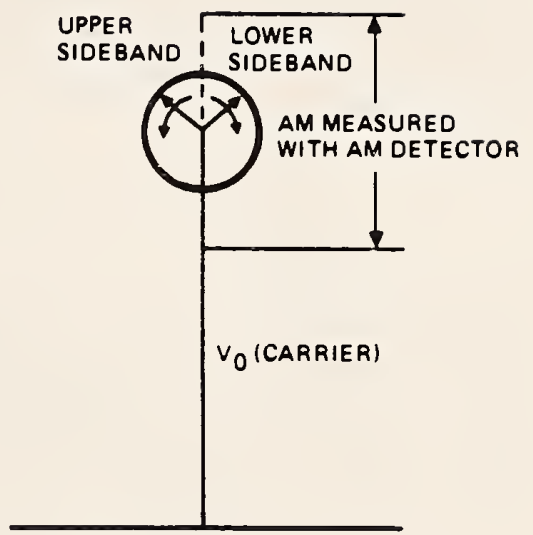

(b)

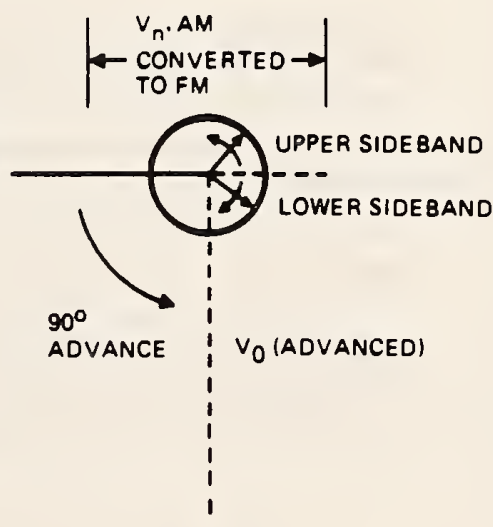

(d)

FIG. 3 (a) Relationships of the FM signal to the carrier: (b) relationship of the AM signal to the carrier; (c) carrier advanced $90^{\circ}$ to obtain FM-AM conversion ; (d) AM-FM conversion.

descriptions of twelve defined spectral densities and the relationships among them were given by Shoaf et al. (1973) and Halford et al. (1973).

Recall that in the perturbed area of the sine wave in Fig. 1 the frequencies are being produced for a given instant of time. This amount of time the signal spends in producing another frequency is referred to as the probability. density of the generated frequencies relative to $v_{0}$. The frequency domain plot is illustrated in Fig. 4. A graph of these probability densities over a period of time produces a continuous line and is called the Power spectral density. 


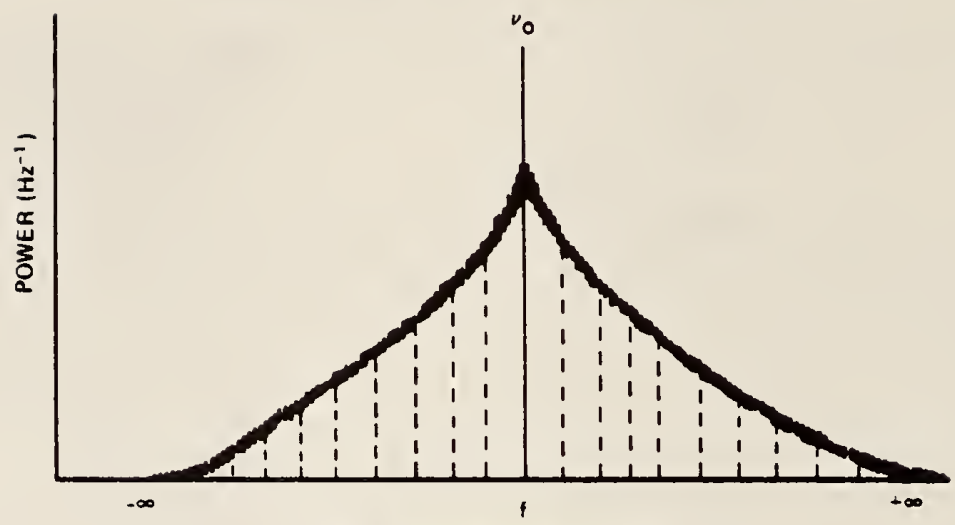

Fig. 4 A power density plot.

The spectral density is the distribution of total variance over frequency. The units of power spectral density are power per hert $z$ : therefore. a plot of power spectral density obtained from amplitude (voltage) measurements requires that the voltage measurements be squared.

The spectral density of power versus frequency, shown in Fig. 4, is a twosided spectral density because the range of Fourier frequencies $f$ is from minus infinity to plus infinity.

The notation $S_{g}(f)$ represents the two-sided spectral density of fuctations of any specified time-dependent quantity $g(t)$. Because the frequency band is defined by the two limit frequencies of minus infinity and plus infinity, the total mean-square fluctuation of that quantity is defined by

$$
G_{\text {sideband }}=\int_{-\infty}^{+\infty} S_{g}(f) d f \text {. }
$$

Two-sided spectral densities are useful mainly in pure mathematical analysis involving Fourier transformations.

Similarly, for the one-sided spectral density,

$$
G_{\text {sideband }}=\int_{0}^{+\infty} S_{g}(f) d f .
$$

The two-sided and one-sided spectral densities are related as follows:

$$
\int_{-\infty}^{+\infty} S_{g_{2}} d f=2 \int_{0}^{+\infty} S_{g_{2}} d f=\int_{0}^{+\infty} S_{g_{1}} d f
$$

where $g_{1}$ indicates one-sided and $g_{2}$ two-sided spectral densities. It is noted that the one-sided density is twice as large as the corresponding two-sided 
spectral density. The terminology for single-sideband versus double-sideband signals is totally distinct from the one-sided spectral density versus twosided spectral terminology. They are totally different concepts. The definitions and concepts of spectral density are set forth in NBS Technical Note 632 (Shoaf et al., 1973).

\section{Spectral Densities of Phase Fluctuations in the FREQUENCY DOMAIN}

The spectral density $S_{y}(f)$ of the instantaneous fractional frequency fluctuations $y(t)$ is defined as a measure of frequency stability, as set forth by Barnes et al. (1970). $S_{y}(f)$ is the one-sided spectral density of frequency fluctuations on a "per hertz" basis, i.e., the dimensionality is $\mathrm{Hz}^{-1}$. The range of Fourier frequency $f$ is from zero to infinity. $S_{\delta v}(f)$, in hertz squared per hertz. is the spectral density of frequency fluctuations $\delta v$. It is calculated as

$$
S_{\delta v}(f)=\frac{\left(\delta v_{\mathrm{rms}}\right)^{2}}{\text { bandwidth used in the measurement of } \delta v_{\mathrm{rms}}} .
$$

The range of the Fourier frequency $f$ is from zero to infinity.

The spectral density of phase fluctuations is a normalized frequency domain measure of phase fluctuation sidebands. $S_{\delta \phi}(f)$, in radians squared per hertz. is the one-sided spectral density of the phase fluctuations on a "per hertz" basis:

$$
S_{\delta \phi}(f)=\frac{\delta \phi_{\mathrm{rms}}}{\text { bandwidth used in the measurement of } \delta \phi_{\mathrm{rms}}} .
$$

The power spectral densities of phase and frequency fluctuation are related by

$$
S_{\delta \phi}(f)=\left(v_{0}^{2} / f\right) S_{y}(f) .
$$

The range of the Fourier frequency $f$ is from zero to infinity:

$S_{\delta \Omega}(f)$. in radians squared Hertz squared per hertz is the spectral density of angular frequency fluctuations $\delta \Omega$ :

$$
S_{\delta \Omega}(f)=(2 \pi)^{2} S_{\delta v}(f) .
$$

The defined spectral densities have the following relationships:

$$
\begin{aligned}
& S_{\delta v}(f)=v_{0}^{2} S_{y}(f)=(1 / 2 \pi)^{2} S_{\delta \Omega}(f)=f^{2} S_{\delta \phi}(f) ; \\
& S_{\delta \phi}(f)=(1 / \omega)^{2} S_{\delta \Omega}(f)=\left(v_{0} / f\right)^{2} S_{y}(f)=\left[S_{\delta v}(f) / f^{2}\right] .
\end{aligned}
$$

Note that Eq. (20) is hertz squared per hertz, whereas Eq. (21) is in radians squared per hertz.

The term $S_{\sqrt{r F P}}(v)$, in watts per hertz, is the spectral density of the (square

* See Appendix Note \# 30 
root of radio frequency power $P$. The power of a signal is dispersed over the frequency spectrum owing to noise, instability, and modulation. This concept is similar to the concept of spectral density of voltage fluctuations $S_{\delta v}(f)$. Typically, $S_{\delta v}(f)$ is more convenient for characterizing a baseband signal where voltage, rather than power, is relevant. $S_{\sqrt{r f P}}(v)$ is typically more convenient for characterizing the dispersion of the signal power in the vicinity of the nominal carrier frequency $v_{0}$. To relate the two spectral densities, it is necessary to specify the impedance associated with the signal.

A definition of frequency stability that relates the actual sideband power of phase fluctuations with respect to the carrier power level, discussed by Glaze (1970), is called $\mathscr{L}(f)$. For a signal with $\mathrm{PM}$ and with no $\mathrm{AM}, \mathscr{L}(f)$ is the normalized version of $S_{\sqrt{r P}}(v)$, with its frequency parameter $f$ referenced to the signal's average frequency $v_{0}$ as the origin such that $f$ equals $v-v_{0}$. If the signal also has $\mathrm{AM}, \mathscr{L}(f)$ is the normalized version of those portions of $S_{\sqrt{\mathrm{rP} P}}(v)$ that are phase-modulation sidebands.

Because $f$ is the Fourier frequency difference $\left(v-v_{0}\right)$, the range of $f$ is from minus $v_{0}$ to plus infinity. Since $\mathscr{L}(f)$ is a normalized density (phase noise sideband power),

$$
\int_{-v_{0}}^{+\infty} \mathscr{L}(f) d f=1
$$

$\mathscr{L}(f)$ is defined as the ratio of the power in one sideband, referred to the input carrier frequency on a per hertz of bandwidth spectral density basis, to the total signal power, at Fourier frequency difference $f$ from the carrier, per one device. It is a normalized frequency domain measure of phase fluctuation sidebands, expressed in decibels relative to the carrier per hertz:

$$
\mathscr{L}(f)=\frac{\text { power density (one phase modulation sideband) }}{\text { carrier power }} .
$$

For the types of signals under consideration, by definition the two phasenoise sidebands (lower sideband and upper sideband, at $-f$ and $f$ from $v_{0}$, respectively) of a signal are approximately coherent with each other, and they are of approximately equal intensity.

It was previously show that the measurement of phase fluctuations (phase noise) required driving a double-balanced mixer with two signals in phase quadrature so the FM-AM conversion resulted in voltage fluctuations at the mixer output that were analogous to the phase fluctuations. The operation of the mixer when it is driven at quadrature is such that the amplitudes of the two phase sidebands are added linearly in the output of the mixer, resulting in four times as much power in the output as would be present if only one of the phase sidebands were allowed to contribute to the output

* See Appendix Note \# 31 
of the mixer. Hence, for $|f|<v_{0}$, and considering only the phase modulation portion of the spectral density of the (square root of) power, we obtain

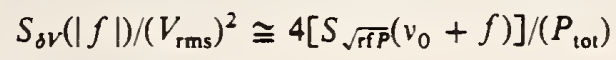

and, using the definition of $\mathscr{L}(f)$,

$$
\mathscr{L}(f) \equiv\left[S_{\sqrt{\mathrm{rPP}}}\left(v_{0}+f\right)\right] /\left(P_{\text {tot }}\right) \cong \frac{1}{2} S_{\delta \phi}(|f|) .
$$

Therefore, for the condition that the phase fluctuations occurring at rates $(f)$ and faster are small compared to one radian, a good approximation in radians squared per hertz for one unit is

$$
\mathscr{L}(f)=\frac{1}{2} S_{\delta \phi}(f) \text {. }
$$

If the small angle condition is not met, Bessel-function algebra must be used to relate $\mathscr{L}(f)$ to $S_{\delta \phi}(f)$.

The NBS-defined spectral density is usually expressed in decibels relative to the carrier per hertz and is calculated for one unit as

$$
\mathscr{L}(f)=10 \log \left[\frac{1}{2} S_{\delta \phi}(f)\right] .
$$

It is very important to note that the theory, definitions, and equations previously set forth relate to a single device.

\section{Modllation Theory and Spectral Density RELATIONSHIPS}

Applying a sinusoidal frequency modulation $f_{\mathrm{m}}$ to a sinusoidal carrier frequency $v_{0}$ produces a wave that is sinusoidally advanced and retarded in phase as a function of times. The instantaneous voltage is expressed as,

$$
V(t)=V_{0} \sin \left(2 \pi v_{0} t+\Delta \phi \sin 2 \pi f_{\mathrm{m}} t\right) .
$$

where $\Delta \phi$ is the peak phase deviation caused by the modulation signal.

The first term inside the parentheses represents the linearly progressing phase of the carrier. The second term is the phase variation (advancing and retarded) from the linearly progressing wave. The effects of modulation can be expressed as residual $f_{\mathrm{m}}$ noise or as single-sideband phase noise. For modulation by a single sinusoidal signal, the peak-frequency deviation of the carrier $\left(v_{0}\right)$ is

$$
\begin{aligned}
\Delta v_{0} & =\Delta \phi \cdot f_{\mathrm{m}}, \\
\Delta \phi & =\Delta v_{0} / f_{\mathrm{m}},
\end{aligned}
$$

where $f_{m}$ is the modulation frequency. This ratio of peak frequency deviation to modulation frequency is called modulation index $m$ so that $\Delta \phi=m$ and

$$
m=\Delta v_{0} / f_{m} \text {. }
$$


The frequency spectrum of the modulated carrier contains frequency components (sidebands) other than the carrier. For small values of modulation index $(m \ll 1)$, as is the case with random phase noise, only the carrier and first upper and lower sidebands are significantly high in energy. The ratio of the amplitude of either single sideband to the amplitude of the carrier is

$$
V_{\mathrm{sb}} / V_{0}=m / 2
$$

This ratio is expressed in decibels below the carrier and is referred to as $\mathrm{dBc}$ for the given band width $B$ :

$$
\begin{aligned}
V_{\mathrm{sb}} / V_{0} & =20 \log (m / 2)=20 \log \left(\Delta v_{0} / 2 f_{\mathrm{m}}\right) \\
& =10 \log (m / 2)^{2}=10 \log \left(\Delta v_{0} / 2 f_{\mathrm{m}}\right)^{2} .
\end{aligned}
$$

If the frequency deviation is given in terms of its rms value, then

$$
\Delta v_{\mathrm{rms}}=\Delta v_{0} / \sqrt{2}
$$

Equation (33) now becomes

$$
\begin{aligned}
V_{\mathrm{sb}} / V_{0} & =\mathscr{L}(f)=20 \log \left(\Delta v_{\mathrm{rms}} / \sqrt{2 f_{\mathrm{m}}}\right) \\
& =10 \log \left(\Delta v_{\mathrm{rms}} / \sqrt{2 f_{\mathrm{m}}}\right)^{2} .
\end{aligned}
$$

The ratio of single sideband to carrier power in decibels (carrier) per hertz is

$$
\mathscr{L}(f)=20 \log \left(\Delta v_{\text {rms }} / f_{\mathrm{m}}\right)-3
$$

and, in decibels relative to one squared radian per hertz,

$$
S_{\delta \phi}(f)=20 \log \left(\Delta v_{s m s} / f_{m}\right)
$$

The interrelationships of modulation index, peak frequency deviation, rms frequency, and spectral density of phase fluctuations can be found from the following:

$$
\begin{aligned}
\frac{1}{2} m & =\Delta v_{0} / 2 f_{\mathrm{m}}=\Delta v_{\mathrm{rms}} / \sqrt{2 f_{\mathrm{m}}}, \\
& =10 \exp (\mathscr{L}(f) / 10)=\frac{1}{2} S_{\delta \phi}(f):
\end{aligned}
$$

or

$$
\frac{1}{2} m=\Delta v_{\mathrm{rms}} / \sqrt{2 f_{\mathrm{m}}}=\sqrt{10 \exp (\mathscr{L}(f) / 10)}=\sqrt{\frac{1}{2} S_{\delta \phi}(f)}
$$

and

$$
\begin{aligned}
m & =\Delta v_{0} / f_{\mathrm{m}}=2 \Delta v_{\mathrm{rms}} / \sqrt{2 f_{\mathrm{m}}} \\
& =2 \sqrt{10 \exp (\mathscr{L}(f) / 10)}=2 \sqrt{\frac{1}{2} S_{\delta \phi}(f)} .
\end{aligned}
$$

The basic relationships are plotted in Fig. 5. 


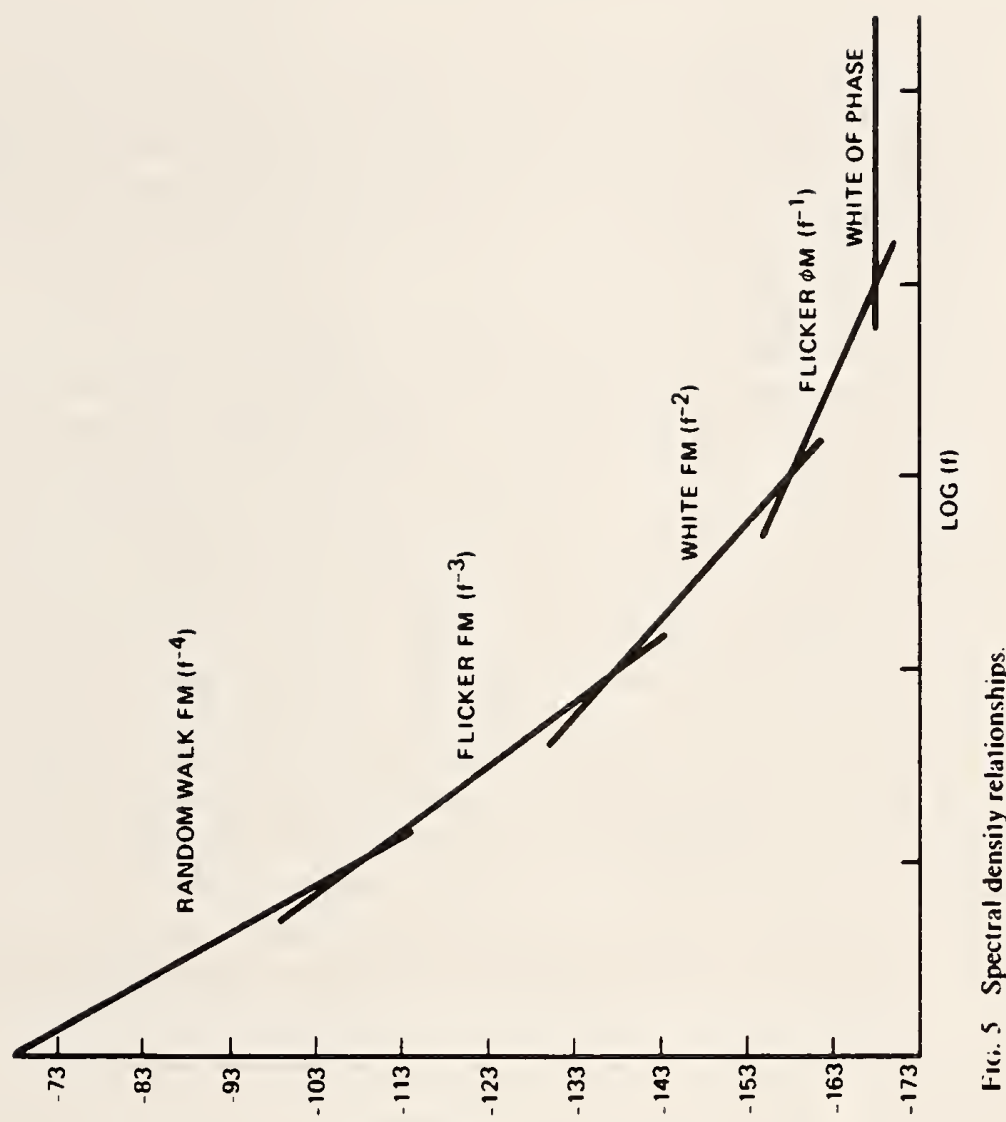

$(2 \mathrm{H} / \mathrm{PPP})(\mathrm{t}) \mathrm{J}$

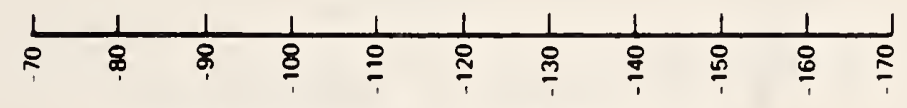

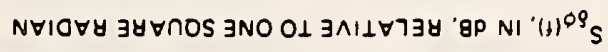

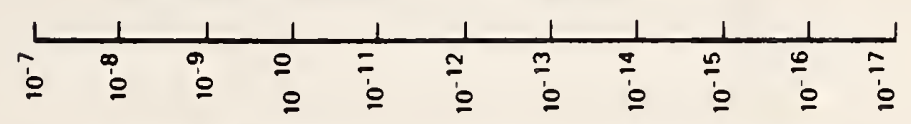

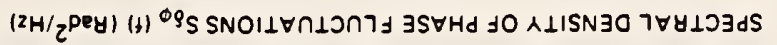

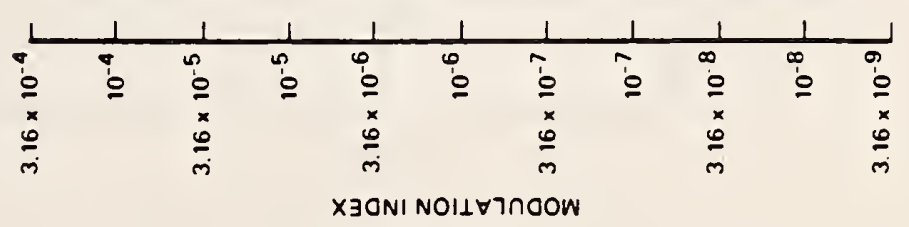




\section{E. NoIse Processes}

The spectral density plot of a typical oscillator's output is usually a combination of different noise processes. It is very useful and meaningful to categorize these processes because the first job in evaluating a spectral density plot is to determine which type of noise exists for the particular range of Fourier frequencies.

The two basic categories are the discrete-frequency noise and the power-law noise process. Discrete-frequency noise is a type of noise in which there is a dominant observable probability, i.e., deterministic in that they can usually be related to the mean frequency, power-line frequency, vibration frequencies, or ac magnetic fields, or to Fourier components of the nominal frequency. Discrete-frequency noise is illustrated in the frequency domain plot of Fig. 6. These frequencies can have their own spectral density plots, which can be defined as noise on noise.

Power-law noise processes are types of noise that produce a certain slope on the one-sided spectral density plot. They are characterized by their dependence on frequency. The spectral density plot of a typical oscillator output is usually a combination of the various power-law processes.

In general, we can classify the power-law noise processes into five categories. These five processes are illustrated in Fig. 5 , which can be referred to with respect to the following description of each process.

(1) Random walk FM (random walk of frequency). The plot goes down as $1 / f^{4}$. This noise is usually very close to the carrier and is difficult to measure. It is usually related to the oscillator's physical environment (mechanical shock, vibration, temperature, or other environmental effects).

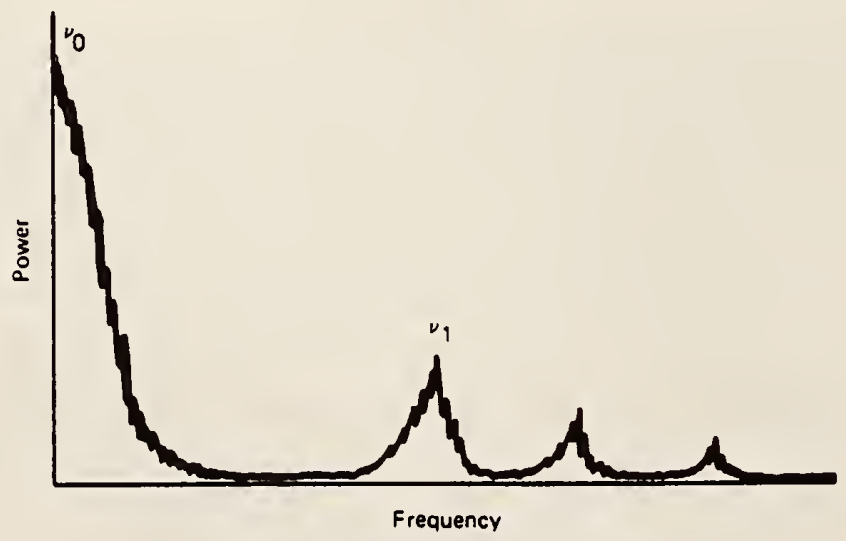

FiG. 6 A basic discrete-frequency signal display. 
(2) Flicker $F M$ (flicker of frequency). The plot goes down as $1 / f^{3}$. This noise is typically related to the physical resonance mechanism of the active oscillator or the design or choice of parts used for the electronic or power supply, or even environmental properties. The time domain frequency stability over extended periods is constant. In high-quality oscillators, this noise may be marked by white $\mathrm{FM}\left(1 / f^{2}\right)$ or flicker phase modulation $\phi M(1 / f)$. It may be masked by drift in low-quality oscillators.

(3) White $F M$ (white frequency, random walk of phase). The plot goes down as $1 / f^{2}$. A common type of noise found in passive-resonator frequency standards. Cesium and rubidium frequency standards have white FM noise characteristic because the oscillator (usually quartz) is locked to the resonance feature of these devices. This noise gets better as a function of time until it (usually) becomes flicker FM $\left(1 / f^{3}\right)$ noise.

(4) Flicker $\phi M$ (flicker modulation of phase). The plot goes down as $1 / f$. This noise may relate to the physical resonance mechanism in an oscillator. It is common in the highest-quality oscillators. This noise can be introduced by noisy electronics-amplifiers necessary to bring the signal amplitude up to a usable level - and frequency multipliers. This noise can be reduced by careful design and by hand-selecting all components.

(5) White $\phi M$ (white phase). White phase noise plot is flat $f^{\circ}$. Broadband phase noise is generally produced in the same way as flicker $\phi M(1 / f)$. Late stages of amplification are usually responsible. This noise can be kept low by careful selection of components and by narrow-band filtering at the output.

The power-law processes are illustrated in Fig. 5.

\section{F. Integrated Phase Noise}

The integrated phase noise is a measure of the phase-noise contribution (rms radians, rms degrees) over a designated range of Fourier frequencies. The integration is a process of summation that must be performed on the measured spectral density within the actual IF bandwidth $(B)$ used in the measurement of $\delta \phi_{\mathrm{rms}}$. Therefore, the spectral density $S_{\delta \phi}(f)$ must be unnormalized to the particular bandwidth used in the measurement. Define $S_{u}(f)$, in radians squared, as the unnormalized spectral density:

$$
S_{\mathrm{u}}(f)=2[10 \exp (\mathscr{L}(f)+10 \log B) / 10] .
$$

Then, the integrated phase noise over the band of Fourier frequencies $\left(f_{1}\right.$ to $\left.f_{n}\right)$ where measurements are performed using a constant IF bandwidth, in radians squared, is

$$
S_{\mathrm{B}}\left(f_{1} \text { to } f_{n}\right)=\int_{f_{1}}^{f_{n}} S_{\mathrm{u}}(f) d f
$$


or, in rms radians,

$$
S_{\mathrm{B}}\left(f_{1} \text { to } f_{n}\right)=\sqrt{\sum S_{\mathrm{u}}\left(f_{1}\right)+S_{\mathrm{u}}\left(f_{2}\right)=\cdots=S_{\mathrm{u}}\left(f_{n}\right)},
$$

and the integrated phase noise in rms degrees is calculated as

$$
S_{\mathrm{B}}(360 / 2 \pi) \text {. }
$$

The integrated phase noise in decibels relative to the carrier is calculated as

$$
S_{\mathrm{B}}=10 \log \left(\frac{1}{2} S_{\mathrm{B}}^{2}\right) \text {. }
$$

The previous calculations correspond to the illustration in Fig. 7, which includes two bandwidths (B1 and B2) over two ranges of Fourier frequencies.

In the measurement program, different IF bandwidths are used as set forth by Lance et al. (1977). The total integrated phase noise over the different ranges of Fourier frequencies, which are measured at constant bandwidths as illustrated, is calculated in rms radians as follows:

$$
S_{\mathrm{Btot}}=\sqrt{\left(S_{\mathrm{B} 1}\right)^{2}+\left(S_{\mathrm{B} 2}\right)^{2}+\cdots+\left(S_{\mathrm{B} n}\right)^{2}},
$$

where it is recalled that the summation is performed in terms of radians squared.

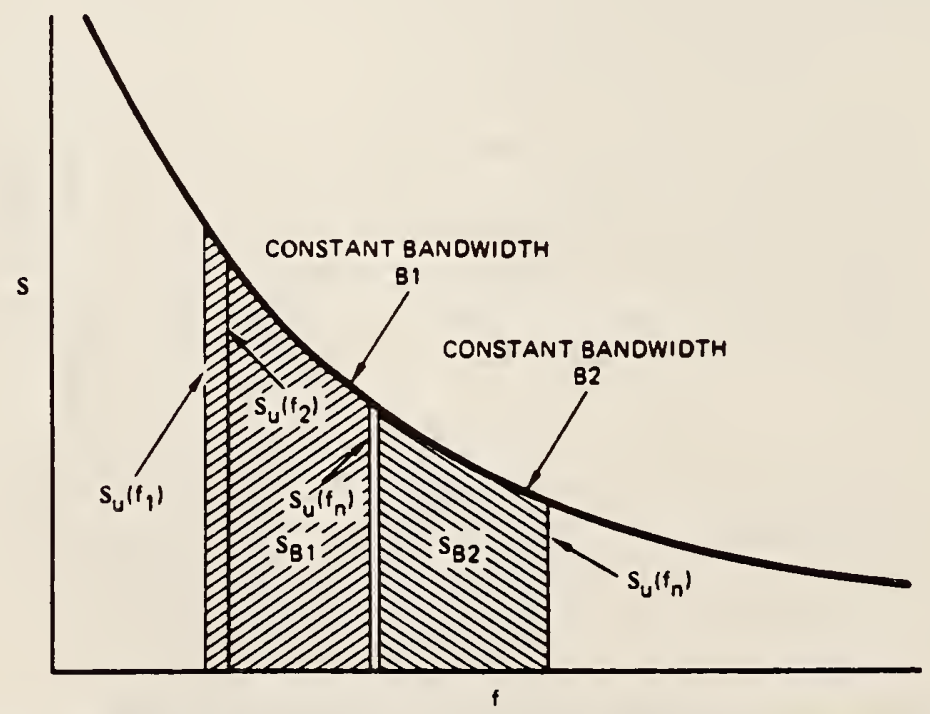

FIG. 7 Integrated phase noise over Fourier frequency ranges at which measurements were performed using constant bandwidth. 


\section{G. AM NoISE IN THE FReQuenCy Domain}

The spectral density of AM fluctuations of a signal follows the same general derivation previously given for the spectral density of phase fluctations. Amplitude fluctuations $\delta \varepsilon$ of the signal under test produces voltage fluctuations $\delta A$ at the output of the mixer. Interpretating the mean-square fluctuations $\delta \varepsilon$ and $\delta$ in spectal density fashion, we obtain $S_{\delta \varepsilon}(f)$, the spectral density of amplitude fluctuations $\delta \varepsilon$ of a signal in volts squared per hertz:

$$
S_{\delta \varepsilon}(f)=\left(\frac{1}{2} V_{0}\right)^{2}\left[S_{\delta A}(f) /\left(A_{\mathrm{rms}}\right)^{2}\right] .
$$

The term $m(f)$ is the normalized version of the amplitude modulation (AM)

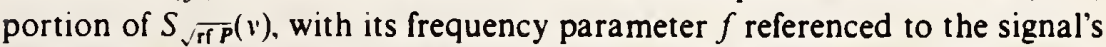
average frequency $v_{0}$, taken as the origin such that the difference frequency $f$ equals $v-v_{0}$. The range of Fourier frequency difference $f$ is from minus $v_{0}$ to plus infinity.

The term $m(f)$ is defined as the ratio of the spectral density of one amplitudemodulated sideband to the total signal power, at Fourier frequency difference $f$ from the signal's a verage frequency $v_{0}$, for a single specified signal or device. The dimensionality is per hertz. $\mathscr{L}(f)$ and $m(f)$ are similar functions; the former is a measure of phase-modulated (PM) sidebands, the later is a corresponding measure of amplitude-modulated (AM) sidebands. We introduce the symbol $m(f)$ to have useful terminology for the important concept of normalized AM sideband power.

For the types of signals under consideration, by definition the two amplitude-fluctuation sidebands (lower sideband and upper sideband, at $-f$ $f$ from $v_{0}$, respectively) of a signal are coherent with each other. Also, they are of equal intensity. The operation of the mixer when it is driven at colinear phase is such that the amplitudes of the two AM sidebands are added linearly in the output of the mixer, resulting in four times as much power in the output as would be present if only one of the AM sidebands were allowed to contribute to the output of the mixer. Hence, for $|f|<v_{0}$,

$$
S_{\mathrm{A}}(|f|) /\left(\mathrm{A}_{\mathrm{rms}}\right)^{2}=4\left[S_{\sqrt{\mathrm{rFP}}}\left(v_{0}+f\right)\right] / P_{\text {tot }},
$$

and, using the definition

$$
m(f) \equiv\left[S_{\sqrt{\mathrm{rfP}}}\left(v_{0}+f\right)\right] / P_{\mathrm{tol}},
$$

we find, in decibels (carrier) per hertz,

$$
m(f)=\left(1 / 2 V_{0}^{2}\right) S_{\delta \varepsilon}(|f|) .
$$




\section{Phase-Noise Measurements Using the Two-Oscillator Technique}

A functional block diagram of the two-oscillator system for measuring phase noise is shown in Fig. 8. NBS has performed phase noise measurements since 1967 using this basic system. The signal level and sideband levels can be measured in terms of voltage or power. The low-pass filter prevents local oscillator leakage power from overloading the spectrum analyzer when baseband measurements are performed at the Fourier (offset) frequencies of interest. Leakage signals will interfere with autoranging and with the dynamic range of the spectrum analyzer.

The low-noise, high-gain preamplifier provides additional system sensitivity by amplyfying the noise signals to be measured. Also, because spectrum analyzers usually have high values of noise figure, this amplifier is very desirable. As an example, if the high-gain preamplifier had a noise figure of $3 \mathrm{~dB}$ and the spectrum analyzer had a noise figure of $18 \mathrm{~dB}$, the system sensitivity at this point has been improved by $15 \mathrm{~dB}$. The overall system sensitivity would not necessarily be improved $15 \mathrm{~dB}$ in all cases, because the limiting sensitivity could have been imposed by a noisy mixer.

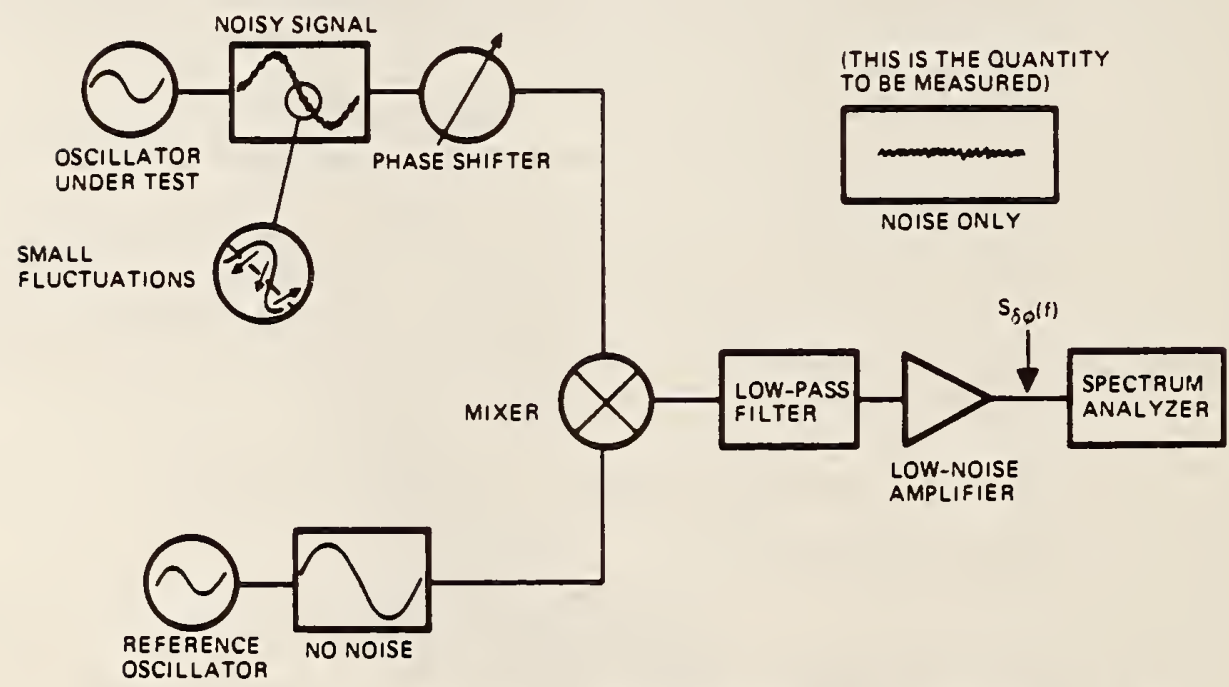

FIG. 8 The two-oscillator technique for measuring phase noise. Small fluctuations from nominal voltage are equivalent to phase variations. The phase shifter adjusts the two signals to quadrature in the mixer, which cancels carriers and converts phase noise to fluctuating dc voltage.

- See Appendix Note \# 6 
Assume that the reference oscillator is perfect (no phase noise), and that it can be adjusted in frequency. Also, assume that both oscillators are extremely stable. so that phase quadrature can be maintained without the use of an external phase-locked loop or reference. The double-balanced mixer acts as a phase detector so that when two input signals are identical in frequency and are in phase quadrature, the output is a small fluctuating voltage. This represents the phase-modulated (PM) sideband component of the signal because, due to the quadrature of the signals at the mixer input, the mixer converts the amplitude-modulated (AM) sideband components to FM, and at the same time it converts the PM sideband components to AM. These AM components can be detected with an amplitude detector, as shown in Fig. 3.

If the two oscillator signals applied to the double-balanced mixer of Fig. 8 are slightly out of zero beat, a slow sinusoidal voltage with a peak-to-peak voltage $V_{\text {ptp }}$ can be measured at the mixer output. If these same signals are returned to zero beat and adjusted for phase quadrature, the output of the mixer is a small fluctuating voltage $(\delta v)$ centered at zero volts. If the fluctuating voltage is small compared to $\frac{1}{2} V_{\text {pip }}$, the phase quadrature condition is being closely maintained and the "small angle" condition is being met. Phase fluctuations in radians between the test and reference signals (phases) are

$$
\delta \phi=\delta\left(\phi_{r}-\phi_{r}\right)
$$

These phase fluctuations produce voltage fluctuations at the output of the mixer,

$$
\delta v=\frac{1}{2} V_{\text {ptp }} \delta \phi,
$$

where phase angles are in radian measure and $\sin \delta \phi=\delta \phi$ for small $\delta \phi$ ( $\delta \phi \ll 1 \mathrm{rad}$ ). Solving for $\delta \phi$, squaring both sides, and taking a time average gives

$$
\left\langle(\delta \phi)^{2}\right\rangle=4\left\langle(\delta v)^{2}\right\rangle /\left(V_{\mathrm{ptp}}\right)^{2},
$$

where the angle brackets represent the time average.

For the sinusoidal beat signal,

$$
\left(V_{\mathrm{ptp}}\right)^{2}=8\left(V_{\mathrm{rms}}\right)^{2}
$$

The mean-square fluctuations of phase $\delta \phi$ and voltage $\delta v$ interpreted in a spectral density fashion gives the following in radians squared per hertz:

$$
S_{\delta \phi}(f)=S_{\delta v}(f) / 2\left(V_{\mathrm{rms}}\right)^{2} .
$$

Here, $S_{\delta r}(f)$, in volts squared per hertz, is the spectral density of the voltage fluctuations at the mixer output. Because the spectrum analyzer measures 
rms voltage, the noise voltage is in units of volts per square root hertz, which means volts per square root bandwidth. Therefore,

$$
S_{\delta v}(f)=\left[\delta v_{\mathrm{rms} /} / \bar{B}\right]=\left(\delta v_{\mathrm{rms}}\right)^{2}, B,
$$

where $B$ is the noise power bandwidth used in the measurement.

Because it was assumed that the reference oscillator did not contribute any noise, the voltage fluctuations $v_{\text {rms }}$ represent the oscillator under test, and the spectral density of the phase fluctuations in terms of the voltage measurements performed with the spectrum analyzer, in radians squared per hertz, is

$$
S_{\delta \phi}(f)=\frac{1}{2}\left[\left(\delta v_{\mathrm{rms}}\right)^{2} / B\left(V_{\mathrm{rms}}\right)^{2}\right] .
$$

Equation (46) is sometimes expressed as

$$
S_{\delta \phi}(f)=S_{\delta \varepsilon}(f) / K^{2} \text {. }
$$

where $K$ is the calibration factor in volts per radian. For sinusoidal beat signals, the peak voltage of the signal equals the slope of the zero crossing in volts per radian. Therefore, $\left(V_{\mathrm{p}}\right)^{2}=2\left(V_{\mathrm{rms}}\right)^{2}$, which is the same as the denominator in Eq. (56).

The term $S_{\delta \Phi}(f)$ can be expressed in decibels relative to one square radian per hertz by calculating $10 \log S_{\delta \phi}(f)$ of the previous equation:

$$
S_{\delta \phi}(f)=20 \log \left(\delta v_{\mathrm{rms}}\right)-20 \log \left(V_{\mathrm{rms}}\right)-10 \log (B)-3 .
$$

A correction of 2.5 is required for the tracking spectrum analyzer used in these measurement systems. $\mathscr{L}(f)$ differs by $3 \mathrm{~dB}$ and is expressed in decibels (carrier) per hertz as

$$
\mathscr{L}(f)=20 \log \left(\delta v_{\text {rms }}\right)-20 \log \left(V_{\text {rms }}\right)-10 \log (\mathrm{B})-6 .
$$

\section{A. Two NoIsy Oscillators}

The measurement system of Fig. 6 yields the output noise from both oscillators. If the reference oscillator is superior in performance as assumed in the previous discussions, then one obtains a direct measure of the noise characteristics of the oscillator under test.

If the reference and test oscillators are the same type, a useful approximation is to assume that the measured noise power is twice that associated with one noisy oscillator. This approximation is in error by no more than $3 \mathrm{~dB}$ for the noisier oscillator, even if one oscillator is the major source of noise. The equation for the spectral density of measured phase fluctuations in radians squared per hertz is

$$
\left.S_{\delta \phi}(f)\right|_{* 1}+\left.S_{\delta \phi}(f)\right|_{\# 2}=\left|S_{\delta r}(f)\right|_{(\text {(wo devices) }} \div 2\left(V_{\mathrm{rmm}}\right)^{2} \doteq\left|2 S_{\delta \phi}(f)\right|_{\text {(one device) }}
$$


The measured value is therefore divided by two to obtain the value for the single oscillator. A determination of the noise of each oscillator can be made if one has three oscillators that can be measured in all pair combinations. The phase noise of each source 1,2, and 3 is calculated as follows:

$$
\begin{aligned}
& \mathscr{L}_{1}(f)=10 \log \left[\frac{1}{2}\left(10^{\mathscr{L}_{12}(f) / 10}+10^{\mathscr{L}_{13(f) / 10}}-10^{\mathscr{L}_{23(f) / 10}}\right)\right], \\
& \mathscr{L}^{2}(f)=10 \log \left[\frac{1}{2}\left(10^{\mathscr{L}_{12}(f) / 10}+10^{\mathscr{L}_{23}(f) / 10}-10^{\mathscr{L}_{13}(f) / 10}\right)\right], \\
& \mathscr{L}^{3}(f)=10 \log \left[\frac{1}{2}\left(10^{\mathscr{L}_{13}(f) / 10}+10^{\mathscr{L}_{23(f) / 10}}-10^{\left.\mathscr{L}_{12(f) / 10}\right)}\right] .\right.
\end{aligned}
$$

\section{B. Automated Phase-Noise Measurements Using the} TWo-OsCillator TECHNiQue

The automated phase-noise measurement system is shown in Fig. 9. It is controlled by a programmable calculator. Each step of the calibration and measurement sequence is included in the program. The software program controls frequency slection, bandwidth settings, settling time, amplitude ranging. measurements, calculations, graphics, and data plotting. Normally, the system is used to obtain a direct plot of $\mathscr{L}(f)$. The integrated phase noise can be calculated for any selected range of Fourier frequencies.

A quasi-continuous plot of phase noise performance $\mathscr{L}(f)$ is obtained by performing measurements at Fourier frequencies separated by the IF bandwidth of the spectrum analyzer used during the measurement. Plots of other defined parameters can be obtained and plotted as desired.

The IF bandwidth settings for the Fourier (offset) frequency-range selections are shown in the following tabulation:

\begin{tabular}{cccc}
\hline $\begin{array}{c}\text { IF } \\
\text { bandwidth } \\
(\mathrm{Hz})\end{array}$ & $\begin{array}{c}\text { Fourier } \\
\text { frequency } \\
(\mathrm{kHz})\end{array}$ & $\begin{array}{c}\text { IF } \\
\text { bandwidth } \\
(\mathrm{kHz})\end{array}$ & $\begin{array}{c}\text { Fourier } \\
\text { frequency } \\
(\mathrm{kHz})\end{array}$ \\
\hline 3 & $0.001-0.4$ & 1 & $40-100$ \\
10 & $0.4-1$ & 3 & $100-400$ \\
30 & $1-4$ & 10 & $400-1300$ \\
100 & $4-10$ & & \\
200 & $10-40$ & & \\
\hline
\end{tabular}

The particular range of Fourier frequencies is limited by the particular spectrum analyzer used in the system. A fast Fourier analyzer (FFT) is also incorporated in the system to measure phase noise from submillihertz to $25 \mathrm{kHz}$.

High-quality sources can be measured without multiplication to enhance the phase noise prior to downconverting and measuring at baseband frequencies. The measurements are not completely automated because the calibration sequence requires several manual operations. 


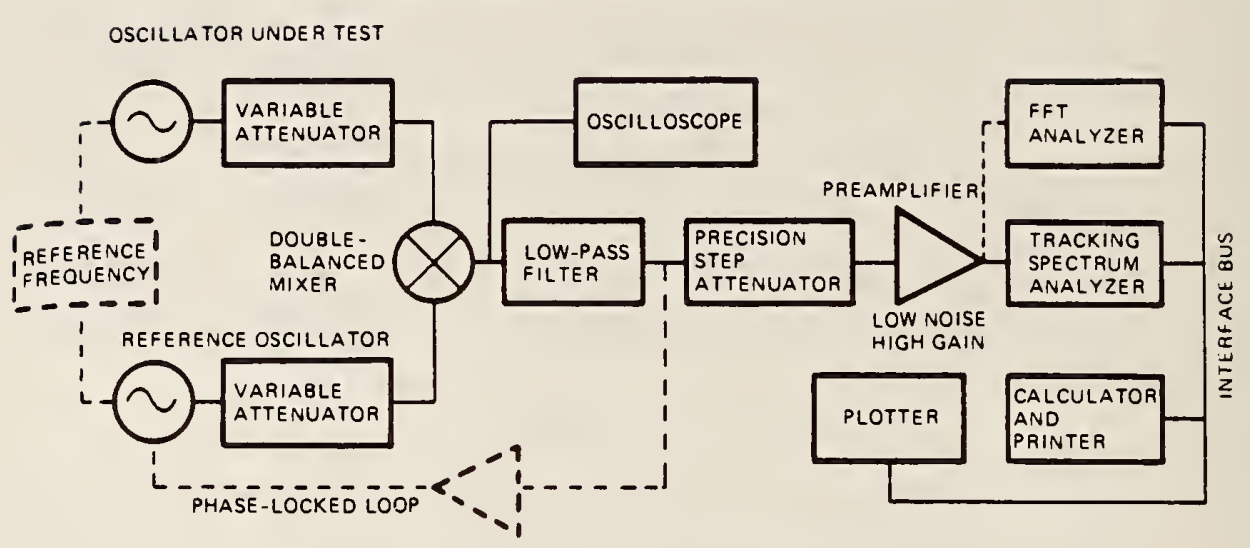

FIG. 9 An automated phase-noise measurement system.

\section{Calibration and Measurements Using the TWO-OSCILLATOR SYSTEM}

$\mathscr{L}(f)$ is a normalized frequency domain measure of phase-fluctuation sideband power. The noise power is measured relative to the carrier power level. Correction must be applied because of the type of measurement and the characteristics of the measurement equipment. The general procedure for the calibration and measurement sequence includes the following: measuring the noise power bandwidth for each IF bandwidth setting on the Tracking Spectrum Analyzer (Section III.C.1); establishing a carrier reference power level referenced to the output of the mixer (Section III.C.2); obtaining phase quadrature of the two signals applied to the mixer (Section III.C.3); measuring the noise power at the selected Fourier frequencies (Section III.C.4): performing the calculations and plotting the data (Section III.C.5); and measuring the system noise floor characteristics, usually referred to as the system sensitivity.

\section{Voise-Power Bandwidth}

Approximations of analyzer-noise bandwidths are not adequate for phase noise measurements and calculations. The IF noise-power bandwidth of the tracking spectrum analyzer must be known and used in the calculations of phase noise parameters. Figure 10 shows the results of measurements performed using automated techniques. For example, with a $1-\mathrm{MHz}$ signal input to the tracking spectrum analyzer, the desired incremental frequency changes covering the IF bandwidth are set by calculator control. 


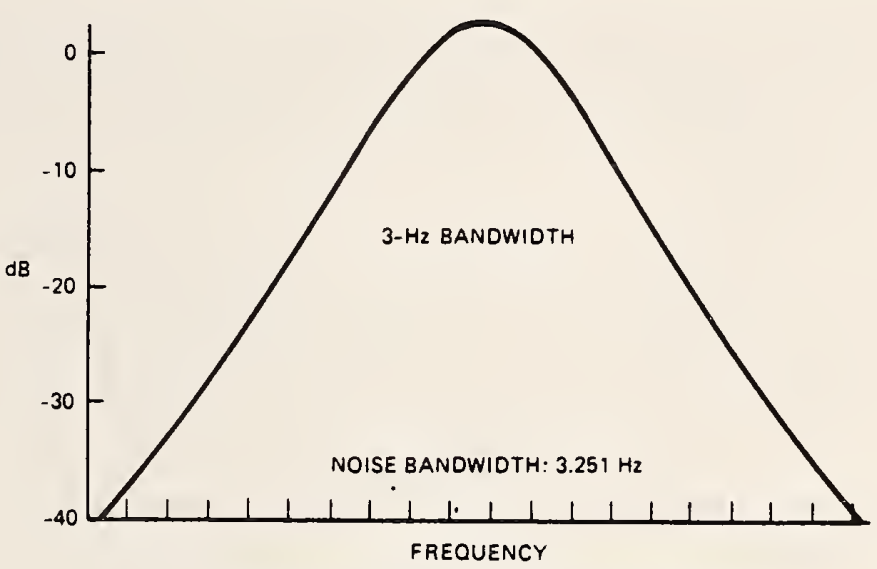

FIG. 10 Plot of automated noise-power bandwidth.

The spectrum analyzer power output is recorded for each frequency setting over the range, as illustrated in Fig. 10. The 40-dB level and the 100 increments in frequency are not the minimum permissible values. The recorded can be plotted for each IF bandwidth, as illustrated, and the noise-power bandwidth is calculated in hertz as

$$
\text { noise power bandwidth }=\frac{\left(P_{1}+P_{2}+P_{3}+\cdots+P^{100}\right) \Delta f}{\text { peak power reading }},
$$

where $\Delta f$ is the frequency increment in hertz and the peak power is the maximum measured point obtained during the measurements. All power values are in watts.

\section{Setting the Carrier Power Reference Level}

Recall from Section III. 6 that for sinusoidal signals the peak voltage of the signal equals the slope of the zero crossing, in volts per radian. A frequency offset is established, and the peak power of the difference frequency is measured as the carrier-power reference level; this establishes the calibration factor of the mixer in volts per radian.

Because the precision IF attenuator is used in the calibration process, one must be aware that the impedance looking back into the mixer should be $50 \Omega$. Also, the mixer output signal should be sinusoidal. Fischer (1978) discussed the mixer as the "critical element" in the measurement system. It is advisable to drive the mixer so that the sinusoidal signal is obtained at the mixer output. In most of the TRW systems, the mixer drive levels are $10 \mathrm{dBm}$ for the reference signal and about zero $\mathrm{dBm}$ for the unit under test. 
System sensitivity can be increased by driving the mixer with high-level signals that lower the mixer output impedance to a few ohms. This presents a problem in establishing the calibration factor of the mixer, because it might be necessary to calibrate the mixer for different Fourier frequency ranges.

The equation sensitivity $=$ slope $=$ beat-note amplitude does not hold if the output of the mixer is not a sine wave. The Hewlett-Packard 3047 automated phase noise measurement system allows accurate calibration of the phase-detector sensitivity even with high-level inputs by using the derivative of the Fourier representation of the signal (the fundamental and its harmonics). The slope at $\phi \cong 0$ radians is given by

$$
\begin{aligned}
A \sin \phi-B \sin 3 \phi+C \sin 5 \phi & =A \cos \phi-3 B \cos 3 \phi+5 C \cos 5 \phi \\
& =A-3 B+5 C+\cdots .
\end{aligned}
$$

Referring to Fig. 9, the carrier-power reference level is obtained as follows.

(1) The precision IF step attenuator is set to a high value to prevent overloading the spectrum analyzer (assume $50 \mathrm{~dB}$ as our example).

(2) The reference and test signals at the mixer inputs are set to approximately $10 \mathrm{dBm}$ and $0 \mathrm{dBm}$, as previously discussed.

(3) If the frequency of one of the oscillators can be adjusted, adjust its frequency for an IF output frequency in the range of 10 to $20 \mathrm{kHz}$. If neither oscillator is adjustable, replace the oscillator under test with one that can be adjusted as required and that can be set to the identical power level of the oscillator under test.

(4) The resulting IF power level is measured by the spectrum analyzer, and the measured value is corrected for the attentuator setting, which was assumed to be $50 \mathrm{~dB}$. The correction is necessary because this attenuator will be set to its zero decibel indication during the measurements of noise power. Assuming a spectrum analyzer reading of $-40 \mathrm{dBm}$, the carrierpower reference level is calculated as

$$
\text { carrier power reference level }=50 \mathrm{~dB}-40 \mathrm{dBm}=10 \mathrm{dBm} \text {. }
$$

\section{Phase Quadrature of the Mixer Input Signals}

After the carrier-power reference has been established, the oscillator under test and the reference oscillator are tuned to the same frequency, and the original reference levels that were used during calibration are reestablished. The quadrature adjustment depends on the type of system used. Three possibilities, illustrated in Fig. 9, are described here.

(1) If the oscillators are very stable, have high-resolution tuning, and are not phase-locked, the frequency of one oscillator is adjusted for zero $d c$ 
voltage output of the mixer as indicated by the sensitive oscilloscope. Note: Experience has shown that the quadrature setting is not critical if the sources have low AM noise characteristics. As an example, experiments performed using two HP 3335 synthesizers showed that degradation of the phase-noise measurement became noticeable with a phase-quadrature offset of 16 degrees.

(2) If the common reference frequency is used, as illustrated in Fig. 9, then it is necessary to include a phase shifter in the line between one of the oscillators and the mixer (preferably between the attenuator and mixer). The phase shifter is adjusted to obtain and maintain zero volts dc at the mixer output. A correction for a nonzero dc value can be applied as exemplified by the HP 3047 automated phase-noise measurement system.

(3) If one oscillator is phase-locked using a phase-locked loop, as shown dotted in on Fig. 9, the frequency of the unit under test is adjusted for zero dc output of the mixer as indicated on the oscilloscope.

A phase-locked loop is a feedback system whose function is to force a voltage-controlled oscillator (VCO) to be coherent with a certain frequency, i.e., it is highly correlated in both frequency and phase. The phase detector is a mixer circuit that mixes the input signal with the VCO signal. The mixer output is $v_{i} \pm v_{0}$. when the loop is locked, the VCO duplicates the input frequency so that the difference frequency is zero, and the output is a dc voltage proportional to the phase difference. The low-pass filter removes the sum frequency component but passes the dc component to control the VCO. The time constant of the loop can be adjusted as needed by varying amplifier gain and RC filtering within the loop.

A loose phase-locked loop is characterized by the following.

(1) The correction voltage varies as phase (in the short term) and phase variations are therefore observed directly.

(2) The bandwidth of the servo response is small compared with the Fourier frequency to be measured.

(3) The response time is very slow.

A tight phase-locked loop is characterized by the following.

(1) The correction voltage of the servo loop varies as frequency.

(2) The bandwidth of the servo response is relatively large.

(3) The response time is much smaller than the smallest time interval $\tau$ at which measurements are performed.

Figure 11 shows the phase-noise characteristics of the H.P. 8640B synthesizer measured at $512 \mathrm{MHz}$. The phase-locked-loop attenuation characteristics extend to $10 \mathrm{kHz}$. The internal-oscillator-source characteristics are plotted at Fourier frequencies beyond the loop-bandwidth cutoff at $10 \mathrm{kHz}$. 


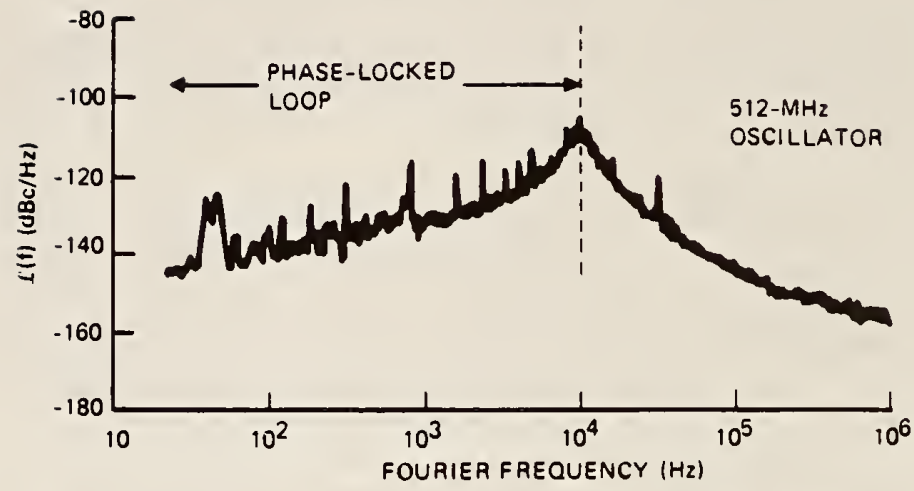

Fig. II Phase-locked-loop characteristics of the H.P. 8640B signal generator, showing the normalized phase-noise sideband power spectral density.

\section{Measurements, Calculations, and Data Plots}

The measurement sequence is automated except for the case where manual adjustments are required to maintain phase quadrature of the signals. After phase quadrature of the signals into the mixer is established, the IF attenuator is returned to the zero-decibel reference setting. This attenuator is set to a high value [assumed to be $50 \mathrm{~dB}$ in Eq. (65)] to prevent saturation of the spectrum analyzer during the calibration process.

The automated measurements are executed, and the direct measurement and data plot of $\mathscr{L}(f)$ is obtained in decibels (carrier) per hertz using the equation

$$
\begin{aligned}
\mathscr{L}(f)= & -[\text { carrier power level }-(\text { noise power level }-6+2.5 \\
& -10 \log \mathrm{B}-3)] .
\end{aligned}
$$

The noise power $(\mathrm{dBm})$ is measured relative to the carrier-power level $(\mathrm{dBm})$, and the remaining terms of the equation represent corrections that must be applied because of the type of measurement and the characteristics of the measurement equipment, as follows.

(1) The measurement of noise sidebands with the signals in phase quadrature requires the $-6-\mathrm{dB}$ correction that is noted in Eq. (69).

(2) The nonlinearity of the spectrum analyzer's logarithmic IF amplifier results in compression of the noise peaks which, when average-detected, require the $2.5-\mathrm{dB}$ correction.

(3) The bandwidth correction is required because the spectrum analyzer measurements of random or white noise are a function of the particular bandwidth used in the measurement. 
(4) The $-3-\mathrm{dB}$ correction is required because this is a direct measure of $\mathscr{L}(f)$ of two oscillators, assuming that the oscillators are of a similar type and that the noise contribution is the same for each oscillator. If one oscillator is sufficiently superior to the other, this correction is not required.

Other defined spectral densities can be calculated and plotted as desired. The plotted or stored value of the spectral density of phase fluctuations in decibels relative to one square radian $\left(\mathrm{dBc} \operatorname{rad}^{2} / \mathrm{Hz}\right)$ is calculated as

$$
S_{\delta \phi}(f)=\mathscr{L}(f)+3 \text {. }
$$

The spectral density of phase fluctuations, in radians squared per hertz, is calculated as

$$
S_{\delta \phi}(f)=10 \exp \left(S_{\delta \phi}(f) / 10\right)
$$

The spectral density of frequency fluctuations, in hertz squared per hertz, is

$$
S_{\delta v}(f)=f^{2} S_{\delta \phi}(f) \text {. }
$$

where $S_{\delta \phi}(F)$ is in decibels with respect to 1 radian.

\section{System Noise Floor Verification}

A plot of the system noise floor (sensitivity) is obtained by repeating the automated measurement procedures with the system modified as shown in Fig. 12. Accurate measurements can be obtained using the configuration shown in Fig. 12a. The reference source supplies $10 \mathrm{dBm}$ to one side of the mixer and $0 \mathrm{dBm}$ to the other mixer input through equal path lengths; phase quadrature is maintained with the phase shifter.

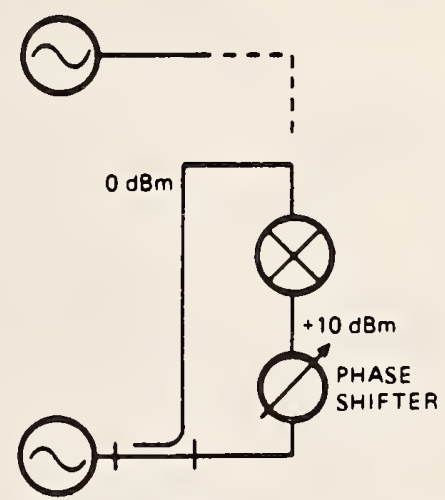

(a)

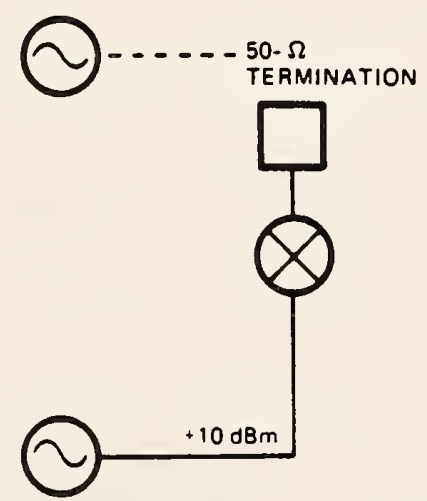

(b)

FIG. 12 System configurations for measuring the system noise floor (sensitivity): (a) configuration used for accurate measurements: (b) alternate configuration sometımes used. 
The configuration shown in Fig. $12 \mathrm{~b}$ is sometimes used and does not greatly degrade the noise floor because the reference signal of $10 \mathrm{dBm}$ is larger than the signal frequency. See Sections IV.B and IV.C. 4 for additional discussions related to system sensitivity and recommended system evaluation.

Proper selection of drive and output termination of the double-balanced mixer can result in improvement by 15 to $25 \mathrm{~dB}$ in the performance of phasenoise measurements, as discussed by Walls et al. (1976). The beat frequency between the two oscillators can be a sine wave, as previously mentioned, with proper low drive levels. This requires a proper terminating impedance for the mixer. With high drive levels, the mixer output waveform will be clipped. The slope of the clipped waveform at the zero crossings, illustrated by Walls et al. (1976), is twice the slope of the sine wave and therefore improves the noise floor sensitivity by $6 \mathrm{~dB}$, i.e., the output signal, proportional to the phase fluctuations, increases with drive level. This condition of clipping requires characterization over the Fourier frequency range, as previously mentioned for the Hewlett-Packard 3047 phase noise measurement system. An amplifier can be used to increase the mixer drive levels for devices that have insufficient output power to drive the double-balanced mixers.

Lower noise floors can be achieved using high-level mixers when available drive levels are sufficient. A step-up transformer can be used to increase the mixer drive voltage because the signal and noise power increase in the same ratio, and the spectral density of phase of the device under test is unchanged, but the noise floor of the measurement system is reduced.

Walls et al. (1976) used a correlation technique that consisted primarily of two phase-noise measurement systems. At TRW the technique is used as shown in Fig. 13. The cross spectrum is obtained with the fast Fourier transform (FFT) analyzer that performs the product of the Fourier trans-form of one signal and the complex conjugate of the Fourier transform of

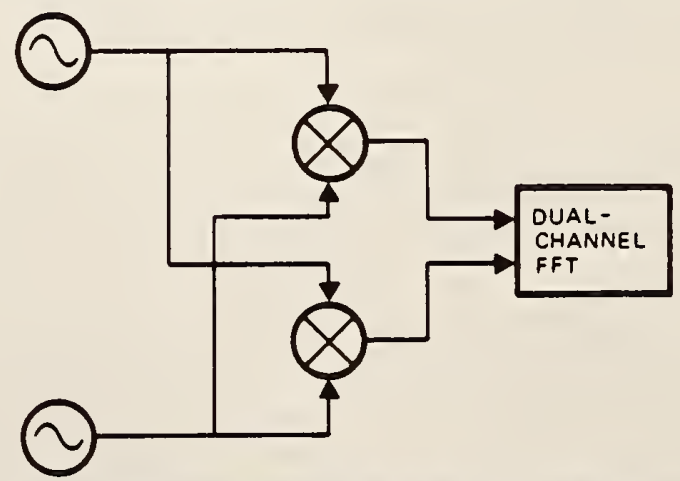

FiG. 13 Cross-spectrum measurement using the two-oscillator technique. 
the second signal. This cross spectrum, which is a phase-sensitive characteristic, gives a phase and amplitude sensitivity measure directly. A signalto-noise enhancement greater than $20 \mathrm{~dB}$ can be achieved.

If the double-balanced phase noise measurement system does not provide a noise floor sufficient for measuring a high-quality source. frequency multiplier chains can be used if their inherent noise is 10-20 dB below the measurement system noise. In frequency multiplication the noise increases according to

$10 \log ($ final frequency/original frequency).

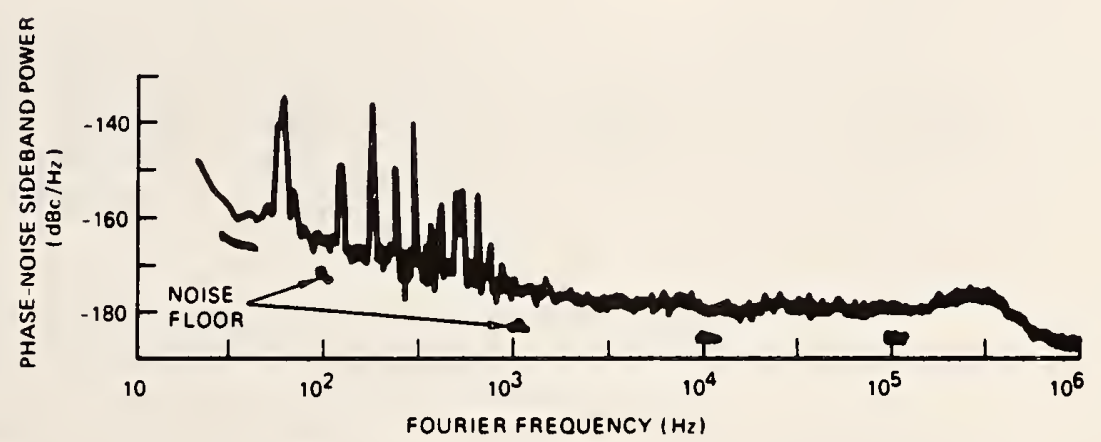

(a)

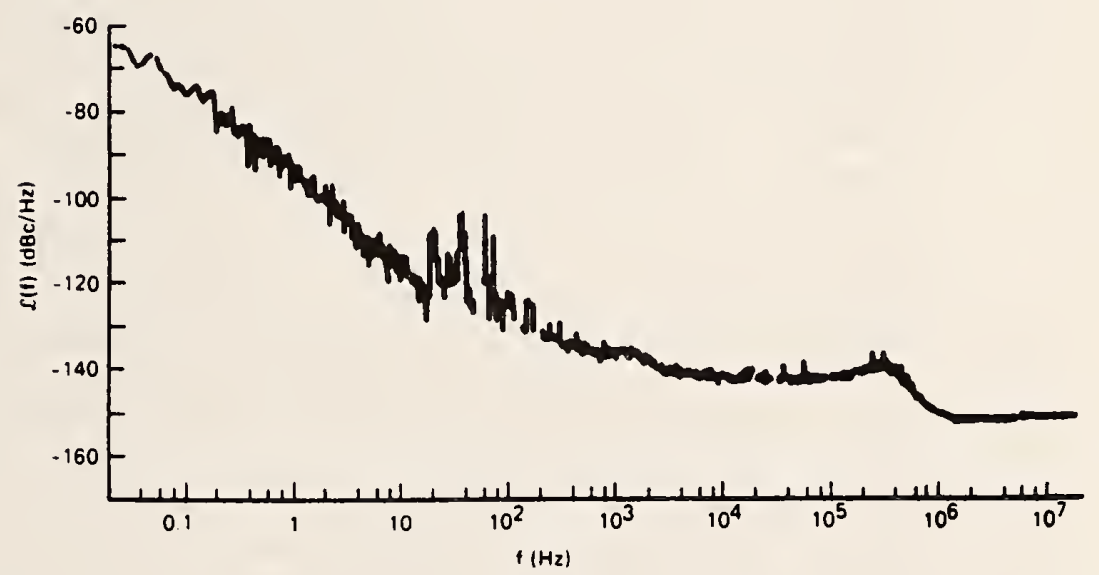

(b)

F1G. 14 Data plots of the automated phase-noise measurement system: (a) a high quality 5-MHz quartz oscillator; (b) combined noise of two H.P. 8662A synthesizers (subtract $3 \mathrm{~dB}$ for a single unit).

- See Appendix Note \# 32 
The following equation is used to correct for noise-floor contribution $P_{\mathrm{nf}}$, in $\mathrm{dBc} / \mathrm{Hz}$, if desired or necessary:

$$
\mathscr{L}(f)(\text { corrected })=-\mathscr{L}(f)+10 \log \left[\frac{P_{\mathscr{L}(f)}-P_{\mathrm{nf}}}{P_{\mathscr{P}(f)}}\right]
$$

The correction for noise-floor contribution can also be obtained by using the measurement of $S_{\delta v}(f)$ of Eq. (57). Measurement of $S_{\delta v}(f)$ of the oscillator plus floor is obtained, then $S_{\delta v}(f)$ is obtained for the noise floor only. Then,

$$
\left.S_{\delta v}(f)\right|_{\text {cor }}=\left.S_{\delta v}(f)\right|_{(\cos +\mathrm{nf})}-\left.S_{\delta v}(f)\right|_{\mathrm{nf}}
$$

Figure $14 \mathrm{a}$ shows a phase noise plot of a very high-quality $(5-\mathrm{MHz})$ quartz oscillator, measured by the two-oscillator technique. The sharp peaks below $1000 \mathrm{~Hz}$ represent the $60-\mathrm{Hz}$ line frequency of the power supply and its harmonics and are not part of the oscillator phase noise. Figure $14 \mathrm{~b}$ shows measurements to $0.02 \mathrm{~Hz}$ of the carrier at a frequency of $20 \mathrm{MHz}$.

\section{Single-Oscillator Phase-Noise Measurement Systems and Techniques}

The phase-noise measurements of a single-oscillator are based on the measurement of frequency fluctuations using discriminator techniques. The practical discriminator acts as a filter with finite bandwidth that suppresses the carrier and the sidebands on both sides of the carrier. The ideal carriersuppression filter would provide infinite attentuation of the carrier and zero attenutation of all other frequencies. The effective $Q$ of the practical discriminator determines how much the signals are attenuated.

Frequency discrimination at very high frequencies (VHF) has been obtained using slope detectors and ratio detectors, by use of lumped circuit elements of inductance and capacitance. At ultrahigh frequencies (UHF) between the VHF and microwave regions, measurements can be performed by beating, or heterodyning, the UHF signal with a local oscillator to obtain a VHF signal that is analyzed with a discriminator in the VHF frequency range. Those techniques provide a means for rejecting residual amplitudemodulated $(\mathrm{AM})$ noise on the signal under test. The VHF discriminators usually employ a limiter or ratio detector.

Ashley et al. (1968) and Ondria (1968) have discussed the microwave cavity discriminator that rejects AM noise, suppresses the carrier so that the input level can be increased, and provides a high discriminated output to improve the signal-to-noise floor ratio. The delay line used as an FM discriminator has been discussed by Tykulsky (1966), Halford (1975), and

* See Appendix Note \# 6 


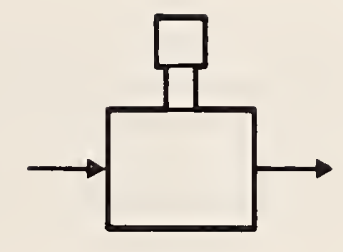

(a)

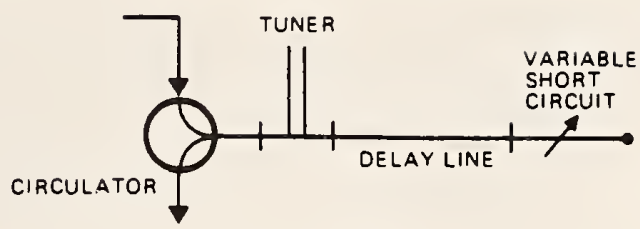

(b)

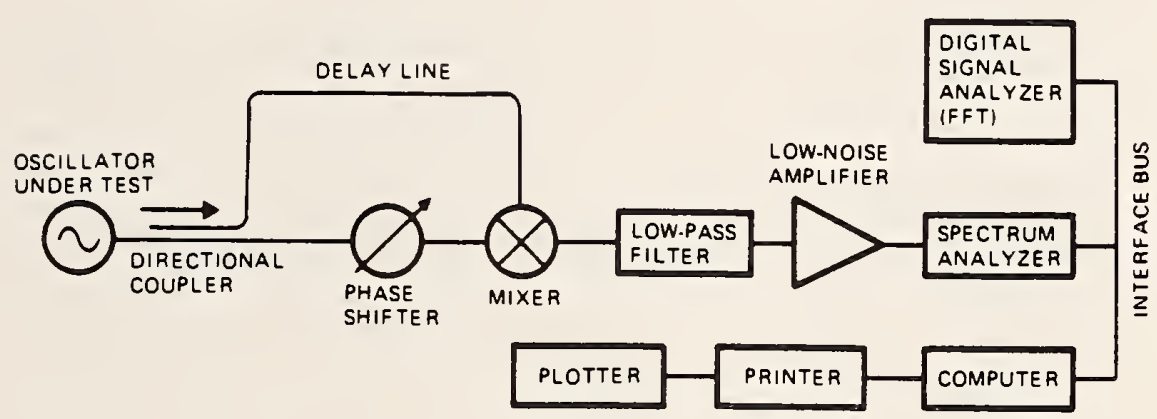

(c)

FIG. 15 Single-oscillator phase-noise measurement techniques: (a) cavity discriminator; (b) reflective-type delay-lıne discriminator; (c) one-way delay line.

Ashley et al. (1968). Ashley et al. (1968) proposed the reflective-type delayline discriminator shown in Fig. 15b. The cavity can also be used to replace delay line. The one-way delay line shown in Fig. $15 \mathrm{c}$ is implemented in the TRW measurement systems. The theory and applications set forth in this section are based on a system of this particular type.

\section{A. The Delay Line as an FM Discriminator}

1. The Single-Oscillator Measurement System

The single-oscillator signal is split into two channels in the system shown in Fig. 15. One channel is called the nondelay or reference channel. It is also referred to as the local-oscillator (LO) channel because the signal in this channel drives the mixer at the prescribed impedance level (the usual LO drive). The signal in the second channel arrives at the mixer through a delay line. The two signals are adjusted for phase quadrature with the phase shifter, and the output of the mixer is a fluctuating voltage, analogous to the frequency fluctuations of the source, centered on approximately zero dc volts.

The delay line yields a phase shift by the time the signal arrives at the balanced mixer. The phase shift depends on the instantaneous frequency of 
the signal. The presence of frequency modulation (FM) on the signal gives rise to differential phase modulation (PM) at the output of the differential delay and its associated (nondelay) reference line. This relationship is linear if the delay $\tau_{d}$ is nondispersive. This is the property that allows the delay line to be used as an FM discriminator. In general, the conversion factors are a function of the delay $\left(\tau_{\mathrm{d}}\right)$ and the Fourier frequency $f$ but not of the carrier frequency.

The differential phase shift of the nominal frequency $v_{0}$ caused by the delay line is

$$
\Delta \phi=2 \pi v_{0} \tau_{\mathrm{d}},
$$

where $\tau_{d}$ is the time delay.

The phase fluctuations at the mixer are related to the frequency fluctuations (at the rate $f$ ) by

$$
\delta \phi=2 \pi \tau_{\mathrm{d}} \delta v(f) .
$$

The spectral density relationships are

$$
\left.S_{\delta \phi}(f)\right|_{\text {mixer }}=\left.\left(2 \pi \tau_{\mathrm{d}}\right)^{2} S_{\delta v}(f)\right|_{\text {osc }}
$$

and

$$
S_{\delta v}(f)=f^{2} S_{\delta \phi}(f)
$$

Then,

$$
\left.S_{\delta \phi}(f)\right|_{\mathrm{dlm}}=\left.\left(2 \pi f \tau_{\mathrm{d}}\right)^{2} S_{\delta \phi}(f)\right|_{\text {osc }}
$$

where the subscript dlm indicates delay-line method. From Eq. (56), the spectral density of phase for the two-oscillator technique, in radians squared per hertz, is

$$
S_{\delta \phi}(f)=4 \frac{S_{\delta v}(f)}{\left(V_{\mathrm{ptp}}\right)^{2}}=\frac{S_{\delta v}(f)}{2\left(V_{\mathrm{rms}}\right)^{2}}=\left[\frac{\left(\dot{\delta} v_{\mathrm{rms}}\right)^{2}}{2\left(V_{\mathrm{rms}}\right) B}\right]
$$

because

$$
\left(V_{\mathrm{ptp}}\right)^{2}=8\left(v_{\mathrm{rms}}\right)^{2}=4\left(V_{\mathrm{p}}\right)^{2}=4\left[2\left(v_{\mathrm{rms}}\right)^{2}\right]
$$

and

$$
\mathscr{L}(f)=2\left(S_{\delta v}(f) /\left(V_{\mathrm{ptp}}\right)^{2}\right)=\left(\delta v_{\mathrm{rms}}\right)^{2} / 4\left(V_{\mathrm{rms}}\right)^{2} B
$$

per hertz. 
The sensitivity (noise floor) of the two-oscillator measurement system includes the thermal and shot noise of the mixer and the noise of the baseband preamplifier (referred to its input). This noise floor is measured with the oscillator under test inoperative. The measurement system sensitivity of the two-oscillator system, on a per hertz density basis $(\mathrm{dBc} / \mathrm{Hz})$ is

$$
\mathscr{L}(f)_{\mathrm{nf}}=10 \log \left[2\left(\delta v_{n}\right)^{2} /\left(V_{\mathrm{ptp}}\right)^{2}\right],
$$

where $\delta r_{n}$ is the rms noise voltage measured in a one-hertz bandwidth.

The two-oscillator system therefore yields the output noise from both oscillators. If the reference oscillator is superior in performance, as assumed in the previous discussions, then one obtains a direct measure of the noise characteristics of the oscillator under test. If the reference and test oscillators are the same type, a useful approximation is to assume that the measured noise power is twice that associated with one noisy oscillator. This approximation is in error by no more than $3 \mathrm{~dB}$ for the noisier oscillator. Substituting in Eq. (80) and using the relationships in Eq. (56), we have, per hertz,

$$
\left.\mathscr{L}(f)\right|_{\mathrm{dlm}}=2\left[\left(\delta v_{\mathrm{rms}}\right)^{2} /\left(V_{\mathrm{plp}}\right)^{2}\right]\left(2 \pi f \tau_{\mathrm{d}}\right)^{2}
$$

Examination of this equation reveals the following.

(1) The term in the brackets represents the two-oscillator response. Note that this term represents the noise floor of the two-oscillator method. Therefore, adoption of the delay-line method results in a higher noise by the factor $\left(2 \pi f \tau_{d}\right)^{2}$ when compared with the two-oscillator measurement method. The sensitivity (noise floor) for delay lines with different values of time delay are illustrated in Fig. 17.

(2) Equation (84) also indicates that the measured value of $\mathscr{L}(f)$ is periodic in $\omega=2 \pi f$. This is shown in Fig. 21. The first null in the responses is at the Fourier frequency $f=1 / \tau_{\mathrm{d}}$. The periodicity indicates that the calibration range of the discriminator is limited and that valid measurements occur only in the indicated range. as verified by the discriminator slope shown in Fig. 16. (See Fig. 23.)

(3) The maximum value of $\left(2 \pi f \tau_{d}\right)^{2}$ can be greater than unity (it is 4 at $f=12 \tau_{\mathrm{d}}$ ). This $6-\mathrm{dB}$ advantage is utilized in the noise-floor measurement. However, it is beyond the valid calibration range of the delay-line system. The 6- $\mathrm{dB}$ advantage is offset by the line attenuation at microwa ve frequencies, as discussed by Halford (1975).

The delay-line discriminator system has been analyzed in terms of a powerlimited system (a particular idealized system in which the choice of power oscillator voltage, the attenuator of the delay line, and the conversion loss 
of the mixer are limited by the capability of the mixer) by Tykulsky (1966), Halford (1975), and Ashley et al. (1977). For this particular case. Eq. (83) indicates that an increase in the length of the delay line (to increase $\tau_{d}$ for decorrelation of Fourier frequencies closer to the carrier) results in an increase in attenuation of the line, which causes a corresponding decrease in $V_{\text {ptp }}$. The optimum length occurs where $\tau_{d}$ is such that the decrease in $V_{\text {ptp }}$ is approximately compensated by the increase in $\left(2 \pi f \tau_{d}\right)$, i.e., where

$$
\frac{d}{d \tau_{d}} \frac{2 \pi f \tau_{d}}{V_{p t p}}=0
$$

This condition occurs where the attenuation of the delay line is $1 \mathrm{~Np}$ $(8.686 \mathrm{~dB})$. However, when the system is not power limited, the attenuation of the delay line is not limited, because the in put power to the delay line can be adjusted to maintain $V_{\text {ptp }}$ at the desired value. The optimum delay-line length is determined at a particular selectable frequency. However, since the attenuation varies slowly (approximately proportional to the square root of frequency), this characteristic allows near-optimum operation over a considerable frequency range without appreciable degradation in the measurements.

A practical view of the time delay $\left(\tau_{d}\right)$ and Fourier-frequency functional relationship can be obtained by reviewing the basic concepts of the dualchannel time-delay measurement system discussed by Lance (1964). If the differential delay between the two channels is zero, there is no phase difference at the detector output when a swept-frequency $\mathrm{cw}$ signal is applied to the system. Figure 16 shows the detected output interference display when a swept-frequency cw signal (zero to $4 \mathrm{MHz}$ ) is applied to a system that has

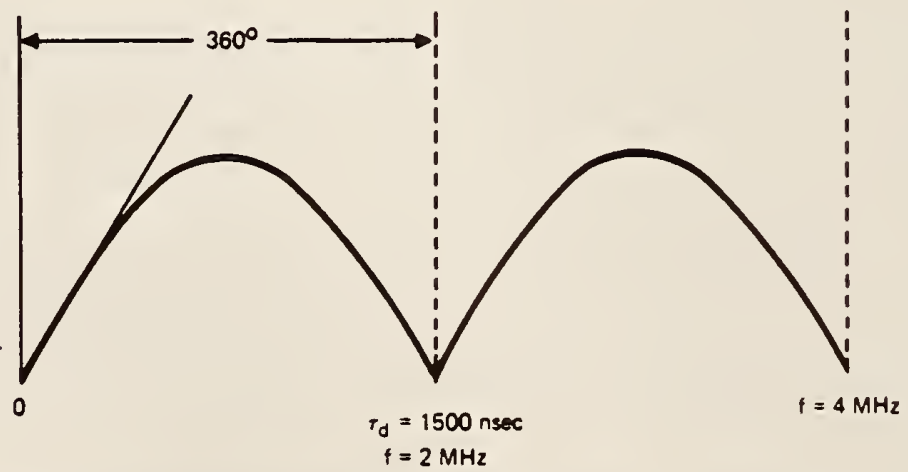

Fig. 16 Swept-frequency interference display at the output of a dual-channel system with a differential delay of $500 \mathrm{nsec}$. 
a differential delay of $500 \mathrm{nsec}$ between the two channels. The signal amplitudes are assumed to be almost equal, thus producing the familiar voltagestanding-wave pattern or interference display. Because this is a two-channel system, there is a null every $360^{\circ}$, as shown.

\section{System Sensitivity (Noise Floor) When Using the \\ Differential Delay-Line Technique}

Halford (1975) has shown that the sensitivity (noise floor) of the singleoscillator differential delay-line technique is reduced relative to the twooscillator techniques. The sensitivity is modified by the factor

$$
S_{\mathrm{d}}=2\left(1-\cos 2 \pi f_{\tau_{\mathrm{d}}}\right) \text {. }
$$

For $\omega \tau_{\mathrm{d}}=2 \pi d \tau_{\mathrm{d}} \ll 1$ a good approximation is

$$
S_{\mathrm{J}}^{2}=2\left(1-\cos 2 \pi \tau_{\mathrm{d}}\right)=\left(\omega \tau_{\mathrm{d}}\right)^{2}\left[1-\frac{1}{12}\left(\omega \tau_{\mathrm{d}}\right)^{2}\right]=\left(2 \pi f \tau_{\mathrm{d}}\right)^{2}=\theta^{2},
$$

where $\theta$ is the phase delay of the differential delay line evaluated at the frequency $f$. Figure 17 shows the relative sensitivity (noise floor) of the twooscillator technique and the single-oscillator technique with different delay-line lengths. The $f^{-2}$ slope is noted at Fourier frequencies beyond

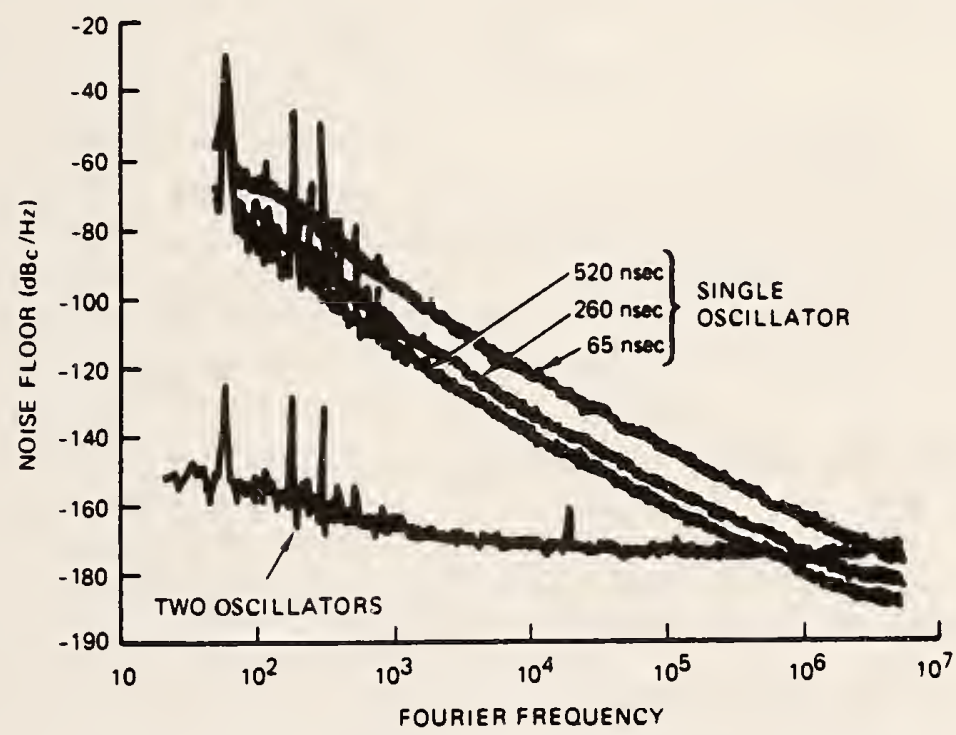

FIG. 17 Relative sensitivity (noise floor) of single-oscillator and two-oscillator phasenoise measurement systems. 
about $1 \mathrm{kHz}$. For Fourier frequencies closer to the carrier, the slope is $f^{-3}$. i.e., the sum of the $f^{-2}$ slope of Eq. (87) and the $f^{-1}$ flicker noise.

Phase-locked sources have phase-noise characteristics that cannot be measured at close-in Fourier frequencies using this basic system. The relative sensitivity of the system can be improved by using a dual (twochannel) delay-line system and performing cross-spectrum analysis, which will be presented in this chapter.

Labaar (1982) developed the delay-line of bridge configuration shown in Fig. 18. At microwave frequencies where a high-gain amplifier is available, suppression of the carrier by the of bridge allows amplification of the noise going into the mixer. A relative sensitivity improvement of $35 \mathrm{~dB}$ has been obtained without difficulty. The limitations of the technique depend on the available $\mathrm{rf}$ power and the carrier suppression by the bridge. Naturally, if the if input to the bridge is high one must use the technique with adequate precautions to prevent mixer damage that can occur by an accidental bridge unbalance. Labaar (1982) indicated the added advantage of using the of bridge carrier-suppression technique when attempting to measure phase noise close to the carrier when AM noise is present. Figure 19 shows the

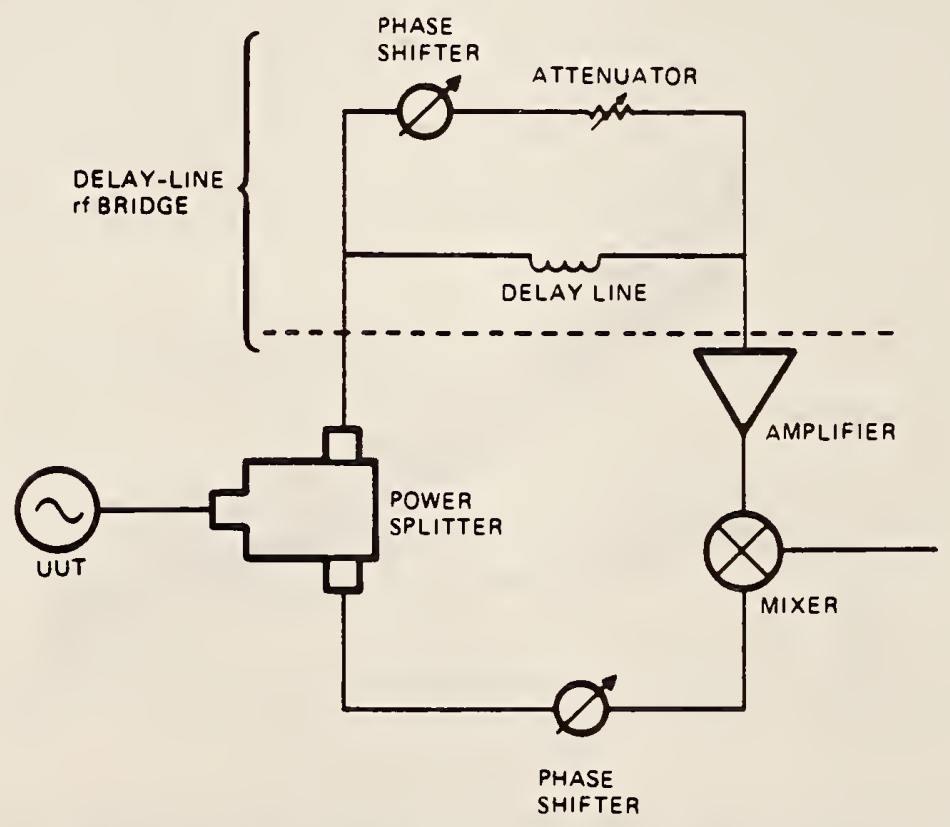

FIG. 18 Carrier suppression using an rf bridge to increase relative sensitivity. (Courtesy Instrument Society of America.) 


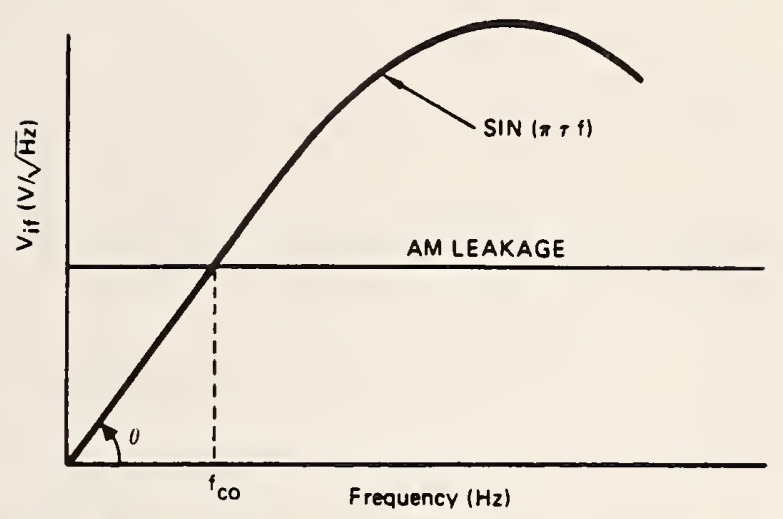

FiG. 19 Phase detector output (AM-PM crossover); $\tau$, delay time.

mixer output for phase (PM) and amplitude (AM) noise in the singleoscillator delay-line FM discriminator system. It is noted that the phase noise and AM noise intersect and that the AM will therefore limit the measurement accuracy near the carrier. Even though AM noise is much lower than phase noise in most sources, and even though the AM is normally suppressed about $20 \mathrm{~dB}$, there is still $\mathrm{AM}$ at the mixer output. This output is $A M$ leakage and is caused by the finite isolation between the mixer ports. The two-oscillator technique does not experience this problem to this extent because the phase noise and AM noise maintain their relative relationships at the mixer output independent of the offset frequency from the carrier.

\section{B. Calibration and Measurements Using the Delay LINE AS AN FM DISCRIMINATOR}

The block diagram of a practical single-oscillator phase noise measurement system is shown in Fig. 20. The signals in the delay-line channel of the system experience the one-way delay of the line. With adequate source power, the system is not limited to the optimum $1 \mathrm{~Np}(8.686 \mathrm{~dB})$ previously discussed for a power-limited system. Measurements are performed using the following operational procedures.

(1) Measure the tracking spectrum analyzer IF bandwidths as set forth in Section III.C.1.

(2) Establish the system power levels (Section IV.B.1).

(3) Establish the discriminator calibration factor (Section IV.B.2).

(4) Measure and plot the oscillator characteristics in the automatic system used (Section IV.B.3).

(5) Measure the system noise floor (sensitivity) (Section IV.B.4). 


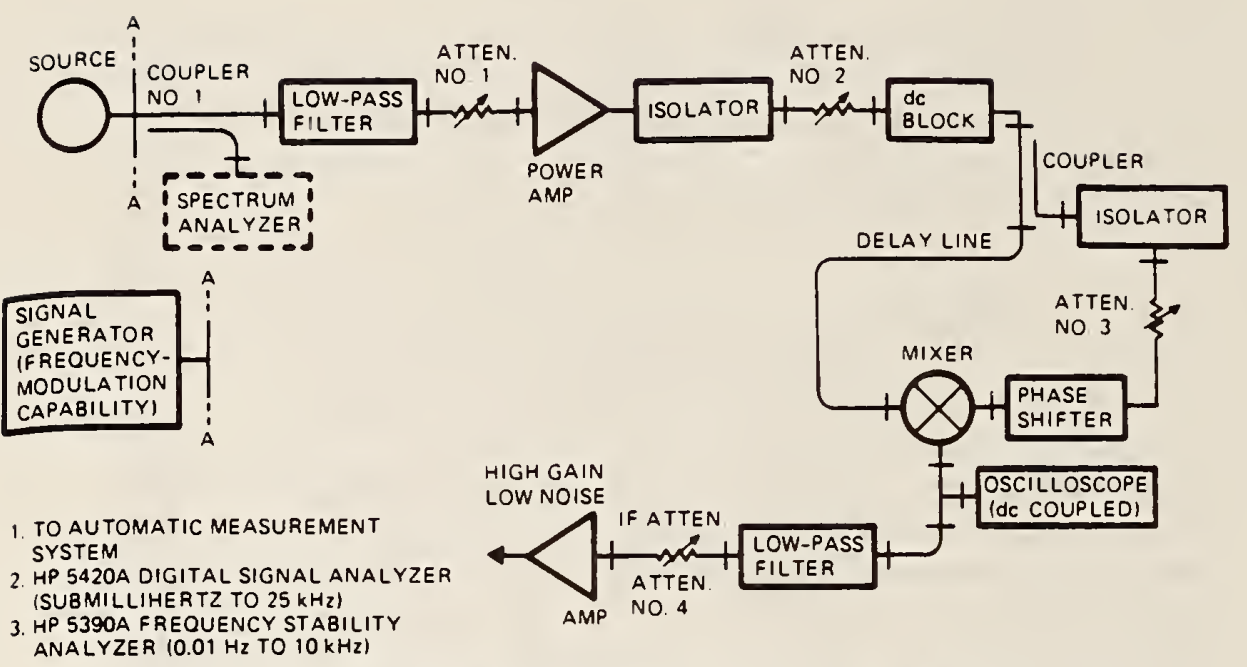

Fig. 20 Single-oscillator phase noise measurement system using the delay line as an FM discriminator. (From Lance et al., 1977a.)

\section{System Power Levels}

The system power levels are set using attenuators, as shown in Fig. 20. Because the characteristic impedance of attenuator No. 4 is $50 \Omega$, mismatch errors will occur if the mixer output impedance is not $50 \Omega$. As previously discussed, the mixer drive levels are set so that the mixer output signal, as observed during calibration, is sinusoidal. This has been accomplished in TRW systems with a reference (LO) signal level of $10 \mathrm{dBm}$ and a mixer input level of about $0 \mathrm{dBm}$ from the delay line.

A power amplifier can be used to increase the source signal to the measurement system. This amplifier must not contribute appreciable additional noise to the signal.

\section{Discriminator Calibration}

The discriminator characteristics are measured as a function of frequency and voltage. The hertz-per-volt sensitivity of the discriminator is defined as the calibration factor (CF). The calibration process involves measuring the effects of intentional modulation of the source (carrier) frequency. A known modulation index must be obtained to calculate the calibration factors of the discriminator. The modulation index is obtained by using amplitude modulation to establish the carrier-to-sideband ratio when there is considerable instability of the source or when the source cannot be frequency modulated. 
It is convenient to consider the system equations and calibration techniques in terms of frequency modulation of stable sources. If the source to be measured cannot be frequency modulated, it must be replaced, during the calibration process, with a modulatable source. The calibration process will be described using a modulatable source and a $20-\mathrm{kHz}$ modulation frequency. However, other modulation frequencies can be used. The calibration factor of this type discriminator has been found to be constant over the usable Fourier frequency range, within the resolution of the measuring technique. The calibration factor of the discriminator is established after the system power levels have been set with the unit under test as the source.

The discriminator calibration procedures are as follows.

(1) Set attenuator No. 4 (Fig. 20) to $50 \mathrm{~dB}$.

(2) Replace the oscillator under test with a signal generator or oscillator that can be frequency modulated. The power output and operating frequency of the generator must be set to the same precise frequency and amplitude values that the oscillator under test will present to the system during the measurement process.

(3) Select a modulation frequency of $20 \mathrm{kHz}$ and increase the modulation until the carrier is reduced to the first Bessel null, as indicated on the spectrum analyzer connected to coupler No. 1 . This establishes a modulation index $(m=2.405)$.

(4) Adjust the phase shifter for zero volts dc at the output of the mixer, as indicated on the oscilloscope connected as shown in Fig. 20. This establishes the quadrature condition for the two inputs to the mixer. This quadrature condition is continuously monitored and is adjusted if necessary.

(5) Tune the tracking spectrum analyzer to the modulation frequency of $20 \mathrm{kHz}$. The power reading at this frequency is recorded in the program and is corrected for the 50-dB setting of attenuator No. 4, which will be set to zero decibel indication during the automated measurements.

$$
P(\mathrm{dBm})=(-\mathrm{dBm} \text { power reading })+50 \mathrm{~dB}
$$

This power level is converted to the equivalent rms voltage that the spectrum analyzer would have read if the total signal had been applied:

$$
V_{\mathrm{rms}}=\sqrt{10^{P / 10} / 1000+R} \text {. }
$$

(6) The discriminator calibration factor can now be calculated because this power in $\mathrm{dBm}$ can be converted to the corresponding rms voltage using the following equation:

$$
V_{\mathrm{rms}}=\sqrt{\left(10^{\text {P.10}} / 1000\right) \times R}
$$

where $R=50 \Omega$ in this system. 
(7) The discriminator calibration factor is calculated in hertz per volt as

$$
C F=m f_{\mathrm{m}} / v^{2} V_{\mathrm{rms}}=2.405 f_{\mathrm{m}} / \sqrt{2} V_{\mathrm{rms}} \text {. }
$$

The modulation index $m$ for the first Bessel null as used in this technique is 2.405 . The modulation frequency is $f_{\mathrm{m}}$.

\section{Measurement and Data Plotting}

After the discriminator is calibrated, the modulated signal source is replaced with the frequency source to be measured. Quadrature of the signals into the mixer is reestablished, attenuator No. 4 (Fig. 20) is set to $0 \mathrm{~dB}$, and the measurement process can begin.

The measurements, calculations, and data plotting are completely automated. The calculator program selects the Fourier frequency, performs autoranging, and sets the band width, and measurements of Fourier frequency power are performed by the tracking spectrum analyzer. Each Fourier frequency noise-power reading $P_{\mathrm{n}}(\mathrm{dBm})$ is converted to the corresponding rms voltage by

$$
v_{1 \mathrm{rms}}=\sqrt{10^{\left(P_{n}+2.5\right) / 10} / 1000 \times R} .
$$

The rms frequency fluctuations are calculated as

$$
\delta v_{\mathrm{rms}}=v_{1 \mathrm{rms}} \times \mathrm{CF} .
$$

The spectral density of frequency fluctuations in hertz squared per hertz is calculated as

$$
S_{i v}(f)=\left(\delta v_{\mathrm{rms}}\right)^{2} / B
$$

where $B$ is the measured IF noise-power bandwidth of the spectrum analyzer. The spectral density of phase fluctuations in radians squared per hertz is calculated as

$$
S_{\delta \phi}(f)=S_{\delta v}(f) / f^{2} .
$$

The NBS-designated spectral density in decibels (carrier) per hertz is calculated as

$$
\mathscr{L}(f)_{\mathrm{dB}}=10 \log \frac{1}{2} S_{\delta \phi}(f) .
$$

Spectral density is plotted in real time in our program. However, the data can be stored and the desired spectral density can be plotted in other forms. Integrated phase noise can be obtained as desired. 


\section{Noise Floor Measurements}

The relative sensitivity (noise floor) of the single-oscillator measurement system is measured as shown in Fig. 12a for the two-oscillator technique. The delay line must be removed and equal channel lengths constructed, as in Fig. (12a). The same power levels used in the original calibration and measurements are reestablished, and the noise floor is measured at specific Fourier frequencies, using the same calibration-measurement technique, or by repeating the automated measurement sequence.

A correction for the noise floor requires a measurement of the rms voltage of the oscillator $\left(v_{1 \mathrm{rms}}\right)$ and a measurement of the noise floor rms voltage $\left(v_{2 \mathrm{rms}}\right)$. These voltages are used in the following equation to obtain the corrected value:

$$
v_{\text {rms }}=\sqrt{\left(v_{1 \mathrm{rms}}\right)^{2}-\left(v_{2 \mathrm{rms}}\right)^{2}} .
$$

The value $t_{\text {rms }}$ is then used in the calculation of frequency fluctuations. If adequate memory is available, each value of $v_{1 \mathrm{rms}}$ can be stored and used after the other set of measurements are performed at the same Fourier frequencies.

The following technique was developed by Labaar (1982). Carrier suppression is obtained using the rf bridge illustrated in Fig. 18. One can easily improve sensitivity more than $40 \mathrm{~dB}$. At 2.0 and $3.0 \mathrm{GHz} 70-\mathrm{dB}$ carrier suppression was realized. In general, the improvement in sensitivity will depend on the availability of an amplifier or adequate input power.

Figure 21 shows the different noise floors in a delay-line bridge discriminator. It is good measurement discipline to always determine these noise floors; also, the measurements, displayed in Fig. 21, give a quick understanding of the physical process involved. The first trace is obtained by terminating the input of the baseband spectrum analyzer. The measured output noise power is then a direct measure of the spectrum analyzer's noise figure (NF). The input noise is thermal noise and is usually indicated by "KTB," which is short for "the thermal noise power at absolute temperature of $T$ degrees $\mathrm{K}$ (elvin) per one hertz bandwidth (B). This $\mathrm{KTB}$ number is, at $18^{\circ} \mathrm{C}$, about $-174 \mathrm{dBm} / \mathrm{Hz}$.

Figure 21 a shows that trace number 1 for frequencies above about $1 \mathrm{kHz}$ is level with a value of about $-150 \mathrm{dBm}=-(174-24) \mathrm{dBm}$, which means that the spectrum analyzer has an NF of $24 \mathrm{~dB}$. At $20 \mathrm{~Hz}$ the NF has gone up to about $48 \mathrm{~dB}$. To improve the NF, a low-noise (NF, 2dB). low-frequency $(10 \mathrm{~Hz}-10 \mathrm{MHz})$ amplifier is inserted as a preamplifier. Terminating its input now results in trace number 2 . At the high frequency end, the measured power goes up by about $12-13 \mathrm{~dB}$, and the amplifiers gain is $34 \mathrm{~dB}$. This means that the NF is improved by $34-12-13=21-22 \mathrm{~dB}$, which is an 


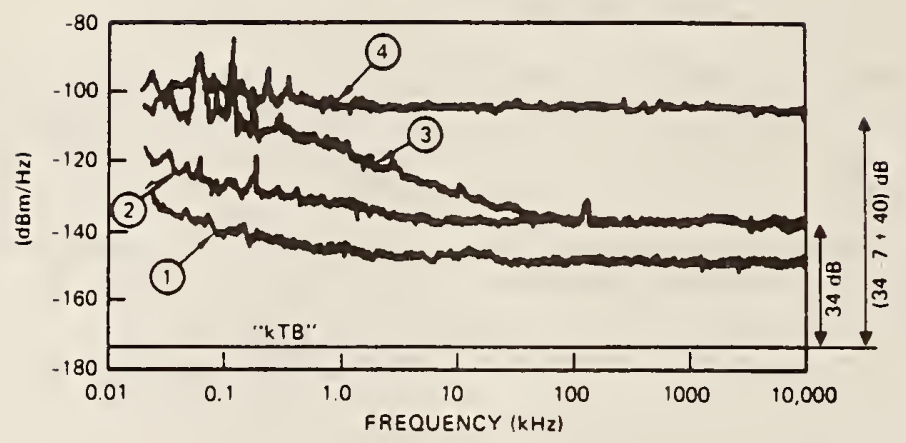

(a)

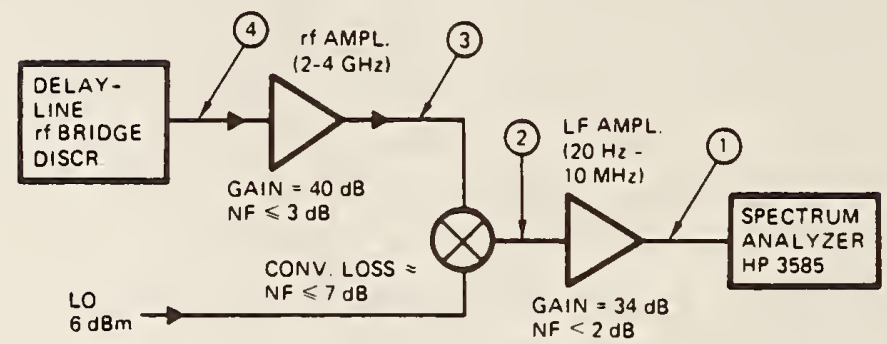

(b)

FIG. 21 (a) Noise contribution analysis: (b) phase noise test setup using a delay-line if bridge discriminator $(\mathrm{r} f=2.8 \mathrm{G} \mathrm{Hz}$; $O$. termination points. NF, nosse figure: $L F$. low frequency. (From Seal and Lance. 1981.)

$\mathrm{NF} \simeq 2-3 \mathrm{~dB}$ as expected, i.e., the first stage noise predominates. The lowfrequency end at $20 \mathrm{~Hz}$ gives an NF of $26 \mathrm{~dB}$, which overall is quite an improvement.

In trace number 3 the mixer is included with it's of (signal) port terminated. It is clear from this trace that certainly up to $100 \mathrm{kHz}$, the noise generated by the mixer diodes being "pumped" by the LO signal dominates. This case represents the "classic" delay-line discriminator. The last trace (number 4) includes the low-noise, high-gain of amplifier that can be used because the carrier is suppressed in the delay-line of bridge discriminator, in contrast to the classic delay-line discriminator case. This trace shows that from $\mathrm{lkHz}$ on up the measured output power is flat, representing a 2-3-dB NF.

At about $20-40 \mathrm{~Hz}$, trace numbers 3 and 4 begin nearing their crossover floor. In this particular case, which is discussed in full by Labaar (1982), the measurement systems noise floor (resolution) has been improved by $40 \mathrm{~dB}$.

Figure 22 shows plots of phase noise as measured at two frequencies using delay lines of different lengths. The delay line used measure at $600 \mathrm{MHz}$ 


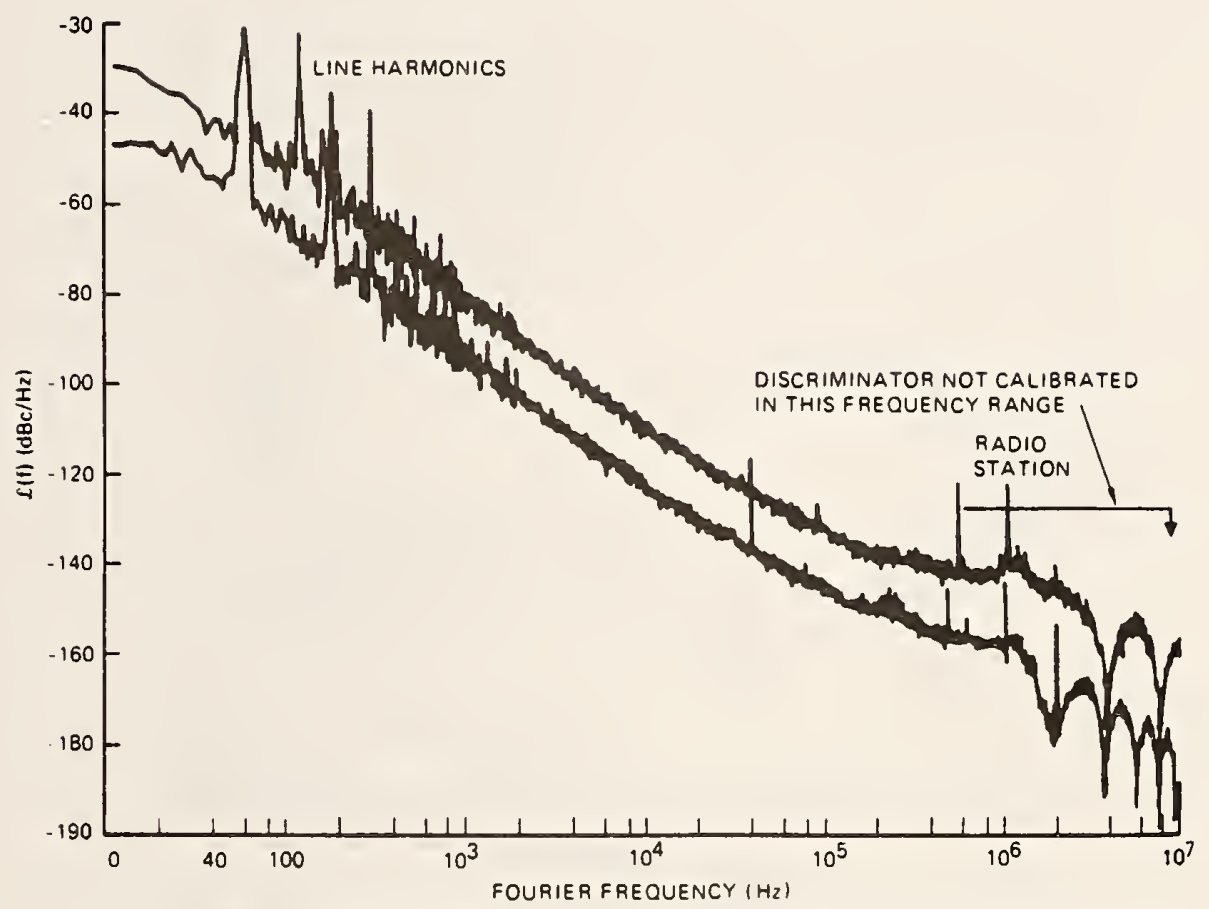

FIG. 22 Phase noise of $600 \cdot \mathrm{MHz}$ oscillator multiplied to $2.4 \mathrm{GHz}$ (From Lance et al., 1977a.)

was about $500 \mathrm{nsec}$ long, as noted by the first null, i.e., the reciprocal of the Fourier frequency of $2 \mathrm{MHz}$ is the approximate differential time delay. Note that a shorter delay line (approximately $250 \mathrm{nsec}$ differential) is used to measure the higher frequency because the delay-line discriminator calibration is valid only to a Fourier frequency at approximately $35 \%$ of the Fourier frequency at which the first null occurs, if a linear transfer function is assumed.

The actual transfer function of a delay-line discriminator (classic and if bridge types) is sinusoidal, as shown in Fig. 23a. The baseband spectrum analyzer measures power in a finite bandwidth, and as a consequence it is possible to measure through a transfer-function null if the noise power does not change substantially over a spectrum-analyzer bandwidth. The following power relations then hold:

$$
P_{\text {meas }}(\omega)=1 / \Delta \omega \int_{\omega-\Delta \omega 2}^{\omega+\Delta \omega \cdot 2} P\left(\omega^{\prime}\right) d \omega^{\prime} \simeq \frac{P(\omega)}{\Delta \omega} \int_{\omega-\Delta \omega \cdot 2}^{\omega+\Delta \omega: 2} d \omega^{\prime}=P(\omega)
$$



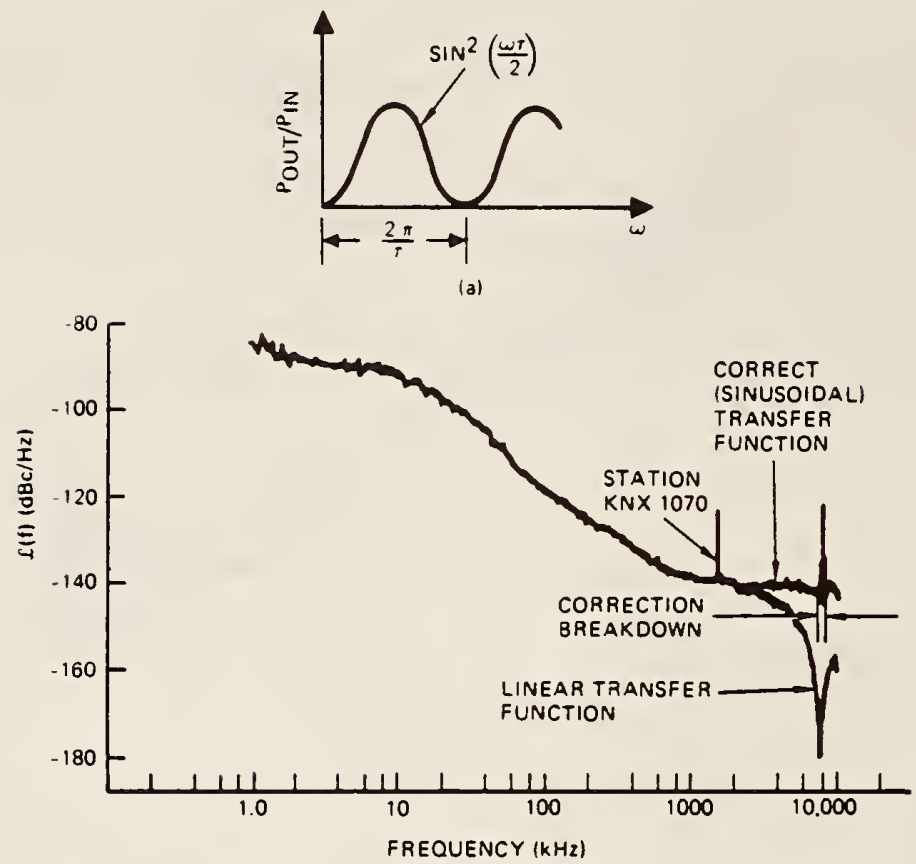

(b)

Fig. 23 Transfer functions for a delay-line if bridge discriminator: (a) actual; (b) approximate (linear) and "correct" (sinusoidal). Phase noise: H.P. 8672A at $2.4 \mathrm{GHz}$.

Figure 23b shows the results using a linear approximation and the "correct" transfer function for a delay-line rf bridge discriminator. The correct transfer function breaks down close to the null because the signal level drops below the system's noise floor, as explained by Labaar (1982).

Using the sinusoidal transfer function in the calculator software gives correct results barring frequency intervals of 5 to 10 spectrum analyzer's bandwidths $(10 \times 30=300 \mathrm{kHz})$ centered at the transfer function nulls. These particular data were selected to illustrate the characteristics of the system. Recall that one can easily make the noise floor $40 \mathrm{~dB}$ lower using the $\mathrm{rf}$ bridge shown in Fig. 18.

\section{Dual Delay-Line Discriminator}

\section{Phase Noise Measurements}

The dual delay-line discriminator is shown in Fig. 24. This system was suggested by Halford (1975) as a technique for lowering the noise floor of the delay-line phase noise measurement system. The system consists of 


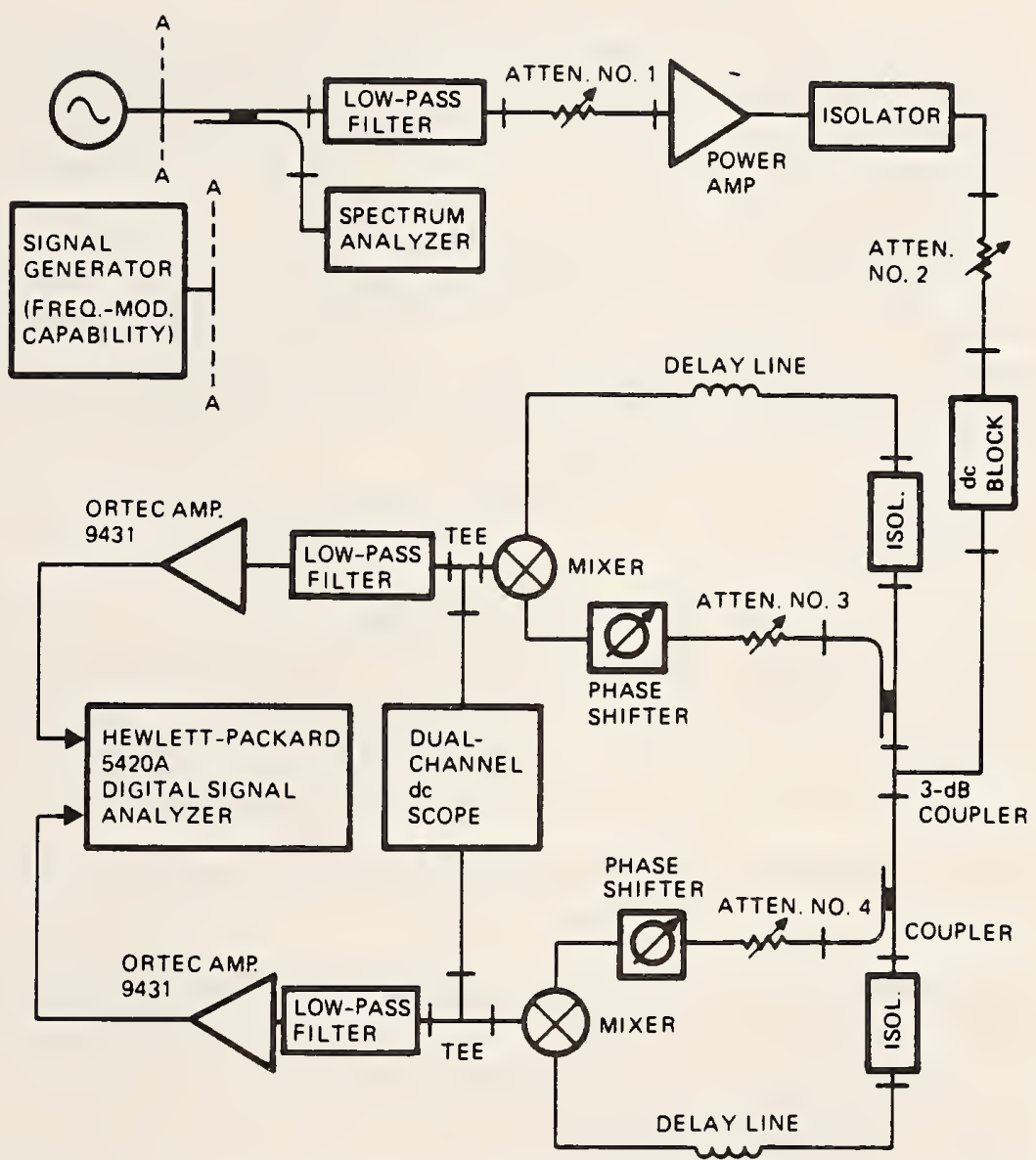

FIG. 24 A dual delay-line phase noise measurement system. (Courtesy Instrument Society of America.)

two differential delay-line systems. The single-oscillator signal is applied to both systems and cross-spectrum analysis is performed on the signal output from the two delay-line systems. Signal processing is performed with the Hewlett-Packard 5420A digital signal analyzer. The cross spectrum is obtained by taking the product of the Fourier transform of one signal and the complex conjugate of the Fourier transform of a second signal. It is a phase-sensitive characteristic resulting in a complex product that serves as a measurement of the relative phase of two signals. Cross spectrum gives a phase- and amplitude-sensitive measurement directly. By performing the product $S y(f) \cdot S x(f)^{*}$, a cert ain signal-to-noise enhancement is achieved. 
The low-noise amplifiers preceding the digital signal analyzer are used when performing measurements at Fourier frequencies from $1 \mathrm{~Hz}$ to $25 \mathrm{kHz}$. The amplifiers are not used when performing measurements below the Fourier frequency of $1 \mathrm{~Hz}$.

\section{Calibrating the Dual Delay-Line System}

Each delay line in the system is calibrated separately following the same basic procedure set forth in Section IV.B. The Hewlett-Packard 5420 measures the one-sided spectral density of frequency fluctuations in hertz squared per hertz. The spectral density of phase fluctuation in radians squared per hertz can be calculated as

$$
S_{\delta \phi}(f)=S_{\delta v}(f) / f^{2}
$$

and

$$
\mathscr{L}(f)=S_{\delta v}(f) / 2 f^{2},
$$

per hertz. The Hewlett-Packard 5420 measurement of $S_{\delta v}(f)$ in $\mathrm{Hz}^{2} / \mathrm{Hz}$ must, therefore, be corrected by $1 / 2 f^{2}$. However, the $f^{2}$ correction must be entered in terms of radian frequency $(\omega=2 \pi f)$. This conversion is accomplished by

$$
\mathscr{L}(f)=S_{\delta v}(f)\left(1 / 2 f^{2}\right)\left(4 \pi^{2} / 4 \pi^{2}\right)=\left[2 \pi^{2} S_{\delta v}(f)\right] /(\omega)^{2}
$$

per hertz since Eq. (100) can be stated in the following terms:

$$
\left[2 \pi^{2} S_{\delta v}(f)\right] / 4 \pi^{2} f^{2} \text {. }
$$

Signal-to-noise enhancement greater than $20 \mathrm{~dB}$ has been obtained using the dual-channel delay-line system.

\section{Millimeter-Wave Phase-Noise Measurements}

\section{Spectral Density of Phase Fluctuations}

The delay line used as an FM discriminator is based, in principle, on a nondispersive delay line. However, a waveguide can be used as the delay line because the Fourier frequency range of interest is a small percentage of the operating bandwidth (seldom over $100 \mathrm{MHz}$ ), and the dispersion can be considered negligible.

The calibration and measurement are performed as set forth in Section IV.B. The modulation index $m$ is usually established using the carrier-tosideband ratio that uses amplitude modulation because millimeter sources are either unstable or cannot be modulated. The two approaches to measurements at millimeter frequencies are shown in Figs. 25 and 26. Figure 25 shows the direct measurement using a waveguide delay line. This system offers improved sensitivity if adequate input power is a vailable. The rf bridge and delay-line portion of the system differs from Fig. 18 because pre- and 


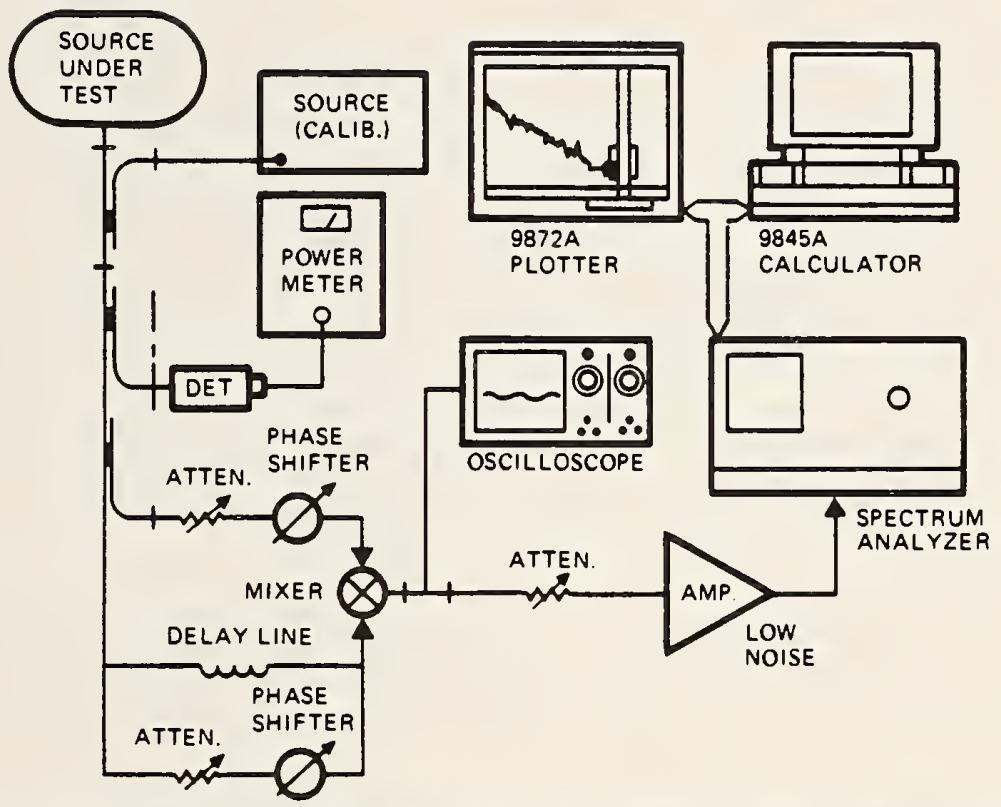

FiG. 25 Millimeter-wave phase noise measurements using a waveguide delay line. (From Seal and Lance. 1981.)

post-bridge amplifiers with appropriate gain are not available, so the sensitivity can equal the amount of carrier suppression.

Figure 26 shows the use of a harmonic mixer to downconvert to the convenient lower frequency where post-bridge amplifiers are available. The relatively low sensitivity to frequency drift that is characteristic of delayline discriminators becomes an advantage here. A separate calibration generator is required, as shown in Fig. 25, and a power meter is used to assure proper power levels during the calibration process.

\section{Spectral Density of Amplitude Fluctuations}

AM noise measurements require equal electrical length in the two channels that supply the signals to the mixer. The delay line must be replaced with the necessary length of transmission line to establish the equal-length condition when the systems shown in Figs. 25 and 26 are used. The AM noise measurement system is calibrated and the noise measurements are performed directly in units of power for a direct measurement of $m(f)$ in $\mathrm{dBc} / \mathrm{Hz} . m(f)$ is the spectral density of one modulation sideband divided by the total signal power at a Fourier frequency difference $f$ from the signal's average frequency $v_{0}$. The system calibration establishes the detection characteristics in terms of total power output at the IF port of the mixer (detector). 


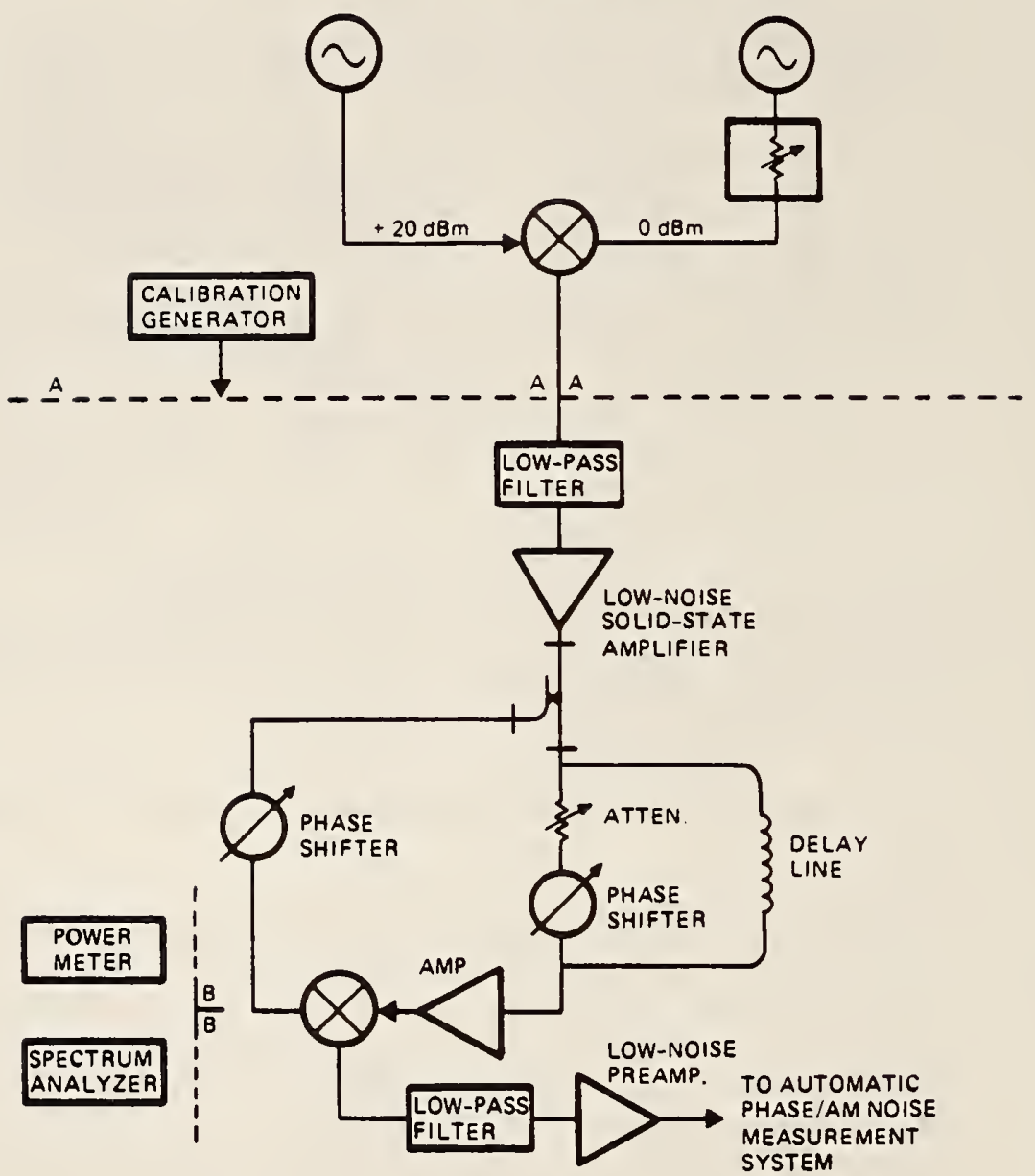

FiG. 26 A millimeter-wave hybrid phase noise measurement system that produces IF frequency and uses a delay-line discriminator at IF frequency. (From Seal and Lance, 1981.)

The AM noise measurements are performed according to the following.

(1) A known AM modulation (carrier-sideband ratio) must be established to calibrate this detector in terms of total power output at the IF port. The modulation must be low enough so that the sidebands are at least $20 \mathrm{~dB}$ below the carrier. This is to keep the total added power due to the modulation small enough to cause an insignificant change in the detector characteristics.

(2) The rf power levels are adjusted for levels of approximately $10 \mathrm{dBm}$ at the reference port and $0 \mathrm{dBm}$ at the test port of the mixer. 
(3) Approximately $40 \mathrm{~dB}$ is set in the precision IF attenuator. The system is adjusted for an out-of-phase quadrature condition.

(4) The modulation frequency and power level are measured by the automatic baseband spectrum analyzer. The total carrier-power reference level is measured power, plus the carrier-sideband modulation ratio, plus the IF attenuator setting.

(5) The AM modulation is removed. the IF attenuator set to $0 \mathrm{~dB}$, and the system re-checked to verify the out-of-phase quadrature (maximum dc output from the mixer IF port). Noise $\left(V_{n}\right)$ is measured at the selected Fourier frequencies. A direct calculation of $m(f)$ in $\mathrm{dBc} / \mathrm{Hz}$ is

$$
\begin{aligned}
m(f)= & {[\text { (modulation power }(\mathrm{dBm})+\text { carrier-sideband ratio }(\mathrm{dB})} \\
& +\mathrm{IF} \text { attenuation }(\mathrm{dB})-\text { noise power }(\mathrm{dBm})+2.5 \mathrm{~dB} \\
& -10 \log (\mathrm{BW})] .
\end{aligned}
$$

Figure 27 illustrates the measurements of $A M$ and phase noise of two GUNN oscillators that were offset in frequency by $1 \mathrm{GHz}$. The measurements were performed using the coaxial delay-line system.

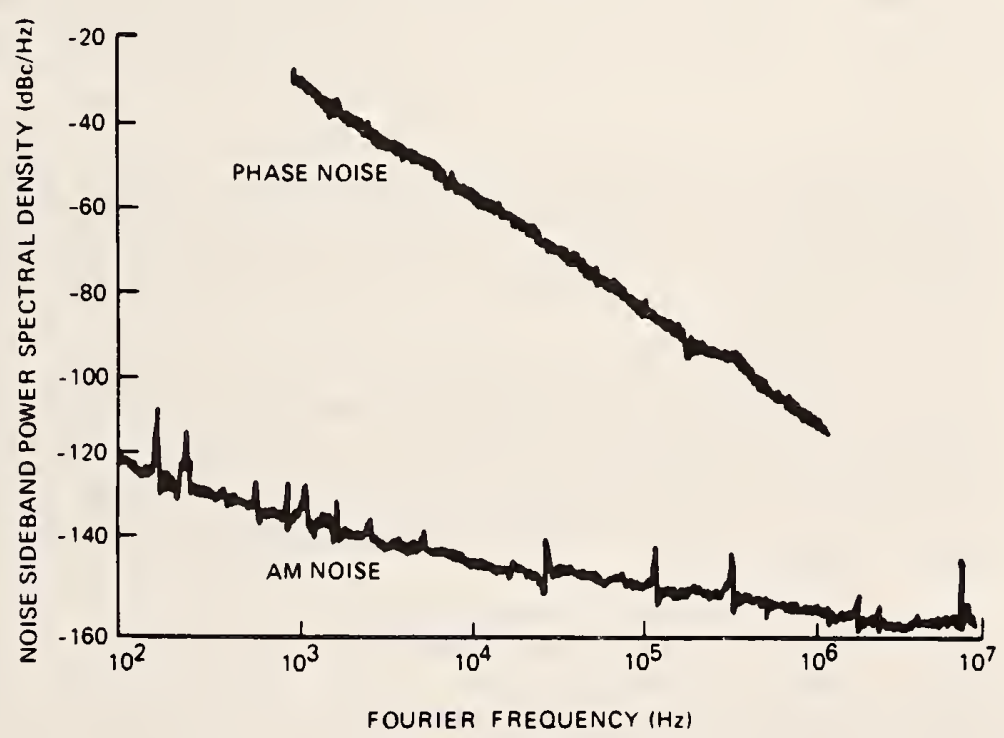

FiG. 27 Phase noise and AM noise of 40- and 4I-GHz Gunn oscillators. (From Seal and Lance. 1981.) 


\section{Conclusion}

The fundamentals and techniques for measurement of phase noise have been set forth for two basic systems. The two-oscillator technique provides the capability for measuring high-performance $\mathrm{cw}$ sources. The system sensitivity is superior to the single-oscillator technique for measuring phase noise very close to the carrier.

High-stability sources such as those used in frequency standards applications can be measured without using phase-locked loops. However, most microwave sources exhibit frequency instability that requires phase-locked loops to maintain the necessary quadrature conditions. The characteristics of the phase-locked loops must be evaluated to obtain the source phase noise characteristics. Also, in principle, one must have three sources at the same frequency to characterize a given source. If three sources are not available, one must assume that either one source is superior in performance or that they have equal phase noise contributions.

The single-oscillator technique employing the delay line as an FM discriminator has adequate sensitivity for measuring most microwave sources. The economic advantages of using this system include the fact that only one source is required, phase-locked loops are not required, system configuration is relatively inexpensive, and the system is inherently insensitive to oscillator frequency drift.

The single-oscillator technique using the delay-line discriminator can be adapted to measure the phase noise of pulsed sources. Pulsed sources have been measured at $94 \mathrm{GHz}$ by F. Labaar at TRW, Redondo Beach, California.

\section{ACKNOWLEDGMENTS}

Our intial preparations for developing a phase noise measurement capability were the result of discussions with Dr. Jorg Raue of TRW. Our first phase noise development effort was assisting in the evaluation of phase noise measurement systems designed and developed by Bill Hook of TRW (Hook. 1973). The efforts of Don Leavitt of TRW were vital in initiating the measurement program.

We are very grateful to Dr. Donald Halford of the National Bureau of Standards in Boulder. Colorado, for his interest, consultations, and valuable suggestions during the development of the phase noise measurement systems at TRW.

We appreciate the measurement cross-checks performed by C. Reynolds. J. Oliverio, and H. Cole of the Hewlett-Packard Company, Dr. J. Robert Ashley of the University of Colorado, Colorado Springs, and G. Rast of the U.S. Army Missile Command, Redstone Arsenal, Huntsville, Alabama.

\section{REFERENCES}

Allen, D. W. (1966). Proc. IEEE S4, 221-230.

Ashley, J. R., Searles, C. B., and Palka, F. M. (1968). IEEE Trans. Microwave Theory Tech. MTT-16(9), 753-760. 
Ashley. J. R.. Barley, T. A., and Rast, G. J. (1977). IEEE Trans. Microwave Theory Tech. MTT-25(4), 294-318.

Baghdady, E. J., Lincoln, R. N., and Nelin, B. D. (1965). Proc. IEEE 53, 704-722.

Barnes. J. A. (1969). "Tables of Bias Functions, $B_{1}$ and $B_{2}$, for Variances Based on Finite Samples of Processes with Power Law Spectral Densities," National Bureau of Standards Technical Note 375.

Barnes, J. A., Chie, A. R., and Cutler, L. S. (1970). "Characterization of Frequency Stability," National Bureau of Standards Technical Note 394; also published in IEEE Trans. Instrum. Meas. IM-20(2), 105-120 (1971).

Brandenberger, H., Hadorn, F., Halford, D., and Shoaf, J. H. (1971). Proc. 25th Ann. Symp. Freq. Control, Fort Monmouth, New Jersey, pp. 226-230.

Culter, L. S.. and Searle, C. L. (1966). Proc. IEEE 54, 136-154.

Fisher. M. C. (1978). "Frequency Domain Measurement Systems," Paper presented at the 10th Annual Precise Time and Time Interval Applications and Planning Meeting, Goddard Space Flight Center, Greenbelt, Maryland.

Halford, D. (1968). Proc. IEEE 56(2), 251-258.

Halford, D. (1975). "The Delay Line Discriminator," National Bureau of Standards Technical Note 10, pp. 19-38.

Halford, D., Wainwright, A. E., and Barnes, J. A. (1968). Proc. 22nd Ann. Symp. Freq. Control, Fort Monmouth, New Jersey, 340-341.

Halford. D., Shoaf, J. H., and Risley, A. S. (1973). Proc. 27 th Ann. Symp. Freq. Control, Cherry Hill. New Jersey, pp. $421-430$.

Hook. W. R. (1973). "Phase Noise Measurement Techniques for Sources Having Extremely Low Phase Noise." TRW Defense and Space Systems Group Internal Document, Redondo Beach, California.

Hewlett-Packard Company (1965). "Frequency and Time Standards," Application Note 52. Hewlett-Packard Co., Palo Alto, California.

Hewlet1-Packard Company (1970). "Computing Counter Applications Library," Application Notes 7, 22. 27, and 29. Hewlett-Packard Co.. Palo Alto, California.

Hewlett-Packard Company (1976). "Understanding and Measuring Phase Noise in the Frequency Domain." Application Note 207. Hewlett-Packard Co., Loveland. Colorado.

Labaar. F. (1982). "New Discriminator Boosts Phase Noise Testing." Microwaves 21(3), 65-69.

Lance, A. L. (1964). "Introduction to Microwave Theory and Measurements." McGraw-Hill, New York.

Lance, A. L., Seal, W. D., Mendoza, F. G., and Hudson, N. W. (1977a). Microwate J. 20(6). 87-103.

Lance, A. L., Seal. W. D., Mendoza, F. G., and Hudson, N. W. (1977b). Proc. 31st Annu. Symp. Freq. Control. Atlantic City, New Jersey, pp. 463-483.

Lance. A. L.. Seal, W. D., Halford. D., Hudson, N., and Mendoza. F. (1978). "Phase Noise Measurements Using Cross-Spectrum Analysis." Paper presented at the IEEE Conference on Electromagnetic Measurements. Ottawa, Canada.

Lance. A. L., Seal, W. D., and Labaar, F. (1982). ISA Trans. 21(4). 37-44.

Meyer, D. G. (1970). IEEE Trans. IM-19(4), 215-227.

Ondria, J. (1968). IEEE Trans. Microwate Theory Tech. MTT-16(9), 767-781.

Ondria, J. G. (1980). IEEE-MTT-S Int. Microwave Symp. Dig., pp. 24-25.

Payne. J. B., III (1976). Microwate System New's 6(2), 118-128.

Scherer, D. (1979). "Design Principles and Measurement Low Phase Noise RF and Microwave Sources." Hewlett-Packard Co.. Palo Alto. Californa.

Seal. W. D., and Lance, A. L. (1981). Microware System New's 11(7), 54-61.

Shoaf. J. H.. Halford. D.. and Risley. A. S. (1973). “Frequency Stability Specifications and Measurement." National Bureau of Standards Technical Note 632.

* See Appendix Note \# 33 
Tykulsky, A. (1966). Proc. IEEE 54(2), 306.

Van Duzer, V. (1965). Proc. IEEE-NASA Symp. Definition Meas. Short-Term Freq. Stability. NASA SP-80, 269-272.

Walls, F. L., and Stein, S. R. (1977). Proc. 31st Ann. Symp. Freq. Control, Atlantic City, New Jersey, pp. 335-343.

Walls, F. L., Stein, S. R., Gray, J. E., Glaze. D. J., and Allen, D. W. (1976). Proc. 30th Ann. Simp. Freq. Control, Atlantic City, New Jersey, p. 269. 


\section{PERFORMANCE OF AN AUTOMATED HIGH ACCURACY PHASE MEASUREMENT SYSTEM}

S. Stein, D. Glaze, J. Levine, J. Gray, D. Hilliard, D. Howe

Time and Frequency Division

National Bureau of Standards

Boulder, Colorado 80303

and

L. Erb

ERBTEC Engineering Inc.

Boulder, Colorado

\section{Summary}

A fully automated measurement system has been developed that combines many properties previously realized with separate techniques. This system is an extension of the dual mixer time difference technique, and maintains its important features: zero dead time, absolute phase difference measurement, very high precision, the ability to measure oscillators of equal frequency and the ability to make measurements at the time of the operator's choice. For one set of design parameters, the theoretical resolution is $0.2 \mathrm{ps}$, the measurement noise is 2 os rms and measurements may be made within $0.1 \mathrm{~s}$ of any selected time. The dual mixer technique has been extended by adding scalers which remove the cycle ambiguity experienced in previous realizations. In this respect, the system functions like a divider plus clock, storing the epoch of each device under test in hardware.

The automation is based on the ANSI/IEEE583 (CAMAC) interface standard. ${ }^{2}$ Each measurement channel consists of a mixer, zero-crossing detector, scaler and time interval counter. Four channels fit in a double width CAMAC module which in turn is installed in a standard CAMAC crate. Controllers are available to interface with a wide variety of computers as well as any IEEE-488 compatible device. Two systems have been in operation for several months. One operates 24 hours a day, taking data from 15 clocks for the NBS time scale, and the other is used for short duration laboratory experiments.

\section{Review of the Dual Mixer Time Difference Technique.}

It is advantageous to measure time directly rather than time fluctuations, frequency or frequency fluctuationns. These measurements constitute a hierarchy in which the subsequently listed quantities may always be calculated from the previous ones. However, the reverse is not true when there are gaps in the measurements. In the past, frequency was usually not derived from time measurements for short sample times because time interval measurements could not be performed with adequate precision. The dual mixer technique, illustrated in Figure 1 , made it possible to realize the precision of the beat frequency technique in time interval measurements.

The signals from two oscillators (clocks) are applied to two ports of a pair of double balanced mixers. Another signal synthesized from one of the oscillators is applied to the remaining two ports of the mixer pair. The input signals may be represented in the usual fashion

$$
\begin{aligned}
& v_{1}(t)=v_{10} \sin \left[2 \pi v_{10} t+\phi_{1}(t)\right], \\
& v_{2}(t)=v_{20} \sin \left[2 \pi v_{20} t+\phi_{2}(t)\right] \text { and } \\
& v_{s}(t)=v_{s 0} \cos \left[2 \pi v_{50} t+\phi_{s}(t)\right]
\end{aligned}
$$

where $v=v_{1}(1-1 / R)$ and $R$ is a constant usually Ealled the heterodyne factor. are The low passed outputs of the two mixers

$$
\begin{aligned}
& V_{B 1}=V_{B 10} \sin \left[\Phi_{1}(t)-\Phi_{s}(t)\right] \text { and } \\
& V_{B 2}=V_{B 20} \sin \left[\Phi_{2}(t)-\Phi_{s}(t)\right] \text { where } \\
& \Phi(t)=2 \pi v_{0} t+\phi(t) .
\end{aligned}
$$

The time interval counter starts at time $t_{M}$ when $V_{B}$ crosses zero in the positive direction and slops at time $t_{N}$, the time of the very next positive zero cros'sing of $\mathrm{V}_{\mathrm{B} 2}$. Thus

$$
\begin{aligned}
& \dot{\phi}_{1}\left(t_{H}\right)-\Phi_{S}\left(t_{M}\right)=2 N \pi \text { and } \\
& \Phi_{2}\left(t_{N}\right)-\Phi_{S}\left(t_{N}\right)=2 N \pi \text { where }
\end{aligned}
$$

$N$ and $M$ are integers.

Subtracting the two equations in order to compare the phases of oscillators 1 and 2 , one obtains

$$
\Phi_{2}\left(t_{N}\right)-\Phi_{1}\left(t_{M}\right)=\Phi_{S}\left(t_{N}\right)-\Phi_{S}\left(t_{M}\right)+2(N-M) \pi \text {. }
$$

The phase of an oscillator at time $t_{N}$ may be written in terms of its phase at $t_{M}$ and its 
average frequency over the intervai $t_{M}<t_{N}$

$$
\phi\left(t_{N}\right)=\phi\left(t_{M}\right)+2 \pi\left[\bar{v}\left(t_{M} ; t_{N}\right)\right]\left(t_{N}-t_{M}\right) \text { and }
$$

when we apply this equation to both $\phi_{2}$ and $\Phi_{S}$ we find

$\Phi_{2}\left(t_{M}\right)-\Phi_{1}\left(t_{M}\right)=2(N-M) \pi$

$$
-2 \pi\left[\bar{v}_{B 2}\left(t_{M} ; t_{N}\right)\right]\left(t_{N}-t_{M}\right)
$$

where $v_{B 2}=v_{2}-v_{S}$.

Since $M$ and $N$ are not measureable with the equipment in Figure 1 , the dual mixer technique has heretofore only been used to measure the phase difference between two oscillators modulo $2 \pi$. We denote the period of the time interval counter time base by $\tau$ and the number of counts recorded in a measurenfent by $P$. Then the phase difference between the two oscillators is given by

$$
\left[\Phi_{2}\left(t_{M}\right)-\Phi_{1}\left(t_{M}\right)\right] \bmod 2 \pi=-2 \pi\left[\bar{v}_{B 2}\left(t_{M} ; t_{N}\right)\right] \tau_{c}{ }^{p}
$$

Figure 2 illustrates the output of the measurement system over a period of time. If a measurement begins and ends without the time interval counter making a transition between zero and its maximum value, e.g., $t<t_{M}<t_{N}<$ $t_{b}$, then the phase difference can Be calculaled from the data. If $t<t_{M}<t_{b}<t_{N}<t_{\text {, }}$, then the data must be corrected by $2 \pi$ to cafculate the phase difference. Experience has shown that there are many measurement situations for which the number of transitions of the time interval counter which occur between $t_{M}$ and $t_{y}$ cannot be known. For this reason, a modification has been developed which removes the ambiguity by measuring $M$ and $N$.

Extended Dual Mixer Time Difference Measurement Technique

In order to configure the system to acquire complete phase information, two scalers are added to count the zero crossings of each mixer. Figure 3 is the block diagram of a two channel system. It is constructed from identical circuit modules and therefore contains an unused time interval counter. However, this design permits very straightforward, and inexpensive extension to the comparison of an arbitrarily large number of oscillators with no need for switching any signals.

The counter outputs are combined to form the phase difference between oscillators.

$$
\begin{aligned}
\Phi_{2}\left(t_{M}\right)-\Phi_{1}\left(t_{M}\right)= & 2\left(N_{0}-M_{0}\right) \pi+2(N-M) \pi \\
& -2 \pi\left[\bar{v}_{B 2}\left(t_{M} ; t_{N}\right)\right] \tau_{C} P
\end{aligned}
$$

The first term is a constant which represents the choice of the time origin and can be $\mathrm{ig}^{-}$ nored. The last two terms and their sum are plotted in Figure 4.
The average teat frequency $\vec{v}_{B 2}\left(t_{M} ; t_{M}\right)$ cannot be known exactly. However, ift may be estimated with sufficient precision from the previous pair of measurements designated ' and ". The average frequency is approximately

$\bar{v}_{B 2}\left(t_{M} ; t_{N}\right) \cong\left(N^{\prime \prime}-N^{\prime}\right) /\left[R\left(M^{\prime \prime}-M^{\prime}\right) / v_{10}+\tau_{C}\left(P^{\prime \prime}-p^{\prime}\right)\right]$

provided that it changes sufficiently slowly compared to the interval $t_{M}<t_{N}$. A typical value for this error will be given in the following section.

\section{Hardware Implementation}

All measurement channels consist of a mixer, zero-crossing detector, scaler and time interval counter. Four such circuits can be built in a double width CAMAC module. The system is easily expanded to compare many oscillators and a complete system for making phase comparisons among four clocks is shown in Figure 5. We have chosen parameters which are reasonable for comparing state-of-the-art atomic standards. Thus, the synthesizer is offsegt $10 \mathrm{~Hz}$ below oscillator \# $I$ and $R=5 \times 10^{\circ}$. The outputs from both mixers are approximately $10 \mathrm{~Hz}$. The noise bandwidth is $100 \mathrm{~Hz}$. The time interval counter is twice the frequency of oscillator \#1 or approximately $10 \mathrm{MHz}$. The quantization error is $1 / 2 R=10^{-6}$ cycle or $0.2 \mathrm{ps}$ which is a factor of ten smaller than the measurement noise. As stated earlier, an error will result from frequency changes which violate the constancy assumption used to estimate $v$. A change in $v_{2}$ by $10^{-10}$ during the intervaj between two measurements will result in a time deviation error of 10ps. Thus, one must make more closely spaced measurements for oscillators which have large dynamic frequency changes than for more stable devices. Two other sources of inaccuracy are the sensitivities to the amplitude and phase of the common oscillator. Figure 6 shows the measured value of $x=\phi / 2 \pi v$ as a function of the amplitude of the input signal and the phase of the synthesizer.

The new measurement system has many desirable features and properties:

(1) It has very high resolution, limited by the internal counters to 0.2 ps and by noise to approximately 2 ps.

(2) It has much lower noise than divider based measurement systems. However compromises made to achieve low cost, low power, small size and automatic operation degrade the performance compared to state-of-the-art systems for comparing 2 oscillators.

(3) The operation is fully automatic.

(4) NBS has developed a detailed operzating manual for the equipment and software. 
(5) All oscillators in the range of $5 \mathrm{MHz} \pm 5$ $\mathrm{Hz}$ may be compared. Other carrier frequencies such as $1 \mathrm{MHz}, 5.115 \mathrm{MHz}, 10 \mathrm{MHz}$ and $10.23 \mathrm{MHz}$ are also usable. However, different carrier frequencies may not be mixed on the same system. The system has been successfully tested with an oscillator of $f$ set $4.6 \mathrm{~Hz}$ from nominal $5 \mathrm{MHz}$. Measurements were made at intervals of 2 hours between which the system had to accumulate approximately $2 \times 10^{6} \pi$. The system has also begn tested with an oscillator of fset $4 \times 10^{-5}$, and no errors were detected during a period of 40 days.

(E) All sampling times in the range of 1 second to 16 days with a resolution of 0.1 second are possible. Measurements may be made on command or in a preprogrammed sequence.

(7) Measurements are synchronized precisely, i.e. at the picosecond level, with the reference clock. They may therefore be synchronized with important user system events, such as the switching times of a FSK or PSK system.

(8) All oscillators are compared synchronously and all measurements are performed within a maximum interval of 0.1 second. As a result, the phase of any oscillator needs to be interpolated to the chosen measurement time for an interval of 0.1 second maximum. This capability, which is not present in either single heterodyne measurement systems or switched measurement systems eliminates a source of "measurement" error which is generally much larger than the noise induced errors. For example, interpolation of the phase of a high performance Cs clock $\left(\sigma_{\sim} \sim 10^{-11} / \tau^{\frac{1}{2}}\right)$ over a period of 3 hours would produce approximately 1.5 ns phase uncertainty. To maintain 4 ps accuracy requires measurements simultaneous to $0.1 s$.

(9) There are no phase errors due to the switching of $r f$ signals since there is no switching anywhere in the analog measurement system.

(10) No appreciable phase errors are introduced when it is necessary to change the reference clock since, as shown in Figure 6 , the peak error due to changes in synthesizer phase is 20 ps.

(11) The measurement system is capable of measuring its own phase noise when the same signal is applied to two input ports. Figure 7 shows the phase deviations between two such channels over a period of 75,000 seconds and Figure 8 is the corresponding Allan variance plot. Figure 9 shows the phase deviations between 2 input channels over a period of 40 days.

(12) Since the IEEE-583 (CAMAC) interface standard has been followed for all the custom hardware, the system may be easily interfaced to almost any instrument controller. NBS has aiready tested the system using a large minicomputer, a small minicomputer and a desk top calculator. Interfaces between IEEE-583 and IEEE-488 controllers are available and have been used successfully.

(13) The system is capable of camparing a very large number of oscillators at a reasonable cost per device.

There are also disadvantages to this measurement system. The most important are:

(1) The complexity of the hardware is greater than for some systems. It is possible that this will reduce reliability.

(2) A high level of redundancy is difficult to achieve. The system design stresses size, power, convenience and cost, resulting in an increase in the number of possible single point failure mechanisms compared to some other techniques. For example, a CAMAC power supply failure will result in a loss of data for all devices being measured.

(3) A substantial committment is required in both specialized hardware and sof tware.

(4) If an oscillator under test experiences a phase jump which exceeds 1 cycle, the measurement system records a jump with incorrect absolute magnitude. As a result, it may not be applicable to signals which are frequency modulated with discontinuous phase steps larger than $2 \pi$.

\section{Conclusions}

We have demonstrated a new phase measurement system with very desirable properties: All oscillators in the range of $5 \mathrm{MHz} \pm 5 \mathrm{~Hz}$ may be measured directly. The sampling times are only restricted by the requirement that they exceed one second. The noise floor is $\sigma_{y}(2, \tau)=3 x$ $10^{-12} / \tau$ in short term and the tine deviations are less than 100 ps. All circuitry is designed as modules which allows expansion at modest cost. Compatibility with a variety of computers is insured through the use of the IEEE-583 interface and adapters are available to permit use with an IEEE-488 controller. The system makes it feasible to make completely automated phase measurements at predetermined times on large numbers of atomic clocks. It's own noise is one-hundred times less than the state-of-theart in clock performance. It will be used in the near future to make all measurement needed to compute NBS atomic time, but it will also be very valuable for any laboratory which uses three or more atomic clocks.

\section{References}

1. D. W. Allan, "The measurement of frequency 
and frequency stablitity of precision oscillators," NBS Tech. Note 669

(1975).

2. "CAMAC instrumentation and interface standards," Institute of Electrical and Electronic Engineers, Inc., 345 E. 47 th St. New York, NY 10017.

3. D. J. Giaze and S. R. Stein, "Picosecond time difference measurements utilizing $C A-$ MAC based ANSI/IEEE -488 data acquisition hardware", NBS Tech Note 1056 (in preparation).

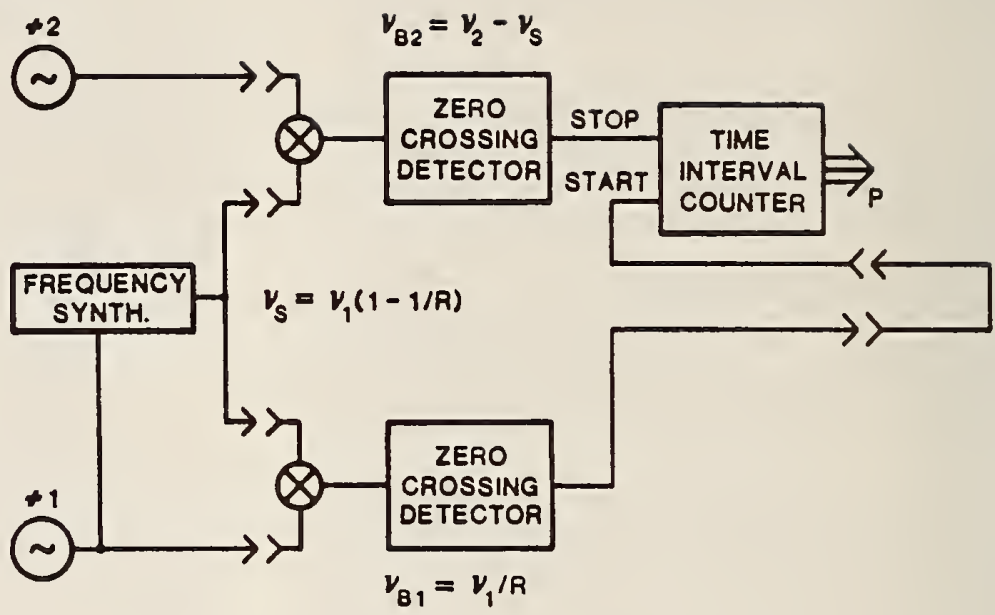

Figure 1. Dual Mixer Time Difference Measurement System

$\left[\Phi_{2}\left(t_{H}\right)-\Phi_{1}\left(t_{H}\right)\right] \bmod 2 \pi=-2 \pi\left[\bar{v}_{B 2}\left(t_{M} ; t_{N}\right)\right] \tau_{C} P$

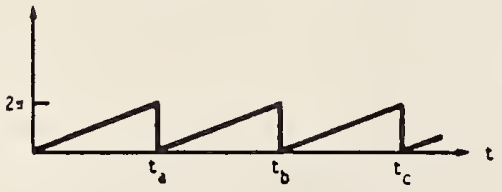

Figure 2. Dual Mixer Data

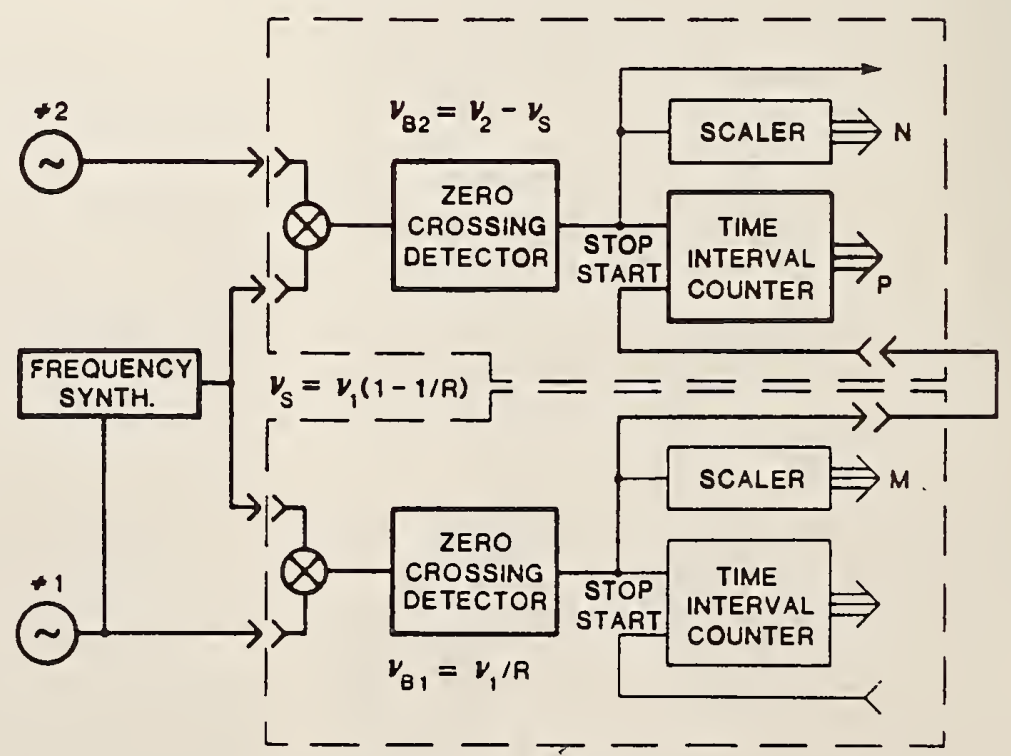

Figure 3. Extended Dual Mixer Time Difference Measurement System 


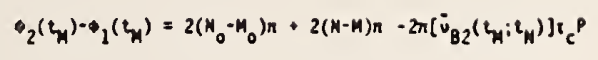

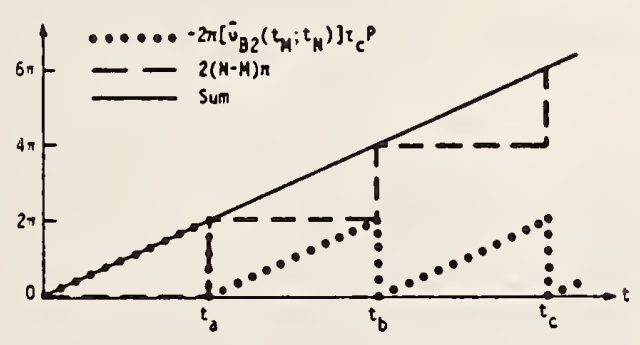

Figure 4. Extended Dual Mixer Data

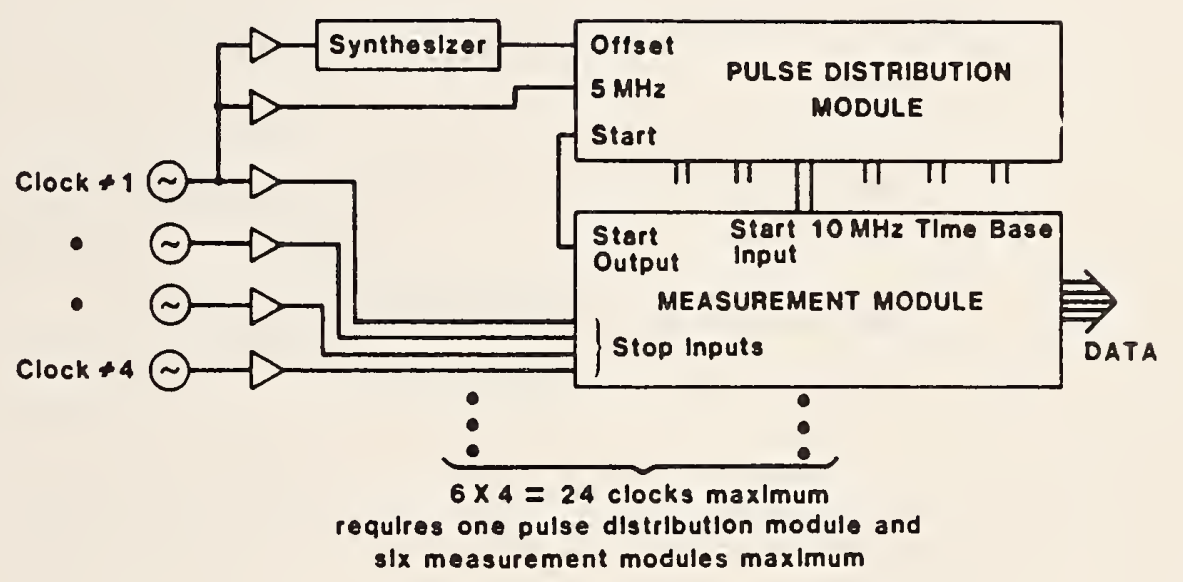

Figure 5. System Block Diagram
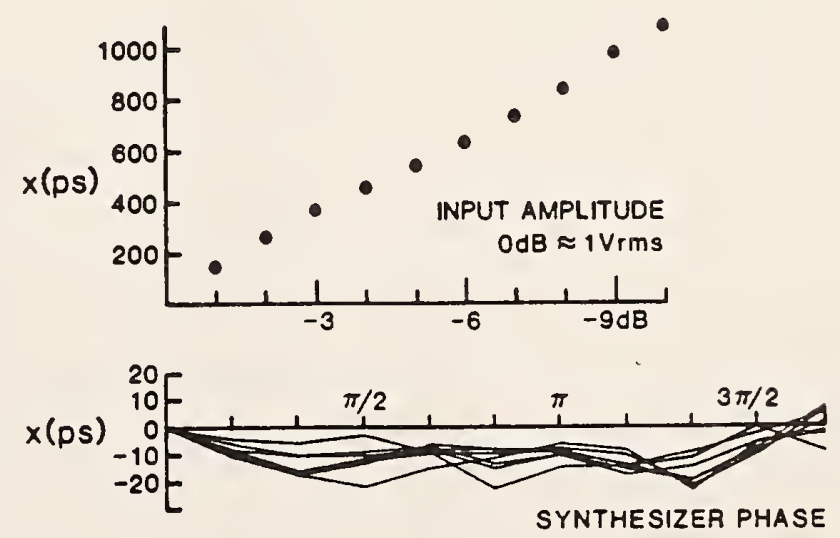

Figure 6. Measured Time Difference vs. Input Amplitude and Synthesizer Phase 


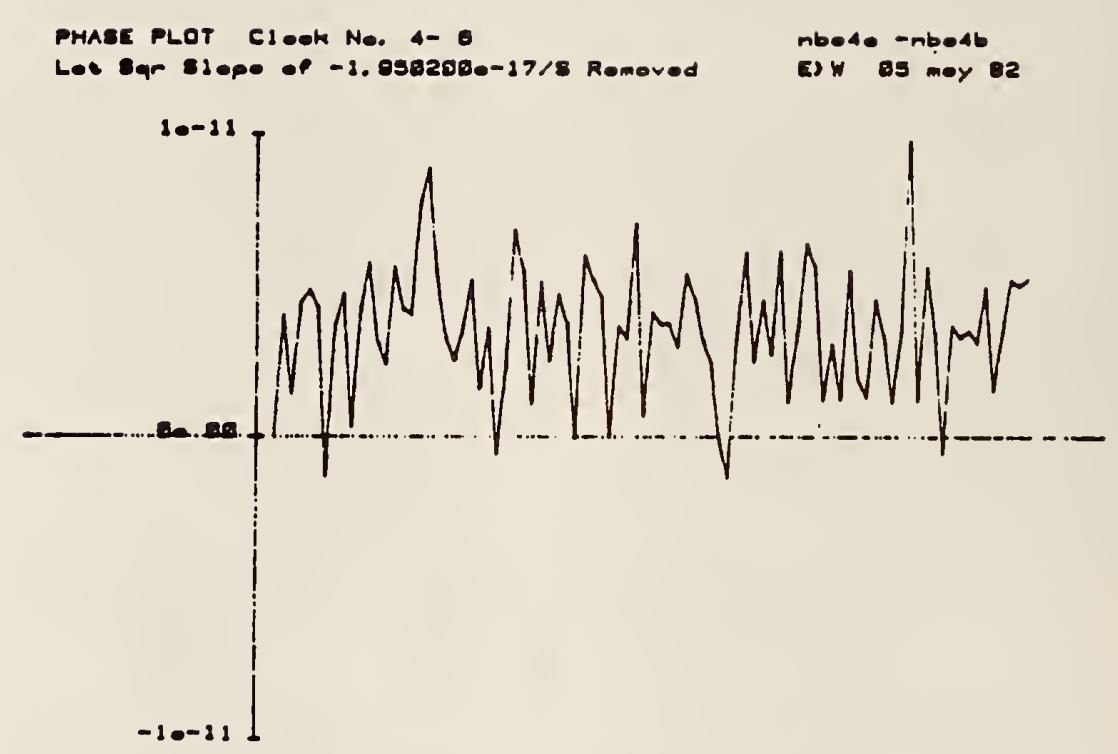

$T=74790$ SECONDS

Figure 7. Raw Phase Data for Two Channels Driven from the Same Source

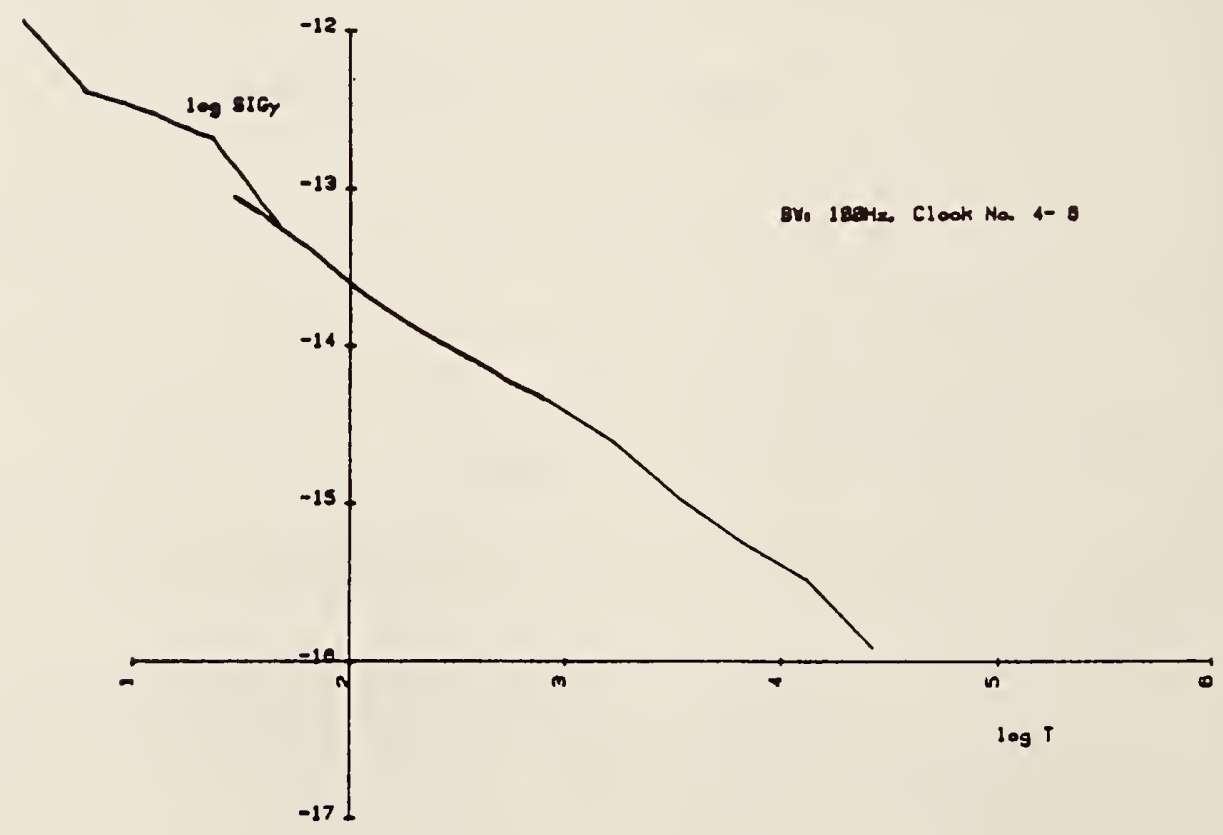

Figure 8. Noise Floor of Measurement System 


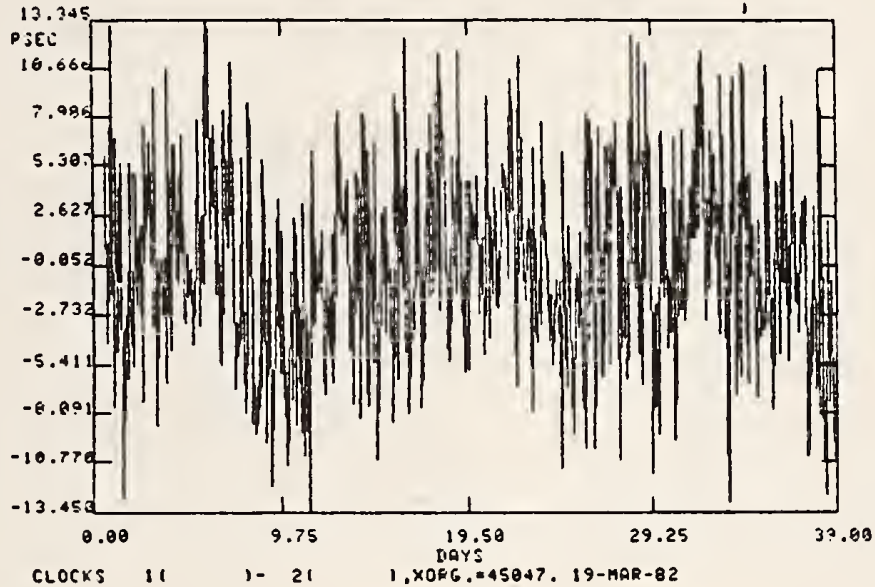

Figure 9. Raw Phase Data for Two Channels Driven from the Same Source 


\title{
BIASES AND VARIANCES OF SEVERAL FFT SPECTRAL ESTIYIATORS AS A FL:YCIION OF NOISE TYPE A.ND NLMBER OF S.A.MPLES
}

\author{
F. L. Walls, Time and Frequency Division. \\ National Institute of Standards and Technology, Boulder, CO 80303 \\ D. B. Percival, Applied Physics Laboratory, \\ University of Washington, Seattle, WA 98106 \\ From: Proceedings of the \\ W. R. Irelan, Irelan Electronics, \\ 412 Janet, Tahlequah, OK 74464 \\ 43rd Annual Symposium on \\ Frequency Control, 1989.
}

\begin{abstract}
We theoretically and experimentally investigate the biases and the variances of Fast Fourier transform (FFT) spectral estimates with different windows (data tapers) when used to analyze power-law noise types $f^{0}, f^{-2}, f^{-3}$ and $f^{-4}$. There is a wide body of literature for white noise but virtually no investigation of biases and variances of spectral estimates for power-law noise spectra commonly seen in oscillators. amplifers, mixers, etc. Biases (errors) in some cases exceed $30 \mathrm{~dB}$. The experimental techniques introduced here permit one to analyze the performance of virtually any window for any power-law noise. This makes it possible to determine the level of a particular noise type to a specified statistical accuracy for a particular window.
\end{abstract}

\section{Introduction}

Fast Fourier transform (FFT) spectrum analyzers are very commonly used to estimate the spectral density of noise. These instruments often have several different windows (data tapers) available for analyzing different types of spectra. For example, in some applications spectral resolution is important; in others, the precise amplitude of a widely resolved line is important; and in still other applications, noise analysis is important. These diverse applications require different types of windows.

We theoretically and experimentally investigate the biases and variances of FFT spectral estimates with different windows when used to analyze a number of common power-law noise types. There is a wide body of literature for white noise but virtually no investigation of these effects for the types of power-law noise spectra commonly seen in oscillators, amplifiers, mixers, etc. Specifically, we present theoretical results for the biases associated with two common windows - the uniform and Hanning windows - when applied to power-law spectra varying as $f^{0}, f^{-2}$ and $f^{-4}$. We then introduce experimeatal tech. niques for accurately determining the biases of any window and use them to evaluate the biases of three different windows for power-law spectra varying as $f^{0}, f^{-2}, f^{-3}$ and $f^{-4}$. As an example we find with $f^{-4}$ noise that the uniform window can have errors ranging from a few $\mathrm{dB}$ to over $30 \mathrm{~dB}$, depending on the length of span of the $f^{-1}$ noise.

We have also theoretically investigated the variances of FFT spectral estimates with the uniform and Hanning windows (confidence of the estimates) as a function of the power-law noise type and as a function of the amount $\alpha$ data. We introduce experimental techniques that make it relatively easy to independently determine the variance of the spectral estimate for virtually any window on any FFT spectrum anajyzer. The variance that is realized on a particular instrument depends not only on the window but on the specific implementation in both hardware and software. We find that the variance of the spectral density estimates for white noise, $f^{0}$, is very similar for three specific windows available on one instrument and almost identical to that obtained by standard statistical analysis. The variances for spectral density estimates of $f^{-4}$ noise are only $4 \%$ bigher than that of $f^{0}$ noise for two of the windows studied. The third window - the uniform window - does not yield usable results for either $f^{-3}$ or $f^{-4}$ noise.

Based on this work it is now possible to determine the minimum number of samples necessary to determine the level of a particular noise type to a specified statistical accuracy as a function of the window. To our knowledge this was previously possible only for white noise - although the traditional results are generally valid for noise that varied as $f^{-\beta}$, where $\beta$ was equal to or less than 4.

\section{Spectrum Analyzer Basics}

The spectrum andyzer which was used in the experimental work reported here is fairly typical of a number $\alpha$ such instruments currently available from various manufacturers. The basic measurement process generally consists of taking a string of $N_{1}=1024$ digital samples of the input wave form, which we represent here by $X_{1}, X_{2}, \ldots, X_{N_{1}}$. The basic measurement period was $4 \mathrm{~ms}$. This yields a sampling time $\Delta t=3.90625 \mu \mathrm{s}$. Associated with the FFT of a time series with.$\dot{V}$, data points. there are usually $(N, / 2)+1=513$ frequencies

$$
f_{,}=\frac{j}{N_{\Delta} \Delta t}, \quad j=0,1, \ldots, . v_{\bullet} / 2 \text {. }
$$

The fundamental frequency $f_{1}$ is $250 \mathrm{~Hz}$, and the Nyquist frequency $f_{N, / 2}$ is $128 \mathrm{kHz}$. Since the spectrum analyzer uses an anti-aliasing filter which sigaificantly distorts the high frequency portion of the spectrum, the instrument only displays the measured spectrum for the lowest 400 nonzero frequencies, namely, $f_{1}=250 \mathrm{~Hz}, f_{2}=500 \mathrm{~Hz}, \ldots, f_{100}=100 \mathrm{kHz}$.

The exact details of how the spectrum analyzer estimates the spectrum for $X_{1}, \ldots, X_{N}$ are unfortunately not provided in the documentation supplied by the manufacturer, so the following must be regarded only as a reasonable guess on our part a to its operation (see [1] for a good discussion on the basic ideas behind a spectrum analyzer; two good general references for spectral analysis are [2] and [4]). The sample mean.

$$
\bar{X} \equiv \frac{1}{N_{t}} \sum_{t=1}^{N_{t}} X_{t},
$$

is subtracted from each of the samples, and each of these "demeaned" samples is multipled by a window $h_{t}$ (sometimes called a data taper) to produce

$$
X_{t}^{(h)}=h_{t}\left(X_{t}-\bar{X}\right) .
$$

The spectral estimate,

$$
\dot{S}_{1}\left(f_{j}\right)=\Delta t\left|\sum_{t=1}^{N_{1}} X_{t}^{(h)} e^{-t 2 \times f_{j} t \Delta t}\right|^{2}, \quad j=0.1 \ldots . . v_{t} / 2 .
$$


is then computed using an FFT aigorithm.

The subscript " 1 " on $S_{1}(f$,$) indicates that this is the spec-$ tral estimate formed from the first block of $V$. samples. A similar spectral estimate $\dot{S}_{2}(f$,$) is then formed from the second$ block of contiguous data $X_{N_{0}+1}, X_{N_{0}+2}, \ldots, X_{2 N_{0}}$. In all, there are $N_{b}$ different spectral estimates from $N_{b}$ contiguous blocks, and the spectrum analyzer averages these together to form

$$
\hat{S}(f,) \equiv \frac{1}{N_{b}} \sum_{t=1}^{N_{b}} \hat{S}_{k}(f,) .
$$

It is the statistical properties of $\hat{S}(f)$ witb which we are concerned in this paper.

Unfortunately some important aspects of the windows are not provided in the documentation for the instrument. One im. portant detail is the manner in which the window is normalized. There are two common normalizations:

$$
\sum_{t=1}^{N_{t}}\left[h_{t}\left(X_{t}-\bar{X}\right)\right]^{2}=\frac{1}{N_{t}} \sum_{t=1}^{N_{t}}\left(X_{t}-\bar{X}\right)^{2}
$$

and

$$
\sum_{t=1}^{N} h_{t}^{2}=1
$$

The first of these is common in engineering applications because it ensures that the power in the windowed samples $X_{t}^{(h)}$ is the same as in the original demeaned samples; the second is equivalent to the first in expectation and is computationaly more convenient, but it can result in small discrepancies in power levels. Either normalization affects only the level of the spectral estimate and not its shape.

There are three windows built into the spectrum analyzer used bere. The first is the uniform (rectangular, default) window $h_{t}^{(U)}=1 / \sqrt{N}$. The second is the Hanning data window, for which there are several slightly different definitions in the literature. In lieu of specific details, we assume the following symmetric definition:

$$
\begin{aligned}
h_{t}^{(H)} & =C^{(H)}\left(1-\cos \frac{2 \pi(t-0.5)}{N_{0}}\right), \quad 1 \leq t \leq \frac{N_{0}}{2}, \\
& =h_{N_{0}-t+1}^{(H)}, \quad \frac{N_{0}}{2}+1 \leq t \leq N_{0} ;
\end{aligned}
$$

here $C^{(H)}$ is a constant which forces the normalization in Equa. tion (2). The third window is a proprietary "liattened peak" window, about which little specific information is avalable (it is evidently designed to accurately measure the beights of peaks in a spectrum).

\section{Expected Value and Bias of Spectral Estimates}

\section{III.A. Theoretical Analysio}

We need to assume a noise model for the $X_{i}$ 's in order to determine the statistical properties of $\bar{S}\left(f_{j}\right)$ in Equation (1). We consider three different models, each of which is represented in terms of a Gaussian white noise process $\epsilon_{t}$ with mean zero and variance $\sigma_{e}^{2}$. The second-order properties of each model are given by a spectral density function $S(\cdot)$ defined over the interval $[-1 /(2 \Delta t), 1 /(2 \Delta t)]$ in cycles/ $\Delta t$. The first model is a discrete parameter, white noise process ( $f^{\circ}$ noise):

$$
X_{1}=\epsilon_{t} \text { and } S(f)=\sigma_{l}^{2} \Delta t .
$$

The second model is a discrete-parameter. ranciom-waik process (nominally $f^{-2}$ noise):

$$
X_{t}=\sum_{t=1}^{t} \epsilon_{0} \text { and } S(f)=\frac{\sigma_{t}^{2} \Delta t}{4 \sin ^{2}(\pi f \Delta t)}
$$

The third model is a discrete-parameter, random-run process (nominally $f^{-4}$ noise):

$$
X_{t}=\sum_{t=1}^{t} \sum_{0=1}^{r} \epsilon_{0} \text { and } S(f)=\frac{\sigma_{l}^{2} \Delta t}{16 \sin ^{4}(\pi f \Delta t)} .
$$

Continuous parameter versions of these three models have been used extensively in the literature as models for noise commoniy seen in oscillators.

For each of the three models we have derived expressions for $E\{\hat{S}(f)\}$, the expected value of $\hat{S}(f)$. These expressions depend on the window $h_{t}$, the number of samples.$\dot{H}$, in each block and - in the case of a random-run process - the number of blocks $N_{b}$. The details behind these calculations will be reported elsewhere [3]; bere we merely summarize our conclusions for the three models in combination with the uniform and Hanning wiodows and $N_{0}=1024$.

First, for a white noise process,

$$
E\left\{\dot{S}\left(f_{2}\right)\right\}=S\left(f_{2}\right), \quad j=1,2, \ldots, 512,
$$

when the uniform window is used. For the Hanning window, the above equality also bolds to a very good approximation for $2 \leq j \leq 511$ and to within $0.8 \mathrm{~dB}$ for $j=1$ and 512 (the latter is of no practical importance since the higbest frequency index given by the spectrum analyzer is $j=400$ ). These theoretical calculations agree with our experimeatal data except at $f_{l}$ (see Table 1).

Second, for a random-walk process,

$$
E\{\hat{S}(f,)\}=2 S(f,), \quad j=1,2, \ldots, 512,
$$

when the uniform window is used, i.e., the expected value is twice what it should be at all frequencies. This theoretical result bas been verified by Monte Carlo simulations, but it does not agree with our experimental data, which shows no signifcant level shift in the estimated spectrum. The source of this discrepancy is currently under investigation, but it may be due to either (a) factors in the experimental data which effectively make it band-limited, random-walk noise, i.e., its spectral shape is markedly different from $f^{-2}$ for, say, $0<f<f_{1}$ or (b) an incorrect guess on our part as to how the spectral estimate is normalized by the spectrum analyzer. For the Hanaing window, we found that

$$
E\{\hat{S}(f,)\}= \begin{cases}1.08 S\left(f_{1}\right) & j=1 \\ 1.48 S(f,) & j=2 \\ 1.15 S\left(f_{1}\right) & j=3 \\ 1.07 S\left(f_{1}\right) & j=4 ; \\ 1.04 S\left(f_{1}\right) & j=5 \\ S\left(f_{3}\right) & 6 \leq j \leq 511 \text { to within } 3 \% .\end{cases}
$$

i.e., $\hat{S}\left(f_{j}\right)$ is essentially an unbiased spectral estimate except for the lowest few frequencies. This theoretical result has been verified by Monte Carlo simulations and also agrees in general with our experimeatal data.

Third, for a random-run process,

$$
E\{\hat{S}(f,)\}=C_{N_{0}} f^{-2}, \quad 1 \leq j \leq 400 .
$$


to a good appoximation when the uniform wrodow is used. where $C_{N_{0}}$ is a constant which depends on the number of blocks $\dot{V}_{6}$ and increases as $V_{b}$ increases. Thus the shape of $E\left\{\dot{S}\left(f_{2}\right)\right\}$ follows that of a random-walk process $\left(f^{-2}\right)$ ratber than that of a random-run process $\left(f^{-4}\right)$. This shape has been verified experimentally (see the next subsection), but the dependence of the level on $V_{b}$ has not. The increase in level of $E\{\hat{S}(f)$,$\} as$ $\therefore$ increases is due to the fact that the expected value of the sample variance of a block of $N$, samples increases with time - by contrast. it is constant with time for the white noise and random-walk cases. For the Hanning window, we found that

$$
E\{\dot{S}(f,)\}=C_{N_{0}}^{\prime} S\left(f_{j}\right), \quad 4 \leq j \leq 400,
$$

to a good approximation, where again $C_{N}^{\prime}$ is a constant different from $C_{N_{b}}$ - which depends on the number of blocks $i_{b}$ and increases as $N_{b}$ increases. For frequencies less than $f_{4}$ the theoretical results indicate significant (greater than $4 \%$ ) distortion in the shape, but these do not agree in detail with the experimental values reported in Table 1 . For $f_{3} \geq f_{4}$ the shape has been verified experimentally, but the dependence of the level on $V_{b}$ has not. The discrepancy in level between the theoretical and experimental results is yet to be resolved, but it is probably due to a mismatch between the assumed random-run model and the true spectrum for the data (possibly band-limited random. run).

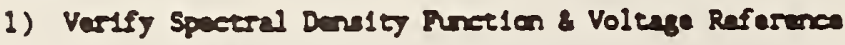

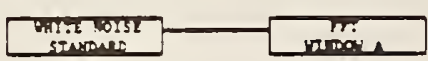

2) Hearto Spectre Ralativ to No1es Source Requires Mulple Scone

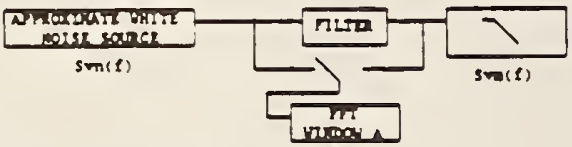

\section{3) Mearo Fller Trenser Rnction $h(\xi)$}

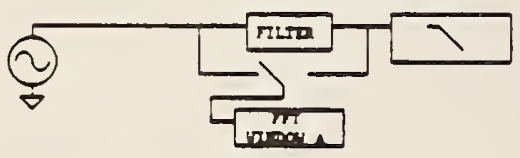

4) Celavire Bless

$$
B(\xi)=\sin (\xi) \cdot h^{2}(\xi) \sin (\xi)
$$

Figure 1. Outline of measurement procedure for determining the biases in spectral eatimators.

\section{III.B. Experimental Determination}

The following procedure can be used to experimentally determine the bias in the spectral estimate of any noise spectrum using any window in a particular instrument. The basic concept is to implement a flter that, when applied to white noise, mimics the approximate noise spectra of interest and then measures the level of the white noise and the filter transfer function in a way which has bigh precision and accuracy as illustrated in Figure 1. First, the level of a known white noise is messured over a convenient range. The higher the frequency span the faster that this is accomplished. Obviously, the chosen range must be one over which the noise source is accurate. To obtain a precision of order $0.2 \mathrm{~dB}$ generally requres 1000 samples Th:s measurement verifies that the spectral density function and the internal reference voltage of the FFT are accurately calibrated and working properly. Virtually all of the windows accurately determine the value of white noise if the first few channels are ignored as explained above. Figure 2 shows the measurement of a noise source, which has been independently determined to have a noise spectral density of $99.8 \mathrm{dBV} / \mathrm{Hz}$ by the three windows. (Appendix A shows the circuit diagram for this noise source which has an accuracy of better than $0.2 \mathrm{~dB}$ for frequencies from 20 to $20 \mathrm{kHz}$.)

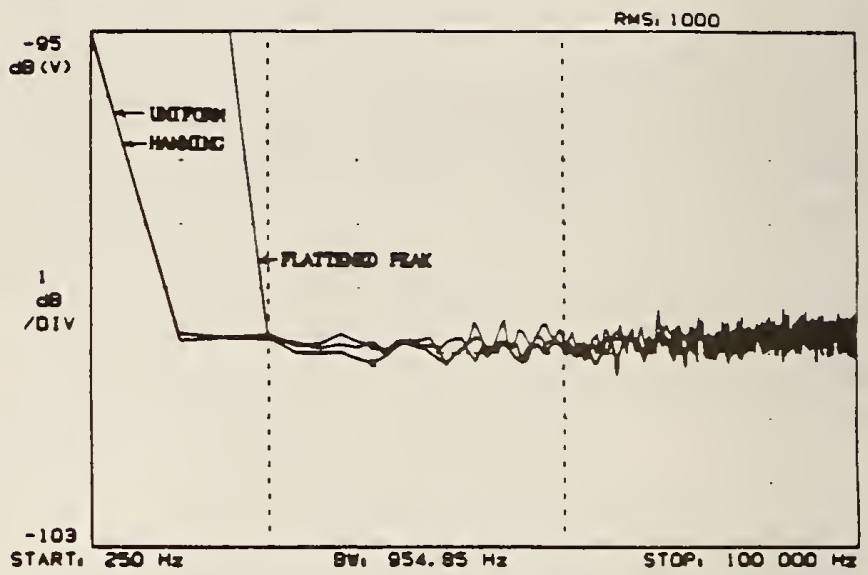

Figure 2. Spectral estimation of a white noise standard us ing the uniform, Hanning and the proprietary "flattened peak" windows.

Second, an approximately flat spectrum is measured over the frequency range of interest. It is not important if there are small variations in the level that change slowly over the frequency span. Third, the transfer function of the filter is determined for the frequencies of interest using a very narow spectral source (typically an audio oscillator is sufficient). The very narrow source is accurately measured by the window since there is no problem with either high frequency or low frequency noise biasing the estimate. The use of a window with a lattened peak response is helpful but not necessary if the frequency source is sufficiently stable. This transfer function is then applied to the measured white noise spectrum in step two above. This yields a very accurate value for the "true" spectral density of the white noise source as measured through the filter. This "true value" is then compared to that obtained by the FFT analyzer. The difference between that mearured in steps two and three and that mearured directly with the FFT is the bias in the spectral estimate for that particular window and noise type. The accuracy of this approsch comes from the fact that the calibration bas been broken up into steps that can individually be determined with high precision and very small bias. The primary assumption is that the FFT analyzer is linear. Even this assumption can be checked by using precision attenuators. If the known white noise in step one does not extend to the frequencies of interest, then there is an additional assumption that the FFT is flat with frequency. This assumption is nearly always good except perhaps near the last few channels where the effect of the antialiasing filter might cause small inaccuracies.

Figures $3 a$ and $3 b$ show the "true" spectral estimate and the estimates as measured on a particular instrument using the uniform, Hanoing, and the instrument's proprietary 'flattened peak" windows for noise that varies as $f^{-1}$ over much of the 

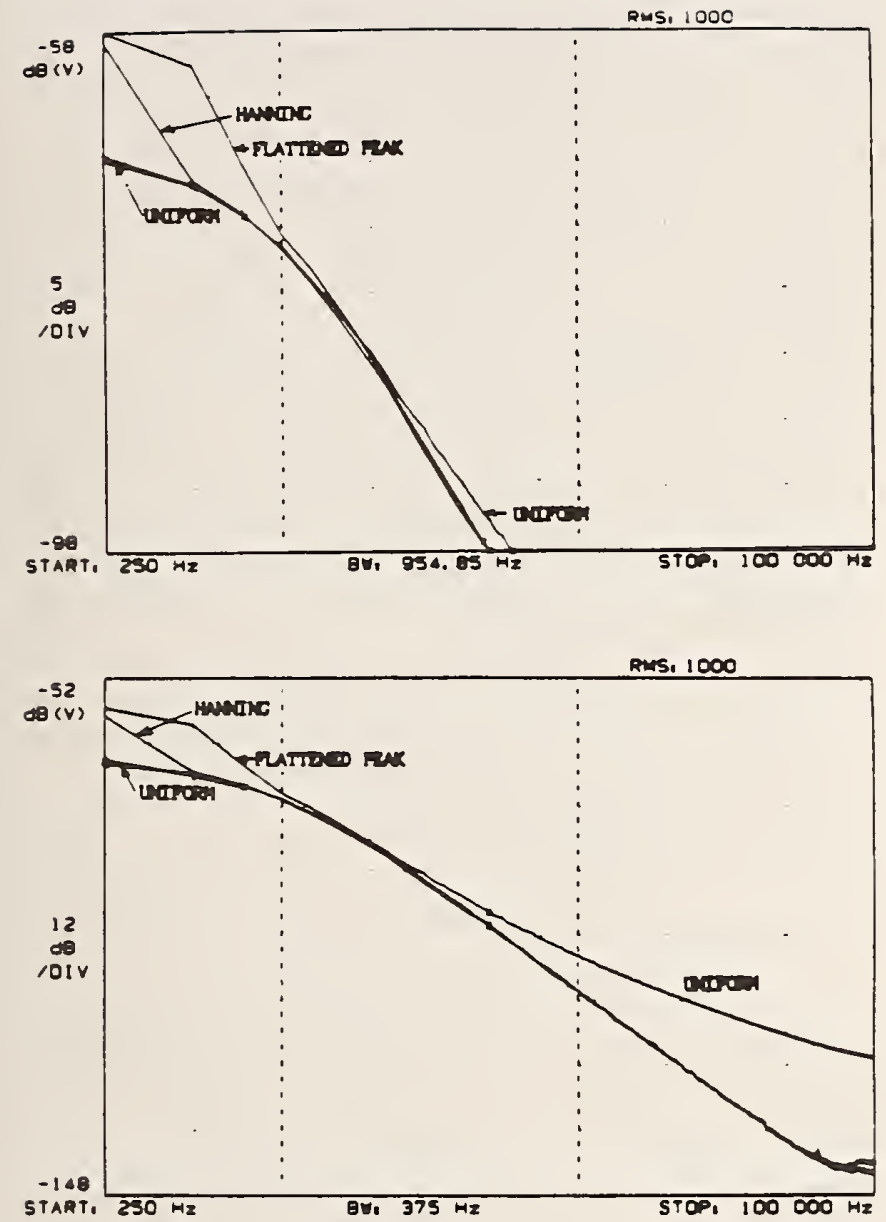

Figures 3a (top) and $3 b$ (bottom). Difference between the true spectrum (top) which varies approxirately as $f^{-4}$ and that estimated by the uniform, Hanning and proprietary tattened peak" windows (bot tom).

range from $1 \mathrm{kHz}$ to $100 \mathrm{kHz}$. The scan is 0 to $100 \mathrm{kHz}$, and 1000 samples were taken for all curves. Note the considerable difference between the spectral estimates for channels 1 to 3 for the Hanning and proprietary "tiattened peak" windows. These results confirm the theoretical calculations above showing that, for the Hanning window, the first 3 channels should be ignored. For the "lattened peak" window, the first 14 channels should be ignored. For botb $f^{-3}$ and $f^{-4}$ noise, the uniform window does not yield usable spectral estimates over any portion of the scan. Note in this example that at frequencies above $80 \mathrm{kHz}$ there is a small step in the spectral estimates. This is due to digitizing errors of the signal due to quantization. If the digitizer bad more bits, these errors would not occur. This problem of dyamic range is common whenever the spectrum of interest covers many decades. The usual solution is to use filters to divide the spectrum into various frequency range segments which are rutable for the dyaamic range of the FFT.

Table 1 summarizes the measured experimental biases in the spectral estimates of a particular instrument with three different windows for power-law noise types varying from $f^{\circ}$ to $f^{-4}$. This covers most of the random types of noise found in oscillators and signal processing equipment. We do not advocate using the biases reported in this table to correct data - they
Table 1. Approximate Biases in FFT Spectra Estrmates

\begin{tabular}{cccc}
\hline \multicolumn{4}{c}{ norse type $f^{0}$} \\
channel \# & uniform & Hanning & flatiened peak \\
\hline 1 & $19.6 \mathrm{~dB}$ & $19.6 \mathrm{~dB}$ & $20.1 \mathrm{~dB}$ \\
2 & small & small & $16.7 \mathrm{~dB}$ \\
3 & $\downarrow$ & $!$ & $7.2 \mathrm{~dB}$ \\
4 & & & small \\
5 & & & $\downarrow$ \\
\hline
\end{tabular}

noise type $f^{-4}$

\begin{tabular}{cccc} 
channel \# & uniform & Hanning & lattened peak \\
\hline 1 & unusable & $8.6 \mathrm{~dB}$ & $10.0 \mathrm{~dB}$ \\
2 & $0.4 \mathrm{~dB}$ & $9.1 \mathrm{~dB}$ \\
3 & $0.4 \mathrm{~dB}$ & $4.0 \mathrm{~dB}$ \\
4 & small & $1.2 \mathrm{~dB}$ \\
5 & $\downarrow$ & $1.1 \mathrm{~dB}$ \\
6 & & $1.1 \mathrm{~dB}$ \\
7 & & $1.0 \mathrm{~dB}$ \\
8 & & $0.8 \mathrm{~dB}$ \\
9 & & $0.6 \mathrm{~dB}$ \\
10 & & $0.6 \mathrm{~dB}$ \\
11 & & $0.5 \mathrm{~dB}$ \\
12 & & $0.4 \mathrm{~dB}$ \\
13 & & $0.4 \mathrm{~dB}$ \\
14 & & small \\
15 & & $!$ \\
\hline
\end{tabular}

only indicate which channels should not be relied upon for data analysis.

\section{Variances of Spectral Estimates}

\section{IV.A. Theoretical Analysis}

We have derived expressions for var $\{\hat{S}(f)\}$ - the variance of $\dot{S}(f)$ - for each of the three models considered in Section III.A. These expressions depend primarily on the number of blocks $N_{b}$. Again, the details behind these calculations will be reported elsewhere [3].

First, for a white noise process, the uniform window yields

$$
\operatorname{var}\left\{\hat{S}\left(f_{3}\right)\right\}=S^{2}\left(f_{3}\right) / N_{b}, \quad 1 \leq j \leq 511
$$

while the Hanning window yields

$$
\operatorname{var}\left\{\hat{S}\left(f_{3}\right)\right\}= \begin{cases}0.69 S^{2}\left(f_{3}\right) / N_{b}, & j=1 \\ S^{2}\left(f_{3}\right) / N_{b}, & 2 \leq j \leq 510 \\ 1.03 S^{2}\left(f_{3}\right) / N_{b}, & j=511\end{cases}
$$

These results are consistent with our experimental results and with standard statistical theory.

Second, for a random-walk process, the uniform findow yields

$$
\operatorname{var}\{\dot{S}(f,)\}=5 S^{2}(f,) / \dot{N}_{b} . \quad 1 \leq \jmath \leq 511 .
$$


while the Hanning wndow yreids

$$
\operatorname{var}\left\{\dot{S}\left(f_{j}\right)\right\}= \begin{cases}1.30 S^{2}\left(f_{j}\right) / V_{b}, & j=1 ; \\ 2.20 S^{2}\left(f_{j}\right) / N_{b}, & j=2 \\ 1.31 S^{2}\left(f_{j}\right) / N_{b}, & j=3 \\ 1.15 S^{2}\left(f_{3}\right) / N_{b}, & j=4 \\ 1.09 S^{2}\left(f_{j}\right) / N_{b}, & j=5 \\ 1.06 S^{2}\left(f_{j}\right) / N_{b}, & j=6 \\ 1.04 S^{2}\left(f_{j}\right) / N_{b}, & j=7 \\ S^{2}\left(f_{3}\right) / N_{b}, & 8 \leq j \leq 511 \text { to within } 3 \% .\end{cases}
$$

Except for the few lowest frequencies, the results for the Hanning window afree with our experimental results and with standard statistical theory; however, the factor of five in the variance for the uniform window disagrees with our experiments and with standard theory (although it has been verified by Monte Carlo techniques). The cause of this discrepancy is under investigation, but we think it is due to the band-limited aature of the experimental data.

Third, for a random-run process, the variance computations are not useful since the variance is dominated by the fact that the expected value of the sample variance for each block of samples increases with time. The agreement which we found between standard statistical theory and our experimental results on the $1 / N_{b}$ rate of decrease of variance is undoubtedly due to the band-limited ature of the experimental data. We will attempt to verify these conclusions in the future using Monte Carlo techniques.

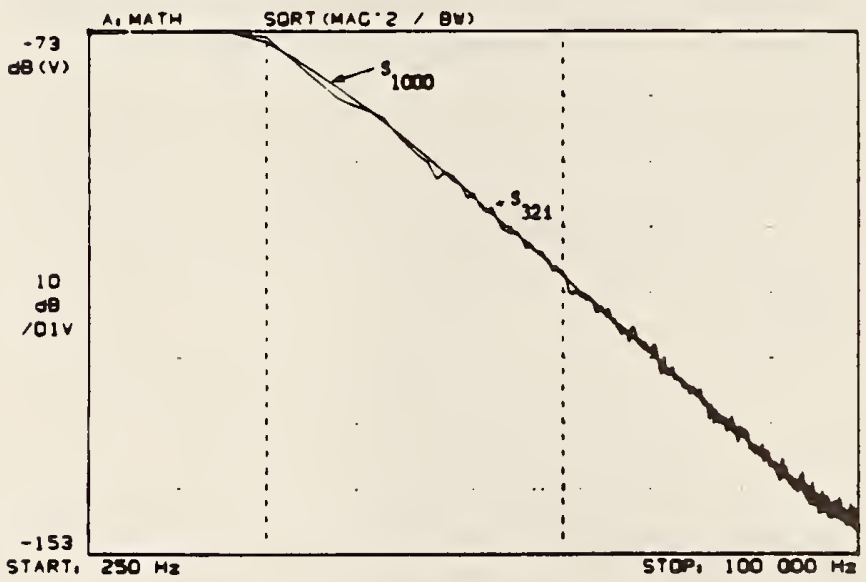

Figure 4. Comparison of the spectral estimate of $f^{-4}$ powerlaw noise with 1000 samples with that obtained with 32 samples. The text explain how these two curves are used to obtain the fractional RMS confidence of the spectral extimate for 32 samples.

\section{IV.B. Experimental Determination}

The following procedure can be used to experimentally determine the variance of the spectral estimates of virtually any type of noise spectrum with any type of window for a particular instrument. Since the spectral density of interest is in general nonwhite, we must determine both the "true value" and a way to normalize the fractional error of the estimate as a function of the number of samples. This can be done by making use of the above theoretical analysis that shows that the variance should decrease as the square root of the number of samples since they are approximately statistically independent (in fact, exactly $s 0$ in the cases of white and random-walk noise). As an example we bave choven to take $\nu_{b}=1000$ blocks of the :arous power-law noise types examined in III. $B$ above and compare the value of the spectral estimate writh that obtained from $V_{b}=32$ blocks (see Figure 4). Since the variance of the 1000 block data is about 32 times smaller than that of the 32 block data. it can serve as an accurate estimate of the "true value." Let $\hat{S}_{1000}(f)$ represent this quantity at the $j$-th channel (frequency). By subtracting the 1000 block data from the 32 block data at the $J$-th channel, we then have one estimate of the error for the 32 block data; by repesting this procedure over $N_{c}$ different channels and $N_{r}$ different replications, we can obtain accurate estimates of the variance for the 32 block data. Let $\hat{S}_{32,}\left(f_{j}\right)$ represent the spectral eatimate for the 32 block data at the $j$-th channel and the $i$-th replication. To compensate for the variation in the level of the spectral estimates with channel, it is necessary to divide the error at the $j$-th channel by the "true value" $\hat{S}_{1000}(f$,$) . The$ mean square fractional error of the 32 block data for the noise type under study is given by

$$
\begin{aligned}
\sigma_{32}^{2} & =\frac{1}{N_{r} N_{c}} \sum_{1=1}^{N_{p}} \sum_{J}\left(\frac{\hat{S}_{321}\left(f_{j}\right)-\hat{S}_{1000}\left(f_{J}\right)}{\dot{S}_{1000}\left(f_{3}\right)}\right)^{2} \\
& \approx \frac{\operatorname{var}\left\{\hat{S}_{321}\left(f_{g}\right)\right\}}{S^{2}\left(f_{g}\right)} .
\end{aligned}
$$

It is assumed tbat all channels with bias - as indicated in Table 1 - have been excluded in the summation over $j$. It is aiso important that the changes in the spectral density not exceed the dyamic range of the digitizer because under this condition the quantization errors - in addition to causing biases in the spectral estimates as discussed earlier - can lead to situations where the variance does not improve as $N_{b}$ increases. These val. ues can be scaled to any number of blocks $N_{\mathrm{b}}$ if care is taken to avoid these quantization errors. Upper and lower approximate $67 \%$ confidence limits for $S\left(f_{j}\right)$ - the true spectral density at channel $j$ - using Hanning, uniform and the proprietary Hat tened peak" windows for $N_{b}$ approximately independent blocks are given by

$$
\hat{S}(f,)\left(1 \pm V\left(\alpha, N_{b}\right)\right)
$$

where $\hat{S}(f$,$) is the spectral estimate given by Equation (1) and$ $V\left(\alpha, N_{b}\right)$ is the fractional variance given in Table 2 for $f^{\circ}$ and $a=0,-2,-3$ and -4 (these results were obtained by averaging over $N_{r} N_{c}=1200$ channels). The variances obtained are very close to those obtained from standard statistical analysis for white noise, i.e.,

$$
\hat{S}(f,)\left(1 \pm \frac{1}{\sqrt{N_{b}}}\right) \text {. }
$$

Table 2. Confidence Intervals for FFT Spectral Estimates

\begin{tabular}{cccc}
\hline \hline $\begin{array}{l}\text { power law } \\
\text { noise type }\end{array}$ & uniform & $\begin{array}{l}\text { window } \\
\text { Banning }\end{array}$ & Gattened peak \\
\hline$f^{0}$ & $1.02 / \sqrt{N_{b}}$ & $0.98 / \sqrt{N_{b}}$ & $0.98 / \sqrt{N_{b}}$ \\
$f^{-2}$ & $1.02 / \sqrt{N_{b}}$ & $1.04 / \sqrt{N_{b}}$ & $1.04 / \sqrt{N_{b}}$ \\
$f^{-3}$ & unusable & $1.04 / \sqrt{N_{b}}$ & $1.04 / \sqrt{N_{b}}$ \\
$f^{-4}$ & unusable & $1.04 / \sqrt{N_{b}}$ & $1.04 / \sqrt{N_{b}}$ \\
\hline
\end{tabular}

\section{Conclusions}

We have introduced experimental techniques to evaluate the statistical properties of FFT spectral estimates for common noise types found in occillators, amplifiers. mixers and similar 
devices, and we bave corpared ihese anth ibeorencai cajcuatrons Wie bave used bese tecinouques to study ibe biases and variances of FFI spectral estimates using the urform. Hanning. and a propretary flattened peak" unadow. The theo retical analysis was greatly hampered because the instrument manufacturer does not disclose the exact form of the Hattened peak" window or the normalization procedure for the otber windows. Nevertheless. we obtanned fair agreement between the theoretical and the experimental analysis. The variances of the spectral estimation were virually identical to a few percent for $f^{0}$ to $f^{-4}$ Doise except for the uniform window which is incapable of measuring noise which falls of faster than $f^{-2}$. There was a very large diference in the bises $\alpha$ the first few chansels for the three windows. The Hanaing wiodow showed significant biases in the Grat 3 channels while the proprietary "Gattened peak" window showed large biases for $f^{-4}$ noise even up to chansel 13. The Hanaing window therefore yields useful information over three umes wider frequency range than the proprietary Hattened peak" window. In the particular instrument studied. the proprietary tattened peak" window is the best choice for estimating the beight of a narrow band source, while the Hanning window is by far the best cboice for spectral analysis of common noise types found in acillators. amplifers. mixers, etc. We have also shown that the $67 \%$ confidence levels for spectral estimation as a function of the number of contiguous nonoverlapping biocks. $V_{6}$. is approximateiy given by

$$
S=S_{m}\left(1=\frac{0.3 \bar{\Sigma}}{v \cdot \bar{T}_{0}}\right)
$$

for white noise $\left(f^{\circ}\right)$ and by

$$
S=S_{m}\left(i \pm \frac{i .04}{\sqrt{. T_{b}}}\right)
$$

for noise types $f^{-2}$ to $f^{-4}$. This agrees to within $4 \%$ of that found by standard statistical analysis for white noise. Using this data one can now determue the aumber of samples necessary to estimate - to a gren level of statistical uncertanty - the spectrum of the various noise types commody found in oscillators, amplifers, mixers. etc.

\section{References}

[1] D. Babiteb and J. Oliverio. "Pbace Noise of Various OscilLators at Very Low Fourier Frequencies." in Proceedings of the 28th Annual Symposium on Frequency Control. 1974. Pp. $150-159$.

[2] A. V. Oppenheim and R. W. Schafer, Dwerte-Time Signal Processing. Englewood Clifs. New Jersey: Preatice Hall. 1989, cb. 11, pp. 695-767.

[3] D. B. Percival and F. L. Walls. "Spectral Analysis of PowerLaw Proceases with a Spectrum Analyzer," manuscript in preparation, 1989.

[4] M. B. Priestley, Spectral Anelysw and Time Seres. London: Acsdemic Press, 1981, ch. 7, pp. 502-612.
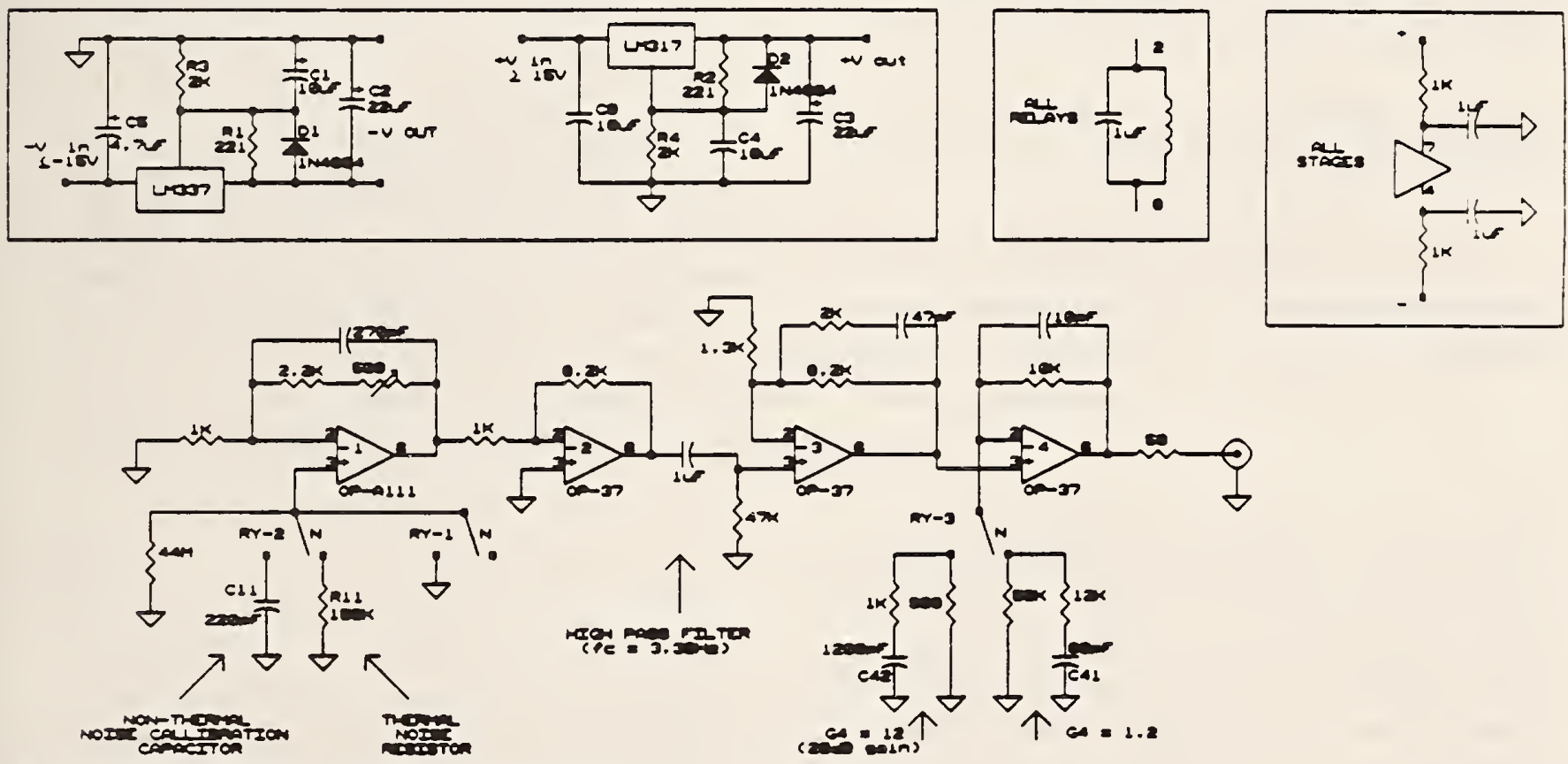

Figure 5. Cireuit diagram of a precision noise source."

\section{Appendix. Precision Noise Source}

Figure 5 shows the circuit diegram of 1 precirion noise source whose spectral density can be determined from first prin. ciples to $\pm 0.2 \mathrm{~dB}$ over the frequency range from $20 \mathrm{Bz}$ to $20 \mathrm{kHz}$. The spectral density is basically given by the Jobnson noise of the $10^{5}$ obm resistor. $V_{n}^{2}=4 k T R$, where $T$ is in Kelvin, and $k$ is Boltzmann's constant. Corrections due to the input noise voltage and noise curreat of the amplifier amount to about $0.2 \mathrm{~dB}$ for the eircuit elements shown. All resistors are precision: 7 metal film resistors. The output level cas be switehed from $-100 \mathrm{dBV} / \mathrm{Hz}$ to $-80 \mathrm{dBV} / \mathrm{Hz}$. By adjusting the noise-gair. capacitors one can make the noise spectrum lat to witbun $0.3 \mathrm{~dB}$ out to $200 \mathrm{kHz}$. There is also provision to measure the :aput Doise voltage of the amplifier by shorting the input to grourd or the combined noise voltage and noise current by sritchusg a $220 \mathrm{pF}$ eapacitor into the input instead of the $10^{5}$ obm noise resistor. 
Proc. 35th Ann. Freq. Control Symposium, USAERADCOH, Ft. Monmouth, IN 07703, May 1981

\section{A MODIFIED "ALLAN VARIANCE" WITH INCREASED OSCILLATOR CHARACTERIZATION ABILITY}

David W. Allan and James A. Barnes

Time and Frequency Division

National Bureau of Standards

Boulder, Colorado 80303

\begin{abstract}
Summary
Heretofore, the "Allan Variance, " $\sigma^{2}(\tau)$, has become the de facto standard for measuryng oscillator instability in the time-domain. Often oscillator frequency instabilities are resonably modelable with a power law spectrum: $S_{f}(f) \sim f^{\alpha}$, where $y$ is the normalized frequency, $y_{f}$ is the Fourier frequency, and $\alpha$ is a constant over some range of Fourier frequencies. It has been shown that for power law spectrum $\sigma^{2}(\tau) \sim \tau^{\mu}$, and that $\mu=-\alpha-1$ for $-3<\alpha \leq+1$, where $\tau$ is the nomimal sample time over which each value of $y$ is measured. The modified "Allan Variance" developed in this paper yields $\mu \cong-\alpha-1$ for all $\alpha$ in the range $-3<\alpha$, which removes the previous ambiguity: $\mu=-2$ for $+1<\alpha$. In other words, with the modified "Allan Variance" one can easily distinguish between white phase noise $(\alpha=+2)$ and flicker phase noise $(\alpha=+1)--$ commonly occurring for the short term instabilities of quartz crystal oscillators and active hydrogen masers.
\end{abstract}

Key Words. Flicker Noise; Frequency Stability; Oscillator Noise Modeling; Power Law Spectrum; Time-Domain Stability; White Noise.

\section{Introduction}

The random fluctuations in precision oscillators may often be characterized by a power law spectrum:

$$
s_{y}(f)=h_{\alpha} f^{\alpha},
$$

where $y$ is the normalized frequency deviation, $f$ is the Fourier frequency, $h_{\alpha}$ is the intensity of the particular noise process, and $\alpha$ is constant over some range of $f$. The typical values of $\alpha$ are: +2 (white noise phase modulation, $P H$ ) $;+1$ (flicker noise $P M$ ); 0 (white noise frequency modulation, FM); -1 (flicker noise $F M$ ); and -2 (random walk FM). The Allan variance, as it has come to be known, ${ }^{1}$ has been demonstrated as a very useful statistical tool for characterizing these various random processes with the exception that if $\alpha=+1$ or +2 , the dependence on $\tau$ is nominally the same, i.e., $\sim \tau^{-2}$. It is not at all uncommon for white $P M$ and flicker PM to occur in precision oscillators for $\tau$ of the order of one second and shorter. The modified Allan variance, as developed in this paper, depends as $\tau^{-2}$ for $\alpha=+1$ and as $\tau^{-3}$ for $\alpha=+2$. This yields a clear distinction in the time domain between these heretofore somewhat ambiguous processes.

\section{Definition of "Allan Variance"} and Related Concepts

Define $y$, the normalized frequency deviation, as

$$
y(t)=\frac{v(t)-v_{0}}{v_{0}}
$$

where $v(t)$ is the output frequency of the oscillator being studied, and $v_{0}$ is nominally the same frequency, but of a reference oscillator assumed for the moment without loss of generality to be better than the test oscillator. The time deviation from some arbitrary origin $(t=0)$ is the integral of the frequency deviations (from that origin):

$$
x(t)=\int_{0}^{t} y\left(t^{\prime}\right) \cdot d t^{\prime}
$$

The $i$ th average frequency deviation over an interval, $\tau$, is

$$
\bar{y}_{i}=\frac{x_{i+1}-x_{i}}{\tau}
$$

where the assumption is made that the time deviation measurements are nominally spaced $\tau$ apart. 
The "Allan Variance" is defined as:

$$
\sigma_{y}^{2}(\tau)=\frac{1}{2}\left\langle\left(\bar{y}_{i+1}-\bar{y}_{i}\right)^{2}\right\rangle \text {, }
$$

where the brackets " $<>$ " denote infinite time average. Using equation (4), one may write:

$$
\sigma_{y}^{2}(\tau)=\frac{1}{2 \tau^{2}}\left\langle\left(x_{i+2}-2 x_{i+1}+x_{i}\right)^{2}\right\rangle .
$$

It has been shown that typically $\sigma_{y}{ }^{2}(\tau)$ varies as $\tau^{\mu}$, and that $\mu=-\alpha-1$ for $-3 \leq \alpha \leq+1.1,2$ Hence, we see one of the dimensions of usefulness of $\sigma_{y}{ }^{2}(\tau)$; i.e., ascertaining the dependence on $\tau$ allows an estimate of $\alpha$ (the power law spectral type of noise). However, if $\alpha \geq+1$, then $\mu \cong-2$, and the $\tau$ dependence becomes somewhat ambiguous as to the type of noise in this region. It is interesting to note that in the region $\alpha \geq+1, \sigma_{y}{ }^{2}(\tau)$ is banowidth $\left(f_{h}\right)$ dependent; i.e., the banowidth of the measurement system will affect the value of $\sigma_{y}(\tau)$, and furthermore, one may use the bandwidth dependence ${ }^{3}$ to determine the value of $\alpha$ (see also Appendix Ref. 2).

\section{Development of the Modified Allan Variance}

One may also write $\sigma_{y}{ }^{2}(\tau)$ in terms of a generalized autocovariance function:

$$
\sigma_{y}^{2}(\tau)=\frac{1}{2 \tau^{2}}\left[4 U_{x}(\tau)-U_{x}(2 \tau)\right],
$$

where

$$
U_{x}(\tau)=2\left[R_{x}(0)-R_{x}(\tau)\right],
$$

and where

$$
R_{x}(\tau)=\langle x(t+\tau) \cdot x(t)\rangle,
$$

the classical autocovariance function of $x(t)$. Using the fourier transforms of generalized functions, one may determine the coefficients relating the power spectral density to $\sigma_{y}{ }^{2}(\tau)$. Ref. 1 gives these relationships. It is of interest to note that $U_{x}(\tau)$ has the following approximate form in the region $\alpha \geq+1$ (see Appendix Ref. 2):

$$
U_{x}(\tau) \sim a(\alpha)\left[\left|\frac{1}{2 \pi f_{h}}\right|^{-\alpha+1}-|\tau|^{-\alpha+1}\right]
$$

Hence, one notes that by changing the reciprocal bandwidth as well as $\tau$, one affects $\sigma_{y}{ }^{2}(\tau)$ in similar ways, depending on the value of $\alpha$. From this, one should be able to deduce the value of $\alpha$, since the bandwith dependence becomes stronger for a moving positive from +1 , and the $\tau$ dependence becomes stronger as $\alpha$ moves negative from +1 . One can change the bandwidth in the hardware or in the software. In the past, it has typically been done in the hardware. ${ }^{3}$ James snyder ${ }^{4}$ has shown that it is relatively easy to change the bandwidth in the data processing by a clever technique and we have followed his lead. In particular, we have chosen a new variance analysis scheme which coincides with the Allan variance at the minimum sample time, $\tau_{0}$, (i.e., minimum data spacing), but which changes the bandwidth in the software as the sample time, $\tau$, is changed.

Each reading of the time deviation, $x_{j}$, has associated with it an intrinsic nominal (hardware) measurement system bandwidth, $f_{h}$. Define $\tau_{h}=\frac{1}{2 \pi f}$; and similarly we may define a software bandwidth, $f_{s}=f_{h} / n$, which is $1 / n$ times narrower than the hardware bandwidth. This software bandwidth can be realized by averaging $n$ adjacent $x_{j}{ }^{\prime} s ; \tau_{s}=n \tau_{h}$, where $\tau_{s}=1 / f_{s}$. We have defined a modified Allan variance which allows the reciprocal software pandwidth to change linearly with the sample time, $\tau$ :

$\operatorname{Mod} \sigma_{y}{ }^{2}(\tau)=\frac{1}{2 \tau^{2}}\left\langle\left[\frac{1}{n} \sum_{i=1}^{n}\left(x_{i+2 n}-2 x_{i+n}+x_{i}\right)\right]^{2}\right\rangle$

where $\tau=n \tau_{0}$. Eq. 11 clearly coincides with Eq. 6 for $n=1$. One can see that, in general, we have formed a second difference of three time readings with each of the three being an average of $n$ of the $x_{i}$ 's (with non-overlapping averages). As $n$ increases, the (software) bandwidth decreases and this bandwidth varies just as $f_{s}=f_{h} / n$.

For a finite data set of $N$ Ireadings of $x_{i}$ $(i=1$ to $N)$, we may write an estimate: 
$\operatorname{Mod} \sigma_{y}{ }^{2}(\tau)=\frac{1}{2 \tau^{2} n^{2}(N-3 n+1)}$

$$
\sum_{j=1}^{N-3 n+1}\left[\sum_{i=j}^{n+j-1}\left(x_{i+2 n}-2 x_{i+n}+x_{i}\right)\right]^{2}
$$

Eq. 12 is easy to program, but takes more time to compute than for $\sigma_{y}(r)$. This is only of significance for the smaller computer or handheld calculator.

\section{Comparisons, Tests, and Examples of Usage}

\section{of the Modified Allan Variance}

We simulated various power law noise processes, and applied Eq. 12. Shown in Fig. 1 are the resulting $\tau$-dependences of the modified Allan variances for $\alpha=-2,-1,0,+1$, and +2 . The solid lines drawn are the anticipated or theoretical slopes for the particular noise process. One sees excellent agreement for white noise PM and for flicker noise PM, and nominal agreement for the others.

One can express Eq. 11 in terms of the generalized autocovariance function:

$$
\begin{aligned}
\operatorname{Mod} \sigma_{y}^{2}(\tau) & =\frac{1}{2 \tau^{2} n^{2}}\left\{\left[4 U_{x}(\tau)-U_{x}(2 \tau)\right] \cdot n\right. \\
& +\sum_{i-1}^{n-1}(n-i)\left[-6 U_{x}\left(i \tau_{0}\right)+4 U_{x}\left((n+i) \tau_{0}\right)\right. \\
& +4 U_{x}\left((n-i) \tau_{0}\right)-U_{x}\left((2 n+i) \tau_{0}\right) \\
& \left.\left.-U_{x}\left((2 n-i) \tau_{0}\right)\right]\right\} .
\end{aligned}
$$

In the range $-3 \leq \alpha \leq+1$, one may write:

$$
U_{x}(\tau)=a(\alpha) \cdot \tau^{-\alpha+1} \text {, }
$$

which when substituted in Eq. 13, and using Eq. 7, yields
$\operatorname{Mod} \sigma_{y}{ }^{2}(\tau)=\sigma_{y}{ }^{2}(\tau)\left\{\frac{1}{n}+\frac{1}{n^{2} 4 n^{-\alpha+1}-(2 n)^{-\alpha+1}}\right.$.

$$
\begin{aligned}
& \sum_{i=1}^{n-1}(n-i) \cdot\left[-6 i^{-\alpha+1}+4(n+i)^{-\alpha+1}\right. \\
& \left.\left.-(2 n+i)^{-\alpha+1}+4(n-i)^{-\alpha+1}-(2 n-i)^{-\alpha+1}\right]\right\}
\end{aligned}
$$

Since we know that $\sigma_{y}{ }^{2}(\tau)$ is well behaved in this range and $\mu=-\alpha-1$, it is of interest to look at the ratio:

$$
R(n)=\operatorname{Mod} \sigma_{y}^{2}(\tau) / \sigma_{y}{ }^{2}(\tau)
$$

As stated before, at $n=1\left(\tau=\tau_{0}\right)$ the ratio is unity. One can evaluate Eq. 15 with a computer. A reasonable empirical fit may be formed, which is good to $0.5 \%$ or better of Eq. 15:

\begin{tabular}{|c|c|c|c|c|c|c|}
\hline \multicolumn{2}{|l|}{ Notse Type } & \multirow[b]{2}{*}{ p } & \multirow[b]{2}{*}{$a$} & \multirow[b]{2}{*}{ E } & \multirow[b]{2}{*}{ pit } & \multirow{2}{*}{$\operatorname{Mod} \sigma_{y}(\tau) / \sigma_{y}(\tau)$} \\
\hline Naw & a & & & & & \\
\hline White $F M$ & 0 & 1 & 2 & 2 & Derfect & .707 \\
\hline Flicker FM & -1 & 99.9 & 248 & 2.35 & $4 x$ & .821 \\
\hline Random Walk FM & -2 & 33 & 40 & 2.35 & $<4$ & 908 \\
\hline Flicker Walk FM & -3 & 1 & 1 & -- & perfect & 1 \\
\hline
\end{tabular}

$$
R(n)=\frac{q+p n^{E}-p}{q n^{E}}
$$

which approaches $p / q$ asymptotically as $n$ approaches infinity, and is within 18 of $p / q$ for $n \geq 8$. Listed in Table 1 are the empirical values of $p, q$, and $E$ and the quality of fit for the appropriate power law noise processes.

TABLE 1

The results of Table 1 are in reasonable agreement with simulated results of Fig. 1(a) through $1(e)$. The last row in Table 1 , "flicker walk" frequency modulation, is out of the range of applicability of $\alpha$, but the ratio, $R(n)$, is still convergent. 
The $U_{x}(\tau)$ function for flicker noise $P M$ is extremely complicated and has not been developed, but one can arrive at an empirical value for $i t$. The $U_{x}(\tau)$ function is derivable for the other power law spectral processes. Table 2 gives the relationships between the time domain measure Mod $\sigma_{y}{ }^{2}(\tau)$ and its power law spectral counterpart, given in Eq. 1. Also listed in the right hand column of Table 2 are the asymptotic values of $R(n)$ :

TABLE 2

\begin{tabular}{|c|c|c|c|c|}
\hline \multicolumn{2}{|l|}{ Notse Type } & \multirow{2}{*}{$\operatorname{Mod} a_{y}{ }^{2}(t)$} & \multirow{2}{*}{ Coment } & \multirow{2}{*}{$R(n)$} \\
\hline Hane & $a$ & & & \\
\hline White PM & +2 & $h_{2} \cdot \frac{3 r_{h}}{(2 \pi)^{2} n^{2}}$ & Exact & 1 \\
\hline Flicker PW & +1 & $n_{1} \cdot \frac{1.038+3 \ln \left(w_{n}{ }^{2}\right)}{(2 \pi)^{2} \tau^{2}}$ & Eapirical & 1 \\
\hline White FM & 0 & $n_{0} \cdot \frac{R(n)}{2 \pi}$ & Exac? & 0.5 \\
\hline Flicker FM & -3 & $n_{-1} 2 \ln (2) \cdot R(n)$ & $\begin{array}{l}\text { Enofrical; Exact } \\
\text { Avatlable }\end{array}$ & 0.674 \\
\hline Randoe Walk FM & -2 & $n_{-2} \cdot \frac{(2 \pi)^{2} x}{6} \cdot R(n)$ & $\begin{array}{l}\text { Empirical; Exact } \\
\text { Avallable }\end{array}$ & 0.824 \\
\hline
\end{tabular}

It is clear from Table 2 that $\operatorname{Mod} \sigma_{y}{ }^{2}(\tau)$ is very useful for white $P M$ and flicker noise $P M$, but for $\alpha<+1$ the conventional Allan variance, $\sigma_{y}{ }^{2}(\tau)$, gives both an easier-to-interpret and an easier-tocalculate measure of stability.

It is interesting to make a graph of a versus $\mu$ for both the ordinary Allan variance and the modified Allan variance. Shown in Fig. 2.is such a graph. This graph allows one to determine power law spectra for non-interger as well as interger values of $\alpha$. The dashed line for the modified Allan variance has been intentionally moved to the left in Fig. 2 because for small values of $n$ the value of $\mu$ will appear to be slightly more negative that for $\sigma_{y}{ }^{2}(\tau)$, even though for large $n$, they both approach the same slope (i.e., the same values of $\mu$ ). In fact, in the asymtotic limit, the equation relating $\mu$ and $\alpha$ for the modified Allan variance is

$$
\alpha=-\mu-1 \text {, for }-3<\alpha<+3 \text {. }
$$

The value of $\mu=-4$ for $\alpha=+3$ was verified empirically with simulated data, and it appears that for $\alpha>+3, \mu$ remains at -4 .

A direct application for using the modified Allan variance recently arose in the analysis of atomic clock data as received from a Global Positioning System (GPS) satellite. We were interested in knowing the short-term characteristics of the newly developed, high-accuracy NBS/GPS receiver, as well as the propagation fluctuations. Fig. 3 shows both $\sigma_{y}{ }^{2}(\tau)$ and $\operatorname{Mod} \sigma_{y}{ }^{2}(\tau)$ for comparison. Using Mod $\sigma_{y}{ }^{2}(\tau)$, we can tell that the fundamental limiting noise process involved in the system is white noise PM with the exciting result that averaging for four minutes can allow one to ascertain time difference to better than one nanosecond excluding other systematic effects.

\section{Conclusion}

We have developed a supplemental measure, the "Modified Allan Variance" (Mod $\sigma_{y}{ }^{2}(\tau)$ ), which has very useful properties when analyzing oscillator or signal stability in the presence of white noise phase modulation or flicker noise phase modulation. It also works reasonably well as a stability measure for other commonly occuring noise processes in precision oscillators.

We would recommend that for most time domain analysis, $\sigma_{y}{ }^{2}(\tau)$ should be the first choice. If $\sigma_{y}{ }^{2}(\tau)$ depends on $\tau$ as $\tau^{-1}$, then the modified Allan variance can be used as a substitute to help remove the ambiguity as to the noise processes.

\section{Acknowledgments}

The authors are deeply grateful for stimulating discussions with Dr. James J. Snyder, and for the useful comments of Dr. Robert Kamper and Mr. David A. Howe.

\section{References}

1. James A. Barnes, Andrew R. Chi, Leonard $S$. Cutler, Daniel J. Healey, David B. Leeson, Tomas E. McGunical, James A. Mullen, Jr., Warren L. Smith, Richard L. Sydnor, Robert F. C. Vessot, and Gernot M. R. Winkler, Proc. IEEE Trans. Instrum. Meas. IM-20, 105 (1971); also published as NBS Tech. Note 394 (1971).

2. David W. Allan, Proc. IEEE 54, 221 (1966).

3. R. F. C. Vessot, Proc. IEEE 54, 199 (1966).

4. James J. Snyder, Proc. 35th Annual Symp. on Freq. Control (1981), to be published. 
(a)

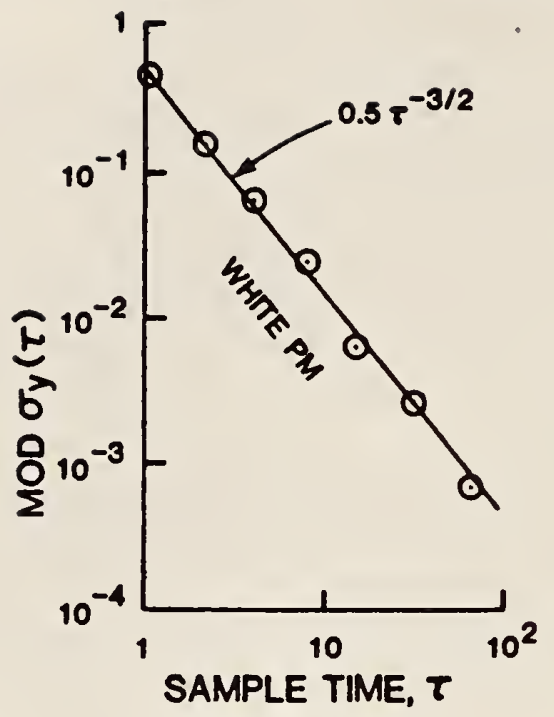

(c)

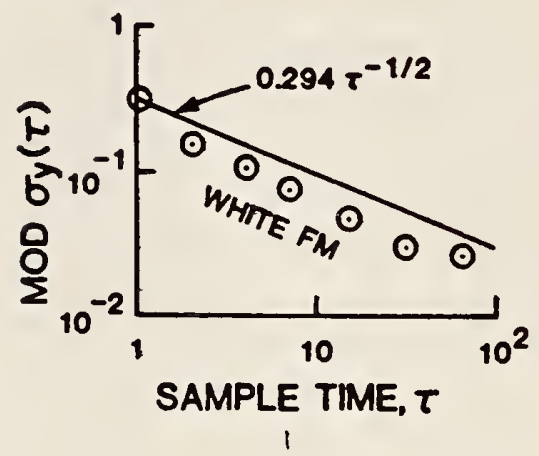

(b)

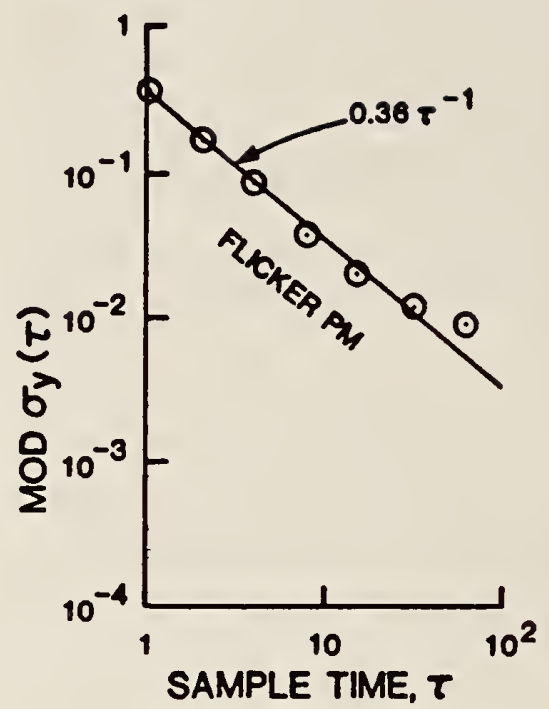

(d)

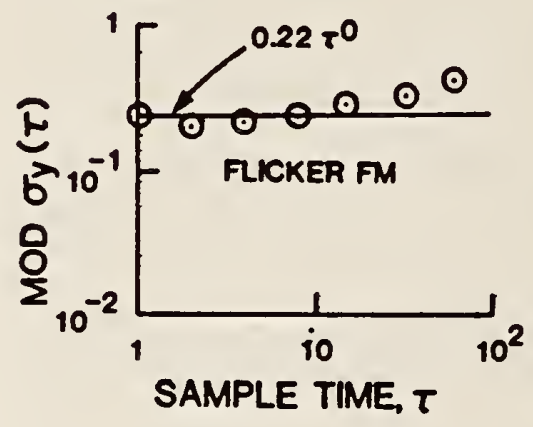

(e)

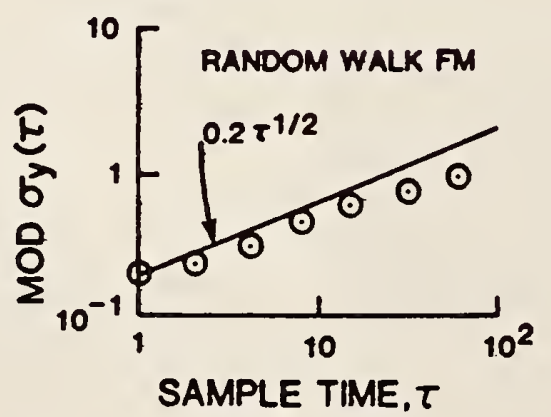

Fig la-e. Mod $\sigma_{y}(\tau)$ using Eq. 12 was calculated for different sample times for independently generated and simulated noise processes, which were white phase noise, flicker phase noise, white frequency noise, flicker frequency noise, and random walk frequency noise, respectively. Mod $\sigma_{y}(\tau)$ was computed for 399 data points in each case. One sees the excellent fit to the theory for white phase noise and flicker phase noise, an important new contribution in the ability to characterize oscillators having these noise processes. 
Copyright 1984 IEEE. Reprinted, with permission, from IEEE Transactions on Instrumentation and Measurements, Vol. IM-33, No. 4, pp. 332-336, December 1984.

Characterization of Frequency Stability: Analysis of the Modified Allan Variance and Properties of Its Estimate

PAUL LESAGE AND THÉOPHANE AYI

Abstroct-An analytical expression for the modified Allan variance is given for each component of the model usually considered to describe the frequency or phase fluctuations in frequency standards. The relation between the Allan variance and the modified Allan variance is pecified and compared with that of a previously published analysis. The uncertainty on the estimate of the modified Allan variance calculated from a finite set of measurement data is discussed.

Manuscript received December 8, 1983; revised February 17, 1984.

The authors are with the Laboratoire de l'Horloge Atomique, Equipe de Recherche du CNRS, associée à l'Université Paris-Sud, 91405 Orsay, France. 


\section{INTRODUCTION}

Many works [1]-[5] have been devoted to the characterization of the frequency stability of ultrastable frequency sources and have shown that the frequency noise of a generator can be easily characterized by means of the "two-sample variance" [2] of frequency fluctuations, which is also known as the "Allan variance" [2] in the special case where the dead time between samples is zero.

An algorithm for frequency measurements has been developed by J. J. Snyder [6], [7]. It increases the resolution of frequency meters, in the presence of white phase noise. It has been considered in detail by D.W. Allan and J. A. Barnes [8]. They have defined a function called the "modified Allan variance" and they have analyzed its properties for the commonly encountered components of phase or frequency fluctuations [3]. For that purpose, the authors of [8] have expressed the modified Allan variance in terms of the autocorrelation of the phase fluctuations. For each noise component, they have computed the modified Allan variance and deduced an empirical expression for the ratio between the modified Allan variance and the Allan variance.

In this paper, we show that the analytical expression of this ratio can be obtained directly, even for the noise components for which the autocorrelation of phase functions is not defined from the mathematical point of view. We give the theoretical expressions and compare them with those published in [8].

The precision of the estimate of the modified Allan variance is discussed and results related to white phase and white frequency noises are presented.

\section{BACKGROUND AND DEFINITIONS}

In the time domain, the characterization of frequency stability is currently achieved by means of the two-sample variance [2] $\left\langle\sigma_{y}^{2}(2, T, \tau)\right\rangle$ of fractional frequency fluctuations. It is defined as

$$
\left\langle\sigma_{y}^{2}(2, T, \tau)\right\rangle=\frac{1}{2}\left\langle\left(\bar{y}_{k+1}-\bar{y}_{k}\right)^{2}\right\rangle
$$

where the quantity $\bar{y}_{k}$ is the average value of the fractional frequency fluctuations $y(t)$ over the time interval $\left(t_{k}, t_{k}+\tau\right)$ such that

$$
\bar{y}_{k}=\frac{1}{\tau} \int_{t_{k}}^{t_{k}+\tau} y(t) d t .
$$

In (2), $t_{k}$ represents the moment at which the $k$ th observation time interval starts. We have

$$
t_{k}=t_{0}+k T, \quad T \geqslant \tau
$$

where $t_{0}$ is an arbitrary time origin, $k$ is a positive integer, and $T$ is the time interval between the beginning of two successive observations.

In all the following, we assume that the dead time between samples is zero. We then have

$$
T=\tau \text {. }
$$

In this special case, the two-sample variance is well known as the Allan variance $\sigma_{y}^{2}(\tau)$

$$
\sigma_{y}^{2}(\tau)=\left\langle\sigma_{y}^{2}(2, \tau, \tau)\right\rangle
$$

The relation between the Allan variance and $y(t)$ can be expressed as

$$
\sigma_{y}^{2}(\tau)=\frac{1}{2 \tau^{2}}\left\langle\left\{\int_{t_{k}+\tau}^{t_{k}+2 \tau} y(t) d t-\int_{t_{k}}^{t_{k}+\tau} y(t) d t\right\}^{2}\right\rangle .
$$

Equation (6) shows that $\sigma_{y}^{2}(\tau)$ is proportional to the true variance of the output of a linear filter with input signal $y(t)$ and impulse response $h_{1}(t)$ in Fig. 1 .

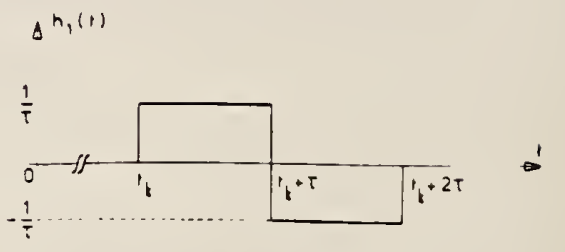

Fig. 1. Variations with time of the linear filter impulse response which represents the signal processing for the Allan variance calculation.

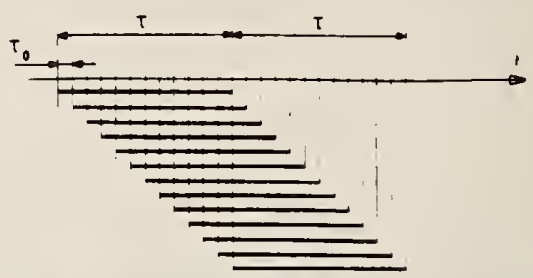

Fig. 2. Illustration of the algorithm considered for the measurement of periodic signal frequency.

The fractional frequency fluctuations $y(t)$ are actually well described by a conventional model which consists of a set of five independent noise processes [2]. Taking in to account the finite bandwidth of the processed signal and assuming a single pole filter, the one-sided power spectral density $S_{y}(f)$ of $y(t)$ can be written as

$$
S_{y}(f)=h_{\alpha} \frac{f^{\alpha}}{1+\left(\frac{f}{f_{c}}\right)^{2}}
$$

where coefficients $h_{\alpha}$ do not depend on $f$. The integer $\alpha$ equals $2,1,0,-1$, and -2 . $f_{c}$ is the $3-\mathrm{dB}$ bandwidth of the hardware filter.

\section{THE Modified Allan Variance}

The main property of the algorithm developed by J. J. Snyder is to increase the precision on the measure of periodic signal frequency, in presence of white phase noise [7]. It consists in dividing a time interval $\tau$ into $n$ cycles of clock period $\tau_{0}$ such as

$$
\tau=n \tau_{0} .
$$

Therefore, from a given observation time interval of duration $2 \tau, n$ overlapping time intervals of duration $\tau$ can be obtained, as depicted in Fig. 2. Another property of this algorithm is to reduce the total observation time by a factor $n / 2$.

Following this way, Allan and Barnes have introduced the "modified Allan variance" [8] such as

$$
\begin{aligned}
\operatorname{Mod} \sigma_{y}^{2}(\tau)= & \frac{1}{2 \tau^{2}}\left\langle\left[\frac { 1 } { n } \sum _ { i = 1 } ^ { n } \left\{\int_{t_{0}+(i+n) \tau_{0}}^{t_{0}+(i+2 n) \tau_{0}} y(t) d t\right.\right.\right. \\
& \left.\left.\left.-\int_{t_{0}+i_{0}}^{t_{0}+(i+n) \tau_{0}} y(t) d t\right\}\right]^{2}\right\rangle
\end{aligned}
$$

It can be easily seen from (9) that the calculation of each statistical sample involved in the definition of $\operatorname{Mod} \sigma_{y}^{2}(\tau)$ requires a signal observation of duration $3 \tau$.

The impulse response $h_{n}(t)$ of the equivalent linear filter consists in finite sum of $n$ shifted impulse responses $h_{1}(t)$. We have

$$
h_{n}(t)=\frac{1}{n} \sum_{i=1}^{n} h_{1}\left(t-i \tau_{0}\right)
$$


TABLE I

ANALYTICAL EXPRESSION for the MODIfIEd ALlan VARIANCE WITHIN CONDITION $2 \pi f_{c} T_{0} \gg 1$

\begin{tabular}{|c|c|c|}
\hline NOISE TYPE & $\alpha$ & $\operatorname{Mod} \sigma_{y}^{2}(-)$ \\
\hline WHITE P M & 2 & $\frac{3 n_{2} f_{c}}{8 n+?^{2}}$ \\
\hline FLICKER P M & 1 & $\frac{n_{1}}{4 \pi^{2} n^{2} \tau^{2}}\left[3 n 2 n\left(2 \pi f c^{T}\right)+\sum_{k=1}^{n-1}(n-k)\left\{4 \ln \left(\frac{n^{2}}{k^{2}}-1\right)-\ln \left(\frac{4 n^{2}}{k^{2}}-1\right)\right\}\right]$ \\
\hline WHITE F M & 0 & $\frac{n_{0}}{2-} \times \frac{n^{2}+1}{2 n^{2}}$ \\
\hline FLICKER F M & -1 & $\begin{array}{l}\frac{2 n-1^{2 n 2}}{n^{2}}\left[\frac{4 n^{2}-3 n+1}{2}+\frac{1}{n^{2} l n 2} \times \sum_{k=1}^{n-1}(n-k) \times\left\{\frac{n}{2}[(k+2 n) \ln (k+2 n)-(k-2 n) \ln (2 n-k)]\right.\right. \\
+\frac{1}{2}(k+n)(k-2 n) \ln (k+n)+\frac{1}{2}(k-n)(k+2 n) \ln \mid k-n+3 k^{2} \ln k-k\left[(n+2 k) \ln \left(k+\frac{n}{2}\right)\right. \\
\left.\left.\left.\left.-(n-2 k) \ln \mid k-\frac{n}{2}\right]\right]\right\}\right]\end{array}$ \\
\hline RANDOM WALK F $M$ & -2 & $\frac{33}{40}+\frac{1}{8 n^{2}}+\frac{1}{20 n^{4}}$ \\
\hline
\end{tabular}

In order to illustrate $(10)$, variations with time of the shifted functions $h_{1}\left(t-i \tau_{0}\right)$ and of the impulse response $h_{n}(t)$ are represented in Fig. 3(a) and (b), respectively, for $n=10$.

For $n=1$, the Allan variance and the modified one are equal. We have

$$
\operatorname{Mod} \sigma_{y}^{2}(\tau)=\sigma_{y}^{2}(\tau)
$$

One can express (9) in terms of the spectral density $S_{y}(f)$. We have

$$
\begin{aligned}
\operatorname{Mod} \sigma_{y}^{2}(\tau)= & \frac{2}{n^{2} \pi^{2} \tau^{2}} \cdot\left\{n \int_{0}^{\infty} \frac{1}{f^{2}} S_{y}(f) \sin ^{4}\left(\pi f n \tau_{0}\right) d f\right. \\
& +2 \sum_{k=1}^{n-1}(n-k) \int_{0}^{\infty} \frac{1}{f^{2}} S_{y}(f) \cos \left(2 \pi f k \tau_{0}\right) \\
& \left.\cdot \sin ^{4}\left(\pi f n \tau_{0}\right) d f\right\} .
\end{aligned}
$$

It should be noted that the integrals involved in (12) are convergent for each noise component. The analytical expression for the modified Allan variance can therefore be deduced directly from this equation.

In the following, it is assumed that the condition $2 \pi f_{c} \tau_{0} \gg 1$ is fulfilled. This means that the hardware bandwidth of the measurement system must be much larger than the reference clock frequency.

We have calculated the modified Allan variance for each noise component. Results are reported in Table I. It appears that the analytical expression for Mod $\sigma_{y}^{2}(\tau)$ is relatively simple except for flicker phase and flicker frequency noises where it is given as a finite sum of functions depending on $n$. In order to compare the Allan variance with the modified one, we

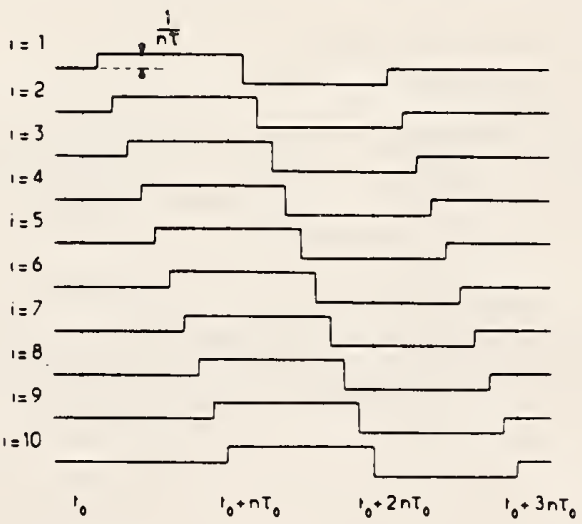

(a)

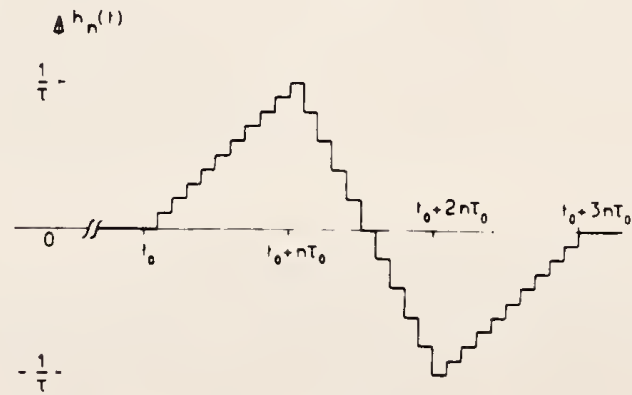

(b)

Fig. 3. (a) The impulse response $h_{n}(t)$, associated with the modified Allan variance calculation, represented as a sum of $n$ shifted impulse response $h_{1}(t)$. It is assumed $n=10$. (b) Variations with time of the impulse response $h_{n}(t)$, in the special case where $n=10$.

\footnotetext{
* See Appendix Note \# 35
} 
TABLE II

Avalytical Expressions and Asymptotical VALUS for $R(n)$

(Results Are Valid within Condition $2 \pi f_{c} \tau_{0}>>1$ )

\begin{tabular}{|c|c|c|}
\hline$\alpha$ & $R(n)$ & $\lim _{n \rightarrow \infty} R(n)$ \\
\hline 2 & $\frac{1}{n}$ & 0 \\
\hline 1 & $\frac{1}{n^{2}}\left[n+\frac{1}{32 n\left(2+f c^{n+}\right)} \times \underset{k=1}{n=1}(n-k)\left\{4 \ln \left(\frac{n^{2}}{k^{2}}-:\right)-i n\left(\frac{4 n^{2}}{k^{2}}-1\right)\right\}\right]$ & 0 \\
\hline 0 & 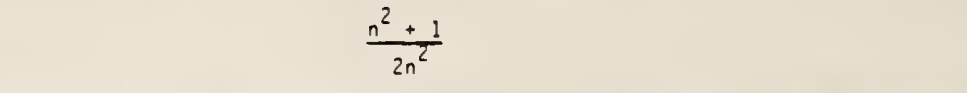 & 0.5 \\
\hline-1 & $\begin{array}{l}\frac{1}{n^{2}}\left[\frac{4 n^{2}-3 n+1}{2}+\frac{1}{n^{2} 2 n^{2}} \times{\underset{k=1}{n-1}}_{k=1}^{=}(n-k) \times\left\{\frac{n}{2}[(k+2 n) \ln (k+2 n)-(k-2 n) \ln (2 n-k)]\right.\right. \\
+\frac{1}{2}(k+n)(k-2 n) 2 n(k+n)+\frac{i}{2}(k-n)(k+2 n) 2 n, k-n+3 k^{2} \ln k-k\left[(n+2 k) \ln \left(k+\frac{n}{2}\right)\right. \\
\left.\left.\left.=(n-2 k) 2 n k-\frac{n}{2}\right]\right\}\right]\end{array}$ & 0.787 \\
\hline-2 & $\frac{33}{40}+\frac{1}{8 n^{2}}+\frac{1}{20 n^{4}}$ & 0.825 \\
\hline
\end{tabular}

consider the ratio $R(n)$ defined in [8] as

$$
R(n)=\operatorname{Mod} \sigma_{y}^{2}(\tau) / \sigma_{y}^{2}(\tau)
$$

The analytical expressions for $R(n)$, deduced from Table I, are reported in Table II. One can see that $R(n)$ does not depend on the product $f_{c} \tau_{0}$, except for flicker phase noise modulation. The asymptotic values of $R(n)$ are also listed in Table II.

Fig. 4 depicts the variations of $R(n)$ with $n$. It shows that, for large values of $n$, white phase and flicker phase noise modulations have different dependences. As outlined in [8], this gives a means to easily distinguish these two noise processes, in the time domain. For large $n$, and for $\alpha=0,-1,-2$, $R(n)$ remains a constant. Consequently the Allan variance can be deduced from the modified one, for these noise processes.

A comparison with results of [8] shows a good agreement for $\alpha=2,0$ and -2 . But, for $\alpha=1$ and -1 , our expressions for the modified Allan variance and ratio $R(n)$ disagree, especially for flicker phase noise modulation. This discrepancy might be due to the fact that in [8], Mod $\sigma_{y}^{2}(\tau)$ is expressed in terms of the autocorrelation function of phase fluctuations which is not defined for $\alpha=1$.

\section{UNCERTAINTY ON THE ESTIMATE OF THE MODIFIED ALLAN VARIANCE}

Equation (9) shows that the definition of the modified Allan variance theoretically implies an infinite set of time intervals.

Practically, one can only estimate this quantity from a finite set of $m$ successive cycles similar to the one depicted in Fig. 3(b).

Let $\operatorname{Mod} \hat{\sigma}_{y}^{2}(\tau)$ be the estimated modified Allan variance (EMAV) such as

$$
\text { Mod } \hat{\sigma}_{y}^{2}(\tau)=\frac{1}{2 \tau^{2} n^{2}} \times \frac{1}{m} \sum_{k=1}^{m}\left\{\sum_{i=1}^{n} A_{i, k}\right\}^{2}
$$

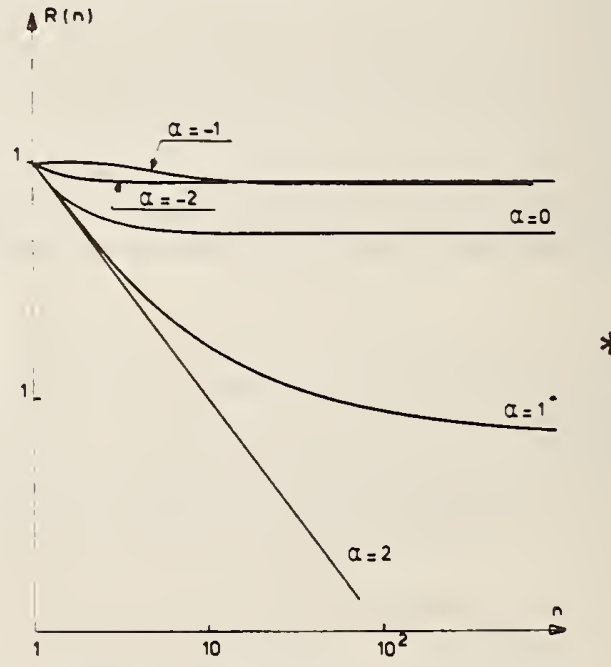

Fig. 4. Variations with $n$ of the ratio $R(n)$, for fractional frequency fluctuations with power law spectrum $S_{y}(f)=h_{\alpha} \cdot\left(1 / 1+\left(f / f_{c}\right)^{2}\right) f^{\alpha}$, within condition $2 \pi f_{c} r_{0} \gg 1$. ("For $\alpha=+1, R(n)$ is a function of $f_{c}$ and $\tau_{0}$. The reported variations are for $2 \pi f_{c} \tau_{0}=10^{4}$.)

where

$$
\begin{aligned}
A_{l, k}= & \int_{t_{0}+(i+n) \tau_{0}+(k-1) 3 n \tau_{0}}^{t_{0}+(i+2 n) \tau_{0}+(k-1) 3 n \tau_{0}} y(t) d t \\
& -\int_{t_{0}+i \tau_{0}+(k-1) 3 n \tau_{0}}^{t_{0}+(i+n) \tau_{0}+(k-1) 3 n \tau_{0}} y(t) d t .
\end{aligned}
$$

* See Appendix Note \# 35

TN-262 
The EMAV is a random function of $m$. Its calculation requires an observation time of duration $3 m \tau$.

We consider $\epsilon$, the fractional deviation of the EMAV relative to the modified Allan variance defined as follows:

$$
\epsilon=\frac{\operatorname{Mod} \hat{\sigma}_{y}^{2}(\tau)-\operatorname{Mod} \sigma_{y}^{2}(\tau)}{\operatorname{Mod} \sigma_{y}^{2}(\tau)} .
$$

The standard deviation $\sigma(\epsilon)$ of $\epsilon$ defines the relative uncertainty on the measurement of the modified Allan variance, due to the finite number of averaging cycles. We have

$$
\sigma(\epsilon)=\frac{1}{\operatorname{Mod} \sigma_{y}^{2}(\tau)}\left\{\sigma^{2}\left[\operatorname{Mod} \hat{\sigma}_{y}^{2}(\tau)\right]\right\}^{1 / 2}
$$

where $\sigma^{2}\left[\operatorname{Mod} \hat{\sigma}_{y}^{2}(\tau)\right]$ denotes the true variance of the EMAV such as

$$
\sigma^{2}\left[\operatorname{Mod} \hat{\sigma}_{y}^{2}(\tau)\right]=\left\langle\left[\operatorname{Mod} \hat{\sigma}_{y}^{2}(\tau)\right]^{2}\right\rangle-\left[\operatorname{Mod} \sigma_{y}^{2}(\tau)\right]^{2} .
$$

We assume that the fluctuations $y(t)$ are normally distributed $[10]$. One can therefore express $\left\langle\left[\operatorname{Mod} \hat{\sigma}_{y}^{2}(\tau)\right]^{2}\right\rangle$ as

$$
\begin{aligned}
m^{2}\left\langle\left[\operatorname{Mod} \hat{\sigma}_{y}^{2}(\tau)\right]^{2}\right\rangle \\
=\left(m^{2}+2 m\right)\left[\operatorname{Mod} \sigma_{y}^{2}(\tau)\right]^{2} \\
\quad+4 \sum_{p=1}^{m-1}(m-p)\left\{2 \sum_{i=1}^{n-1}(n-i) I_{n}+n I_{0}\right\}^{2}
\end{aligned}
$$

where $I_{n}$ are integrals which depend on $n$ and on the noise process. We have

$$
\begin{aligned}
8 \pi^{2} \tau^{2} n^{2} I_{n}= & \int_{0}^{\infty} \frac{S_{y}(f)}{f^{2}} \cos 6 \pi n p f \tau_{0} \\
& \times\left\{6 \cos 2 \pi f \tau_{0} i-4 \cos 2 \pi f \tau_{0}(i+n)\right. \\
& -4 \cos 2 \pi f \tau_{0}(i-n)+\cos 2 \pi f \tau_{0}(i+2 n) \\
& \left.+\cos 2 \pi f \tau_{0}(i-2 n)\right\} d f .
\end{aligned}
$$

For each noise component, the expression for $\sigma(\epsilon)$ can be deduced from the calculation of integrals involved in (20). These expressions are generally lengthy and complicated except for white phase and white frequency noise modulations, where integrals $I_{n}$ equal zero. We have limited the present analysis to these two noise components. We get for $\sigma(\epsilon)$

$$
\sigma(\epsilon)=\frac{2}{m}, \quad \text { for } \alpha=2 \text { and } 0 .
$$

We now compare (21) with previously published results related to the estimate of the Allan variance [5]. For a given time observation of duration $3 m \tau$, it can be easily deduced from [5] that the relative uncertainty on the estimate of the Allan variance varies asymptotically as $1.14 m^{-1 / 2}$ and $1.0 m^{-1 / 2}$ for $\alpha=2$ and 0 , respectively. For these two noise components, the uncertainty on the EMAV is larger than the uncertainty on the estimated Allan variance, but of the same order of magnitude.

\section{CONCLUSION}

We have calculated the analytical expression for the modified Allan variance for each component of the model usually considered to characterize random frequency fluctuations in precision oscillators. These expressions have been compared with previously published results and the link between the Allan variance and the modified Allan variance has been specified.

The uncertainty on the estimate of the modified Allan vari- ance has been studied and numerical values have been reported for white phase and white frequency noise modulations.

In conclusion, the modified Allan variance appears to be well suited for removing the ambiguity between white and flicker phase noise modulation. Nevertheless, the calculation of the modified Allan variance requires signal processing which is complicated, compared to the Allan variance. In the presence of white or flicker phase noise, the Allan variance cannot be easily deduced from the modified Allan variance. Furthermore, for a given source exhibiting different noise components, the determination of the Allan variance from the modified one is difficult to perform. For most of time-domain measurements, the use of the Allan variance is preferred.

\section{ACKNOWLEDGMENT}

The authors would like to express their thanks to Dr. Claude Audoin for constructive discussions and valuable comments on the manuscript.

\section{REFERENCES}

[1] D. W. Allan, "Statistics of atomic frequency standards," Proc. IEEE, vol. 54, pp. 221-230, Feb. 1966

[2] J. A. Barnes et al., "Characterization of frequency stability," IEEE Trens. Instrum. Meos., vol. IM-20, pp. 105-120, May 1971.

(3) L. S. Cutler and C. L. Searle, "Some aspects of the theory and measurement of frequency fluctuations in frequency standards," Proc. IEEE, vol 54, pp. 136-154, Feb. 1966.

[4] J. Rutman, "Characterization of phase and frequency instabilities in precision frequency sources: Fifteen years of progress," Proc. IEEE, vol 66, pp. 1048-1075, Sept. 1978.

15] P. Lesage and C. Audoin, "Effect of dead-time on the estimation of the two-sample variance," IEEE Trans. Instrum. Meas., vol. IM-28, pp. 6-10, Mar. 1979.

16] J. J. Snyder, "Algorithm for fast digital analysis of interference fringes," Appl Opt., vol 19, pp. 1223-1225, Apr. 1980.

[7] -, "An ultra-high resolution frequency meter," in Proc. 35th Annu. Symp. Frequency Control (Fort Monmouth, NJ), 1981, pp. 464-469.

18] D. W. Allan and J. A. Barnes, "A modified Allan variance with increased oscillator characterization ability," in Proc. 35th Annu. Symp. Frequency Control (Fort Monmouth, NJ), 1981, pp. 470474.

[9] B. Picinbono, "Processus de diffusion et stationnarité," C.R. Acad. Sci., vol 271, pp. 661-664, Oct. 1970.

[10] P. Lesage and C. Audoin, "Characterization of frequency stability: Uncertainty due to the finite number of measurements," IEEE Trans. Instrum. Meas., vol IM-22, pp. 157-161, June 1973. 
From: Proceedings of the 15th Annual PTTI Meeting, 1983.

THE MEASUREMENT OF LINEAR FREQUENCY DRIFT IN OSCILLATORS

\author{
James A. Barnes \\ Alstron, Inc., Austin, Texas
}

\begin{abstract}
A linear drift in frequency is an important element in most stochastic models of oscillator performance. Quartz crystal osclllators often have drifts in excess of a part in ten to the tenth power per day. Even commercial ceslum beam devices often show drifts of a few parts in ten to the thirteenth per year. There are many ways to estimate the drift rates from data samples (e.g., regress the phase on a quadratic; regress the frequency on a linear; compute the simple mean of the first difference of frequency; use Kalman filters with a drift term as one element in the state vector; and others). Although most of these estimators are unblased, they vary in efficiency (1.e., confidence intervals). Further, the est1mation of confidence intervals using the standard analysis of variance (typically associated with the specific estimation technique) can give amazingly optimistic results. The source of these problems is not an error in, say, the regressions techniques, but rather the problems arise from correlations within the residuals. That is, the oscillator model is often not consistent with constraints on the analysis technique or, in other words, some specific analysis techniques are often inappropriate for the task at hand.

The appropriateness of a specific analysis technique is critlcally dependent on the oscillator model and can of ten be checked with a simple "whiteness" test on the residuals. Following a brief review of linear regression techniques, the paper provides guidelines for appropriate drift estimation for various oscillator models, including estimation of realistic confidence intervals for the drift.
\end{abstract}

\title{
I. INTRODUCTION
}

Almost all oscillators display a superposition of random and deterministic variations in frequency and phase. The most typical model used is [1]:

$$
X(t)=a+b \cdot t+D r \cdot t^{2} / 2+\phi(t)
$$

where $X(t)$ is the time (phase) error of the oscillator (or clock) relati:e to some standard; $a, b$, and $\mathrm{Dr}$ are constants for the particular clock; and $\psi(t)$ is the random part. $X(t)$ is a random variable by virtue of its dependence on $\phi(t)$.

* See Appendix Note \# 36 
Even though one cannot predict future values of $X(t)$ exactly, there are often significant autocorrelations within the random parts of the model. These correlations allow forecasts which can significantly reduce clock errors. Errors in each element of the model (Eq. 1) contribute their own uncertainties to the prediction. These time uncertainties depend on the duration of the forecast interval, $\tau$, as shown below in Table 1 :

TABLE 1. GROWTH OF TIME ERRORS

$\frac{\begin{array}{l}\text { MODEL ELEMENT } \\ \text { NAME }\end{array}}{\text { Initial Time Error }}$
Initial Freq Error
Frequency Drift
Random Variations

\author{
CLOCK \\ PARAMETER
}

a

b

$\mathrm{Dr}$

$\phi(t)$

\section{RMS TIME \\ ERROR}

Constant

$\sim \tau$

$\sim \tau^{2}$

$\sim \tau^{3 / 2 \star}$

*The growth of time uncertainties due to the random component, $\phi(t)$, can have various time dependencies. The three-halves power-law shown here is a "worst case" model. [2]

One of the most significant points provided by Table 1 is that eventually, the linear drift term in the model over-powers all other uncertainties for sufficiently long forecast intervals! While one can certainly measure (1.e., estimate) the drift coefficient, $\mathrm{Dr}$, and make corrections, there must always remain some uncertainty in the value used. That is, the effect of a drift correction based on a measurement of. Dr, is to reduce (hopefully!) the magnitude of the drift error, but not remove it. Thus, even with drift corrections, the drift term eventually dominates all time uncertainties in the model.

As with any random process, one wants not only the point estimate of a parameter, but one also wants the confidence interval. For example, one might be happy to know that a particular value (e.g., clock time error) can be estimated without bias, he may still want to know how large an error range he should expect. Clearly, an error in the drift estimate (see Eq. 1) leads directly to a time error and hence the drift confidence interval leads directly to a confidence interval for the forecast time.

\section{LEAST SQUARES REGRESSION OF PHASE ON A QUADRATIC}

A conventional least squares regression of oscillator phase data on a quadratic function reveals a great deal about the general problems. A slight modification of Eq. 1 provides a conventional model used in regression analysis[3]:

$$
X(t)=a+b \cdot t+c \cdot t^{2}+\phi(t)
$$


where $c=D r / 2$. In regression analysis, it is customary to use the symbol "Y" as the dependent variable and " $X$ " as the independent variable. This is in conflict with usage in time and frequency where " $X$ " and " $Y$ " ( $t$ Ime error and frequency error, respectively) are dependent on a coarse measure of $t$ ime, $t$, the independent variable. This paper will follow the time and frequency custom even though this may cause some confusion in the use of regression analysis text books.

The model given by Eq. 2 is complete if the random component, $\phi(t)$, is a white nolse (i.e., random, uncorrelated, normally distributed, zero mean, and finite variance).

\section{EXAMPLE}

One must emphasize here that ALL results regarding parameter error magnitudes and their distributions are totally dependent on the adequacy of the model. A primary source of errors is of ten autocorrelation of the residuals (contrary to the explicit model assumptions). While simple visual inspection of the residuals is often sufficient to recognize the autocorrelation problem, "whiteness tests" can be more objective and precise.

This section analyzes a set of 94 hourly values of the time difference between two oscillators. Figure 1 is a plot of the time difference (measured in microseconds) between the two oscillators. The general curve of the data along with the general expectation of frequency drift in crystal oscillators leads one to try the quadratic behavior (Model \#1; models $\# 2$ and $\# 3$ discussed below). While it is not common to find white phase nolse on oscillators at levels indicated on the plot, that assumption will be made temporarily. The results of the regression are summarized in a conventional Analysis of Variance, Table 2.

\section{TABLE 2. ANALYSIS OF VARIANCE QUADRATIC FIT TO PHASE} (Units: seconds squared)

SOURCE

Regression

Residuals

Total
SUM OF SQUARES

$2.32 E-9$

$4 \cdot 26 E-12$

$2.329 E-9$

Coefficient of simple determination

Parameters:

$$
\begin{array}{lll}
\hat{a}=1.10795 \mathrm{E}-5 & (\text { seconds }) & t \text {-ratio }=161.98 \\
\hat{b}=1.4034 \mathrm{E}-10(\mathrm{sec} / \mathrm{sec}) & t \text {-ratio }=152.02 \\
\hat{c}=-3.7534 \mathrm{E}-16\left(\mathrm{sec} / \mathrm{sec}^{2}\right) & t-\mathrm{ratio}=-143.51
\end{array}
$$




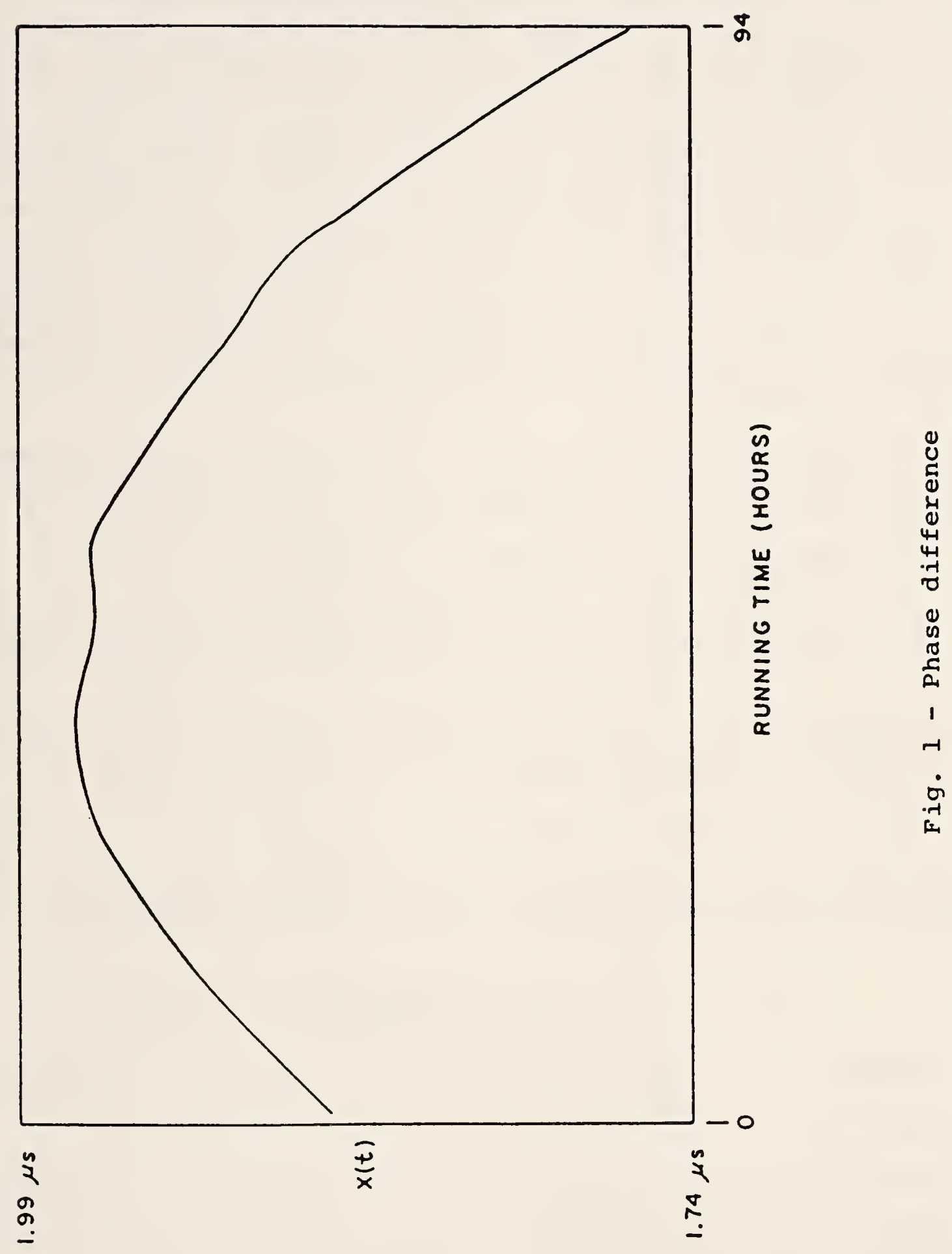




\section{$\hat{D r}=2 \hat{c}=-7.507 \mathrm{E}-16$ (about $-6.5 \mathrm{E}-11$ per day) \\ Std. Error $=0.05231 \mathrm{E}-16$}

(Note: $\hat{a}$ is the value estimated for the a-parameter, etc.)

The Analysis of Variance, Table 2, above suggests an impressive fit of the data to a quadratic function, with $99.71 \%$ of the variations in the data "explained" by the regression. The estimated drift coefficient, $\mathrm{Dr}$, is $-7.507 \mathrm{E}-16\left(\mathrm{sec}_{\mathrm{sec}}{ }^{2}\right)$ or about $-6.5 \mathrm{E}-11$ per day --- 143 times the indicated standard error of the estimate. However, Figure 2, a plot of the residuals, reveals significant autocorrelations even visually and without sensitive tests. (The autocorrelations can be recognized by the essentially smooth variations in the plot. See Fig. 5 as an example of a more nearly white data set.) It is true that the regression reduced the peak-to-peak deviations from about 18 microseconds to less than one microsecond. It is also true that the drift rate is an unbiased estimate of the actual drift rate, but the model assumptions are NOT consistent with the autocorrelation visible in Fig. 2. This means that the confidence intervals for the parameters are not reliable. In fact, the analysis to follow will show just how extremely optimistic these intervals really are.

At this point we can consider at least two other simple analysis schemes which might provide more realistic estimates of the drift rate and its variance. Each of the two analysis schemes has its own implicit model; they are:

(2) Regress the beat frequency on a straight line.

(Model: Linear frequency drift and white FM.)

(3) Remove a simple average from the second difference of the phase.

(Model: Linear frequency drift and random walk FM.)

Continuing with scheme 2 , above, the (average) frequency, $\bar{Y}(t)$, is the first difference of the phase data divided by the time interval between successive data points. The regression model is:

$$
\bar{Y}(t)=b+D r \cdot t+\varepsilon(t)
$$

where $\varepsilon(t)=\left[\phi\left(t+\tau_{0}\right)-\phi(t)\right] / \tau_{0}$. Following standard regression procedures as before, the results are summarized in another Analysis of Variance Table, Table 3 .

TABLE 3. ANALYSIS OF VARIANCE LINEAR FIT TO FREQUENCY (Units: $\sec ^{2} / \sec ^{2}$ )

SOURCE

Regression

Res1duals

Total

(Note:

SUM OF SQUARES

$1.879 E-18$

$2.084 E-20$

$1.899 E-18$ d.f.

2

91

93

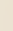

MEAN SQUARE

$2.29 E-22$

$2.042 E-20$

Taking the first differences of the original data set reduces the number of data points from 94 to 93. )

* See Appendix Note \# 37 


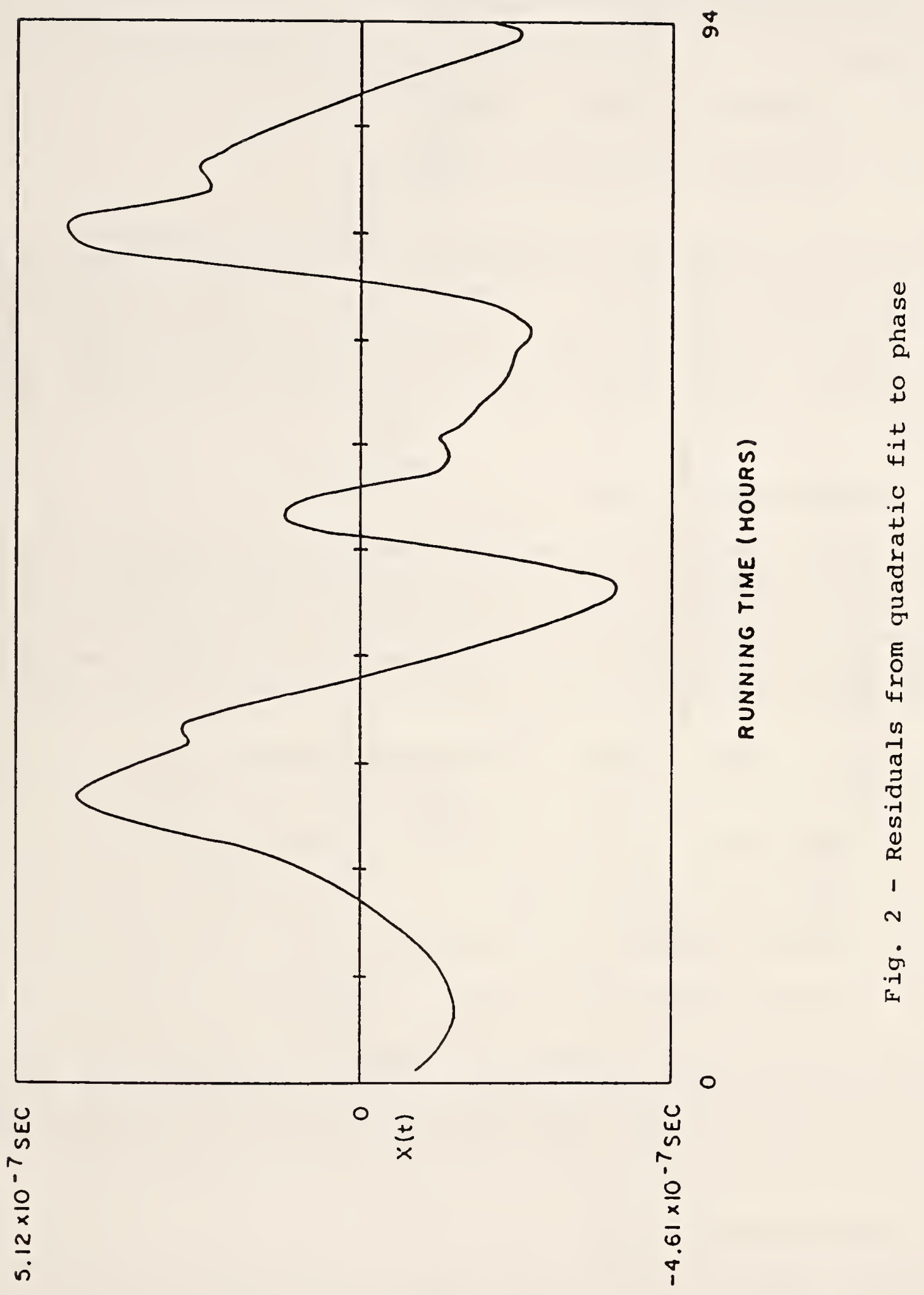


Parameters :

$$
\begin{aligned}
\hat{b}= & 1.049 \mathrm{E}-10(\mathrm{sec} / \mathrm{sec}) \quad t-\operatorname{rat10}=3.32 \\
\hat{\mathrm{D} r}= & -7.635 \mathrm{E}-16\left(\mathrm{sec} / \mathrm{sec}^{2}\right) \quad t-\operatorname{rat10}=-4.70 \\
& \text { Std. Error }=0.1624 \mathrm{E}-16 \quad\left(\mathrm{sec}^{\mathrm{sec}} \mathrm{sec}^{2}\right)
\end{aligned}
$$

While the drift rate estimates for the two regressions are comparable in value $(-7.507 E-16$ and $-7.635 E-16)$, the standard errors of the drift estimates have gone from $0.052 \mathrm{E}-16$ to $0.162 \mathrm{E}-16$ (a factor of 3 ). The linear regression's coefficlent of simple determination 1 s $96.05 \%$ compared to $99.17 \%$ for the quadratic f1t. Figure 3 shows the residuals from the linear fit and they appear more nearly white. A cumulative perlodogram[4] is a more objective test of whiteness, however. The perlodogram, F1g. 4, does not find the residuals acceptable at all.

\section{DRIFT AND RANDOM WALR FM}

In the absence of nolse, the second difference of the phase would be a constant, $\mathrm{Dr}^{\cdot} \tau_{\mathrm{O}}{ }^{2}$. If one assumes that the second difference of the noise part is white, then one has the classic problem of estimating a constant (the drift term), in the presence of white noise (the second difference of the phase noise). Of course, the optimum estimate of the drift term is just the simple mean of the second difference divided by $\tau_{0}{ }^{2}$. The results are sumarized below, Table 4:

\section{TABLE 4. SIMPLE MEAN OF SECOND DIFFERENCE PHASE}

$$
\begin{aligned}
& \text { Simple mean } \hat{\mathrm{D} r}=-6.709 \mathrm{E}-16 \quad \text { t-rat1o }=-2.45 \\
& \text { Degrees of Freedom }=91 \\
& \text { Standard Deviation } \hat{\mathrm{s}}=26.2405 \mathrm{E}-16 \\
& \text { Standard Deviation of the Mean }=2.7358 \mathrm{E}-16
\end{aligned}
$$

Figure 5 shows the second difference of the phase after the mean was subtracted. Visually, the data appear reasonably white, and the periodogram, Fig. 6, cannot reject the null hypothesis of whiteness. Now the standard error of the drift term is $2.735 \mathrm{E}-16,52$ times larger than that computed for the quadratic fit! Indeed, the estimated drift term is only 2.45 times 1ts standard error.

\section{SUMMARY OF TESTS}

The analyses reported above were all performed on a single data set. In order to verify any conclusions, all three analyses used above were performed a total of four times on four different data sets from the same pair of oscillators. Table 5 summarizes the results: 


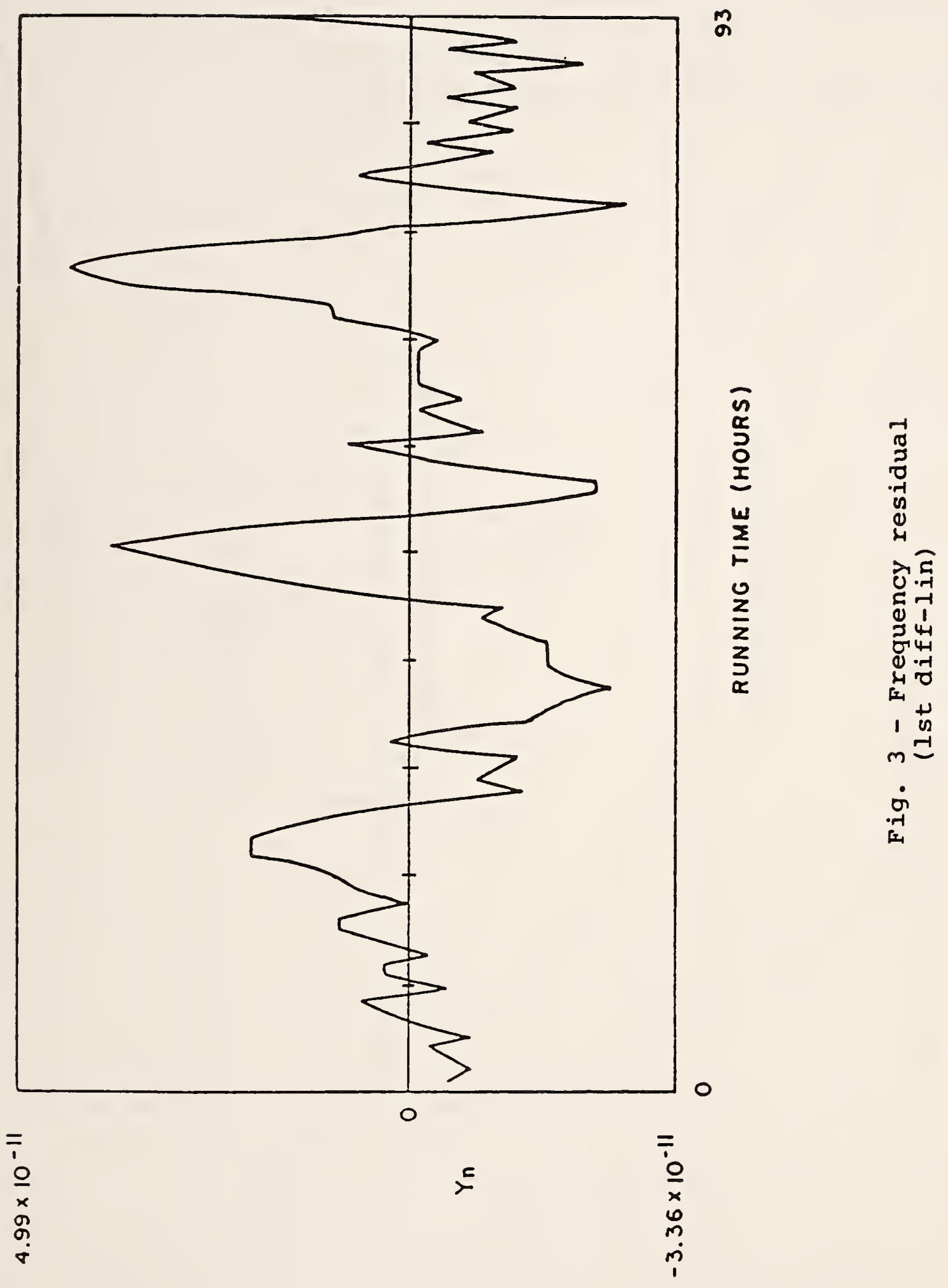




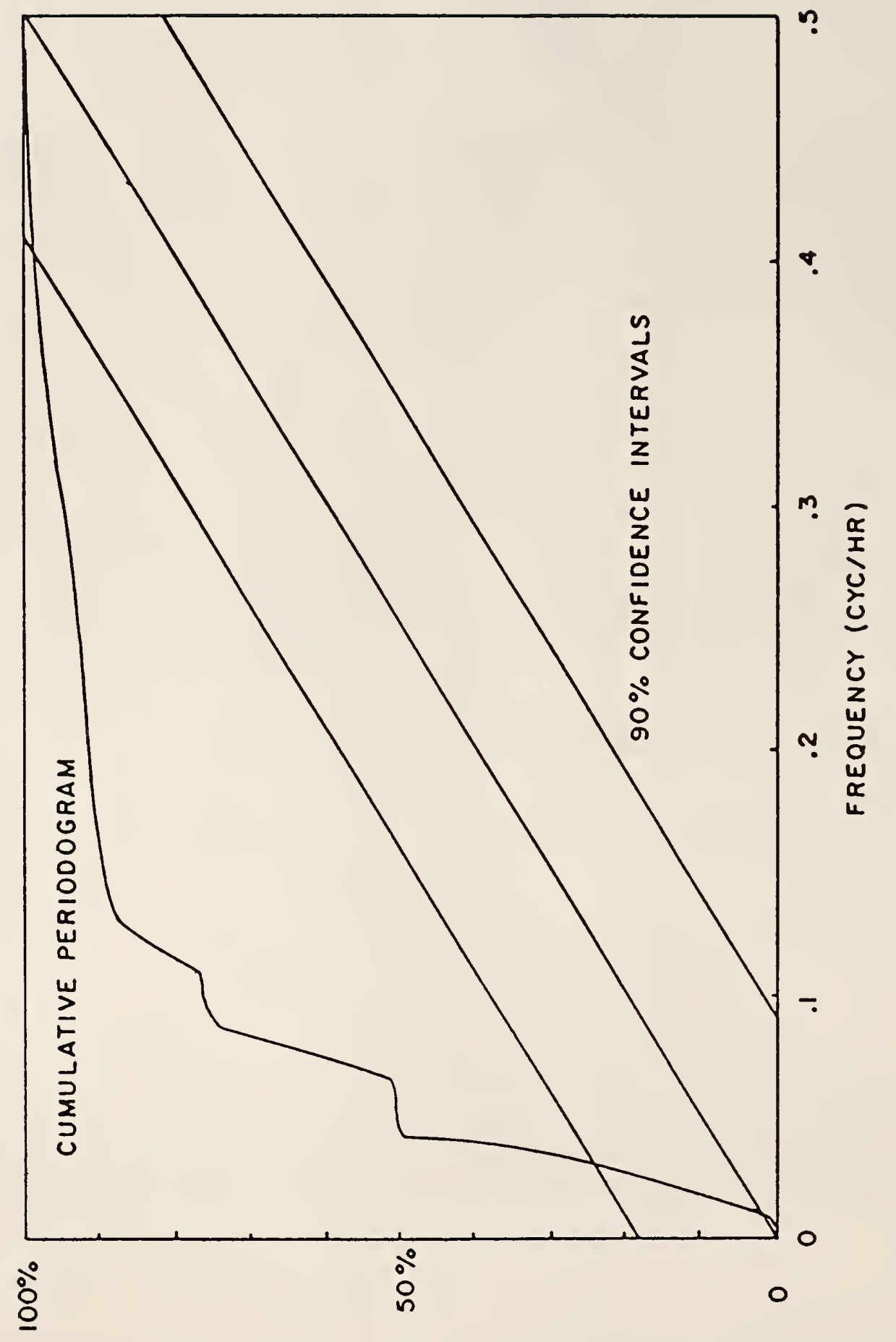

 


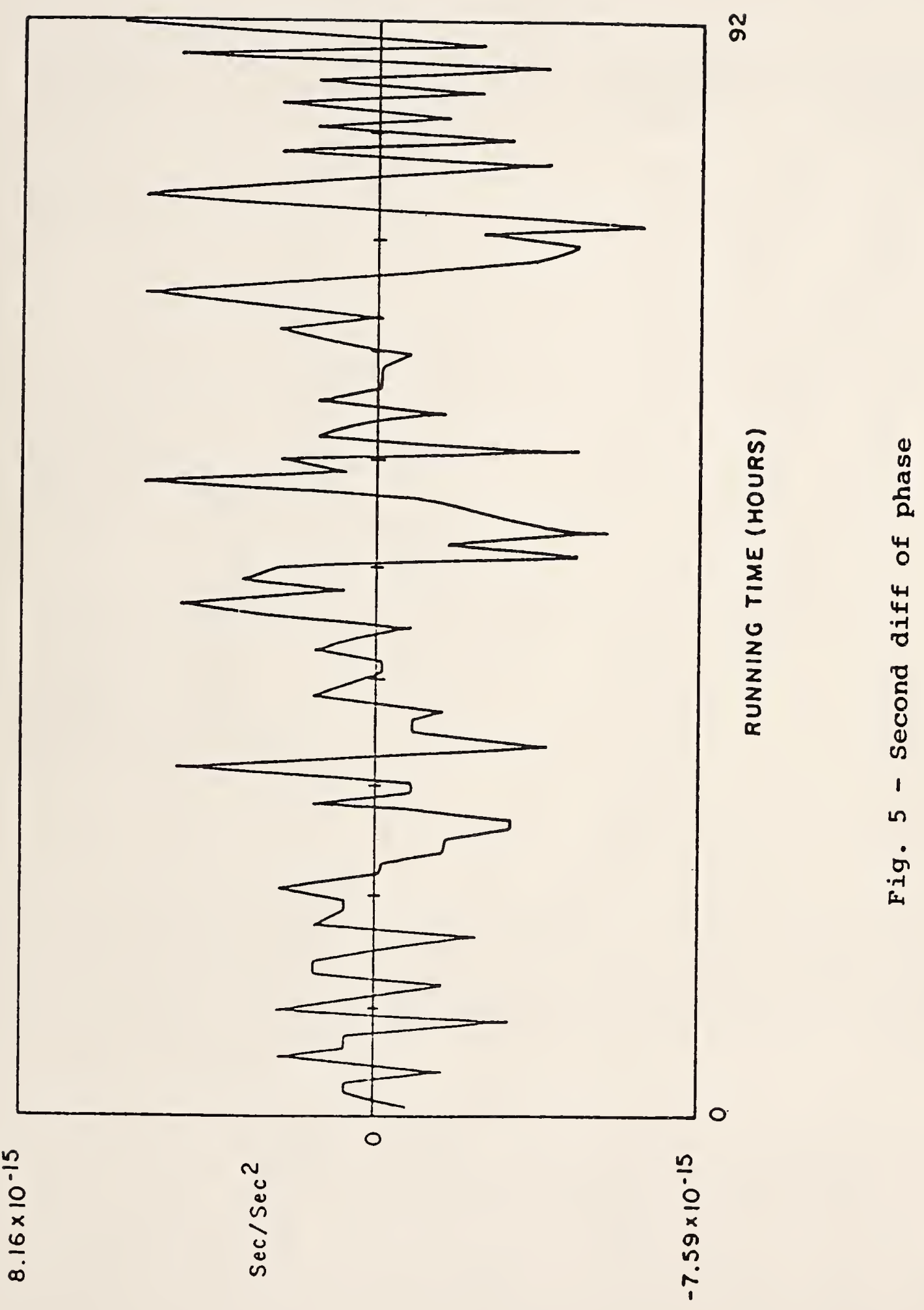




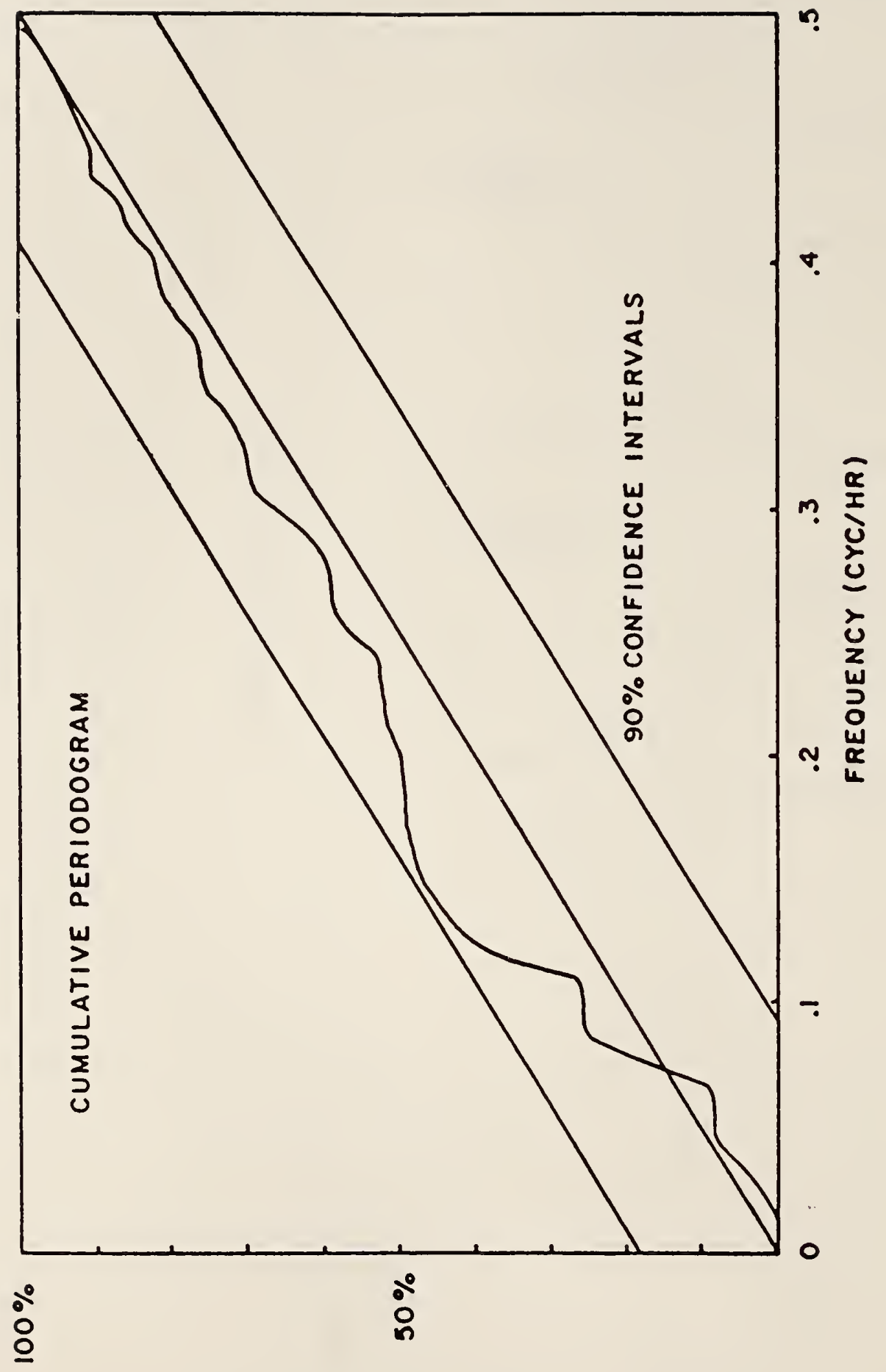

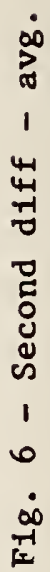


TABLE 5. SUMMARY OF DRIFT ESTIMATES

(Units of $1 . \mathrm{E}-16 \mathrm{sec} / \mathrm{sec}^{2}$ )

\begin{tabular}{|c|c|c|c|}
\hline $\begin{array}{l}\text { ESTIMATION } \\
\text { PROCEDURE } \\
\& \text { MODEL } \\
\end{array}$ & $\begin{array}{c}\text { DRIFT } \\
\text { ESTIMATE } \\
\end{array}$ & $\begin{array}{c}\text { COMPUTED } \\
\text { STANDARD } \\
\text { ERROR } \\
\end{array}$ & $\begin{array}{c}\text { PASS } \\
\text { WHITENESS } \\
\text { TESTS? } \\
\end{array}$ \\
\hline $\begin{array}{l}\text { Quad Fit } \\
\text { (White PM } \\
\text { and Drift) }\end{array}$ & $\begin{array}{l}-7.507 \\
-8.746 \\
-6.479 \\
-6.468\end{array}$ & $\begin{array}{l}.0523 \\
.0493 \\
.0645 \\
.0880\end{array}$ & $\begin{array}{l}\text { No } \\
\text { No } \\
\text { No } \\
\text { No }\end{array}$ \\
\hline $\begin{array}{l}\text { lst Difference } \\
\text { Linear Fit } \\
\text { (White FM } \\
\text { and Drift) }\end{array}$ & $\begin{array}{l}-7.635 \\
-8.558 \\
-6.443 \\
-6.253\end{array}$ & $\begin{array}{l}.162 \\
.206 \\
.192 \\
.295\end{array}$ & $\begin{array}{l}\text { No } \\
\text { No } \\
\text { No } \\
\text { No }\end{array}$ \\
\hline $\begin{array}{l}\text { Second Difference } \\
\text { Less Mean } \\
\text { (Random Walk } \\
\text { FM and Drift) }\end{array}$ & $\begin{array}{l}-6.710 \\
-7.462 \\
-6.870\end{array}$ & $\begin{array}{r}2.736 \\
9.335 \\
3.424\end{array}$ & $\begin{array}{l}\text { Yes } \\
\text { No } \\
\text { Yes }\end{array}$ \\
\hline & -6.412 & 3.543 & Yes \\
\hline
\end{tabular}

One can calculate the sample means and variances of the drift estimates for each of the three procedures listed in Table 5, and compare these "external" estimates with those values listed in the table under "Computed Standard Error," the "Internal" estimates. Of course the sample size is small and we do not expect high precision in the results, but some conclusions can be drawn. The comparisons are shown in Table 6.

It is clear that the quadratic fit to the data displays a very optimistic internal estimate for the standard deviation of the drift rate. Other conclusions are not so clear cut, but still some things can be sald. Considering Table 5, the "2nd Diff - Mean" residuals passed the whiteness test three times out of four. The external estimate of the drift standard deviation lies between the internal estimates based on the first and second differences. Since the oscillators under test were crystal oscillators, one expects flicker FM to be present at some level. One also expects the flicker FM behavior to lie between white FM and random walk FM. This may be the explanation of the observed standard deviations, noted in Table 6. 
PROCEDURE

(Mode 1)

Quad Fit (White PM)

lst Diff - Lin

(White FM)

2nd Diff - Mean

(Rand WIk FM)
EXTERNAL ESTIMATE

(Std. Dev. of Drift Estimates from Col. \#2, Table 5)

1.08

1.08

0.44
INTERNAL ESTIMATE (RMS Computed Std. Dev. Col. 非3, Table 5)

0.065

0.203

\section{DISCUSSION}

In all three of the analysis procedures used above, more parameters than just the frequency drift rate were estimated. Indeed, this is generally the case. The estimated parameters included the drift rate, the variance of the random (white) noise component, and other parameters appropriate to the specific model (e.g., the initial frequency offset for the first two models). If these other parameters could be known precisely by some other means, then methods exist to exploit this knowledge and get even better estimates of the drift rate. The real problems, however, seem to require the estimate of several parameters in addition to the drift rate, and it is not appropriate to just ignore unknown model parameters.

To this point, we have considered only three, rather ideal oscillator models, and seldom does one encounter such simpliclty. Typical models for commercial cesium beam frequency standards include white FM, random walks FM, and frequency drift. Unfortunately, none of the three estimation routines discussed above are appropriate to such a model. This problem has been solved in some of the recent work of Jones and Tryon $[5],[6]$. Their estimation routines are based on Kalman Filters and maximum likelihood estimators and these methods are appropriate for the more complex models. For detalls, the reader is referred to the works of Jones and Tryon.

Still left untreated are the models which, in addition to drift and other noises, incorporate flicker noises, elther in PM or FM or both. In principle, the methods of Jones and Tryon could be applied to Kalman Filters which incorporate empirical flicker models[7]. To the author's knowledgi, however, no such analyses have been reported.

\section{CONCLUSIONS}

There are two primary conclusions to be drawn:

(1) The estimation of the linear frequency drift rates of oscillators and the inclusion of realistic confidence intervals for these 
estimates are critically dependent on the adequacy of the model used and, hence the adequacy of the analysis procedures.

(2) The estimation of the drift rate must be carrled along with the estimation of any and all other. model parameters which are not known precisely from other considerations (e.g., initial frequency and time offsets, phase noise types, etc.)

More and more, sclentists and englneers require clocks which can be relied on to maintain accuracy relative to some master clock. Not only is it important to know that on the average the clock runs well, but it is essential to have some measure of time 1mprecision as the clock ages. For example, the uncertainties mlght be expressed as, say,, $90 \%$ certain that the clock will be within 5 microseconds of the master two weeks after synchronization. Such measures are what statisticians call "Interval estimates" (In contrast to point estimates) and their estimations require interval estimates of the clock's model parameters. Clearly, the parameter estlmation routines must be reliable and based on sound measurement practices. Some inappropriate estimation routines can be applied to clocks and oscillators and give dangerously optimistic forecasts of performance. 
APPENDIX A

\section{REGRESSION ANALYSIS}

(Equally Spaced Data)

We begin with the continuous model equation:

$$
X(t)=a+b \cdot t+c \cdot t^{2}+\phi(t)
$$

We assume that the data is in the form of discrete readings of the dependent variable $X(t)$ at the regular intervals given by:

$$
t=n \tau_{0}
$$

Equation (Al) can then be written in the obvious form:

$$
x_{n}=a+\tau_{0} b \cdot n+\tau_{0}^{2} c \cdot n^{2}+\phi\left(n \tau_{0}\right)
$$

for $n=1,2,3 \ldots, N$.

Next, we define the matrices:

$$
\begin{aligned}
& \underline{N}=\left[\begin{array}{ccc}
1 & 1 & 1 \\
1 & 2 & 4 \\
1 & 3 & 9 \\
\cdot & \cdot & \cdot \\
\cdot & \cdot & \cdot \\
1 & N & N^{2}
\end{array}\right] \quad \underline{x}=\left[\begin{array}{c}
x_{1} \\
x_{2} \\
x_{3} \\
\cdot \\
\cdots \\
x_{N}
\end{array}\right] \\
& \underline{T}=\left[\begin{array}{ccc}
1 & 0 & 0 \\
0 & \tau_{0} & 0 \\
0 & 0 & \tau_{0}^{2}
\end{array}\right]
\end{aligned}
$$

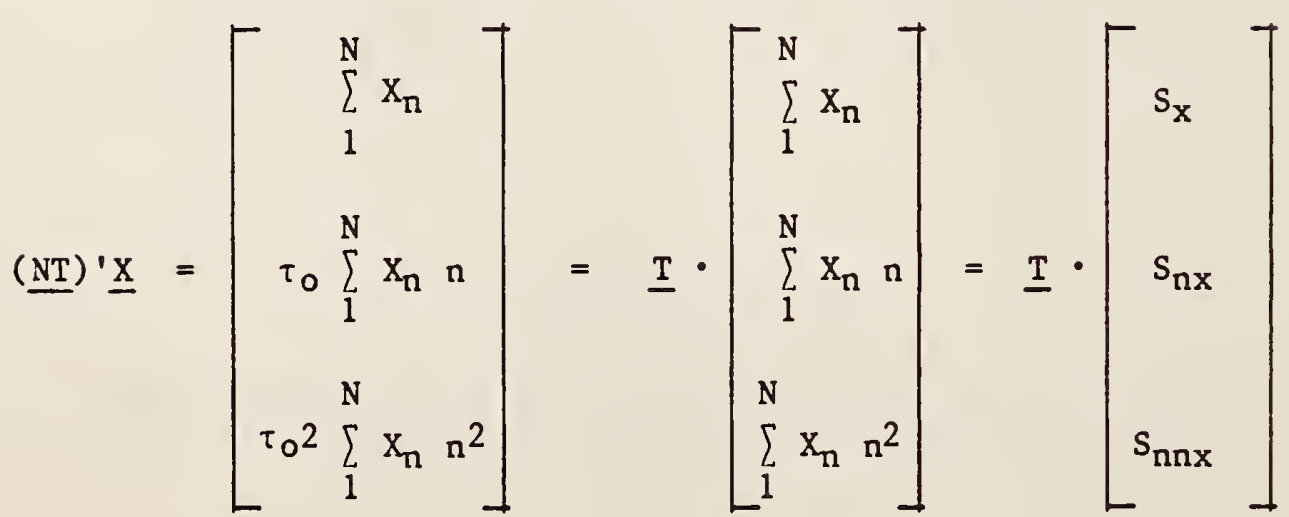


where four quantities must be calculated from the data:

$$
\begin{array}{ll}
s_{x}=\sum_{n=1}^{N} x_{n} & s_{n n x}=\sum_{n=1}^{N} x_{n} n^{2} \\
s_{n x}=\sum_{n=1}^{N} x_{n} n & s_{x x}=\sum_{n=1}^{N} x_{n}^{2}
\end{array}
$$

Define

$$
\underline{B}=\left[\begin{array}{l}
a \\
b \\
c
\end{array}\right]
$$

With these definitions, Eq. A3 can be rewritten in the matrix form:

$$
\underline{X}=\underline{N} \underline{T} \underline{B}+\underline{\varepsilon}
$$

and the coefficients, B, which minimize the squared errors are given by:

$$
\underline{\hat{B}}=\left|\begin{array}{c}
\hat{a} \\
\hat{b} \\
\hat{c}
\end{array}\right|=\underline{T} \cdot\left(\underline{N^{\prime} N}\right)^{-1} \cdot\left(N^{\prime} X\right)
$$

The advantage of evenly spaced data for these regressions is that, with a bit of algebra, the matrix, $\left(\underline{N^{\prime} N}\right)^{-1}$, can be written down in closed form:

$$
\left(N^{\prime} N\right)^{-1}=\left[\begin{array}{lll}
A & B & C \\
B & D & E \\
C & E & F
\end{array}\right] \cdot 1 / G
$$

where

$$
\begin{aligned}
& A=3[3(N+1)+2] \\
& B=-18(2 N+1) \\
& C=30 \\
& D=12(2 N+1)(8 N+11) /[(N+1)(N+2)] \\
& E=-180 /(N+2) \\
& F=180 /[(N+1)(N+2)]
\end{aligned}
$$


and

$$
G=N(N-1)(N-2)
$$

Also, the inverse of $T$ is just:

$$
\underline{T}^{-1}=\left[\begin{array}{ccc}
1 & 0 & 0 \\
0 & 1 / \tau_{0} & 0 \\
0 & 0 & 1 / \tau_{0}{ }^{2}
\end{array}\right]
$$

The complete solution for the regression parameters can be summarized as follows:

There are four quantities which must calculate from the data:

$$
\begin{array}{ll}
s_{x}=\sum_{n=1}^{N} x_{n} & s_{n n x}=\sum_{n=1}^{N} x_{n} n^{2} \\
s_{n x}=\sum_{n=1}^{N} x_{n} n & s_{x x}=\sum_{n=1}^{N} x_{n}{ }^{2}
\end{array}
$$

for $n=1,2,3, \ldots, N$. Based on these four quantities, the regression parameters are calculated from the seven following equations:

$$
\begin{aligned}
& \hat{a}=\left(A S_{x}+\tau_{0} B S_{n x}+\tau_{0}{ }^{2} C S_{n n x}\right) / G \\
& \hat{b}=\left(B S_{x}+\tau_{0} D S_{n x}+\tau_{0}{ }^{2} E S_{n n x}\right) /\left(G \tau_{0}\right) \\
& \hat{c}=\left(C S_{x}+\tau_{0} E S_{n x}+\tau_{0}{ }^{2} F S_{n n x}\right) /\left(G \tau_{0}{ }^{2}\right) \\
& \hat{\sigma}^{2}=\left(S_{x x}-\hat{a} S_{x}-\tau_{0} \hat{b} S_{n x}-\tau_{0}{ }^{2} \hat{c} S_{n n x}\right) /(N-3) \\
& \hat{\sigma}_{a}{ }^{2}=\hat{\sigma}^{2} A / G \\
& \hat{\sigma}_{b}^{2}=\hat{\sigma}^{2} D /\left(G \tau_{0}{ }^{2}\right) \\
& \hat{\sigma}_{c}^{2}=\hat{\sigma}^{2} F /\left(G \tau_{0}{ }^{4}\right)
\end{aligned}
$$


where the coefficients $A, B, C$, etc., are given by:

$$
\begin{aligned}
& A=3[3 N(N+1)+2] \\
& B=-18(2 N+1) \\
& C=30 \\
& D=12(2 N+1)(8 N+11) /[(N+1)(N+2)] \\
& E=-180 /(N+2) \\
& F=180 /[(N+1)(N+2)]
\end{aligned}
$$

and

$$
G=N(N-1)(N-2) \text {. }
$$

In matrix form, the error variance for forecast values is:

$$
\operatorname{Var}\left(\hat{X}_{k}\right)=\hat{\sigma}^{2}\left[1+\underline{N_{k}}\left(\underline{N^{\prime} N}\right)^{-1} \underline{N_{k}}\right]
$$

where $N_{k}{ }^{\prime}=\left[\begin{array}{lll}1 & n_{0} & n_{0}\end{array}\right]$ and $\tau_{0} n_{0}$ is the date for the forecast point, $\hat{x}_{k}$. That 1s, $n_{0}=N+K$ and $K$ is the number of lags past the last data point at lag $N$. 
The matrixes $\left(N^{\prime} N\right)^{-1}$ for the linear fit and cubic fit, which correspond to Eq. A6 in Appendix $\mathrm{A}$ are as follows:

For the linear fit:

$$
\left(N^{\prime} N\right)^{-1}=\left[\begin{array}{ll}
A & B \\
B & C
\end{array}\right] 1 / D
$$

where

$$
\begin{aligned}
& A=2(2 \mathrm{~N}+1) \\
& B=-6 \\
& C=12 /(\mathrm{N}+1)
\end{aligned}
$$

and

$$
D=N(N-1)
$$

For the cubic fit:

$$
\left(N^{\prime} N\right)^{-1}=\left[\begin{array}{llll}
\text { A } & \text { B } & \text { C } & \text { D } \\
\text { B } & \text { E } & \text { F } & \text { G } \\
\text { C } & \text { F } & \text { H } & \text { I } \\
\text { D } & \text { G } & \text { I } & \text { J }
\end{array}\right] 1 / K
$$

where

$$
\begin{aligned}
& A=8(2 N+1)(N+N+3) \\
& B=-20\left(6 N^{2}+6 N+5\right) \\
& C=120(2 N+1) \\
& D=-140 \\
& E=200\left(6 N^{4}+27 N^{3}+42 N^{2}+30 N+11\right) / L \\
& F=-300(N+1)(3 N+5)(3 N+2) / L \\
& G=280\left(6 N^{2}+15 N+11\right) / L
\end{aligned}
$$




$$
\begin{aligned}
& \mathrm{H}=360(2 \mathrm{~N}+1)(9 \mathrm{~N}+13) / \mathrm{L} \\
& \mathrm{I}=-4200(\mathrm{~N}+1) / \mathrm{L} \\
& J=2800 / \mathrm{L}
\end{aligned}
$$

and

$$
\begin{aligned}
& K=N(N-1)(N-2)(N-3) \\
& L=(N+1)(N+2)(N+3)
\end{aligned}
$$

The restrictions on these equations are that the data is evenly spaced beginning with $n=1$ to $n=N$, and no missing values. For error estimates (and their distributions) to be valid, the residuals must be random, uncorrelated, (1.e., white). 


\section{REPERENCES}

1. D.W. Allan, "The Measurement of Frequency and Frequency Stability of Precision Oscillators," Proc. 6th PTTI Planning Meeting, pl09 (1975).

2. J.A. Barnes, et al., "Characterization of Frequency Stability," Proc IEEE Trans. on Inst. and Meas., IM-20, p105 (1971).

3. N.R. Draper and H. Smith, "Applied Regression Analysis," John Wiley and Sons, N.Y., (1966).

4. G.E.P. Box and G.M. Jenkins, "Time Series Analysis," Holden-Day, San Francisco, p294, (1970).

5. P.V. Tryon and R.H. Jones, "Estimation of Parameters in Models for Cesium Beam Atomlc Clocks," NBS Journal of Research, Vol. 88, No. 1, Jan-Feb. 1983.

6. R.H. Jones and P.V. Tryon, "Estimating Time from Atomic Clocks," NBS Journal of Research, Vol. 88, No. 1, Jan-Feb. 1983.

7. J.A. Barnes and S. Jarvis, Jr., "Efficient Numerical and Analog Modeling of Flicker Noise Processes," NBS Tech. Note 604, (1971). 


$$
71
$$




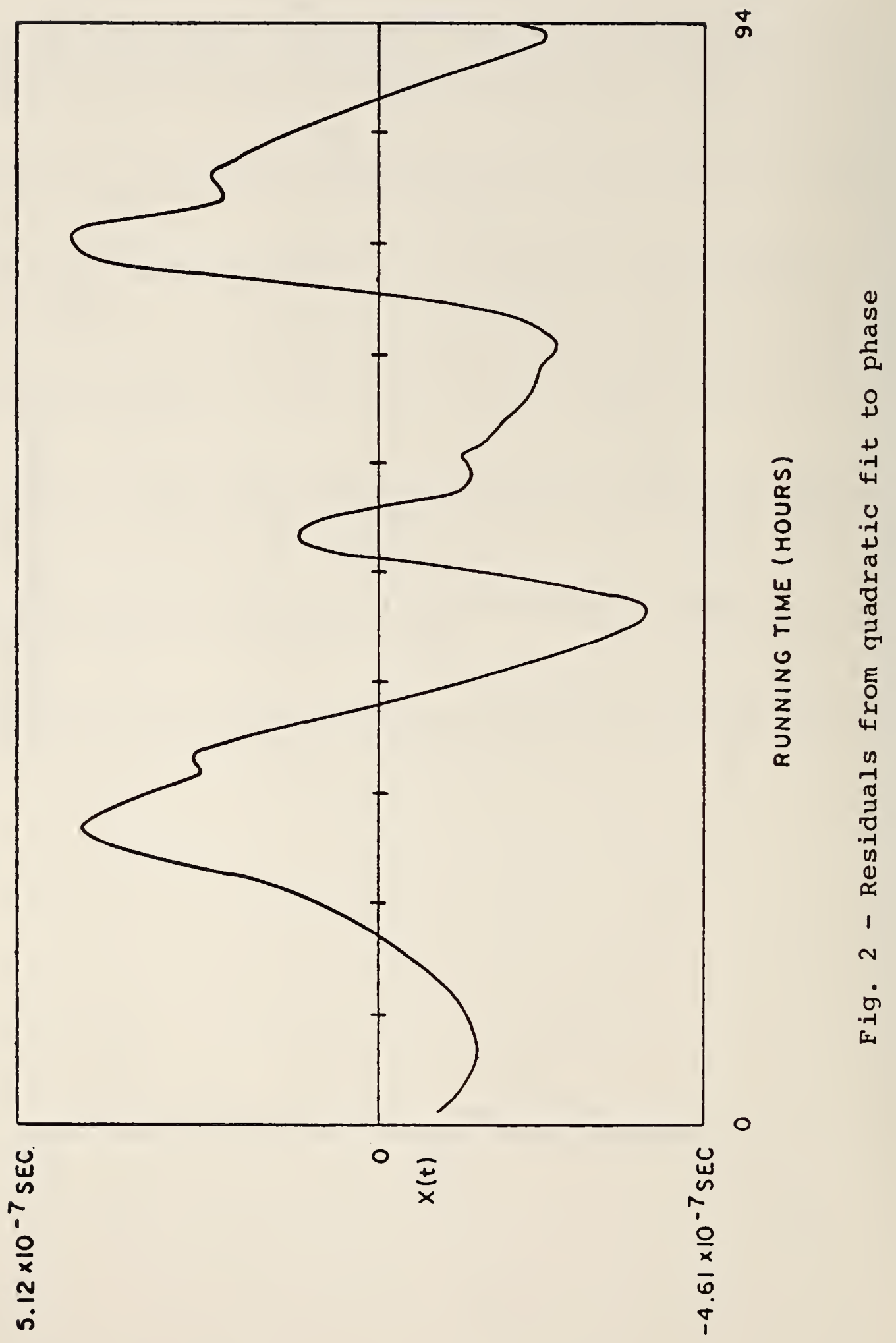




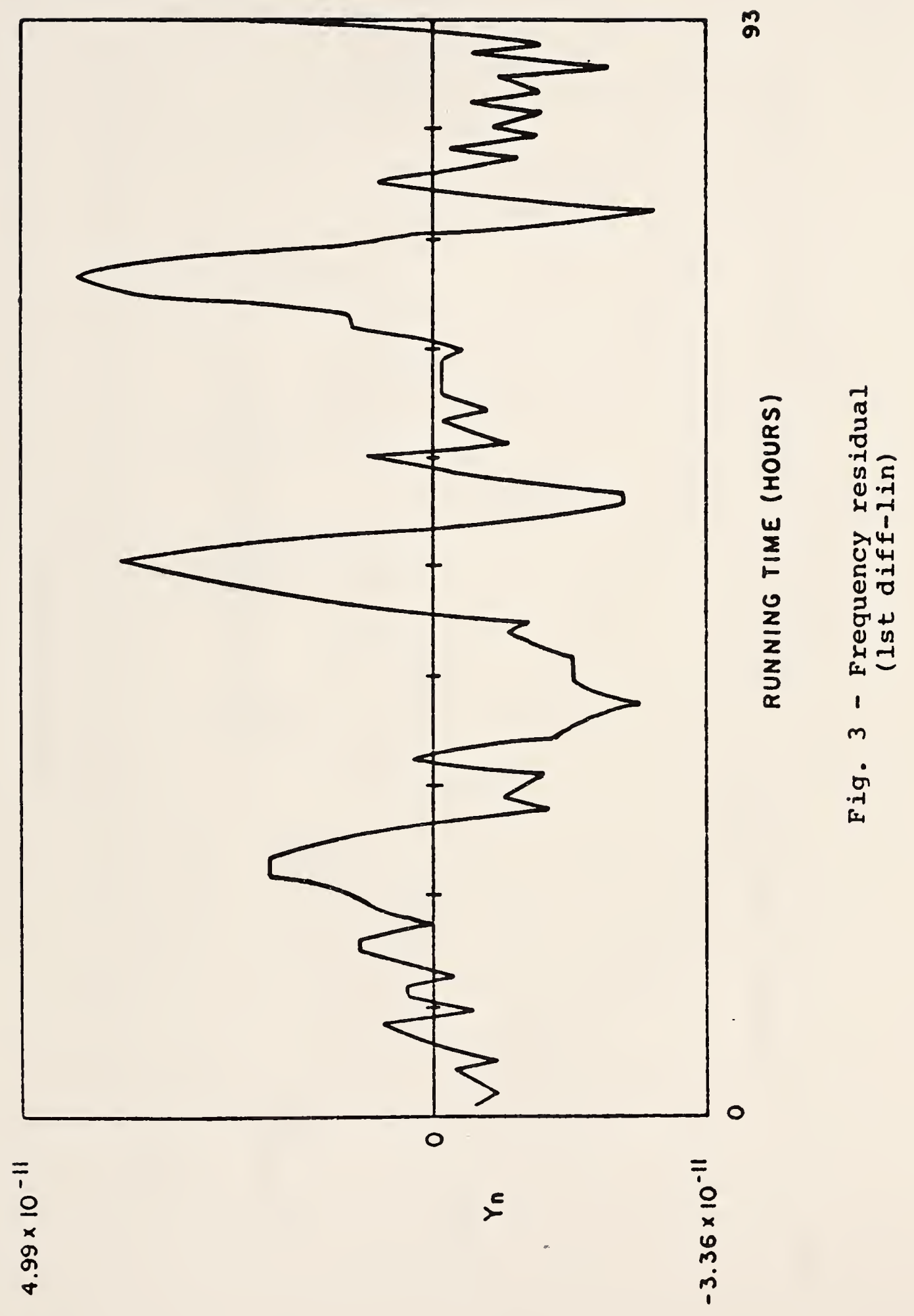




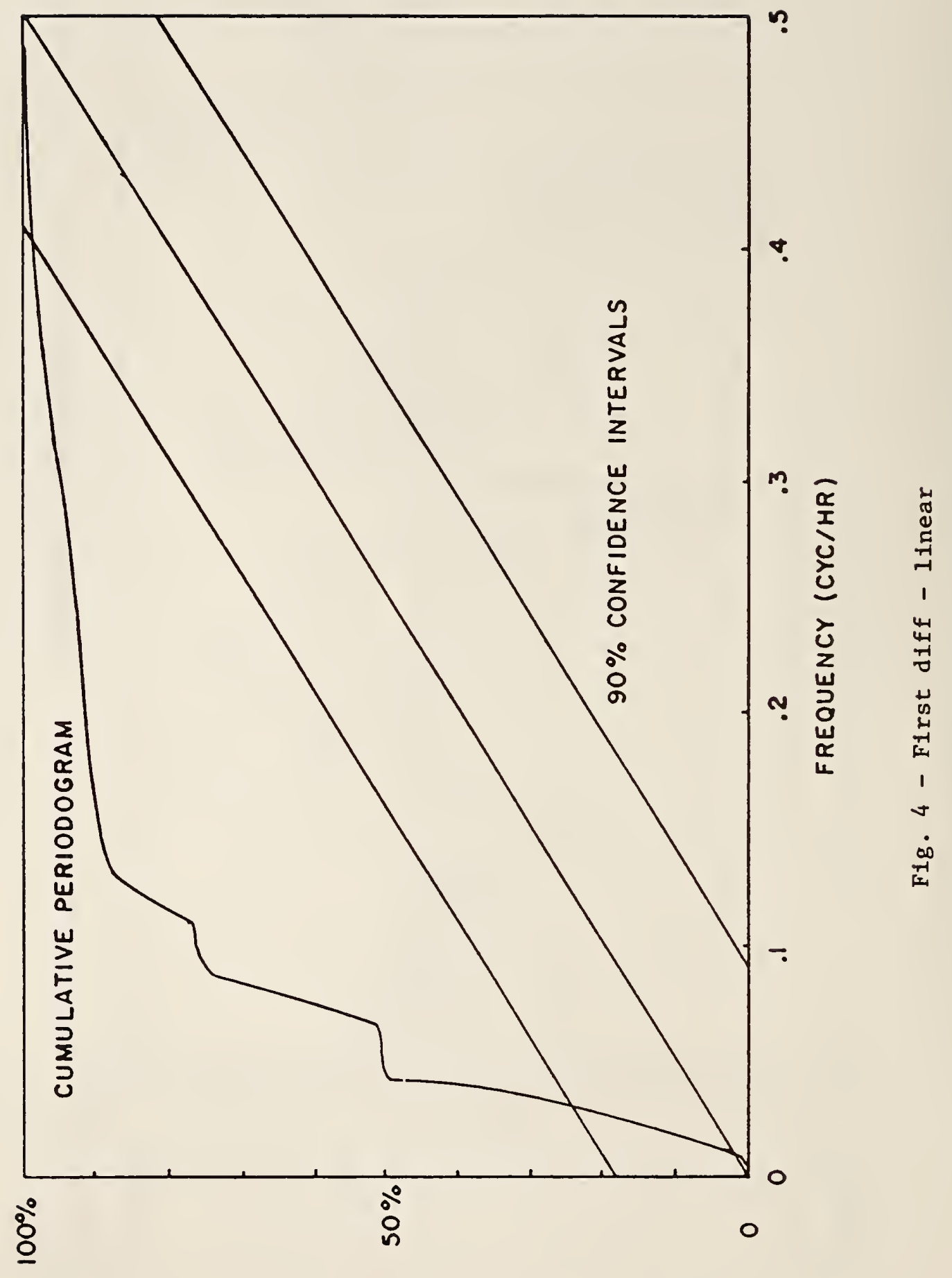




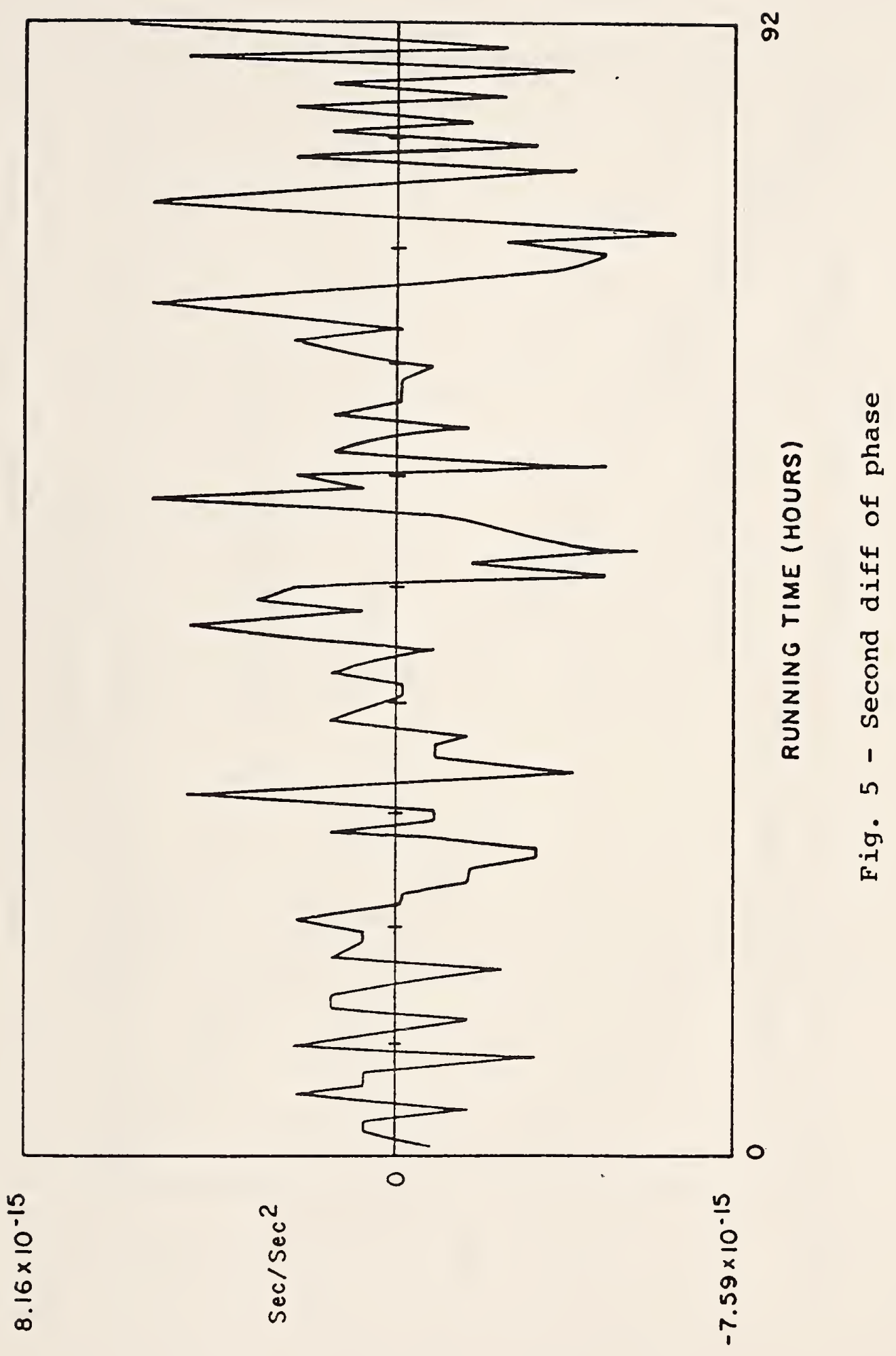




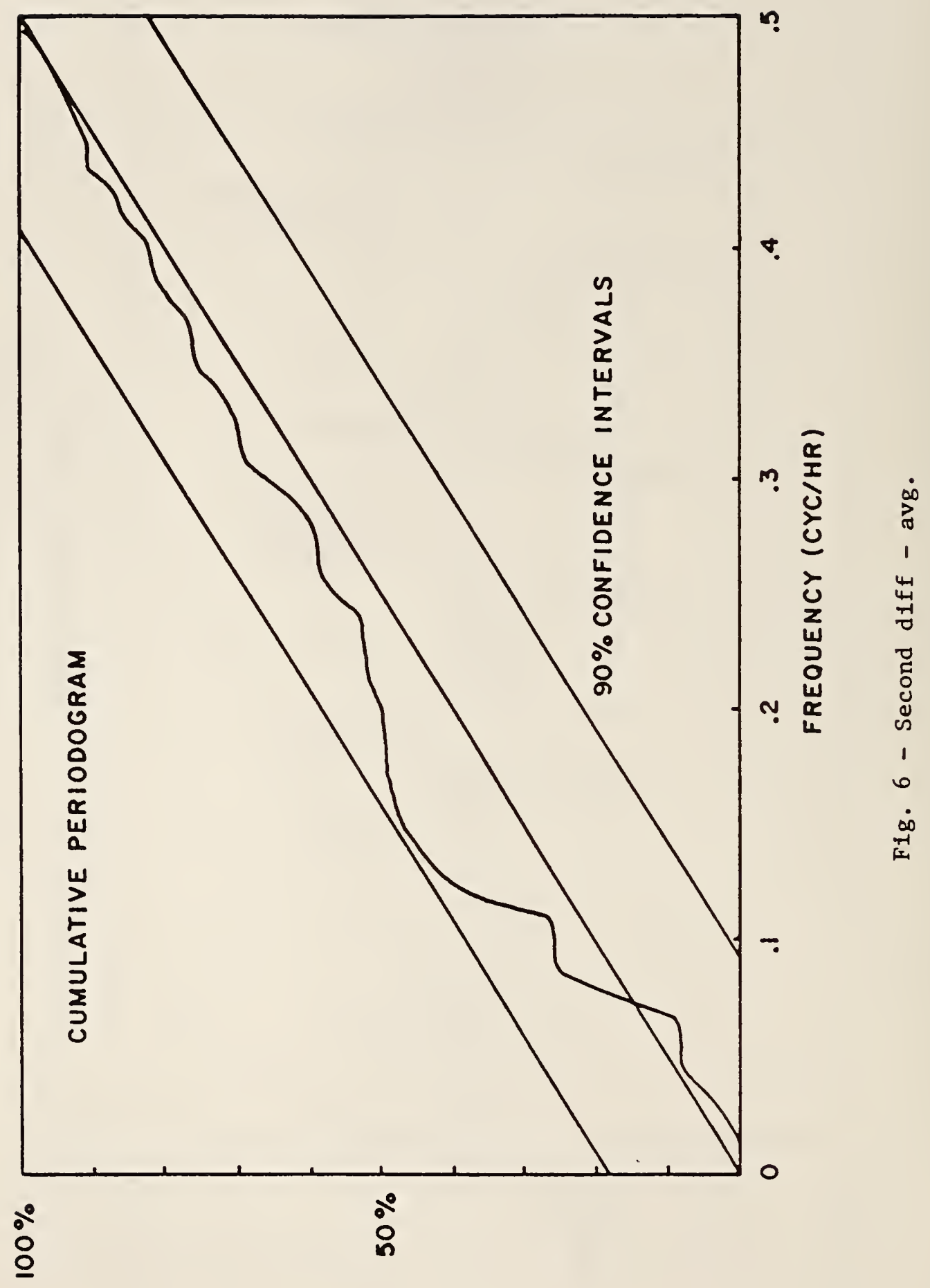




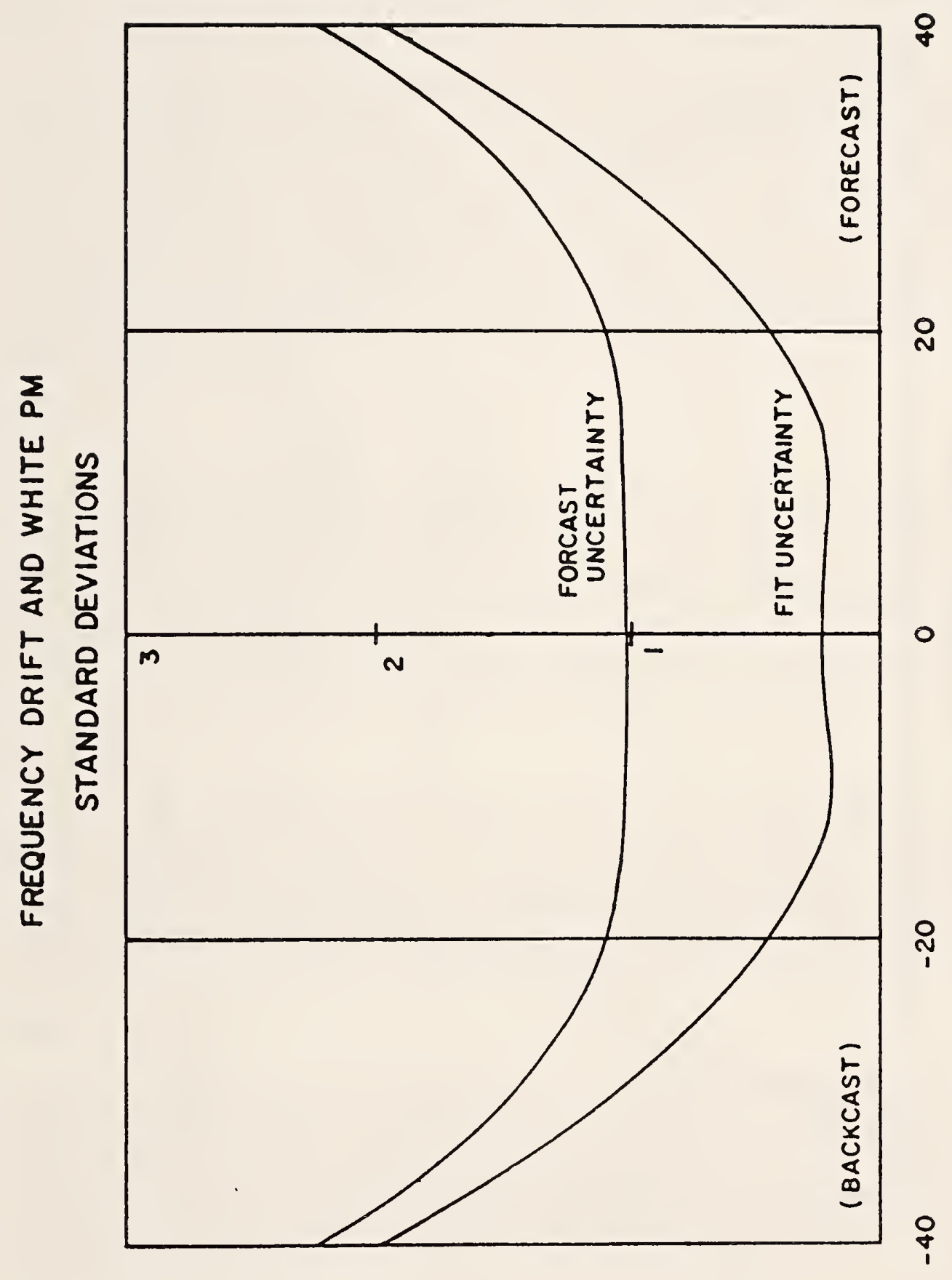




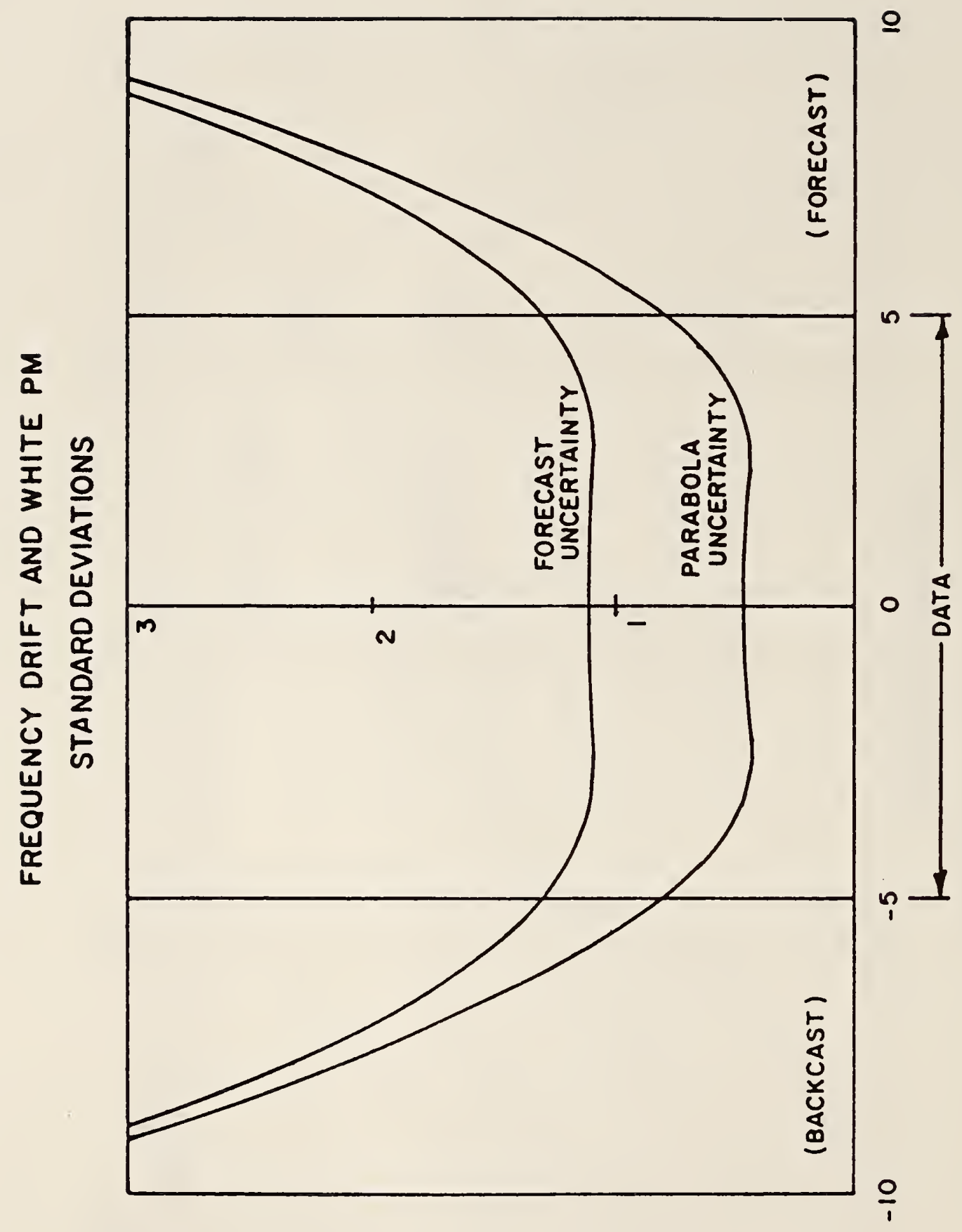




\section{QUESTIONS AND ANSWERS}

MR. ALLAN:

We have found the second difference drift estimator to be useful for the random walk FM process. One has to be careful when you apply it, because if you use overlapping second differences, for example, if you have a cesium beam, and you have white noise FM out to several days and then random walk FM beyond that point, and you are making hourly or 2 hour measurements, if you use the mean of the second differences, you can show that all the middle terms cancel, and in fact you are looking at the frequency at the beginning of the run and the frequency at the end of the run to compute the frequency drift and that's very poor.

DR. BARNES :

My comment would be: There again the problem is in the model, and not in the arithmatic. The models applied here were 3 very simple models, very simple, simpler than you will run into in life. It was pure random walk plus a drift or pure frequency noise plus a drift, or pure random walk of frequency noise plus a drift and it did not approach at all any of the noise complex models where you would have both white frequency and random walk frequency and a drift. That's got to be handled separately, I'm not even totally sure how to perform it in all cases at this point.

MR. MCCASKILL:

I would like to know if you would comment on the value of the sample time and the reason why, of course, is that the mean second difference does depend on the sample time? For instance, if you wanted to estimate the aging rate or change in linear change of frequency, what value of sample time would you use in order to make that correction. So, really, the question is, how does the sample time enter into your calculations?

DR. BARNES :

At least I will try to answer in part. I don't know in all cases, I'm sure, but if you have a complex noise process where you have at short term different noise behavior than in long term, it may benefit you to take a longer sampling time and effectively not look at the short term, and then one of the simple models might apply. I honestly haven't looked in great detall at how to choose the sample time. It is an interesting question. 
MR. MCCASKILL:

We1l, let me go further, because we had the benefit of being out at NBS and talking with Dr. Allan earlier and he suggested that we use the mean second difference, and the only problem is if we want to calculate or correct for our aging rate at a tau of five days or ten days, and you calculate the mean second difference, you come up with exactly the Allan Variance. What appears is that in order to come up with the number for the aging rate, you have to calculate the mean second difference using a sample time whenever you take your differences of longer than, let's say, ten days sample time. So we use, of course, the regression model, but we use on the order of two or three weeks in order to calculate an aging rate correction for, say, something like the rubidium in the NAVSTAR 3 clock. It looks like in order to come up with a valid value for the Allan Variance of five or ten day sample time you have to calculate the aging rate at a longer, maybe two or three times longer sample time.

DR. BARNES :

Dave Allan, do you think you can answer that?

MR. ALLAN :

Not to go into details, but if you assume that in the longer term you have random walk frequency modulation as the predominant noise process in a clock, which seems to be true for rubidium, cesium and hydrogen, you can do a very simple thing. You can take the full data length and take the time at the beginning, the time in the middle and the time in the end and construct a second difference, and that's your drift.

There is still the issue of the confidence interval on that. If you really want to verify your confidence interval, you have to have enough data to do a regression. You need enough data to test to be sure the model is good.

DR. WINKIER:

That argument is fourteen years old, because we have been critized here. I still believe that for a practical case where you depend on measurements which are contaminated and maybe even contaminated by arbitrarily large errors if they are digital. These, theoretical advantages that you have outlined may not be as important as the benefits which you get when you make a least square regression. In this case you can immediately identify the wrong data. Otherwise, if you put the data into an algorithm you may not know how much your data is contaminated. So for practical applications, the first model even though theoretically it is poor, it still gives reasonable estimates of the drift and you have residuals which let you identify wrong phase values immediately. 
DR. BARNES :

I think that's true and you may have different reasons to do regression analysis, if your purpose is to measure a drift and understand the confidence intervals, then I think what has been presented is reasonable, if you have as your purpose to look--to see if there are indications of funny behavior in a curve that has such strong curvature or drift that you can't get it on graph paper without doing that, I think it is a very reasonable thing to do. I think looking at the data is one of the healthiest things any analyst can do. 
NIST Technical Note 1318, 1990.

VARIANCES BASED ON DATA WITH DEAD TIME BETWEEN THE MEASUREMENTS

James A. Barnes

Austron, Inc.

Boulder, Colorado 80301

and

David W. Allan

Time and Frequency Division

National Institute of Standards and Technology

Boulder, Colorado 80303

The accepted definition of frequency stability in the time domain is the two-sample variance (or Allan variance). It is based on the measurement of average frequencies over adjacent time intervals, with no "dead time" between the intervals. The primary advantages of the Allan variance are that (1) it is convergent for many encountered noise models for which the conventional variance is divergent; (2) it can distinguish between many important and different spectral noise types; (3) the two-sample approach relates to many practical implementations; for example, the rms change of an oscillator's frequency from one period to the next; and (4) Allan variances can be easily estimated at integer multiples of the sample interval.

In 1974 a table of bias functions which related variance estimates with various configurations of number of samples and dead time to the Allan variance was published [1]. The tables were based on noises with pure power-law spectral densities.

Often situations occur that unavoidably have dead time between measurements, but still the conventional variances are not convergent. Some of these applications are outside of the time-andfrequency field. Also, the dead times are often distributed throughout a given average, and this distributed dead time is not treated in the 1974 tables.

This paper reviews the bias functions $B_{1}(N, r, \mu)$, and $B_{2}(r, \mu)$ and introduces a new bias function, $B_{3}(2, M, r, \mu)$, to handle the commonly occurring cases of the effect of distributed dead time on the computed variances. Some convenient and easy-to-interpret asymptotic limits are reported. A set of tables for the bias functions are included at the end of this paper.

Key words: Allan variance; bias functions; data sampling and dead time; dead time between the measurement; definition of frequency stability; distributed dead time; two-sample variance 


\section{Introduction}

The sample mean and variance indicate respectively the approximate magnitude of a quantity and its uncertainty. For many situations a continuous function of time is sampled, or measured, at fairly regular intervals. Sampling is not always instantaneous. It takes a finite time and provides an "average reading." If the underlying process (or noise) is random and uncorrelated in time, then the fluctuations are said to be "white" noise. In this situation, the sample mean and variance calculated by the conventional formulas,

$$
\begin{aligned}
m & =\frac{1}{N} \sum_{n=1}^{N} \bar{y}_{n}, \\
s^{2} & =\frac{1}{N-1} \sum_{n=1}^{N}\left(\bar{y}_{n}-m\right)^{2},
\end{aligned}
$$

provide the needed information. The "bar" over the $y$ in eq (1) above denotes the average over a finite time interval. In time and frequency work, $y$ is defined as the average fractional (or normalized) frequency deviation from nominal over an interval $\tau$ and at some specified measurement time. As in science generally, the physical model determines the appropriate mathematical model. For the white noise model, the sample mean and variance are the mainstays of most analyses.

Although white noise is a common model for many physical processes, more general noise models are being identified and used. In precise time and frequency measurement, for example, there are two quantities of great interest: instantaneous frequency and phase. These two quantities by definition are exactly related by a differential. (We are NOT considering Fourier frequencies at this point.) That is, the instantaneous frequency is the time rate of change of phase. Thus, if we were employing a model of white frequency-modulation (white FM) noise, then the phase noise is the integral of the white FM noise, commonly called a Brownian motion or random walk. Therefore, depending on whether we are currently interested in phase or frequency, the sample mean and variance may or may not be appropriate. 
By definition, white noise has a power spectral density (PSD) that is constant with Fourier frequency. Since random walk noise is the integral of white noise, the power spectral density of a random walk varies as $1 / f^{2}$ (where $f$ is the Fourier frequency) [2]. We encounter noise models whose power spectral densities are various power laws of their Fourier frequencies. Flicker noise is very common and is defined as a noise whose power spectral density varies as $1 / f$ over a relevant spectral range. If an oscillator's instantaneous frequency is well modeled by flicker noise, then its phase would be the integral of the flicker noise. It would have a PSD which varied as $1 / f^{3}$.

Noise models whose PSD's are power laws of the Fourier frequency but not integer exponents are possible as well but not as common. This paper considers power-law PSD's of a quantity $y(t) ; y(t)$ is a continuous sample function which can be measured at regular intervals. For noises whose PSD's vary as $f^{\alpha}$ with $\alpha<-1$ at low frequencies, the conventional sample mean and variance given in eq (1) do not converge as $N$ gets large [2, 3]. This lack of convergence renders the sample mean and variance ineffective and often misleading in some situations.

Although the sample mean and variance have limitations, other time-domain statistics can be convergent and quite useful. The quantities that we consider in this paper depend significantly on the details of the sampling procedures. Indeed, each sampling scheme has its own bias, and this is the motivation for the bias functions discussed in this paper.

\section{The Allan Variance}

Recognizing that for particular types of noise, the conventional sample variance fails to converge as the number of samples, $\mathrm{N}$, grows, Allan suggested that we set $N=2$ and average many of these two-sample variances to get a convergent and stable measure of the spread of the quantity in question [3]. This is what has come to be called the Allan variance. 
More specifically let us consider a sample function of time as indicated in figure 1. A measurement consists of averaging $y(t)$ over the interval $\tau$. The next measurement begins at a time $\mathrm{T}$ after the beginning of the previous measurement interval. There is no logical reason why $\mathrm{T}$ must be as large as $\tau$ or larger--if $T<\tau$, then the second measurement begins before the first is completed, which is unusual but possible. When $T=\tau$, there is no dead time between measurements.

The accepted definition of the Allan variance is the expected value of a twosample variance with no dead time between successive measurements. In symbols, the Allan variance is given by

$$
\sigma_{\mathrm{y}}^{2}(\tau)=\frac{1}{2} E\left[\left(\bar{y}_{n+1}-\bar{y}_{n}\right)^{2}\right]
$$

where there is no dead time between the two sample averages for the Allan variance and the $E[\cdot]$ denotes the expectation operator.

\section{The Bias Function $B_{1}(N, r, \mu)$}

Define $N$ to be the number of sample averages of $y(t)$ used in eq (1) to estimate a sample variance ( $N=2$ for an Allan variance). Also define $r$ to be the ratio of $\mathrm{T}$ to $\tau$ ( $\mathrm{r}=1$ when there is no dead time between measurements). The parameter $\mu$ is related to the exponent of the power law of the PSD of the process $y(t)$. If $\alpha$ is the exponent in the power-law spectrum for $y(t)$, then the Allan variance varies as $\tau$ raised to the $\mu$ power, where $\alpha$ and $\mu$ are related as shown in figure 2 [2-4]. We can use estimates of $\mu$ to infer $\alpha$, the spectral type. The ambiguity in $\alpha$ for $\mu=-2$ has been resolved by using a modified $\sigma_{\mathrm{y}}^{2}(\tau)[5-7]$.

Often data cannot be taken without dead time between sample averages, and it is useful to consider other than two-sample variances. We will define the bias function $B_{1}(N, r, \mu)$ by the ratio,

$$
B_{1}(N, r, \mu)=\frac{\sigma^{2}(N, T, \tau)}{\sigma^{2}(2, T, \tau)}
$$


where $\sigma^{2}(\mathrm{~N}, \mathrm{~T}, \tau)$ is the expected sample variance given in eq (1) and based on $\mathrm{N}$ measurements at intervals $T$ and averaged over a time $\tau$ and $r=T / \tau$. In words, $\mathrm{B}_{1}(\mathrm{~N}, \mathrm{r}, \mu)$ is the ratio of the expected variance for $\mathrm{N}$ measurements to the expected variance for two samples (everything else held constant). The variances on the right in eq (3) depend implicitly on the noise type even though $\mu$ or $\alpha$ are not shown as independent variables. The noise-type parameter, $\mu$, is shown as an independent variable for all of the bias functions in this paper, because the values of the ratio of these variances explicitly depend on $\mu$ as will be derived later in the paper. Allan showed that if $\mathrm{N}$ and $\mathrm{r}$ are held constant, then the $\alpha, \mu$ relationship shown in figure 2 is the same; that is, we can still infer the spectral type from the $\tau$ dependence using the equation $\alpha=-\mu-1,-2 \leq \mu<2$ [3].

\section{The Bias Function $B_{2}(r, \mu)$}

The bias function $B_{2}(r, \mu)$ is defined in [1] by the relation,

$$
\mathrm{B}_{2}(\mathrm{r}, \mu)=\frac{\sigma^{2}(2, \mathrm{~T}, \tau)}{\sigma^{2}(2, \tau, \tau)}=\frac{\sigma^{2}(2, \mathrm{~T}, \tau)}{\sigma_{\mathrm{y}}^{2}(\tau)}
$$

In words, $B_{2}(r, \mu)$ is the ratio of the expected two-sample variance with dead time to that without dead time (with $\mathrm{N}=2$ and $\tau$ the same for both variances). A plot of the $B_{2}(r, \mu)$ function is shown in figure 3 . The bias functions $B_{1}$ and $B_{2}$ represent biases relative to $N=2$ rather than infinity; that is, the ratio of the $N$ sample variance (with or without dead time) to the Allan variance and the ratio of the two-sample dead-time variance to the Allan variance respectively.

\section{The Bias Function $B_{3}(N, M, r, \mu)$}

Consider the case where a great many measurements are available with dead time between each pair of measurements $\left(\mathrm{T}_{0}>\tau_{0}\right)$. The measurements are averaged over the time interval $\tau_{0}$, the spacing between the beginning of one measurement to the next is $\mathrm{T}_{0}$, and it may not be convenient to retake the data. We might want to estimate the Allan variance at, say, multiples $M$ of 
the averaging time $\tau_{0}$. If we average groups of the measurements of $y(t)$, then the dead times between the original measurements are distributed periodically throughout the new average measurements (see figure 4). Define

$$
\overline{\bar{y}}_{i}=\frac{1}{M} \sum_{n=i}^{M+i-1} \bar{y}_{n},
$$

where $\bar{y}_{i}$ are the raw or original measurements based on dead time $T_{0}-\tau_{0}$.

Also define the two-sample variance with distributed dead time as

$$
\sigma^{2}(2, \mathrm{M}, \mathrm{T}, \tau)=3 / 2 \mathrm{E}\left[\left(\overline{\overline{\mathrm{y}}}_{\mathrm{i}}-\overline{\overline{\mathrm{y}}}_{\mathrm{i}}+\mathrm{M}\right)^{2}\right]
$$

with $\tau=\mathrm{M} \tau_{0}$ and $\mathrm{T}=\mathrm{MT}_{0}$.

We can now define $B_{3}$ as the ratio of the $\mathrm{N}$-sample variance with distributed dead time to the $\mathrm{N}$-sample variance with dead time accumulated at the end as in figure 1:

$$
B_{3}(N, M, r, \mu)=\frac{\sigma^{2}(N, M, T, \tau)}{\sigma^{2}(N, T, \tau)} .
$$

Although $B_{3}(N, M, r, \mu)$ is defined for general $N$, the tables in the Appendix confine treatment to the case where $N=2$. There is little value in extending the tables to include general $N$. Though the variances on the right in eq (7) depend explicitly on $N, T$ and $\tau$, the ratio $B_{3}(N, M, r, \mu)$ depends on the ratio $\mathrm{r}=\mathrm{T} / \tau$, and on $\mu$ as developed later in this paper.

In words, $B_{3}(2, M, r, \mu)$ is the ratio of the expected two-sample variance with periodically distributed dead time, as shown in figure 4, to the expected twosample variance with all the dead time grouped together as shown in figure 1. Both the numerator and the denominator have the same total averaging time and dead time, but they are apportioned differently. The product $B_{2}(r, \mu) \cdot$ $B_{3}(2, M, r, \mu)$ is the distributed dead-time variance over the Allan variance for a particular $\mathrm{T}, \tau, \mathrm{M}$ and $\mu$. 
Some useful asymptotic forms of $B_{3}$ can be found. In the case of large $M$ and $M>r$, we may write that

$$
\begin{array}{ll}
B_{3} \simeq \frac{1+\mu}{3}, & 1 \leq \mu \leq 2, \\
B_{3} \simeq \frac{4 \ln (2)}{2 \ln (r)+3}, & \mu=0 .
\end{array}
$$

One simple and important conclusion from these two equations is that for the cases of flicker FM noise and random-walk FM noise, the $\boldsymbol{r}^{\mu}$ dependence for large $\tau$ is the same whether or not there is periodically distributed dead time. The values of the variances differ only by a constant, and in the latter case the constant is 1 . This conclusion is also true for white FM noise, and in this case the constant is also 1.

In the cases $\mathrm{r} \gg 1$ and $-2 \leq \mu \leq-1$, we may write for the asymptotic behavior of $\mathrm{B}_{3}$

$$
B_{3} \simeq M^{\alpha}, \alpha=-\mu-1 \text {, }
$$

as was determined empirically. In this region of power-law spectrum the $B_{3}$ function has an $\mathrm{M}^{\alpha}$ dependence for an $\mathrm{f}^{\alpha}$ spectrum.

\section{The Bias Functions}

The bias functions can be written fairly simply by first defining the function,

$$
F(A)=2 A^{\mu+2}-(A+1)^{\mu+2}-|(A-1)|^{\mu+2}
$$

The bias functions become

$$
B_{1}(N, r, \mu)=\frac{1+\sum_{n=1}^{N-1} \frac{N-n}{N(N-1)} \cdot F(n r)}{1+\frac{1}{K} F(r)},
$$




$$
B_{2}(r, \mu)=\frac{1+3 / 2 F(r)}{2\left(1-2^{\mu}\right)}
$$

as given in [1], and

$B_{3}(2, M, r, \mu)=\frac{2 M+M \cdot F(M r)-\sum_{n=1}^{M-1}(M-n)[2 F(n r)-F((M+n) r)-F((M-n) r)]}{\left(M^{\mu+2}\right)[F(r)+2]}$,

as indicated in the appendix.

For $\mu=0$, eqs (11), (12), and (13) are the indeterminate form $0 / 0$ and must be evaluated by l'Hôpital's rule. Special attention must also be given when expressions of the form $0^{0}$ arise. We verified a random sampling of the table entries using noise simulation and Monte Carlo techniques. No errors were detected. The results in this paper differ some from those in [8], which suggests that there may be some mistakes. Tables for the three bias functions are listed at the end of the paper (note that the computer print-out did not have a symbol for Greek $\mathrm{mu} \equiv \mu$ ).

\section{Examples of the Use of the Bias Functions}

The spectral type, that is, the value of $\mu$, may be inferred by varying $\tau$, the sample time. However, another useful way of determining the value of $\mu$ is by using $\mathrm{B}_{1}(\mathrm{~N}, \mathrm{r}, \mu)$ as follows: calculate an estimate of $\sigma_{\mathrm{y}}^{2}(\mathrm{~N}, \mathrm{~T}, \tau)$ and $\sigma_{\mathrm{y}}^{2}(2, \mathrm{~T}, \tau)$ and hence $B_{1}(N, r, \mu)$; then use the tables to infer the value of $\mu$.

Suppose one has an experimental value for $\sigma_{y}^{2}\left(\mathrm{~N}_{1}, \mathrm{~T}_{1}, \tau_{1}\right)$ and its spectral type is known, that is, $\mu$ is known. Suppose also that one wishes to know the variance at some other set of measurement parameters, $\mathrm{N}_{2}, \mathrm{~T}_{2}, \tau_{2}$. An unbiased estimate of $\sigma_{\mathrm{y}}^{2}\left(\mathrm{~N}_{2}, \mathrm{~T}_{2}, \tau_{2}\right)$ may be calculated by the equation:

$$
\sigma_{\mathrm{y}}^{2}\left(\mathrm{~N}_{2}, \mathrm{~T}_{2}, \tau_{2}\right)=\left(\frac{\tau_{2}}{\tau_{1}}\right)^{\mu}\left(\frac{\mathrm{B}_{1}\left(\mathrm{~N}_{2}, \mathrm{r}_{2}, \mu\right) \mathrm{B}_{2}\left(\mathrm{r}_{2}, \mu\right)}{\mathrm{B}_{1}\left(\mathrm{~N}_{1}, \mathrm{r}_{1}, \mu\right) \mathrm{B}_{2}\left(\mathrm{r}_{1}, \mu\right)}\right) \sigma_{\mathrm{y}}^{2}\left(\mathrm{~N}_{1}, \mathrm{~T}_{1}, \tau_{1}\right)
$$

where $\mathrm{r}_{1}=\mathrm{T}_{1} / \tau_{1}$ and $\mathrm{r}_{2}=\mathrm{T}_{2} / \tau_{2}$. 
Since the time-domain definition for frequency stability is the Allan variance, it behooves us, where possible, to relate other variances to the Allan variance. If we have an $\mathrm{N}$-sample variance on data with dead-time $\mathrm{T}-\tau$ and we know the power-law spectral type (the value of $\mu$ ), then we may write

$$
\sigma_{\mathrm{y}}^{2}(\tau)=\frac{\sigma_{\mathrm{y}}^{2}(\mathrm{~N}, \mathrm{~T}, \tau)}{\mathrm{B}_{1}(\mathrm{~N}, \mathrm{r}, \mu) \mathrm{B}_{2}(\mathrm{r}, \mu)} .
$$

If we have an $\mathrm{N}$-sample variance where each data entry is an average of $\mathrm{M}$ samples with distributed dead time, then we may write

$$
\sigma_{\mathrm{y}}^{2}(\tau)=\frac{\sigma_{\mathrm{y}}^{2}(\mathrm{~N}, \mathrm{M}, \mathrm{T}, \tau)}{\mathrm{B}_{1}(\mathrm{~N}, \mathrm{r}, \mu) \mathrm{B}_{2}(\mathrm{r}, \mu) \mathrm{B}_{3}(\mathrm{~N}, \mathrm{M}, \mathrm{r}, \mu)}
$$

\section{Conclusion}

For some important power-law spectral density models often used in characterizing precision oscillators $\left(S_{y}(f) \sim f^{\alpha}, \alpha=-2,-1,0,+1,+2\right)$, we have studied the effects on variances when there is dead time between the frequency samples, and the frequency samples are averaged to increase the integration time. Since dead time between measurements is a common problem throughout metrology, the analysis here has broader applicability than just to time and frequency. Specifically, this kind of analysis has been used with gage blocks and standard volt cells--showing that the classical variance may be non-convergent in some cases [9].

Heretofore, the Allan variance has been shown to have some convenient theoretical properties in relation to power-law spectra as the integration or sample time is varied (if $\sigma_{\mathrm{y}}{ }^{2}(\tau) \sim \tau^{\mu}$, then $\alpha=-\mu-1,-2<\mu \leq 2$ ). Since $\sigma_{\mathrm{y}}(\tau)$, by definition, is estimated from data with no dead time, the sample or integration time can be unambiguously changed to investigate the $\tau$ dependence. From our analysis, we have concluded that for the asymptotic limit of several samples being averaged with dead time present in the data, the $\tau$ dependence of the variances is the same. The $\alpha=-\mu-1$ relationship still remains valid for white FM noise $(\mu=-1, \alpha=0)$, flicker FM noise $(\mu=0, \alpha=-1)$, and for 
random-walk FM noise $(\mu=+1, \alpha=-2)$. The asymptotic limit is approached as the product of number of samples averaged and the initial data sample time, $r_{0}$, becomes larger than the dead time $(M>r)$. The variances so obtained differ only by a constant, which can be calculated as given in this paper.

A knowledge of the appropriate power-law spectral model is required to translate a distributed dead-time variance to the corresponding value of the Allan variance. In principle, the power-law spectral model can be estimated from the $\tau^{\mu}$ dependence, using the variance analysis on the data as outlined above.

\section{References}

[1] J.A. Barnes, "Tables of Bias Functions, $B_{1}$ and $B_{2}$, for Variances Based on Finite Samples of Processes with Power Law Spectral Densities," NBS Tech. Note 375 (1969).

[2] J.A. Barnes, "Atomic Timekeeping and the Statistics of Precision Signal Generation," IEEE Proc. 54, No. 2, pp. 207-220, Feb. 1966.

[3] D.W. Allan, "Statistics of Atomic Frequency Standards, "IEEE Proc. 54, No. 2, pp. 221-230, Feb. 1966.

[4] RFC Vessot, L. Mueller, and J. Vanier, "The Specification of Oscillator Characteristics from Measurements Made in the Frequency Domain," IEEE Proc. 54, No. 2, pp. 199-207, Feb. 1966.

[5] D.W. Allan and J.A. Barnes, "A Modified "Allan Variance" With Increased Oscillator Characterization Ability," Proc. 35th Annual Frequency Control Symposium, USAERADCOM, Ft. Monmouth, NJ, May 1981, pp. 470-475.

[6] D.W. Allan, "Time and Frequency (Time-Domain) Characterization, Estimation, and Prediction of Precision Clocks and Oscillators," IEEE Transactions on UFFC, November 1987.

[7] P. Lesage and T. Ayi, "Characterization of Frequency Stability: Analysis of the Modified Allan Variance and Properties of its Estimate," IEEE Trans. Instrum. Meas., IM-33, no. 4, pp. 332-336, Dec. 1984.

[8] N.D. Faulkner and E.V.I. Mestre, "Time-Domain Analysis of Frequency 
Stability Using Non-zero Dead-Time Counter Techniques," IEEE Trans. on Instrum. \& Meas., IM-34, 144-151 (1985).

[9] D.W. Allan, "Should the Classical Variance Be Used as a Basic Measure in Standards Metrology?" IEEE Trans. on Instrumentation and Measurement, IM-36, 646-654, 1987. 
Table 1. Table of some bias function identities

$$
\begin{aligned}
& \mathrm{B}_{1}(2, r, \mu) \quad=1 \\
& \mathrm{~B}_{1}(\mathrm{~N}, \mathrm{r}, 2) \quad=(\mathrm{N}(\mathrm{N}+1)) / 6 \\
& \mathrm{~B}_{1}(\mathrm{~N}, 1,1) \quad=\mathrm{N} / 2 \\
& \mathrm{~B}_{1}(\mathrm{~N}, 1, \mu) \quad=\left(\mathrm{N}\left(1-\mathrm{N}^{\mu}\right)\right) /\left[\left(2(\mathrm{~N}-1)\left(1-2^{\mu}\right)\right] \text { for } \mu \neq 0\right. \\
& =\mathrm{N} \ln (\mathrm{N}) /[2(\mathrm{~N}-1) \ln (2)] \text { for } \mu=0 \\
& \mathrm{~B}_{1}(\mathrm{~N}, 1, \mu) \quad=1 \text { for } \mu<0 \\
& =\left[2 /(N(N-1)] \sum_{n=1}^{N-1}(N-n) \cdot n^{\mu} \text { for } \mu>0\right. \\
& \mathrm{B}_{1}(\mathrm{~N}, \mathrm{r},-1) \quad=1 \text { if } \mathrm{r} \geq 1 \\
& \mathrm{~B}_{1}(\mathrm{~N}, \mathrm{r},-2) \quad=1 \text { if } r \neq 1 \text { or } 0 \\
& \mathrm{~B}_{2}(0, \mu) \quad=0 \\
& \mathrm{~B}_{2}(1, \mu) \quad=1 \\
& \mathrm{~B}_{2}(r, 2) \quad=r^{2} \\
& \mathrm{~B}_{2}(\mathrm{r}, 1) \quad=(3 \mathrm{r}-1) / 2 \text { if } \mathrm{r} \geq 1 \\
& \mathrm{~B}_{2}(r,-1) \quad=r \text { if } 0 \leq r \leq 1 \\
& =1 \text { if } r \geq 1 \\
& B_{2}(r,-2) \quad=0 \text { if } r=0 \\
& =1 \text { if } \mathrm{r}=1 \\
& =2 / 3 \text { otherwise } \\
& \mathrm{B}_{3}(2, \mathrm{M}, 1, \mu) \quad=1 \\
& \mathrm{~B}_{3}(2, \mathrm{M}, \mathrm{r},-2) \quad=\mathrm{M} \\
& \mathrm{B}_{3}(2, \mathrm{r}, \mu) \quad=1 \\
& \mathrm{~B}_{3}(2, \mathrm{M}, \mathrm{r}, 2) \quad=1 \\
& B_{3}(2, M, r,-1) \quad=1 \text { for } r \geq 1
\end{aligned}
$$




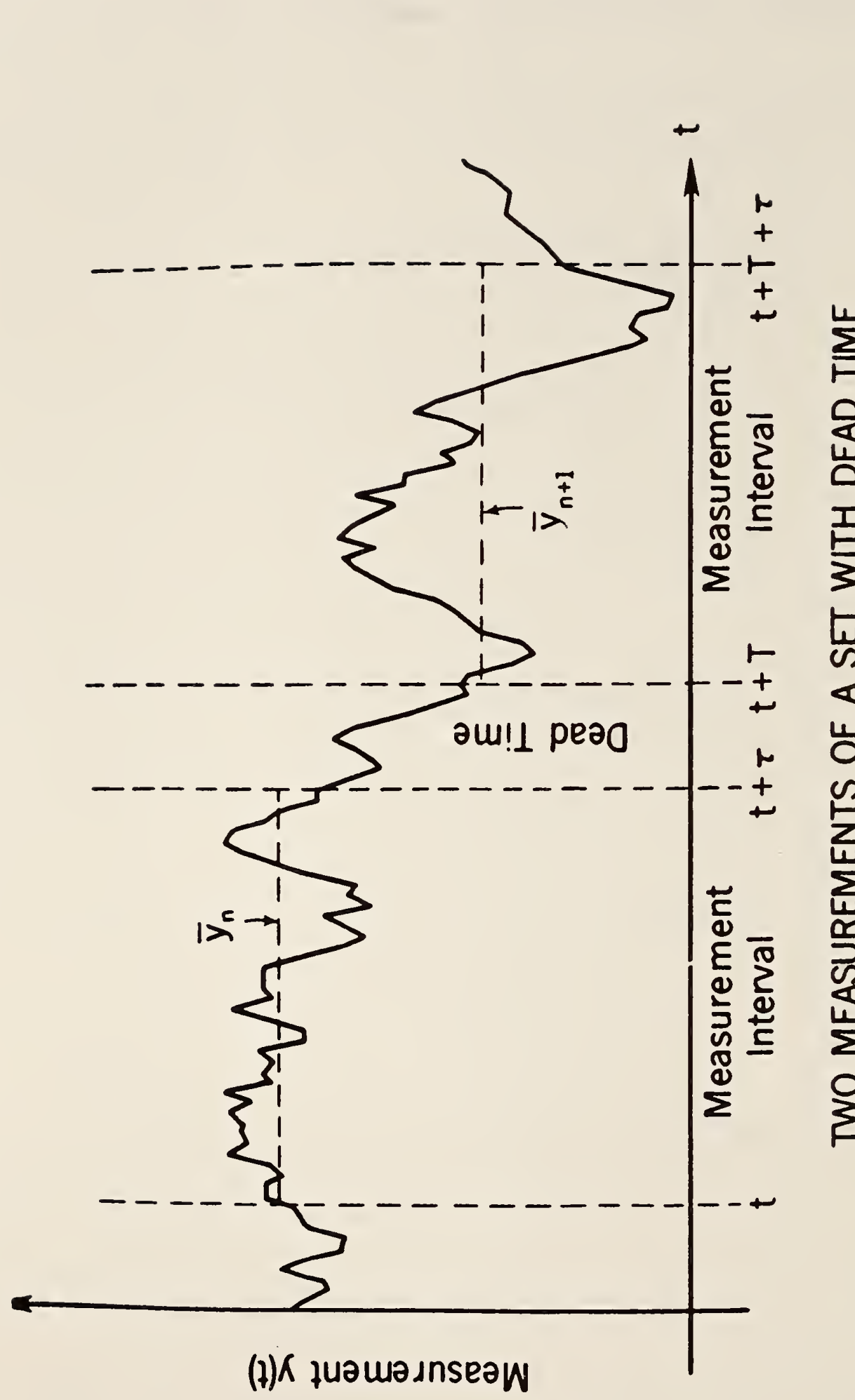




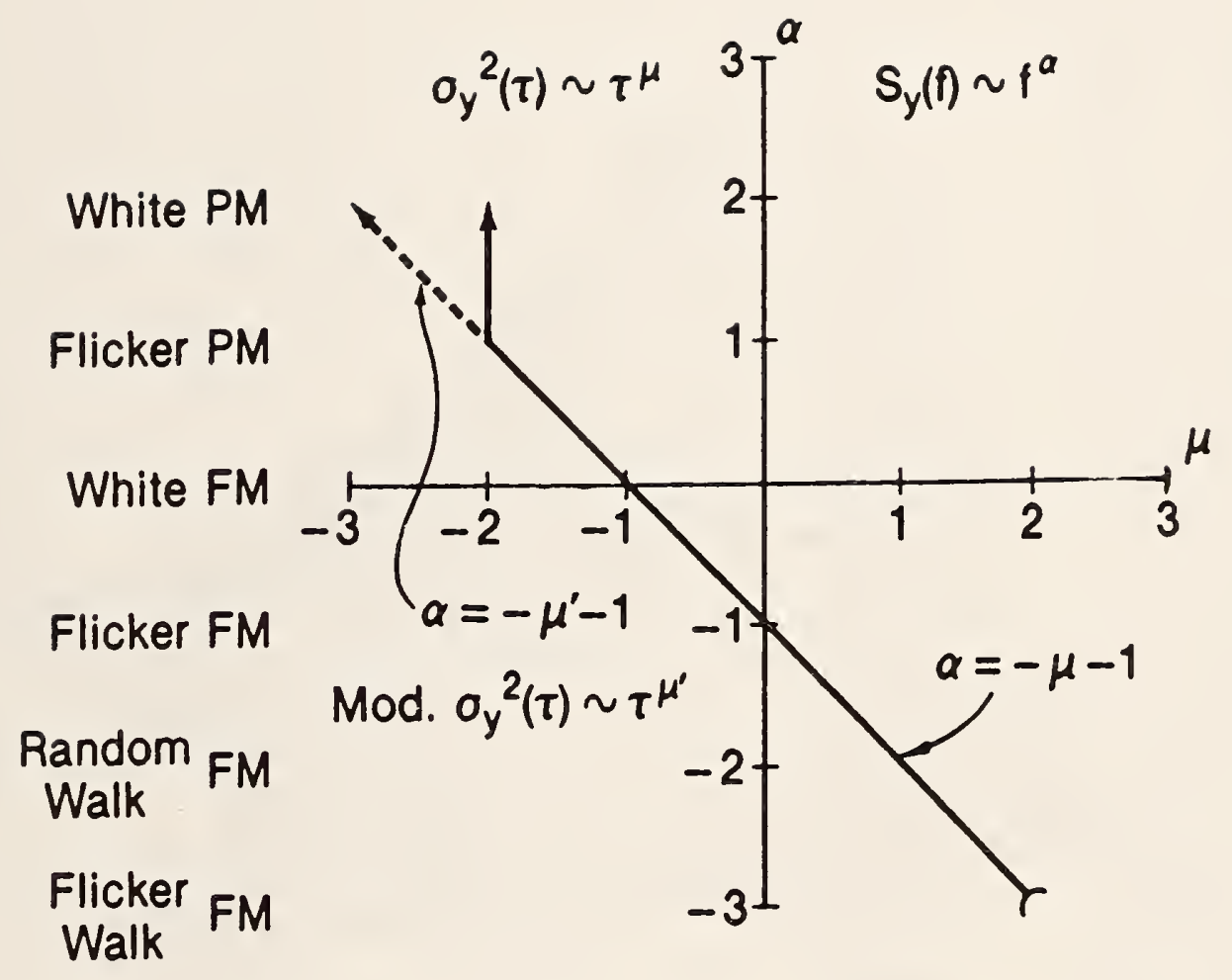

Figure 2. A plot of the relationship between the frequency-domain power-law spectral-density exponent $\alpha$ and the time-domain two-sample Allan variance exponent $\mu(\alpha=-\mu-1,-2 \leq \mu<2$ and $\alpha \geq 1$ for $\mu=-2)$. Also shown is the similar relationship between $\alpha$ and the modified Allan variance with exponent on $\tau$ of $\mu^{\prime}\left(\alpha=-\mu^{\prime}-1,-4 \leq \mu^{\prime}<2\right)$. The pointing arrows indicate the mualpha relationship ( $\alpha$ vs. $\mu$ or $\mu^{\prime}$ ) for which the particular variance applies. 


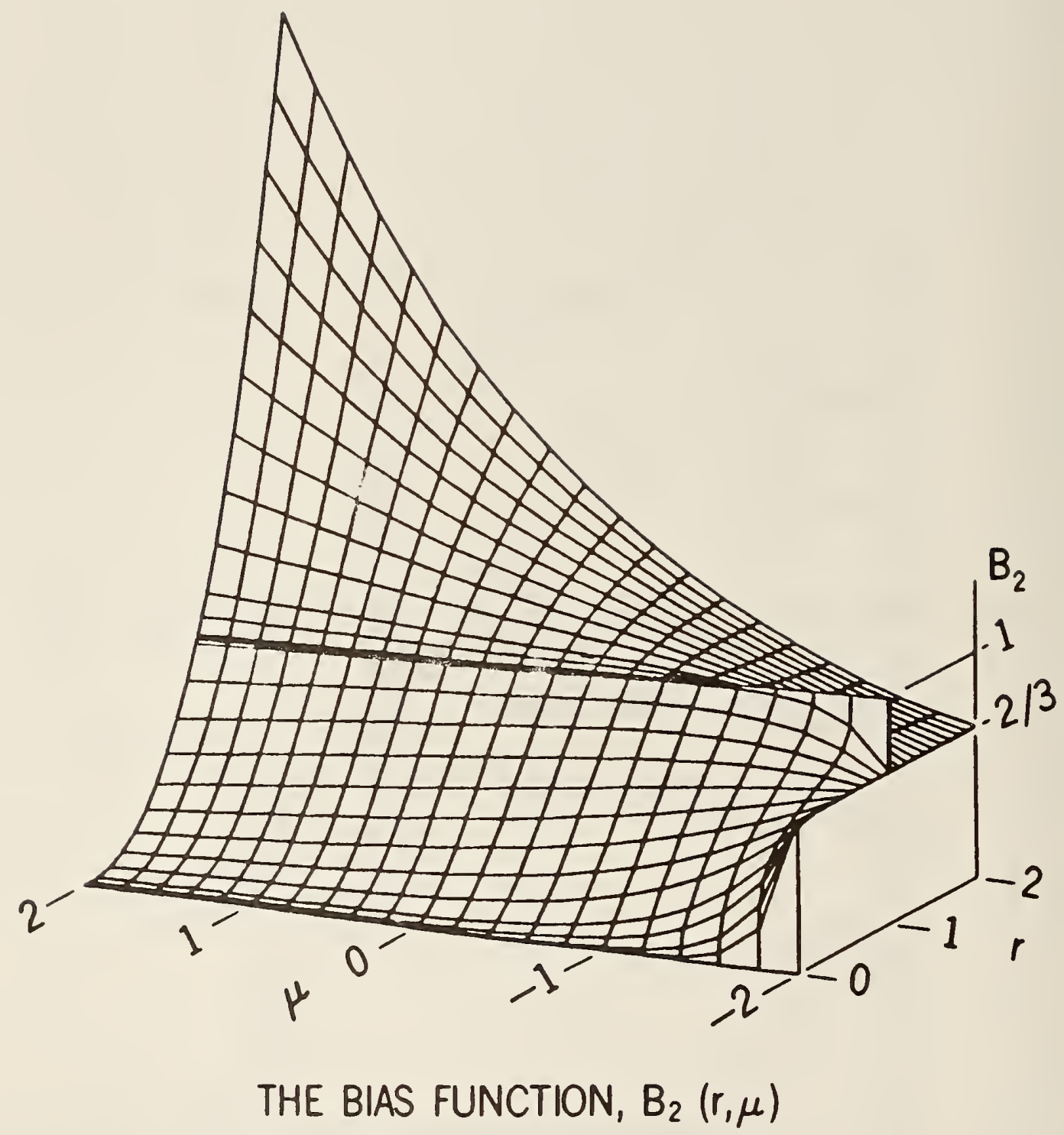

Figure 3. A three dimensional plot of the bias function $B_{2}(r, \mu)$, where $r=T / \tau$, and the dead time is $T-\tau$. The "fin" at $r=1$ and $\mu=-2$ approaches zero width as the measurement bandwidth approaches infinity (see appendix ref. [3]). 


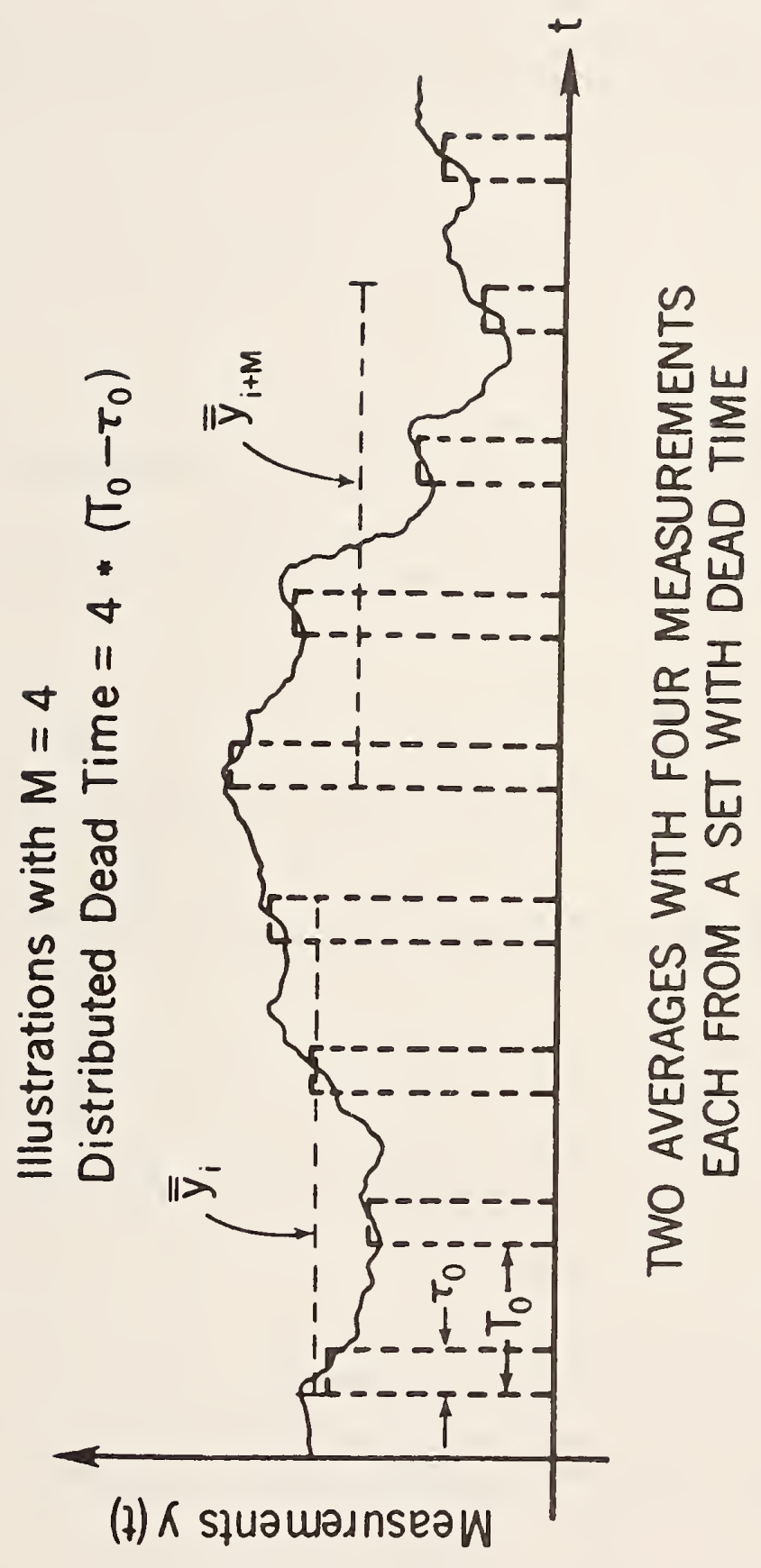

๑. ด ปี ᄃ 0 o

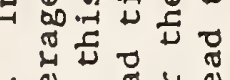
i 류

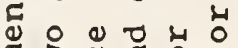
E 3 E 议

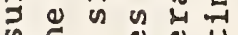
ज

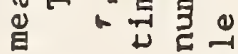

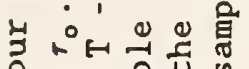
फ 1 11 虽至

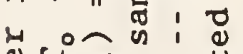
$\overbrace{}^{0}$ ○ \& 10 ธี त्र

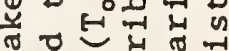
क $\checkmark$ 出 $>0$

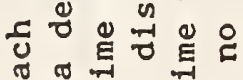
ช

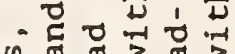
ग $\infty_{\infty} 0$ वाँ \% ¿ ¿ ฟ $\circ$ 而范 उ出幽。 0 을 겅 d ह के ㄱ

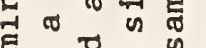
है 0 U $u$ ?

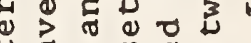

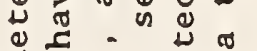
का फ - ठㅇㅓㅔ

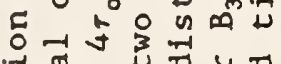

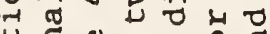
㫕 4 an. $4-10$ 岁形实员 ง

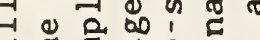

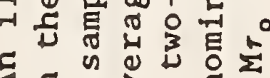
క . 武造 0 。 -

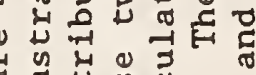
氠出 की

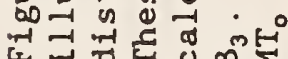




\section{Appendix}

With reference to figure 1 , the frequency sampling window has an equivalent phase sampling window. The intent is to evaluate the variance, $S(M)$, of the sampled phase function in terms of the phase autocorrelation function, $R(\tau)$. The process here is to correctly account for terms and cross-terms coming from squaring and averaging the samples for each $M$. The $B_{3}(2, M, r, \mu)$ function can then be obtained from the relation,

$$
B_{3}(2, M, r, \mu)=\frac{S(M)}{S(1) \cdot M^{\mu+2}},
$$

for appropriate $M, r$, and $\mu$. The denominator is just the two-sample variance with dead time for MT and Mr (in accordance with the definition of $\left.B_{3}(2, M, r, \mu)\right)$. The factors common to the numerator and denominator are ignored in the following.

For $M=1$, the variance $S(1)$ is just

$$
S(1)=4 \cdot R(0)-4 \cdot R(\tau)-4 \cdot R(T)+2 \cdot R(T+\tau)+2 R(T-\tau)
$$

where use has been made of the definition of the autocorrelation function,

$$
R(T)=E[\phi(t) \cdot \phi(t+T)]
$$

It is convenient to define a function $G(T)$ as

$$
G(T)=2 \cdot R(T)-R(T+\tau)-R(T-\tau)
$$

Similarly, $S(2)$ can now be written in the form,

$$
S(2)=8 \cdot R(0)-8 \cdot R(\tau)+2 \cdot G(T)-4 \cdot G(2 T)-2 \cdot G(3 T) \text {. }
$$

Following this procedure, we can verify that the general $S(M)$ is just 


$$
\begin{aligned}
S(M) & =4 \cdot M \cdot R(0)-4 \cdot M \cdot R(\tau)-2 \cdot M \cdot G(M T) \\
& +2 \sum_{n=1}^{M-1}(M-n)[2 \cdot G(n T)-G((M+n) T)-G((M-n) T)] .
\end{aligned}
$$

Following the work of Barnes and Allan $[2,3]$, we can define the function $U(\tau)$ by the relation,

$$
\mathrm{U}(\tau)=2 \cdot \mathrm{R}(0)-2 \cdot \mathrm{R}(\tau)
$$

and also define

$$
F(n r)=G(n T) / U(\tau)
$$

where $\mathrm{r}=\mathrm{T} / \tau$. The function $\mathrm{U}(\tau)$ for power-law power spectral densities has the form,

$$
U(\tau)=\frac{|\tau|^{\mu+2}}{4-2^{\mu+2}}
$$

which yields

$$
F(n r)=2 \cdot(n r)^{\mu+2}-(n r+1)^{\mu+2}-|n r-1|^{\mu+2} .
$$

Finally, the working relation can be written as

$$
B_{3}(2, M, r, \mu)=\frac{2 \cdot M+M \cdot F(M r) \frac{-\sum_{n=1}^{M-1}(M-n)[2 \cdot F(n r)-F((M+n) r)-F((M-n) r)]}{[2+F(r)] \cdot M^{\mu+2}} .}{.}
$$


$B 1(N, r, w)$ for $r=.01$

\begin{tabular}{|c|c|c|c|c|c|c|c|c|c|c|c|}
\hline He 1 & $N=$ & 4 & 8 & 16 & 32 & 64 & 128 & 256 & 512 & 1024 & INF \\
\hline 2 & : & $1.000 E+\infty 0$ & $1.000 E+\infty 0$ & $1.000 E+\infty 0$ & $1.000 E+\infty 0$ & 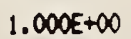 & $1.000 E+\infty$ & $1.000 E+\infty 0$ & $1.000 E+\infty 0$ & $1.000 E+\infty 0$ & $1.000 E+\infty$ \\
\hline 1.8 & : & $1.091 E+\infty 0$ & $1.210 E+\infty$ & $1.360 E+\infty$ & $1.545 E+\infty$ & 1. $772 E+\infty$ & $2.089 \varepsilon+\infty$ & $2.349 E+\infty 0$ & $2.456 E+\infty 0$ & $2.494 \varepsilon+\infty$ & $2.512 E+\infty$ \\
\hline 1.6 & : & $1.199 E+\infty 0$ & $1.487 E+\infty$ & $1.893 E+\infty$ & $2.45 E+\infty 0$ & $3.239 E+\infty 0$ & $4.440 E+\infty 0$ & $5.505 E+\infty$ & $6.00 X E+\infty$ & $6.198 E+00$ & $6.309 E+00$ \\
\hline 1.4 & $:$ & $1.328 E+\infty 0$ & $1.856 E+\infty 0$ & $2.688 E+\infty 0$ & $3.991 E+\infty 0$ & $6.054 E+\infty 0$ & $9.500 E+\infty 0$ & $1.282 E+01$ & $1.456 E+01$ & $1.532 E+01$ & $1.585 E+01$ \\
\hline 1.2 & : & $1.482 E+\infty 0$ & $2.346 E+\infty$ & $3.880 E+\infty$ & $6.598 \varepsilon+00$ & $1.145 E+01$ & $2.026 E+01$ & $2.947 E+01$ & $3.486 E+01$ & 3. $754 E+01$ & 3. $980 E+01$ \\
\hline 1 & : & $1.667 \mathrm{E}+\infty 0$ & 3. $000 E+\infty 0$ & $5.667 E+\infty$ & $1.100 E+01$ & $2.167 E+01$ & $4.255 E+01$ & $6.628 E+01$ & $8.190 E+01$ & $9.064 E+01$ & $1.000 E+02$ \\
\hline .8 & : & $1.884 E+\infty 0$ & $3.860 E+00$ & $8.303 E+\infty 0$ & $1.828 E+01$ & 4.041E+01 & $8.691 E+01$ & $1.443 E+02$ & $1.866 \varepsilon+02$ & 2. $137 E+02$ & $2.519 E+02$ \\
\hline .6 & : & $2.134 E+\infty 0$ & $4.959 E+\infty$ & $1.205 E+01$ & $2.977 \mathrm{E}+01$ & $7.296 E+01$ & $1.697 E+02$ & $2.995 E+02$ & $4.076 E+02$ & $4.851 E+02$ & $6.423 E+02$ \\
\hline .4 & : & $2.407 E+\infty 0$ & $6.279 E+\infty 0$ & $1.703 E+01$ & $4.655 E+01$ & $1.247 E+02$ & $3.106 E+02$ & $5.814 E+02$ & 8. $35 \pi+02$ & $1.043 E+03$ & $1.715 E+03$ \\
\hline-.2 & : & $2.677 E+\infty$ & $7.714 E+\infty 0$ & $2.296 E+01$ & $6.836 E+01$ & $1.976 E+02$ & $5.220 E+02$ & $1.036 E+03$ & $1.580 E+03$ & $2.084 E+03$ & $5.581 E+03$ \\
\hline 0 & : & $2.912 E+\infty$ & $9.075 E+\infty 0$ & $2.909 E+01$ & 9. 282E+01 & $2.857 E+02$ & $7.951 E+02$ & $1.672 E+03$ & $2.719 E+03$ & $3.826 E+03$ & \\
\hline .2 & : & $3.009 E+00$ & $1.018 E+01$ & 3. $448 E+01$ & $1.16 \geq E+02$ & $3.763 E+02$ & $1.097 E+03$ & $2.446 E+03$ & $4.263 E+03$ & $6.453 E+03$ & \\
\hline .4 & : & $3.203 E+00$ & $1.095 E+01$ & $3.857 E+01$ & 1. $354 E+02$ & $4.57 Z \mathrm{E}+02$ & $1.390 E+03$ & $3.287 E+03$ & $6.172 E+03$ & $1.014 E+04$ & \\
\hline .6 & : & $3.268 E+00$ & $1.143 E+01$ & 4.133E+01 & $1.495 E+02$ & $5.221 E+02$ & $1.649 E+03$ & $4.142 E+03$ & $8.419 E+03$ & $1.514 E+04$ & \\
\hline .8 & : & 3. $302 E+00$ & $1.170 E+01$ & 4. $303 E+01$ & $1.590 E+02$ & $5.700 E+02$ & $1.869 E+03$ & $4.991 E+03$ & $1.104 E+04$ & 2. $18 \% E+04$ & \\
\hline 1 & : & $3.319 E+0 n$ & $1.185 E+01$ & $4.403 E+01$ & $1.653 E+02$ & $6.066 E+02$ & $2.055 E+03$ & $5.842 E+03$ & $1.412 E+04$ & 3.109E+04 & \\
\hline 1.2 & : & $3.32 \pi E+\infty D$ & $1.192 E+01$ & $4.461 E+01$ & $1.693 E+02$ & $6.330 E+02$ & $2.215 E+03$ & $6.71 \pi E+03$ & $1.782 E+04$ & $4.383 E+04$ & \\
\hline 1.4 & : & $3.330 E+\infty 0$ & $1.196 E+01$ & 4.494E+01 & $1.720 E+02$ & $6.530 E+02$ & 2.359E+03 & $7.640 E+03$ & $2.234 E+04$ & $6.169 E+04$ & \\
\hline 1.6 & : & $3.332 E+(100$ & $1.198 E+01$ & $4.514 E+01$ & $1.738 \varepsilon+02$ & $6.688 E+02$ & $2.493 E+03$ & $8.637 E+03$ & $2.793 E+04$ & 8.697E+0.4 & \\
\hline 1.8 & : & $3.333 E+100$ & $1.199 E+01$ & $4.526 E+01$ & 1. $750 E+02$ & $6.819 E+02$ & $2.623 E+03$ & $9.737 E+03$ & $3.493 E+04$ & $1.231 E+05$ & \\
\hline 2 & : & 3. 333E+00 & 1.200E+01 & 4.533E+01 & $1.760 E+02$ & $6.933 E+02$ & $2.75 Z \mathbf{E}+0.3$ & $1.097 E+04$ & $4.378 E+04$ & $1.749 E+05$ & \\
\hline
\end{tabular}

$B 1(N, r, \ldots u)$ for $r=.03$

\begin{tabular}{|c|c|c|c|c|c|c|c|c|c|c|c|}
\hline Mu 1 & $N=$ & 4 & 8 & 16 & 32 & 64 & 128 & 256 & 512 & 1024 & INF \\
\hline 2 & : & $1.000 E+\infty 0$ & $1.000 E+\infty$ & $1.000 E+\infty 0$ & 1. $000 E+\infty$ & $1.000 E+\infty$ & $1.000 E+\infty 0$ & 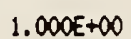 & 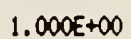 & $1.000 E+00$ & $1.000 E+\infty$ \\
\hline 1.8 & $:$ & $1.091 E+00$ & $1.211 E+\infty$ & $1.366 E+\infty 00$ & $1.573 E+\infty 0$ & $1.827 E+\infty$ & $1.950 E+\infty 0$ & $1.995 E+00$ & 2. $010 E+00$ & $2.015 x+00$ & $2.016 E+\infty 0$ \\
\hline 1.6 & : & $1.199 E+00$ & $1.491 E+\infty$ & $1.910 \varepsilon+\infty$ & $2.532 E+00$ & 3. $341 E+\infty 0$ & $3.782 E+\infty 0$ & $3.963 E+\infty 0$ & $4.030 E+\infty 0$ & $4.053 E+00$ & $4.064 E+\infty 0$ \\
\hline 1.4 & $:$ & $1.329 E+\infty 0$ & $1.863 E+\infty 0$ & $2.719 E+00$ & $4.133 E+\infty$ & $6.000 E+\infty 0$ & $7.268 E+\infty 0$ & $7.815 E+\infty 0$ & $8.044 E+00$ & $8.135 E+00$ & 8.191E+OO \\
\hline 1.2 & : & $1.484 E+00$ & $2.355 E+00$ & $3.918 E+\infty$ & $6.768 E+\infty 00$ & $1.093 E+01$ & $1.378 E+01$ & $1.525 E+01$ & $1.595 E+01$ & $1.626 E+01$ & $1.651 E+01$ \\
\hline 1 & : & 1. $(6 \in \pi E+\infty)$ & 3. $000 E+\infty)$ & $5.66 \pi \mathrm{E}+\infty$ & $1.100 E+01$ & $1.928 E+01$ & $2.560 E+01$ & $2.929 E+01$ & $3.12 \pi+01$ & 3. 229E+01 & 3. $333 E+01$ \\
\hline .8 & : & $1.878 E+\infty 0$ & $3.825 E+\infty$ & $8.141 E+00$ & 1. $753 E+01$ & 3. $304 E+01$ & $4.626 E+01$ & $5.499 E+01$ & $6.029 E+01$ & 6. $341 E+01$ & $6.770 E+01$ \\
\hline .6 & : & $2.114 E+\infty 0$ & $4.838 \varepsilon+\infty .00$ & 1.147E+01 & $2.706 E+01$ & $5.441 E+01$ & 8. $049 E+01$ & $9.997 E+01$ & $1.134 E+02$ & 1. $225 E+02$ & $1.403 E+02$ \\
\hline .4 & : & $2.362 E+\infty 0$ & $6.005 E+\infty$ & $1.566 E+01$ & $3.993 E+01$ & $8.524 E+01$ & $1.335 E+02$ & $1.744 E+02$ & $2.066 E+02$ & $2.314 E+02$ & $3.099 E+02$ \\
\hline-.2 & : & $2.6014 E+00$ & $7.242 E+\infty 00$ & $2.047 E+01$ & $5.581 E+01$ & $1.259 E+02$ & $2.094 E+02$ & $2.897 E+02$ & $3.619 E+0 ?$ & $4.256 E+02$ & $8.571 E+02$ \\
\hline 0 & : & $2.819 E+\infty$ & $8.430 E+00$ & $2.546 E+01$ & $7.348 E+01$ & $1.748 E+02$ & $3.096 E+02$ & $4.568 E+02$ & $6.08 Z E+02$ & $7.611 \mathrm{E}+02$ & \\
\hline .2 & : & $2.992 E+00$ & $9.460 E+\infty$ & $3.013 E+01$ & $9.127 E+01$ & 2. $2865+02$ & 4.324E+02 & $6.856 E+02$ & $9.834 E+02$ & $1.32 x E+03$ & \\
\hline .4 & : & $3.118 E+00$ & $1.027 E+01$ & $3.412 E+01$ & $1.077 E+02$ & $2.835 E+02$ & 5. $750 E+02$ & $9.868 E+02$ & $1.541 E+03$ & $2.27 \pi E+03$ & \\
\hline .6 & : & $3.203 E+\infty 0$ & $1.087 E+01$ & $3.728 E+01$ & 1.220E+02 & 3. $37 Z E+02$ & $7.357 \mathrm{E}+02$ & 1. $376 E+03$ & 2. $364 E+03$ & $3.868 \varepsilon+03$ & \\
\hline .8 & : & $3.257 E+\infty 0$ & $1.128 E+01$ & $3.966 E+01$ & $1.339 E+02$ & $3.88 \pi E+02$ & $9.152 E+02$ & $1.879 E+03$ & $3.580 E+03$ & $6.554 E+03$ & \\
\hline 1 & : & $3.290 E+00$ & $1.155 E+01$ & $4.141 E+01$ & $1.437 E+02$ & 4. $382 E+02$ & $1.117 E+03$ & $2.533 E+03$ & 5. $395 E+03$ & $1.114 E+04$ & \\
\hline 1.2 & : & $3.309 E+\infty 0$ & $1.17 X E+01$ & $4.267 E+01$ & $1.518 \varepsilon+02$ & $4.867 E+02$ & $1.348 E+03$ & $3.3922 \times 03$ & $8.122 E+03$ & $1.903 E+04$ & \\
\hline 1.4 & : & $3.320 E+\infty 0$ & $1.184 E+01$ & $4.360 E+01$ & $1.5888+02$ & $5.352 \mp+02$ & $1.615 E+03$ & $4.535 E+03$ & $1.228 x+04$ & $3.276 E+04$ & \\
\hline 1.6 & : & $3.327 E+\infty$ & $1.191 E+01$ & $4.431 E+01$ & $1.650 E+02$ & 5. $851 E+02$ & $1.930 E+03$ & $6.068 E+03$ & $1.866 E+04$ & $5.684 E+0.4$ & \\
\hline 1.8 & : & $3.331 E+\infty 0$ & $1.196 E+01$ & $4.487 E+01$ & $1.706 E+02$ & $6.374 E+02$ & 2.304E+03 & $8.142 E+03$ & $2.849 E+04$ & $9.936 E+04$ & \\
\hline 2 & : & $3.33 .3 E+\infty 0$ & 1. $200 E+01$ & $4.533 E+01$ & $1.760 E+02$ & $6.933 E+02$ & $2.75 x E+03$ & $1.097 E+04$ & $4.378 E+04$ & $1.749 E+05$ & \\
\hline
\end{tabular}


$B 1(N, r, m)$ for $r=.1$

\begin{tabular}{|c|c|c|c|c|c|c|c|c|c|c|c|}
\hline 41 & $N=$ & 4 & 8 & 16 & 32 & 64 & 128 & 256 & 512 & 1024 & IN \\
\hline-2 & : & $1.000 E+\infty 0$ & 1. $000 E+\infty$ & $1.000 E+\infty$ & 1.000E+ +00 & 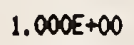 & $1.000 E+\infty 0$ & $1.000 E+\infty 0$ & $1.000 E+\infty$ & $1.000 E+\infty 0$ & $1.000 E+\infty 0$ \\
\hline 1.8 & : & $1.093 E+00$ & 1. $226 E+\infty$ & $1.438 E+\infty 0$ & $1.547 E+\infty$ & $1.579 E+\infty 0$ & $1.586 E+\infty 0$ & $1.586 E+\infty$ & $1.585 E+00$ & $1.584 E+\infty 0$ & $1.5835+\infty$ \\
\hline 1.6 & : & $1.205 E+\infty 0$ & $1.522 E+\infty 0$ & $2.021 E+\infty 0$ & $2.329 E+\infty 0$ & $2.449 E+\infty 0$ & $2.490 E+\infty 0$ & $2.50 \mathbb{Z E}+\infty 0$ & $2.505 E+\infty 0$ & $2.505 E+00$ & $2.504 E+\infty 0$ \\
\hline 1.4 & $:$ & 1. $33 \pi t+\infty$ & $1.907 E+\infty$ & $2.832 E+\infty$ & $3.477 E+\infty$ & 3. $772 E+00$ & $3.892 E+\infty 0$ & $3.938 \varepsilon+\infty$ & $3.954 E+00$ & $3.960 E+100$ & $3.962 E+100$ \\
\hline 1.2 & : & $1.491 E+\infty$ & $2.396 E+00$ & $3.931 E+\infty$ & $5.125 E+\infty 0$ & 5. $751 E+\infty 0$ & $6.046 E+\infty 0$ & $6.177 E+\infty$ & $6.235 E+\infty 0$ & $6.260 E+\infty 0$ & $6.278 E+\infty 0$ \\
\hline & $:$ & $1.66 \pi \mathrm{E}+\infty$ & $.000 E+\infty$ & $5.375 E+\infty$ & $7.42 \%+\infty$ & $8.653 E+\infty 0$ & $9.312 E+\infty 0$ & $9.652 E+\infty$ & $9.825 E+\infty$ & $9.912 E+100$ & $1.000 E+101$ \\
\hline .8 & : & $1.860 E+\infty 0$ & $3.720 E+\infty 0$ & $7.204 E+\infty$ & 1.055E+01 & 1.281E+01 & 1.419E+01 & $1.502 E+01$ & $1.550 E+01$ & $1.577 E+01$ & $1.616 E+01$ \\
\hline .6 & : & $2.065 E+\infty 0$ & $4.540 E+\infty$ & $9.423 E+\infty$ & $1.461 E+01$ & $1.85 \% E+01$ & $2.137 E+01$ & $2.326 E+01$ & $2.452 E+01$ & $2.537 E+01$ & $2.702 E+01$ \\
\hline-.4 & $:$ & $2.273 E+\infty 0$ & $-430 E+\infty 0$ & $1.199 E+01$ & $1.971 E+01$ & $2.642 E+01$ & 3.177E+01 & $3.592 \mathbf{E}+01$ & $3.910 E+01$ & 4. $153 E+01$ & $4.922 E+01$ \\
\hline-.2 & : & $2.472 E+\infty$ & $344 E+C$ & $1.481 E+01$ & $2.585 E+01$ & $3.673 E+01$ & $664 E+01$ & $5.542 E+01$ & $6.314 E+01$ & $6.989 E+01$ & $1.156 E+02$ \\
\hline 0 & : & $2.653 E+\infty 0$ & $7.236 E+\infty 0$ & 1. $779 E+01$ & 3. $300 E+01$ & $5.003 E+01$ & $6.771 E+01$ & $8.566 E+01$ & $1.037 E+02$ & $1.219 E+02$ & \\
\hline .2 & $:$ & $2.810 E+00$ & $8.065 E+00$ & $2.080 E+01$ & $4.111 E+01$ & $6.690 E+01$ & $9.750 E+01$ & $1.331 E+02$ & $1.741 E+02$ & $2.214 E+02$ & \\
\hline .4 & : & $2.940 E+\infty 0$ & $8.804 E+\infty 0$ & $2.376 E+01$ & $5.015 E+01$ & $8.816 E+01$ & 1.39TE+02 & $2.084 E+02$ & $2.993 x+02$ & $4.194 E+02$ & \\
\hline .6 & $:$ & $3.043 E+\infty$ & $9.445 E+\infty$ & $2.663 E+01$ & $6.014 E+01$ & $1.149 E+02$ & 1. $999 E+02$ & $3.295 E+02$ & $5.265 E+02$ & 8. $254 E+02$ & \\
\hline .8 & : & $3.123 E+00$ & $9.989 E+00$ & $2.938 E+01$ & $7.118 E+01$ & $1.487 E+02$ & $2.862 E+02$ & $5.26 \% E+02$ & $9.466 E+02$ & $1.678 E+03$ & \\
\hline 1 & : & $3.184 E+00$ & $1.045 E+01$ & $3.204 E+01$ & 8. $347 E+01$ & $1.918 E+02$ & $4.114 E+02$ & $8.523 E+02$ & $1.735 E+03$ & $3.500 E+103$ & \\
\hline 1.2 & : & 3. $230 E+\infty 0$ & $34 E+01$ & $3.463 E+01$ & $9.728 E+01$ & $2.470 E+02$ & $5.943 E+02$ & $1.394 E+03$ & $3.232 E+03$ & $7.453 E+03$ & \\
\hline 1.4 & : & $3.265 E+\infty 0$ & $.118 E+01$ & $3.721 E+01$ & 1. $130 E+02$ & 3. $183 E+02$ & $8.636 E+02$ & $2.304 E+03$ & $6.103 E+03$ & $1.613 E+04$ & \\
\hline 1.6 & : & $3.29 S E+00$ & $1.147 E+01$ & $3.982 E+01$ & 1. $309 E+02$ & 4.113E+02 & $1.263 E+03$ & $3.843 E+03$ & $1.166 E+04$ & 3. $535 E+04$ & \\
\hline 1.8 & : & $3.315 E+\infty)$ & $1.174 E+01$ & $4.252 E+01$ & $1.51 T E+02$ & 5. $330 E+102$ & $1.858 E+03$ & $6.467 E+0.3$ & 2. $250 E+04$ & $7.830 E+04$ & \\
\hline 2 & : & $3.333 E+\infty 0$ & $1.200 E+1) 1$ & $4.533 E+01$ & $1.760 E+02$ & 6. $.933 E+02$ & $2.75 x E+0 ?$ & $1.09 \pi+04$ & $4.378 E+i 4$ & $1.749 E+05$ & \\
\hline
\end{tabular}

$B 1(N, r, m)$ for $r=.3$

\begin{tabular}{|c|c|c|c|c|c|c|c|c|c|c|c|}
\hline Mu 1 & $N=$ & 4 & 8 & 16 & 32 & 64 & 128 & 256 & 512 & 1024 & INF \\
\hline-2 & : & 1. $000 E+\infty O$ & $1.000 E+00$ & $1.000 E+\infty$ & $1.000 E+00$ & $1.000 E+00$ & $1.000 E+\infty 0$ & $1.000 E+00$ & 1.000E+CO & 1.000E+00 & $1.000 E+00$ \\
\hline-1.8 & : & 1. $124 E+\infty 0$ & $1.226 E+\infty 0$ & 1. $259 E+\infty)$ & $1.265 E+00$ & $1.265 E+\infty 0$ & $1.263 E+00$ & $1.262 E+\infty$ & $1.261 E+00$ & $1.261 E+\infty)$ & $1.260 E+00$ \\
\hline-1.6 & : & 1. $254 E+\infty$ & $1.479 E+\infty 0$ & $1.564 E+O D$ & $1.58 \% E+00$ & $1.594 E+\infty 0$ & $1.594 E+\infty 0$ & $1.593 E+\infty$ & $1.591 E+() 0$ & $1.591 E+\infty 0$ & $1.590 E+00$ \\
\hline-1.4 & $:$ & 1. $388 E+(0 ;)$ & $1.75 \% E+\infty 0$ & $1.925 E+00$ & $1.988 E+\infty 0$ & $2.009 E+\infty 0$ & $2.014 E+\infty 0$ & $2.015 E+00$ & $2.015 E+00$ & $2.014 E+100$ & $2.013 E+00$ \\
\hline 1.2 & : & $1.527 E+00$ & $2.068 E+00$ & $2.351 E+\infty 0$ & $2.479 E+\infty 00$ & $2.534 E+\infty 0$ & $2.556 E+\infty 0$ & $2.565 E+(10$ & $2.569 E+\infty 0$ & $2.570 E+00$ & $2.571 E+00$ \\
\hline-1 & : & $1.66 T E+00$ & $2.405 E+\infty 0$ & $2.850 E+00$ & $3.087 E+(n)$ & $3.209 E+\infty 0$ & $3.271 E+\infty 0$ & $3.302 E+00$ & $3.318 E+\infty 0$ & $3.326 E+00$ & $3.333 E+00$ \\
\hline-.8 & : & $1.806 E+00$ & $2.769 E+00$ & $3.435 E+00$ & $3.847 E+\infty 0$ & $4.053 E+\infty 0$ & $4.239 E+00$ & $4.325 E+\infty 0$ & $4.376 E+\infty 0$ & $4.405 E+00$ & 4. $447 E+(10)$ \\
\hline .6 & $:$ & $1.943 E+00$ & $3.160 E+C 0$ & 4. $122 E+\infty 0$ & $4.804 E+\infty 0$ & $5.275 E+\infty 0$ & $5.595 E+00$ & $5.812 E+00$ & $5.958 E+00$ & $6.056 E+000$ & $0.252 E+00$ \\
\hline .4 & : & $2.076 E+00$ & $3.577 E+\infty D$ & $4.927 E+00$ & $6.024 E+00$ & $6.889 E+00$ & $7.564 E+\infty 0$ & $8.086 E+\infty 0$ & 8. $48 \pi E+00$ & $8.795 E+(x)$ & $9.7765+00$ \\
\hline .2 & : & $2.203 E+100$ & $4.021 E+00$ & $5.877 E+00$ & $7.59 \pi E+00$ & $9.147 E+100$ & $1.053 E+01$ & $1.175 E+01$ & 1. $282 E+101$ & $1.377 E+01$ & $2.017 E+01$ \\
\hline 0 & $:$ & $2.325 E+00$ & $4.492 E+\infty 0$ & $7.000 E+\infty 00$ & $9.651 E+00$ & $1.238 E+01$ & $1.515 E+01$ & $1.796 E+01$ & $2.079 E+01$ & $2.363 E+01$ & \\
\hline .2 & : & $2.440 E+00$ & $4.994 E+00$ & $8.3385+\infty 0$ & $1.236 E+01$ & $1.710 E+01$ & $2.260 E+01$ & $2.897 E+01$ & $3.632 E+01$ & $4.479 E+01$ & \\
\hline .4 & $:$ & $2.550 E+00$ & $5.530 E+\infty 0$ & $9.940 E+(0)$ & $1.599 E+01$ & $2.411 E+01$ & $3.493 E+01$ & $4.926 E+01$ & $6.823 E+01$ & 9. $330 E+01$ & \\
\hline .6 & : & $2.654 E+00$ & $6.106 E+00$ & $1.187 E+01$ & $2.089 E+01$ & $3.472 E+01$ & $5.581 E+01$ & $8.786 E+01$ & 1. $365 E+02$ & $2.103 E+02$ & \\
\hline .8 & : & 2. $754 E+C D$ & $6.729 E+\infty 0$ & 1. 422E+01 & $2.756 E+01$ & $5.098 E+01$ & $9.187 E+01$ & $1.632 E+02$ & $2.874 E+02$ & $5.03 T E+02$ & \\
\hline 1 & $:$ & $2.852 E+(0) 0$ & $7.406 E+00$ & $1.708 E+01$ & $3.673 E+01$ & $7.618 \varepsilon+01$ & 1. $552 E+02$ & $3.132 E+02$ & $6.292 E+02$ & $1.261 E+0.3$ & \\
\hline 1.2 & $:$ & $2.947 E+00$ & 8. $147 E+\infty 0$ & $2.059 E+01$ & $4.942 E+01$ & $1.157 E+02$ & $2.67 \notin+02$ & $6.170 E+02$ & $1.419 E+03$ & $3.261 E+03$ & \\
\hline 1.4 & : & $3.04 Z E+\infty$ & $8.963 E+100$ & $2.493 E+01$ & $6.709 E+01$ & $1.781 E+02$ & $4.703 E+02$ & $1.241 E+03$ & $3.272 E+03$ & $8.631 E+103$ & \\
\hline 1.6 & : & $3.137 E+00$ & $9.868 E+\infty 0$ & 3. $030 E+01$ & $9.185 E+01$ & $2.774 E+02$ & $8.381 E+02$ & $2.535 x+03$ & $7.673 E+03$ & $2.324 E+04$ & \\
\hline 1.8 & : & $3.234 E+00$ & $1.08 \pi E+01$ & $3.699 \mathrm{E}+01$ & $1.26 \pi+02$ & 4. $366 E+02$ & $1.511 E+03$ & $5.245 E+03$ & $1.823 E+04$ & $6.343 E+104$ & \\
\hline 2 & : & $3.333 E+\infty 0$ & 1. $200 E+01$ & $4.533 x+01$ & $1.760 E+02$ & $6.933 E+02$ & $2.752 E+03$ & $1.097 E+0.4$ & $4.378 E+04$ & $1.74 \% E+05$ & \\
\hline
\end{tabular}


$B 1(N, r, w)$ for $r=1$

\begin{tabular}{|c|c|c|c|c|c|c|c|c|c|c|c|}
\hline Hus 1 & $N=$ & 4 & 8 & 16 & 32 & 64 & 128 & 256 & 512 & 1024 & INF \\
\hline-2 & : & 8. 333E-01 & $7.500 E-01$ & $7.083 E-01$ & $6.875 E-01$ & $6.771 E-01$ & $6.719-01$ & $6.693 E-01$ & $6.680 E-01$ & $6.673 E-01$ & 6. $667 E-01$ \\
\hline-1.8 & $:$ & $8.581 E-01$ & $7.827 \mathrm{E}-01$ & $7.431 E-01$ & $7.226 E-01$ & $7.122 E-01$ & $7.068 E-01$ & $7.042 E-01$ & $7.028 E-01$ & $7.021 E-01$ & $7.014 E-01$ \\
\hline-1.6 & : & $8.866 E-01$ & 8. $221 E-01$ & $7.864 E-01$ & $7.672 E-01$ & $7.570 E-01$ & 7.517E-01 & $7.490 E-01$ & $7.476 E-01$ & 7. $468 E-01$ & $7.461 E-01$ \\
\hline-1.4 & $:$ & $9.193 E-01$ & $8.700 E-01$ & $8.410 E-01$ & $8.245 E-01$ & $8.154 E-01$ & $8.105 E-01$ & $8.07 \%-01$ & 8.065E-01 & 8.058E-01 & 8.051E-01 \\
\hline-1.2 & : & $9.56 \% E-01$ & $9.284 E-01$ & $9.105 E-01$ & $8.997 E-01$ & $8.9335-01$ & $8.897 \mathrm{E}-01$ & 8. $877 E-01$ & $8.866 E-01$ & $8.860 E-01$ & 8.854E-01 \\
\hline-1 & : & $1.000 E+\infty$ & $1.000 E+\infty$ & 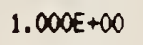 & $1.000 E+\infty 0$ & 1.000E+00 & $1.000 E+\infty 0$ & $1.000 E+\infty 0$ & $1.000 E+\infty 0$ & 1.000E $+\infty 0$ & 1.000E+DO \\
\hline-.8 & $:$ & $1.050 E+00$ & 1. $0088 E+\infty$ & $1.117 E+\infty$ & $1.137 E+\infty$ & $1.150 E+\infty 0$ & $1.160 E+00$ & $1.165 E+\infty 0$ & $1.16 \% E+00$ & $1.171 E+\infty$ & $1.175 E+\infty$ \\
\hline-.6 & $:$ & $1.107 E+\infty$ & $1.197 \mathrm{E}+$ & $1.271 E+$ & $1.327 E+\infty$ & $1.370 E+\infty 0$ & $1.401 E+\infty$ & $1.42 Z E+\infty 0$ & $1.438 E+00$ & $1.448 E+\infty 0$ & $1.470 E+00$ \\
\hline-.4 & : & $1.172 E+\infty 0$ & $1.333 E+\infty$ & $1.476 E+\infty 0$ & $1.59 \%+\infty 0$ & $1.700 E+00$ & $1.782 E+\infty$ & $1.847 E+00$ & $1.898 E+00$ & $1.938 E+\infty 0$ & $2.065 E+\infty$ \\
\hline-.2 & : & $1.247 E+00$ & $1.50 z E+\infty$ & $1.754 E+00$ & $1.994 E+00$ & $2.216 E+\infty 0$ & $2.418 E+\infty$ & $2.599 E+\infty 0$ & 2. $759 E+\infty$ & 2. $900 E+00$ & $3.863 E+00$ \\
\hline 0 & : & $1.333 E+\infty$ & $1.714 E+\infty 0$ & $2.133 E+00$ & $2.581 E+\infty$ & $3.048 E+00$ & $3.528 E+\infty 0$ & $4.016 E+00$ & $4.509 E+00$ & $5.005 E+\infty 0$ & \\
\hline .2 & : & $1.432 E+00$ & $1.98 \mathrm{E}+\infty$ & $2.658 \varepsilon+00$ & $3.471 E+\infty 0$ & $4.432 E+\infty 0$ & $5.555 E+00$ & $6.858 E+00$ & $8.363 E+\infty 0$ & $1.010 E+01$ & \\
\hline .4 & $:$ & $1.546 E+\infty 0$ & $2.320 E+00$ & $3.391 E+\infty 0$ & $4.846 E+\infty 0$ & $6.801 E+\infty 0$ & $9.407 E+\infty 0$ & 1. $287 E+01$ & $1.744 E+01$ & $2.350 E+01$ & \\
\hline .6 & $:$ & $1.677 E+\infty$ & $2.750 E+\infty 0$ & $4.424 E+00$ & $7.006 E+\infty 0$ & $1.096 E+01$ & $1.698 E+01$ & $2.614 E+01$ & $4.005 E+01$ & $6.114 E+01$ & \\
\hline .8 & $:$ & $1.827 E+\infty$ & 3. $299 E+\infty 0$ & $5.894 E+\infty$ & $1.045 E+01$ & $1.841 E+01$ & 3. $230 E+01$ & $5.652 E+01$ & $9.872 E+01$ & $1.722 E+02$ & \\
\hline 1 & : & $2.000 E+\infty 0$ & $4.000 E+00$ & $8.000 E+00$ & $1.600 E+01$ & $3.200 E+01$ & $6.400 E+01$ & 1. $280 E+02$ & $2.560 E+02$ & $5.120 E+02$ & \\
\hline 1.2 & : & $2.19 B E+00$ & $4.900 E+\infty 0$ & $1.104 E+01$ & $2.506 E+01$ & $5.717 E+01$ & $1.308 E+02$ & $2.999 E+02$ & $6.881 E+02$ & $1.580 E+0.3$ & \\
\hline 1.4 & $:$ & $2.426 E+100$ & $6.059 E+00$ & $1.546 E+01$ & $3.999 E+01$ & $1.044 E+02$ & $2.738 E+02$ & $7.202 E+02$ & $1.897 E+03$ & $5.003 E+03$ & \\
\hline 1.6 & : & $2.68 B E+100$ & $7.555 E+00$ & $2.191 E+01$ & $6.479 E+01$ & $1.938 E+02$ & $5.833 E+02$ & $1.762 E+03$ & $5.331 E+03$ & $1.615 k+04$ & \\
\hline 1.8 & : & $2.988 E+100$ & $9.490 E+00$ & $3.138 E+01$ & $1.063 E+02$ & $3.646 E+02$ & 1. $260 E+03$ & $4.372 E+03$ & $1.519 E+04$ & $5.286 E+0.4$ & \\
\hline 2 & $:$ & $3.33 .3 E+\infty 0$ & 1. $200 E+01$ & $4.533 E+01$ & $1.760 E+02$ & $6.933 E+02$ & $2.752 E+03$ & $1.097 E+04$ & 4. $378 E+04$ & $1.749 E+05$ & \\
\hline
\end{tabular}

B1(N, $r, n u)$ for $r=1.01$

\begin{tabular}{|c|c|c|c|c|c|c|c|c|c|c|c|}
\hline Mu 1 & $N=$ & 4 & 8 & 16 & 32 & 64 & 128 & 256 & 512 & 1024 & INF \\
\hline-2 & : & 1.000E+O0 & 1.000E+00 & $1.000 E+100$ & 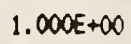 & $1.000 E+\infty$ & 1. $000 E+\infty$ & 1.000E+00 & $1.000 E+00$ & $1.000 E+00$ & $1.000 E+00$ \\
\hline-1.8 & $:$ & $9.156 E-01$ & $8.682 E-01$ & $8.425 E-0.1$ & 8. $2808 E-01$ & $8.217 E-01$ & $8.181 E-01$ & $8.162 E-01$ & $8.153 E-01$ & $8.148 E-01$ & $8.143 E-01$ \\
\hline-1.6 & : & $9.0985-01$ & $8.566 E-01$ & $8.264 E-01$ & $8.098 E-01$ & $8.009 E-01$ & $7.963 E-01$ & $7 ., 938 E-01$ & $7.926 E-01$ & $7.920 E-01$ & 7.913E-01 \\
\hline-1.4 & : & $9.286 E-(11$ & $8.840 E-01$ & $8.573 x-01$ & 8.419E-01 & 8. $333 E-01$ & 8.287E-01 & $8.262 E-01$ & $8.249 E-01$ & $8.242 E-01$ & $8.235 E-01$ \\
\hline-1.2 & : & 9.599E-01 & $9.331 E-01$ & $9.160 E-01$ & $9.056 E-01$ & 8. $995 E-01$ & $8.960 E-01$ & $8.940 E-01$ & $8.929 E-01$ & $8.924 E-01$ & $8.917 \mathrm{E}-01$ \\
\hline-1 & : & 1. $000 E+\infty 0$ & 1. $000 E+00$ & $1.000 E+\infty 00$ & $1.000 E+00$ & $1.000 E+00$ & 1. $000 E+\infty$ & $1.000 E+\infty 0$ & $1.000 E+\infty$ & 1.000E +00 & $1.000 E+00$ \\
\hline.$- \varepsilon$ & : & 1. $048 E+00$ & $1.005 E+00$ & $1.113 E+\infty 0$ & $1.133 E+\infty 0$ & $1.147 E+\infty 0$ & $1.155 E+100$ & $1.161 E+00$ & $1.165 E+\infty 0$ & $1.167 E+00$ & $1.170 E+\infty 0$ \\
\hline-.6 & : & $1.104 E+00$ & $1.193 E+100$ & $1.265 E+00$ & $1.321 E+00$ & $1.362 E$ & $1.303 E+00$ & $1.414 E+00$ & $1.42 \% E+00$ & $1.440 E+00$ & $1.461 E+00$ \\
\hline-.4 & : & 1. $168 E+00$ & 1. 327E+Co & $1.468 E+\infty 0$ & $1.589 E+\infty 0$ & $1.689 E+00$ & $1.770 E+00$ & $1.835 E+00$ & $1.885 E+100$ & $1.924 E+\infty 0$ & $2.050 E+00$ \\
\hline-.2 & $:$ & $1.243 E+100$ & $1.495 E+00$ & $1.743 E+00$ & $1.980 E+\infty 0$ & $2.200 E+00$ & $2.400 E+00$ & $2.579, E+\infty 0$ & $2.737 E+00$ & $2.877 E+Q 0$ & $3.829 E+\infty 0$ \\
\hline 0 & : & 1. $329 E+\infty 0$ & $1.706 E+\infty 0$ & $2.120 E+\infty 0$ & $2.563 E+00$ & $3.025 E+00$ & $3.500 E+00$ & $3.984 E+00$ & $4.472 E+00$ & $4.9635+00$ & \\
\hline .2 & $:$ & $1.428 E+\infty 0$ & $1.972 E+00$ & $2.642 E+00$ & $3.447 E+\infty 0$ & 4. $400 E+00$ & $5.512 E+00$ & $6.804 E+00$ & $8.296 E+00$ & $1.002 E+01$ & \\
\hline .4 & : & $1.541 E+\infty$ & 2. $30 \% E+\infty 0$ & $3.371 E+\infty 0$ & $4.814 E+\infty 0$ & $6.754 E+\infty 0$ & $9.340 E+00$ & $1.277 E+01$ & $1.731 E+01$ & $2.332 E+01$ & \\
\hline.$t$ & $:$ & $1.672 E+00$ & $2.738 E+\infty 0$ & $4.400 E+00$ & $6.963 E+00$ & $1.089 E+01$ & $1.687 E+01$ & $2.597 E+01$ & $3.978 E+01$ & $6.073 E+01$ & \\
\hline .8 & $:$ & $1.822 E+(M)$ & 3. $28.5 E+\infty 0$ & $5.864 E+a 0$ & $1.039 E+01$ & $1.830 E+01$ & $3.212 E+01$ & $5.61 \%+01$ & $9.814 E+01$ & $1.712 E+02$ & \\
\hline 1 & : & $1.995 E+00$ & $3.985 E+00$ & $7.966 E+00$ & $1.593 E+01$ & $3.185 E+01$ & $6.369 E+01$ & $1.274 E+02$ & $2.547 E+02$ & $5.095 E+02$ & \\
\hline 1.2 & : & $2.194 E+00$ & $4.885 E+00$ & 1. $100 E+01$ & $2.497 E+01$ & $5.695 E+01$ & $1.303 E+02$ & $2.987 E+02$ & $6.854 E+02$ & $1.573 E+03$ & \\
\hline 1.4 & : & $2.422 E+00$ & $6.045 E+\infty 0$ & $1.542 E+01$ & $3.988 E+01$ & $1.041 E+02$ & $2.730 E+02$ & $7.180 E+02$ & $1.892 E+03$ & $4.988 E+03$ & \\
\hline 1.6 & : & $2.685 E+00$ & $7.542 E+00$ & $2.187 E+01$ & $6.466 E+01$ & $1.934 E+02$ & $5.82 Z E+02$ & 1. $758 E+03$ & $5.321 E+03$ & $1.611 E+04$ & \\
\hline 1.8 & : & $2.986 \mathrm{E}+00$ & $9.482 E+00$ & $3.135 E+01$ & $1.062 E+02$ & $3.643 E+02$ & 1. $25 \% E+03$ & $4.367 E+03$ & $1.518 E+04$ & $5.280 E+04$ & \\
\hline 2 & $:$ & 3. $33.3 E+00$ & 1. $200 E+01$ & $4.533 E+01$ & $1.760 E+02$ & $6.933 E+02$ & $2.752 E+03$ & $1.097 E+04$ & 4. $378 E+04$ & $1.749 E+05$ & \\
\hline
\end{tabular}


$B 1(N, r, m)$ for $r=1.1$

\begin{tabular}{|c|c|c|c|c|c|c|c|c|c|c|c|}
\hline u 1 & $N=$ & 4 & 8 & 16 & 32 & 64 & 128 & 256 & 512 & 1024 & INF \\
\hline-2 & : & $1.000 E+00$ & $1.000 E+00$ & $1.000 E+00$ & $1.000 E+00$ & 1.000E+00 & $1.000 E+00$ & $1.000 E+00$ & $1.000 E+00$ & 1. $000 E E+00$ & 1. $000 E+00$ \\
\hline-1.8 & : & $9.527 E-01$ & $9.243 E-01$ & $9.082 E-01$ & $8.994 E-01$ & $3.948 E-01$ & $8.924 E-01$ & $8.911 E-01$ & $8.905 E-01$ & $8.902 E-01$ & $8.898 E-01$ \\
\hline-1.6 & : & $9.415 E-01$ & $9.048 E-01$ & 8.831E-01 & 8.709E-01 & $8.64 \geq \mathbf{E}-01$ & $8.607 E-01$ & $8.588 \varepsilon-01$ & $8.578 E-01$ & $8.574 E-01$ & $8.5695-01$ \\
\hline-1.4 & : & $9.493 E-01$ & $9.157 \mathrm{E}-01$ & $8.948 E-01$ & $8.825 E-01$ & 8. $755 E-01$ & $8.717 E-01$ & $8.696 E-01$ & $8.665 E-01$ & 8.680E-01 & $8.674 E-01$ \\
\hline-1.2 & : & $9.696 E-01$ & $9.482 E-01$ & $9.341 E-01$ & $9.254 E-01$ & $9.201 E-01$ & $9.171 E-01$ & $9.154 E-01$ & $9.145 E-01$ & 9.140E-01 & 9.134E-01 \\
\hline-1 & $:$ & $1.000 E+00$ & $1.000 E+\infty 0$ & $1.000 E+\infty 0$ & $1.000 E+00$ & 1.000E+00 & $1.000 E+\infty 0$ & $1.000 E+\infty$ & $1.000 E+\infty 0$ & $1.000 E+\infty$ & $1.000 E+00$ \\
\hline-.8 & : & 1. $040 E+00$ & $1.072 E+00$ & $1.096 E+00$ & $1.114 E+\infty 0$ & $1.126 E+00$ & $1.134 E+\infty 0$ & $1.139 E+00$ & $1.14 \mathbb{E}+\infty 0$ & $1.144 E+00$ & $1.147 E+\infty O$ \\
\hline .6 & : & $1.088 E+\infty$ & $1.166 E+\infty 0$ & $1.230 E+00$ & $1.281 E+\infty$ & $1.319 E+\infty 0$ & $1.346 E+00$ & $1.366 E+00$ & $1.379 E+\infty$ & 1. $399 E+00$ & $1.408 E+00$ \\
\hline-.4 & : & $1.147 E+00$ & $.268 E+\infty$ & $1.416 E+\infty$ & $1.526 E+00$ & $1.617 E+00$ & $1.692 E+00$ & $1.751 E+\infty 0$ & $1.797 E+\infty$ & $1.832 \mathbb{E}+00$ & $1.948 E+00$ \\
\hline-.2 & $:$ & $1.217 E+00$ & $1.444 E+00$ & $1.671 E+00$ & $1.889 E+00$ & $2.091 E+00$ & $2.275 E+\infty 0$ & $2.440 E+\infty 0$ & $2.586 E+00$ & $2.714 E+00$ & $3.593 E+\infty 0$ \\
\hline 0 & : & 1. $298 E+00$ & $1.643 E+\infty 0$ & $2.026 E+00$ & $2.435 E+00$ & $2.863 E+00$ & 3. $304 E+\infty 0$ & 3. $752 E+\infty 0$ & $4.205 E+00$ & $4.661 E+\infty$ & \\
\hline .2 & : & $1.393 E+00$ & $1.898 E+00$ & $2.521 E+00$ & $3.272 E+00$ & $4.160 E+00$ & $5.199 \varepsilon+00$ & $6.404 E+00$ & $7.797 E+\infty$ & $9.40 Z E+00$ & \\
\hline .4 & $:$ & $1.504 E+\infty$ & 2. $223 E+\infty 0$ & $3.219 E+\infty 0$ & $4.575 E+\infty$ & $6.398 E+\infty$ & $8.828 E+00$ & $1.205 E+01$ & $1.632 E+01$ & $2.197 \mathrm{E}+01$ & \\
\hline .6 & : & $1.633 E+\infty$ & $.640 E+\infty 0$ & $4.213 E+\infty$ & $6.639 E+00$ & $1.035 E+01$ & $1.602 E+01$ & $2.463 E+01$ & 3. $771 E+01$ & 5. $754 E+01$ & \\
\hline .8 & : & $1.783 E+00$ & $177 E+00$ & $5.637 E+\infty 0$ & $9.954 E+00$ & 1. $750 E+01$ & 3. $068 E+01$ & $5.365 E+01$ & $9.366 E+01$ & $1.634 E+02$ & \\
\hline 1 & $:$ & $1.957 E+\infty$ & $3.870 E+\infty 0$ & $7.696 E+\infty 0$ & $1.535 E+01$ & 3. $065 E+01$ & $6.126 E+01$ & $1.225 E+02$ & $2.449+02$ & $4.898 E+02$ & \\
\hline 1.2 & : & $2.158 E+\infty$ & 1.7GbE+00? & $1.069 E+01$ & $2.422 E+01$ & $5.521 E+01$ & $1.263 E+02$ & $2.894 \varepsilon+02$ & $6.640 E+02$ & $1.524 E+03$ & \\
\hline 1.4 & : & $2.391 E+10$ & $5.929 E+(10)$ & $1.508 E+01$ & 3. $897 E+01$ & $1.016 E+02$ & $2.666 E+02$ & $7.011 E+02$ & $1.847 E+03$ & $4.870 E+03$ & \\
\hline 1.6 & : & 2. $660 E+00$ & $7.443 E+00$ & $2.154 E+01$ & $6.366 E+01$ & $1.904 E+02$ & $5.730 E+02$ & $1.731 E+03$ & $5.237 E+03$ & $1.586 E+04$ & \\
\hline 1.8 & : & $2.97 Z E+00$ & $9.418 E+00$ & $3.111 \mathrm{E}+01$ & $1.053 E+02$ & $3.614 E+02$ & $1.249 E+03$ & 4. $333 E+03$ & $1.506 E+04$ & $5.238 E+04$ & \\
\hline 2 & : & 3. $323 E+100$ & $1.200 E+01$ & $4.533 E+01$ & $1.760 E+02$ & $6.933 E+02$ & $2.752 E+03$ & $1.097 \mathrm{E}+04$ & $4.378 E+04$ & $1.749 E+05$ & \\
\hline
\end{tabular}

$B 1(N, r, m)$ for $r=$ ?

\begin{tabular}{|c|c|c|c|c|c|c|c|c|c|c|c|}
\hline Hu 1 & $N=$ & 4 & 8 & 16 & 32 & 64 & 128 & $25 t$ & 512 & 1024 & INF \\
\hline-2 & : & 1.00DE +00 & 1. $000 E+00$ & $1.000 E+\infty 0$ & $1.000 E+\infty 0$ & $1.000 E+\infty 0$ & $1.000 E+00$ & $1.000 E+00$ & $1.000 E+00$ & $1.000 E+00$ & $1.000 E+00$ \\
\hline-1.8 & $:$ & $9.901 E-01$ & $9.836 E-01$ & $9.796 E-01$ & 9. $774 E-01$ & $9.761 E-01$ & $9.755 E-01$ & $9.752 E-01$ & $9.750 E-01$ & $9.749 E-01$ & $9.748 E-01$ \\
\hline-1.6 & $:$ & $9.845 E-01$ & $9.737 E-01$ & $9.669 E-01$ & $9.630=-01$ & $9.607 \mathrm{E}-01$ & $9.595 E-01$ & $9.589 E-01$ & $9.586 E-01$ & $9.584 E-01$ & $9.582 \mathrm{E}-01$ \\
\hline-1.4 & : & $9.837 E-01$ & $9.719 E-01$ & $9.641 E-01$ & $9.593 E-01$ & $9.565 E-01$ & $9.550 E-01$ & $9.541 \mathrm{E}-01$ & $9.537 \mathrm{E}-01$ & $9.534 E-01$ & $9.532 E-01$ \\
\hline-1.2 & : & $9.886 E-01$ & $9.790 E-01$ & $9.738 E-01$ & $9.699 E-01$ & $9.675 E-01$ & 9. b61E-01 & $9.653 E-01$ & $9.648 E-01$ & $9.646 E-01$ & $9.643 E-01$ \\
\hline-1 & $:$ & 1. $000 E+C O D$ & 1.000E+(0) & $1.000 E+00$ & 1. $000 E+00$ & $1.000 E+00$ & $1.000 E+00$ & $1.000 E+00$ & $1.000 E+00$ & $1.000 E+00$ & $1.000 t+00$ \\
\hline .8 & : & $1.019 E+0 n$ & $1.035 E+00$ & 1. $048 E+00$ & $1.058 E+00$ & $1.064 E+00$ & $1.069 E+00$ & $1.072 \mathbf{E}+00$ & $1.074 E+\infty 0$ & $1.075 E+00$ & $1.077 E+00$ \\
\hline-.6 & $:$ & $1.046 E+00$ & $1.090 E+00$ & $1.126 E+00$ & $1.156 E+00$ & $1.178 E+00$ & $1.195 E+00$ & $1.207 E+00$ & $1.215 E+00$ & $1.221 E+00$ & 1. $233 E+00$ \\
\hline-.4 & $:$ & $1.084 E+00$ & $1.168 E+00$ & $1.246 E+00$ & $1.315 E+00$ & $1.373 E+\infty$ & $1.420 E+00$ & $1.457 \mathrm{E}+\infty$ & $1.487 E+00$ & $1.509 E+00$ & $1.583 E+00$ \\
\hline-.2 & $:$ & $1.133 E+00$ & $1.277 E+(n)$ & $1.425 E+00$ & $1.568 E+00$ & $1.702 E+C 0$ & $1.824 E+0.0$ & $1.934 E+00$ & $2.031 E+00$ & $2.117 E+00$ & $2.704 E+C$ \\
\hline 0 & : & $1.195 E+00$ & $1.427 E+00$ & $1.6835+00$ & $1.971 E+00$ & $2.267 E+00$ & $2.573 E+\infty$ & $2.884 E+\infty$ & $3.198 E+00$ & $3.515 E+00$ & \\
\hline .2 & $:$ & $1.273 E+100$ & $1.629 E+00$ & $2.075 E+10$ & $2.615 E+00$ & $3.256 E+00$ & $4.005 E+00$ & $4.876 E+\infty 0$ & $5.882 E+(10)$ & $7.042 E+00$ & \\
\hline .4 & $:$ & $1.369 E+00$ & $1.901 E+00$ & $2.644 E+C D$ & $3.659 E+00$ & $5.025 E+\infty 0$ & $6.847 E+00$ & $9.267 E+\infty 0$ & $1.247 E+01$ & $1.670 E+01$ & \\
\hline.$b$ & : & $1.486 E+00$ & $\therefore 264 E+00$ & $3.485 E+\infty 0$ & $5.371 E+\infty$ & $8.262 E+\infty 0$ & $1.267 E+01$ & $1.937 E+01$ & $2.955 E+01$ & $4.498 E+01$ & \\
\hline .8 & : & $1.628 E+00$ & 2. $750 E+\infty 0$ & $4.733 E+\infty$ & 8. $215 E+\infty$ & $1.431 E+01$ & $2.494 E+01$ & 4. $347 E+01$ & $7.576 E+01$ & $1.320 E+02$ & \\
\hline 1 & : & $1.800 E+00$ & $3.400 E+00$ & $6.600 E+00$ & 1. $300 E+01$ & $2.580 E+01$ & $5.140 E+01$ & $1.026 E+02$ & $2.050 E+02$ & $4.098 E+02$ & \\
\hline 1.2 & : & $2.007 E+00$ & $4.271 E++0$ & $9.409 E+00$ & $2.114 E+01$ & $4.800 E+01$ & $1.096 E+02$ & $2.510 E+02$ & $5.756 E+02$ & $1.321 E+03$ & \\
\hline 1.4 & : & $2.255 E+\infty 0$ & $5.439 E+\infty 0$ & $1.366 E+01$ & $3.512 E+01$ & $9.142 E+01$ & $2.395 E+02$ & $6.299 E+02$ & $1.65 \% E+03$ & $4.374 E+03$ & \\
\hline $1 . t$ & $:$ & $2.552 E+00$ & $7.012 E+00$ & $2.014 E+01$ & $5.935 E+01$ & $1.773 E+02$ & $5.334 E+02$ & $1.611 E+03$ & $4.874 E+03$ & $1.476 E+04$ & \\
\hline 1.8 & : & $2.908 E+00$ & $9.132 E+00$ & $3.006 E+01$ & $1.017 E+02$ & $3.487 \mathrm{E}+02$ & $1.205 E+03$ & 4.179E+03 & $1.453 E+04$ & $5.053 E+04$ & \\
\hline 2 & : & $3.333 E+10$ & 1. $200 E+01$ & $4.5335+01$ & $1.7 t O E+02$ & $6.933 E+02$ & 2. $752 E+03$ & $1.097 \mathrm{E}+04$ & $4.378 E+04$ & $1.749 \varepsilon+05$ & \\
\hline
\end{tabular}


$B 1(N, r, \infty u)$ for $r=4$

\begin{tabular}{|c|c|c|c|c|c|c|c|c|c|c|c|}
\hline An 1 & $N=$ & 4 & 8 & 16 & 32 & 64 & 128 & 256 & 512 & 1024 & INF \\
\hline-2 & $:$ & $1.000 E+\infty$ & $1.000 E+\infty$ & $1.000 E+\infty$ & $1.000 E+\infty$ & 1. $000 E+\infty$ & $1.000 E+\infty 0$ & 1.000E+- & 1.000E+00 & $1.000 E+00$ & $1.000 E+\infty$ \\
\hline-1.8 & : & $9.974 E-01$ & $9.957 E-01$ & $9.946 E-01$ & $9.940 E-01$ & $9.936 E-01$ & $9.935 E-01$ & $9.934 E-01$ & $9.933 E-01$ & $9.933 E-01$ & $9.933 E-01$ \\
\hline-1.6 & : & $9.95 x-01$ & $9.918 E-01$ & $9.896 E-01$ & $9.884 E-01$ & $9.876 E-01$ & $9.873 E-01$ & $9.870 E-01$ & $9.869 E-01$ & $9.86 \%-01$ & $9.868 E-01$ \\
\hline 1.4 & : & $9.941 E-01$ & $9.898 E-01$ & $9.86 \% E-01$ & $9.851 E-01$ & $9.840 E-01$ & $9.834 E-01$ & $9.831 E-01$ & $9.829 E-01$ & $9.82 \%-01$ & $9.828 E-01$ \\
\hline-1.2 & : & $9.953 E-01$ & $9.916 E-01$ & $9.890 E-01$ & $9.873 E-01$ & $9.862 E-01$ & $9.856 E-01$ & $9.853 E-01$ & $9.851 E-01$ & $9.850 E-01$ & $9.849 E-01$ \\
\hline-1 & : & $1.000 E+\infty$ & $1.000 E+\infty$ & $1.000 E+00$ & $1.000 E+\infty 0$ & $1.000 E+\infty 0$ & 1. $000 E+\infty$ & 1.000E++00 & $1.000 E+00$ & $1.000 E+00$ & $1.000 E+\infty 0$ \\
\hline-.8 & : & $1.010 E+\infty$ & $1.019 E+\infty 0$ & $1.026 E+00$ & $1.031 E+\infty 0$ & $1.035 E+00$ & $1.037 E+00$ & $1.039 E+00$ & $1.040 E+00$ & $1.041 E+00$ & $1.04 x \pm+00$ \\
\hline .6 & : & $1.027 E+\infty$ & $1.053 E+\infty$ & $1.075 E+00$ & $1.093 E+00$ & $1.107 E+\infty 0$ & $1.117 E+\infty 0$ & $1.124 E+00$ & $1.129 E+00$ & $1.132 E+00$ & $1.140 E+00$ \\
\hline-.4 & : & $1.054 E+\infty 0$ & 1. $109 E+00$ & $1.160 E+00$ & $1.205 x+00$ & $1.243 E+00$ & $1.274 E+00$ & $1.299+00$ & $1.318 E+\infty 0$ & $1.333 E+00$ & $1.38 z E+00$ \\
\hline-.2 & : & $1.092 E+00$ & $1.194 E+\infty 0$ & $1.298 E+\infty 0$ & $1.399 E+00$ & $1.494 E+\infty 0$ & $1.580 E+\infty 0$ & $1.658 E+\infty 0$ & $1.727 E+\infty 0$ & $1.788 \varepsilon+00$ & $2.204 E+\infty 0$ \\
\hline 0 & : & $1.144 E+\infty$ & $1.318 E+00$ & $1.514 E+00$ & $1.726 E+00$ & $1.949 E+00$ & $2.179 E+00$ & $2.414 E+00$ & $2.651 E+00$ & $2.890 E+00$ & \\
\hline .2 & : & $1.214 E+\infty 0$ & $1.495 x+\infty$ & $1.848 E+00$ & $2.275 E+00$ & $2.783 E+00$ & 3. $377 E+\infty 0$ & $4.067 E+00$ & $4.865 E+\infty 0$ & $5.784 E+00$ & \\
\hline .4 & : & 1. $303 E+00$ & $1.742 E+\infty$ & $2.356 E+\infty 0$ & $3.196 E+00$ & $4.326 E+\infty 0$ & $5.834 E+00$ & $7.837 E+\infty 0$ & 1. $049 E+01$ & $1.399 E+01$ & \\
\hline .6 & : & $1.415 E+00$ & $2.082 E+\infty 0$ & $3.129 E+\infty 0$ & $4.748 E+\infty 0$ & $7.229 E+\infty$ & $1.101 E+01$ & $1.676 E+01$ & $2.550 E+01$ & $3.875 E+01$ & \\
\hline .8 & $:$ & $1.555 E+\infty$ & $2.548 E+\infty 0$ & 4. $303 E+\infty 0$ & $7.385 E+00$ & $1.278 E+01$ & $2.219 E+01$ & $3.860 E+01$ & $6.718 E+01$ & $1.170 E+02$ & \\
\hline 1 & $:$ & $1.72 \pi E+\infty$ & $3.182 E+00$ & $6.091 E+00$ & $1.191 E+01$ & $2.355 E+01$ & $4.682 E+01$ & $9.336 E+01$ & $1.865 E+02$ & $3.726 E+02$ & \\
\hline 1.2 & : & $1.938 E+00$ & $4.045 E+00$ & $8.826 E+100$ & $1.974 E+01$ & $4.472 E+01$ & $1.020 E+02$ & $2.336 E+02$ & $5.356 E+02$ & 1.229E+03 & \\
\hline 1.4 & : & $2.194 E+100$ & $5.219 E+0 D$ & $1.303 E+01$ & 3. $340 E+01$ & $8.683 E+01$ & $2.275 E+02$ & $5.981 E+02$ & $1.575 E+03$ & $4.154 E+1.3$ & \\
\hline 1.6 & : & $2.504 E+\infty 0$ & $6.819 E+00$ & $1.952 E+01$ & $5.745 E+01$ & $1.715 E+02$ & 5. $160 E+02$ & $1.558 E+03$ & $4.714 E+03$ & $1.428 E+04$ & \\
\hline 1.8 & : & $2.879 E+00$ & $9.006 E+00$ & $2.960 E+01$ & $1.000 E+02$ & $3.431 E+02$ & $1.186 E+03$ & 4.112E+03 & 1. $429 E+04$ & $4.972 E+04$ & \\
\hline 2 & : & $3.323 E+\infty 0$ & 1. $200 E+01$ & $4.533 E+01$ & $1.760 E+02$ & $6.933 E+02$ & $2.752 E+03$ & $1.097 E+04$ & $4.378 E+04$ & $1.749 E+05$ & \\
\hline
\end{tabular}

$B 1(N, r, m u)$ for $r=8$

\begin{tabular}{|c|c|c|c|c|c|c|c|c|c|c|c|}
\hline Mu $i$ & $N=$ & 4 & 8 & 16 & 32 & 64 & 128 & 256 & 512 & 1024 & INF \\
\hline-2 & : & 1.000E+100 & $1.000 E+101)$ & $1.000 E+00$ & $1.000 E+\infty$ & $1.000 E+00$ & 1. $000 E+00$ & $1.000 E+00$ & $1.000 E+\infty 00$ & $1.000 E+00$ & $1.000 E+00$ \\
\hline-1.8 & $:$ & $9.993 \mathrm{E}-01$ & $9.988 E-01$ & $9.985 E-01$ & $9.983 E-01$ & $9.982 E-01$ & $9.981 E-01$ & $9.981 E-01$ & $9.981 E-01$ & $9.981 E-01$ & $9.981 E-01$ \\
\hline-1.6 & : & $9.984 E-01$ & $9.973 E-01$ & $9.966 E-01$ & $9 ., \div \in 2 E-01$ & $9.960 E-01$ & $9.958 E-01$ & $9.958 E-01$ & $9.957 E-01$ & $9.957 E-01$ & $9.957 E-01$ \\
\hline-1.4 & : & $9.978 E-01$ & $9.961 E-01$ & $9.950 E-01$ & $9.944 E-01$ & $9.940 E-01$ & $9.937 \mathrm{E}-01$ & $9.936 E-01$ & $9.936 E-01$ & $9.935 E-01$ & $9.935 E-0.1$ \\
\hline-1.2 & : & 9. QBOE-01 & $9.963 E-01$ & $9.952 E-01$ & $9.945 E-01$ & $9.940 E-01$ & $9.930 E-01$ & $9.936 E-01$ & $9.935 E-01$ & $9.935 E-01$ & $9.934 E-01$ \\
\hline-1 & : & $1.000 E+00$ & $1.000 E+(10)$ & $1.000 E+\infty$ & $1.000 E+\infty 0$ & $1.000 E+00$ & $1.000 E+\infty$ & $1.000 E+00$ & $1.000 E+00$ & $1.000 E+00$ & $1.000 E+0 x$ \\
\hline-.8 & : & $1.006 E+(0,0)$ & $1.011 E+\infty 0$ & $1.014 E+\infty 0$ & $1.017 E+00$ & $1.020 E+00$ & $1.021 E+\infty$ & $1.022 E+\infty 00$ & $1.022 E+\infty 0$ & $1.023 E+\infty$ & $1.023 E+00$ \\
\hline-.6 & $:$ & $1.017 E+(00)$ & $1.033 E+00$ & $1.04 \pi E+\infty$ & $1.058 E+\infty 0$ & $1.067 E+00$ & $1.073 E+\infty$ & $1.078 E+\infty 0$ & $1.081 E+\infty$ & $1.083 E+00$ & $1.088 E+\infty 0$ \\
\hline-.4 & : & $1.03 \pi E+00$ & $1.075 E+60$ & $1.110 E+\infty$ & $1.142 E+\infty 0$ & $x+\infty$ & $1.189 E+00$ & $1.207 E+\infty$ & $1.220 E+\infty$ & $1.231 E+\infty 0$ & $1.264 E+00$ \\
\hline-.2 & $:$ & $1.06 \% E+010$ & $1.145 E+00$ & $1.223 E+\infty$ & 1. $299 E+100$ & $1.371 E+\infty 0$ & $1.436 E+00$ & $1.494 E+\infty 0$ & $1.546 E+\infty 0$ & $1.592 E+100$ & $1.905 E+00$ \\
\hline 0 & : & $1.116 E+\infty$ & $1.255 E+\infty$ & $1.413 E+\infty$ & $1.584 E+\infty 0$ & $E+\infty$ & $1.949 E+00$ & $2.13 \pi E+\infty 0$ & $2.32 B E+\infty 0$ & $2.520 E+C D$ & \\
\hline .2 & : & $1.181 E+(h)$ & $1.420 E+010$ & $1.720 E+00$ & $2.083 E+(0)$ & $2.514 E+\infty 0$ & $3.019 E+00$ & $3.606 E+00$ & $4.284 E+00$ & $5.066 E+00$ & \\
\hline .4 & : & $1.268 E+\infty 0$ & $1.657 E+\infty$ & $2.202 E+\infty 0$ & $2.946 E+\infty 0$ & $3.949 E+\infty 0$ & $5.226 E+00$ & $7.062 E+00$ & $9.414 E+\infty 0$ & $1.252 E+01$ & \\
\hline .6 & : & $1.380 E+010$ & $1.991 E+00$ & $2.950 E+\infty 0$ & $4.433 E+00$ & $6.706 E+0.0$ & $1.017 E+01$ & $1.544 E+01$ & $2.345 E+01$ & $3.559 E+01$ & \\
\hline .8 & : & $1.521 E+\infty$ & $2.453 E+\infty 0$ & 4.101E+00 & $6.996 E+00$ & $1.206 E+01$ & $2.090 E+01$ & $3.630 E+01$ & $6.315 E+01$ & $1.099 E+02$ & \\
\hline 1 & $:$ & $1.696 E+00$ & 3. $067 E+\infty 0$ & $5.870 E+\infty 0$ & $1.143 E+01$ & $2.257 E+01$ & $4.483 E+01$ & $8.935 E+01$ & $1.784 E+02$ & $3.565 E+02$ & \\
\hline 1.2 & : & $1.910 E+\infty 0$ & $3.953 E+00$ & $8.590 E+00$ & $1.917 E+01$ & 4. $341 E+01$ & $9.898 E+01$ & $2.265 E+02$ & $5.195 E+02$ & $1.192 E+03$ & \\
\hline 1.4 & : & $2.170 E+00$ & $5.135 E+\infty 0$ & $1.279 E+01$ & $3.276 E+01$ & $8.515 E+01$ & $2.230 E+02$ & $5.862 E+02$ & $1.544 E+03$ & $4.071 E+03$ & \\
\hline 1.6 & : & $2.486 E+(0)$ & $6.751 E+\infty$ & $1.930 E+01$ & $5.678 E+01$ & $1.695 E+02$ & $5.099 E+02$ & $1.540 E+03$ & $4.658 E+03$ & $1.411 E+04$ & \\
\hline 1.8 & : & $2.870 E+00$ & $8.963 E+\infty 0$ & $2.945 E+01$ & $9.951 E+01$ & $3.413 E+02$ & $1.179 E+03$ & $4.090 E+03$ & $1.421 E+04$ & $4.945 E+04$ & \\
\hline 2 & : & $3.3335+00$ & 1. $200 E+01$ & $4.533 E+01$ & $1.760 E+02$ & $6.933 E+02$ & $2.752 E+03$ & $1.097 E+04$ & 4. $378 E+04$ & $1.749 E+05$ & \\
\hline
\end{tabular}


$81(N, r$, mu $)$ for $r=16$

\begin{tabular}{|c|c|c|c|c|c|c|c|c|c|c|c|}
\hline w 1 & $N=$ & 4 & 8 & 16 & 32 & 64 & 128 & 256 & 512 & 1024 & INF \\
\hline-2 & : & $1.000 E+00$ & $1.000 E+\infty 0$ & $1.000 E+00$ & $1.000 E+00$ & $1.000 E+\infty 0$ & $1.000 E+\infty$ & $1.000 E+\infty$ & $1.000 E+\infty$ & $1.000 E+\infty 0$ & $1.000 E+\infty$ \\
\hline-1.8 & : & $9.998 E-01$ & $9.997 \mathrm{E}-01$ & $9.996 E-01$ & $9.995 E-01$ & $9.995 E-01$ & $995 E-01$ & $9.995 E-01$ & $9.995 E-01$ & $9.995 E-01$ & $9.9955-01$ \\
\hline-1.6 & : & $9.995 E-01$ & $9.991 E-01$ & $9.989 E-01$ & $9.987 \mathrm{E}-01$ & $9.987 E-01$ & $9.986 E-01$ & $9.986 E-01$ & $9.986 E-01$ & $9.986 E-01$ & $9.986 E-01$ \\
\hline-1.4 & : & $9.992 E-01$ & $9.985 x-01$ & $9.981 E-01$ & $9.979 E-01$ & $9.977 E-01$ & $9.976 E-01$ & $9.976 E-01$ & $9.976 E-01$ & $9.975 E-01$ & $9.975 E-01$ \\
\hline-1.2 & $:$ & $9.991 E-01$ & $9.984 E-01$ & 9.979E-01 & $9.976 E-01$ & $9.974 E-01$ & $9.97 x-01$ & $9.972 E-01$ & $9.972 \varepsilon-01$ & $9.972 E-01$ & $9.971 E-01$ \\
\hline-1 & : & $1.000 E+\infty 0$ & $1.000 E+\infty 0$ & $1.000 E+\infty$ & $1.000 E+\infty$ & $1.000 E+\infty 0$ & $1.000 E+\infty$ & $1.000 E+\infty 0$ & $1.000 E+\infty$ & $1.000 E+\infty$ & 1.000E+00 \\
\hline-.8 & : & $1.003 E+00$ & $1.006 E+\infty$ & $1.008 E+\infty 0$ & $1.010 E+00$ & $1.011 E+\infty$ & $1.012 E+\infty$ & $1.012 E+\infty 0$ & $1.013 x+\infty$ & $1.013 E+\infty$ & $1.013 E+00$ \\
\hline-.6 & : & $1.011 E+\infty 0$ & $1.021 E+\infty 0$ & $1.030 E+\infty$ & $1.037 E+\infty 0$ & $1.043 E+\infty 0$ & $1.047 E+\infty 0$ & $1.050 E+\infty 0$ & $1.052 E+\infty$ & $1.053 E+\infty 0$ & $1.056 E+100$ \\
\hline-.4 & : & $1.026 E+\infty$ & $1.0535+\infty$ & $1.078 E+\infty$ & $1.101 E+\infty 0$ & $1.119+\infty$ & $1.135 E+\infty$ & $1.147 E+00$ & $1.157 E+\infty 0$ & $1.164 E+\infty 0$ & $1.188 \varepsilon+00$ \\
\hline-.2 & $:$ & $1.054 E+\infty$ & $1.113 E+\infty 0$ & $1.174 E+\infty 0$ & $1.233 E+\infty$ & 1.289 $+\infty$ & 1. $33 \%+\infty$ & 1. $385 E+\infty 0$ & $1.425 E+\infty$ & $1.461 E+\infty$ & $1.705 E+\infty 0$ \\
\hline 0 & : & $1.097 E+\infty 0$ & $1.214 E+\infty 0$ & $1.346 E+00$ & $1.489 \varepsilon+\infty$ & $1.639 E+\infty 0$ & $1.794 E+\infty 0$ & $1.952 E+\infty 0$ & $2.112 E+\infty$ & $2.273 E+\infty 0$ & \\
\hline .2 & : & $1.160 E+00$ & $1.37 x+\infty 0$ & $1.637 E+\infty$ & $1.958 E+\infty 0$ & $2.340 E+\infty 0$ & $2.787 E+\infty 0$ & $3.307 E+\infty 0$ & $3.907 E+\infty$ & $4.599 E+\infty 0$ & \\
\hline .4 & : & $1.247 E+\infty 0$ & $1.606 E+00$ & $2.108 E+\infty 0$ & $2.794 E+00$ & $3.717 E+\infty 0$ & $4.950 E+\infty$ & $6.587 E+\infty 0$ & 8. $754 E+\infty$ & $1.162 \mathcal{E}+01$ & \\
\hline.$t$ & $:$ & $1.360 E+\infty 0$ & 1.93 & $2.848 E+\infty$ & 4. 254E & $6.409 E+00$ & $9.696 E+00$ & 1. $469 E+01$ & $2.228 E+01$ & 3. $379 E+01$ & \\
\hline .8 & : & $1.504 E+00$ & $2.404 E+00$ & $3.997 E+\infty 0$ & $6.794 E+00$ & 1.169E+01 & $2.023 E+01$ & $3.512 E+01$ & $6.106 E+01$ & $1.062 E+02$ & \\
\hline 1 & $:$ & $1.681 E+\infty 0$ & $3.0435+00$ & $5.766 E+00$ & $1.121 E+01$ & $2.211 E+01$ & 4. $389 E+01$ & $8.747 E+01$ & $1.746 E+02$ & $3.489 E+02$ & \\
\hline 1.2 & : & $1.898 E+\infty 0$ & $3.914 E+\infty 0$ & $8.491 E+\infty 0$ & $1.894 E+01$ & $4.285 E+01$ & $9.76 \% E+01$ & 2. $236 E+02$ & $5.127 E+02$ & $1.177 E+03$ & \\
\hline 1.4 & : & $2.161 E+100$ & $5.104 E+\infty 0$ & $1.270 E+01$ & 3. $251 E+01$ & $8.450 E+01$ & 2. $21 x+02$ & $5.817 E+02$ & $1.532 E+03$ & $4.039 E+03$ & \\
\hline 1.6 & : & $2.480 E+\infty$ & $.127+4$ & $1.923 E+01$ & $5.654 E+01$ & $1.688 E+02$ & $5.077 E+02$ & $1.533 E+03$ & $4.639 E+03$ & 1. $405 E+04$ & \\
\hline 1.8 & $:$ & $2.866 E+00$ & $8.949 E+00$ & $2.940 E+01$ & $9.934 E+01$ & 3. $406 E+02$ & $1.177 E+03$ & $4.083 E+03$ & $1.419 E+04$ & $4.936 E+104$ & \\
\hline 2 & : & $3.33 .3 E+0.0$ & $1.200 E+01$ & $4.533 E+01$ & $1.760 E+02$ & $6.9335+02$ & 2. $752 E+03$ & $1.097 E+04$ & $4.378 E+04$ & $1.749 E+05$ & \\
\hline
\end{tabular}

$B 1(N, r, m)$ for $r=32$

\begin{tabular}{|c|c|c|c|c|c|c|c|c|c|c|c|}
\hline Hus 1 & $N=$ & 4 & 8 & 16 & 32 & 64 & 128 & 256 & 512 & 1024 & INF \\
\hline-2 & $:$ & $1.000 E+00$ & $1.000 E+00$ & $1.000 E+00$ & $1.000 E+00$ & $1.000 E+\infty$ & $1.000 E+00$ & $1.000 E+\infty 0$ & $1.000 E+\infty$ & 1.000E +00 & $1.000 E+00$ \\
\hline-1.8 & : & $9.999 \mathrm{E}-01$ & $9.999 \varepsilon-01$ & $9.999 E-01$ & $9.999 E-01$ & $9.999 E-01$ & $9.998 E-01$ & $9.998 E-01$ & $9.998 E-01$ & $9.998 E-01$ & $9.998 E-01$ \\
\hline-1.6 & : & $9.998 E-01$ & $9.097 E-01$ & $9.996 E-01$ & $9.996 \mathrm{E}-01$ & $9.996 E-01$ & $9.995 E-01$ & $9.995 E-01$ & $9.995 E-01$ & $9.995 E-01$ & $9.995-01$ \\
\hline-1.4 & : & $9.997 E-01$ & $9.994 E-01$ & $9.993 x-01$ & $9.992 E-01$ & $9.991 E-01$ & $9.991 E-01$ & $9.991 E-01$ & $9.991 E-01$ & $9.991 E-01$ & $9.991 E-01$ \\
\hline-1.2 & : & $9.996 \mathrm{E}-01$ & $9.993 E-01$ & $9.991 E-01$ & $9.9905-01$ & $9.989 E-01$ & $9.988 E-01$ & $9.988 E-01$ & $9.988 E-01$ & $9.988 E-01$ & $9.988 E-01$ \\
\hline-1 & : & $1.000 E+00$ & $1.000 E+00$ & 1. $000 E+C D$ & $1.000 E+\infty$ & $1.000 E+\infty 1$ & $1.000 E+\infty$ & $1.000 E+\infty 0$ & $1.000 E+\infty$ & 1.000E +00 & $1.000 E+00$ \\
\hline-.8 & $:$ & $1.002 E+00$ & $1.003 E+\infty 0$ & $1.005 E+00$ & $1.006 E+\infty 0$ & 1. $006 E+00$ & $1.007 E+00$ & 1.007E+00 & $1.007 E+\infty$ & $1.007 E+\infty 00$ & 1. $000 E+\infty$ \\
\hline-.6 & $:$ & $1.007 E+(x)$ & $1.014 E+00$ & $1.019 E+\infty 0$ & $1.024 E+\infty 0$ & $1.028 E+\infty 0$ & $1.030 E+00$ & $1.032 E+00$ & $1.033 \varepsilon+\infty$ & $1.034 E+00$ & $1.036 E+100$ \\
\hline-.4 & : & $1.019 E+\infty 0$ & $1.039 E+00$ & $1.057 E+\infty$ & $1.073 E+\infty$ & $1.087 E+\infty 0$ & $1.098 E+00$ & 1. $107 E+\infty$ & $1.114 E+00$ & $1.119 E+00$ & $1.136 E+\infty$ \\
\hline-.2 & $:$ & $1.043 E+00$ & $1.090 E+00$ & $1.139 E+\infty 0$ & $1.186 E+\infty$ & 1. $230 E+\infty$ & $1.271 E+00$ & 1. $307 E+00$ & $1.339 E+\infty 0$ & $1.368 E+\infty 0$ & $1.563 E+\infty 0$ \\
\hline 0 & $:$ & 1. $083 E+\infty 0$ & 1. $184 E+i 0$ & 1. $x 97 E+\infty 0$ & $1.420 E+\infty$ & $1.550 E+00$ & $1.683 E+\infty 0$ & $1.819 E+\infty$ & $1.957 E+\infty$ & $2.095 E+\infty 0$ & \\
\hline .2 & : & $1.146 E+000$ & 1. $338 E+10$ & 1.57\% $+\infty$ & $1.871 E+00$ & $2.218 E+\infty$ & $2.625 E+00$ & $3.097 E+\infty$ & $3.643 E+\infty 0$ & $4.272 E+\infty 0$ & \\
\hline .4 & $:$ & $1.233 E+\infty 0$ & $1.572 E+\infty 0$ & $2.046 E+\infty 0$ & $2.693 E+00$ & $3.5655+\infty$ & $4.729 E+00$ & $6.275 E+00$ & $8.320 E+\infty 0$ & $1.102 E+01$ & \\
\hline .6 & $:$ & $1.348 E+00$ & $1.908 E+\infty 0$ & $2.787 E+\infty 0$ & $4.147 E+\infty 0$ & $6.231 E+00$ & $9.408 E+\infty 0$ & $1.424 E+01$ & $2.158 E+01$ & 3.270E+01 & \\
\hline .8 & $:$ & $1.494 E+00$ & $2.378 E+\infty 0$ & $3.940 E+\infty$ & $6.685 t+00$ & $1.149 E+01$ & $1.986 E+01$ & $3.447 E+01$ & $5.992 \varepsilon+01$ & $1.042 E+02$ & \\
\hline 1 & $:$ & $1.674 E+\infty 0$ & $3.021 E+100$ & $5.716 E+00$ & 1. $111 E+01$ & $2.188 \varepsilon+01$ & 4. $344 E+01$ & $8.656 E+01$ & $1.728 E+02$ & $3.453 E+02$ & \\
\hline 1.2 & $:$ & $1.893 E+\infty 0$ & $3.898 E+00$ & $8.448 E+00$ & 1. $883 E+01$ & 4. $261 E+01$ & $9.714 E+01$ & $2.223 E+02$ & $5.097 E+02$ & $1.170 E+03$ & \\
\hline 1.4 & $:$ & $2.158 E+0.0$ & $5.091 E+1) 0$ & $1.266 E+01$ & 3. $242 E+101$ & $8.425 E+01$ & $2.206 E+02$ & $5.799 E+02$ & $1.527 E+03$ & $4.027 E+03$ & \\
\hline 1.6 & : & $2.47 E E+00$ & $6.719 E+\infty 0$ & $1.920 E+01$ & $5.646 E+01$ & $1.685 E+02$ & $5.070 E+02$ & $1.531 E+03$ & $4.632 E+03$ & 1. $403 E+04$ & \\
\hline 1.8 & : & $2.865 E+100$ & $8.945 E+\infty 0$ & $2.938 E+01$ & $9.928 E+01$ & 3. $405 E+02$ & $1.176 E+03$ & $4.081 E+03$ & $1.418 E+04$ & $4.934 E+04$ & \\
\hline 2 & : & $3.333 E+\infty 0$ & $1.200 E+01$ & $4 \cdot 533 E+01$ & $1.760 E+02$ & $6.933 E+02$ & $2.752 E+03$ & $1.097 E+04$ & $4.37 \& \varepsilon+04$ & $1.749 E+05$ & \\
\hline
\end{tabular}


$B 1(N, r, w)$ for $r=64$

\begin{tabular}{|c|c|c|c|c|c|c|c|c|c|c|c|}
\hline m 1 & $N=$ & 4 & 8 & 16 & 32 & 64 & 128 & 256 & 512 & 1024 & INF \\
\hline-2 & : & $1.000 E+\infty 0$ & $1.000 E+00$ & $1.000 E+\infty$ & $1.000 E+00$ & $1.000 E+\infty 0$ & 1.000E $+\infty 0$ & $1.000 E+\infty 0$ & $1.000 E+00$ & $1.000 E+\infty 0$ & $1.000 E+00$ \\
\hline-1.8 & : & $1.000 E+\infty 0$ & $.000 E+00$ & $1.000 E+\infty$ & $1.000 E+\infty 0$ & $1.000 E+\infty 0$ & $1.000 E+00$ & $1.000 E+00$ & $1.000 E+00$ & 1. $000 E+00$ & $1.000 E+00$ \\
\hline-1.6 & : & $9.999 E-01$ & $9.999 E-01$ & $9.999 E-01$ & $9.999 E-01$ & $9.999 E-01$ & 9.999E-01 & $9.998 E-01$ & $9.99 \% \varepsilon-01$ & $9.999 E-01$ & $9.998<-01$ \\
\hline-1.4 & : & $9.999 \mathrm{E}-01$ & $9.998 c-01$ & $9.997 \mathrm{~T}-01$ & $9.997 E-01$ & $9.997 E-01$ & $9.997 E-01$ & $9.997 E-01$ & $9.996 E-01$ & $9.996 E-01$ & $9.996 E-01$ \\
\hline 1.2 & : & $9.998<-01$ & $9.997 \mathrm{E}-01$ & $9.996 E-01$ & $9.995 E-01$ & $9.995 E-01$ & $9.9955-01$ & $9.995 E-01$ & $9.995 E-01$ & $9.995 E-01$ & $9.995 E-01$ \\
\hline-1 & : & $1.000 E+\infty$ & 1. $000 E+\infty$ & $1.000 E+\infty$ & $1.000 E+00$ & $1.000 E+\infty 0$ & $1.000 E+\infty$ & $1.000 E+00$ & $1.000 E+\infty$ & $1.000 E+00$ & $1.000 E+00$ \\
\hline-.8 & : & $1.001 E+\infty$ & $1.002 E+00$ & $1.003 E+\infty$ & $1.003 E+\infty$ & $1.004 E+\infty$ & $1.004 E+00$ & $1.004 E+00$ & $1.004 E+\infty 0$ & 1.004E+00 & $1.004 E+00$ \\
\hline-.6 & : & $1.005 E+00$ & $1.009 E+00$ & $1.013 E+\infty$ & $1.016 E+00$ & $1.018 E+\infty$ & $1.020 E+00$ & $1.021 E+00$ & $1.022 E+00$ & $1.022 E+00$ & $1.024 E+00$ \\
\hline-.4 & : & $1.014 E+00$ & $1.028 E+\infty 0$ & $1.042 E+\infty$ & $1.054 E+\infty$ & $1.064 E+\infty 00$ & $1.072 \mathbb{E}+\infty$ & $1.078 \varepsilon+\infty$ & $1.083 x+\infty$ & $1.08 \pi E+\infty 0$ & $1.100 E+00$ \\
\hline-.2 & : & $1.035 E+00$ & $1.073 E+\infty$ & $1.112 E+\infty$ & $1.151 E+\infty$ & $1.187 E+\infty$ & 1. $220 E+\infty 0$ & $1.249 E+00$ & $1.275 E+\infty$ & $1.298 E+00$ & $1.456 E+\infty 0$ \\
\hline 0 & : & $1.073 E+00$ & $1.161 E+\infty$ & $1.261 E+\infty$ & $1.369 E+\infty 0$ & $1.482 E+\infty 0$ & $1.600 E+00$ & $1.719 E+00$ & $1.840 E+\infty 0$ & $1.961 E+00$ & \\
\hline .2 & : & $1.135 E+00$ & $1.313 E+\infty 0$ & $1.536 E+\infty$ & $1.808 E+\infty$ & $2.129 E+\infty 0$ & $2.506 E+\infty 0$ & $2.944 E+00$ & $3.450 E+\infty 0$ & $4.033 E+00$ & \\
\hline .4 & : & 1. $224 E+\infty 0$ & $1.548 E+00$ & $2.003 E+\infty 0$ & $2.624 E+\infty 0$ & $3.461 E+\infty 0$ & $4.578 \varepsilon+\infty$ & $6.060 E+\infty 0$ & $8.023 E+\infty 0$ & $1.06 x+01$ & \\
\hline .6 & $:$ & $1.341 E+\infty$ & $1.889 E+100$ & $2.749 E+\infty 0$ & 4. $0800 E+00$ & $6.119 E+00$ & $9.229+\infty 0$ & $1.396 E+01$ & $2.114 E+01$ & $3.203 E+01$ & \\
\hline .8 & : & $1.489 E+00$ & $2.363 E+00$ & $3.909 E+\infty$ & $6.624 E+00$ & 1.13TE+01 & $1.966 E+01$ & $3.411 E+01$ & $5.929 E+01$ & $1.031 E+02$ & \\
\hline 1 & : & $1.670 E+00$ & $3.010 E+100$ & $5.691 E+\infty$ & $1.105 E+01$ & $2.177 E+01$ & $4.322 E+01$ & $8.611 E+01$ & $1.719 E+02$ & $3.435 E+02$ & \\
\hline 1.2 & $:$ & $1.890 E+00$ & $3.891 E+\infty$ & $8.429 E+\infty$ & $1.879 E+01$ & $4.251 E+01$ & $9.690 E+01$ & $2.218 E+02$ & $5.065 E+02$ & $1.16 \pi E+03$ & \\
\hline 1.4 & $:$ & $2.156 E+100$ & $5.08 \pi t+00$ & $1.265 E+01$ & 3. $238 E+01$ & $8.415 E+01$ & 2. $204 E+02$ & $5.793 E+02$ & $1.526 E+03$ & $4.022 E+03$ & \\
\hline 1.6 & : & $2.477 E+00$ & $6.716 E+00$ & $1.919 E+01$ & $5.644 E+01$ & $1.6655+02$ & $5.067 E+02$ & $1.530 E+03$ & $4.630 E+03$ & $1.402 E+04$ & \\
\hline 1.8 & : & $2.865 E+00$ & $8.943 E+C 0$ & $2.938 E+01$ & $9.92 \pi t+01$ & $3.404 E+02$ & $1.176 E+03$ & 4.080E +03 & $1.418 E+04$ & $4.933 E+04$ & \\
\hline 2 & : & $3.323 E+00$ & 1.200E+01 & $4.533 E+01$ & $1.760 E+02$ & $6.933 E+02$ & 2. $752 E+03$ & $1.097 E+04$ & 4. $378 E+04$ & $1.749 E+05$ & \\
\hline
\end{tabular}

$B 1(N, r, m)$ for $r=128$

\begin{tabular}{|c|c|c|c|c|c|c|c|c|c|c|c|}
\hline Thu 1 & $N=$ & 4 & 8 & 16 & 32 & 64 & 128 & 256 & 512 & 1024 & INF \\
\hline-2 & : & $1.000 E+\infty 0$ & $1.000 E+00$ & $1.000 E+\infty 0$ & $1.000 E+00$ & $1.000 E+\infty 0$ & $1.000 E+00$ & $1.000 E+00$ & $1.000 E+00$ & $1.000 E+00$ & 1. $000 E+00$ \\
\hline-1.8 & : & $1.000 E+00$ & $1.000 E+\infty$ & $1.000 E+00$ & 1.000E+D0 & $1.000 E+00$ & 1. $000 E+(0)$ & $1.000 E+00$ & $1.000 E+\infty 0$ & 1.00OE+00) & 1.000E +00 \\
\hline-1.6 & : & $1.000 E+(00)$ & $1.000 E+\infty \times$ & $1.000 E+\infty$ & $1.000 E+\infty 0$ & $1.000 E+\infty$ & $1.000 E+00$ & $9.999 E-01$ & $9.999 E-01$ & $9.999 E-01$ & $9.00,0 E-01$ \\
\hline-1.4 & : & $1.000 E+0.0$ & $9.90,9 E-01$ & $9.999 E-01$ & $9.909 E-01$ & $9.999 E-01$ & $9.999 E-01$ & $9.999 E-01$ & $9.999 E-01$ & $9.999 E-01$ & 9.9P,PE-01 \\
\hline-1.2 & : & $9.999 E-01$ & $9.909 E-01$ & $9.998 E-01$ & $9.998 E-01$ & 9.998E-01 & $9.998 E-01$ & $9.998 E-01$ & $9.998 E-01$ & $9.99,8 \varepsilon-01$ & $9.998 E-01$ \\
\hline-1 & : & 1. $000 E+\infty)$ & $1.000 E+(0)$ & $1.000 E+(10$ & $1.000 E+00)$ & $1.000 E+00$ & 1. $000 E+\infty$ & 1.000E+00 & 1. $000 E+100$ & 1. $000 E+00$ & $1.000 E+00$ \\
\hline-.8 & : & $1.001 E+\infty \times$ & $1.001 E+\infty$ & $1.002 E+00$ & $1.002 E+\infty 0$ & $1.002 E+\infty 0$ & $1.002 E+00$ & $1.002 E+\infty$ & $1.002 E+\infty$ & $1.002 E+\infty 0$ & $1.002 E+\infty$ \\
\hline-.6 & : & $1.003 E+00$ & $1.006 E+\infty 0$ & $1.008 E+\infty 0$ & $1.010 E+00$ & $1.012 E+(00$ & $1.013 E+00$ & $1.014 E+\infty 0$ & $1.014 E+\infty$ & $1.015 E+00$ & $1.015 E+00$ \\
\hline-.4 & : & 1. $010 E+00$ & $1.021 E+\infty 0$ & $1.031 E+\infty$ & $1.040 E+00$ & $1.047 E+\infty$ & $1.053 E+\infty$ & $1.058 E+00$ & $1.0625+\infty$ & $1.06 .5 E+00$ & $1.074 E+00$ \\
\hline-.2 & : & $1.029 E+019$ & $1.060 E+00$ & $1.092 E+\infty$ & $1.124 E+\infty 0$ & $1.153 E+\infty$ & $1.181 E+\infty 0$ & 1. $205 E+\infty 0$ & $1.226 E+00$ & $1.245 E+00$ & $1.375 E+00$ \\
\hline 0 & : & 1. $0 t 5 E+\infty$ & $1.144 E+00$ & 1. $232 E+i 0$ & $1.329 E+00$ & $1.430 E+\infty 0$ & $1.534 E+00$ & $1.640 E+00$ & $1.748 E+00$ & $1.856 E+\infty 0$ & \\
\hline .2 & : & $1.12 \pi E+00$ & $1.294 E+00$ & $1.504 E+00$ & 1. $75 \% E+\infty$ & 2. $062 E+\infty 0$ & $2.416 E+\infty 0$ & $2.827 E+\infty 0$ & $3.303 E+\infty 0$ & $3.851 E+00$ & \\
\hline .4 & : & $1.217 E+00$ & $1.532 E+00$ & $1.973 E+00$ & $2.576 E+\infty 0$ & 3. $388 E+\infty 0$ & $4.471 E+\infty$ & $5.909 E+00$ & $7.813 E+00$ & $1.033 E+01$ & \\
\hline.$t$ & : & $1.336 E+00$ & $1.877 E+\infty$ & $2.725 E+00$ & $4.03 \pi E+\infty$ & $6.048 E+00$ & $9.115 E+\infty 0$ & $1.378 E+01$ & $2.086 E+01$ & $3.160 E+01$ & \\
\hline .8 & : & $1.486 E+\infty 0$ & $2.354 E+0.0$ & $3.891 E+\infty 0$ & $6.589 E+\infty$ & $1.131 E+01$ & $1.955 E+01$ & $3.391 E+01$ & $5.893 E+01$ & $1.025 E+02$ & \\
\hline 1 & : & $1.6+B E+(00)$ & $3.005 E+0.1$ & $5.679 E+\infty 0$ & $1.103 E+01$ & $2.172 E+01$ & 4. $311 E+01$ & $8.589 E+01$ & $1.714 E+02$ & $3.426 E+02$ & \\
\hline 1.2 & : & $1.889 E+00$ & $3.887 E+\infty 0$ & $8.421 E+00$ & $1.877 E+01$ & $4.246 E+01$ & $9.680 E+01$ & $2.215 E+02$ & $5.079 E+02$ & $1.166 E+03$ & \\
\hline 1.4 & : & $2.156 E+0.0$ & $5.085 E+00$ & $1.265 E+01$ & 3. $237 E+01$ & $8.411 E+01$ & 2. $203 E+02$ & $5.790 E+02$ & $1.525 E+03$ & $4.021 E+03$ & \\
\hline 1.6 & : & $2.477 E+00$ & $6.715 E+00$ & $1.919 E+01$ & $5.643 E+01$ & $1.684 E+02$ & $5.06 \pi+02$ & $1.530 E+03$ & $4.629 E+03$ & $1.402 E+04$ & \\
\hline 1.8 & : & $2.865 E+100$ & $8.943 E+00$ & $2.938 E+01$ & $9.926 E+01$ & $3.404 E+02$ & $1.176 E+03$ & $4.080 E+03$ & $1.418 E+04$ & $4.932 \mathrm{E}+04$ & \\
\hline 2 & : & $3.353 E+00$ & $1.200 E+01$ & $4.533 E+01$ & $1.760 E+02$ & $6.933 E+02$ & $2.752 E+03$ & $1.097 \mathrm{E}+04$ & $4.378 E+04$ & $1.749 E+05$ & \\
\hline
\end{tabular}


$B 1(N, r, m)$ for $r=256$

\begin{tabular}{|c|c|c|c|c|c|c|c|c|c|c|c|}
\hline Hus 1 & $N=$ & 4 & 8 & 16 & 32 & 64 & 128 & 256 & 512 & 1024 & INF \\
\hline & & & & & & & & & & & \\
\hline-2 & $:$ & 1. $000 E+00$ & $1.000 E+00$ & $1.000 E+\infty 0$ & $1.000 E+\infty 0$ & $1.000 E+\infty 0$ & $1.000 E+00$ & $1.000 E+\infty 00$ & $1.000 E+\infty 0$ & $1.000 E+\infty 0$ & $1.000 E+\infty 0$ \\
\hline-1.8 & $:$ & $1.000 E+00$ & $1.000 E+00$ & 1. $000 E+\infty 0$ & 1. $000 E+\infty$ & $1.000 E+\infty$ & $1.000 E+00$ & $1.000 E+00$ & 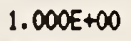 & 1. $000 E+00$ & $1.000 E+00$ \\
\hline-1.6 & $:$ & 1. $000 E+\infty 0$ & $1.000 E+\infty 0$ & 1. $000 E+\infty$ & 1. $000 E+\infty$ & $1.000 E+\infty$ & $1.000 E+00$ & $1.000 E+\infty 0$ & $1.000 E+\infty$ & 1. $000 E+\infty 0$ & 1.000E+00 \\
\hline-1.4 & : & $1.000 E+\infty 0$ & $1.000 E+00$ & 1. $000 E+\infty 0$ & $1.000 E+\infty$ & $1.000 E+00$ & $1.000 E+\infty 0$ & $1.000 E+00$ & $9.9996-01$ & $9.999 E-01$ & $9.999 E-01$ \\
\hline-1.2 & : & $1.000 E+00$ & $9.999 E-01$ & $9.999 E-01$ & $9.999 E-01$ & $9.999 E-01$ & $9.999 E-01$ & $9.99 \%-01$ & $9.999 E-01$ & $9.999 E-01$ & $9.999 E-01$ \\
\hline-1 & : & $1.000 E+00$ & 1. $000 E+\infty 0$ & $1.000 E+\infty 0$ & 1. $000 E+\infty 0$ & $1.000 E+00$ & $1.000 E+00$ & $1.000 E+00$ & 1.000E++0 & $1.000 E+\infty 0$ & $1.000 E+\infty 0$ \\
\hline-.8 & $:$ & $1.000 E+00$ & $1.001 E+\infty$ & 1. $001 E+\infty$ & $1.001 E+\infty$ & $1.001 E+\infty 0$ & $1.001 E+\infty 0$ & $1.001 E+\infty$ & $1.001 E+\infty$ & $1.001 E+\infty 0$ & $1.001 E+00$ \\
\hline-.6 & $:$ & $1.002 E+00$ & $1.004 E+\infty 0$ & $1.005 E+00$ & $1.007 E+\infty 0$ & $1.008 E+00$ & $1.008 E+00$ & $1.009 E+\infty 00$ & 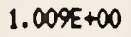 & $1.010 E+\infty$ & $1.010 E+00$ \\
\hline-.4 & $:$ & 1. $008 E+00$ & $1.016 E+\infty$ & $1.023 E+00$ & $1.029 E+\infty 0$ & $1.035 E+\infty$ & $1.039 E+00$ & $1.043 E+00$ & $1.046 E+00$ & $1.048 E+00$ & $1.055+00$ \\
\hline-.2 & : & $1.024 E+\infty 0$ & $1.050 E+\infty 0$ & 1.077E+D0 & 1. $103 E+\infty$ & 1. 127E+OD & $1.150 E+00$ & $1.170 \varepsilon+00$ & $1.188 E+\infty 0$ & $1.204 E+\infty 0$ & $1.311 E+\infty 0$ \\
\hline 0 & $:$ & $1.059 E+00$ & $1.130 E+\infty 0$ & $1.210 E+\infty 0$ & $1.296 E+00$ & $1.3885+00$ & $1.482 E+00$ & $1.577 E+00$ & $1.674 E+\infty 0$ & 1. $772 \leq+\infty$ & \\
\hline .2 & : & $1.121 E+\infty 0$ & $1.280 E+\infty 0$ & $1.479 E+00$ & $1.722 E+00$ & $2.009 E+00$ & $2.346 E+00$ & $2.737 E+00$ & $3.189 E+00$ & $3.710 E+\infty 0$ & \\
\hline .4 & $:$ & $1.212 E+\infty 0$ & $1.520 E+\infty 0$ & $1.952 E+\infty 0$ & $2.541 E+\infty 0$ & $3.335 E+\infty 0$ & $4.394 E+00$ & $5.800 E+00$ & $7.662 \varepsilon+\infty 0$ & $1.01 \geq E+01$ & \\
\hline .6 & : & $1.333 E+\infty 0$ & $1.86 \% E+\infty 0$ & $2.709 E+\infty 0$ & $4.010 E+\infty 0$ & $6.003 E+00$ & $9.042 E+\infty 0$ & $1.366 E+01$ & $2.068 E+01$ & $3.132 E+101$ & \\
\hline .8 & $:$ & $1.484 E+\infty 0$ & $2.350 E+\infty$ & $3.881 \mathrm{E}+\infty 0$ & $6.570 E+\infty$ & $1.127 E+01$ & $1.948 E+01$ & 3. 3.0E+01 & $5.873 E+01$ & $1.022 E+02$ & \\
\hline 1 & $:$ & $1.668 E+00$ & $3.003 E+\infty 0$ & $5.673 E+\infty 0$ & $1.101 E+01$ & $2.169 E+01$ & $4.305 E+01$ & $8.578 E+01$ & $1.71 Z E+02$ & $3.421 E+02$ & \\
\hline 1.2 & $:$ & $1.88 \%+00$ & $3.886 E+\infty 0$ & $8.418 E+\infty 0$ & $1.876 E+01$ & $4.244 E+01$ & $9.675 E+01$ & $2.214 E+0 \hat{2}$ & $5.07 T E+02$ & $1.165 E+03$ & \\
\hline 1.4 & $:$ & $2.156 E+00$ & $5.084 E+\infty 0$ & $1.26-4 E+(01)$ & $3.236 E+01$ & $8.410 E+01$ & $2.20 Z E+02$ & $5.789 E+02$ & $1.525 E+03$ & $4.020 E+0.3$ & \\
\hline 1.6 & $:$ & $2.477 E+\infty 0$ & $6.715 E+00$ & $1.919 E+01$ & $5.642 E+01$ & $1.684 E+02$ & $5.066 E+02$ & $1.530 E+03$ & $4.629 E+03$ & $1.402 E+0.4$ & \\
\hline 1.8 & $:$ & $2.865 E+00$ & $8.943 E+00$ & $2.938 E+01$ & $9.926 E+01$ & $3.404 E+02$ & $1.176 E+03$ & $4.080 E+03$ & $1.418 E+104$ & $4.932 E+0.4$ & \\
\hline 2 & : & 3. $333 E+00$ & $1.200 E+01$ & $4.533 E+01$ & $1.760 E+02$ & $6.93 .3 E+02$ & $2.752 E+0.3$ & $1.097 E+04$ & $4.378 E+04$ & 1.749E+05 & \\
\hline
\end{tabular}

$B !(N, r, m u)$ for $r=512$

\begin{tabular}{|c|c|c|c|c|c|c|c|c|c|c|c|}
\hline Hus 1 & $N=$ & 4 & 8 & 16 & 32 & 64 & 128 & 256 & 512 & 1024 & INF \\
\hline & & & & & & & & & & & \\
\hline-2 & : & 1. $0005+00$ & 1. $000 E+(1)$ & $1.000 E+00$ & 1.000E $+\infty 0$ & 1.000E +00 & $1.000 E+00$ & 1.000E+00 & $1.000 E+010$ & $1.000 E+00$ & 1. $(w 0)+\infty)$ \\
\hline-1.8 & : & $1.000 E+00$ & 1. $000 E+00$ & $1.000 E+00$ & $1.000 E+00$ & $1.000 E+000$ & 1. OOOHE $+\infty 0$ & $1.000 E+00$ & $1.000 E+00$ & $1.000 E+100$ & 1. OOOE +1$) 0$ \\
\hline 1.6 & : & $1.000 E+00$ & 1.000E+00 & . 000E+ORS & $1.000 E+00$ & $1.000 E+\infty)$ & 1. $000 E+\infty$ & $1.000 E+00$ & $1.000 E+00$ & $1.000 E+00$ & $1.000 E+00$ \\
\hline-1.4 & : & $1.000 E+00$ & $1.000 E+010$ & 1.000E+00 & 1.000E+101! & 1.000E+00 & $1.000 E+\infty$ & $1.000 E+\infty 0$ & 1.000E+10? & 1. $000 E+100$ & 1. $000 E+(100)$ \\
\hline-1.2 & : & $1.000 \bar{E}+00$ & $1.000 E+00$ & $.000 E+C O$ & $1.000 t+00$ & 1.000E+00 & 1. $000 E+\infty 0$ & 1. $000 E+10$ & 1. $000 E+00$ & $5+00$ & 1.000E+00 \\
\hline-1 & : & 1.000E+00 & $1.000 E+00$ & $1.000 E+00$ & $1.0005+100$ & $1.000 E+00$ & 1. $000 E+\infty D$ & $1.000 E+00$ & $1.000 E+00$ & $E+100$ & $E+i x$ \\
\hline-.8 & : & 1. $(x) E+(x)$ & 1. $000 E+00$ & $1.001 E+00$ & $1.001 E+00$ & 1. $001 E+\infty)$ & $1.001 E+\infty 0$ & 1. $M 01 E+\infty)$ & $1.001 E+00$ & $E+\phi \hat{N}$ & $E+00$ \\
\hline.$- t$ & : & 1.001E+00 & $1.003 E+00$ & $1.004 E+00$ & 1.004E+000 & $1.005 E+00$ & $1.006 E+001$ & 1. OChEE +00) & $1.006 E+00$ & $t E+\infty$ & 1. $\alpha_{1}>E+100$ \\
\hline-.4 & : & 1.006E+0 & $1.012 E+i)$ & 1.017E+00 & $1.022 E+00$ & $1.026 E+00$ & $1.030 E+00$ & $E+00$ & $1.034 E+060$ & $1.036 E+00$ & $1.041 \equiv+00$ \\
\hline-.2 & : & $1.0205+100$ & $1.042 E+00$ & $1.064 E+(00)$ & $1.086 E+00$ & $1.107 E+00$ & $1.125 E+00$ & $E+\infty$ & $1.157 E+(0)$ & $1.170 E+00$ & $1.261 E+100$ \\
\hline 0 & : & $1.054 E+0.0$ & $1.118 E+O O$ & $1.191 E+00$ & $1.270 E+00$ & 1. $353 E+00$ & $1.438 E+00$ & $E+\infty$ & $1.614 E+00$ & $1.703 E+100$ & \\
\hline .2 & : & $1.116 E+(n)$ & $1.268 E+100$ & 1. $460 E+(10)$ & $1.692 E+00$ & $1.967 E+00$ & $E+00$ & $E+(1)$ & $3.098 E+00$ & $3.598 E+00$ & \\
\hline .4 & : & 1. $\left.20^{\circ} E+O O O\right)$ & $1.512 E+00$ & $1.936 E+0 \hat{0}$ & $2.516 E+00$ & $3.296 E+100$ & $4.338 E+00$ & $5.721 E+\infty 0$ & $7.553 E+00$ & $9.973 E+00$ & \\
\hline .6 & $:$ & $1.331 E+00$ & $1.864 E+(00)$ & $2.699 E+100$ & $3.992 E+(100$ & $5.973 E+00$ & $8.994 E+00$ & $1.359 E+01$ & $2.056 E+[1]$ & $3.114 E+C 1$ & \\
\hline .8 & $:$ & 1.48\%t+i & $2.347 E+O 0)$ & $3.875 E+00$ & $6.558 E+00$ & $1.125 E+(01$ & $1.945 E+01$ & $3.373 E+01$ & $5.86 .2 E+(01$ & $1.020 E+0 i$ & \\
\hline 1 & : & $1.66 .7 E+0.0$ & $3.001 E+00$ & $5.670 E+00$ & $1.101 E+01$ & $2.168 E+01$ & $4.30 .3 E+01$ & $8.572 E+01$ & $1.711 E+02$ & $3.419 E+02$ & \\
\hline 1.2 & $:$ & $1.889 E+00$ & 3. $8.5 E+00$ & $8.416 E+(00)$ & $1.87 t E+(0) !$ & $4.243 E+01$ & $9.673 \varepsilon+01$ & $2.214 E+02$ & $5.076 E+102$ & $1.165 E+1.3$ & \\
\hline 1.4 & : & $2.156 E+00$ & $5.084 E+00$ & $1.264 E+01$ & $3.236 E+01$ & $8.410 E+01$ & $2.202 E+02$ & $5.789 E+02$ & $1.525 E+03$ & $4.020 E+03$ & \\
\hline 1.6 & $:$ & $2.477 E+00$ & $6.714 E+00$ & $1.919 E+01$ & $5.642 E+01$ & $1.684 E+02$ & $5.066 E+02$ & $1.530 E+0 ?$ & $4.628 E+03$ & $1.402 E+04$ & \\
\hline 1.8 & $:$ & $2.86 .5 E+100$ & $8.943 x+(10)$ & $2.938 E+(01$ & $9.926 E+01$ & $3.404 E+02$ & $1.176 E+0 ?$ & 4. $080 E+03$ & $1.418 E+04$ & $4.932 E+0.4$ & \\
\hline 2 & : & $3.3 .3 E+(x)$ & $1.200 E+01$ & $4.533 E+01$ & $1.760 E+02$ & $6.933 \mathrm{EE}+0 \mathrm{i}$ & $2.752 E+03$ & $1.097 E+0.4$ & $4.3785+04$ & $1.749 E+0.5$ & \\
\hline
\end{tabular}


$B 1(N, r, m)$ for $r=1024$

\begin{tabular}{|c|c|c|c|c|c|c|c|c|c|c|c|}
\hline 1 & $N=$ & 4 & 8 & 16 & 32 & 64 & 128 & 256 & 512 & 1024 & INF \\
\hline-2 & : & $1.000 E+\infty$ & $1.000 E+00$ & $1.000 E+\infty 0$ & $1.000 E+\infty$ & $1.000 E+\infty$ & $1.000 E+\infty$ & $1.000 E+\infty$ & $1.000 E+\infty 0$ & $1.000 E+\infty$ & $1.000 E+\infty 0$ \\
\hline-1.8 & $:$ & $1.000 E+00$ & $1.000 E+\infty 0$ & $1.000 E+\infty 0$ & $1.000 E+\infty 0$ & $1.000 E+\infty$ & $1.000 E+\infty$ & $1.000 E+\infty$ & $1.000 E+\infty$ & $1.000 E+\infty 0$ & 1. $000 E+\infty$ \\
\hline-1.6 & : & 1. $000 E+\infty$ & $1.000 E+\infty$ & $1.000 E+00$ & $1.000 E+\infty 0$ & $1.000 E+\infty$ & $1.000 E+\infty$ & $1.000 E+\infty 0$ & $1.000 E+\infty$ & $1.000 E+\infty$ & $1.000 E+\infty 0$ \\
\hline-1.4 & : & $1.000 E+\infty 0$ & $1.000 E+00$ & $1.000 E+\infty 0$ & 1. $000 E+\infty 0$ & $1.000 E+\infty 0$ & $1.000 E+\infty 0$ & 1. $000 E+\infty$ & $1.000 E+\infty$ & $1.000 E+\infty 0$ & 1. $000 E+00$ \\
\hline-1.2 & : & 1. $000 E+\infty 0$ & $1.000 E+00$ & $1.000 E+00$ & $1.000 E+\infty 0$ & $1.000 E+\infty$ & $1.000 E+\infty$ & $1.000 E+00$ & $1.000 E+\infty$ & 1. $000 E+\infty$ & $1.000 E+\infty$ \\
\hline-1 & : & $1.000 E+(10$ & $1.000 E+00$ & $1.000 E+00$ & $1.000 E+00$ & $1.000 E+\infty 0$ & $1.000 E+\infty$ & $1.000 E+\infty 0$ & $1.000 E+\infty$ & 1. $000 E+\infty$ & $1.000 E+00$ \\
\hline-.8 & : & $1.000 E+\infty 0$ & $1.000 E+\infty 0$ & $1.000 E+\infty 0$ & $1.000 E+\infty 0$ & 1. $000 E+\infty 0$ & $1.000 E+\infty 0$ & $1.000 E+\infty 0$ & $1.000 E+\infty 00$ & $1.000 E+\infty 0$ & $1.000 E+\infty$ \\
\hline-.6 & : & $1.001 E+\infty 0$ & $1.00 \mathbb{E}+\infty$ & $1.00 Z E+\infty 0$ & $1.003 E+\infty 0$ & $1.003 E+\infty 0$ & $1.004 E+\infty 0$ & $1.004 E+\infty 0$ & $1.004 E+\infty$ & $1.004 E+\infty$ & $1.004 E+\infty$ \\
\hline-.4 & : & $1.004 E+00$ & 1. $00 \% E+\infty$ & $1.013 E+\infty$ & $1.017 E+\infty 0$ & $1.020 E+\infty 0$ & $1.022 E+\infty 0$ & $1.024 E+\infty 0$ & $1.026 E+\infty 0$ & $1.027 E+\infty 0$ & $1.031 E+\infty$ \\
\hline-.2 & : & $1.017 E+00$ & $1.035 E+\infty 0$ & $1.054 E+00$ & $1.073 E+\infty 0$ & $1.090 E+\infty$ & $1.106 E+\infty 0$ & $1.120 E+\infty 0$ & $1.132 E+\infty$ & $1.144 E+\infty$ & $1.220 E+\infty$ \\
\hline 0 & : & 1.049E+00 & $1.108 E+\infty 0$ & $1.175 E+\infty$ & $1.248 E+00$ & $1.324 E+\infty$ & $1.402 E+\infty 0$ & $1.483 E+\infty 0$ & $1.564 E+\infty 0$ & $1.645 E+\infty$ & \\
\hline .2 & : & $1.112 \mathbb{E}+\infty$ & 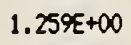 & $1.444 E+00$ & $1.668 E+00$ & $1.934 E+\infty 0$ & $2.245 E+\infty 0$ & $2.60 \pi \mathrm{E}+\infty$ & $3.025 E+\infty$ & $3.507 E+\infty$ & \\
\hline .4 & : & $1.206 E+\infty 0$ & $1.505 E+\infty 0$ & $1.924 E+\infty 0$ & $2.497 E+\infty 0$ & $3.268 \varepsilon+\infty 0$ & 4. $29 \pi t+\infty$ & $5.663 E+\infty 0$ & $7.472 E+\infty 0$ & $9.862 \mathbb{E}+\infty 0$ & \\
\hline .6 & $:$ & 1. $330 E+\infty$ & $1.860 E+\infty 0$ & $2.693 E+\infty 0$ & $3.980 E+\infty 0$ & $5.953 E+(10)$ & $8.963 E+\infty 0$ & $1.354 E+01$ & $2.049 E+01$ & 3.10XE +01 & \\
\hline .8 & : & $1.482 E+\infty 0$ & $2.345 E+\infty 0$ & $3.872 \mathbb{E}+\infty$ & $6.552 E+\infty 0$ & $1.124 E+01$ & $1.942 E+01$ & $3.369 E+01$ & 5. $855 x+01$ & $1.018 E+02$ & \\
\hline 1 & : & $1.667 E+100$ & $3.001 E+\infty$ & $5.668 E+00$ & $1.100 E+01$ & $2.167 E+01$ & 4.301E+01 & $8.56 \% E+01$ & $1.711 E+02$ & $3.418 \varepsilon+02$ & \\
\hline 1.2 & $:$ & $1.889 E+\infty 0$ & $3.885 E+00$ & $8.416 E+\infty 0$ & $1.876 E+01$ & 4. $243 E+01$ & $9.67 x+01$ & $2.213 E+02$ & $5.075 E+02$ & $1.165 E+03$ & \\
\hline 1.4 & : & $2.156 E+\infty 0$ & $5.084 E+\infty 0$ & $1.264 E+01$ & $3.236 E+01$ & 8. $409 E+01$ & $2.202 E+02$ & $5.789 E+02$ & $1.525 E+03$ & $4.020 E+03$ & \\
\hline 1.6 & $:$ & $2.477 E+00$ & $6.714 E+\infty 0$ & $1.919 E+01$ & $5.642 E+01$ & $1.684 E+02$ & $5.066 E+02$ & $1.530 E+03$ & $4.628 E+03$ & $1.40 \mathbb{Z}+04$ & \\
\hline 1.8 & $:$ & $2.865 E+00$ & $8.943 E+100$ & $2.938 E+01$ & $9.926 E+01$ & 3. $404 E+02$ & $1.176 E+03$ & $4.080 E+03$ & $1.418 E+04$ & $4.932 E+04$ & \\
\hline 2 & $:$ & 3. $333 E+00$ & 1.200E+01 & $4.533 E+01$ & $1.760 E+02$ & $6.933 E+02$ & 2. $752 E+0 ?$ & $1.097 E+04$ & $4.378 E+04$ & $1.749 E+05$ & \\
\hline
\end{tabular}

$B 1(N, r, m)$ for $r=2048$

\begin{tabular}{|c|c|c|c|c|c|c|c|c|c|c|c|}
\hline mu 1 & $N=$ & 4 & 8 & 16 & 32 & 64 & 128 & 256 & 512 & 1024 & INF \\
\hline-2 & : & 1. $000 n E+\infty$ & 1. $100 E+00$ & $1.000 E+\infty$ & 1.000E+D0 & $1.000 E+\infty 0$ & $1.000 E+00$ & $1.000 E+\infty 0$ & 1. $000 E+\infty 0$ & $1.000 E+\infty 0$ & 1. $000 E+\infty$ \\
\hline-1.8 & : & 1.000E +00 & $1.000 E+\infty$ & $1.000 E+\infty$ & 1.000E + $+\infty$ & $1.000 E+00$ & $1.000 E+\infty$ & $1.000 E+00$ & $1.000 E+\infty 0$ & $1.000 E+00$ & 1. $000 E+00$ \\
\hline-1.6 & : & $1.000 E+\infty 0$ & $1.000 E+00$ & 1. $000 E+\infty O$ & $1.000 E+\infty$ & $1.000 E+00$ & $1.000 E+00$ & 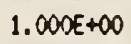 & $1.000 E+\infty$ & $1.000 E+\infty$ & 1. $000 E+\infty$ \\
\hline-1.4 & : & 1.000E+00 & $1.000 E+\infty 0$ & 1.000E+00 & $1.000 \Sigma+\infty$ & $1.000 E+\infty 0$ & $1.000 E+\infty$ & $1.000 E+\infty 0$ & $1.000 E+\infty 0$ & $1.000 E+00$ & $1.000 E+00$ \\
\hline-1.2 & : & $1.000 E+\infty 0$ & 1.000E+CO & $1.000 E+\infty 0$ & $1.000 E+\infty$ & $1.000 E+\infty 0$ & $1.000 E+\infty 0$ & $1.000 E+00$ & $1.000 E+\infty$ & $1.000 E+\infty 0$ & $1.000 E+00$ \\
\hline-1 & : & $1.000 E+100$ & $1.000 E+0.0$ & 1.000E+00 & $1.0000 E+\infty$ & 1.000E $+\infty 0$ & $1.000 E+00$ & $1.000 E+\infty$ & 1.000E+00 & $1.000 E+\infty 0$ & $1.000 E+00$ \\
\hline-.8 & : & $1.000 E+\infty 0$ & $1.000 E+00$ & $1.000 E+\infty 0$ & $1.000 E+\infty$ & $1.000 E+\infty 0$ & $1.000 E+\infty$ & 1. $000 E+\infty 0$ & $1.000 E+\infty 0$ & $1.000 E+00$ & $1.000 E+00$ \\
\hline-.6 & : & $1.001 E+\infty 0$ & $1.001 E+\infty 0$ & $1.002 E+\infty$ & $1.002 E+\infty 0$ & $1.002 E+00$ & $1.002 E+00$ & $1.003 E+00$ & $1.003 E+\infty 0$ & $1.003 E+00$ & $1.003 E+00$ \\
\hline-.4 & : & $1.003 E+\infty 0$ & $1.007 E+\infty 0$ & $1.010 E$ & 1.012 & $1.015 E+\infty 0$ & $1.017 E+\infty 0$ & $1.018 E+\infty$ & $1.019 E+\infty 0$ & $1.020 E+\infty 0$ & $1.023 E+00$ \\
\hline-.2 & : & $1.014 E+\infty 0$ & $1.030 \mathrm{E}+$ & $1.046 E$ & 1.061 & $1.0765+00$ & $1.089 E+00$ & $1.101 E+00$ & $1.112 E+01$ & $1.122 E+\infty 0$ & $1.186 E+00$ \\
\hline 0 & : & $1.045 E+00$ & $1.100 E+00$ & $1.162 E+\infty 0$ & $1.229 E+\infty 0$ & 1. $299 E+00$ & $1.372 E+\infty 0$ & $1.46 E+\infty 00$ & $1.521 E+00$ & $1.596 E+00$ & \\
\hline .2 & : & $1.108 E+\infty 0$ & $1.251 E+\infty$ & $1.431 E+\infty$ & $1.648 E+\infty 0$ & $1.906 E+\infty 00$ & 2. $209 E+00$ & $2.560 E+00$ & $2.966 E+00$ & $3.434 E+\infty 0$ & \\
\hline .4 & : & $1.204 E+\infty 0$ & $1.501 E+\infty 0$ & $1.916 E+\infty 0$ & $2.483 E+\infty 0$ & $3.247 E+\infty 0$ & $4.266 E+00$ & $5.620 E+00$ & $7.41 X E+\infty$ & $9.780 E+00$ & \\
\hline .6 & : & $1.329 E+\infty 0$ & $1.858 \varepsilon+00$ & $2.6888+\infty 0$ & $3.972 E+\infty$ & $5.941 E+\infty$ & $8.942 E+\infty 0$ & $1.351 E+01$ & $2.044 E+01$ & $3.095 E+01$ & \\
\hline .8 & : & 1. $48 z E+00$ & $2.345 E+100$ & $3.870 E+\infty 0$ & $6.548 \varepsilon+00$ & $1.123 E+01$ & $1.941 E+01$ & $3.367 E+01$ & $5.851 E+01$ & $1.018 \varepsilon+02$ & \\
\hline 1 & : & $1.667 E+00$ & $3.000 E+00$ & $5.667 E+00$ & 1. $100 E+01$ & $2.167 E+01$ & 4. $301 E+01$ & $8.568 E+01$ & $1.710 E+02$ & $3.417 E+02$ & \\
\hline 1.2 & : & $1.889 E+\infty 0$ & $3.85 E+\infty 0$ & $8.415 E+00$ & $1.875 E+01$ & $4.243 E+01$ & $9.67 \geq E+01$ & $2.213 E+02$ & $5.075 E+02$ & $1.165 E+03$ & \\
\hline 1.4 & : & $2.156 E+\infty 0$ & $5.084 E+\infty 0$ & $1.264 E+01$ & 3. $236 E+01$ & 8. $409 E+01$ & 2. $202 E+02$ & $5.789 E+02$ & $1.525 E+03$ & $4.020 E+03$ & \\
\hline 1.6 & : & $2.477 E+\infty)$ & $6.714 E+\infty 00$ & $1.91 \%+01$ & $5.642 E+01$ & $1.684 E+02$ & $5.066 E+02$ & $1.530 E+03$ & $4.628 E+03$ & $1.402 E+04$ & \\
\hline 1.8 & : & $2.8 E .5 E+(n)$ & $8.943 E+\infty$ & $2.938 E+01$ & $9.926 E+01$ & 3. $404 E+02$ & $1.176 E+03$ & $4.000 E+03$ & $1.418 E+04$ & $4.932 E+04$ & \\
\hline 2 & : & 3. $33 E+00$ & 1. $200 E+01$ & $4.533 E+01$ & $1.760 E+02$ & $6.933 E+02$ & $2.752 E+03$ & $1.097 E+04$ & $4.378 E+04$ & $1.749 E+05$ & \\
\hline
\end{tabular}


$B 1(N, r, \ldots)$ for $r=I N I N I T Y$

\begin{tabular}{|c|c|c|c|c|c|c|c|c|c|c|c|}
\hline Ho 1 & $N=$ & 4 & 8 & 16 & 32 & 64 & 128 & 256 & 512 & 1024 & INF \\
\hline-2 & : & $1.000 E+\infty 0$ & 1.000E +00 & 1.000E +00 & $1.000 x+\infty 0$ & $1.000 E+\infty$ & $1.000 E+\infty$ & $1.000 E+00$ & $1.000 E+00$ & $1.000 E+\infty 0$ & $1.000 E+0$ \\
\hline-1.8 & $:$ & 1. $000 E+\infty$ & $1.000 E+00$ & 1.000E+00 & $1.000 E+00$ & $1.000 E+\infty 0$ & $1.000 E+00$ & 1.000E+00 & 1.000E+00 & $1.000 E+\infty$ & 1.000E+00 \\
\hline 1.5 & $:$ & $1.000 E+100$ & $1.000 E+00$ & $1.000 E+\infty 00$ & $1.000 E+\infty 0$ & $1.000 E+\infty 0$ & $1.000 E+00$ & $1.000 E+00$ & $1.000 E+00$ & $1.000 E+00$ & $1.000 E+00$ \\
\hline-1.4 & $:$ & $1.000 E+\infty 0$ & $1.000 E+00$ & $1.000 E+00$ & 1.000E+00 & 1.000E+00 & $1.000 E+00$ & $1.000 E+00$ & $1.000 E+00$ & $1.000 E+00$ & 1.000E+00 \\
\hline-1.2 & : & 1.000E+1)0 & $1.000 E+00$ & $1.000 E+\infty 0$ & 1. $000 E+00$ & 1. $000 E+00$ & $1.000 E+\infty 0$ & $1.000 E+00$ & $1.000 E+00$ & 1.000E+00 & $1.000 E+C$ \\
\hline-1 & : & 1.000E+CD & $1.000 E+\infty 0$ & $1.000 E+\infty 0$ & $1.000 E+\infty 0$ & $1.000 E+\infty 0$ & $1.000 E+00$ & $1.000 E+00$ & $1.000 E+1010$ & $1.000 E+\infty 0$ & 1.000E+1 \\
\hline-.8 & : & 1. $000 E+00$ & $1.000 E+\infty$ & $1.000 E+00$ & $1.000 E+00$ & $1.000 E+\infty 0$ & $1.000 E+00$ & $1.000 E+00$ & $1.000 E+00$ & $1.000 E+\infty 0$ & $1.000 t+1$ \\
\hline-.6 & : & $200 E+00$ & $1.000 E+00$ & $1.000 E+00$ & 1. $000 E+100$ & $1.000 E+\infty 0$ & $1.000 E+00$ & $1.000 E+00$ & $1.000 E+\infty$ & $1.000 E+\infty D$ & $1.000 t+$ \\
\hline-.4 & : & $1.000 E+00$ & 1.000E+00 & $1.000 E+00$ & 1.000E+c0 & $1.000 E+\infty$ & 1.000E +00 & 1. $000 E+00$ & 1.000E+00: & 1. $000 E+\infty 0$ & $1.000 E+C$ \\
\hline-.2 & : & $1.000 E+000$ & $1.000 E+00$ & $1.000 E+00$ & $1.000 E+\infty D$ & $1.000 E+00$ & 1.000E+00 & $1.0000+00$ & $1.000 E+00$ & $1.000 E+\infty 0$ & 1.000E+ \\
\hline 0 & : & $1.000 E+00$ & $1.000 E+00$ & $1.000 E+00$ & $1.000 E+\infty 0$ & $1.000 E+00$ & $1.000 E+\infty 0$ & $1.000 E+00$ & $1.000 E+00$ & 1. $000 E+00$ & \\
\hline .2 & : & $1.091 E+(00$ & $1.210 E+00$ & $1.360 E+00$ & $\therefore .541 E+\infty 0$ & 1. $757 E+\infty 0$ & $2.009 E+00$ & $2.303 E+00$ & $2.642 E+00$ & $3.033 E+\infty 0$ & \\
\hline .4 & : & $1.198 E+C 0$ & 1. $487 E+i j$ & $1.8 \% 0 E+\infty$ & $2.441 E+\infty 0$ & $3.184 E+\infty 0$ & $4.174 E+\infty 0$ & $5.489 E+00$ & $7.231 E+00$ & $9.533 x+00$ & \\
\hline .6 & : & $1.327 \mathrm{E}+00$ & $1.854 E+00$ & $2.680 E+00$ & $3.958 E+00$ & $5.916 E+00$ & $8.903 E+\infty 0$ & 1. $344 E+01$ & $2.034 E+01$ & 3. $080 E+01$ & \\
\hline .8 & : & 1. $4 \varepsilon 2 E+00$ & $2.34 .3 E+00$ & $3.867 E+00$ & $6.543 E+00$ & $1.123 E+01$ & $1.940 E+01$ & 3. $364 E+01$ & $5.846 E+01$ & $1.01 \pi E+02$ & \\
\hline 1 & : & $1.667 E+00$ & 3. $000 E+00$ & $5.667 E+\infty 0$ & $1.100 E+01$ & $2.167 E+01$ & $4.300 E+01$ & $8.567 E+01$ & $1.710 E+02$ & $3.417 E+02$ & \\
\hline 1.2 & : & $1.889 E+00$ & $3.885 E+00$ & $8.415 E+00$ & $1.875 E+01$ & $4.243 E+01$ & $9.67 Z \mathbb{E}+01$ & $2.213 E+02$ & $5.075 E+02$ & $1.165 E+03$ & \\
\hline 1.4 & : & $2.156 E+\infty 0$ & $5.084 E+00$ & $1.264 E+01$ & $3.236 \mathrm{E}+01$ & $8.409 E+01$ & $2.202 E+02$ & $5.789 E+02$ & $1.525 E+03$ & $4.020 E+03$ & \\
\hline 1.6 & : & $2.477 E+\infty 0$ & $6.714 E+\infty 0$ & $1.919 E+01$ & $5.642 E+01$ & $1.684 E+02$ & $5.066 E+02$ & $1.530 \varepsilon+03$ & $4.628 E+03$ & $1.402 E+04$ & \\
\hline 1.8 & : & $2.865 E+00$ & $8.943 E+\infty 0$ & $2.938 E+(1) 1$ & $9.926 E+01$ & $3.404 E+02$ & $1.176 E+03$ & $4.080 E+(1) 3$ & $1.418 E+04$ & $4.932 E+04$ & \\
\hline 2 & : & $3.33 .3 E+00$ & $1.200 E+01$ & $4.533 E+01$ & $1.760 E+02$ & $6.933 E+02$ & $2.752 E+03$ & $1.097 E+0.4$ & $4.37 \notin E+0.4$ & $1.749 E+05$ & \\
\hline
\end{tabular}


$B 2(r, w)$

\begin{tabular}{|c|c|c|c|c|c|c|c|c|c|c|c|}
\hline 1 & & .0001 & .0003 & .001 & .003 & .01 & .03 & .1 & .3 & .5 & .7 \\
\hline & $:$ & $6.66 \pi E-01$ & $6.66 \pi-01$ & $6.66 \pi E-01$ & $6.667 E-01$ & $6.66 \pi-01$ & $6.66 \pi t-01$ & $6.66 \pi E-01$ & $6.66 \pi t-01$ & $6.66 \pi E-01$ & \\
\hline & : & $1.11 \geq \mathbf{x}-01$ & $1.305 x-01$ & $1.762 x-01$ & $2.195 t-01$ & $2.793 E-01$ & $3.47 \% E-01$ & $4.431 E-01$ & $5.566 E-01$ & $6.264 E-01$ & $6.899 E-01$ \\
\hline & : & $1.874 E-02$ & $2.900 E-02$ & 4. $708<-02$ & $7.306 E-02$ & $1.185 E-01$ & $1.836 E-01$ & $2.97 \% E-01$ & $4.693 E-01$ & 5.901E-01 & $.013 E-01$ \\
\hline & : & 3. $205 x-03$ & $6.196 E-03$ & $1.276 E-02$ & 2. $467 E-02$ & $5.081 E-02$ & $9.82 \times E-02$ & $2.032 E-01$ & $3.9 \% E-01$ & $; .57 x-01$ & \\
\hline & : & $5.5865-04$ & $1.345 E-03$ & $3.525 x-03$ & $8.489 \div-03$ & $2.225 E-02$ & $5.36 x-02$ & $1.410 E-01$ & 3. $444 E-01$ & 5.273E-01 & $7.052 \mathbf{E}-01$ \\
\hline & $:$ & 1.000E-04 & 3.000E-04 & $1.000 E-03$ & $3.000 E-03$ & $1.000 E-02$ & $3.000 E-02$ & 1.000E-01 & 3. $000 E-01$ & $5.000 E-01$ & $7.000 E-01$ \\
\hline & : & $1.86 x \leq-05$ & $6.956 E-05$ & $2.949 E-04$ & $1.101 E-03$ & $4.66 x-03$ & $1.735 t-02$ & $7.271 E-02$ & $2.64 x-01$ & $4.7495-01$ & $6.916 E-01$ \\
\hline & $:$ & $3.687 E-06$ & $1.715 x-\infty$ & $.231 E-05$ & 4.200E-04 & 2. $28 x<-03$ & $04 \pi E-02$ & 5. $430 E-02$ & $2.351 E-01$ & $4.51 \pi E-01$ & 6.80cE-01 \\
\hline & $:$ & 8. 121E-07 & $4.678 E-06$ & 3.174E-05 & 1. 809E-04 & $1.204 E-03$ & $6.664 E-03$ & $4.195 x-02$ & $2.112 \leq-01$ & $4.303 E-01$ & $6.6805-01$ \\
\hline & $:$ & IE-07 & $1.510<-\infty$ & $1.260 E-05$ & $8.606 E-05$ & $6.921 E-04$ & $4.507 t-03$ & 3. $340 E-02$ & $1.915 E-01$ & $4.103 E-01$ & $6.546 E-01$ \\
\hline & : & $7.726 E-\infty 8$ & $6.240 E-07$ & $6.065 E-06$ & $4.745 E-05$ & 4. 404E-04 & 3. $250 \mathrm{E}-03$ & $2.74 \geq-02$ & 1. $750 \leq-01$ & $15 \bar{E}-01$ & $6.401 E-01$ \\
\hline 2 & : & $3.906<-08$ & 3. $397 \mathrm{E}-07$ & 3. $994 E-06$ & 3.048E-05 & 3. $100 E-04$ & $2.494 E-03$ & 16E-02 & $11 E-01$ & ACE-01 & $6.251 E-01$ \\
\hline & $:$ & $2.590 E-08$ & $2.311 E-07$ & $2.530 E-06$ & 2.220E-05 & 2. $381 E-04$ & $2.020 E-03$ & $2.006 E-02$ & $1.49 z-01$ & $74 E-01$ & $E-01$ \\
\hline & : & $2.013 E-\infty 8$ & $1.800 E-07$ & $2.001 E-\infty 6$ & $1.788 E-05$ & $1.955 E-04$ & $1.706 E-03$ & $1.773 E-02$ & $1.388 z-01$ & $.416 E-01$ & $5.9435-01$ \\
\hline & : & $1.700 E-08$ & $1.52 \%-07$ & $1.69 \pi E-06$ & $1.524 E-05$ & $1.680 E-04$ & $1.493 E-03$ & $1.593 E-02$ & $1.297 t-01$ & $3.267 E-01$ & $5.789 E-01$ \\
\hline & : & $1.500 E-\infty 8$ & $1.350 E-07$ & $1.500 E-06$ & $1.349-05$ & $1.495 z-04$ & $1.33 \pi-03$ & $1.450 E-02$ & $1.215 \pi-01$ & $3.125 E-01$ & $5.635 x-01$ \\
\hline & : & $1.35 \pi-\infty$ & $1.221 E-07$ & $1.3565-06$ & $1.221 E-05$ & $1.355 E-04$ & $1.216 E-03$ & $1.333 E-02$ & 1. 141E-01 & $2.99 \%-01$ & $5.483 x-01$ \\
\hline & : & $1.245 E-\infty 8$ & 1.120E-07 & $1.245 t-06$ & $1.120 \varepsilon-05$ & $1.244 E-04$ & $1.11 \& E-03$ & $1.233 E-02$ & $1.074 E-01$ & $2.85 \% E-01$ & $5.333 E-01$ \\
\hline & : & $1.15 X-\infty$ & $1.037 E-07$ & $1.15 x-06$ & $1.037 E-05$ & $1.15 x-04$ & $1.0365-03$ & $1.14 \pi \mathrm{E}-02$ & $1.012 \boldsymbol{E}-01$ & $2.735 E-01$ & 5. $186 E-01$ \\
\hline & : & $1.07 x-08$ & $9.645 z-08$ & $1.072 E-06$ & $9.645 E-06$ & $1.07 \geq-04$ & $9.642 E-04$ & $1.070<-02$ & $9.541 E-02$ & $2.615 E-01$ & $5.04 x-01$ \\
\hline & : & $1.000 E-08$ & $9.000 E-08$ & $1.000 E-06$ & $9.000 E-06$ & $1.000 E-04$ & $9.000 E-04$ & $1.000 E-02$ & $9.000 E-02$ & $2.500 E-01$ & $4.900 E-01$ \\
\hline
\end{tabular}


$B 2(r, w)$

\begin{tabular}{|c|c|c|c|c|c|c|c|c|c|c|c|}
\hline 1 & $r$ & 1 & 1.01 & 1.1 & 2 & 4 & 8 & 16 & 32 & 64 & 128 \\
\hline 2 & $:$ & $1.000 E+\infty 0$ & $6.667 E-01$ & $6.66 \pi=01$ & $6.667 E-01$ & $6.66 \pi-01$ & $6.66 \pi-01$ & $6.667 E-01$ & & & \\
\hline 1.8 & $:$ & $1.000 E+\infty$ & $8.614 E-01$ & $7.833 E-01$ & $7.196 E-01$ & $7.06 x-01$ & $7.028 E-01$ & $7.018 \varepsilon-01$ & $7.01 \mathbf{E}-01$ & $7.015-01$ & $7.014 E-01$ \\
\hline 1.6 & : & 1. $000 E+\infty$ & $9.429 E-01$ & $8.708 E-01$ & $7.787 \mathrm{E}-01$ & $7.561 E-01$ & $7.494 E-01$ & $7.47 \geq-01$ & $7.465-01$ & $7.462 x-01$ & $7.462 \varepsilon-01$ \\
\hline 1 & : & 1. $000 E+\infty$ & $9.776 \varepsilon-01$ & $9.281 E-01$ & $8.446 E-01$ & $8.192 \mathbb{E}-01$ & 8. $103 E-01$ & 8.071E-01 & $8.056 E-01$ & $8.0535-01$ & $8.05 z x-01$ \\
\hline 1.2 & $:$ & 1. $000 E+\infty 0$ & $9.92 \%-01$ & $9.693 E-01$ & $.181 E-01$ & $990-01$ & $.912 x-01$ & $E-01$ & $.865 t=01$ & & $8565-01$ \\
\hline & : & $1.000 E+\infty$ & $1.000 E+\infty$ & $.000 E+\infty 0$ & $.000 E+\infty 0$ & $100 E+\infty$ & $.000 E+\infty$ & $=\infty$ & $.000 \varepsilon+\infty$ & $0,00 E+\infty$ & $1.000 E+\infty 0$ \\
\hline .8 & : & $1.000 E+\infty 0$ & $1.004 E+00$ & $.024 E+00$ & $.091 E+\infty$ & $28 E+\infty$ & cotw & 100 & $:+\infty$ & $:+\infty$ & $E+\infty$ \\
\hline .6 & : & $1.000<+\infty 0$ & $.006 E+\infty 0$ & - 0iservo & - IJ & $c=00$ & $=0$ & & $+\infty$ & $\varepsilon+\infty$ & $E+\infty$ \\
\hline .4 & : & $1.000 E+\infty 0$ & $+\infty$ & - oderin & odnor & tenvo & 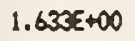 & $\infty$ & 0 & $E+\infty$ & $E+\infty$ \\
\hline .2 & : & 1. $000 E+\infty 0$ & $+\infty$ & $.075 E+\infty 0$ & $182 x+100$ & $0<x+\infty$ & L/E+W0 & w & $=\infty$ & $=+\infty$ & $E+\infty$ \\
\hline 0 & : & $D E+\infty$ & $0 E+\infty$ & 1. $08 \% E+\infty$ & $1.000<\ln$ & $1 \varepsilon E+\infty 0$ & & & $:+\infty$ & $E+\infty$ & $E+\infty$ \\
\hline .2 & : & 1. $000 E+\infty$ & $:+\infty$ & $.10 x+00$ & 7100 & $2.48 \% E+\infty 0$ & IE & & $E+\infty$ & $E+\infty$ & $E+\infty$ \\
\hline .4 & $:$ & 1. $000 E+\infty$ & $x+\infty$ & $.114 E+00$ & nace & A & & & & & $1.674 E+01$ \\
\hline .6 & : & $1.000 E+\infty 0$ & & & & a iener & & & & & 3. $609 E+01$ \\
\hline .8 & $:$ & $1.000 E+\infty 0$ & $14 E+\infty$ & $1.138 E+\infty 0$ & 6.2130 .00 & $4.4 / x+100$ & $8.297 E+\infty$ & & $2.65 x \varepsilon+01$ & $4.669 E+01$ & $8.179 E+01$ \\
\hline 1 & $:$ & $1.000 E+\infty 0$ & $1.015+00$ & $1.150 E+\infty 0$ & $2.500 E+00$ & & & & & $9.550 E+01$ & $1.915+02$ \\
\hline 1.2 & $:$ & $1.000 E+\infty 0$ & $1.016 E+\infty 0$ & $1.16 x+00$ & $2.747 E+\infty 0$ & & $1.607 E+01$ & & & $1.991 E+02$ & $4.579 E+02$ \\
\hline 1.4 & : & $1.000 E+00$ & $1.017 E+\infty$ & $1.174 E+00$ & $3.018 \varepsilon+\infty 0$ & & & & $1.590 E+02$ & $4.201 E+02$ & 1. $10 \% E+0$ \\
\hline 1.6 & $:$ & $1.000 E+\infty 0$ & $1.018 \varepsilon+\infty 0$ & $1.186 E+\infty 0$ & $3.316 E+\infty 0$ & $1.03 \%+01$ & 3. $188 \varepsilon+01$ & $9.706 E+01$ & $2.947 \mathrm{E}+02$ & $8.937 E+02$ & $2.710 E+03$ \\
\hline 1.8 & : & $1.000 E \sqcap \times 0$ & $1.019 E+\infty 0$ & $1.19 \%<+\infty 0$ & $3.642 E+00$ & & & $1.574 E+02$ & 5. $485 E+02$ & $1.910 E+03$ & $6.6535+03$ \\
\hline 2 & $:$ & 1. $000 E+\infty$ & $1.020=+\infty 0$ & $1.210 E \div 00$ & $4.000 E+00$ & $1.600 E+01$ & 6. $400 E+01$ & $2.560 E+02$ & $1.024 E+03$ & $4.096 E+03$ & $1.638 x+10$ \\
\hline
\end{tabular}

\begin{tabular}{|c|c|c|c|c|c|c|c|}
\hline $\mathrm{m} \backslash$ & $r$ & 256 & 512 & 1024 & 2048 & 4096 & INF \\
\hline-2 & : & $6.667 E-01$ & $6.667 E-01$ & $6.667 E-01$ & $6.667 E-01$ & $6.6 \in \pi E-01$ & $6.667 E-01$ \\
\hline-1.8 & $:$ & $7.014 E-01$ & $7.014 E-01$ & $7.014 E-01$ & $7.014 E-01$ & $7.014 E-01$ & $7.014 E-01$ \\
\hline-1.6 & $:$ & $7.461 E-01$ & $7.461 E-01$ & $7.461 E-01$ & $7.461 E-01$ & $7.461 E-01$ & $7,461 E-01$ \\
\hline-1.4 & : & $8.051 E-01$ & $8.051 E-01$ & $8.051 E-01$ & $8.051 E-01$ & $8.051 E-01$ & $8.051 E-01$ \\
\hline-1.2 & $:$ & $8.855 E-01$ & $8.854 E-01$ & $8.854 E-01$ & $8.854 E-01$ & $8.854 E-01$ & $8.8545-01$ \\
\hline-1 & : & 1. $\cos E+\infty$ & 1.000E+100 & 1. $000 E+\infty 0$ & $1.000 E+00$ & 1. $000 E+i j 0$ & $1.000 E+100$ \\
\hline-.8 & : & $1.173 E$ minj & $1.174 E+00$ & $1.174 E+\infty .00$ & $1.174 E+\infty$ & $1.174 E+\infty 0$ & $1.175 E+00$ \\
\hline-.6 & : & $1.455 E+(x)$ & $1.480 E+100$ & $1.463 E+\infty 0$ & $1.465 E+\infty$ & $1.46 \pi E+\infty 0$ & $1.470 E+00$ \\
\hline-.4 & : & $1.957 E+100$ & $1.983 E+(x)$ & $2.003 E+\infty 0$ & $2.018 E+\infty$ & $2.029 E+100$ & $2.065 E+\infty 0$ \\
\hline.$- \hat{2}$ & : & $2.945 x+00$ & $3.064 E+00$ & $3.167 E+\infty$ & $3.257 \mathrm{E}+00$ & $3.336 E+100$ & $3.8635+00$ \\
\hline 0 & $:$ & $5.08 \times 5+10$ & $5.582 E+\infty 0$ & $6.082 E+\infty)$ & $6.582 E+00$ & $7.082 E+1) 0$ & \\
\hline.$\overline{2}$ & : & $1.00 \div E+01$ & $1.219 E+01$ & $1.439 E+01$ & $1.703 E+01$ & $2.006 E+01$ & \\
\hline .4 & : & $2.25 \% E+01$ & $3.031 E+01$ & 4. $050 E+01$ & 5. $394 E+01$ & $7.167 E+01$ & \\
\hline .6 & : & $5.521 E \phi(1) 1$ & $8.418 E+01$ & 1. $281 E+02$ & $1.947 E+02$ & $2.955 E+02$ & \\
\hline .8 & : & 1. 429E-U2 & $2.493 E+02$ & $4.346 E+02$ & $7.571 E+02$ & $1.319 E+03$ & \\
\hline 1 & $:$ & $3.8356+02$ & $7.675 E+02$ & $1.536 E+03$ & $3.072 E+03$ & $6.144 E+03$ & \\
\hline 1.2 & : & $1.052 E+103$ & $2.418 E \mapsto 03$ & $5.5565+03$ & $1.27 \pi E+124$ & $2.933 E+04$ & \\
\hline 1.4 & : & $2.92 x+103$ & $7.727 E+03$ & $2.03 \% \div+04$ & $5.3825+04$ & 1. $420 E+05$ & \\
\hline 1.6 & : & $8.215 E+03$ & $2.490 E+04$ & $7.549 E+04$ & $2.288 \varepsilon+05$ & $6.937 E+05$ & \\
\hline 1.8 & : & $2.317 E \div 04$ & $8.067 E+04$ & $2.80 x E+05$ & $9.782 E+05$ & $3.406 E+06$ & \\
\hline 2 & : & $6.551 E+04$ & $2.621 E+05$ & $1.049 E+06$ & $4.194 E+06$ & $1.678 \varepsilon+07$ & \\
\hline
\end{tabular}


$23(2, H, r, \ldots)$ for $r=.01$

\begin{tabular}{|c|c|c|c|c|c|c|c|c|c|c|c|c|}
\hline 11 & $H=$ & 1 & 2 & 4 & 8 & 16 & 32 & 64 & 128 & 256 & 512 & 1024 \\
\hline 2 & $:$ & $1.000 E+\infty 0$ & $2.000 E+\infty 0$ & $4.000 E+00$ & $8.000 E+00$ & $1.600 E+01$ & $3.200 E+01$ & $6.400 E+01$ & 1. $200 E+02$ & $2.560 \varepsilon+02$ & $5.120 E+02$ & $1.024 E+03$ \\
\hline 1.8 & $:$ & $1.000 E+00$ & $214 E+\infty$ & $5.842 E+\infty 0$ & $1.829 E+01$ & $6.50 x E+01$ & $2.536 E+02$ & $1.173 E+03$ & $3.30<\varepsilon+03$ & $4.8045+03$ & $6.0000=03$ & $7.145 E+03$ \\
\hline 1.6 & $:$ & $000 E+\infty 0$ & $419+\infty$ & $.398 E+\infty 0$ & $2.630 E+01$ & $1.01 \& E+02$ & $4.151 E+02$ & $1.800+\infty+\infty$ & $5.075 E+03$ & $7.2225+\infty 3$ & $8.748 E+\infty 3$ & $9: 882 E+03$ \\
\hline 1.4 & $:$ & $1.000 E+00$ & $.61 \pi E+\infty$ & $8.745 E+\infty 0$ & $3.295 E+01$ & $1.306 E+02$ & $5.358 \varepsilon+02$ & $2.342 E+03$ & $5.964 E+03$ & $8.263 E+03$ & $9.700 E+03$ & $1.063 E+04$ \\
\hline 1.2 & : & $1.000 E+\infty 0$ & $.811 E+\infty 0$ & $9.93 \pi+00$ & $3.843 E+01$ & $1.534 E+02$ & $6.247 E+02$ & $2.595 x+03$ & 6. $253 E+03$ & $8.449 E+03$ & $9.671 E+03$ & $1.036 E+04$ \\
\hline , & $:$ & 1.000E+-00 & 3. $000 E+00$ & 1. $100 E+01$ & 4.300E +01 & $1.710 E+02$ & $6.830 E+02$ & $2.674 E+03$ & $6.094 E+03$ & $8.04 \pi+03$ & $9.024 E+03$ & $9.512 \mathrm{E}+03$ \\
\hline 8 & : & 1.000 & $.180<+00$ & & 4.65 & $1.823 E+02$ & $7.065+02$ & $2.588 \varepsilon+03$ & $5.57 \notin \varepsilon+\infty 3$ & $+\infty 3$ & $7.950 E+03$ & 8.28 \\
\hline 6 & : & $1.000 E+\infty 0$ & $+\infty$ & & 4.848 & $1.851 E+02$ & $6.8 \pi T E+02$ & $2.342 E+03$ & 4. $\pi 75+03$ & $6.045 E+03$ & $6.577 E+03$ & 6.79 \\
\hline .4 & : & $1.000 E+\infty 0$ & $3.444 E+00$ & +01 & 4.812 & $1.766 \varepsilon+02$ & $6.215 E+02$ & $1.95 \pi+03$ & $3.774 E+03$ & $4.6865+03$ & $5.041 E+03$ & $5.171 E+03$ \\
\hline .2 & $:$ & $1.000 E+00$ & $3.463 E+00$ & & 4.480 & 1. $55 \pi+02$ & $5.131 E+02$ & $1.486 E+03$ & $2.71 \simeq \mathbb{E}+03$ & 3. $304 E+03$ & $3.521 E+03$ & $3.594 E+03$ \\
\hline 0 & : & 1.000 & $+\infty$ & & 3.866 & 1. $253 E+02$ & $3.826 E+02$ & $1.015 E+03$ & $1.7535+03$ & 2.09 & $\varepsilon+03$ & $2.254 E+03$ \\
\hline .2 & : & $1.000 E$ & $+\infty$ & & 3.000 & $9.213 E+01$ & $2.579 E+02$ & $6.25 x E+02$ & $1.021 E+03$ & 1. $200 E+03$ & $1.25 \%+03$ & $1.277 E+03$ \\
\hline .4 & : & $1.000 E+\infty 0$ & $2.883 E+\infty 0$ & $E+\infty$ & $2.314 E+01$ & $6.266 E+01$ & $1.5 \% 5 E+02$ & $3.521 E+02$ & $5.442 E+02$ & $6.280 E+02$ & $6.549 E+02$ & $6.62 \pi+02$ \\
\hline .6 & : & $1.000 E+\infty 00$ & $2.574 E+00$ & $+\infty$ & 1.651 & $4.018 E+01$ & $9.228 E+01$ & $1.851 E+02$ & $2.709 E+02$ & $\$ 02$ & $3.183 E+02$ & $3.215 E+02$ \\
\hline .8 & : & $1.000 \varepsilon+00$ & $2.270 E+00$ & $\varepsilon+\infty$ & 1.141 & $2.475 E+01$ & $5.098 E+01$ & $9.280 E+01$ & $1.285 E+02$ & +02 & $1.476 E+02$ & 1. $488 \varepsilon+02$ \\
\hline 1 & : & 1.000 & $1.989 E+00$ & & $7.728 \varepsilon+\infty 0$ & $1.486 E+01$ & $2.731 E+01$ & $4.505 E+01$ & $5.900 E+01$ & $6.464 E+01$ & $6.62 \% E+01$ & $6.673 E+01$ \\
\hline 1.2 & & $1.000 E+\infty 0$ & $1.73 \pi t+00$ & 3. $008 \varepsilon+00$ & $5.177 E+\infty 0$ & 8. $775 E+\infty 0$ & $1.435 E+01$ & $2.142 E+01$ & $2.660 E+01$ & $2.85 \%+01$ & $2.91 \pi+01$ & $2.933 \varepsilon+01$ \\
\hline & & $1.000 E+00$ & $1.514 E+\infty$ & 2.289E+00 & $3.446 E+\infty 00$ & $5.133 E+\infty$ & $7.440 E+\infty 0$ & $1.005 E+01$ & $1.18 Z \mathbf{E}+01$ & $1.248 E+01$ & $1.26 \pi E+01$ & $1.27 Z E+01$ \\
\hline 1. & & 1. $000 E+\infty 0$ & $1.319 E+\infty 0$ & $1.738 \varepsilon+\infty 0$ & $2.285 E+00$ & $2.98 x++00$ & $3.828 \varepsilon+00$ & $4.678 \varepsilon+\infty 0$ & $5.211 E+\infty 0$ & $5.405 E+\infty 0$ & $5.45 \%+00$ & $5.473 E+\infty$ \\
\hline 1.8 & & $1.000 E+\infty 0$ & 1. $149 E+\infty$ & & $1.513 E+\infty$ & $1.72 \% E+100$ & $1.960 E+00$ & $2.166 E+00$ & $2.286 E+\infty 0$ & $2.328 E+\infty 00$ & $2.340 E+05$ & $2.343 E+\infty$ \\
\hline 2 & : & $1.000 E+0,0$ & $1.000 E+\infty$ & $1.000 E+00$ & $1.000 E+00$ & $1.000 E+\infty$ & $1.000 E+00$ & $1.000 E+\infty 0$ & $1.000 E+\infty$ & $1.000 E+\infty$ & 1. $000 E+\infty$ & $1.000 E+00$ \\
\hline
\end{tabular}

$83(2, H, r, w)$ for $r=.03$

\begin{tabular}{|c|c|c|c|c|c|c|c|c|c|c|c|c|}
\hline sis & $H=$ & 1 & 2 & 4 & 8 & 16 & 32 & 64 & 128 & 256 & 512 & 1024 \\
\hline 2 & $:$ & 1. $000 E+\infty 0$ & $2.000 E+\infty 0$ & 4. $000 E+00$ & $8.000 E+00$ & 1. $600 E+01$ & 3. $200 E+01$ & 6. $400 E+01$ & 1. $280 \mathrm{E}+02$ & $2.560 E+02$ & $5.120 E+02$ & $1.024 E+03$ \\
\hline 1.8 & : & $1.000 E+\infty 0$ & $2.216 E+\infty 0$ & $5.866 E+00$ & $1.865 E+01$ & $7.138 E+01$ & $2.812 \boldsymbol{E}+02$ & $4.880 E+02$ & $6.534 E+02$ & $8.075 E+02$ & $9.647 E+02$ & $1.143 E+03$ \\
\hline 1.6 & : & $1.000 E+\infty 0$ & $2.42 z+\infty 0$ & $7.49 E+100$ & $2.705 E+01$ & $1.11 \%+02$ & 4. $27 \% E+02$ & $7.195 E+02$ & $9.170 E+02$ & $1.06 x+03$ & 1. $1800 E+03$ & $1.274 E+03$ \\
\hline 1.4 & : & 1.000E+MO & $.623 E+00$ & $8.81 \pi E+\infty$ & $3.378 E+01$ & $1.411 E+02$ & $5.073 E+02$ & 8. $285 E+02$ & $1.022 E+03$ & $1.147 E+03$ & $1.229+03$ & 1. $283 E+03$ \\
\hline 1.2 & : & 1.00ne + & $2.816 E+0 \dot{0}$ & 1.000E+01 & $3.910 E+01$ & $1.60 \pi+02$ & 5. 386E+02 & $8.548 E+02$ & $1.02 \pi E+03$ & $1.125 \bar{t}+03$ & $1.180 E+03$ & $1.21 \mathbb{x}+03$ \\
\hline 1 & : & $1.000 E+\infty 0$ & $3.000 E+00$ & 1.100E+01 & 4. 300E+1)1 & 1. $710 E+D 2$ & $5.3 z * \varepsilon+02$ & $8.221 E+02$ & $9.667 E+02$ & $1.039 E+03$ & $1.075 E+03$ & $1.093 E+03$ \\
\hline .8 & : & $1.000=+\infty 0$ & $3.164 E+\infty \dot{U}$ & $1.175 E+01$ & $23 E+0 i 1$ & 1.71 & $4.971 E+02$ & $7.455+02$ & $8.606 E+02$ & $9.122 E+02$ & $50 E+02$ & 9.45 \\
\hline 6 & : & $1.000 E+00$ & $=+\infty 0$ & $\pi+0 \mid$ & (3E+1)1 & $1.632 E+02$ & $4.376 E+02$ & $6.378 E+02$ & $7.247 E+02$ & $7.600 E+02$ & $7.740 E+02$ & $7.794 E+02$ \\
\hline .4 & : & $1.000 E+\infty 0$ & $3.356 E+00$ & $3 E+01$ & 4. $331 E+01$ & $1.45 x+02$ & $3.618 E+02$ & $5.126 E+02$ & $5.743 E+02$ & $5.974 E+02$ & $6.057 E+02$ & $6.086 E+02$ \\
\hline .2 & : & $1.000 E+00$ & 3. $33 \%, E+(1)$ & $5 T E+01$ & $3.896 E+01$ & $1.214 E+02$ & $2.79 \pi \in+02$ & $3.851 E+02$ & $4.262 E+02$ & $4.405 E+02$ & $4.452 E+02$ & $4.468 \varepsilon+02$ \\
\hline 0 & : & $1.000 E+00$ & $3.22 \times \varepsilon+\infty 0$ & $E+01$ & 3.29 & $9.462 E+01$ & $2.019 E+02$ & $x+02$ & $2.95 \pi+02$ & $3.040 E+02$ & $3.0 x \leq E+02$ & $3.074 E+102$ \\
\hline .2 & $:$ & 1. $000 E+00$ & $3.036 E+100$ & $E+\infty$ & 2.635 & 6.912 & 1.366 & 1. $\pi 6 E+02$ & $1.924 E+02$ & $1.970 E+02$ & 1. $983 E+02$ & $1.987 E+02$ \\
\hline .4 & : & 1. $000 E+\infty$ & $2.787 E+\infty 0$ & $7.640 E+00$ & $2.00 \geq E+01$ & $4.74 E+01$ & 8. $73 \pi E+01$ & $1.104 E+02$ & $1.184 E+02$ & $1.20 \% E+02$ & $1.215 x+02$ & $1.21 \pi+02$ \\
\hline .6 & : & 1.DOOE+ 00 & $2.510 E+\infty 0$ & $6.186 E+00$ & 1. $461 E \oplus 01$ & 3. $153 \mathrm{E}+01$ & 5. $345+01$ & $6.564 E+01$ & $6.975 E+01$ & $7.095 x+01$ & $7.12 \approx E+01$ & $7.1365+01$ \\
\hline .8 & : & 1.000E+00) & 2. $232 x+\infty$ & $4.89 \pi+\infty 0$ & $1.035 E+01$ & $2.013 E+01$ & 3.16X $1+01$ & 3. $\pi \approx E+01$ & $3.973 \varepsilon+01$ & 4. $030 E+01$ & $4.045 E+01$ & $4.04 \%+01$ \\
\hline 1 & $:$ & $1.000 E+100$ & $1.96 \pi+00$ & $3.818 E+\infty 0$ & & 1. $254 E+01$ & & & $2.210 E+01$ & $2.236 E+01$ & $2.242 E+01$ & $2.244 E+01$ \\
\hline 1.2 & & 1. $000 E+00$ & $1.725 x+\infty 0$ & $2.946 E+00$ & 4. $90 \pi E+00$ & $7.688 \varepsilon+\infty 0$ & $1.03 \pi+01$ & $1.168 E+01$ & $1.20 \% E+01$ & $1.220 E \div 01$ & 1. $223 E+01$ & $1.224 E+01$ \\
\hline 1. & & $1.000 E+00$ & $1.508 \varepsilon+00$ & $2.259 E+00$ & 3. $322 E+00$ & $4.658 E+00$ & $5.82 \%+00$ & $6.371 E+\infty 0$ & $6.538 E+\infty 0$ & $6.583 E+00$ & $6.595 E+00$ & $6.59 \% \varepsilon+\infty$ \\
\hline 1. & & $1.000 E+00$ & $1.316 E+00$ & $1.725 E+00$ & $2.234 E+00$ & $2.801 E+\infty 0$ & $3.251 E+\infty 0$ & 3. $450 E+00$ & $3.511 E+\infty 0$ & $3.527 E+00$ & $3.531 E+00$ & $3.53 \geq E+\infty$ \\
\hline & & $1.000 E+\infty 0$ & $1.148 E+00$ & $1.314 E+\infty 0$ & & $1.676 E+\infty 0$ & $1.805 E+\infty 0$ & $1.860 E+00$ & $1.876 E+\infty 0$ & $1.881 E+\infty$ & $1.882 E+\infty 0$ & $1.882 E+00$ \\
\hline 2 & : & 1. $000 E+00$ & 1.000E+00 & 1.000E+OC & $1.000 E+00$ & $1.000 E+00$ & $1.000 E+\infty 00$ & $1.000 E+\infty$ & $1.000 E+\infty 0$ & $1.000 E+\infty 0$ & $1.000 E+\infty 00$ & 1. $000 E+\infty$ \\
\hline
\end{tabular}


$B(2, H, r, \ldots)$ for $r=.1$

\begin{tabular}{|c|c|c|c|c|c|c|c|c|c|c|c|c|}
\hline An 1 & $\omega=$ & 1 & 2 & 4 & 8 & 16 & 32 & 64 & 128 & 256 & 512 & 1024 \\
\hline-2 & : & $1.000 E+\infty 0$ & 2. $000 E+\infty 0$ & $4.000 E+\infty 00$ & 8. $000 \varepsilon+\infty$ & $1.600 E+01$ & $3.200 E+01$ & 6. $4005+01$ & 1. $200 E+02$ & $2.560 E+02$ & $5.120 \varepsilon+02$ & $1.024 E+03$ \\
\hline-1.8 & : & $1.000 E+\infty$ & 2. $22 \% E+00$ & $6.118 \varepsilon+\infty$ & $2.32 \pi \mathrm{E}+01$ & $4.41 \%=01$ & 5. $757 \mathrm{E}+01$ & $6.871 E+01$ & $7.801 E+01$ & $8.665 E+01$ & $9.393 E+01$ & $1.003 E+02$ \\
\hline-1.6 & : & 1. $000 E+\infty 0$ & 2. $448 E+00$ & $7.8665+00$ & 3. $138 E+01$ & $6.09 \pi E+01$ & $7.934 E+01$ & $9.26 \pi+01$ & $1.026 E+02$ & $1.101 E+02$ & $1.15 E E+02$ & $1.201 E+02$ \\
\hline-1.4 & : & $1.000 E+00$ & $2.654 E+00$ & $9.270 E+\infty 0$ & $3.626 E+01$ & $6.919 E+01$ & $8.832 E+01$ & $1.005 E+02$ & 1. Coret02 & $1.136 E+02$ & $1.170 E+02$ & $1.193 E+02$ \\
\hline-1.2 & : & $1.000 E+\infty 0$ & $2.8425+00$ & $1.032 \leq+01$ & 3. 85$\} E+01$ & $7.135 E+01$ & $8.911 E+01$ & $9.90 \%+01$ & $1.048 E+02$ & 1. $000 E+02$ & $1.09 \%=02$ & 1. $10 \%=02$ \\
\hline-1 & $:$ & $1.000 E+\infty 0$ & $3.000 E+00$ & $1.100 E+01$ & $3.8635+01$ & $6.9065+01$ & $8.453 E+01$ & $9.22 \pi+01$ & $9.613 E+01$ & $80 T E+01$ & $9.903 E+01$ & $9.952 E+01$ \\
\hline-.8 & : & $000 E+\infty$ & $3.11 \pi=00$ & $1.127 E+01$ & $.694 E+01$ & $6.365 \mathrm{E}+01$ & $7.645 E+01$ & $8.222 \times+01$ & $478 E+01$ & $591 E+01$ & $8.640 E+01$ & $8.662 E+01$ \\
\hline-.6 & : & $1.000 E+\infty$ & 3. $180 E+00$ & $E+01$ & $E+01$ & $5.61 \pi+01$ & $\mathbb{E}+01$ & $7.04 \% E+01$ & $7.215+01$ & $7.2005+01$ & $7.305 E+01$ & $14 E+01$ \\
\hline-.4 & $:$ & 1.000E+00 & $3.17 \%+\infty 0$ & $E+01$ & $E+01$ & $E+01$ & $5.532 \varepsilon+01$ & $\mathbf{E}+01$ & $5.931 E+01$ & $5.968 \varepsilon+01$ & $5.981 E+01$ & $5.985 E+01$ \\
\hline-.2 & : & $1.000 E+00$ & $3.111 E+\infty 0$ & $9.757 \mathrm{E}+\infty 0$ & $2.528 E \leftarrow 01$ & 3.884 & 4. $47 E+01$ & $\pi \neq 01$ & $4.714 E+01$ & $4.735 E+01$ & $4.742 E+01$ & $4 E+01$ \\
\hline 0 & : & 1.000E+00 & $2.980 E+00$ & $8.683 E+00$ & $2.06 \% 5+01$ & $3.05 \%+01$ & 3. $455 E+01$ & $E+01$ & $3.62 \%=01$ & $3.641 E+01$ & $3.645 E+01$ & $6 E+01$ \\
\hline .2 & : & 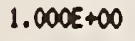 & $2.79 \% \div+00$ & $7.502 \leq+00$ & $1.642 E+01$ & $2.365 \times 401$ & 2. $603 E+01$ & $2.68 \%=01$ & $2.714 E+01$ & $2.721 E \multimap 01$ & 2. $723 E+01$ & $2.724 E+01$ \\
\hline .4 & : & $1.000 E+\infty$ & $2.584 E+00$ & $6.31 \pi+\infty$ & $1.26 \%+01$ & $1.73 \pi+01$ & $1.910 E \div 01$ & $1.964 E+01$ & $1.97 \%+01$ & $1.9045+01$ & $1.965 \times+01$ & $1.985 E+01$ \\
\hline .8 & $:$ & $1.000 E+00$ & $2.116 E+00$ & $4.221 E+\infty 0$ & $7.125 E+\infty$ & $9.013 E+\infty$ & $9.685 \div+00$ & $9.87 \varepsilon E+\infty 0$ & $1 E+\infty$ & $9.944 E+\infty 00$ & $9.94 \varepsilon \varepsilon+\infty 0$ & $19=+\infty$ \\
\hline 1 & : & $1.000 E+00$ & $1.888 E+00$ & $3.377 E+\infty$ & $5.223 E+\infty 0$ & $6.354 E+00$ & $6.745 E+00$ & $6.85 \pi t+00$ & $6.8865+\infty 0$ & $6.894 E+00$ & $6.896 E+00$ & $6.8965+00$ \\
\hline & : & $1.000 E+\infty$ & $1.674 E+00$ & $2.676 E+00$ & $3.791 E+\infty 0$ & $4.435 x+00$ & $4.6535+\infty$ & $4.714 E+00$ & $4.730 E+\infty$ & 4. $734 E+00$ & $4.735 E+\infty$ & $4.735 E+00$ \\
\hline & : & $1.000 E+\infty 0$ & $1.477 E+00$ & $2.105 E+\infty$ & $2.732 E+\infty$ & $3.074 E+00$ & 3. $18 \pi E+\infty$ & $3.218 \varepsilon+\infty 0$ & $3.226 E+\infty 0$ & $3.228 \varepsilon+\infty$ & $3.228 E+00$ & $3.229+10$ \\
\hline & $:$ & $1.000 E+\infty 0$ & $1.29 \%+00$ & $1.64 \pi+\infty$ & $1.960 E+\infty 0$ & $2.1205+\infty 0$ & $2.172 \pm+\infty$ & $2.1865+00$ & $2.189+00$ & 2. $190 E+\infty$ & $2.191 E+\infty$ & $2.191 E+\infty 0$ \\
\hline & : & $1.000 E+\infty 0$ & $1.141 E+\infty$ & $1.285 E+\infty 0$ & $1.401 E+\infty$ & $1.457 E+\infty 0$ & $1.475 \pi+\infty 0$ & $1.480 E+\infty)$ & $1.481 E+00$ & $1.481 E+\infty 0$ & $1.482 E+00$ & $1.4825+00$ \\
\hline 2 & $:$ & $1.000 E+\infty 0$ & $1.000 E+00$ & $1.000 E+\infty 0$ & 1.000E+-00 & 1. $000 E+00$ & $1.000 E+\infty$ & $1.000 E+\infty 0$ & $1.000 E+\infty 0$ & $1.000 E+\infty 0$ & $1.000 E+00$ & 1. $000 E+\infty$ \\
\hline
\end{tabular}

$B 3(2, H, r, 2 u)$ for $r=.3$

\begin{tabular}{|c|c|c|c|c|c|c|c|c|c|c|c|c|}
\hline nus 1 & $H=$ & 1 & 2 & 4 & 8 & 16 & 32 & 64 & 128 & 256 & 512 & 1024 \\
\hline-2 & : & $1.000 E+\infty 0$ & $2.000 E+00$ & $4.000 E+00$ & $8.000=+00$ & $1.600=01$ & $3.200=01$ & $6.4005+01$ & $200<+02$ & $2.560<+02$ & $120 E+02$ & $1.024 E+103$ \\
\hline-1.8 & : & $1.000 E+\infty$ & $2.388 E+\infty 0$ & $5.582 \varepsilon+00$ & $9.024 E+\infty 0$ & $1.341 E \div 01$ & $1.980 E+01$ & $2.987 E+01$ & $4.6495+01$ & $7.464 E+01$ & 1. $230 E+02$ & $2.0655+02$ \\
\hline-1.6 & : & $1.000 E+\infty 0$ & $2.66 \%++\infty$ & $6.503 E+\infty 0$ & $9.614 E+\infty)$ & $1.23 \pi E+01$ & $1.521 E+01$ & $1.854 E+01$ & $2.285 E+01$ & $2.882 E+01$ & $3.7465+01$ & $5.023 E+01$ \\
\hline-1.4 & $:$ & $1.000 E+00$ & $2.85 \pi+\infty$ & $6.93 \pi+00$ & 9. $770=000$ & $1.171 E+01$ & $1.31 \pi+(1) 1$ & $1.440 E+01$ & $1.557 E+01$ & $1.681 E+01$ & $1.825 E+01$ & $2.00 X E+01$ \\
\hline-1.2 & : & 1. $000 E+\infty$ & $2.964 E+00$ & $7.013 E+\infty$ & $9.562 E+100$ & $1.10 x \leq+01$ & $1.18 x+01$ & $1.242 E+01$ & $1.27 \notin+01$ & $1.305 x+01$ & 1. $328 \varepsilon+01$ & 1. $348 E+01$ \\
\hline-1 & $:$ & 1. $000=+\infty$ & $3.000 E+\infty 0$ & $6.833 E+00$ & $9.0838+\infty 0$ & $1.021 E \div 01$ & $1.077 E+101$ & $1.105 E+01$ & $1.11 \%+01$ & $1.126 E+01$ & $1.130 E+01$ & 1. $132 X E+01$ \\
\hline-.8 & : & $1.000 \leqslant+\infty$ & $2.976 E+\infty 0$ & $6.477 E+00$ & $8.422 E+00$ & 9. $300 E+00$ & $9.686 E+00$ & $9.850 E+00$ & $9.91 \varepsilon+\infty$ & $9.944 E+00$ & $9.952 E+\infty 0$ & $9.9535+00$ \\
\hline. .6 & $:$ & $1.000=+\infty 0$ & $2.902 E+\infty 0$ & $6.008 E+100$ & $7.655 E+\infty 0$ & $8.339 E+\infty 0$ & $8.610 E+\infty$ & $8.713 \leq+\infty 0$ & $8.750 E+\infty 0$ & $8.7635+00$ & $8.766 E+00$ & $8.766 E+00$ \\
\hline-.4 & $:$ & $1.000 E+\infty 0$ & $2.789 E+00$ & $5.478 \varepsilon+\infty 0$ & $6.844 E+\infty 0$ & $7.374 E+\infty$ & $7.566 \varepsilon+00$ & $7.6335+\infty 0$ & $7.655 E+00$ & $7.662 \varepsilon+\infty 0$ & $7.664 E+00$ & $7.664 E+\infty 0$ \\
\hline-.2 & : & $1.000 E+00$ & $2.648 E+00$ & $4.923 E+00$ & $6.036 E+00$ & $6.443 E+00$ & $6.580 E+00$ & $6.624 E+\infty 0$ & $6.638 E+\infty 0$ & $6.642 E+00$ & $6.643 E+00$ & $6.643 x+00$ \\
\hline 0 & : & 1. $\cos E=00$ & $2.487 E+00$ & $4.374 E+\infty 00$ & $5.264 E+\infty 0$ & $5.573 E+\infty 0$ & $5.671 E+\infty 0$ & $5.701 E+\infty 0$ & $5.70 \%+\infty 0$ & $5.712 \varepsilon+\infty$ & $5.712 \varepsilon+\infty$ & $5.713 \varepsilon+\infty$ \\
\hline .2 & : & $1.000 E+00$ & $2.316 E+\infty$ & $3.850 E+00$ & $4.548 E+\infty$ & $4.781 E+\infty 0$ & $4.851 E+\infty$ & $4.871 E+\infty 0$ & $4.876 E+\infty$ & $4.878 E+\infty 0$ & $4.878 \varepsilon+\infty$ & $4.87 \% \Leftarrow+00$ \\
\hline .4 & : & $1.000 E+\infty 0$ & $2.140 E+00$ & 3. $362 \varepsilon+\infty$ & 3. $900 E+\infty$ & $4.073 x+\infty 0$ & $4.123 E+\infty$ & 4. $13 \pi E+\infty$ & $4.140 E+\infty$ & $4.141 E+\infty 0$ & $4.141 E+\infty$ & $4.142 E+\infty$ \\
\hline .8 & : & $1.000 E+00$ & $1.799 E+\infty 0$ & $2.522 x+\infty$ & $2.820 \varepsilon+\infty 0$ & $x+\infty$ & $2.935+\infty$ & $2 E+\infty$ & $943 E+00$ & $2.944 E+\infty 0$ & $2.944 E+00$ & $2.944 E+00$ \\
\hline 1 & $:$ & $1.000 E+00$ & $1.6395+00$ & $2.171 E+\infty$ & $2.303 E+\infty 0$ & 2.446 & 2. $463 E+\infty 0$ & $2.468 \varepsilon+00$ & $2.46 \%+\infty$ & $2.46 \%+\infty 0$ & $2.46 \% E+\infty 0$ & $2.46 \%+00$ \\
\hline 1.2 & : & $1.000 E+00$ & $1.489 E+\infty 0$ & $1.86+E+00$ & 2. $009 E+\infty$ & $2.051 E+00$ & $2.062 E+\infty$ & $2.065+00$ & $2.066 E+\infty 0$ & $2.066 \varepsilon+\infty 0$ & $2.0665+00$ & $2.066 E+\infty 0$ \\
\hline 1.4 & : & $1.000 E+\infty$ & $1.350 E+\infty 0$ & $1.597 E+\infty$ & $1.690 E+\infty$ & $1.7165+00$ & $1.723 E+\infty$ & $1.725 x+\infty$ & $1.726 \varepsilon+\infty$ & 1. $726 E+\infty$ & 1. $726 E+\infty 0$ & 1. $726 E+\infty$ \\
\hline 1.6 & : & $1.000 \varepsilon+\infty$ & $1.223 E+00$ & $1.36 \pi+\infty 0$ & $1.420 E+\infty$ & $1.434 E+\infty$ & $1.438 \varepsilon+\infty 0$ & $1.439 \varepsilon+\infty$ & 1. $440 E+00$ & $1.440 \varepsilon+\infty$ & $1.40 E+\infty$ & $1.440 E+\infty 0$ \\
\hline 1.8 & : & $1.000 E+\infty 0$ & $1.106 E+\infty 0$ & $1.16 \%+00$ & $1.19 z E+\infty$ & $1.1900+\infty$ & $1.200 E+\infty$ & $1.200 E+\infty$ & $1.200 E+\infty$ & $1.200 E+\infty 0$ & $1.200 E+\infty 0$ & 1. $200 E+00$ \\
\hline 2 & : & $1.000 E+\infty$ & $1.000 E+\infty 0$ & $1.000 E+\infty$ & $1.000 E+\infty 00$ & $1.000 E \div 00$ & $1.000 E+\infty 0$ & $1.000 E+00$ & $1.000 E+\infty$ & $1.000 E+\infty$ & 1. $000 E+\infty$ & $1.000 E+\infty$ \\
\hline
\end{tabular}


$B 3(2, H, r, w)$ for $r=1$

\begin{tabular}{|c|c|c|c|c|c|c|c|c|c|c|c|c|}
\hline 1 & & 1 & 2 & 4 & 8 & 16 & 32 & 64 & 128 & 256 & 512 & 1024 \\
\hline & : & DOE $+\infty 0$ & $D E E+\infty$ & $\infty 0 \leq+\infty$ & $00 E+\infty$ & $\infty 00 E+\infty$ & & $1.000 E+\infty$ & $1.000 E+\infty$ & & & \\
\hline 1.8 & : & & & & & $\infty O D E+\infty$ & $1.000 E+\infty 0$ & & $\infty 00 E+\infty$ & & 1. $000 E+\infty$ & 1. $000 E+00$ \\
\hline 1.6 & : & $\infty O D E+\infty$ & $D O E+\infty$ & $.000 E+\infty$ & & & & & $1.000 E+\infty$ & & & \\
\hline 1.4 & : & $\infty \infty O E+\infty$ & $\infty O 0 E+\infty$ & & $+\infty$ & $+\infty$ & $0 E+\infty$ & $1.000 E+\infty$ & $1.000 E+\infty$ & $1.000 \leq+\infty$ & $1.000 E+\infty$ & $1.000 E+\infty$ \\
\hline 1.2 & : & OOOE $+\infty$ & $000 E+\infty$ & $00 E+\infty$ & $E+\infty$ & $.000 E+\infty$ & $0 E+\infty$ & & $1.000 E+\infty$ & $1.000 E+\infty$ & 1. $000 E+\infty 0$ & $1.000 E+\infty$ \\
\hline & $:$ & 1. $\infty 00 E+\infty$ & $000 E+\infty$ & $1.000 E+\infty$ & 1. $000 E+\infty$ & 1. $000 E+\infty$ & $1.000 E+\infty 0$ & & $1.000 E+\infty$ & 1. $000 E+00$ & $1.000 E+\infty 0$ & 1. $000 E+00$ \\
\hline .8 & : & & $:+\infty$ & & & & & & $+\infty$ & $+\infty$ & $+\infty$ & \\
\hline .6 & : & & $+\infty$ & & & & & & $-\infty$ & $1.000 E+\infty$ & $1.000 E+\infty$ & $E+\infty$ \\
\hline .4 & : & & $=\infty$ & & & & & & & 1. $000 E+\infty$ & $E+\infty$ & $E+\infty$ \\
\hline .2 & : & 1. $000 E+\infty$ & $.000 E+\infty$ & $E+\infty$ & & & & & & $0 E+\infty O$ & $E+\infty$ & $x+\infty$ \\
\hline 0 & : & $1.000 E+00$ & $.000 E+\infty 0$ & $=+\infty$ & & & & & $+\infty$ & +00 & & $E+\infty$ \\
\hline .2 & : & $1 . \infty$ & $0 E+\infty$ & $+\infty$ & & & $+\infty$ & & 1.000 & 1.000 & 1. $000 E+\infty$ & $1.000 E+\infty$ \\
\hline .4 & : & $+\infty$ & $+\infty$ & $+\infty$ & & & $+\infty$ & $+\infty$ & $+\infty$ & 1. $\infty 00 E+\infty$ & $E+\infty$ & $E+\infty$ \\
\hline .6 & : & 1. $000 E+\infty$ & 1. $000 E+\infty$ & $D O E+\infty$ & & & $\infty$ & & $-\infty$ & 1. $000 E+\infty$ & $E+\infty$ & $x+\infty$ \\
\hline .8 & : & $1.000 E+\infty$ & $1.000 E+\infty$ & $1.000 E+\infty$ & 1. $000 E+\infty$ & $1.000 E+\infty$ & $1.000 E+\infty$ & & 1.000 & $1.000=+\infty$ & $1.000 E+\infty 0$ & 1.00 \\
\hline 1 & : & & & & & & & & 1.000 & 1.000 & $E+\infty$ & $E+\infty$ \\
\hline & & & 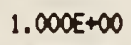 & & & & & & & 1. $000 E+\infty$ & 1. $000 E+\infty$ & 1. $000 E+\infty$ \\
\hline 1. & & 1. $000 E+\infty$ & $1.000 E+\infty$ & $1.000 E+\infty 0$ & & & & & $1.000 E+\infty$ & $1.000 E+\infty$ & $1.000 E+\infty$ & 1. $000 E+\infty$ \\
\hline & & $1.000 E+\infty 0$ & $1.000 E+\infty$ & $1.000 E+\infty$ & 1. $000 E+00$ & 1.000 & $1.000 E+\infty$ & 1.000 & $1.000 E+\infty$ & 1. $000 E+\infty 0$ & 1. $000 E+00$ & 1. $000 E+\infty$ \\
\hline & & & & & & & & & & & 1. $000 E+\infty 0$ & \\
\hline & : & $1.000 E+\infty 0$ & $1.000 E+00$ & $1.000 E+00$ & 1. $000 E+\infty 0$ & 1. $000 E+\infty$ & 1. $000 E+\infty$ & $1.000 E+\infty$ & $1.000 E+\infty$ & 1. $000 E+\infty$ & 1. $000 E+00$ & 1. $000 E+\infty$ \\
\hline
\end{tabular}

$B 3(2, M, r, m)$ for $r=1.01$

\begin{tabular}{|c|c|c|c|c|c|c|c|c|c|c|c|c|}
\hline 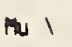 & $H=$ & & & 4 & & 16 & 32 & 64 & 128 & 256 & 512 & \\
\hline 2 & : & 1. $000 \varepsilon+00$ & $2.000 E+00$ & $4.000 E+00$ & $.000 E+00$ & $1.600 E+01$ & 3. $200 E+01$ & $6.400 E+01$ & 1. $280 E+02$ & $2.560 E+02$ & & $1.024 E+103$ \\
\hline 1.8 & : & 1.000E +DON & $300 E+00$ & $771 E+\infty 0$ & $2.545 E+00$ & $3.85 x+00$ & $6.095 E+00$ & $9.968 E+\infty 0$ & $1.66 \%+01$ & $2.836 E+01$ & $4.867 E+11$ & 8. $401 E+01$ \\
\hline 1.6 & : & $D E E+00$ & $105 E+00$ & $234 E+00$ & & $1.650 E+\infty 0$ & 2. $004 E+00$ & $2.532 E+00$ & $3.324 E+00$ & $4.518 \varepsilon+\infty$ & $6.324 E+00$ & \\
\hline 1.4 & : & 1. $000 E+100$ & $037 E+00$ & $.073 E+\infty$ & $=+\infty$ & $1.155 E+100$ & $1.209 E$ & $1.27 \& \propto+\infty 0$ & $.367 E+00$ & $484 E+00$ & $=+\infty$ & $\varepsilon+\infty$ \\
\hline 1.2 & $:$ & & & & & $1.03 x+\infty 0$ & & $1.046 E+00$ & $1.054 E+00$ & $1.063 x+\infty 0$ & $1.073 E+00$ & $1.084 E+00$ \\
\hline & : & 1. $(0 j) E+0 i j$ & $000 E+00$ & 1. $000 E+\infty$ & & $1.000 E+\infty 0$ & $1.000 E+00$ & $1.000 E+00$ & 1. $000 E+\infty 0$ & 1. $000 E+00$ & 1. $000 E+00$ & 1. $\infty 00 E+\infty$ \\
\hline 8 & : & +00 & -01 & -01 & 01 & $|x-0|$ & .01 & $9.905 x-01$ & $9.90 Z E-01$ & E -01 & $9.897 E-01$ & $E-01$ \\
\hline 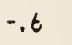 & $:$ & & & & & -01 & 100 & 01 & $26 E-01$ & $\varepsilon-01$ & $E-01$ & $9.884 E-01$ \\
\hline & $:$ & & & & & & & 9.808 & $8 E-01$ & $E-01$ & $E-01$ & $9.827 E-01$ \\
\hline .2 & : & & 01 & & & $96 E-01$ & 9.805 & $9.895 E$ & EE -01 & $E-01$ & $E-01$ & $9.894 E-01$ \\
\hline 0 & $:$ & & & & & $9.904 E-01$ & 9.903 & 9.90 & & & $E-01$ & $E-01$ \\
\hline .2 & : & $-x$ & -01 & & & $9.913 E-01$ & 9.91 & -01 & $2 E-01$ & $x=01$ & $12 E-01$ & $9.912 \mathbf{E}-01$ \\
\hline .4 & : & $+\infty 0$ & -01 & & -01 & $92 x-01$ & $9.922 \mathrm{E}-01$ & $.92 x-01$ & $.922 \varepsilon-01$ & $.922 E-01$ & $9.92 x-01$ & $9.922 E-01$ \\
\hline .6 & $:$ & & & & & $32 E-01$ & & & -01 & $E-01$ & $31 E-01$ & $9.931 E-01$ \\
\hline .8 & : & 1. $000 E+100$ & -01 & & & $9.941 E-01$ & & & $E-01$ & $E-01$ & $E-01$ & $E-01$ \\
\hline 1 & $:$ & 1.00 & & & & & & & & -01 & $E-01$ & $51 E-01$ \\
\hline & & & & & & & & & $1 E-01$ & E-01 & $9.961 E-01$ & $9.961 E-01$ \\
\hline & & 1. $000 E+00$ & & & & $9.970 E-01$ & $9.970 E-01$ & & $9.970 E-01$ & $9.970 E-01$ & $9.970 E-01$ & $9.970 E-01$ \\
\hline 1. & & $1.000 E+\infty 0$ & $9.985 x-01$ & & $9.981 E-01$ & $9.980 E=01$ & $9.980 E-01$ & $9.980 E-01$ & $9.980 E-01$ & $9.980 E-01$ & $9.980 E-01$ & $9.980 \leq-01$ \\
\hline & & $1.000 E+00$ & $9.992 E-01$ & & $9.990 E-01$ & $9.990 E-01$ & $9.990 E-01$ & $9.990 E-01$ & $9.990 E-01$ & $9.990 E-01$ & $9.990 E-01$ & $9.990 E-01$ \\
\hline & : & 1. $000 E+\infty$ & $1.000 E+\infty$ & 1. $000 E+\infty$ & $1.000 E+\infty$ & $1.000 \xi+\infty 0$ & $1.000 E+\infty 0$ & $1.000 E+\infty 0$ & $1.000 E+\infty$ & 1. $000 E+\infty$ & 1. $000 E+\infty$ & $1.000 E+\infty 0$ \\
\hline
\end{tabular}


$B 3(2, H, r$, wu $)$ for $r=1.1$

\begin{tabular}{|c|c|c|c|c|c|c|c|c|c|c|c|c|}
\hline 41 & $r$ & 1 & 2 & 4 & 8 & 16 & 32 & 64 & 128 & 256 & 512 & 1024 \\
\hline & . & & & & & & & & & & & \\
\hline & : & $1.000 E+\infty 0$ & $1.494 E+\infty 0$ & $2.286 E+\infty 0$ & $3.604 E+00$ & $5.845 E+\infty$ & $9.700 E+00$ & $1.637 \mathrm{E}+01$ & & $4.800 E+01$ & & \\
\hline & : & $1.000 E+00$ & $1.11 \%+\infty$ & $.243=+00$ & $1.385 E+\infty 0$ & $1.556 E+\infty$ & 1. $\pi \mathbb{2}+\infty$ & $2.050 E+\infty 0$ & $2.412 E+\infty$ & $2.88 \pi t+\infty$ & $3.51 \geq \pm+\infty$ & 4.33 \\
\hline & : & $1.000 E+00$ & $1.044 E+00$ & $.08 \approx E+\infty 0$ & $1.11 \pi E+\infty 0$ & $1.153 E+\infty$ & 1. $191 E+00$ & 1. $234 E+00$ & $1.28 \approx+00$ & $1.336 E+00$ & $1.390<+\infty$ & $1.470 \varepsilon+\infty 0$ \\
\hline & : & $000 E+00$ & $1.000 E+\infty 0$ & $.000 E+\infty 0$ & $.000 E+\infty 00$ & $1.000 E+00$ & $1.000 E+00$ & 1. $000 E+\infty$ & $1.000 E+\infty 0$ & $1.000 E+\infty$ & $1.000 E+00$ & $1.000 E+00$ \\
\hline & : & $000 E+\infty$ & $E-01$ & $=-01$ & $268 c-01$ & $9.20 x-01$ & $9.160 E-01$ & $x=01$ & $1 E-01$ & $6 E-01$ & $9.00 \pi x-01$ & $9.077 E-01$ \\
\hline & : & $:+\infty$ & $10 \varepsilon-01$ & $E-01$ & $193 E-01$ & $9.145 x-01$ & $19-01$ & $5-01$ & $E-01$ & $O E-01$ & $9.08 \pi-01$ & $9.00 z E-01$ \\
\hline & : & $E+\infty$ & $9.476 E-01$ & $\varepsilon-01$ & $18 \% E-01$ & $56 E-01$ & $9.14 x-01$ & $5-01$ & $E-01$ & $9-01$ & $9.128 E-01$ & $9.12 \alpha E-01$ \\
\hline & : & . $000 E+\infty 00$ & $9.4725-01$ & $=-01$ & $223 E-01$ & $9.200 E-01$ & $22 E-01$ & $x-01$ & $E-01$ & $\pi-01$ & $9.186 E-01$ & $9.186 E-01$ \\
\hline .2 & : & $1.000 E+00$ & $9.4905-01$ & $E-01$ & $9.27 \pi E-01$ & $9.261 E-01$ & $9.256 E-01$ & $4 E-01$ & $E-01$ & $3 E-01$ & $9.253 E-01$ & $E-01$ \\
\hline & $:$ & $1.000 E+00$ & $9.523 E-01$ & $x-01$ & $9.342 E-01$ & $9.331 E-01$ & $E-01$ & $6 E-01$ & $E-01$ & $6 E-01$ & $9.326 E-01$ & $E-01$ \\
\hline & $:$ & $1.000 E+00$ & $9.5665-01$ & -01 & $9.415 E-01$ & $9.406 E-01$ & 9.40 & $3 E-01$ & $E-01$ & $3 E-01$ & $9.403 E-01$ & $\varepsilon-01$ \\
\hline & : & 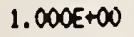 & $9.674 E-01$ & $x-01$ & $9.572 E-01$ & $9.56 \pi-01$ & $9.5666-01$ & $9.565 \varepsilon-01$ & $9.565 E-01$ & $9.565 E-01$ & $9.565 E-01$ & $9.565 E-01$ \\
\hline & : & 1.000E+00 & $9.735 x-01$ & $9.670 E-01$ & $9.655 x-01$ & $9.651 E-01$ & $9.650 E-01$ & $9.6495-01$ & $9.649 E-01$ & $9.6495-01$ & $9.64 \div=-6$ & $0.64 \%-01$ \\
\hline & : & 1. $000 E+00$ & $9.798 E-01$ & $9.751 E-01$ & $0.73 \% E-01$ & $9.736 E-01$ & $9.735 E-01$ & $9.735 x-01$ & $9.735 E-01$ & $9.735 E-01$ & $\because 35 E-01$ & $9.735 x-01$ \\
\hline & $:$ & 1.000E+ION & $9.864 E-01$ & $9.83 X-01$ & $9.825=01$ & $9.823 E-01$ & $9.82 x-01$ & 9.:Z:E-01 & $9.822 x-01$ & $9.822 \leq-01$ & $9.822 E-01$ & $9.822 \mathbf{x}-01$ \\
\hline & : & 1. $0000 E+\infty j$ & $9.931 E-01$ & $9.916 E-01$ & $9.91 Z E-0.1$ & ․ : 15-01 & $9.911 E-01$ & $9.911 E-01$ & $9.911 E-01$ & $9.911 E-01$ & $9.911 E-01$ & $9.911 E-01$ \\
\hline & : & 1. $\cos E=100$ & 1. $000 E+\infty$ & 1. MUE- & $1.000 E+100$ & $1.000 E+\infty$ & $1.000 E+00$ & $1.000=00$ & $1.000 E+\infty 0$ & $1.000 E+00$ & $1.000 E+\infty$ & $1.000 E+00$ \\
\hline
\end{tabular}

$B 3(2, \mu, r, w)$ for $r=2$

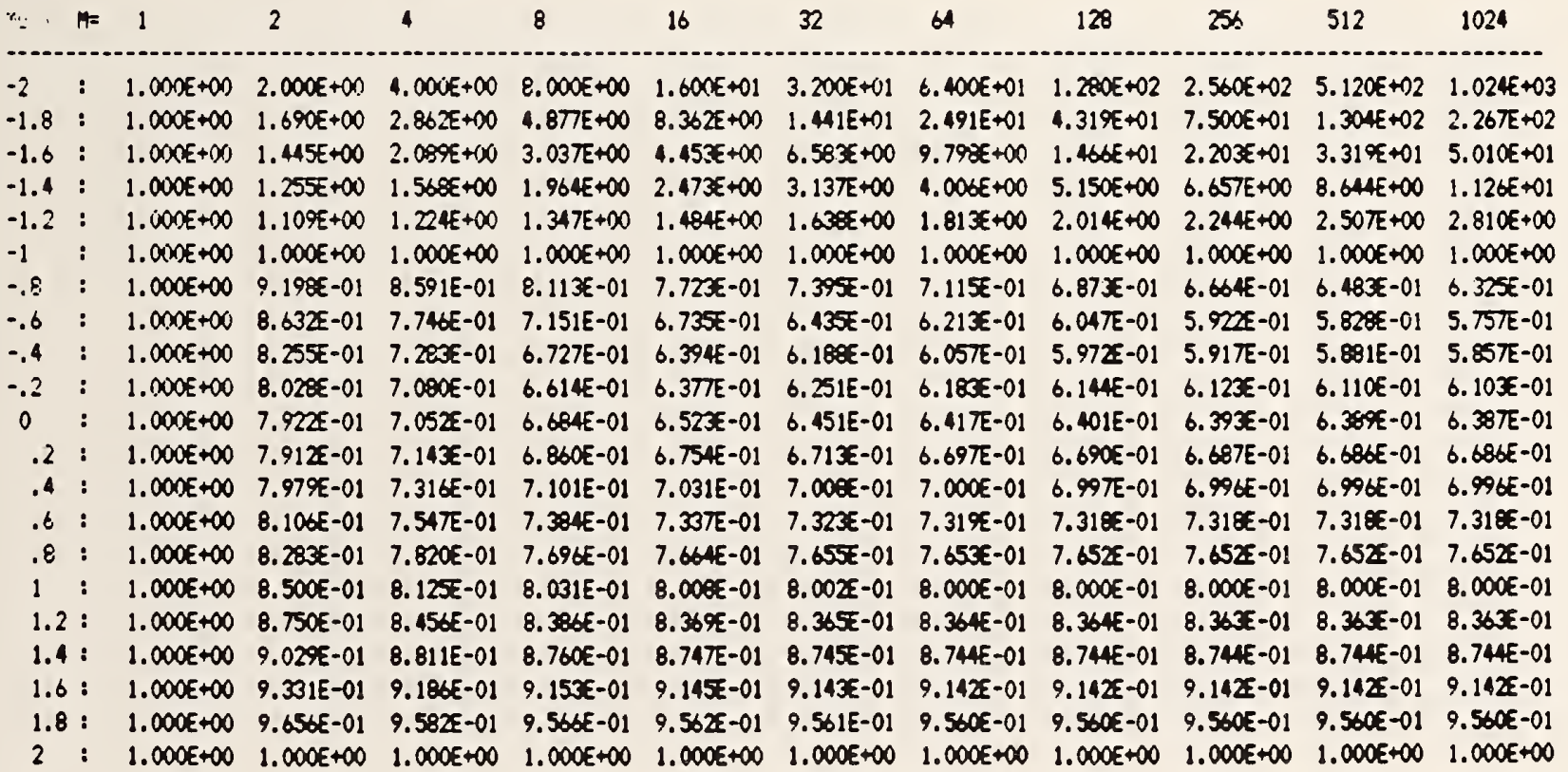


$B 3(2, n, r$, ,us $)$ for $r=4$

\begin{tabular}{|c|c|c|c|c|c|c|c|c|c|c|c|c|}
\hline tus 1 & 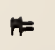 & 1 & 2 & 4 & 8 & 16 & 32 & 4 & 128 & 256 & 512 & 1024 \\
\hline-2 & : & $1.000 E+00$ & $000 E+\infty 0$ & $.000 E+\infty$ & $0,00<+\infty$ & $600 E+01$ & $200 E+01$ & $6.400 E+01$ & $1.200 \leq+02$ & $2.560<+02$ & $5.120 E+02$ & $1.024 E+03$ \\
\hline-1.8 & : & $000 E+\infty$ & $.728 E+\infty 0$ & $2.986 E+\infty 0$ & $170 E+\infty$ & 964E+DO & & $705 \bar{x}+01$ & 4.704E+01 & $8.185 x+01$ & $1.425 E+02$ & $2.490 E+02$ \\
\hline 1.6 & : & $000 E+\infty$ & $1.494 E+00$ & $2.232 E+00$ & $11 E+\infty$ & $5.014 E+\infty$ & $544 E+\infty 0$ & & $1.71 \varepsilon E+01$ & $2.597 E+01$ & $.92 \%+01$ & $5.948 E+01$ \\
\hline 1.4 & : & $\infty 00 E+\infty$ & $1.296 \varepsilon+00$ & $1.678 \varepsilon+\infty$ & $2.174 E+\infty$ & $2.823 E+\infty$ & $3.676 E+\infty$ & 4. $79 \% E+\infty$ & $6.279 E+\infty$ & $8.232 x+\infty$ & $.081 E+01$ & $1.420 E+01$ \\
\hline 1.2 & : & $1.000 \varepsilon+\infty 0$ & $1.13 z+00$ & $1.27 \%++\infty$ & $1.44 E+\infty 0$ & $1.631 E+\infty$ & $1.844 E+\infty 0$ & $2.008<+\infty$ & $2.36 \pi+\infty$ & $2.680 \div+\infty 0$ & $3.05 \pi+\infty$ & 3. $480 E+\infty 0$ \\
\hline & : & $000 E+00$ & $:+\infty$ & $1.000 E+\infty$ & $1.000 E+\infty 0$ & $1.000 E+\infty$ & $1.000 E+\infty$ & $1.000 E+\infty$ & $1.000 E+\infty$ & $.000 E+\infty$ & $1.000 E+\infty$ & $1.000 E+\infty$ \\
\hline .8 & : & $1.000 E+00$ & 01 & $8.123 E-01$ & $7.420 E-01$ & $6.824 E-01$ & $E-01$ & $5.87 x-01$ & $5.48 \% E-01$ & $5.157 \mathrm{E}-01$ & $4.868 E-01$ & $4.616 E-01$ \\
\hline .6 & : & $1.000 E+00$ & & & -01 & $5.370 E-01$ & -01 & $E-01$ & $4.237 E-01$ & $4.028 E-01$ & $3.870 E-01$ & 3. $750 E-01$ \\
\hline .4 & : & $1.000 E+00$ & 01 & 6.2565 & $E-01$ & $4.8495-01$ & 4.502 & & $4.12 z z-01$ & $4.03 z=01$ & $3.968 x-01$ & $3.926 E-01$ \\
\hline .2 & : & $1.000 E+00$ & 01 & 5.946 & -01 & 4. 824E-01 & 4.607 & $E-01$ & $E-01$ & $4.378 E-01$ & $4.355-01$ & $4.34 \geq-01$ \\
\hline 0 & $:$ & $1.000 E+\infty$ & 7.16 & 5.898E-01 & 5. $323 E-01$ & 5. $05 / E-01$ & $4.932 \leq-01$ & $71 E-01$ & $4.841 E-01$ & $826 E-01$ & 4.819E-01 & $4.815 E-01$ \\
\hline .2 & : & $1.000 E+00$ & 7.14 & $6.030 E-01$ & 5. $595 E-01$ & $5.422 x-01$ & 5.351E-01 & $5.320 x-01$ & $5.310 E-01$ & $305 E-01$ & 5. $303 E-01$ & OZE-01 \\
\hline .4 & : & $1.000 \varepsilon+\infty$ & 7.232 & $6.285 x-01$ & $5.96 x-01$ & $5.851 E-01$ & 5.812 & $E-01$ & $E-01$ & $5.79 x-01$ & $5.791 E-01$ & $5.791 E-01$ \\
\hline .6 & : & $1.000 E+00$ & $7.40 x$ & $6.621 E-01$ & 6. 384E-01 & 6.31 జE-01 & 6.291 & $6.284 E-01$ & $6.28 \simeq \leq-01$ & 6. $281 E-01$ & $6.281 E-01$ & $6.281 E-01$ \\
\hline .8 & : & $1.000 E+00$ & 7.65 & & 6. $80 \% E-01$ & $6.794-01$ & 6.779 & 6. T/SE-01 & $6.775-01$ & $6.774 E-01$ & $6.774 E-01$ & $6.774 E-01$ \\
\hline 1 & : & $1.000 E+00$ & $7.955 E-01$ & $7.443 E-01$ & $7.315 E-01$ & 7.283E-01 & $E-01$ & $7.273 E-01$ & $7.273 E-01$ & $7.273 E-01$ & $7.273 \mathrm{E}-01$ & $7.273 E-01$ \\
\hline 1.2 & & $1.000 E+\infty$ & $8.2905-01$ & $7.903 E-01$ & $7.810 E-01$ & $7.788 c-01$ & $7.782 \leq-01$ & $7.781 E-01$ & $7.781 E-01$ & $7.781 E-01$ & $7.781 E-01$ & $7.781 E-01$ \\
\hline 1. & & $1.000 E+\infty$ & $8.679-01$ & 8. $389 x-01$ & 8. 323E-01 & 8.307E-01 & $8.303 E-01$ & $8.303 E-01$ & $8.30 \simeq-01$ & $8.302 E-01$ & $8.30 x-01$ & $8.30<-01$ \\
\hline & & 1.000E+C10 & $9.090 E-01$ & 8.899-01 & $8.856 E-01$ & $8.846 E-01$ & $8.843 E-01$ & $8.843 E-01$ & $8.843 E-01$ & $8.843 E-01$ & $8.843 x-01$ & $8.843 E-01$ \\
\hline 1.8 & & 1. OOOE +OOD & & & & & & $9.407 E-01$ & $9.407 \mathrm{E}-01$ & & $9.40 \pi-01$ & $9.40 \pi-01$ \\
\hline 2 & : & 1. $\operatorname{coc}=5+\infty 0$ & $1.000 E+\infty 0$ & $1.000 E+\infty$ & $1.000 E+\infty$ & $1.000 \leq+\infty$ & $1.000 E+\infty$ & $1.000 E+00$ & $1.000 \varepsilon+00$ & $1.000 E+\infty 0$ & $1.000 E+\infty$ & $1.000 E+00$ \\
\hline
\end{tabular}

$B 3(2, n, r, w)$ for $r=8$

\begin{tabular}{|c|c|c|c|c|c|c|c|c|c|c|c|c|}
\hline An 1 & $k=$ & 1 & 2 & 4 & 8 & 16 & 32 & 64 & 128 & 256 & 512 & 024 \\
\hline & : & $1.000 E+00$ & $O C O E+\infty O$ & $4.000 E+00$ & $8.000 E+00$ & $1.600 E+01$ & $3.200 E+01$ & 6. $400 E+01$ & $1.280 E+02$ & $2.560 E+02$ & $5.120 E+02$ & $1.024 E+03$ \\
\hline 1.8 & : & 1.000E+00 & $1.73 \pi E+\infty 0$ & $3.01 \%+\infty 0$ & $5.247 E+\infty 0$ & $9.126 E+00$ & $.5800 \varepsilon+01$ & $2.763 E+01$ & $4.80 \% \varepsilon+01$ & $8.371 E+01$ & $1.457 E+02$ & $2.537 E+02$ \\
\hline 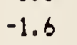 & : & $\infty, 0 E+\infty 0$ & $1.509 E+00$ & $2.276 E+00$ & $3.436 E+00$ & $5.191 E+\infty 0$ & $7.850 E+\infty 0$ & $.1885+01$ & $1.798 E+01$ & $.723 E+01$ & $4.1265+01$ & \\
\hline 1.4 & : & $1.000 E+100$ & $1.311 E+\infty 0$ & $1.71 \pi+\infty 0$ & $2.251 E+00$ & $2.95 x+00$ & $3.877 E+\infty 0$ & $5.096 E+00$ & $6.704 E+00$ & $3.825 E+00$ & $1.16 \mathbb{E}+01$ & $1.532 E+01$ \\
\hline 1.2 & : & $1.000 E+\infty 0$ & $1.142 E+00$ & 1. $30 \geq E+\infty$ & $1.484 E+00$ & $1.693 E+00$ & $1.932 x+00$ & $2.206 E+00$ & $2.520 E+00$ & $2.882 E+00$ & $3.296 \times+\infty 0$ & $3.773 x+\infty 0$ \\
\hline & : & $000 E+100$ & $1.000 E+00$ & $1.000 E+00$ & $1.000 E+\infty$ & $1.000 E+00$ & $1.000 E+\infty 0$ & $1.000 \varepsilon+\infty 0$ & $1.000 E+\infty 0$ & $1.000 E+00$ & $1.000 \varepsilon+00$ & $1.000 E+00$ \\
\hline .8 & : & $1.000 E+00$ & $8.851 E-01$ & $7.835-01$ & $7.059-01$ & $6.351 E-01$ & 5. $73 \%-01$ & $5.20<c-01$ & $4.747 E-01$ & $4.346 E-01$ & $3.99 \pi-01$ & $3.69 x x-01$ \\
\hline 6 & : & 1. $000 E+\infty$ & $7.965 x-01$ & $6.48 \%=-01$ & 5.405E-01 & 4. $600 E-01$ & $3.996 E-01$ & $3.541 E-01$ & 3.19\%E-01 & $2.938 x-01$ & $2.741 E-01$ & $2.59 x-01$ \\
\hline .4 & : & $1.000 E+00$ & $7.331 E-01$ & $5.666 E-01$ & $4.614 E-01$ & $3.93 \%-01$ & $3.50 x=-01$ & $3.216 E-01$ & $3.0 z<E-01$ & 2.904E-01 & $2.823 \varepsilon-01$ & $2.76 \%-01$ \\
\hline .2 & $:$ & $1.000 E+00$ & $6.931 E-01$ & $34 E-01$ & 4.390E-01 & $3.896 E-01$ & $3.620 E-01$ & $=-01$ & 3.374E-01 & $323 E-01$ & $E-01$ & $3.2 \pi \pi-01$ \\
\hline 0 & $:$ & $1.000 E+00$ & $6.73 x x-01$ & -01 & 4.52 & $E-01$ & -01 & 3. $952 E-01$ & $13 E-01$ & $93 E-01$ & $3.884 E-01$ & 3.878 \\
\hline .2 & : & & & & & $4.6 x$ & -01 & $4.534 E-01$ & $18 E-01$ & $E-01$ & $E-01$ & 4.507 \\
\hline .4 & : & $1.000 E+00$ & $6.838 E-01$ & -01 & $5.34 \pi E-01$ & $5.212 E-01$ & 5. $164 E-01$ & $46 E-01$ & $5.140 E-01$ & $3 \pi-01$ & $5.136 E-01$ & $5.1365-01$ \\
\hline .6 & : & $1.000 E+00$ & $7.061 E-01$ & $5 E-01$ & $5.878 E-01$ & $5.793 \varepsilon-01$ & $5.76 \pi \mathrm{E}-01$ & 5. $75 \%-01$ & 5. $\pi(56-01$ & 5. $756 E-01$ & 5. $755 x-01$ & 5. $75 x-01$ \\
\hline .8 & $:$ & $1.000 E+\infty$ & $7.361 E-01$ & -01 & $6.436 E-01$ & $6.382 x-01$ & 6.367 & $6.363 E-01$ & $6.36 x-01$ & $6.361 E-01$ & $6.361 E-01$ & $6.361 E-01$ \\
\hline 1 & : & $1.000 E+00$ & $7.717 E-01$ & $\pi t E-01$ & 7.004E-01 & $6.968 E-01$ & $6.95 \%-01$ & $6.957 E-01$ & $6.957 E-01$ & 6. 95ाE-01 & $6.95 \pi-01$ & $6.957 \mathrm{E}-01$ \\
\hline 1.2 & & $1.000 E+\infty$ & $8.115 E-01$ & $7.600 E-01$ & $7.578<-01$ & $7.554 E-01$ & $7.548 E-01$ & $7.546 E-01$ & $7.546 E-01$ & $7.546 E-01$ & $7.546 E-01$ & $7.546 E-01$ \\
\hline & & $1.000 \varepsilon+\infty$ & $8.546 E-01$ & $8.230 E-01$ & 8.159-01 & 8.14ZE-01 & $8.130<-01$ & $8.13 \pi-01$ & $8.13 \pi-01$ & 8. 137E-01 & $8.13 \pi-01$ & 8. $13 \pi-01$ \\
\hline 1. & & $1.000 E+\infty$ & $9.005 E-01$ & $8.79 \pi-01$ & 8.751E-01 & $8.741 E-01$ & 8. $7385-01$ & $8.73 x E-01$ & 8. $737 E-01$ & $8.73 \pi-01$ & $8.737 E-01$ & $8.737 E-01$ \\
\hline & & $1.000 E+\infty 0$ & $9.499 E-01$ & & & & $9.356 E-01$ & $9.3566-01$ & $9.356 E-01$ & & & \\
\hline 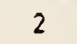 & : & $1.000 E+\infty 0$ & 1.000E+00 & $1.000 E+\infty$ & $1.000 E+\infty$ & $1.000 E+00$ & $1.000 E+\infty$ & $1.000 E+\infty$ & $1.000 E+\infty$ & $1.000 E+\infty$ & $1.000 E+\infty$ & $1.000 E+\infty$ \\
\hline
\end{tabular}


$B 3(2, H, r$, wu $)$ for $r=16$

$-1.8$

$-1.8$

$-1.6$

$-1.2$

$-1$

$-.8$

$-.6$

$-.4$

$-.2$

0

.2

.4

.6

.8

1

1.2

1.4 :

1.6

1.8 :

2 $\begin{array}{llllllllllllllllll}1.000 E+\infty 00 & 2.000 E+\infty 0 & 4.000 E+\infty & 8.000 E+\infty 0 & 1.600 E+01 & 3.200 E+01 & 6.400 E+01 & 1.200 E+02 & 2.560 E+02 & 5.120 E+02 & 1.024 E+03\end{array}$ $\begin{array}{lllllllllllll}1.000 E+\infty 0 & 1.740 E+\infty & 3.028 E+\infty & 5.26 \%+\infty & 9.171 E+\infty & 1.596 E+01 & 2.77 \%+01 & 4.836 E+01 & 8.424 E+01 & 1.467 E+02 & 2.553 E+02\end{array}$

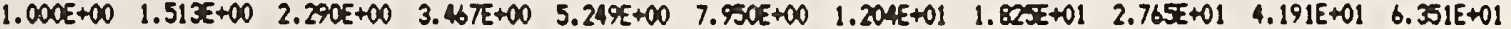
$\begin{array}{llllllllllll}1.000 E+\infty & 1.316 E+\infty & 1.732 E+\infty & 2.260 E+\infty & 3.001 E+\infty & 3.953 E+\infty & 5.20 \%+\infty & 6.865 E+\infty & 9.051 E+\infty & 1.194 E+01 & 1.574 E+\infty 1\end{array}$ $\begin{array}{lllllllllll}1.000 E+\infty & 1.146 E+\infty & 1.312 E+\infty & 1.502 E+\infty & 1.720 E+\infty & 1.970 E+\infty & 2.25 \pi E+\infty & 2.58 \pi E+\infty & 2.966 E+\infty & 3.401 E+\infty & 3.901 E+\infty\end{array}$ $\begin{array}{llllllllllllllllll}1.000 E+\infty & 1.000 E+\infty & 1.000 E+\infty & 1.000 E+\infty & 1.000 E+\infty & 1.000 E+\infty & 1.000 E+\infty & 1.000 E+\infty & 1.000 E+\infty & 1.000 E+\infty & 1.000 E+\infty\end{array}$ $1.000 E+\infty 0$ 8.788E-01 $7.752 E-01 \quad 6.860 E-01 \quad 6.08 \% E-01 \quad 5.420 E-01 \quad 4.83 \%-01 \quad 4.334 E-01 \quad 3.894 E-01 \quad 3.511 E-01 \quad 3.178 E-01$ $1.000 E+\infty 0 \quad 7.326 E-01 \quad 6.220 E-01 \quad 5.026 E-01 \quad 4.132 E-01 \quad 3.45 E E-01 \quad 2.949 E-01 \quad 2.564 E-01 \quad 2.273 E-01 \quad 2.052 E-01 \quad 1.835 E-01$ 1. $000 E+\infty 0 \quad 7.119 E-01 \quad 5.28 \pi E-01 \quad 4.11 Z E-01 \quad 3.350 E-01 \quad 2.853 E-01 \quad 2.52 \pi E-01 \quad 2.313 E-01 \quad 2.172 E-01 \quad 2.078 E-01 \quad 2.017 E-01$

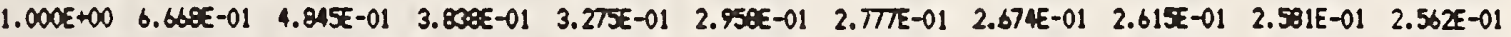
$1.000 E+\infty 00 \quad 6.455 E-01 \quad 4.725 E-01 \quad 3.992 E-01 \quad 3.612 E-01 \quad 3.426 E-01 \quad 3.305 E-01 \quad 3.290 E-01 \quad 3.26 \pi E-01 \quad 3.256 E-01 \quad 3.250 E-01$ $1.000 E+\infty 0 \quad 6.446 E-01 \quad 5.001 E-01 \quad 4.410 E-01 \quad 4.167 E-01 \quad 4.065 E-01 \quad 4.021 E-01 \quad 4.003 E-01 \quad 3.995 E-01 \quad 3.991 E-01 \quad 3.990 E-01$ $\begin{array}{llllllllllll}1.000 E+\infty & 6.597 E-01 & 5.395 E-01 & 4.970 E-01 & 4.81 \% E-01 & 4.7 E E-01 & 4.745 E-01 & 4.73 E E-01 & 4.735 E-01 & 4.734 E-01 & 4.734 E-01\end{array}$ $1.000 E+00 \quad 6.865 E-01 \quad 5.891 E-01 \quad 5.591 E-01 \quad 5.499 E-01 \quad 5.469 E-01 \quad 5.460 E-01 \quad 5.456 E-01 \quad 5.45 T E-01 \quad 5.456 E-01 \quad 5.456 E-01$ $\begin{array}{llllllllllll}1.000 E+\infty & 7.210 E-01 & 6.43 \% E-01 & 6.22 \pi E-01 & 6.16 \% E-01 & 6.154 E-01 & 6.149 E-01 & 6.148 E-01 & 6.148 E-01 & 6.148 E-01 & 6.148 E-01\end{array}$ $\begin{array}{lllllllllllll}1.000 E+00 & 7.606 E-01 & 7.008 E-01 & 6.858 E-01 & 6.821 E-01 & 6.812 E-01 & 6.809 E-01 & 6.809 E-01 & 6.009 E-01 & 6.809 E-01 & 6.809 E-01\end{array}$

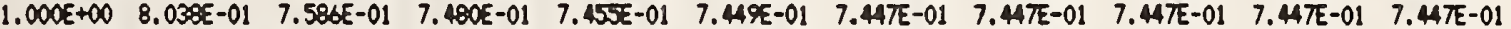
$\begin{array}{llllllllllll}1.000 E+00 & 8.495 E-01 & 8.169 E-01 & 8.096 E-01 & 8.079 E-01 & 8.075 E-01 & 8.074 E-01 & 8.074 E-01 & 8.074 E-01 & 8.074 E-01 & 8.074 E-01\end{array}$ $\begin{array}{lllllllllllll}1.000 E+\infty 0 & 8.974 E-01 & 8.762 E-01 & 8.715 E-01 & 8.704 E-01 & 8.701 E-01 & 8.701 E-01 & 8.701 E-01 & 8.701 E-01 & 8.701 E-01 & 8.701 E-01\end{array}$ $1.000 E+00$ 9.475E-01 $9.370 E-01 \quad 9.346 E-01 \quad 9.341 E-01 \quad 9.340 E-01 \quad 9.339 E-01 \quad 9.339 E-01 \quad 9.339 E-01 \quad 9.339 E-01 \quad 9.339 E-01$ $\begin{array}{llllllllllll}1.000 E+\infty & 1.000 E+\infty & 1.000 E+\infty & 1.000 E+\infty & 1.000 E+\infty & 1.000 E+\infty & 1.000 E+\infty & 1.000 E+\infty & 1.000 E+\infty & 1.000 E+\infty & 1.000 E+\infty\end{array}$

$B 3(2, M, r, \ldots)$ for $r=32$

\begin{tabular}{|c|c|c|c|c|c|c|c|c|c|c|c|c|}
\hline 41 & & 1 & 2 & 4 & 8 & 16 & 32 & 64 & 128 & 256 & 512 & 1024 \\
\hline & : & & 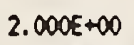 & & & & & & & & & \\
\hline .8 & : & $1.000 E+\infty 0$ & $741 E+\infty$ & & & & & & & & & \\
\hline .6 & : & 1.00DE+00 & $.515 E+\infty 0$ & $2955+\infty 0$ & $477 E+\infty$ & $5.26 \%+\infty$ & $7.984 E+\infty 0$ & $10 E+01$ & $1.834 E+01$ & . $779 E+01$ & $4.212 E+01$ & $6.384 E+01$ \\
\hline .4 & : & $1.000 E+\infty$ & $.31 \varepsilon \varepsilon+\infty 0$ & $738 x+\infty 0$ & $291 E+\infty$ & $3.020 E+00$ & $3.982 E+00$ & & $6.92 \pi+\infty 0$ & $9.13 \pi+\infty$ & $1.205 E+01$ & $590 E+01$ \\
\hline .2 & : & 1. $000 E+00$ & $14 \pi E+\infty$ & $+\infty$ & $\alpha E+\infty$ & 1.7325 & $1.987 E+\infty 0$ & $\alpha E+\infty$ & $2.616 E+\infty 0$ & $3.003 E+\infty$ & 3. $44 \pi+\infty$ & $95 \pi E+\infty$ \\
\hline & : & DOUE +100 & $+\infty$ & 1.000 & $+\infty$ & $\infty$ & $1.000 E+\infty$ & & $1.000 E+\infty$ & $.000 E+\infty 0$ & $.000 E+\infty 0$ & $.000 E+00$ \\
\hline & : & $C O O E+\infty$ & $753 E-01$ & .677 & & 01 & $.240 E-01$ & & $4.100 E-01$ & $.639 E-01$ & $.237 E-01$ & $.887 E-01$ \\
\hline & : & $1.000 E+\infty 0$ & $7.738 \varepsilon-01$ & 6.05 & & -01 & & & & $1.855 E-01$ & $.61 \%-01$ & $1.41 \mathrm{E}-01$ \\
\hline & : & $1.000 E+\infty$ & -01 & & & 1 & & & & & $7 \mathfrak{x}-01$ & $5 E-01$ \\
\hline 2 & : & & & 1 & & & & & & & $=-01$ & \\
\hline 0 & : & $O E+\infty$ & 01 & 01 & & & 2.99 & & 2.84 & $816 E-01$ & $2.804 E-01$ & $2.79 \circ E-01$ \\
\hline .2 & : & $+\infty$ & -01 & 4.71 & -01 & & & & -01 & $34 E-01$ & $3.630 E-01$ & $.628 \varepsilon-01$ \\
\hline .4 & : & $0 E+00$ & $40 E$ & & & & & & & -01 & $9 E-01$ & E-01 \\
\hline .6 & : & $1.000 E+\infty 0$ & $74 \pi$ & & -01 & & & & & & $76 E-01$ & $:-01$ \\
\hline .8 & : & $1.000 E+\infty$ & $128 E-01$ & 6.33 & & 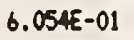 & 6. $038 E-01$ & & $6.0325-$ & $.032 x-01$ & $.031 E-01$ & $6.031 E-01$ \\
\hline 1 & : & & & & -01 & & & & & $E-01$ & $3 T E-01$ & $E-01$ \\
\hline & & & & & $=01$ & & & & & $7.404 E-01$ & $7.404 E-01$ & $7.404 E-01$ \\
\hline & & $1.000 E+00$ & $8.475 E-01$ & & & & 8.051E-01 & & 8. $050 E-01$ & $8.04 \%-01$ & $.049-01$ & 8.049E-01 \\
\hline & & $1.000 E+\infty$ & $8.964 E-01$ & $8.750 E-01$ & $8.702 E-01$ & $8.691 E-01$ & 8.68\%-01 & $88 E-01$ & $8.689 E-01$ & $8.688 E-01$ & $8.688 E-01$ & 8. $688 E-01$ \\
\hline & & $1.000 \varepsilon+\infty$ & $9.471 E-01$ & $9.365 E-01$ & $9.341 E-01$ & $9.336 \mathrm{E}$ & 9.334E-01 & & 9.334E-01 & $9.334 E-01$ & 9.334E-01 & 9. 334E-01 \\
\hline 2 & : & $1.000 E+\infty$ & 1. $000 E+\infty$ & $1.000 E+\infty$ & $1.000 E+\infty 0$ & $1.000 E+\infty 0$ & $1.000 E+\infty$ & $1.000 E+\infty$ & $1.000 E+\infty 0$ & $1.000 E+\infty 0$ & $1.000 E+\infty 0$ & $1.000 E+00$ \\
\hline
\end{tabular}


$B 3(2, H, r, \ldots)$ for $r=64$

\begin{tabular}{|c|c|c|c|c|c|c|c|c|c|c|c|c|}
\hline nu 1 & $\omega^{2}$ & 1 & 2 & 4 & 8 & 16 & 32 & 64 & 128 & 256 & 512 & 1024 \\
\hline$?$ & $:$ & $1.000 E+\infty 0$ & $2.000<+00$ & $4.000 E+00$ & $8.000 E+\infty 0$ & $1.600 E+01$ & $3.200 E+01$ & 6. $400 E+01$ & $.220 E+02$ & $2.560 E+02$ & $5.120 \varepsilon+02$ & $.024 E+03$ \\
\hline & $:$ & $1.000 E+\infty 0$ & $1.741 E+00$ & $3.031 E+00$ & 5. 277E↔ & $9.180 E+\infty 0$ & $1.600 E+01$ & $2.785=+01$ & $4.84 \%+01$ & 8. $43 E+01$ & $1.470 \leq+02$ & 2. $59 E+02$ \\
\hline & $:$ & $1.0005+00$ & $1.515=00$ & $2.2975+00$ & $3.481 E+\infty 0$ & $5.275+00$ & $7.995+\infty$ & $1.212 \varepsilon+01$ & $1.836 E+01$ & 2. $724 E+01$ & $4.219 E+01$ & $6.395 E+01$ \\
\hline & 8 & 1. $000 E+\infty$ & $1.31 \%+\infty$ & $1.740 E+00$ & $2.295 E+00$ & $3.02 \pi++\infty$ & 3. $993 E+\infty 0$ & $5.268 \varepsilon+\infty 0$ & $6.950 E+00$ & $9.170 E+\infty$ & $1.210 E+01$ & $1.596 E+01$ \\
\hline 1.2 & $:$ & $1.000=00$ & $1.148 E+00$ & $1.31 \varepsilon \mathcal{E}+\infty$ & $1.513 E+00$ & $1.73 \pi+\infty$ & $1.994 E+00$ & $2.2 \% 0 \varepsilon+\infty$ & $2.62 \%+00$ & $3.01 \%+\infty$ & $3.46 \pi+\infty$ & $3.981 E+\infty 0$ \\
\hline & : & $1.000=4+00$ & $1.000<+00$ & $1.000=+00$ & 1. $000 E+\infty 0$ & $1.000 E+\infty 0$ & $1.000 E+00$ & $1.000 \varepsilon+\infty$ & $1.000 \leq+\infty 0$ & 1. $000 E+\infty$ & $1.000 \leq+\infty 0$ & $1.000 \leq+00$ \\
\hline & $:$ & $1.000 \leq+\infty$ & 8. $735 E-01$ & $7.635 E-01$ & $6.683 E-01$ & $5.8565-01$ & $5.13 \pi-01$ & $4.51 z-01$ & $3.967 E-01$ & $3.493 E-01$ & 3. $\infty 81 E-01$ & $2.722 x-01$ \\
\hline & : & $1.000=400$ & $7.68<5-01$ & $5.945 t-01$ & $4.63 \pi-01$ & $3.650 E-01$ & 2. $904 E-01$ & $2.340 E-01$ & $1.912 \leq-01$ & $1.50 E-01$ & $1.343 E-01$ & $1.157 E-01$ \\
\hline & : & $1.000 \varepsilon+\infty$ & $6.873 \pm-01$ & 4.84\%-01 & $3.531 E-01$ & $2.668 E-01$ & $2.10 z-01$ & 1. $730 E-01$ & $1.485 E-01$ & $1.323 E-01$ & $1.216 E-01$ & $1.146 E-01$ \\
\hline - & $:$ & $1.000 \leq+00$ & $6.341 E-01$ & $4.2 \%=-01$ & $3.15 x-01$ & $2.504 E-01$ & 2. $135 E-01$ & $1.924 E-01$ & $1.801 E-01$ & 1. $734 E-01$ & $1.695 E-01$ & $1.67 Z \mathbf{E}-01$ \\
\hline 0 & $:$ & $1.000=+00$ & $6.0985-01$ & $4.225-01$ & $3.320 \leq-01$ & $2.880 E-01$ & $2.663 z-01$ & $2.556 \varepsilon-01$ & $2.503 E-01$ & $2.476 E-01$ & 2. 46$\} E-01$ & $2.456 E-01$ \\
\hline .2 & 8 & $1.000 E+\infty$ & $6.116 E-01$ & $4.512 E-01$ & $3.846 E-01$ & $3.568 \varepsilon-01$ & $3.450 E-01$ & 3. $400 E-01$ & $3.378 \varepsilon-01$ & $3.36 \%-01$ & $3.365 E-01$ & $3.363 c-01$ \\
\hline .4 & $:$ & $1.000 \leq+00$ & $6.332=-01$ & $5.021 E-01$ & $4.553 \varepsilon-01$ & $4.381 E-01$ & $4.3235-01$ & $4.300<-01$ & $4.292 x-01$ & $4.28 \%-01$ & $4.280 E-01$ & TE-01 \\
\hline .6 & $:$ & $1.000=+00$ & $6.675 E-01$ & 5.634E-01 & $5.310 \varepsilon-01$ & $5.210 E-01$ & $5.17 \varepsilon \varepsilon-01$ & $5.168 \varepsilon-01$ & $5.165 z-01$ & $5.164 E-01$ & $5.16 A E-01$ & $E-01$ \\
\hline .8 & : & $1.000=+00$ & $7.082 E-01$ & $6.274 E-01$ & $6.051 E-01$ & $5.990 E-01$ & $5.973 \epsilon-01$ & $5.969 E-01$ & $5.967 E-01$ & $5.96 \pi-01$ & $E-01$ & $E-01$ \\
\hline 1 & : & $1.000 \varepsilon+\infty$ & $7.526 E-01$ & $6.905=-01$ & $6.7535-01$ & $6.714 E-01$ & $6.705 E-01$ & $6.702 \leq-01$ & $6.702 E-01$ & $6.70 \mathbb{E}-01$ & $6.70 x-01$ & $\mathbf{x}-01$ \\
\hline & : & $1.000 \leq+00$ & $7.990 E-01$ & $7.527 \mathrm{E}-01$ & $7.419-01$ & $7.394 E-01$ & $7.388 \leq-01$ & $7.386 \varepsilon-01$ & $7.386 \varepsilon-01$ & $7.386 E-01$ & $7.3865-01$ & $6 E-01$ \\
\hline & : & $1.000 \varepsilon+\infty 0$ & $8.468 E-01$ & $8.137 E-01$ & 8. $063 E-01$ & $8.045 E-01$ & $8.041 E-01$ & 8. 040E-01 & $8.040 E-01$ & $8.040 E-01$ & 8. 040E-01 & $8.040 E-01$ \\
\hline & : & 1. $000 \varepsilon+\infty$ & $8.960 E-01$ & $8.745-01$ & $8.698<-01$ & $8.687 E-01$ & $8.684 E-01$ & $8.684 E-01$ & $8.684 E-01$ & $8.684 E-01$ & $8.684 E-01$ & $8.684 E-01$ \\
\hline & : & $1.000 \leq 400$ & $9.470 E-01$ & $9.363 z-01$ & $9.340=-01$ & $9.3345-01$ & $9.303 x-01$ & $9.332=-01$ & $9.332 x-01$ & $9.332 x-01$ & $9.302 \leq-01$ & $9.332 E-01$ \\
\hline 2 & $:$ & $1.000 \varepsilon+\infty$ & $1.000 E+\infty$ & $1.000 \varepsilon+\infty$ & $1.000 \varepsilon+\infty 0$ & $1.000 \varepsilon+\infty$ & 1. $000 E+\infty$ & 1. $000 E+\infty$ & $1.000 E+\infty$ & $1.000 E+\infty$ & $1.000 E+\infty$ & $1.000 E+\infty$ \\
\hline
\end{tabular}

$B(2, n, r, w)$ for $r=128$

\begin{tabular}{|c|c|c|c|c|c|c|c|c|c|c|c|c|}
\hline nu 1 & $H$ & 1 & 2 & 4 & 8 & 16 & 32 & 64 & 128 & 256 & 512 & 1024 \\
\hline-2 & : & 1. $000 E+\infty$ & $2.000 E+00$ & 4.000E+00 & 8. $000 E+\infty 0$ & $1.600 E+01$ & 3. $200 E+01$ & $6.400 E+01$ & $1.280 E+02$ & $2.560 E+02$ & $5.120 E+02$ & $1.024 E+03$ \\
\hline-1.8 & : & $1.000 E+100$ & $1.741 E+\infty 0$ & $3.031 E+\infty 0$ & $5.278 E+\infty 0$ & $9.189 E+00$ & $1.600 E+01$ & $2.786 E+01$ & 4. $850 E+01$ & 8. 44E +01 & $1.470 E+02$ & $2.560 E+02$ \\
\hline-1.6 & $:$ & $1.000 E+\infty$ & $1.516 E+00$ & $2.29 \pi+\infty 0$ & 3. $482 E+\infty 0$ & $5.2 \pi 7 E+\infty 0$ & $7.9 \% \times+\infty 0$ & $1.212 E+01$ & $1.83 \pi+01$ & $2.785 x+01$ & $4.221 E+01$ & 6. $3 \times x+01$ \\
\hline-1.4 & $:$ & 1. $000 E+\infty$ & $1.319 E+\infty 0$ & $1.741 E+\infty 0$ & 2. $296 E+00$ & $3.030 E+\infty 0$ & $3.99 \pi E+D$ & $5.274 E+00$ & $6.95 \%+00$ & $9.182 E+00$ & $1.21 \geq \mp+01$ & $1.599+01$ \\
\hline-1.2 & $:$ & $1.000 z+\infty 0$ & $1.148 E+\infty 0$ & $1.319 E+00$ & $1.515 t+\infty$ & $1.739 x+\infty$ & $1.99 \times 5+00$ & $2.294 E+00$ & $2.635 E+00$ & $3.026 E+00$ & $3.475 x+\infty$ & $3.99 \geq E+\infty$ \\
\hline-1 & $:$ & $1.000 E+\infty$ & $1.000 E+\infty$ & $1.000 E+\infty 0$ & $1.000 \varepsilon+\infty 0$ & $1.000 E+\infty$ & $1.000 E+\infty 0$ & 1. $000 E+\infty$ & $1.000 E+\infty$ & $1.000 E+\infty 0$ & $1.000 E+00$ & $1.000 E+\infty$ \\
\hline-.8 & : & $1.000 E+\infty$ & 8. $721 E-01$ & $7.611 E-01$ & $6.647 E-01$ & $5.808 \varepsilon-01$ & $5.07 \%-01$ & $4.44 E-01$ & $3.891 E-01$ & $3.410 E-01$ & $2.992 E-01$ & $2.62 \pi E-01$ \\
\hline-.6 & : & $1.000 E+\infty$ & $7.647 E-01$ & $5.875 E-01$ & $4.53 \%=-01$ & $3.52 \%-01$ & $2.764 E-01$ & $2.186 E-01$ & $1.747 E-01$ & $1.415 E-01$ & $1.164 E-01$ & $9.729 E-02$ \\
\hline-.4 & : & $1.000 \varepsilon+\infty$ & $6.803 \times-01$ & $4.720=-01$ & 3. 359-01 & $2.46 \pi-01$ & $1.881 E-01$ & $1.494 E-01$ & $1.240 E-01$ & $1.0725-01$ & $9.614 E-02$ & $4 E-02$ \\
\hline-.2 & : & $1.000 E+\infty$ & $6.235 E-01$ & $4.121 E-01$ & $2.928 E-01$ & $2.25 x-01$ & $1.866 E-01$ & $1.646 E-01$ & $1.51 \%-01$ & $1.447 \mathrm{E}-01$ & 1. $405 E-01$ & $1 E-01$ \\
\hline 0 & $:$ & 1. $000 E+\infty$ & $5.978<-01$ & $4.036 E-01$ & $3.094 E-01$ & $2.634 E-01$ & 2. $40 \pi-01$ & $2.2945-01$ & 2. $23 \times<-01$ & 2. $210 E-01$ & $2.196 \varepsilon-01$ & $E-01$ \\
\hline .2 & : & $1.000=(-0)$ & $6.011 E-01$ & $4.355=01$ & $3.665 E-01$ & $3.376 E-01$ & 3. $253 E-01$ & $3.200 E-01$ & $E-01$ & $\xi-01$ & $\varepsilon-01$ & $E-01$ \\
\hline .4 & : & 1. $000 E+\infty$ & $6.256 E-01$ & $4.914 E-01$ & $4.435=-01$ & 4. $259-01$ & 4. $196 \varepsilon-01$ & $4.173 E-01$ & $E-01$ & & $O E-01$ & $E-01$ \\
\hline .6 & $:$ & $1.000 E+\infty$ & $6.626 E-01$ & $5.571 E-01$ & 5. $24 Z E-01$ & 5. $13 \%-01$ & $5.10 \pi-01$ & 5.097E-01 & & & $x-01$ & $\mathbf{E}-01$ \\
\hline .8 & $:$ & 1. $000 E+\infty$ & $7.056 \varepsilon-01$ & $6.240 E-01$ & $6.016 \varepsilon-01$ & 5. $954 E-01$ & $5.93 \pi-01$ & $5.93 \alpha E-01$ & $E-01$ & $0 \varepsilon=01$ & $\varepsilon-01$ & $E-01$ \\
\hline 1 & : & $1.000 E+\infty$ & $7.513 E-01$ & $6.891 E-01$ & $6.736 E-01$ & $6.697 E-01$ & $6.687 E-01$ & $6.685 E-01$ & & & & $E-01$ \\
\hline 1.2 & . & $1.000=+00$ & $7.983 \varepsilon-01$ & $7.520<-01$ & $7.411 E-01$ & $7.3865-01$ & $7.380 E-01$ & $7.37 \varepsilon \varepsilon-01$ & $7.37:=-01$ & $7.378 \leq-01$ & $7.37 \notin E-01$ & $\alpha E-01$ \\
\hline & : & $1.000 E+\infty$ & $8.465 x-01$ & $8.135=-01$ & 8. $05 \% E-01$ & $8.042 E-01$ & $8.03 \div E-01$ & $8.03 \pi-01$ & $8.03 \pi-01$ & 8. $037 E-01$ & 8. $03 \pi-01$ & $7 E-01$ \\
\hline & : & 1. $000 E+\infty 0$ & $8.95 \%-01$ & 8.74EE-01 & $8.696 \leq-01$ & $8.686 E-01$ & $8.6835-01$ & $8.682 \varepsilon-01$ & $8.68 z E-01$ & $8.6825-01$ & $8.682 \varepsilon-01$ & $8.682 E-01$ \\
\hline 1.8 & : & 1. $000 E+\infty$ & $9.46 \%-01$ & $9.363 \varepsilon-01$ & $9.33 \%=-01$ & $9.331 E-01$ & $9.3325-01$ & $9.3325-01$ & $9.332 E-01$ & $9.332 x-01$ & $9.3324-01$ & $9.332 x-01$ \\
\hline 2 & : & $1.000 E+\infty$ & $1.000 E+\infty$ & $1.000 E+00$ & $1.000 \leq+00$ & 1. $000 \varepsilon+00$ & $1.000 E+00$ & 1. $000 E+00$ & $1.000 \varepsilon+\infty$ & $1.000 E+\infty$ & $1.000 E+\infty$ & $1.000 \leq+00$ \\
\hline
\end{tabular}




\begin{tabular}{|c|c|c|c|c|c|c|c|c|c|c|c|c|}
\hline 1 & $n=$ & 1 & 2 & 4 & 8 & 16 & 32 & 64 & 128 & 256 & 512 & 1024 \\
\hline-2 & : & $000 E+\infty$ & & $00 E+\infty$ & $000 E+\infty$ & $600 E+01$ & $3.200 \varepsilon+01$ & $.400 E+01$ & $2006+02$ & $2.560<+02$ & $.120 E+02$ & $1.02 \pi<00$ \\
\hline 1.8 & & & & & $278 E+\infty 0$ & $.189 E+\infty 0$ & & $.7865+01$ & $850 E+01$ & 8.45E+01 & & \\
\hline 1.6 & : & & & & & & & & & & & \\
\hline .4 & : & $\infty$ & & & $\infty$ & $1 E+\infty$ & $\infty$ & & $962 E+\infty 0$ & $-\infty$ & $+0 !$ & \\
\hline 1.2 & & & & & & & & & $.637 E+\infty 0$ & $3.02 \%+\infty+\infty$ & & \\
\hline & : & & & & & $1.000 \leqslant+00$ & $1.000 E+\infty 0$ & $1.000 E+\infty 0$ & $1.000 E+\infty$ & $1.000 E+\infty 0$ & 1.000E +00 & $1.000 E+\infty 0$ \\
\hline 8 & : & & & & & & & $E-01$ & $848 E-01$ & $3.363 E-01$ & $2.941 E-01$ & $.573 E-01$ \\
\hline & : & & & & & & & & & & & \\
\hline .4 & : & & 01 & & & & 1.72 & & $062 x-01$ & $8.89 \%-02$ & $.763 x-02$ & $.014 E-02$ \\
\hline-.2 & : & & & & & & 1.6 & & $296 E-01$ & $1.221 E-01$ & & . 153E-01 \\
\hline 0 & : & & & & & & & & $2.026 E-01$ & -01 & & $.9 T \overline{S E}-01$ \\
\hline .2 & : & & & & & & & & $3.022 E-01$ & & & $3.005 E-01$ \\
\hline .4 & : & & & & & & & & $=-01$ & & & $.067 E=01$ \\
\hline .6 & : & & & & & & & & -01 & $046 E-01$ & & $5.046 E-01$ \\
\hline .8 & : & & & & & -01 & & & $910 E-01$ & $\alpha E-01$ & & $.910 E-01$ \\
\hline 1 & : & & & & & -01 & & & $.676 E-01$ & $75 E-01$ & $.675 E-01$ & $.675 E-01$ \\
\hline 1.2 & & & & & & & & & $7.374 E-01$ & $7.374 E-01$ & & $.374 E-01$ \\
\hline 1. & & $1.000 E$ & $.464 E-01$ & & $.058 E-01$ & $041 E-01$ & $8.036 E-01$ & $E-01$ & $8.035 x-01$ & $8.035 E-01$ & $3.0355-01$ & $.035-01$ \\
\hline 1. & & & 01 & & & & $8.682-01$ & 8.68 & $8.68 x-01$ & $8.6825-01$ & $8.68 z-01$ & $.6825-01$ \\
\hline & & & & & & & & & & & & \\
\hline 2 & : & $1.000 E+00$ & 1.000E $+\infty$ & $1.000 E+\infty 0$ & $1.000 E+\infty$ & $1.000 E+00$ & $1.000 E+\infty$ & $1.000 E+\infty 0$ & 1) & S & $O E+\infty 0$ & $\varepsilon+\infty$ \\
\hline
\end{tabular}

$B 3(2, n, r, \infty)$ for $r=512$

\begin{tabular}{|c|c|c|c|c|c|c|c|c|c|c|c|c|}
\hline & $H=$ & 1 & 2 & 4 & 8 & 16 & 32 & 64 & 128 & 256 & 512 & 1024 \\
\hline & : & $D O E+\infty D$ & $000 E+\infty$ & $000 E+\infty$ & $00 E+\infty 0$ & $600 E+01$ & 3. $200 E+01$ & 6. $400 E+01$ & & & & \\
\hline .8 & : & & & $031 E+\infty 0$ & & $190 E+00$ & $.600 E+01$ & & & & & \\
\hline .6 & : & $1.000 E+\infty$ & & $29 \pi E+\infty$ & $482 E+00$ & $278 E+\infty 0$ & $8.000 E+00$ & $213 E+01$ & $838 E+01$ & $786 E+01$ & $4.222 E \div 01$ & $.400 E+01$ \\
\hline .4 & : & & & & & & & & & & & \\
\hline .2 & : & 0 & & & & & $+\infty$ & $+\infty$ & $+\infty$ & $030 E+\infty$ & & 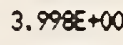 \\
\hline & : & & & & & $=\infty 0$ & $1.000 E+\infty$ & $E+\infty$ & $00 E+\infty$ & $.000 E+\infty$ & & $.000 E+\infty 0$ \\
\hline 8 & : & 1.000 & & & & & $5.026 E-01$ & 4. $383 E-01$ & $.8235-01$ & $3.336 E-01$ & & $2.542 \mathbb{x}-01$ \\
\hline & : & & & & & -01 & & $=-01$ & $570 E-01$ & & & \\
\hline 4 & : & & & & & & & & & & & \\
\hline .2 & $:$ & 1.00 & & & & & & -01 & & & & $.716 E-02$ \\
\hline 0 & : & $1 . \alpha$ & & & & -01 & & $=-01$ & & -01 & $06 E-01$ & $1.799 E-01$ \\
\hline .2 & : & & & & & & & & & & & \\
\hline .4 & : & 1.00 & & & & & & -01 & & & & $.000 E-01$ \\
\hline .6 & : & 1.00 & & & & & & $E-01$ & $8 E-01$ & $E-01$ & $E-01$ & $16 E-01$ \\
\hline .8 & : & & & & & & & $E-01$ & $5.898 \varepsilon=01$ & $5.898 \varepsilon-01$ & $E-01$ & $.898 E-01$ \\
\hline 1 & $:$ & & & & & & & $=-01$ & $1 E-01$ & $E-01$ & & $.671 E-01$ \\
\hline 1. & : & & & & & & & & & & & $.3735-01$ \\
\hline 1. & : & $1.000 E+\infty$ & 8.463 & & & $8.040 E-01$ & 8. $0365-01$ & $8.0355-01$ & $8.035 E=01$ & $8.035 E-01$ & $8.035 t-01$ & $.035 E-01$ \\
\hline & $:$ & 1.000 & 8.958 & 8.7 & 8.69 & $8.685-01$ & $8.6825=01$ & $8.682 x-01$ & $8.681 E-01$ & $8.681 E-01$ & $8.681 E-01$ & $.681 E-01$ \\
\hline & : & 1.000 & & & & & $9.332 E-01$ & $9.332 \leq-01$ & $9.332 E-01$ & $9.332 \leq-01$ & $9.332 \leq-01$ & $9.332 \leq-01$ \\
\hline 2 & : & $1.000 E+\infty 0$ & $1.000 E+\infty$ & $1.000 \varepsilon+\infty$ & $1.000 E+\infty$ & $1.000 E+\infty$ & $1.000 \varepsilon+\infty$ & $1.000 \varepsilon+\infty$ & $1.000 E+\infty$ & $1.000 E+\infty 0$ & $1.000 E+\infty$ & 1. $000 E+\infty$ \\
\hline
\end{tabular}




\begin{tabular}{|c|c|c|c|c|c|c|c|c|c|c|c|c|}
\hline w 1 & $N$ & d & 2 & 4 & 8 & 16 & 32 & 64 & 128 & 256 & 512 & 1024 \\
\hline-2 & : & $1.000 E+\infty 00$ & $2.000 E+\infty 0$ & $\infty 00 E+\infty$ & $000 \varepsilon+\infty$ & $1.600 E+01$ & & & & & & \\
\hline 1.8 & $:$ & $1.000 E+\infty 0$ & & & & & & & $850 E+01$ & 44E+01 & $1.470 E+02$ & \\
\hline 1.6 & : & $1.000 E+\infty 0$ & $1.516 E+\infty$ & $2.29 \pi+\infty$ & $3.482 \leq+\infty 0$ & $5.278 E+\infty$ & $8.000 \varepsilon+\infty 0$ & $1.213 E+01$ & $1.805 E+01$ & $2.786 E+01$ & $4.222 E+01$ & $6.400 E+01$ \\
\hline 1.4 & : & $1.000 E+\infty 0$ & $.31 \%+\infty$ & $1.741 E+\infty 0$ & $229 \pi+\infty 0$ & 3. $031 E+\infty 0$ & $4.000 E+\infty$ & $5.278 E$ & $6.964 E+\infty 00$ & $9.18 \% E+\infty$ & $1.213 E+01$ & $1.600 E+01$ \\
\hline 1.2 & : & $1.000 E+00$ & $-\infty$ & & $1.516 E+\infty 0$ & 1.741 & 2.00 & $x$ & 00 & $-\infty$ & $3.482 E+\infty 0$ & $3.9996+\infty 0$ \\
\hline & : & $1.000 E+\infty$ & & & $\infty$ & $\infty$ & $\infty$ & & $\infty$ & $\infty$ & $-\infty$ & $E+\infty$ \\
\hline .8 & : & $1.000 E+\infty 0$ & & & 6.607 & 5.75 & & & -01 & 01 & & $2.524 E-01$ \\
\hline .6 & : & $1.000 E+\infty$ & 7.59 & & & & & & 1.524E-01 & & $9.210 \varepsilon-02$ & $7.23: 5-02$ \\
\hline .4 & : & $1.000 E+\infty 0$ & 01 & & 3.0 & 2.1 & & & 02 & & & 4.62 \\
\hline .2 & $:$ & $1.000 E+00$ & 01 & & 01 & $1.7 t$ & & & 9.744 & 8.956 & $\varepsilon-02$ & $8.244 E-02$ \\
\hline 0 & : & $1.000 E+\infty 0$ & & & .01 & 2.1 & & & 1.705 & 01 & $E-01$ & $1.65 x-01$ \\
\hline .2 & $:$ & $1.000 E+\infty$ & 01 & & & & & & & & $E-01$ & $E-01$ \\
\hline .4 & : & $1.000 E+\infty$ & 01 & & & & & & 01 & 01 & & 3.95 \\
\hline .6 & $:$ & $1.000 E+\infty 0$ & & & & & & & 4.9\%E & 4.997E-01 & & $4.996 E-01$ \\
\hline .8 & $:$ & $1.000 E+\infty 0$ & $7.0 Z 8 E-01$ & 6.20 & $5.977 \mathrm{E}-01$ & 5.915 & & 5.895 & $5.891 E-01$ & $5.891 E-01$ & $5.891 E-01$ & $5.891 E-01$ \\
\hline 1 & $:$ & $1.000 E+\infty 0$ & $7.502 E-01$ & & 6.721E-01 & 6.682 & 6.67 & 6.67 & $6.66 \%-01$ & $6.66 \%-01$ & $6.66 \%=01$ & $6.669 E-01$ \\
\hline & & $1.000 E+\infty$ & 7.979 & & & & & & $7.37 x-01$ & $7.37 \boldsymbol{x} \leq-01$ & 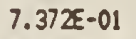 & $7.372 x-01$ \\
\hline & & $1.000 E+\infty 0$ & & & & $8.040 E$ & & & $8.035 E-01$ & $8.035 E-01$ & $8.035 E-01$ & $8.035 E-01$ \\
\hline 1. & & $1.000 E+\infty$ & $8.958<-01$ & & $8.696 E-01$ & $8.685 x-01$ & $8.682 E-01$ & $8.682 E-01$ & $8.681 E-01$ & 8. $681 E-01$ & $8.681 E-01$ & $8.681 E-01$ \\
\hline 1.8 & & $1.000 E+\infty 0$ & $9.46 \%-01$ & $9.36 z E-01$ & 9. 33\%E-01 & $9.333 E-01$ & $9.332 E-01$ & $9.332 \leq-01$ & $9.3325-01$ & $9.332 E-01$ & $9.332 E-01$ & $9.332 x-01$ \\
\hline 2 & : & $1.000 E+\infty 0$ & $1.000 E+\infty 0$ & $1.000 E+00$ & $1.000 E+\infty 0$ & $1.000 E+\infty 0$ & $1.000 E+00$ & 1. $000 E+\infty 0$ & $1.000 E+\infty$ & $1.000 E+\infty$ & $1.000 E+\infty 0$ & $1.000 E+\infty 0$ \\
\hline
\end{tabular}

$83(2, M, r, \ldots)$ for $r=2048$

\begin{tabular}{|c|c|c|c|c|c|c|c|c|c|c|c|c|}
\hline 1 & $H=$ & 1 & 2 & 4 & 8 & 16 & 32 & 64 & 128 & 256 & 512 & 24 \\
\hline-2 & : & $1.000 E+00$ & $2.000 E+\infty 0$ & 4. $000 E+00$ & $000 E+\infty 0$ & $1.600 E+01$ & $200 E+01$ & $6.400 E+01$ & 1. $280 E+02$ & $2.560 E+02$ & $5.120 E+02$ & $1.024 E+03$ \\
\hline-1.8 & : & 1.000E+10) & $.741 E+\infty$ & $.031 E+00$ & $278 E+\infty 0$ & $190 E+\infty 0$ & $.600 E+01$ & $.786 E+01$ & $4.850 E+01$ & $8.45 E+01$ & $1.470 E+122$ & $2.560 \varepsilon+02$ \\
\hline-1.6 & $:$ & 1. $000 E+00$ & $1.516 E+\infty 0$ & 2. $29 \pi+\infty 0$ & $.482 \varepsilon+00$ & $.278 \varepsilon+00$ & & & & $2.786 E+01$ & & \\
\hline 1.4 & $:$ & $1.000 E+00$ & 1. $320 E+\infty 0$ & $1.741 E+\infty$ & $.297 E+\infty 0$ & $.031 E+\infty 0$ & 4. $000 E+00$ & & $6.964 E+00$ & $9.189 E+00$ & & $1.600 E+01$ \\
\hline 1.2 & $:$ & $1.000 E+00$ & 1. $149 E+\infty$ & $1.31 \%+\infty 0$ & $1.516 E+00$ & $1.741 E+\infty 0$ & $2.000 E+\infty 0$ & & $2.63 \%+\infty$ & $3.031 E+\infty 0$ & & 4. $000 E+\infty 0$ \\
\hline-9 & $:$ & 1. OOOE +(0) & $1.000 E+\infty 0$ & $1.000 E+\infty$ & 1. $000 E+\infty 0$ & $1.000 E \div 00$ & $1.000 E+00$ & $1.000 E+00$ & $1.000 E+00$ & $1.000 E+\infty 0$ & $1.000 E+\infty 0$ & $1.000 E+\infty$ \\
\hline. .8 & $:$ & 1. $000 E+\infty$ & 8. $707 E-01$ & $7.58 x-01$ & $.603 E-01$ & 5.751 & 5.009 & & $3.800 E-01$ & 3. 3111 & & $E-01$ \\
\hline$b$ & $:$ & $1.0005+00$ & 7.59 & -01 & -01 & -01 & 2.54 & & $1.494 E-01$ & $1.14 \%-01$ & & $E-02$ \\
\hline-.4 & $:$ & $1.000 \varepsilon+00$ & 6.66 & 4. $468<-01$ & $E-01$ & 2.075 & 1.48 & & $7.628 E-02$ & $5.831 E-02$ & & $3.864 E-02$ \\
\hline-.2 & : & $1.000 E+\infty 0$ & $5.98 \pi-01$ & $3.7065-01$ & $E-01$ & -01 & 1.24 & & $8.565 E-02$ & $7.763 E-02$ & & $7.038 E-02$ \\
\hline 0 & $:$ & 1.000 & $5.681 E-01$ & $3.5705-01$ & $E-01$ & 2.023 & $1 . \pi$ & & -01 & 1.55 & & $E-01$ \\
\hline .2 & : & 1.0000 & 5.768 & 3.9 & -01 & 2.93 & 2.80 & & $2.71 \pi-01$ & & & \\
\hline .4 & : & $1.000 E+00$ & 6.111E-01 & 4. $70 \%$ E-01 & $4.204 E-01$ & $4.021 E-01$ & 3. $953 E-01$ & -01 & $3.920 E-01$ & $3.916 E-01$ & $\bar{x}-01$ & $3.914 E-01$ \\
\hline .6 & $:$ & $1.000 E+00$ & $6.5555-01$ & 5. $475 E-01$ & $E-01$ & 5.032 & 4.99 & & 4. $985 E-01$ & $4.984 E-01$ & $E-01$ & $4.984 E-01$ \\
\hline .8 & : & $1.000 E+00$ & $7.026 E-01$ & 6.201E-01 & -01 & 5.911 & 5.89 & & $5.888 \leq-01$ & $5.88 \pi-01$ & & $5.88 \pi-01$ \\
\hline 1 & : & $1.000 E+\infty 0$ & $7.501 E-01$ & $6.876 E-01$ & 6. $720 E-01$ & 6.681 & 6.671 & & $6.668 E-01$ & $6.668 E-01$ & $68 E-01$ & $6.668 \varepsilon-01$ \\
\hline & & $1.000 E+\infty 0$ & $7.9795-01$ & $7.514 E-01$ & 7. $405 x-01$ & 7.300E-01 & $7.374 E-01$ & $7.37 x-01$ & $7.3725-01$ & $7.372 \varepsilon-01$ & $7.372 \leq-01$ & $7.37 \mathbb{E}-01$ \\
\hline & & $1.000 E+\infty 0$ & $8.463 E-01$ & 8. $131 E-01$ & $8.05 \pi-01$ & $8.040 E-01$ & $8.0365-01$ & $=-01$ & $8.03 x-01$ & 8.034E-01 & 8. $034 E-01$ & $8.034 E-01$ \\
\hline & & 1. $000 E+\infty 0$ & $8.958 E-01$ & & & 8.685 & 8.682 & & $8.681 E-01$ & $8.681 E-01$ & $8.681 E-01$ & $8.681 E-01$ \\
\hline & $:$ & & & & & & $9.332 x-01$ & & $9.332 E-01$ & $9.33 x=-01$ & $9.3325-01$ & $9.332 E-01$ \\
\hline 2 & : & $1.000 n++00$ & $1.000 E+\infty 0$ & $1.000 E+\infty 0$ & $1.000 E+00$ & 1.000E $+\infty 0$ & $1.000 E+00$ & $1.000 E+00$ & $1.000 E+\infty 0$ & $1.000 E+\infty 0$ & $1.000 E+\infty 0$ & $1.000 E+\infty 0$ \\
\hline
\end{tabular}


$83(2, H, r, \ldots u)$ for $r=4096$

\begin{tabular}{|c|c|c|c|c|c|c|c|c|c|c|c|c|}
\hline 1 & $n=$ & 1 & 2 & 4 & 8 & 16 & 32 & 64 & 128 & 256 & 512 & 1024 \\
\hline & : & $000 E+\infty$ & 2. $000 \varepsilon+\infty$ & 4. $000 \varepsilon+\infty$ & $D E+\infty$ & $600 E 401$ & $200 E+01$ & $6.400 E+01$ & $1.200<+02$ & $560 \varepsilon+02$ & $5.120 E+02$ & $1.024 E+c$ \\
\hline .8 & $:$ & & $741 E+\infty$ & WIET & & & & & & & & \\
\hline & : & & $1.516 \varepsilon+\infty$ & $2.29 \pi+\infty$ & & $+\infty$ & $.000 E+\infty$ & $1.213 E$ & 1.0 & $786 E+01$ & 4. $200 E+01$ & $6.400 \varepsilon+01$ \\
\hline .4 & : & & $1.320 E+\infty 0$ & $1.741 E+\infty$ & $2.29 \pi$ & & $4.000 E+00$ & $5.278 E+\infty 0$ & 6.9644 & $.190 \varepsilon+\infty$ & $1.213 E+01$ & $1.600 E+01$ \\
\hline .2 & : & & 1.149 & 1.315 & & & $2.000 E+\infty$ & $2.29 \pi+\infty 0$ & $2.639 E+\infty 0$ & $3.031 E+\infty$ & $3.482 x+\infty 0$ & $4.000 E+\infty$ \\
\hline & : & $1.000 E+\infty$ & $1.000 E+\infty 0$ & 1.000 & & & $1.000 E+\infty 0$ & $1.000 E+\infty 0$ & $1.000 E+\infty$ & $1.000 E+00$ & $1.000 E+00$ & 1. $000 E+00$ \\
\hline 8 & : & & 8.706 & 7.58 & & & & & 3.796 & .01 & $2.879 E-01$ & \\
\hline 6 & : & 1.000 & 7.587 & 5.76 & & & & 1.93 & 1.47 & 1 & $8.665 E-02$ & 6.675 \\
\hline 4 & $:$ & & 6.64 & 4.4 & & & & 9.850 & 7.08 & $5.27 €-02$ & $4.005 \bar{x}-02$ & 3. $29 T E-02$ \\
\hline .2 & $:$ & & $5.95 x$ & 3.6 & & & & 9.007 & 7.590 & 6. TTाE-02 & $6.310 \varepsilon-02$ & $6.042 E-02$ \\
\hline 0 & $:$ & & 5.63 & 3.49 & & & & 1.539 & 1.476 & $1.444-01$ & $1.428 z-01$ & $1.420 E-01$ \\
\hline .2 & $:$ & 1.000 & 5.73 & 3.94 & & & & 2.675 & 2.650 & & $2.634 E-01$ & $2.632 \leq-01$ \\
\hline .4 & $:$ & 1.00 & 6.09 & 4.68 & & & & 3.902 & 3.892 & $3.88 \%$ & 3. $88 \pi \mathrm{E}-01$ & $3.88 \pi-01$ \\
\hline .6 & : & & 6.55 & & & & & 4.990 & $4.9 \pi$ & & $4.975-01$ & $4.9 T 5-01$ \\
\hline .8 & : & 1.000 & 7.024 & 6.19 & & & & $5.88 \pi$ & $5.805 E-01$ & $5.885 E-01$ & $5.285-01$ & $5.25-01$ \\
\hline 1 & $:$ & $1.000 \varepsilon+\infty$ & $7.500 E-01$ & 6.876 & & & & 6.6680 & $6.66 \pi-01$ & $6.66 \pi-01$ & $6.66 \pi-01$ & $6.66 \pi-01$ \\
\hline & & $1.000 E+00$ & $7.979 E-01$ & $7.514 E-01$ & & 7.38 & $7.374 E-01$ & $7.3725-01$ & $7.3725-01$ & $7.372 E-01$ & $7.372 \varepsilon-01$ & $7.37 x-01$ \\
\hline & & $1.000 E$ & 8.463 & 8.13 & & & 8.036 & 8.035 & 8.034 & $8.034 E$ & $8.034 E-01$ & $8.034 E-01$ \\
\hline & & & 8.958 & & & & & & & & $8.681 E-01$ & $8.681 E-01$ \\
\hline & & & $9.46 \%-01$ & & & & & $9.3325-01$ & $9.332 E-01$ & & $9.302 E-01$ & $9.332 E-01$ \\
\hline & $:$ & $1.000 E+00$ & $1.000 E+\infty$ & $1.000 E+\infty$ & $1.000 E+10$ & $1.000 E+\infty$ & 1. $000 E+00$ & $1.000 E+\infty 0$ & $1.000 E+00$ & $1.000 E+\infty 0$ & $1.000 E+00$ & $1.000 E+\infty 0$ \\
\hline
\end{tabular}

$B 3(2, M, r$, mu) for $r=8192$

\begin{tabular}{|c|c|c|c|c|c|c|c|c|c|c|c|c|}
\hline 1 & $F$ & 1 & 2 & 4 & 8 & 16 & 32 & 64 & 128 & 256 & 512 & 1024 \\
\hline & : & $1.000 E+\infty 0$ & $2.000 E+00$ & & & & & & & & & \\
\hline .8 & $:$ & & & & & & & & 01 & & & \\
\hline & : & 1.000E+00 & $.516 E+\infty$ & $29 \pi+\infty$ & 3. $482 E+00$ & $5.278 E+00$ & $.000 E+\infty 0$ & & $1.838 \varepsilon+01$ & $.786 E+01$ & $4.222 E+01$ & $6.400 E+0 !$ \\
\hline .4 & : & $1.000 E+00$ & $.320 E+00$ & $741 E+\infty$ & $2.29 \pi+\infty$ & $3.031 E+00$ & 4. $000 E+\infty 0$ & & $6.964 E+\infty 0$ & $9.190 E+00$ & $1.213 \varepsilon+01$ & $1.600 E+01$ \\
\hline .2 & : & & & & & 1.741 & $\infty$ & & $2.639 E+\infty 0$ & $3.031 E+\infty 0$ & $3.48 x+\infty$ & $4.000 E+\infty$ \\
\hline & : & $1.000 E+\infty 0$ & $-\infty$ & & & 1.000 & 0 & & 1.0005 & $1.000 E+00$ & $1.000 E+\infty 0$ & $1.000 \leqslant+\infty 0$ \\
\hline & : & & & & & 01 & & & $3.793 E-01$ & & & $2.505 E-01$ \\
\hline & : & 1.00 & $!$ & & & & & & $1.461 E-01$ & & & $6.533 E-02$ \\
\hline & $:$ & 1.00 & 6.63 & & & & & & $6.685 t-02$ & & & $2.871 E-02$ \\
\hline 2 & : & 1.00 & 01 & & & & & & 6.7786 & 02 & 5.483 & 5.21 \\
\hline 0 & : & 1.00 & 5.5 & & & 1.8 & 1 & & $1.3835-01$ & $1.351 E-01$ & $1.35 x-01$ & $1.327 E-01$ \\
\hline .2 & $:$ & 1.00 & & & & 2.8 & 1 & & $2.594 E-01$ & $2.583 E-01$ & -01 & $2.576 E-01$ \\
\hline .4 & : & $\infty$ & 1 & & & & & & $3.872 E-01$ & $3.86 E E-01$ & -01 & $3.86 \pi-01$ \\
\hline .6 & : & 1.000 & -01 & & & & & & & $70 E-01$ & -01 & $E-01$ \\
\hline .8 & : & 1. $000 E+\infty$ & $7.0235-01$ & & 5.970 & 5.90 & 5.89 & & 5.884-01 & $5.984 E-01$ & $5.884 E-01$ & $5.884 E-01$ \\
\hline & $:$ & 1.000 & 7.50 & & & & & & & -01 & 01 & $6.66 \pi-01$ \\
\hline & & & & & & & & & & $7.37 \geq-01$ & & $7.3725-01$ \\
\hline & & $1.000 E+\infty 0$ & $8.4635-01$ & & & $8.040 E-01$ & 8.036 & & $8.034 E-01$ & $8.034 E-01$ & $8.034 E-01$ & $8.034 E-01$ \\
\hline & & $1.000 E+\infty$ & $8.958 c-01$ & $8.743 \varepsilon-01$ & $8.696 E-01$ & $8.685 x-01$ & $8.682 x$ & & $8.681 E-01$ & $8.681 E-01$ & $8.681 E-01$ & $8.681 E-01$ \\
\hline & & $1.000=+\infty$ & $9.46 \%-01$ & $9.36 z-01$ & $9.3395-01$ & $9.333 E-01$ & $9.332 E$ & 9.3325 & $9.332 E-01$ & $9.3325=01$ & $9.332 E-01$ & $9.332 E-01$ \\
\hline 2 & $:$ & $1.000 \leqslant+\infty$ & $1.000 E+\infty$ & 1. $000 E+\infty$ & $1.000 E+\infty 0$ & $1.000 E+\infty 0$ & $1.000 E+\infty 0$ & $1.000 E+\infty$ & $1.000 E+\infty$ & $1.000 E+\infty 0$ & 1.000E+00 & $1.000 E+00$ \\
\hline
\end{tabular}




\section{E. APPENDIX - Notes and Errata}

The notes listed below are included to bring attention to notation and other problems. The page number provides reference to the location of the problem or comment.

1. page $\mathrm{TN}-4$

AM noise is especially important in the measurement of the (residual) phase noise added by amplifiers and other signal handling components. Often the driving source for the measurements is a frequency synthesizer that has phase and/or amplitude noise that is comparable or larger than the added noise of the component under test. Most measurement systems are configured so that the phase noise of the source cancels out to a large degree (at high Fourier frequencies decorrelation effects limit the cancellation). In such measurement systems, the AM to PM conversion factor and the AM noise of the source may then set the noise floor. Discussion of the effect of AM noise and AM to PM conversion factors on the accuracy and precision of phase noise measurements is found in "Residual Phase Noise Measurements of vhf, uhf, and Microwave Components" by G. K. Montress, T. E. Parker, and M. J. Loboda Proc. 43rd Annual Symposium on Frequency Control, pp. 349-359 (1989) and in "Accuracy Model for Phase Noise Measurements by F. L. Walls, C. M. Felton, and A. J. D. Clements, 21st Annual Precision Time and Time Interval Meeting (1989). The notation in these papers as well as that in other parts of the literature differs from that given below. Our notation below is drawn from a modest level of consensus among individuals responsible for setting standards. We expect that it will gradually be adopted within standards committees. The following comments are directed specifically at the specification of noise performance.

The total power spectral density in a signal can be approximated by expanding eq $12-5$ of paper B.2 (by Stein) and extending it to include the spectral density of relative amplitude fluctuations, $S_{a}(f)$. The double-sideband density written in single-sideband form is given by

$$
S_{V}(f) \approx \frac{v_{0}^{2}}{2}\left[e^{-I\left(f_{c}\right)} \delta(f)+e^{-I(f)} S_{\phi}(f)+S_{a}(f)\right] \quad 0<f<\infty,
$$

where

$$
I(f) \propto \int_{f}^{\infty} S_{\phi}(f) d f .
$$

$I(f)$ is the integrated phase modulation due to the pedestal and $\delta(f)$ represents the carrier with frequency width $\pm f_{c^{*}}$. The effect of large $S_{a}(f)$ on power in the carrier has not, to our knowledge, been explored. The power spectral density of relative phase fluctuations, $S_{\phi}(f)$, is normalized to one $\operatorname{rad}^{2} / \mathrm{Hz}$ and $\mathrm{S}_{\mathrm{a}}(\mathrm{f})$ is normalized to the carrier voltage, but the total power spectral density, $S_{V}(f)$ is not normalized and has the units of $V^{2} / \mathrm{Hz}$. All of these are single-sided spectral densities. For most measurement purposes, we can disregard the carrier and find that, away from the carrier, the above expression simplifies to

$$
S_{\nu}(f)=\frac{v_{0}^{2}}{2}\left[S_{\phi}(f)+S_{a}(f)\right] \quad \text { for } \quad 0 \leq f \leq \infty .
$$


The more general expression is important only very near the carrier and in certain types of frequency multiplication. The single-sideband, amplitude noise, normalized to the total signal power is given simply by $S_{a}(f)$ for $0 \leq f \leq \infty$. The measurement of added phase and amplitude noise for amplifiers and other signal handling components should specify the signal level since the AM noise level and the contribution due to AM-to-PM conversion depend on the signal level.

2.

page TN-6

For a direct measurement, time accuracy only has meaning when the phase of the timebase oscillator of the frequency counter is known with respect to some time standard. Either it is phase locked or is calibrated with respect to that standard at the time of measurement. The phase of the time-base oscillator can then be measured with respect to the phase of the frequency standard being calibrated (accounting for cable delays, etc.). Except for the cycle ambiguity of the carrier, the phase of the frequency standard being measured can carry time information and have time accuracy. This technique is not common, but is very useful and eliminates divider noise that typically occurs in going from 5 or $10 \mathrm{Mhz}$ to 1 pulse per second. Caution must be exercised to assure that the phase point measured in a sine wave is at a reproducible voltage and impedance so that the cycle ambiguity is an exact integer.

3. page $\mathrm{TN}-35$

A second-order servo loop provides substantially enhanced performance. See, for example, F.L. Walls and S.R. Stein, "Servo techniques in oscillators and measurement systems," NBS Tech. Note 692 (1976).

4. page $\mathrm{TN}-35$

This error can be identified and corrected using the phase modulation scheme described in paper B.4 on page TN-136.

5. page $\mathrm{TN}-36$

Low-noise DC amplifiers have been substantially improved since publication of this paper.

6. pages TN-37, TN-91, TN-130, TN-174, TN-206 and TN-218

The reader is reminded that the discussions of frequency-domain measurements assume incoherent noise processes. Often the phase noise spectrum of a signal will contain bright spectral features (spurious lines) other than the carrier. Frequency-domain measurements are often useful in identifying such features. But if these spurious lines are narrow compared to the measurement bandwidth, statistical measures such as $S_{\phi}(f)$ and $\mathscr{L}(\mathrm{f})$ are not appropriate. It is better to specify the phase deviation in terms of the rms value of the phase deviations, $\phi_{\mathrm{rms}}$ (rms radians), without reference to bandwidth. This specification in rms radians can be related to the Allan variance (see note \# 8 below).

7. page $\mathrm{TN}-51$

Humidity is often an important environmental factor. See, for example, J.E. Gray, H.E. Machlan and D.W. Allan, "The Effect of humidity on commercial cesium beam atomic clocks," 42nd Annual Symp. on Frequency Control, pp. 514-518 (1988) and F.L. Walls, "The 
Influence of Pressure and Humidity on the Medium and Long-Term Frequency Stability of Quartz Oscillators," 42nd Annual Symp. on Frequency Control, pp. 279-283 (1988).

8. page TN-74

For a bright line (one which is narrow compared with the measurement bandwidth), the solution of eq 12-27 simplifies to

$$
\sigma_{y}(\tau)=\frac{\sqrt{2} \phi_{r m s}}{\pi v_{0} \tau} \sin ^{2}(\pi f \tau)
$$

where $\phi_{\mathrm{rms}}$ is the rms value of the phase deviations. The above relationship may be useful where one is trying to determine the effect of a bright line in the time domain. If the bright line is the dominant factor, the plot of $\sigma_{\mathrm{y}}(\tau)$ versus $\tau$ has strong $\sin ^{2}(\pi \mathrm{f} \tau)$ oscillations and it can be ambiguous. In that case, it is better to provide a specification in terms of $\phi_{\mathrm{rms}}$ without reference to bandwidth. Statistical measures such as $\sigma_{\mathrm{y}}(\tau)$ and $\mathrm{S}_{\phi}(\mathrm{f})$ are not meant to be used to describe coherent signals. For further discussion see paper B.1, section 12.2 (page TN-51).

9. page TN-75

A set of brackets, [ ], are missing in eq 12-29. The equation should read

$$
\operatorname{mod\sigma }_{y}^{2}(\tau) \propto \frac{1}{2 \tau^{2} N^{2}(N-3 n+1)} \sum_{j=1}^{N-3 n+1}\left[\sum_{i=j}^{n+j-1}\left(x_{i+2 n}-2 x_{i+n}+x_{i}\right)\right]^{2} .
$$

10. page TN-119

The reference (Walls and DeMarchi, 1975) is listed as being on pages 310-317. The page numbers should be 210-217.

11. page $\mathrm{TN}-121$

Most of the literature uses the expression $\left[V_{0}+\epsilon(t)\right]$ instead of $V_{0}$ as in eq (2). $V_{0}$ is the peak voltage amplitude and $\epsilon(t)$ is the voltage deviation of the amplitude from nominal.

12. page $\mathrm{TN}-122$

In eq (5), most of the literature uses $\mathrm{x}_{1}(\mathrm{t})$ instead of $\epsilon(\mathrm{t}) . \quad \epsilon(\mathrm{t})$ is usually the voltage deviation as described in note 11 above.

13. page $\mathrm{TN}-123$

There is an error in the caption for figure 7. The last portion of that caption should read: "where $f$ is Fourier frequency $\left((\omega=2 \pi f)\right.$ and $\left.S_{y}(f)=\omega^{2} S_{x}(f)\right)$."

14. page $\mathrm{TN}-123$

In the right-hand column, last paragraph, 5 th line, there is an extraneous minus sign. The quantity $\bar{y}_{i}^{-\tau_{0}}$ should read $\bar{y}_{i}^{\tau_{0}^{0}}$. Also, note that the use of supercript $\tau$ and $\tau_{0}$ with $\bar{y}$ is not consistent with the new IEEE standard definitions (see paper C.1, page TN-139). 
15. page $\mathrm{TN}-124$

In eq 9 the subscript, $k-n$, should read $k+n$. That is, the equation should read

$$
\bar{y}_{k}^{\tau}=\frac{1}{n} \sum_{i=k}^{k+n-1} \bar{y}_{i}^{\tau_{0}}=\frac{x_{k+n}-x_{k}}{\tau} .
$$

16. page $\mathrm{TN}-125$

The quantity $\bar{\sigma}_{\mathrm{y}}^{2}(\tau)$ is now commonly known as $\bmod \sigma_{\mathrm{y}}^{2}(\tau)$. This latter form has been recently adopted by IEEE as the standard terminology.

17. page $\mathrm{TN}-139$

IEEE Std 1139-1988, IEEE Standard Definitions of Physical Quantities for Fundamental Frequency and Time Metrology, is an almost exact replica of this paper (D.1). The paper was published during the latter period of the development of the standard. The only substantial difference is that, wherever it occurs, the word "departure" in the paper is replaced in the IEEE standard by "deviation."

18. page $\mathrm{TN}-146$

This widely cited paper provided the de facto standards for terminology and oscillator characterization until the recent adoption of the IEEE standard presented in paper C.1 (page TN-139). For terminology, the latest IEEE standard should always take precedence. This paper (C.2) is fairly consistent with the IEEE standard. One exception is that, in this paper, $\mathrm{N}$ denotes the number of frequency measurements. The symbol $M$ in the IEEE standard is the same as $\mathrm{N}$ in this paper. In the standard, the equation relating $\mathrm{M}$ and $\mathrm{N}$ is $\mathrm{M}=\mathrm{N}-1$.

19. page $\mathrm{TN}-151$

Equation (23) can be substantially simplified as shown, for example, by Stein (page TN-74, eq $(12-27))$, which is

$$
\sigma_{y}^{2}(\tau)=\frac{2}{\left(\pi v_{0} \tau\right)^{2}} \int_{0}^{\infty} S_{\phi}(f) \sin ^{4}(\pi f \tau) d f .
$$

20. page $\mathrm{TN}-154$

In eq 36 the $\mathrm{T}$ outside the brackets should be a $\tau$. The equation should read

$$
\hat{x}\left(t_{0}+\tau\right)=x\left(t_{0}\right)+\tau\left[\frac{x\left(t_{0}\right)-x\left(t_{0}-T\right)}{T}\right] .
$$

21. page $\mathrm{TN}-160$

The 2 expressions listed as eq (95) are in error. They should read

$$
\begin{array}{ll}
\sim h_{-1} \tau^{2}\left[3+2 \ln r-1 /\left(6 r^{2}\right)\right] & r>1 \\
\sim h_{-1} T^{2}[3-2 \ln r] & r<1 .
\end{array}
$$


In eqs (101), (102), (103), (104), and (105), a Greek $\gamma$ was mistakenly replaced by the number 2 . In each of these equations the quantity $\left[2+\ln \left(2 \pi f_{h} \tau\right)\right]$ should be replaced by the quantity $\left[\gamma+\ln \left(2 \pi f_{h} \boldsymbol{\tau}\right)\right]$. $\gamma$, Euler's constant, has the value $0.5772156649 \ldots \ldots . .$.

23. page $\mathrm{TN}-162$

This paper is included in this collection because it presents the internationally accepted terminology and definitions. There are no substantial inconsistencies with the new IEEE standard (paper C.1, page TN-139), but the latest IEEE standard should is considered to be the most up-to-date authority. A new version of this international report should be issued by the CCIR in 1990 .

24. page $\mathrm{TN}-171$

The definitions for symbols used in this paper are fairly consistent with those adopted in the recent IEEE standard (C.1). One exception is that, in this paper, $\mathrm{N}$ denotes the number of frequency measurements. The symbol $M$ in the IEEE standard is the same as $\mathrm{N}$ in this paper. Another is that, while this paper uses $\mu$ as the exponent of $\tau$ in describing the power-law noise processes, the paper adopts the opposite sign convention for $\mu$. $v(t)$ is used where many other papers use $V(t)$ for instantaneous voltage. Some confusion is generated when this small $\mathrm{v}$ is typeset in the equations to look almost identical to the Greek $\nu$, a symbol which is used exclusively to represent frequency. For example, In equation (1) the left-hand quantity is voltage, whereas the $\nu(t)$ and $\nu_{0}$ in equation (4) are clearly frequencies. Finally, the authors of this paper, in equation (2), define $\epsilon(t)$ as the normalized amplitude fluctuations, a very sound choice, but the reader should note that most other papers have not normalized it.

25. page $\mathrm{TN}-175$

For consistency with figure 12 and the text, $\nu(t)$, the left-hand member of eq (11) should probably be $u(t)$.

26. page $\mathrm{TN}-177$

Walls, Percival and Irelan (D.4) have recently addressed the more accurate specification of the quantity $\mathrm{p}$ in eq (12).

27. page $\mathrm{TN}-179$

It is important to note that the expressions in Table 2 in this paper are derived assuming use of a single-pole filter. The calculations can also be done using an ideal (infinitely sharp) filter. The solutions in these two limits are useful because they define the range of practical values (using n-pole filters) for the expressions. Table $I$ in this section is an expansion of Table 2 of Lesage and Audoin providing both the single-pole results as well as the results for an infinitely sharp filter. There are discrepencies in several of the coefficients between terms in Table 2 in the paper and those in Table I on the next page.

28. page TN-180

Barnes and Allan (paper D.8) have recently completed further analysis of the effect of dead time on measurements. 


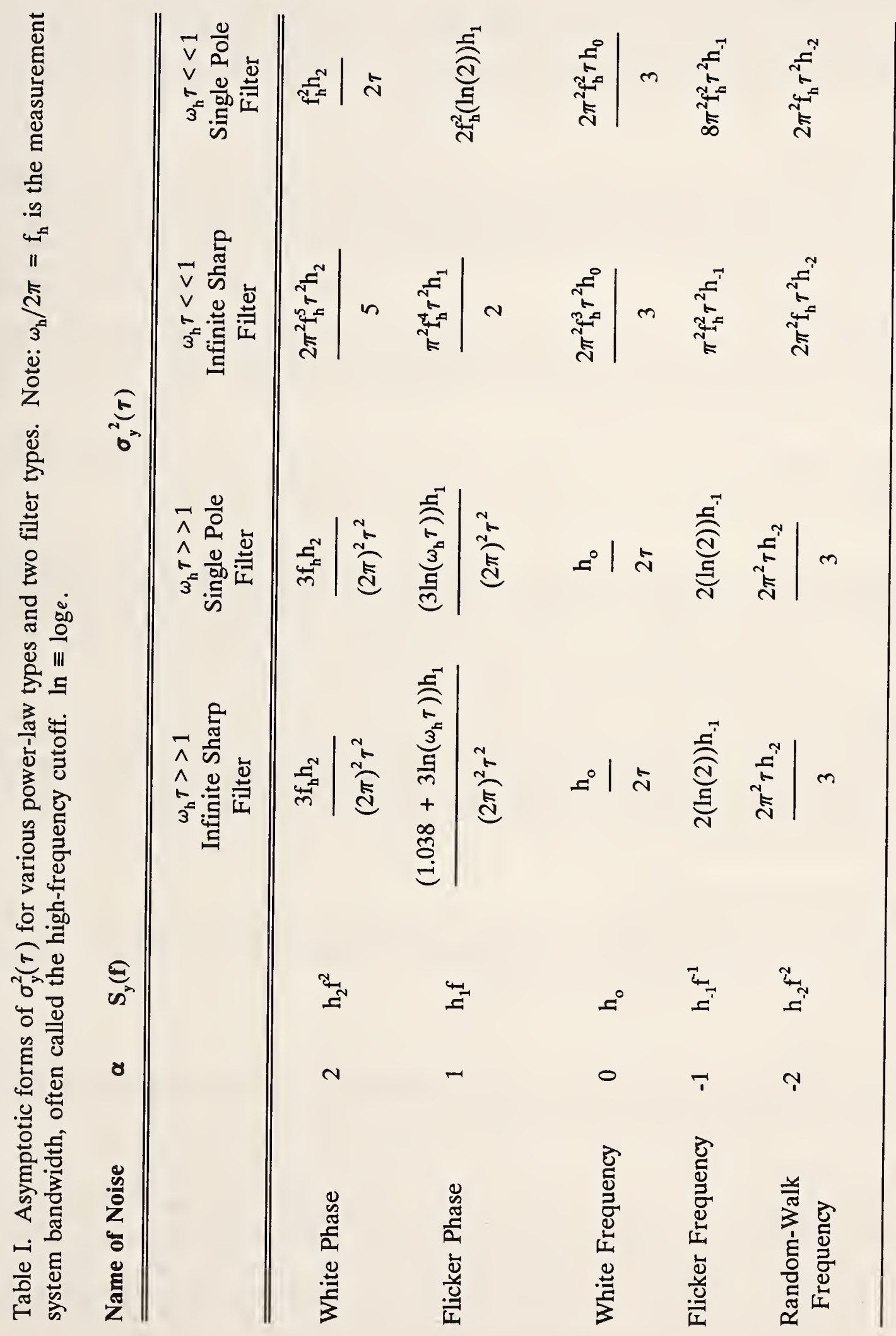


29. page TN-180

If the ratio of $\mathrm{T} / \tau$ is constant and greater than 1 (the usual case), the problem described is eliminated. However, in taking data for a plot of $\sigma_{\mathrm{y}}(\tau)$ versus $\tau$, it is difficult to achieve this in the hardware and not possible to do it with software processing alone. For further discussion see paper D.8 (page TN-296).

30. page $\mathrm{TN}-197$

The most recent definitions and concepts for spectral density are given in a new IEEE standard (paper C.1 on page TN-138). This new standard should be consulted as the latest authority on definitions and terminology.

31. page $\mathrm{TN}-198$

The newly accepted definition of $\mathscr{L}(\mathrm{f})$ is given in paper C.1. This new definition, $\mathscr{L}(\mathrm{f}) \equiv$ $1 / 2 S_{\phi}(f)$, was always valid for Fourier frequencies far from the carrier. It has now been extended to cover all frequencies.

32. page $\mathrm{TN}-217$

Equation (73) should read $20 \log$ (final frequency/original frequency).

33. page $\mathrm{TN}-239$

On page TN-198 the authors refer to a paper by Glaze (1970). The reference, apparently lost in printing, is: Glaze, D.J. (1970). "Improvements in Atomic Beam Frequency Standards at the National Bureau of Standards, "IEEE Trans. Instrum. Meas. IM-19(3), 156-160.

34. page $\mathrm{TN}-257$

There are two errors in Table 2 . Under $R(n)$ the first entry should be $1 / n$ rather than 1 . The second item in the same column is not single valued (1), but takes on different values for different measurement bandwidths. The reader is referred to section A.6 (page TN-9) for a discussion of this topic.

35. pages TN-261 and TN-262

Subsequent work on $\bmod \sigma_{\mathrm{y}}^{2}(\tau)$ and $\mathrm{R}(\mathrm{n})$ is reported in section A.6 (page TN-9) of this report. There are some differences between the results in A.6 and the ones reported in Tables I and II and Figure 4 in this paper.

36. page $\mathrm{TN}-264$

To be consistent with other papers in the literature, $\phi(t)$ in eq (1) should probably be written as $\mathrm{x}_{1}(\mathrm{t})$.

37. page $\mathrm{TN}-268$

The term $\epsilon(\mathrm{t})$ in eq (3) is normally used to represent the amplitude fluctuations in the output voltage of an oscillator. This term might be better designated $Y_{1}(t)$. 


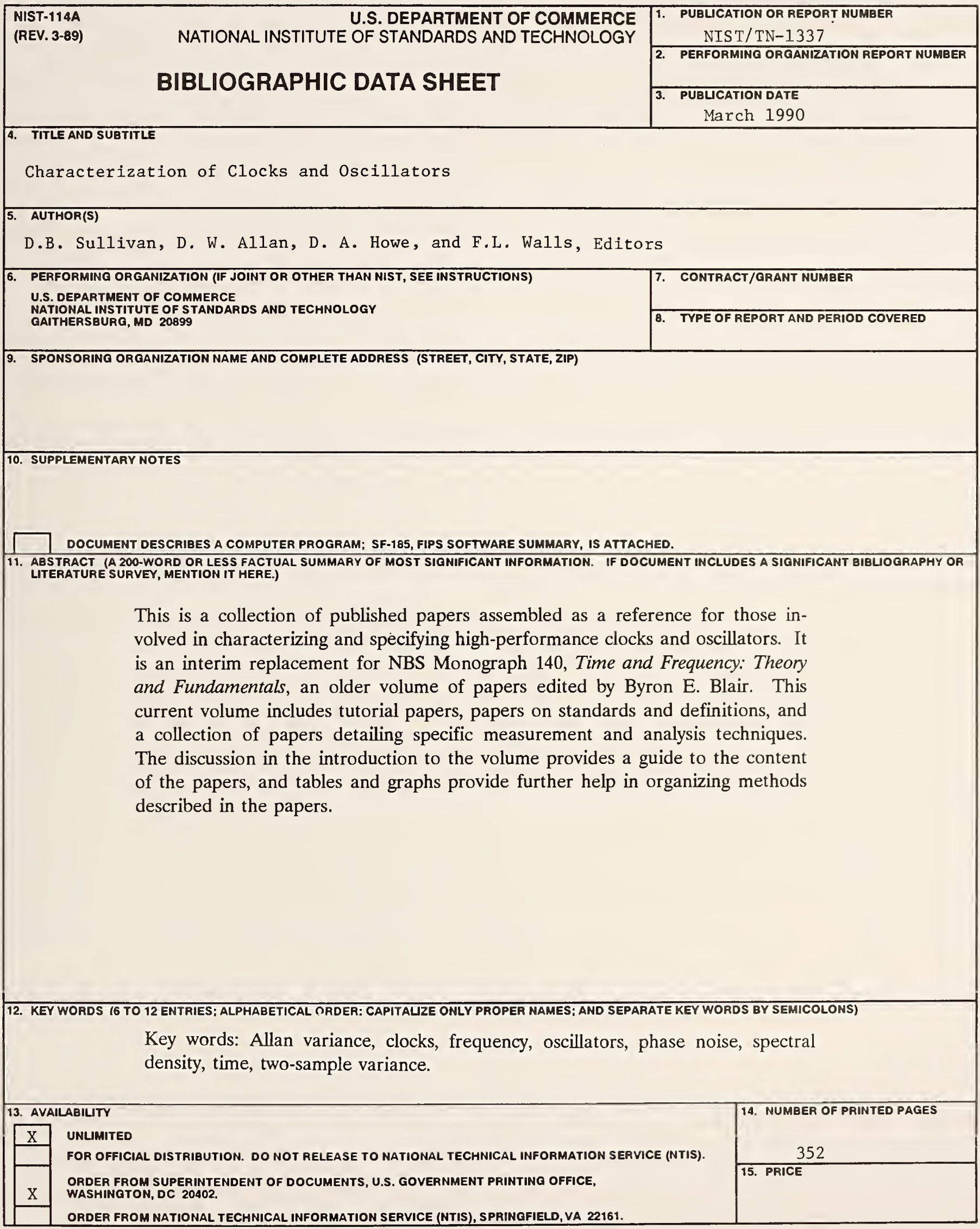

\section{ELECTRONIC FORM}

\&U.S. GOVERNMENT PRINTING OFFICE: $1990 \quad 775-260 / 25098$ 






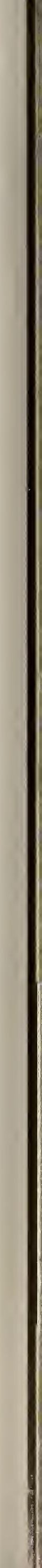




\section{Periodical}

Journal of Research of the National Institute of Standards and Technology-Reports NIST research and development in those disciplines of the physical and engineering sciences in which the Institute is active. These include physics, chemistry, engineering, mathematics, and computer sciences. Papers cover a broad range of subjects, with major emphasis on measurement methodology and the basic technology underlying standardization. Also included from time to time are survey articles on topics closely related to the Institute's technical and scientific programs. Issued six times a year.

\section{Nomperiodicals}

Monographs-Major contributions to the technical literature on various subjects related to the Institute's scientific and technical activities.

Handbooks-Recommended codes of engineering and industrial practice (including safety codes) developed in cooperation with interested industries, professional organizations, and regulatory bodies.

Special Publications-Include proceedings of conferences sponsored by NIST, NIST annual reports, and other special publications appropriate to this grouping such as wall charts, pocket cards, and bibliographies.

Applied Mathematics Serles-Mathematical tables, manuals, and studies of special interest to physicists, engineers, chemists, biologists, mathematicians, computer programmers, and others engaged in scientific and technical work.

National Standard Reference Data Serles-Provides quantitative data on the physical and chemical properties of materials, compiled from the world's literature and critically evaluated. Developed under a worldwide program coordinated by NIST under the authority of the National Standard Data Act (Public Law 90-396). NOTE: The Journal of Physical and Chemical Reference Data (JPCRD) is published quarterly for NIST by the American Chemical Society (ACS) and the American Institute of Physics (AIP). Subscriptions, reprints, and supplements are available from ACS, $1155 \mathrm{Sir}$. teenth St., NW., Washington, DC 20056.

Building Sclence Series-Disseminates technical information developed at the Institute on building materials, components, systems, and whole structures. The series presents research results, test methods, and performance criteria related to the structural and environmental functions and the durability and safety characteristics of building elements and systems.

Technical Notes-Studies or reports which are complete in themselves but restrictive in their treatment of a subject. Analogous to monographs but not so comprehensive in scope or definitive in treatment of the subject area. Often serve as a vehicle for final reports of work performed at NIST under the sponsorship of other government agencies.

Voluntary Product Standards-Developed under procedures published by the Department of Commerce in Part 10, Title 15, of the Code of Federal Regulations. The standards establish nationally recognized requirements for products, and provide all concerned interests with a basis for common understanding of the characteristics of the products. NIST administers this program as a supplement to the activities of the private sector standardizing organizations.

Consumer Information Series-Practical information, based on NIST research and experience, covering areas of interest to the consumer. Easily understandable language and illustrations provide useful background knowledge for shopping in today's technological marketplace.

Order the above NIST publications from: Superintendent of Documents, Government Printing Office, Washington, DC 20402.

Order the following NIST publications-FIPS and NISTIRs-from the Notional Technical Information Service, Springfield, VA 22161.

Federal Information Processing Standards Publications (FIPS PUB)-Publications in this series collectively constitute the Federal Information Processing Standards Register. The Register serves as the official source of information in the Federal Government regarding standards issued by NIST pursuant to the Federal Property and Administrative Services Act of 1949 as amended, Public Law 89-306 (79 Stat. 1127), and as implemented by Executive Order 11717 (38 FR 12315, dated May 11, 1973) and Part 6 of Title 15 CFR (Code of Federal Regulations).

NIST Interagency Reports (NISTIR)-A special series of interim or final reports on work performed by NIST for outside sponsors (both government and non-government). In general, initial distribution is handled by the sponsor; public distribution is by the National Technical Information Service, Springfield, VA 22161, in paper copy or microfiche form. 
U.S. DEPARTMENT OF COMMERCE

National Institute of Standards and Technology

(lormerty National Bureau of Standards)

325 Broadway

Boulder, Colorado $80303-3328$

OFFICLAL BUSINESS

PENALTY FOR PRIVATE USE, $\$ 300$ 